\title{
Chemical Alterations of Dissolved Organic Matter by Permanganate Oxidation
}

\section{Supporting Information}

\author{
Juliana R. Laszakovits*,, Arpad Somogyi, Allison A. MacKay* \\ *Department of Civil, Environmental, and Geodetic Engineering, The Ohio State \\ University \\ \#Campus Chemical Instrument Center: Mass Spectrometry and Proteomics, The Ohio \\ State University \\ ^Corresponding author: Laszakovits.1@buckeyemail.osu.edu
}

Number of Pages: 197

\section{Summary of Contents}

\section{Content}

Table S1: FT-IR Second Derivative Peaks for SRFA

Table S2: FT-IR Second Derivative Peaks for GLSM DOM

Text S1: Instrumental Analysis Details

Text S2: FT-ICR MS Data Analysis and Justification

Figure S1: SRFA Negative Mode ESI Example Mass Spectrum

Figure S2: Raw UV-Vis Absorbance Spectra

$\mathrm{S} 12$

Figure S3: E2/E3 Ratio Changes as a Function of $\mathrm{pH}$

$\mathrm{S} 13$

Figure S4: Comparison of ESI Negative and Positive Modes

$\mathrm{S} 13$

Figure S5: Comparison of LDI Negative and Positive Modes

$\mathrm{S} 14$

Figure S6: Comparison of ESI and LDI

S15

S16

Figure S7: Overall Pre- and Post-Oxidation SRFA and GLSM Van Krevelen $\quad$ S17 Diagrams

Figure S8: ${ }^{13} \mathrm{C}$ NMR of GLSM DOM

$\mathrm{S} 18$

Figure S9: Overall Pre- and Post-Oxidation SRFA and GLSM Van Krevelen

Diagrams Using Negative Mode ES

Figure S10: Comparison of Pre- and Post-Oxidation SRFA and GLSM Using S20 Negative Mode ESI

Figure S11: Overall Pre- and Post-Oxidation SRFA and GLSM Van Krevelen S21

Diagrams Using Negative Mode LDI

Figure S12: Overall Pre- and Post-Oxidation SRFA and GLSM Van Krevelen S22

Diagrams Using Positive Mode LDI

Figure S13: Overall Pre- and Post-Oxidation SRFA and GLSM Van Krevelen S23

Diagrams Using Positive Mode ESI

References

Text S3: Matlab Code 
Table S1: FT-IR Second Derivative Peaks for SRFA.

Summary of observed second derivative peaks in SRFA pre- and post-oxidation samples (contact time $=1$ hour). Categorized broadly according to Pretsch. ${ }^{1}$ Bolded wavenumbers denote shifts in modes where some stretches are retained.

\begin{tabular}{|c|c|c|c|c|c|}
\hline $\begin{array}{c}\text { Functional } \\
\text { Group }\end{array}$ & Mode & $\begin{array}{c}\text { Value/Range } \\
\left(\mathrm{cm}^{-1}\right)\end{array}$ & $\begin{array}{c}\text { Pre-Oxidation } \\
\left(\mathrm{cm}^{-1}\right)\end{array}$ & $\begin{array}{c}\text { Post-Oxidation } \\
\left(\mathrm{cm}^{-1}\right)\end{array}$ & Comment \\
\hline \multirow{10}{*}{ Alcohol } & O-H Stretch & $3200-3650$ & $\begin{array}{c}3430,3452 \\
3473,3493 \\
3514\end{array}$ & $\begin{array}{c}3335,3388,3419 \\
3431,3462,3493, \\
3505,3536,3620 \\
3632\end{array}$ & New peaks \\
\hline & $\begin{array}{l}\text { O-H Stretch, } \\
\text { polymer }\end{array}$ & $3200-3500$ & $\begin{array}{l}3430,3452, \\
3473,3493\end{array}$ & $\begin{array}{l}3335,3388,3419 \\
3431,3462,3493\end{array}$ & $\begin{array}{c}\text { Shift to lower } \\
\text { wavenumbers }\end{array}$ \\
\hline & $\begin{array}{l}\text { O-H Stretch, } \\
\text { free }\end{array}$ & $3650-3590$ & - & 3632,3818 & New peaks \\
\hline & $\begin{array}{l}\text { O-H Stretch, } \\
\text { hydrogen } \\
\text { bonded }\end{array}$ & $3450-3550$ & $\begin{array}{l}3452,3473 \\
3493,3514\end{array}$ & $\begin{array}{c}3462,3493,3505 \\
3536\end{array}$ & $\begin{array}{l}\text { Shift to higher } \\
\text { wavenumbers }\end{array}$ \\
\hline & $\begin{array}{l}\text { O-H Stretch, } \\
\text { enols, chelates }\end{array}$ & $3200-2500$ & $\begin{array}{l}\mathbf{2 5 1 5}, \mathbf{2 5 2 7} \\
\text { 2548, 2570, } \\
2591, \mathbf{2 7 0 0} \\
2722,2743 \\
2764,2775 \\
\mathbf{2 7 8 6 , 2 7 9 7} \\
2807,2818, \\
2850,2860 \\
\mathbf{2 8 8 1}, \mathbf{2 9 2 5}\end{array}$ & $\begin{array}{c}\mathbf{2 5 4 8}, \mathbf{2 5 5 9}, 2591, \\
\mathbf{2 6 3 5}, \mathbf{2 6 7 8}, \mathbf{2 6 9 0}, \\
2721,2732,2743, \\
2764,2775,2807, \\
2818,2849,2861, \\
\mathbf{3 0 7 6}\end{array}$ & $\begin{array}{l}\text { Shift to higher } \\
\text { wavenumbers }\end{array}$ \\
\hline & $\begin{array}{l}\text { O-H In-Plane- } \\
\text { Bend }\end{array}$ & $1200-1450$ & $\begin{array}{l}\mathbf{1 2 0 6}, 1276, \\
1286,1297, \\
\text { 1307, 1318, } \\
\text { 1340, 1360, } \\
\text { 1370, 1379, } \\
\text { 1386, 1392, } \\
1411,1415, \\
1434,1445\end{array}$ & $\begin{array}{l}1243,1255,1275 \\
1286,1297,1307 \\
1340,1379,1385 \\
1393,1411,1415 \\
1424,1435,1445\end{array}$ & $\begin{array}{l}\text { Shift to lower } \\
\text { wavenumbers }\end{array}$ \\
\hline & $\begin{array}{l}\text { C-O Stretch, } \\
\text { primary }\end{array}$ & $1075-1000$ & $\begin{array}{c}\mathbf{1 0 0 4}, 1015, \\
1026,1037 \\
1047,1058, \\
1068\end{array}$ & $\begin{array}{l}1016,1026,1036 \\
1047,1058,1069\end{array}$ & Peak lost \\
\hline & $\begin{array}{l}\text { C-O Stretch, } \\
\text { secondary }\end{array}$ & $1000-1125$ & $\begin{array}{c}1090,1100 \\
1122\end{array}$ & 1099, 1111 & Mixed shifts \\
\hline & $\begin{array}{c}\text { C-O Stretch, } \\
\text { tertiary }\end{array}$ & $1210-1100$ & 1133,1206 & $\begin{array}{c}1127, \begin{array}{l}1164, \\
1197\end{array} \\
\end{array}$ & $\begin{array}{l}\text { Shift to lower } \\
\text { wavenumbers }\end{array}$ \\
\hline & $\begin{array}{l}\text { C-O Stretch, } \\
\text { aromatic }\end{array}$ & $1150-1275$ & - & 1243 & New peak \\
\hline
\end{tabular}




\begin{tabular}{|c|c|c|c|c|c|}
\hline \multirow{8}{*}{$\begin{array}{c}\text { Carboxylic } \\
\text { Acid } \\
\text { (COOH) }\end{array}$} & COO-H Stretch & $2500-3550$ & $\begin{array}{c}\mathbf{2 5 1 5}, \mathbf{2 5 2 7} \\
2548, \mathbf{2 5 7 0} \\
2591, \mathbf{2 7 0 0} \\
2722,2743, \\
2764,2775 \\
\mathbf{2 7 8 6 , 2 7 9 7} \\
2807,2818, \\
2850,2860 \\
\mathbf{2 8 8 1}, \mathbf{2 9 2 5} \\
3430, \mathbf{3 4 5 2} \\
\mathbf{3 4 7 3}, 3493 \\
\mathbf{3 5 1 4}\end{array}$ & $\begin{array}{c}2548,2559,2591, \\
2635,2678,2690, \\
2721,2732,2743, \\
2764,2775,2807, \\
2818,2849,2861, \\
3076,3335,3388, \\
3419,3431,3462, \\
3493,3505\end{array}$ & $\begin{array}{l}\text { Shift to higher } \\
\text { wavenumbers }\end{array}$ \\
\hline & $\begin{array}{c}\text { O-H Stretch, } \\
\text { free }\end{array}$ & $3500-3550$ & 3514 & 3505,3536 & Mixed shifts \\
\hline & $\begin{array}{l}\text { O-H Stretch, } \\
\text { hydrogen } \\
\text { bonded }\end{array}$ & $2500-3300$ & $\begin{array}{l}2515, \mathbf{2 5 2 7} \\
2548,2570 \\
2591,2700 \\
2722,2743 \\
2764,2775 \\
2786,2797 \\
2807,2818 \\
2850,2860 \\
\mathbf{2 8 8 1}, \mathbf{2 9 2 5}\end{array}$ & $\begin{array}{c}2548,2559,2591, \\
\text { 2635, 2678, 2690, } \\
2721, \mathbf{2 7 3 2}, 2743, \\
2764,2775,2807, \\
2818,2849,2861, \\
\mathbf{3 0 7 6}\end{array}$ & $\begin{array}{l}\text { Shift to higher } \\
\text { wavenumbers }\end{array}$ \\
\hline & $C=O$ Stretch & $1650-1800$ & $\begin{array}{l}1703,1745 \\
1775,1786\end{array}$ & $1708,1784,1797$ & $\begin{array}{l}\text { Shift to higher } \\
\text { wavenumbers }\end{array}$ \\
\hline & $\begin{array}{c}\mathrm{C}=\mathrm{O} \text { Stretch, } \\
\text { free and } \\
\text { dicarboxylic } \\
\text { acids }\end{array}$ & $1740-1800$ & $\begin{array}{c}\text { 1745, 1775 } \\
1786\end{array}$ & $1708,1784,1797$ & $\begin{array}{l}\text { Shift to higher } \\
\text { wavenumbers }\end{array}$ \\
\hline & $\begin{array}{c}\mathrm{C}=\mathrm{O} \text { Stretch, } \\
\text { aliphatic }\end{array}$ & $1700-1725$ & 1703 & 1708 & $\begin{array}{l}\text { Shift to higher } \\
\text { wavenumbers }\end{array}$ \\
\hline & $\begin{array}{c}\mathrm{C}=\mathrm{O} \text { Stretch, } \\
\text { alkene }\end{array}$ & $1690-1715$ & 1703 & 1708 & $\begin{array}{l}\text { Shift to higher } \\
\text { wavenumbers }\end{array}$ \\
\hline & $\begin{array}{l}\text { OC-OH Out-Of- } \\
\text { Plane Bend }\end{array}$ & $880-960$ & $898,919,941$ & 888, 900 919, 930 & $\begin{array}{l}\text { Shift to lower } \\
\text { wavenumbers }\end{array}$ \\
\hline \multirow{3}{*}{$\begin{array}{l}\text { Carboxylic } \\
\text { Acid } \\
\text { (COO-) }\end{array}$} & $\begin{array}{l}\text { Asymmetric } \\
\text { Stretch }\end{array}$ & $1550-1610$ & $\begin{array}{c}1562,1574 \\
1608\end{array}$ & 1583,1595 & Mixed shifts \\
\hline & $\begin{array}{l}\text { Symmetric } \\
\text { Stretch }\end{array}$ & $1400-1450$ & $\begin{array}{l}1411,1415 \\
1434,1445\end{array}$ & $\begin{array}{c}1411,1415,1424 \\
1435,1445\end{array}$ & New peak \\
\hline & Bend, acetates & 925 & 919 & 919 & Conserved \\
\hline \multirow{2}{*}{ Alkene } & $\begin{array}{l}\mathrm{C}=\mathrm{C} \text { C-H Out- } \\
\text { Of-Plane Bend, } \\
\text { monosubstituted }\end{array}$ & $985-1005$ & 993,1004 & 0 & Peaks lost \\
\hline & $\begin{array}{l}\mathrm{C}=\mathrm{C} \text { C-H Out- } \\
\text { Of-Plane Bend, } \\
\text { monosubstituted }\end{array}$ & $900-920$ & 919 & 919 & Conserved \\
\hline
\end{tabular}




\begin{tabular}{|c|c|c|c|c|c|}
\hline & $\begin{array}{c}\mathrm{C}=\mathrm{C}-\mathrm{C}=\mathrm{O}, \mathrm{C}-\mathrm{H} \\
\text { Out-Of-Plane } \\
\text { Bend, } \\
\text { monosubstituted }\end{array}$ & 980 & 983 & 984 & Conserved \\
\hline & $\begin{array}{c}\mathrm{C}=\mathrm{C}-\mathrm{O}-\mathrm{C}=\mathrm{O}, \mathrm{C}- \\
\mathrm{H} \text { Out-Of-Plane } \\
\text { Bend }\end{array}$ & 870 & 877 & 875 & Conserved \\
\hline & $\mathrm{C}=\mathrm{C}$ Stretch & $1635-1690$ & - & 1638 & New peak \\
\hline \multirow{3}{*}{ Aromatic } & C-C Stretch & $1575-1625$ & 1608,1625 & 1583, 1595, 1612 & $\begin{array}{l}\text { Shift to lower } \\
\text { wavenumbers }\end{array}$ \\
\hline & C-C Stretch & $1450-1525$ & $\begin{array}{c}1453,1458, \\
1463,1469, \\
1497,1508, \\
\mathbf{1 5 1 9}\end{array}$ & $\begin{array}{c}1454,1457,1464 \\
1469,1487,1498 \\
1509\end{array}$ & $\begin{array}{l}\text { Mostly conserved, } \\
\text { one shift to higher } \\
\text { wavenumber }\end{array}$ \\
\hline & $\begin{array}{l}\text { C-H Out-Of- } \\
\text { Plane Bend }\end{array}$ & $650-900$ & $\begin{array}{c}655,667,700 \\
710,721,731 \\
763,784,838 \\
877,898 \\
\end{array}$ & $\begin{array}{c}665,708,785 \\
\mathbf{8 4 6 , 8 5 8 , 8 7 5} \\
\mathbf{8 8 8}, 900\end{array}$ & $\begin{array}{l}\text { Shifted to higher } \\
\text { wavenumbers }\end{array}$ \\
\hline \multirow{6}{*}{ Aldehyde } & $\begin{array}{l}\text { C-H Combined } \\
\text { Mode, aliphatic }\end{array}$ & $2810-2830$ & 2818 & 2818 & Conserved \\
\hline & $\begin{array}{l}\text { C-H Combined } \\
\text { Mode, aliphatic }\end{array}$ & $2690-2720$ & 2700 & 2690 & $\begin{array}{l}\text { Shift to lower } \\
\text { wavenumbers }\end{array}$ \\
\hline & $\begin{array}{l}\text { C-H Combined } \\
\text { Mode, aromatic }\end{array}$ & $2810-2830$ & 2818 & 2818 & Conserved \\
\hline & $\begin{array}{l}\text { C-H Combined } \\
\text { Mode, aromatic }\end{array}$ & $2720-2750$ & 2722,2743 & $2721,2732,2743$ & New peak \\
\hline & $\mathrm{C}=\mathrm{O}$ Stretch & $1645-1765$ & 1703,1745 & 1708 & Mixed shifts \\
\hline & $\begin{array}{c}\mathrm{C}=\mathrm{O} \text { Stretch } \\
\text { aromatic }\end{array}$ & $1685-1710$ & 1703 & 1708 & $\begin{array}{l}\text { Shift to higher } \\
\text { wavenumbers }\end{array}$ \\
\hline Ketone & $\begin{array}{c}\mathrm{C}=\mathrm{O} \text { Stretch, } \\
\text { aliphatic }\end{array}$ & 1715 & 1723 & 1723 & Conserved \\
\hline \multirow[b]{2}{*}{ Ester } & $C=O$ Stretch & $1790-1650$ & $\begin{array}{l}1703,1745, \\
1775,1786\end{array}$ & 1708,1784 & Peaks lost \\
\hline & C-O Stretch & $1050-1330$ & $\begin{array}{l}1058,1068, \\
1090,1100, \\
\mathbf{1 1 2 2}, \mathbf{1 1 3 3}, \\
\mathbf{1 2 0 6 , 1 2 7 6 ,} \\
1286,1297, \\
1307, \mathbf{1 3 1 8} \\
\end{array}$ & $\begin{array}{c}\text { 1058, 1069, 1079, } \\
1089,1099,1111, \\
1127, \mathbf{1 1 6 4}, 1174, \\
\begin{array}{c}1197,1243,1255 \\
1275, \\
1286,1297, \\
1307\end{array}\end{array}$ & $\begin{array}{l}\text { Shifted to higher } \\
\text { wavenumbers }\end{array}$ \\
\hline
\end{tabular}


Table S2: FT-IR Second Derivative Peaks for GLSM DOM.

Summary of observed second derivative peaks in GLSM pre- and post-oxidation samples (contact time $=1$ hour). Categorized broadly according to Pretsch. ${ }^{1}$ Bolded wavenumbers denote shifts in modes where some stretches are retained.

\begin{tabular}{|c|c|c|c|c|c|}
\hline $\begin{array}{c}\text { Functional } \\
\text { Group }\end{array}$ & Mode & $\begin{array}{c}\text { Value/Range } \\
\left(\mathrm{cm}^{-1}\right)\end{array}$ & $\begin{array}{c}\text { Pre-Oxidation } \\
\left(\mathbf{c m}^{-1}\right)\end{array}$ & $\begin{array}{c}\text { Post-Oxidation } \\
\left(\mathrm{cm}^{-1}\right)\end{array}$ & Comment \\
\hline \multirow{9}{*}{ Alcohol } & $\mathrm{O}-\mathrm{H}$ Stretch & $3200-3650$ & $\begin{array}{l}3335,3430 \\
3462,3494\end{array}$ & 3557 & Peaks lost \\
\hline & $\begin{array}{l}\text { O-H Stretch, } \\
\text { polymer }\end{array}$ & $3200-3500$ & $\begin{array}{l}3335,3430 \\
3462,3494\end{array}$ & 3557 & Peaks lost \\
\hline & $\begin{array}{l}\text { O-H Stretch, } \\
\text { Hydrogen } \\
\text { Bonded }\end{array}$ & $3450-3550$ & $3430,3462,3494$ & - & Peaks lost \\
\hline & $\begin{array}{l}\text { O-H Stretch, } \\
\text { enols, chelates }\end{array}$ & $3200-2500$ & $\begin{array}{l}\text { 2505, 2537, } \\
\text { 2570, 2646, } \\
\text { 2678, 2711, } \\
2722,2732, \\
2743,2754, \\
2764,2775, \\
2786,2807, \\
2818,2850, \\
2871,2881\end{array}$ & $\begin{array}{l}2526,2711,2722, \\
2732,2743,2754, \\
2764,2775,2786, \\
2796,2807,2818, \\
2828,2871,2879, \\
2881\end{array}$ & $\begin{array}{l}\text { Peaks lost or } \\
\text { shifted to } \\
\text { higher } \\
\text { wavenumbers }\end{array}$ \\
\hline & $\begin{array}{l}\text { O-H In-Plane- } \\
\text { Bend }\end{array}$ & $1200-1450$ & $\begin{array}{l}\mathbf{1 2 0 7}, \mathbf{1 2 6 3} \\
\text { 1275, 1286, } \\
\text { 1297, 1307, } \\
\text { 1318, 1339, } \\
\text { 1350, 1361, } \\
\text { 1385, 1392, } \\
1435,1445\end{array}$ & $\begin{array}{c}1254,1276,1286 \\
1297,1307,1318 \\
1340,1349,1378 \\
1385,1401,1434 \\
1445\end{array}$ & $\begin{array}{l}\text { Peaks shifted } \\
\text { to higher } \\
\text { wavenumbers }\end{array}$ \\
\hline & $\begin{array}{l}\text { C-O Stretch, } \\
\text { primary }\end{array}$ & $1075-1000$ & $\begin{array}{l}1004,1015 \\
1025,1037 \\
1047,1058\end{array}$ & $\begin{array}{c}\text { 1004, 1016, 1025, } \\
\begin{array}{c}1037, \\
1047,1058 \\
\mathbf{1 0 6 8}\end{array} \\
\end{array}$ & New peak \\
\hline & $\begin{array}{l}\text { C-O Stretch, } \\
\text { secondary }\end{array}$ & $1000-1125$ & $\begin{array}{l}\text { 1079, 1089, } \\
1100,1111\end{array}$ & $1079,1090,1100$ & New Peak \\
\hline & $\begin{array}{l}\text { C-O Stretch, } \\
\text { tertiary }\end{array}$ & $1210-1100$ & 1195,1207 & 1100 & $\begin{array}{c}\text { Peaks shifted } \\
\text { to lower } \\
\text { wavenumbers }\end{array}$ \\
\hline & $\begin{array}{l}\text { C-O Stretch, } \\
\text { aromatic }\end{array}$ & $1150-1275$ & 1195,1207 & 1254 & $\begin{array}{l}\text { Peaks shifted } \\
\text { to higher } \\
\text { wavenumbers }\end{array}$ \\
\hline
\end{tabular}




\begin{tabular}{|c|c|c|c|c|c|}
\hline \multirow{8}{*}{$\begin{array}{c}\text { Carboxylic } \\
\text { Acid } \\
\text { (COOH) }\end{array}$} & COO-H Stretch & $2500-3550$ & $\begin{array}{l}\text { 2505, 2537, } \\
\text { 2570, 2646, } \\
\text { 2678, 2711, } \\
2722,2732, \\
2743,2754, \\
2764,2775, \\
2786,2807, \\
2818, \mathbf{2 8 5 0} \\
2871,2881, \\
3335, \mathbf{3 4 3 0}, \\
\mathbf{3 4 6 2}, \mathbf{3 4 9 4}\end{array}$ & $\begin{array}{l}\mathbf{2 5 2 6}, 2711,2722 \\
2732,2743,2754 \\
2764,2775,2786 \\
2796,2807,2818 \\
\mathbf{2 8 2 8}, 2871,2881\end{array}$ & $\begin{array}{l}\text { Peaks lost or } \\
\text { shifted to } \\
\text { lower } \\
\text { wavenumbers }\end{array}$ \\
\hline & $\begin{array}{l}\text { COO-H Stretch, } \\
\text { hydrogen } \\
\text { bonded }\end{array}$ & $2500-3300$ & $\begin{array}{l}\text { 2505, 2537, } \\
\text { 2570, 2646, } \\
\text { 2678, 2711, } \\
2722,2732, \\
2743,2754, \\
2764,2775, \\
2786,2807, \\
2818,2850, \\
2871,2881\end{array}$ & $\begin{array}{l}\text { 2526, 2711, 2722, } \\
2732,2743,2754 \\
2764,2775,2786 \\
2796,2807,2818 \\
2828,2871,2881\end{array}$ & $\begin{array}{l}\text { Peaks lost or } \\
\text { shifted to } \\
\text { lower } \\
\text { wavenumbers }\end{array}$ \\
\hline & $\mathrm{C}=\mathrm{O}$ Stretch & $1650-1800$ & $1660,1720,1740$ & $1703,1719,1735$ & $\begin{array}{c}\text { Shifted to } \\
\text { higher } \\
\text { wavenumbers }\end{array}$ \\
\hline & $\begin{array}{c}\mathrm{C}=\mathrm{O} \text { Stretch, } \\
\text { free and } \\
\text { dicarboxylic } \\
\text { acids }\end{array}$ & $1740-1800$ & 1740 & - & Peak lost \\
\hline & $\begin{array}{c}\mathrm{C}=\mathrm{O} \text { Stretch, } \\
\text { hydrogen } \\
\text { bonded, dimer }\end{array}$ & $1650-1740$ & 1660,17201740 & 1703, 1719, 1735 & $\begin{array}{c}\text { Shifted to } \\
\text { higher } \\
\text { wavenumbers }\end{array}$ \\
\hline & $\begin{array}{l}\mathrm{C}=\mathrm{O} \text { Stretch, } \\
\text { aliphatic }\end{array}$ & $1700-1725$ & - & 1703,1719 & New peaks \\
\hline & $\begin{array}{c}\mathrm{C}=\mathrm{O} \text { Stretch, } \\
\text { alkene }\end{array}$ & $1690-1715$ & - & 1703 & New peak \\
\hline & $\begin{array}{l}\text { OC-OH out-of- } \\
\text { plane bend }\end{array}$ & $880-960$ & $\begin{array}{c}898,908,919 \\
930\end{array}$ & $898,908,919,947$ & $\begin{array}{l}\text { Peak shifted } \\
\text { to higher } \\
\text { wavenumber }\end{array}$ \\
\hline \multirow{4}{*}{$\begin{array}{l}\text { Carboxylic } \\
\text { Acid } \\
\text { (COO-) }\end{array}$} & $\begin{array}{l}\text { Asymmetric } \\
\text { Stretch }\end{array}$ & $1550-1610$ & $1551,1572,1584$ & $\begin{array}{c}1551,1563,1572 \\
1584\end{array}$ & New peak \\
\hline & $\begin{array}{l}\text { Symmetric } \\
\text { Stretch }\end{array}$ & $1400-1450$ & 1435,1445 & 1434,1445 & Conserved \\
\hline & Bend, acetates & 925 & 930 & - & Peak lost \\
\hline & $\begin{array}{l}\text { Bend, } \\
\text { benzoates }\end{array}$ & 680 & 678 & 678 & Conserved \\
\hline
\end{tabular}




\begin{tabular}{|c|c|c|c|c|c|}
\hline \multirow{7}{*}{ Alkene } & $\begin{array}{l}\mathrm{C}=\mathrm{C} \mathrm{C}-\mathrm{H} \text { out-of- } \\
\text { plane bend, } \\
\text { monosubstituted }\end{array}$ & $985-1005$ & 993,1004 & 994,1004 & Conserved \\
\hline & $\begin{array}{l}\mathrm{C}=\mathrm{C} \mathrm{C}-\mathrm{H} \text { out-of- } \\
\text { plane bend, } \\
\text { monosubstituted }\end{array}$ & $900-920$ & 908,919 & 908,919 & Conserved \\
\hline & $\begin{array}{l}\mathrm{C}=\mathrm{C}-\mathrm{C}=\mathrm{O}, \mathrm{C}-\mathrm{H} \\
\text { out-of-plane } \\
\text { bend, } \\
\text { monosubstituted }\end{array}$ & 980 & 983 & 983 & Conserved \\
\hline & $\begin{array}{c}\mathrm{C}=\mathrm{C}-\mathrm{C}=\mathrm{O}, \mathrm{C}-\mathrm{H} \\
\text { Out-Of-Plane } \\
\text { Bend, } \\
\text { disubstituted }\end{array}$ & 810 & 805 & - & Peak lost \\
\hline & $\begin{array}{c}\mathrm{C}=\mathrm{C}-\mathrm{O}-\mathrm{C}=\mathrm{O}, \mathrm{C}- \\
\mathrm{H} \text { Out-Of-Plane } \\
\text { Bend, } \\
\text { disubstituted }\end{array}$ & 950 & - & 947 & New peak \\
\hline & $\begin{array}{c}\mathrm{C}=\mathrm{C}-\mathrm{O}-\mathrm{C}=\mathrm{O}, \\
\text { Out-Of-Plane } \\
\text { Bend, } \\
\text { monosubstituted }\end{array}$ & 870 & 867 & 877 & $\begin{array}{l}\text { Peak shifted } \\
\text { to higher } \\
\text { wavenumber }\end{array}$ \\
\hline & $\mathrm{C}=\mathrm{C}$ Stretch & $1635-1690$ & 1647,1660 & - & Peaks lost \\
\hline \multirow[b]{3}{*}{ Aromatic } & C-C Stretch & $1575-1625$ & 1584,1615 & 1584 & Peak lost \\
\hline & C-C Stretch & $1450-1525$ & $\begin{array}{l}1477, \mathbf{1 4 8 8} \\
1498,1519\end{array}$ & $1477,1498,1519$ & Peak lost \\
\hline & $\begin{array}{l}\text { C-H Out-Of- } \\
\text { Plane Bend }\end{array}$ & $650-900$ & $\begin{array}{l}667,678,699 \\
709,721,753, \\
763,785,795 \\
\mathbf{8 0 5}, 837, \mathbf{8 6 7} \\
898\end{array}$ & $\begin{array}{c}667,678, \mathbf{6 8 8}, 699, \\
710,721,731,742, \\
754,763,785,795, \\
837,877,898\end{array}$ & $\begin{array}{l}\text { Peaks shifted } \\
\text { to lower } \\
\text { wavenumbers }\end{array}$ \\
\hline \multirow{6}{*}{ Aldehyde } & $\begin{array}{l}\text { C-H Combined } \\
\text { Mode, aliphatic }\end{array}$ & $2810-2830$ & 2818 & 2818,2828 & New peak \\
\hline & $\begin{array}{l}\text { C-H Combined } \\
\text { Mode, aliphatic }\end{array}$ & $2690-2720$ & 2711 & 2711 & Conserved \\
\hline & $\begin{array}{l}\text { C-H Combined } \\
\text { Mode, aromatic }\end{array}$ & $2810-2830$ & 2818 & 2818,2828 & New peak \\
\hline & $\begin{array}{l}\text { C-H Combined } \\
\text { Mode, aromatic }\end{array}$ & $2720-2750$ & $2722,2732,2743$ & $2722,2732,2743$ & Conserved \\
\hline & $C=O$ Stretch & $1645-1765$ & $\begin{array}{l}1647,1660 \\
1720,1740\end{array}$ & $1703,1719,1735$ & $\begin{array}{c}\text { Shifted to } \\
\text { higher } \\
\text { wavenumbers }\end{array}$ \\
\hline & $\begin{array}{c}\mathrm{C}=\mathrm{O} \text { Stretch } \\
\text { aliphatic }\end{array}$ & $1720-1740$ & - & 1735 & New peak \\
\hline
\end{tabular}




\begin{tabular}{|c|c|c|c|c|c|}
\hline & $\begin{array}{c}\mathrm{C}=\mathrm{O} \text { Stretch } \\
\text { aromatic }\end{array}$ & $1685-1710$ & - & 1703 & New peak \\
\hline & $\begin{array}{c}\mathrm{C}=\mathrm{O} \text { Stretch, } \\
\text { with } \\
\text { intramolecular } \\
\text { hydrogen bonds }\end{array}$ & $1645-1670$ & 1647,1660 & - & Peaks lost \\
\hline Ketone & $\begin{array}{c}\mathrm{C}=\mathrm{O} \text { Stretch, } \\
\text { aliphatic }\end{array}$ & 1715 & 1720 & 1719 & Conserved \\
\hline \multirow{4}{*}{ Ester } & $\begin{array}{c}\mathrm{C}=\mathrm{O} \text { Stretch } \\
\text { aliphatic }\end{array}$ & $1735-1750$ & 1740 & 1735 & $\begin{array}{c}\text { Shifted to } \\
\text { lower } \\
\text { wavenumbers }\end{array}$ \\
\hline & $\begin{array}{c}\mathrm{C}=\mathrm{O} \text { Stretch, } \\
\text { aromatic }\end{array}$ & $1715-1730$ & 1720 & 1719 & Conserved \\
\hline & $\begin{array}{l}\mathrm{C}=\mathrm{O} \text { Stretch, } \\
\text { phenol esters of } \\
\text { aromatic acids }\end{array}$ & 1735 & 1740 & 1735 & $\begin{array}{c}\text { Shifted to } \\
\text { lower } \\
\text { wavenumber }\end{array}$ \\
\hline & C-O Stretch & $1050-1330$ & $\begin{array}{c}1058,1079, \\
1089,1100, \\
1111,1195 \\
1207,1263 \\
1275,1286 \\
1297,1307,1318\end{array}$ & $\begin{array}{c}1058,1068,1079 \\
1090,1100,1254 \\
1276,1286,1297 \\
1307,1318\end{array}$ & $\begin{array}{c}\text { Shifted to } \\
\text { lower } \\
\text { wavenumbers }\end{array}$ \\
\hline
\end{tabular}




\section{Text S1: Instrumental Analysis Details}

TOC. An ASI autosampler was used. Samples were acidified with $1.5 \% 2 \mathrm{M} \mathrm{HCl}$ to adjust the $\mathrm{pH}$. Samples were sparged for 2 minutes prior to analysis. An injection volume of $50 \mu \mathrm{L}$ was used.

UV-Vis. UV-Vis spectra were obtained between $200-600 \mathrm{~nm}$ using a quartz 1-cm path cuvette. The instrument was blanked with air v. air (i.e., no cuvette). A DI blank was measured and manually subtracted from the spectra presented to ensure the cuvette was clean and to account for absorbance by water.

FT-IR. Solid DOM samples were prepared as $\mathrm{KBr}$ pellets where $1 \mathrm{mg} \mathrm{DOM}$ was diluted with $100 \mathrm{mg} \mathrm{KBr}$. The sample cell was purged with $\mathrm{N}_{2}$ gas for 4 minutes prior to analysis. The samples were scanned from 4000 to $450 \mathrm{~cm}^{-1}$. Second derivative plots were generated using SynchronizIR. ${ }^{2}$

${ }^{13} \mathrm{C} \mathrm{NMR}$. Approximately $50 \mathrm{mg}$ of the GLSM DOM solid isolate was packed into a 4 $\mathrm{mm}$ zirconium rotor with a Kel-F cap. Adamantane was used as a secondary external reference for the ${ }^{13} \mathrm{C}$ chemical shifts. The ${ }^{13} \mathrm{C}$ NMR spectra were acquired using the standard CPMAS pulse program with acquisition parameters as follows: sample scans of $32 \mathrm{k}$ per experiment, spinning rate of $12 \mathrm{kHz}$, high power two-pulse phasemodulation-15 (TPPM-15) proton decoupling at $100 \mathrm{kHz}$, cross polarization contact time of $2 \mathrm{~ms}$ using a ramped $1 \mathrm{H}$ pulse during the Hartman-Hahn contact, $2 \mathrm{~s}$ recycle delay, $45 \mathrm{~ms}$ of FID acquisition, and the number of digital sampling points was 2048. The FIDs were processed using zero filling and $30 \mathrm{~Hz}$ line broadening. ${ }^{3}$

FT-ICR MS. For ESI, solid samples were dissolved in 1:1 water acetonitrile and sprayed at a rate of $2.5 \mu \mathrm{L} \mathrm{min}{ }^{-1}$. The FT-ICR-MS is housed in the Ohio State University Mass Spectrometry and Proteomics Campus Chemical Instrument Center and is used daily by a variety of researchers. Because of this, the FT-ICR MS is calibrated daily for ESI using an Agilent calibration standard and any major deviations (error > $1 \mathrm{ppm}$ ) to the calibration are reported the facility director. Prior to data collection, our spectra were calibrated externally using known background contaminants (i.e., fluorotelomers). For $\mathrm{LDI}$, samples were dissolved in methanol and plated on a 384-fold stainless steel MALDI plate. The FT-ICR MS is also routinely used for MALDI imaging analysis and is therefore regularly calibrated. Prior to our data collection, the FT-ICR MS was calibrated externally using a PepMix standard. The methanol was evaporated prior to analysis and a Yag/Nd (351 nm) laser was used to ionize the solid sample with varying laser power. The ESI spectra were internally calibrated using known fatty acids and background contaminants. Chemical formulae for each mass-to-charge ratio obtained were assigned using Bruker's Data Analysis software (version 5.1). A Matlab script developed in house (Text S3) was used to remove unlikely or impossible formulae (Text S2) and subsequently to combine chemical formulae obtained by different ionization 
techniques ${ }^{4-8}$ Van Krevelen diagrams were used to visually compare the chemical composition of the pre- and post-oxidation samples. ${ }^{9-11}$

\section{Text S2: FT-ICR MS Data Analysis and Justification}

Ion formulae were predicted using Bruker's Compass Data Analysis software (v 5.0). Peaks with an absolute intensity greater than 100 , relative base peak intensity greater than $0.05 \%$ and a signal-to-noise $(\mathrm{S} / \mathrm{N})$ ratio greater than 5 were considered. All ions were assumed to have a charge of 1 (singly charged ions). The elemental composition considered by the software was set such that:

$\begin{array}{ll}\mathrm{C} \leq 50 & \text { Equation } \mathrm{S} 1 \\ \mathrm{H} \leq 102 & \text { Equation S2 } \\ \mathrm{N} \leq 25 & \text { Equation S3 } \\ \mathrm{O} \leq 60 & \text { Equation S4 } \\ \mathrm{P} \leq 5 & \text { Equation S5 } \\ \mathrm{S} \leq 10 & \text { Equation S6 }\end{array}$

For positive ion modes, $\mathrm{M}+\mathrm{Na}$ and $\mathrm{M}+\mathrm{H}$ ions were considered; for negative ion modes only $\mathrm{M}-\mathrm{H}$ ions were considered. For LDI, molecular ions were also considered (odd electron configurations). Up to 1000 formulae were considered for every peak. The data was exported in an excel spreadsheet and subsequently formulae were eliminated using a Matlab code (Text S2).

We excluded formulae on the basis of an allowable error for the ICR used ( $\leq 0.5 \mathrm{ppm}$ ). We only considered formulae between $\mathrm{m} / \mathrm{z}=200$ and 700 because of how the instrument was tuned and because we could reliably assign formulae in this mass range. We removed background impurity formulae based on a known background spectrum. Only formulae that met the following criteria were included based on previous work and adjusted $\left({ }^{*}\right)$ to eliminate unlikely chemical formulae $e^{5-8}$ :
$2 \leq \mathrm{H} \leq 2 \mathrm{C}+2$
$\mathrm{O} \leq \mathrm{C}+2$
$\mathrm{O} / \mathrm{C}<1.2$
$0.33 \leq \mathrm{H} / \mathrm{C} \leq 2.25$
$\mathrm{N} / \mathrm{C}<0.5$
$\mathrm{S} / \mathrm{C}<0.2$
$\mathrm{P} / \mathrm{C}<0.05$
$(\mathrm{S}+\mathrm{P}) / \mathrm{C}<0.1$
$(\mathrm{N}+\mathrm{P}) / \mathrm{C}<0.4$
Equation $\mathrm{S7}$
Equation S8
Equation S9
Equation S10
Equation S11
Equation S12
Equation S13
Equation S14
Equation S15
$\mathrm{DBE} \geq 0$ and an integer
$-10 \leq \mathrm{DBE}+\mathrm{O} \leq 10$
Equation S16
$\mathrm{Al}<1$ and a real number*
Equation S17
Equation S18 
Where DBE is the double bond equivalents (Equation S18) and $\mathrm{Al}$ is the aromaticity index (Equation S19) 12, 13:

$$
\begin{gathered}
\text { DBE }=1+\frac{1}{2}(2 \mathrm{C}-\mathrm{H}+\mathrm{N}+\mathrm{P}) \\
\mathrm{Al}=\frac{1+\mathrm{C}-\mathrm{O}-\mathrm{S}-\frac{1}{2}(\mathrm{~N}+\mathrm{P}+\mathrm{H})}{\mathrm{C}-\mathrm{O}-\mathrm{N}-\mathrm{S}-\mathrm{P}}
\end{gathered}
$$

We considered elemental ratios (i.e., $\mathrm{H} / \mathrm{C}, \mathrm{O} / \mathrm{C}, \mathrm{N} / \mathrm{C}$, etc.) rather than number of the element in a formula so that the same constraints could be used for all ionization techniques. Specifically, peptides that contain amines readily ionize in positive mode and as such it is possible to ionize compounds that contain greater than 3 nitrogen atoms, which is a typical constraint for negative mode ESI. ${ }^{6}$ From the elemental ratios, van Krevelen diagrams were constructed and used for comparison. ${ }^{9,11}$ 

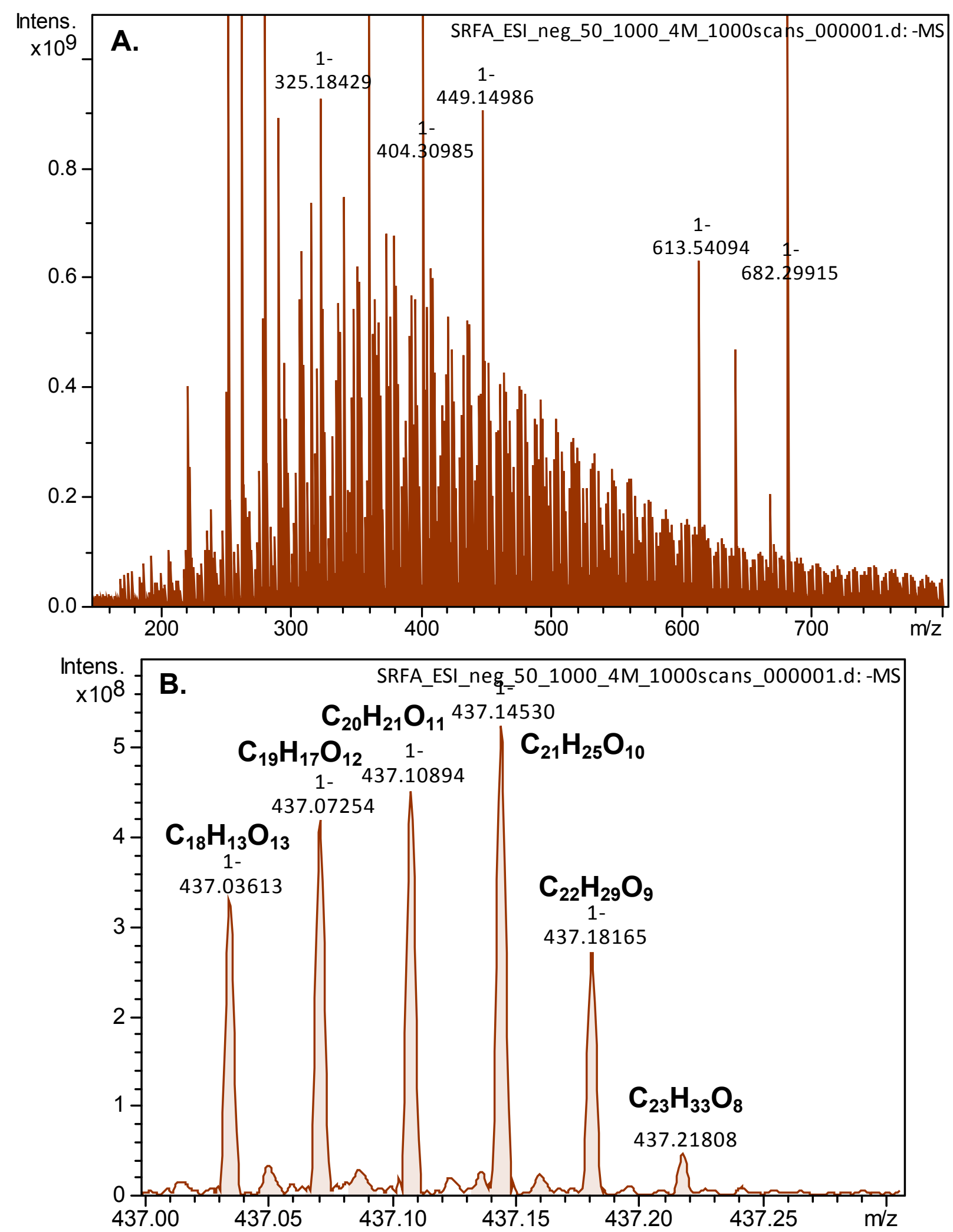

Figure S1: SRFA Negative Mode ESI Example Mass Spectrum A. Full Spectrum, $m / z=150-800$; B. Zoom in around $m / z=437$. 

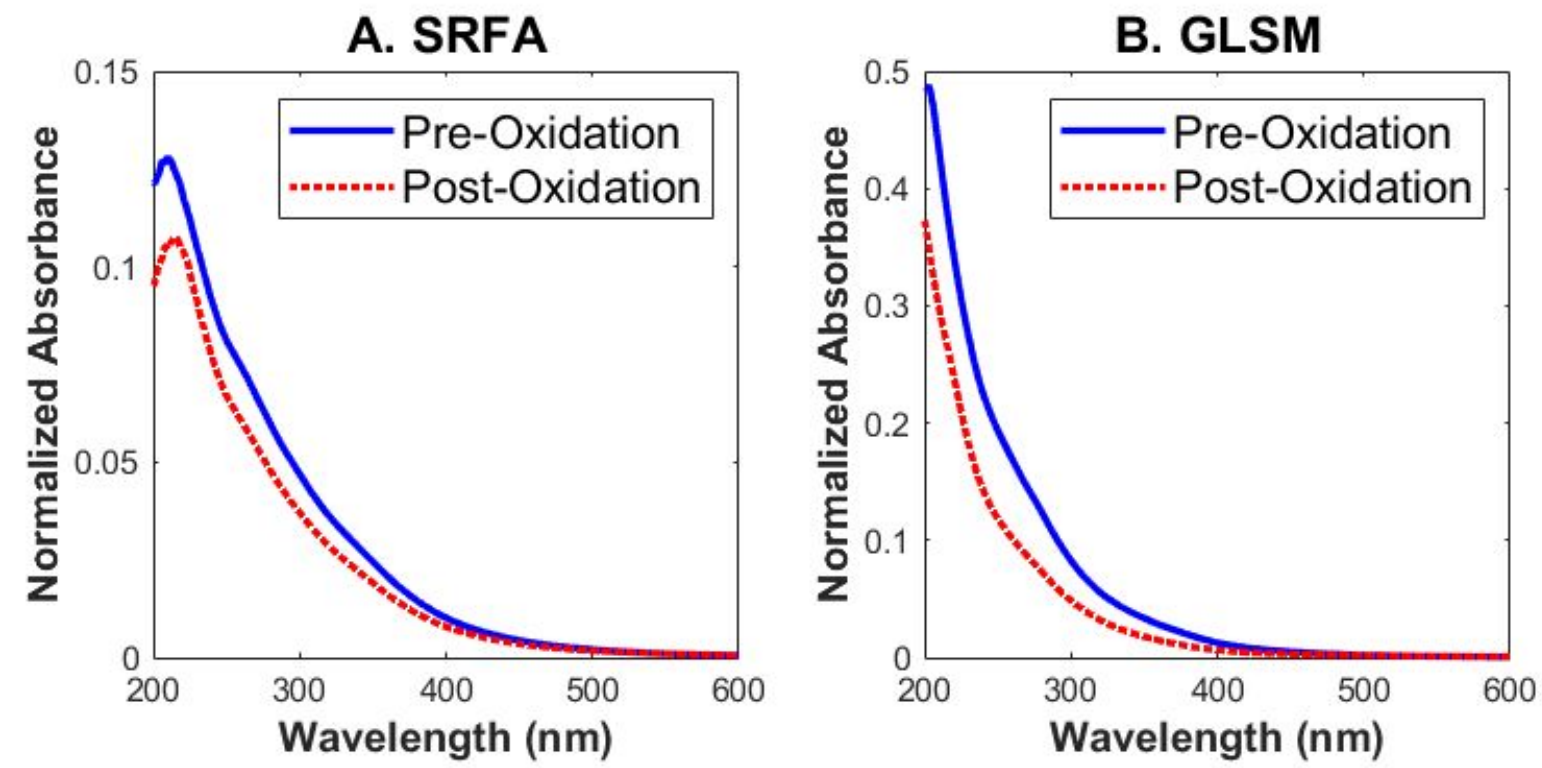

Figure S2: Raw UV-Vis Absorbance Spectra

UV-Vis absorbance spectra measured of pre-oxidation and post-oxidation DOM isolates for SRFA (A) and GLSM DOM (B). The spectra were normalized such that $650 \mathrm{~nm}=$ 0.001 because only small amounts of sample were available and it was not possible to measure identical concentrations.
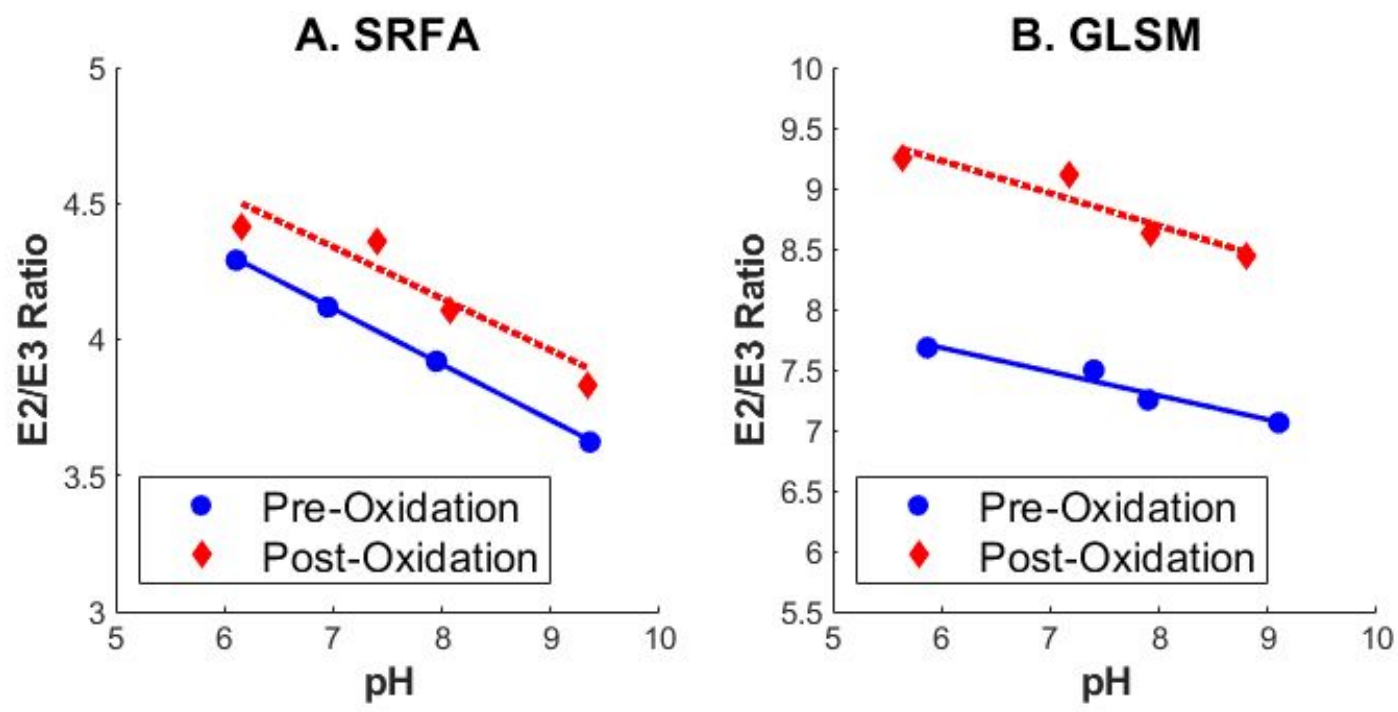

Figure S3: E2/E3 Ratio Changes as a Function of $\mathrm{pH}$

E2/E3 ratio was measured for SRFA (A) and GLSM (B) isolates as a function of $\mathrm{pH}$. E2/E3 ratios are inversely correlated with electron donating capacity (EDC). For both isolates, an increase in E2/E3 ratio is observed regardless of $\mathrm{pH}$. 

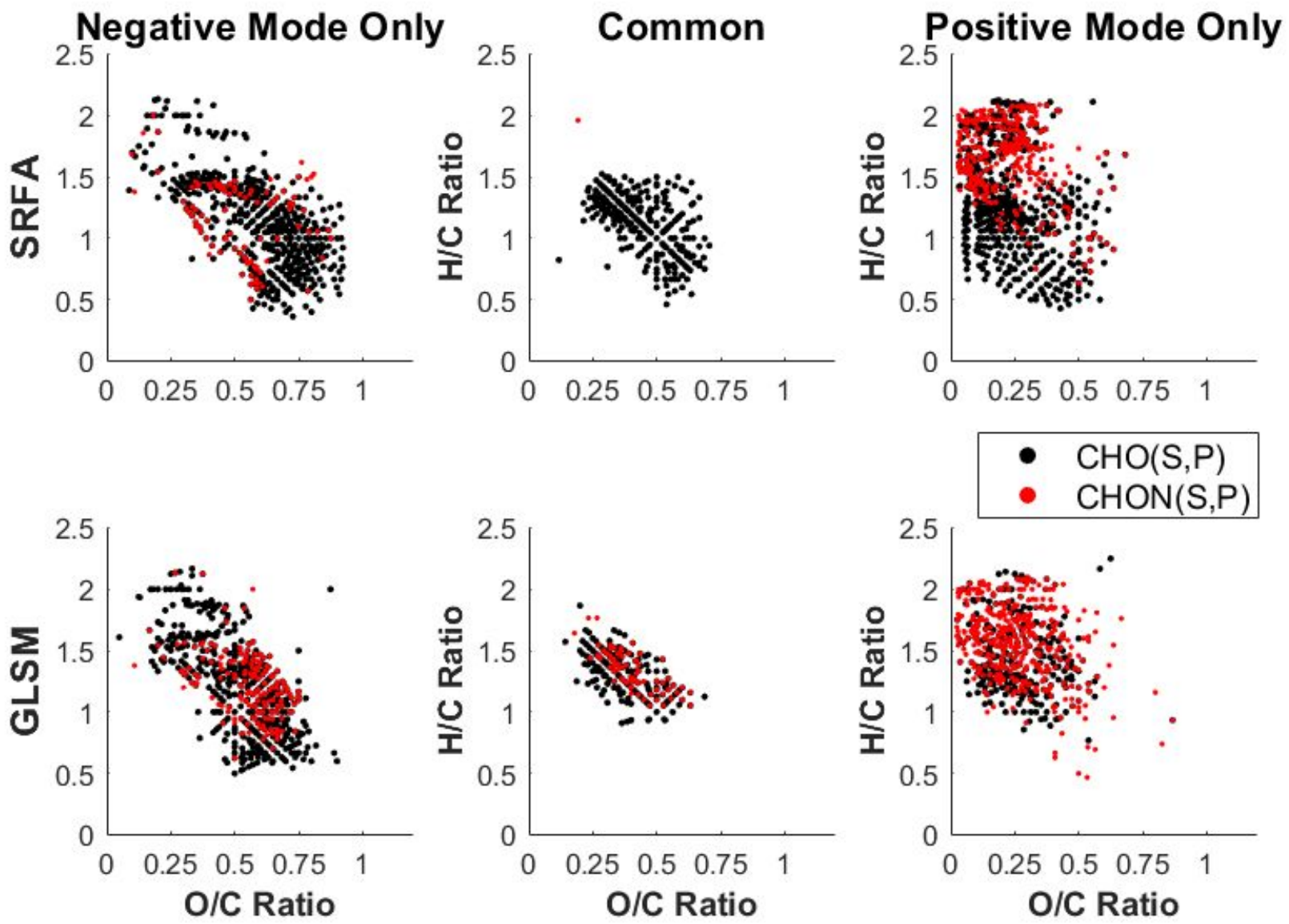

Figure S4: Comparison of ESI Negative and Positive Modes

Components separated into (i) those only ionized by negative ion mode ESI, (ii) those common to both negative and positive ion mode ESI, and (iii) those only ionized by positive ion mode ESI. Positive ion mode ionizes more nitrogen-containing components than negative ion mode. 

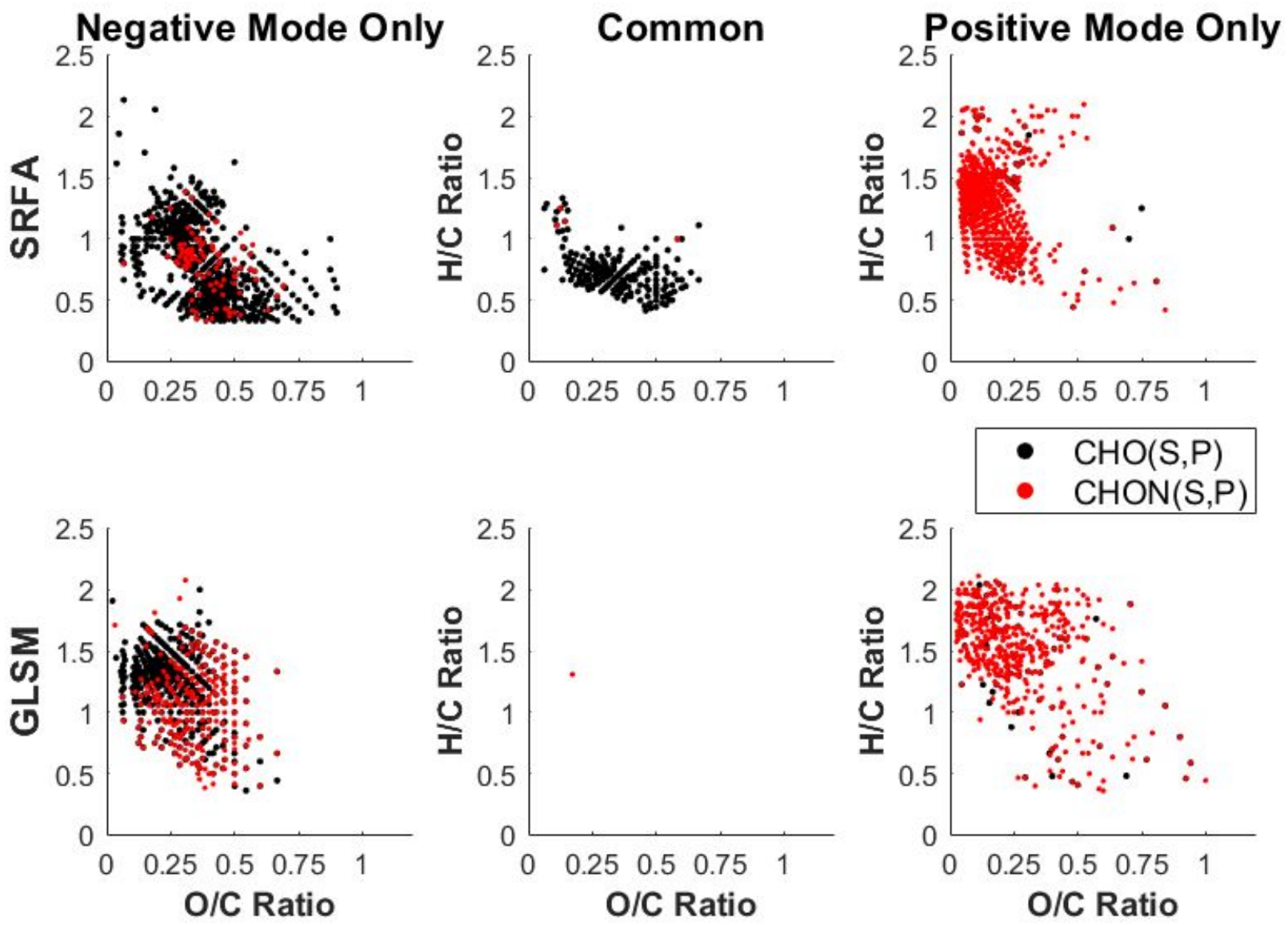

Figure S5: Comparison of LDI Negative and Positive Modes

Components separated into (i) those only ionized by negative ion mode LDI, (ii) those common to both negative and positive ion mode LDI, and (iii) those only ionized by positive ion mode LDI. Positive ion mode ionizes more nitrogen-containing components than negative ion mode. 

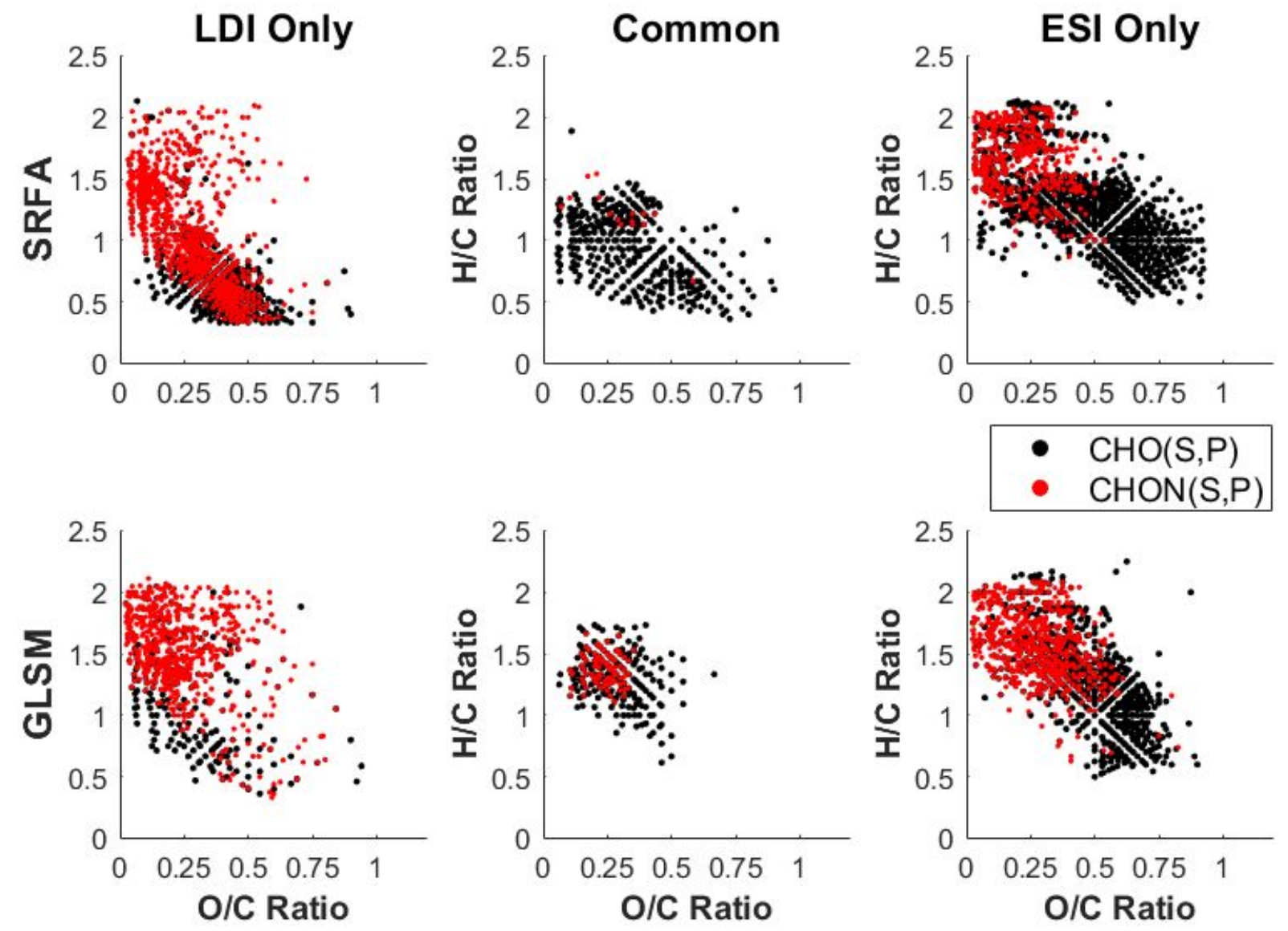

Figure S6: Comparison of ESI and LDI

Components separated into (i) those only ionized by LDI (combined negative and positive ion modes), (ii) those common to both LDI and ESI (regardless of ion mode), and (iii) those only ionized by ESI. LDI ionizes more aromatic-like components while ESI ionizes more aliphatic-like components. 

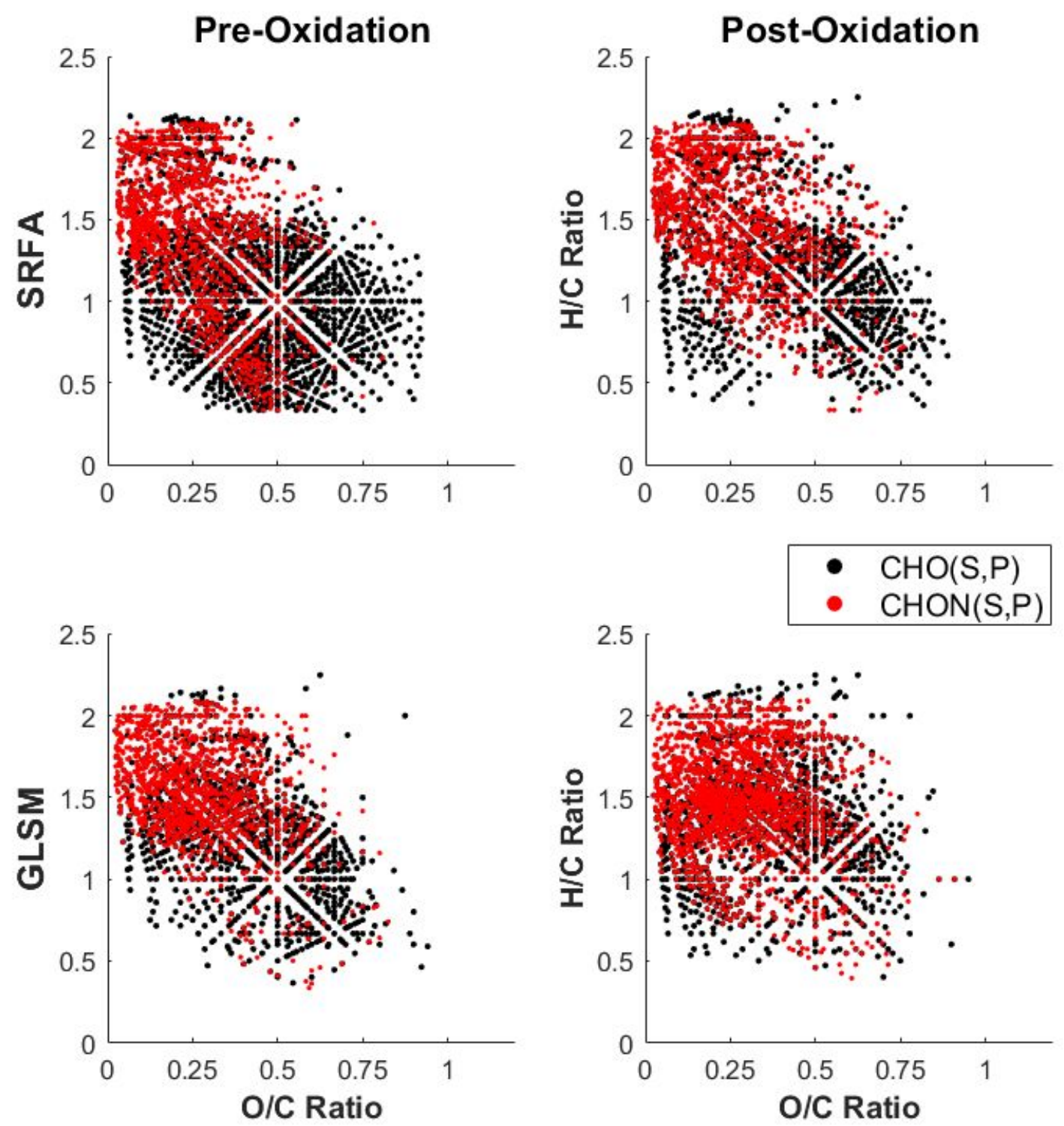

Figure S7: Overall Pre- and Post-Oxidation SRFA and GLSM Van Krevelen Diagrams

Overall van Krevelen diagrams for the pre- and post oxidation samples. Discussed in detail in the main text. 


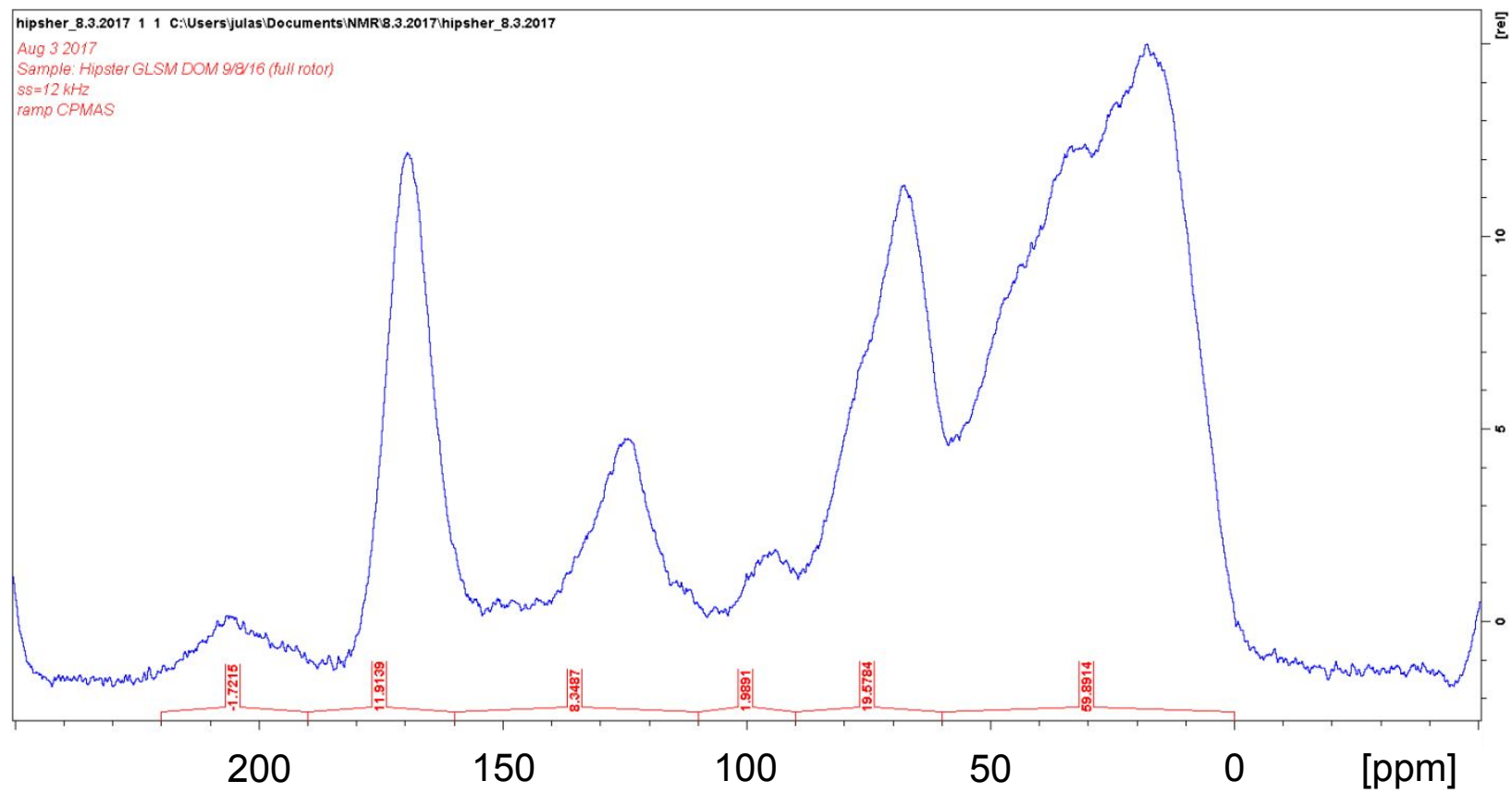

Figure S8: ${ }^{13} \mathrm{C}$ NMR Spectra of GLSM DOM

${ }^{13} \mathrm{C}$ NMR spectra of GLSM DOM, with previously defined carbon types integrated: Aliphatic (60-0ppm, 57.9\%), heteroaliphatic (90-60ppm, 18.9\%), acetal (110-90ppm, $1.9 \%)$, aromatic (165-110ppm, 8.1\%), carboxyl (190-165ppm, 11.5\%), ketone/quinone (220-190ppm, 1.7\%). ${ }^{14}$ 

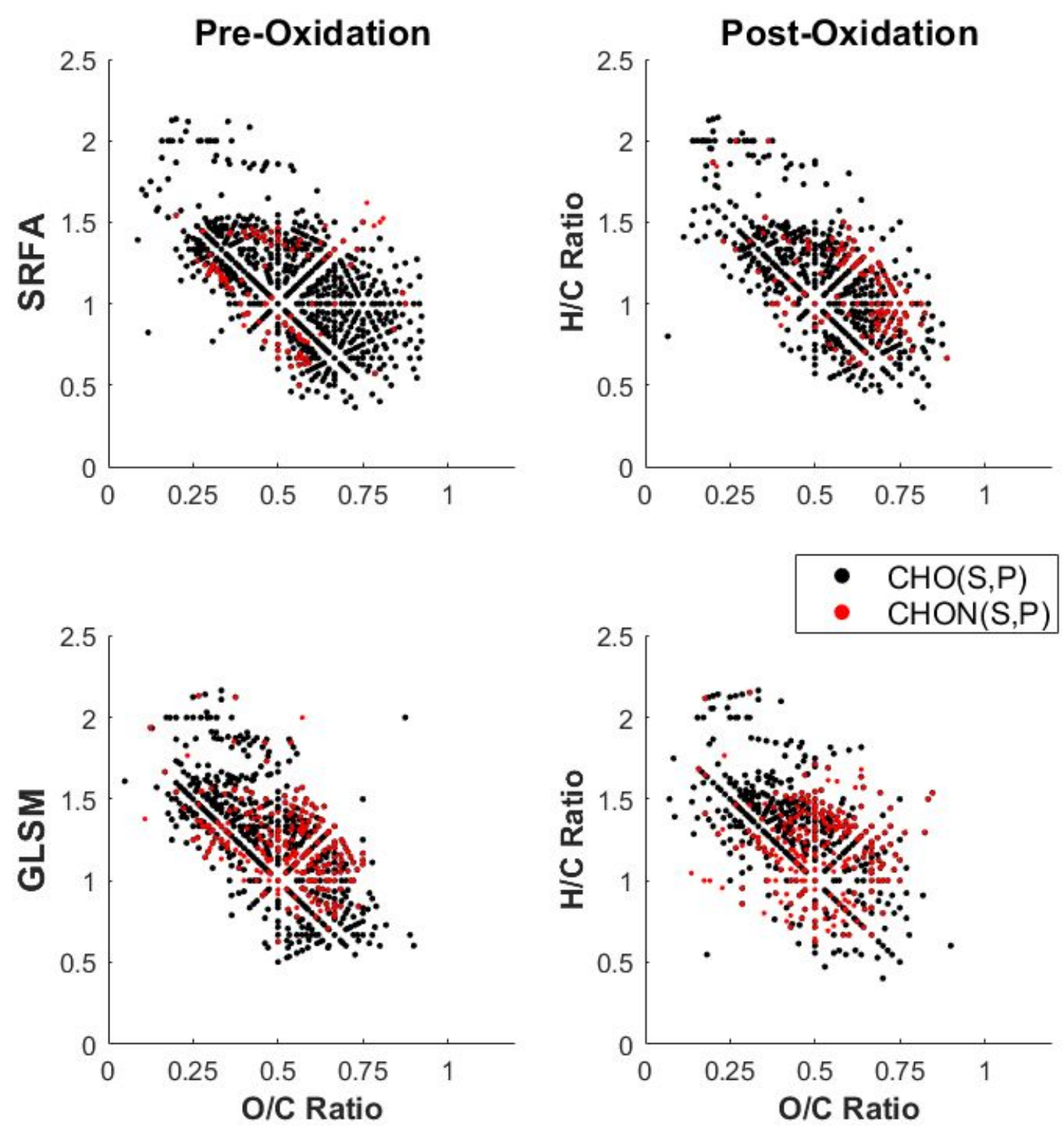

Figure S9: Overall Pre- and Post-Oxidation SRFA and GLSM Van Krevelen Diagrams Using Negative Mode ESI

Van Krevelen diagrams for SRFA and GLSM pre- and post- permanganate oxidation. Little to no shifts in chemical composition were observed. The distribution of components for pre-oxidation SRFA is comparable to what has been reported previously and gives us confidence in our chemical formula assignment. ${ }^{15}$ 

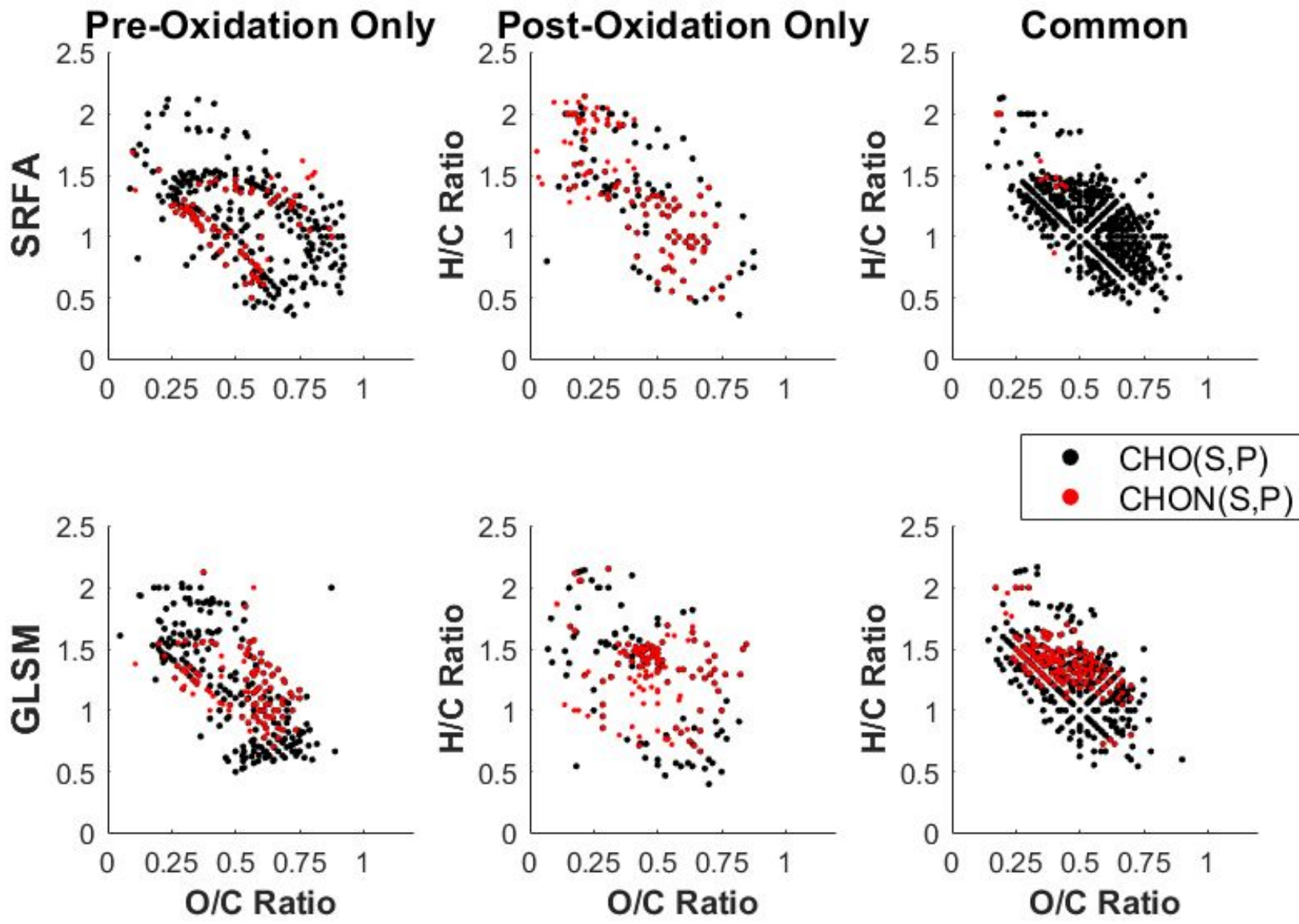

Figure S10: Comparison of Pre- and Post-Oxidation SRFA and GLSM Using Negative Mode ESI

Van Krevelen Diagrams for SRFA and GLSM sorted into formulae observed (i) only initially before permanganate oxidation, (ii) only post-permanganate oxidation, and (iii) common to both samples using negative mode ESI. There are few clear shifts in components observed pre- and post-oxidation. 

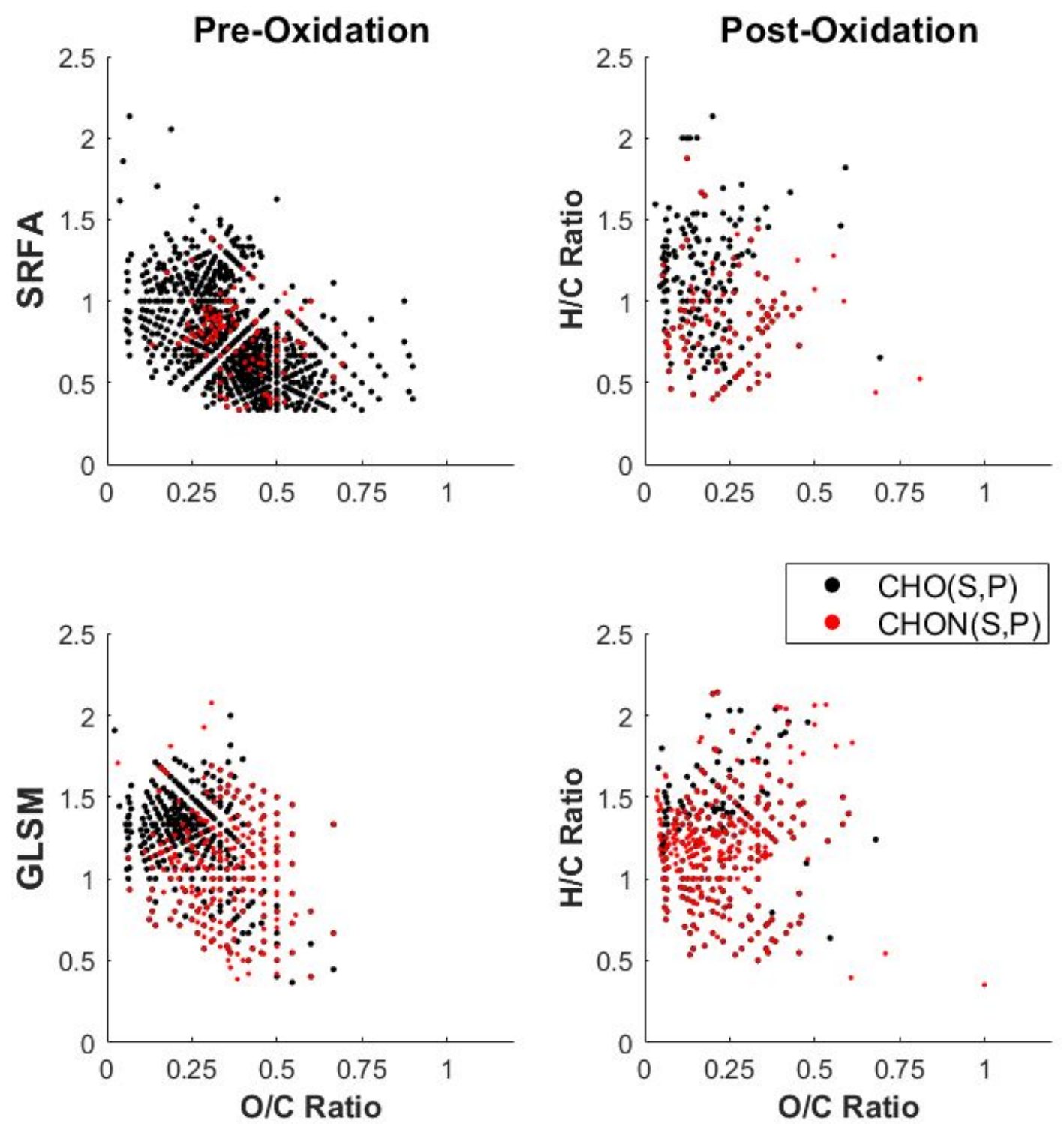

Figure S11: Overall Pre- and Post-Oxidation SRFA and GLSM Van Krevelen Diagrams Using Negative Mode LDI

For SRFA, condensed aromatics are lost following permanganate oxidation. In contrast, few condensed aromatics are observed for GLSM. 

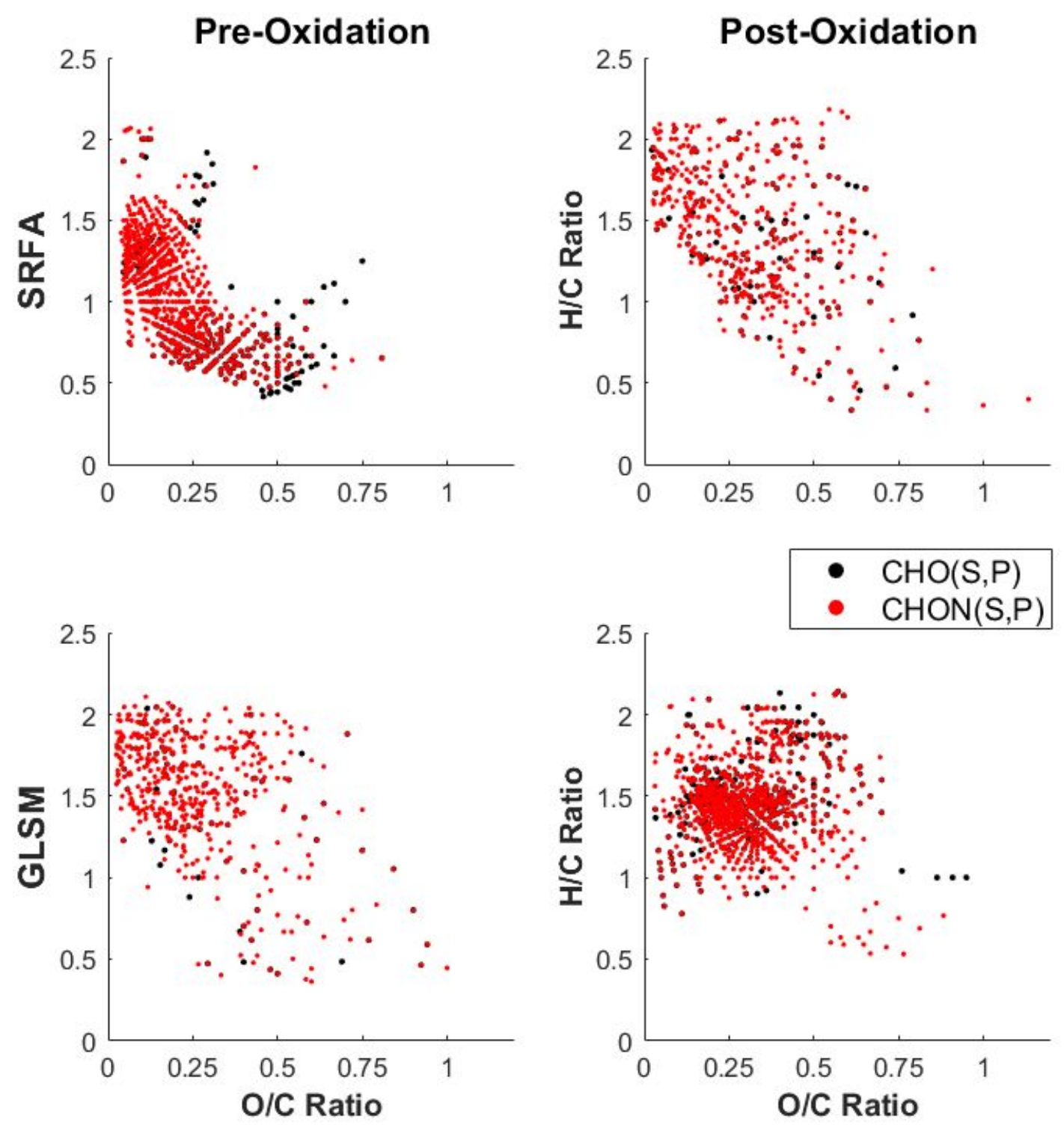

Figure S12: Overall Pre- and Post-Oxidation SRFA and GLSM Van Krevelen Diagrams Using Positive Mode LDI

For SRFA, condensed aromatics are not observed following permanganate oxidation and nitrogen-containing aromatics appear to be more oxidized. The initial GLSM sample was poorly ionized using positive mode LDI. Following oxidation, similar shifts are observed to what was found using positive mode ESI. 

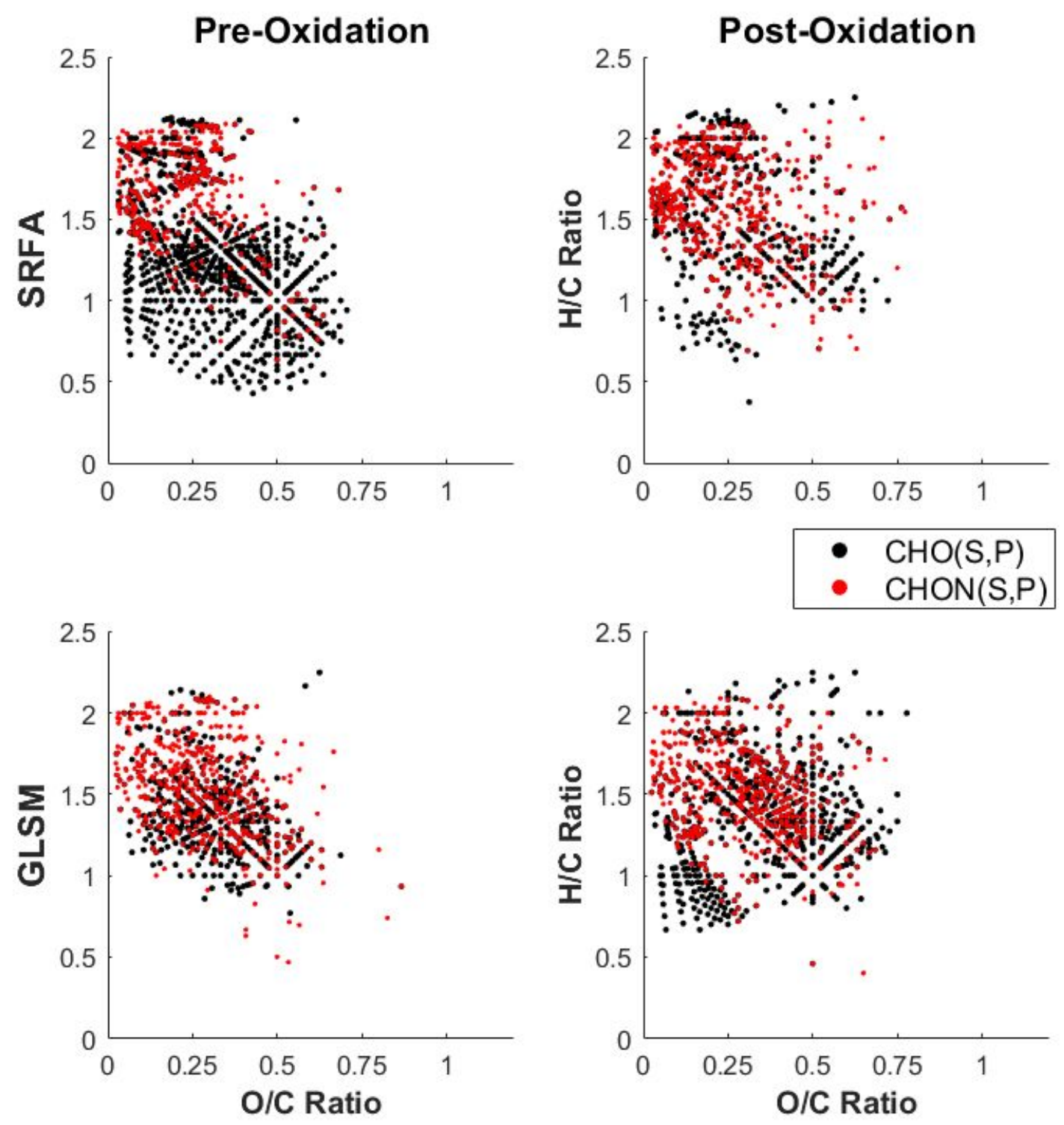

Figure S13: Overall Pre- and Post-Oxidation SRFA and GLSM Van Krevelen Diagrams Using Positive Mode ESI

Many components are observed post-oxidation including more oxidized nitrogencontaining components and polycyclic aromatics, which likely contain benzoic acid moieties. 


\section{References.}

1. Pretsch, E.; Buhlmann, P.; Affolter, C., Structure Determination of Organic Compounds. 4 ed.; Springer: Berlin, Germany, 2009.

2. Abdulla, H. A. N.; Minor, E. C.; Dias, R. F.; Hatcher, P. G., Changes in the compound classes of dissolved organic matter along an estuarine transect: A study using FTIR and 13C NMR. Geochimica et Cosmochimica Acta 2010, 74 (13), 38153838.

3. Panettieri, M.; Knicker, H.; Murillo, J. M.; Madejón, E.; Hatcher, P. G., Soil organic matter degradation in an agricultural chronosequence under different tillage regimes evaluated by organic matter pools, enzymatic activities and CPMAS ${ }^{13} \mathrm{C}$ NMR. Soil Biology and Biochemistry 2014, 78, 170-181.

4. $\quad$ Sleighter, R. L.; Hatcher, P. G., Molecular characterization of dissolved organic matter (DOM) along a river to ocean transect of the lower Chesapeake Bay by ultrahigh resolution electrospray ionization Fourier transform ion cyclotron resonance mass spectrometry. Marine Chemistry 2008, 110, 140-152.

5. $\quad$ Stubbins, A.; Spencer, R. G. M.; Chen, H.; Hatcher, P. G.; Mopper, K.; Hernes, P. J.; Mwamba, V. L.; Mangangu, A. M.; Wabakanghanzi, J. N.; Six, J., Illuminated darkness: molecular signatures of Congo River dissolved organic matter and its photochemical alteration as revealed by ultrahigh precision mass spectrometry. Limnology and Oceanography 2010, 55, 1467-1477.

6. Luek, J. L.; Schmitt-Kopplin, P.; Mouser, P. J.; Petty, W. T.; Richardson, S. D.; Gonsior, M., Halogenated Organic Compounds Identified in Hydraulic Fracturing Wastewaters Using Ultrahigh Resolution Mass Spectrometry. Environmental Science \& Technology 2017, 51 (10), 5377-5385.

7. Herzsprung, P.; Hertkorn, N.; von Tumpling, W.; Harir, M.; Friese, K.; SchmittKopplin, P., Molecular formula assignment for dissolved organic matter (DOM) using high-field FT-ICR-MS: chemical perspective and validation sulphur-rich organic components (CHOS) in pit lake samples. Analytical and Bioanalytical Chemistry 2016, $408,2461-2469$.

8. Herzsprung, P.; Hertkorn, N.; von Tumpling, W.; Harir, M.; Friese, K.; SchmittKopplin, P., Understanding molecular formula assignment of Fourier transform ion cyclotron resonance mass spectrometry data of natural organic matter from a chemical point of view. Analytical and Bioanalytical Chemistry 2014, 406 (30), 7977-87.

9. Van Krevelen, D. W., Graphical-statistical method for the study of structure and reaction process of coal. Fuel 1950, 29, 269-284.

10. Somogyi, A.; Thissen, R.; Orthous-Daunay, F. R.; Vuitton, V., The Role of Ultrahigh Resolution Fourier Transform Mass Spectrometry (FT-MS) in AstrobiologyRelated Research: Analysis of Meteorites and Tholins. International Journal of Molecular Sciences 2016, 17 (4), 439.

11. Kim, S.; Kramer, R. W.; Hatcher, P. G., Graphical Method for Analysis of Ultrahigh-Resolution Broadband Mass Spectra of Natural Organic Matter, the Van Krevelen Diagram. Analytical chemistry 2003, 75 (20), 5336-5344.

12. Koch, B. P.; Dittmar, T., From mass to structure: an aromaticity index for highresolution mass data of natural organic matter. Rapid Communications in Mass Spectrometry 2016, 30 (1), 250-250. 
13. Koch, B. P.; Dittmar, T., From mass to structure: an aromaticity index for highresolution mass data of natural organic matter. Rapid Communications in Mass Spectrometry 2006, 20 (5), 926-932.

14. Thorn, K. A.; Folan, D. W.; MacCarthy, P. Characterization of the International Humic Substances Society standard and reference fulvic and humic acids by solution state carbon-13 (13C) and hydrogen-1 (1H) nuclear magnetic resonance spectrometry; U.S. Geological Survey: Denver, Colorado, 1989.

15. D’Andrilli, J.; Foreman, C. M.; Marshall, A. G.; McKnight, D. M., Characterization of IHSS Pony Lake fulvic acid dissolved organic matter by electrospray ionization Fourier transform ion cyclotron resonance mass spectrometry and fluorescence spectroscopy. Organic Geochemistry 2013, 65, 19-28. 


\section{Text S3: Matlab Code}

응 Code to Analyze Pre- and Post-Permanganate Oxidation SRFA and GLSM oThe following code is organized into the following major sections as follows:

⒈ Pre-oxidation SRFA clean up

\%2. Post-oxidation SREA clean up

⒊ Pre-oxidation GLSM clean up

\%4. Post-oxidation GLSM clean up

응 Initial SRFA Sample

응 Negative Mode ESI: Data Clean Up

oLoading negative mode background impurities

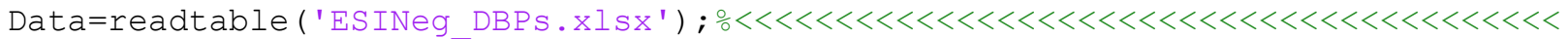

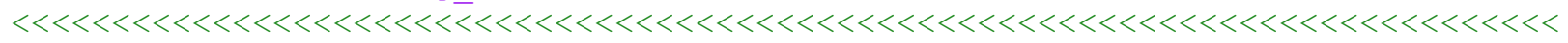
$<<<<<<<<<<<<<<<<<<<<<<<<<<<<<<<<<<~$

Data=unique (Data);

Data. NominalMass=Data. $\mathrm{C} * 12+$ Data $\cdot 0 * 16+$ Data $\cdot H * 1+$ Data $\cdot P * 31+$ Data $\cdot S * 32+D a t a \cdot N * 14$;

Data.KMD $\mathrm{CH} 2=$ Data $\cdot$ NominalMass -

$0.998883 \overline{4} *\left(\right.$ Data. $C * 12+$ Data. $H^{*} 1.007825+$ Data. $N^{*} 14.003074+$ Data. $0 * 15.994915+$ Data.$P$

*30.973763+Data.S*31.972072);

Data.KMD_COO=Data. NominalMass-

1.000231 (Data. C*12+Data.H*1.007825+Data.N*14.003074+Data.0*15.994915+Data.P* $30.973763+$ Data.S*31.972072);

Data.KMD 02=Data. NominalMass-

$1.0003177^{\bar{*}}\left(\right.$ Data. $C * 12+$ Data $. H^{*} 1.007825+$ Data. $N^{*} 14.003074+$ Data. $0 * 15.994915+$ Data. $P *$ $30.973763+$ Data..$* 31.972072)$;

Data.KMD_H2=Data.NominalMass-

$0.992236^{\star}\left(\right.$ Data. $C * 12+$ Data. $H^{*} 1.007825+$ Data. $N^{*} 14.003074+$ Data. $0 * 15.994915+$ Data. $P *$ $30.973763+$ Data. $S * 31.972072)$;

Data.H=Data.H+1;

Data.HC Ratio = Data.H./ Data.C;

Data.oC_Ratio = Data.O./ Data.C;

Data.NC_Ratio = Data.N ./ Data.C;

Data.SC_Ratio = Data.S ./ Data.C;

Data.PC Ratio = Data.P ./ Data.C;

Data. $\mathrm{DBE}=1+0.5 *(2 *$ Data. $\mathrm{C}-$ Data.H + Data. $\mathrm{N}+$ Data.P $)$;

Data.AI $=(1+$ Data.C - Data.O - Data.S - 0.5* (Data.N + Data.P + Data.H))./

(Data.C - Data.O - Data.N - Data.S - Data.P);

Data.AvgCoxstate = 2*Data.oC_Ratio - Data.HC_Ratio;

oRemove data points based on following criteria

ㄴ. Error (allowable: ppm <=0.5)

Data (Data. errPpm>0.5,:) = [];

Data (Data.errPpm<-0.5,:) = [] ;

\%2. Stubbins, 2010 :

$\div \mathrm{A} . \mathrm{DBE}=$ integer, greater than or equal to 0

Data (Data. DBE =round (Data. DBE), :) = [] ;

Data (Data. $\mathrm{DBE}<0,:)=[]$;

ㅇ. $\mathrm{C}<=50$

Data (Data.C $>50,:)=[]$;

$\div \mathrm{C} \cdot \mathrm{H}>=2 \&<=2 * \mathrm{C}+2$

Data (Data. $\mathrm{H}<2,:)=[]$;

Data (Data.H $>($ Data. $C * 2+2),:)=[]$;

$\because \mathrm{D} . \mathrm{O}=<\mathrm{C}+2$

Data $($ Data. O $>($ Data $. C+2),:)=[]$;

E. $O / C<1.2$

Data (Data.oC_Ratio>1.2,:) =[ ] ; 


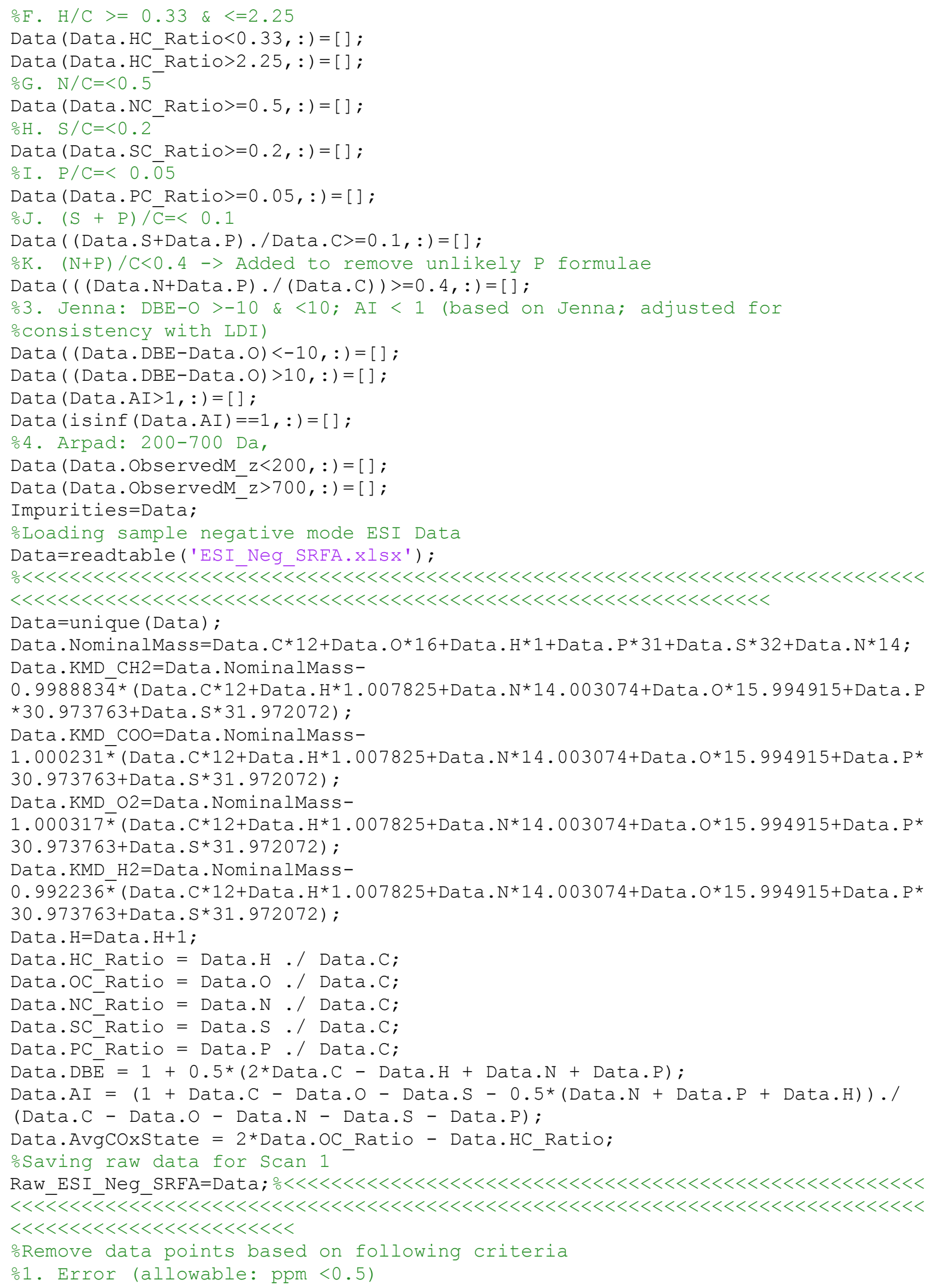




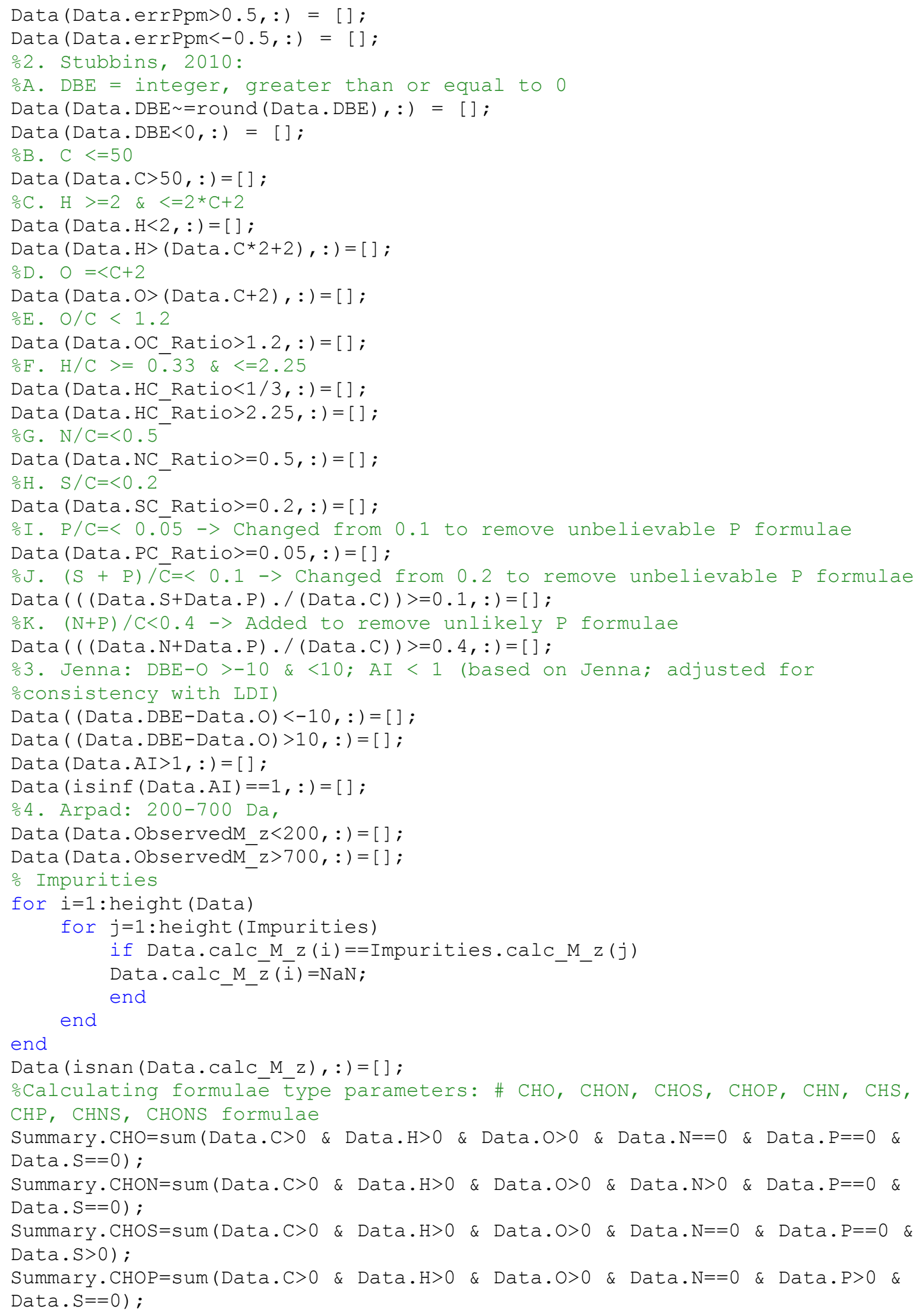


Summary. $\mathrm{CHN}=$ sum $($ Data. $\mathrm{C}>0$ \& Data.H $>0$ \& Data. $\mathrm{O}==0$ \& Data. $\mathrm{N}>0$ \& Data.P==0 \& Data. $\mathrm{S}==0$ );

Summary $\cdot \mathrm{CHS}=\operatorname{sum}($ Data $\cdot \mathrm{C}>0$ \& Data.H $>0$ \& Data. $\mathrm{O}==0$ \& Data. $\mathrm{N}==0$ \& Data. $\mathrm{P}==0$ \& Data. S $>0$ );

Summary $\cdot \mathrm{CHNS}=\operatorname{sum}($ Data. $\mathrm{C}>0$ \& Data. $\mathrm{H}>0$ \& Data. $\mathrm{O}==0$ \& Data. $\mathrm{N}>0$ \& Data. $\mathrm{P}==0$ \& Data. $\mathrm{S}>0$ );

Summary. $\mathrm{CHNP}=\operatorname{sum}($ Data. $\mathrm{C}>0$ \& Data. $\mathrm{H}>0$ \& Data. $\mathrm{O}==0$ \& Data.N $>0$ \& Data.P $>0$ \&

Data. $\mathrm{S}=0$ ) ;

Summary $\cdot \mathrm{CHONS}=\operatorname{sum}($ Data $\cdot \mathrm{C}>0$ \& Data. $\mathrm{H}>0$ \& Data. $\mathrm{O}>0$ \& Data.N $>0$ \& Data.P==0 \&

Data. $\mathrm{S}>0$ );

Summary. $\mathrm{CHONP}=$ sum $($ Data. $\mathrm{C}>0$ \& Data.H $>0$ \& Data. O $>0$ \& Data.N $>0$ \& Data.P $>0$ \&

Data. $\mathrm{S}=0$ ) ;

Summary. $\mathrm{CHONSP}=$ sum $($ Data. $\mathrm{C}>0$ \& Data.H $>0$ \& Data.O $>0$ \& Data.N $>0$ \& Data.P>0 \&

Data. S>0);

ocalculating aromaticity parameters: \# condensed aromatic (AI>=0.67),

aromatic $(A I>=0.5)$,

onon-aromatic $($ A I < 0.5$)$

Summary. CondensedAromatics $=$ sum (Data.AI $>=0.67)$;

Summary. Aromatics=sum (Data.AI $>=0.5)$;

Summary. NonAromatics=sum (Data.AI<0.5);

ocalculating other parameters: total \# of formulae, average molecular

oweight

Summary. TotalFormulae=height (Data);

Summary. AvgMW=mean (Data. NominalMass);

ostoring Data in Tables

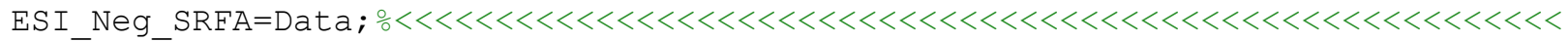

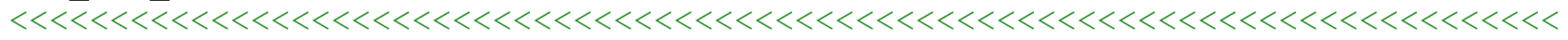
$<<<<<<<<<<<<<<<<<<<<~$

ESI Neg SRFA Summary=struct2table (Summary) ; $\circ<<<<<<<<<<<<<<<<<<<<<<<<<<<<<<<<<<~$ $<<<\overline{<}<<<\overline{<}<<<<\overline{<}<<<<<<<<<<<<<<<<<<<<<<<<<<<<<<<<<<<<<<<<<<<<<<<<<<<<<<<<<<<<<<<<~$

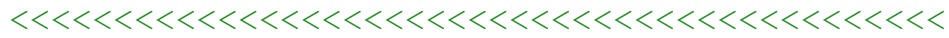

응 Positive Mode ESI: Data Clean Up

oLoading positive mode ESI impurities

Data =readtable ('DBP Control.xlsx');

Data. NominalMass=Data $\cdot \mathrm{C} * 12+$ Data $\cdot 0 * 16+$ Data $\cdot H * 1+$ Data $\cdot P * 31+$ Data $\cdot S * 32+D a t a \cdot N * 14+D$ ata. $\mathrm{Na} * 23$;

Data. KMD CH2=Data. NominalMass -

$0.998883 \overline{4} *($ Data. $C * 12+$ Data. $H * 1.007825+$ Data. $N * 14.003074+$ Data. $0 * 15.994915+$ Data. $P$ *30.973763+Data.S*31.972072+Data.Na*22.98977);

Data.KMD $\mathrm{COO}=$ Data. NominalMass-

$1.000231^{\star}\left(\right.$ Data. C*12+Data.H*1.007825+Data. N $^{*} 14.003074+$ Data. $0 * 15.994915+$ Data.P*

$30.973763+$ Data. $\mathrm{S} * 31.972072+$ Data.Na*22.98977);

Data.KMD $02=$ Data. NominalMass-

$1.000317^{\star}$ (Data. C*12+Data.H*1.007825+Data.N*14.003074+Data.0*15.994915+Data.P* $30.973763+$ Data. $\mathrm{S} * 31.972072+$ Data.Na*22.98977);

Data.KMD_H2=Data.NominalMass-

$0.992236^{\star}$ (Data. C*12+Data.H*1.007825+Data. $N^{*} 14.003074+$ Data. $0 * 15.994915+$ Data.P*

$30.973763+$ Data. S*31.972072+Data.Na*22.98977);

for $i=1$ : height (Data)

if Data.Na(i)==0

Data.H(i)=Data.H (i) -1 ;

end

end

Data.HC Ratio = Data.H ./ Data.C;

Data.OC_Ratio = Data.O ./ Data.C;

Data.NC_Ratio = Data.N ./ Data.C; 


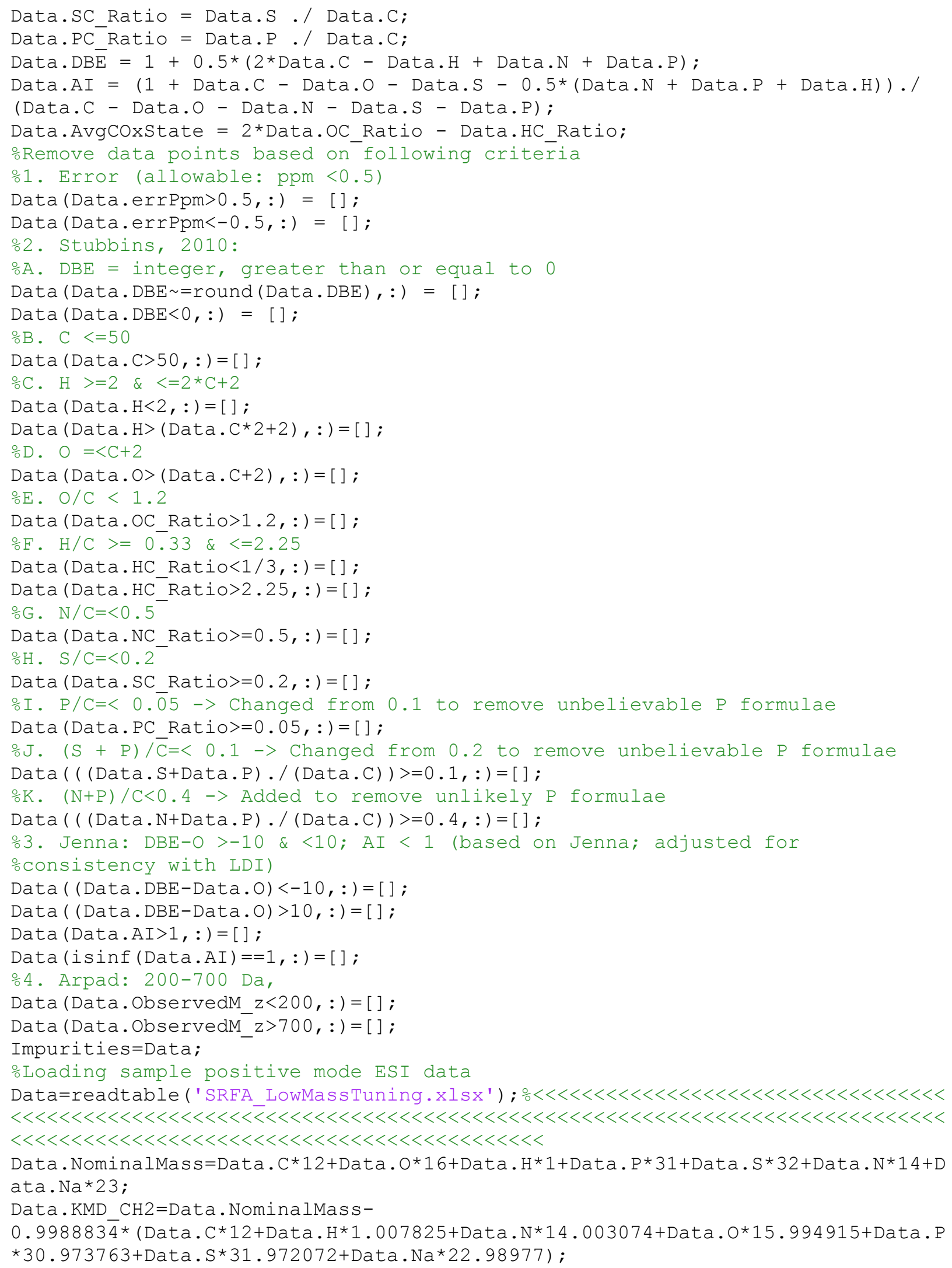




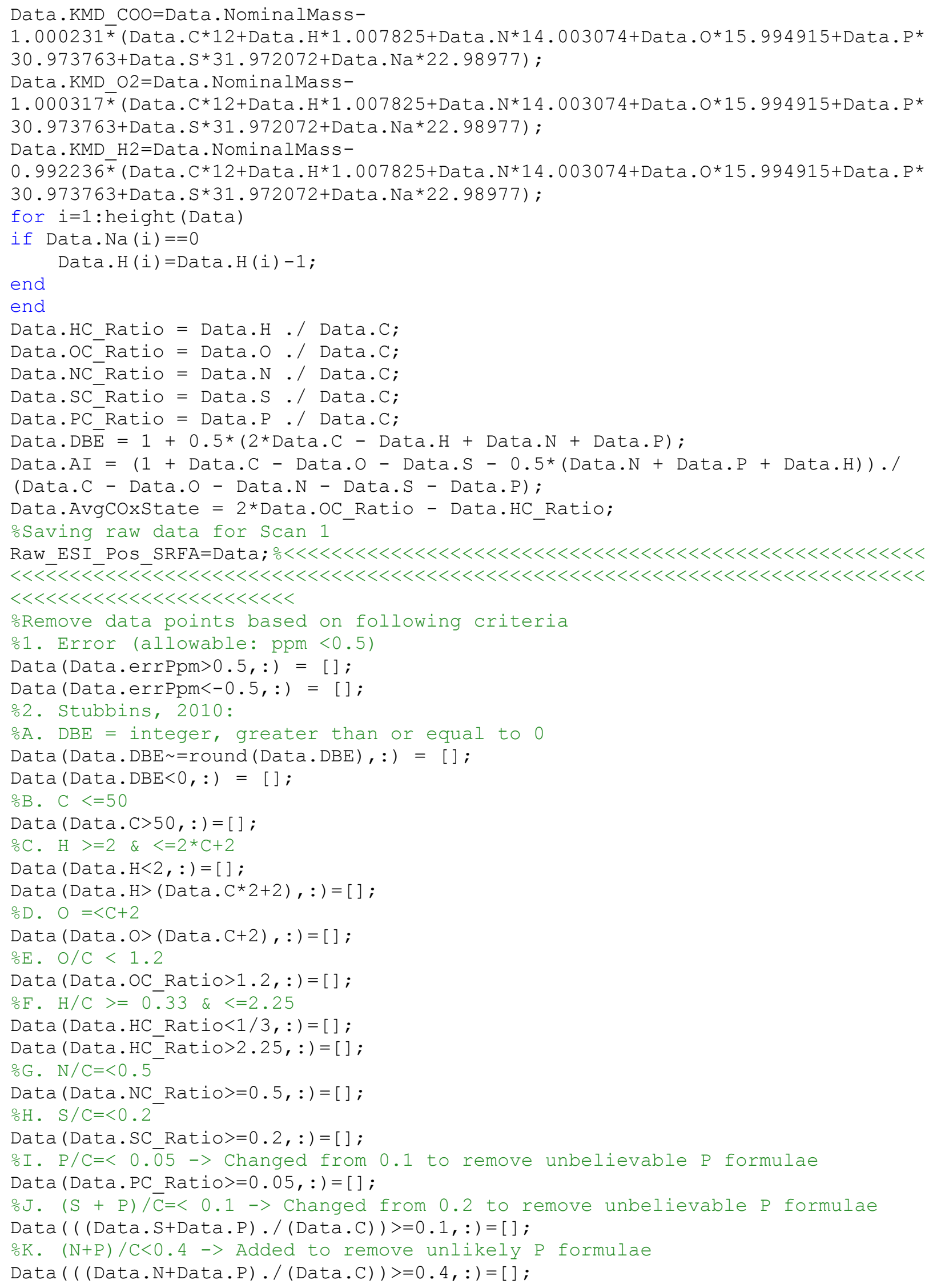




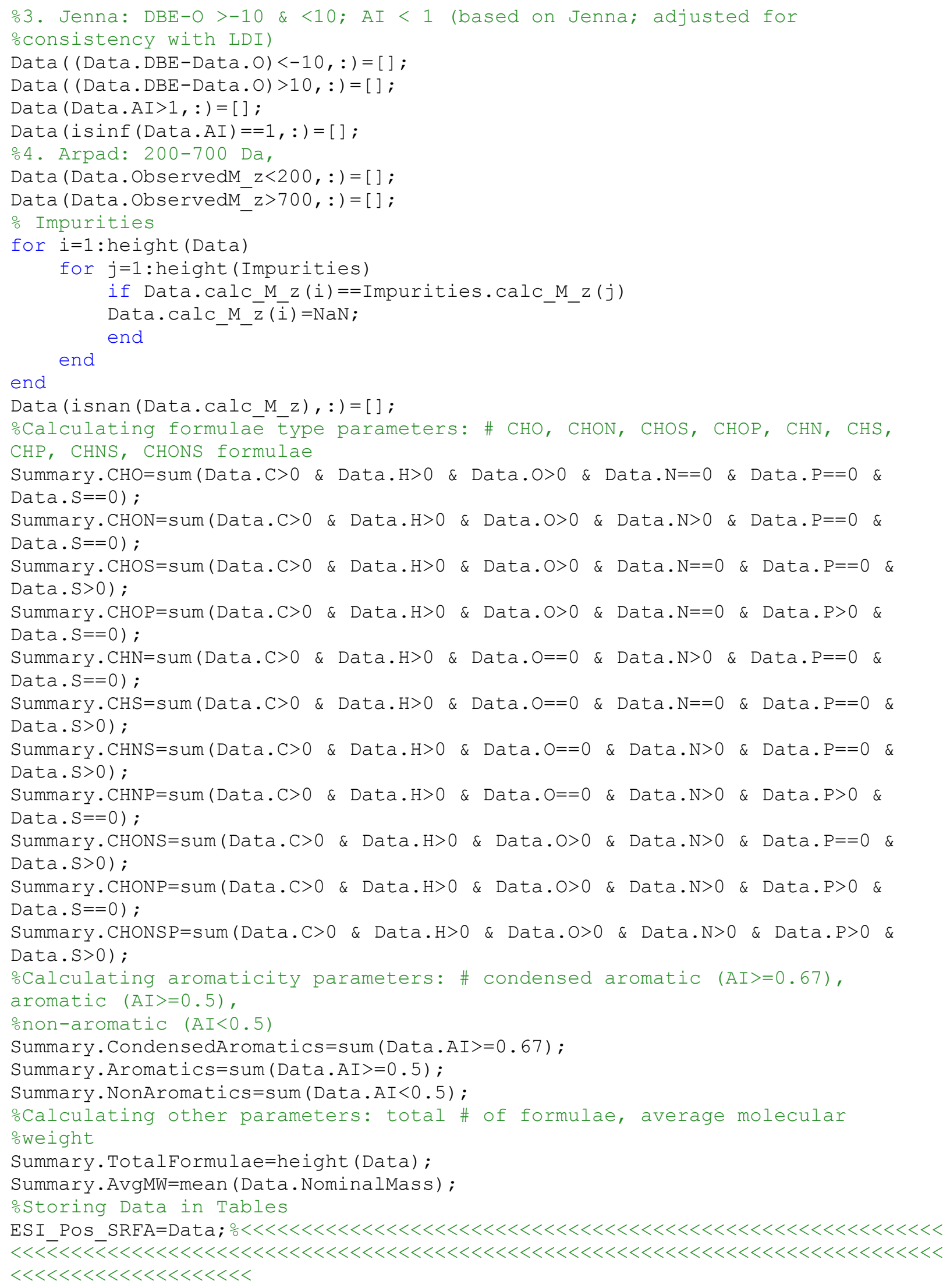




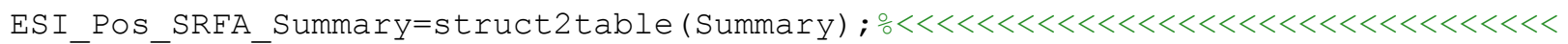

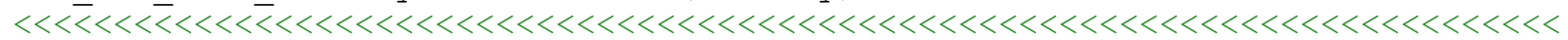

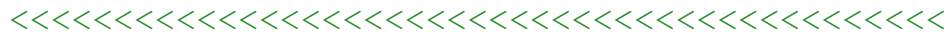

\% Negative Mode LDI: Data Clean Up \%LDI assumed to have no background ions

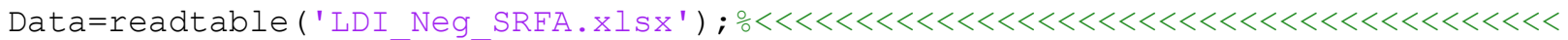

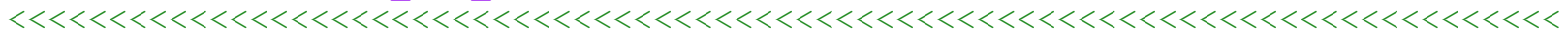

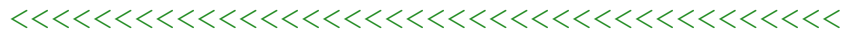

Data=unique (Data);

Data. NominalMass=Data. $\mathrm{C} * 12+$ Data. $0 * 16+$ Data $\cdot H * 1+$ Data. $P * 31+$ Data $. S * 32+D a t a \cdot N * 14 ;$ Data. KMD CH2=Data. NominalMass-

$0.998883 \overline{4} *\left(\right.$ Data. $C * 12+$ Data. $H * 1.007825+$ Data. $N^{*} 14.003074+$ Data. $0 * 15.994915+$ Data. P *30.973763+Data.S*31.972072);

Data.KMD COO=Data. NominalMass -

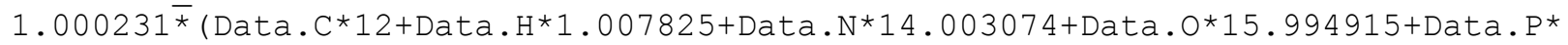
$30.973763+$ Data. $S * 31.972072)$;

Data.KMD O2=Data. NominalMass-

$1.000317^{\bar{*}}\left(\right.$ Data. $\mathrm{C}^{\star} 12+$ Data. $\mathrm{H}^{\star} 1.007825+$ Data. $\mathrm{N}^{\star} 14.003074+\mathrm{Data} .0 * 15.994915+\mathrm{Data} . \mathrm{P} *$ $30.973763+$ Data. S*31.972072);

Data.KMD H2=Data. NominalMass-

$0.992236^{\star}$ (Data.C*12+Data.H*1.007825+Data.N*14.003074+Data. $* 15.994915+$ Data.P* $30.973763+$ Data..$* 31.972072)$;

Data.DBE $=1+0.5 *(2 *$ Data.C - Data.H + Data.N + Data.P $)$;

for $i=1$ : height (Data)

if Data.DBE (i) =round (Data.DBE (i))

Data.H (i) =Data.H(i) + ;

Data. IonType $(i)=0 ; \% H-$ Adduct $s=0$

else

Data.IonType(i)=2; №lecularIons=2 \%Temporary fix until I figure out how

to enter names into a table end

end

Data.HC_Ratio = Data.H ./ Data.C;

Data.OC_Ratio = Data.O./ Data.C;

Data.NC Ratio = Data.N ./ Data.C;

Data.SC_Ratio = Data.S ./ Data.C;

Data.PC Ratio = Data.P ./ Data.C;

Data. $\mathrm{DBE}=1+0.5 *(2 *$ Data.C - Data.H + Data. $\mathrm{N}+$ Data.P $)$;

Data.AI $=(1+$ Data.C - Data.O - Data.S - 0.5* (Data.N + Data.P + Data.H) $) . /$

(Data.C - Data.O - Data.N - Data.S - Data.P);

Data.AvgCoxstate = 2*Data.OC_Ratio - Data.HC_Ratio;

osaving raw data

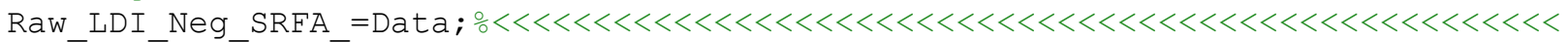

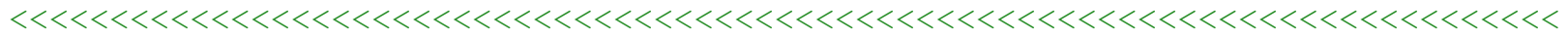
$<<<<<<<<<<<<<<<<<<<<<<<<<<<~$

oRemove data points based on following criteria

\%1. Error (allowable: ppm <0.5)

Data (Data.errPpm>0.5,:) = [];

Data (Data.errPpm<-0.5,:) = [ ] ;

ㄴ. Stubbins, 2010 :

\%A. $\mathrm{DBE}=$ integer, greater than or equal to 0

Data (Data.DBE =round (Data.DBE), : ) = [];

Data (Data. $\mathrm{DBE}<0,:)=[]$;

ㅇ. $\mathrm{C}<=50$

Data (Data.C $>50,:)=[]$;

ㄷ. $\mathrm{H}>=2 \&<=2 * \mathrm{C}+2$ 


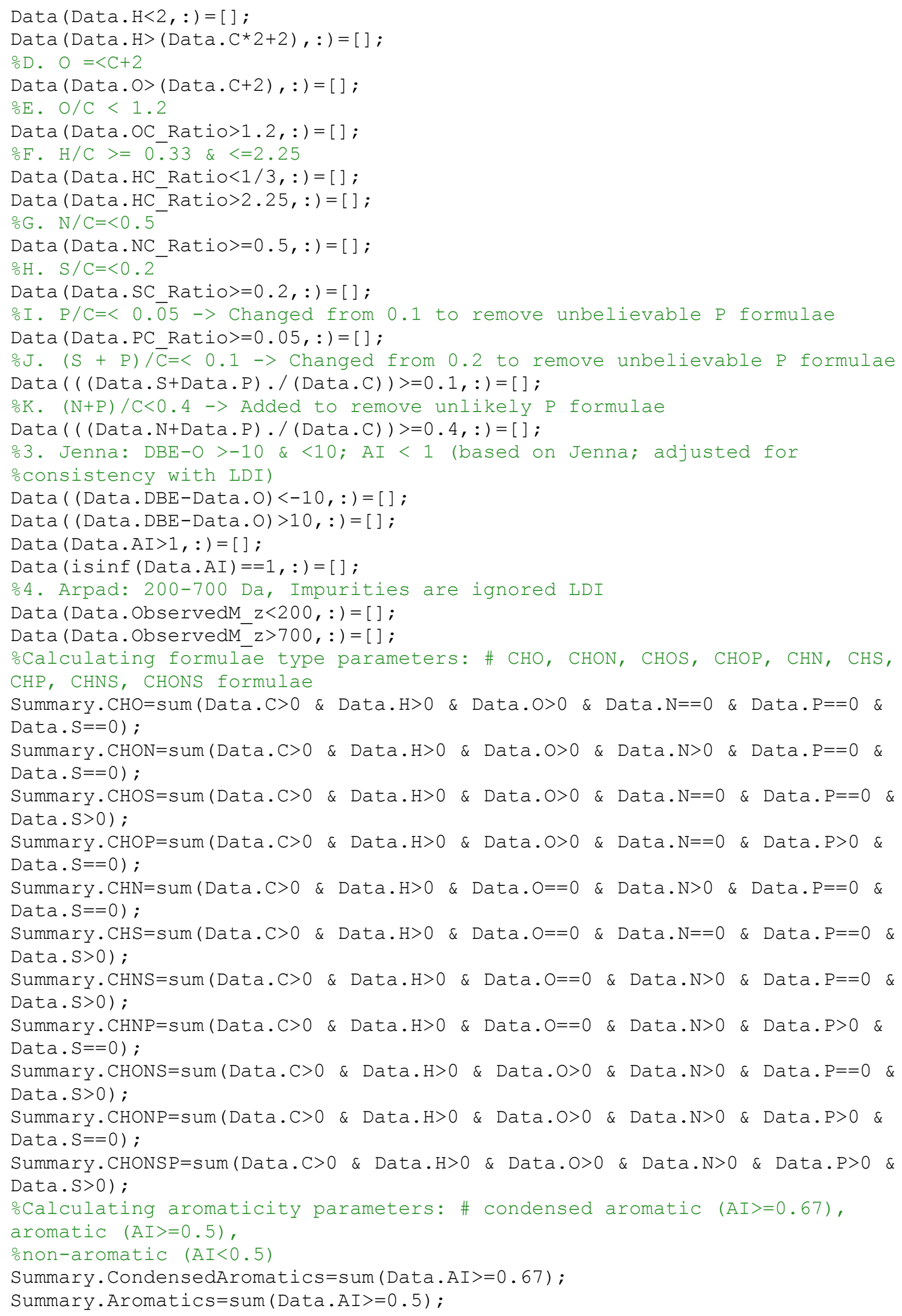


Summary. NonAromatics=sum (Data.AI<0.5);

ocalculating other parameters: total \# of formulae, average molecular

oweight

Summary. TotalFormulae=height (Data);

Summary. AvgMW=mean (Data. NominalMass) ;

ostoring Data in Tables

LDI Neg SRFA=Data; $\circ<<<<<<<<<<<<<<<<<<<<<<<<<<<<<<<<<<<<<<<<<<<<<<<<<<<<<<<<<<<~$

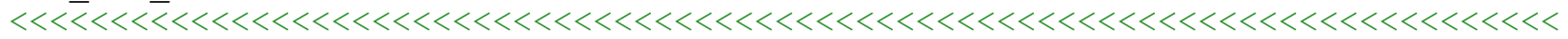
$<<<<<<<<<<<<<<<<<<~$

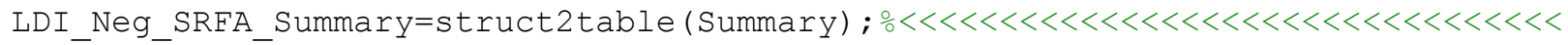

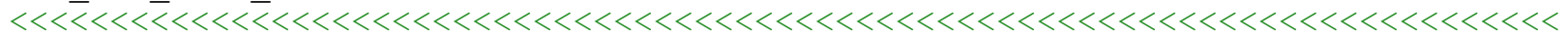

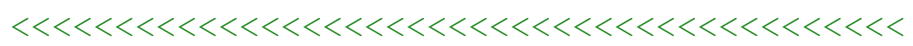

응 Positive Mode LDI: Data Clean Up

\%LDI assumed to have no background ions

oLoading sample positive mode LDI data

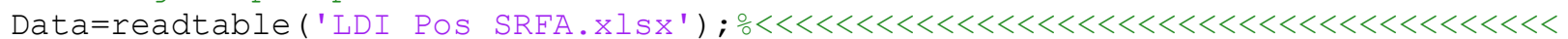

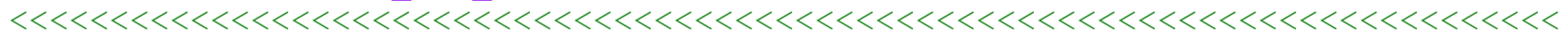
$<<<<<<<<<<<<<<<<<<<<<<<<<<<<<<<<<<<<<<<<<<<<<<~$

Data=unique (Data);

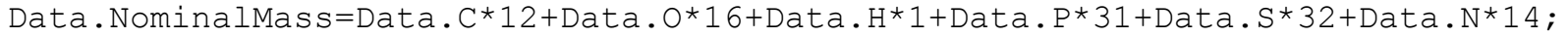

Data.KMD_CH2=Data. NominalMass-

$0.998883 \overline{4} *\left(\right.$ Data. $C * 12+$ Data. $H^{*} 1.007825+$ Data. $N^{*} 14.003074+$ Data. $0 * 15.994915+$ Data.$P$ $\star 30.973763+$ Data. $\mathrm{S} * 31.972072$ );

Data.KMD_COO=Data.NominalMass-

$1.000231^{\star}\left(\right.$ Data. $C * 12+$ Data $. H * 1.007825+$ Data. $N^{*} 14.003074+$ Data. $0 * 15.994915+$ Data. P* $30.973763+$ Data.. S*31.972072);

Data.KMD $02=$ Data. NominalMass-

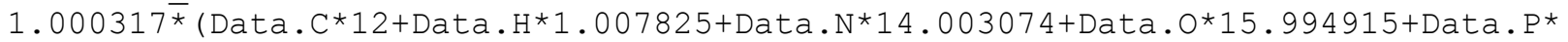
$30.973763+$ Data. $S * 31.972072)$;

Data.KMD_H2=Data.NominalMass-

$0.992236^{\star}\left(\right.$ Data. $C^{*} 12+$ Data $. H^{*} 1.007825+$ Data $. N^{*} 14.003074+$ Data $.0 * 15.994915+$ Data. $P^{*}$

$30.973763+$ Data. $S * 31.972072)$;

Data.DBE $=1+0.5 *(2 *$ Data.C - Data.H + Data.N + Data.P $)$;

for $i=1$ : height (Data)

if Data.DBE(i) =round (Data.DBE(i)) \&\& Data.Na(i)==0

Data.H $(i)=$ Data.H $(i)+1$;

Data. IonType $(i)=0 ; \circ H-$ Adduct $s=0$

elseif Data.DBE(i)==round (Data.DBE(i)) \&\& Data.Na(i) $>0$

Data.IonType $(i)=1 ; \circ \mathrm{Na}$-Adducts else

Data. IonType (i)=2; $\frac{\circ}{\circ}$ MolecularIons=2 \%Temporary fix until I figure out how

to enter names into a table end

end

Data.HC_Ratio = Data.H ./ Data.C;

Data.OC_Ratio = Data.O./ Data.C;

Data.NC_Ratio = Data.N ./ Data.C;

Data.SC Ratio = Data.S./ Data.C;

Data.PC_Ratio = Data.P./ Data.C;

Data.DBE $=1+0.5 *(2 *$ Data.C - Data.H + Data.N + Data.P $)$;

Data.AI $=(1+$ Data.C - Data.O - Data.S - 0.5* (Data.N + Data.P + Data.H) $) \cdot /$

(Data.C - Data.O - Data.N - Data.S - Data.P);

Data.AvgCoxstate = 2*Data.OC_Ratio - Data.HC_Ratio;

oSaving raw data for Scan 1 


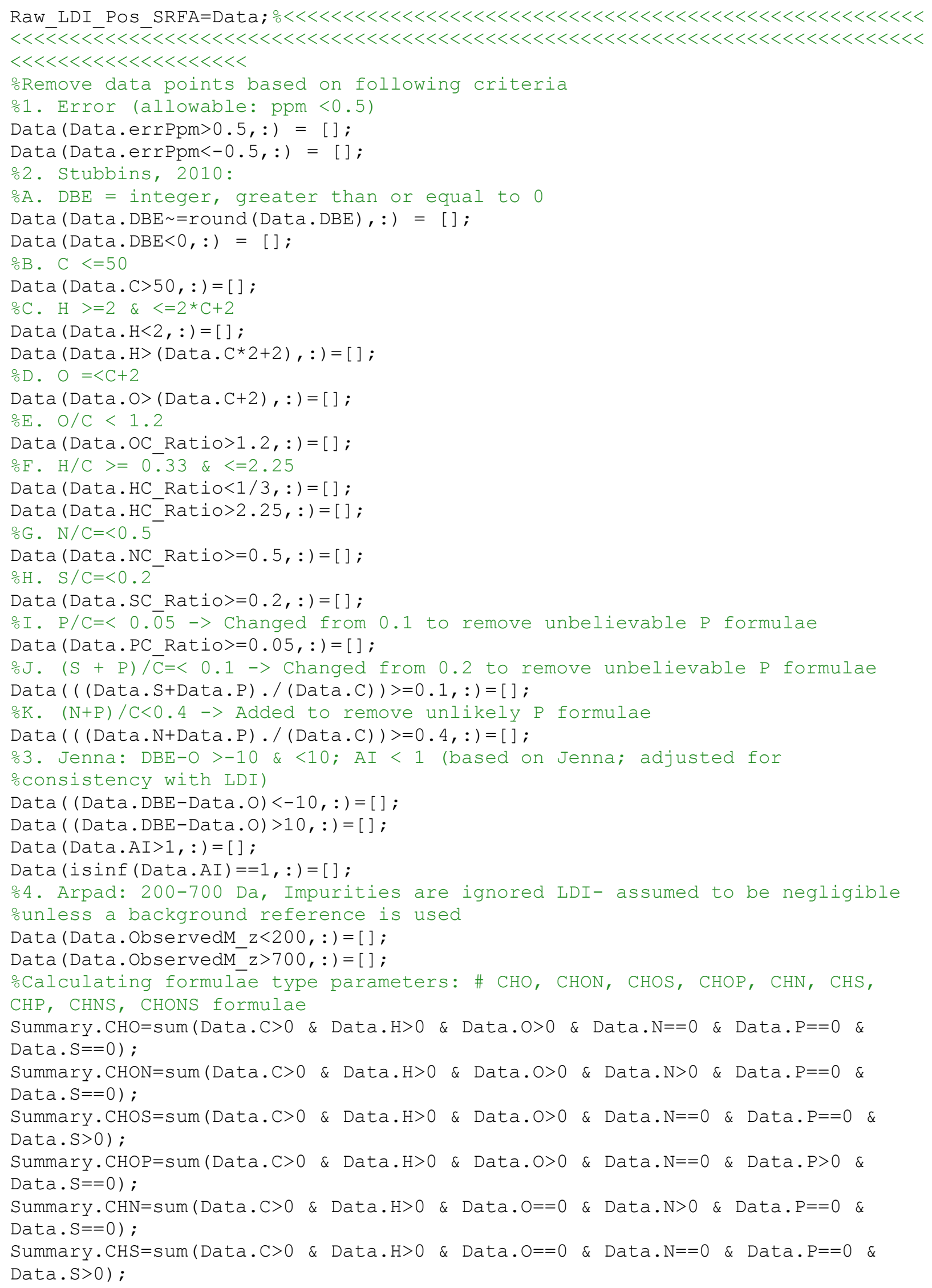




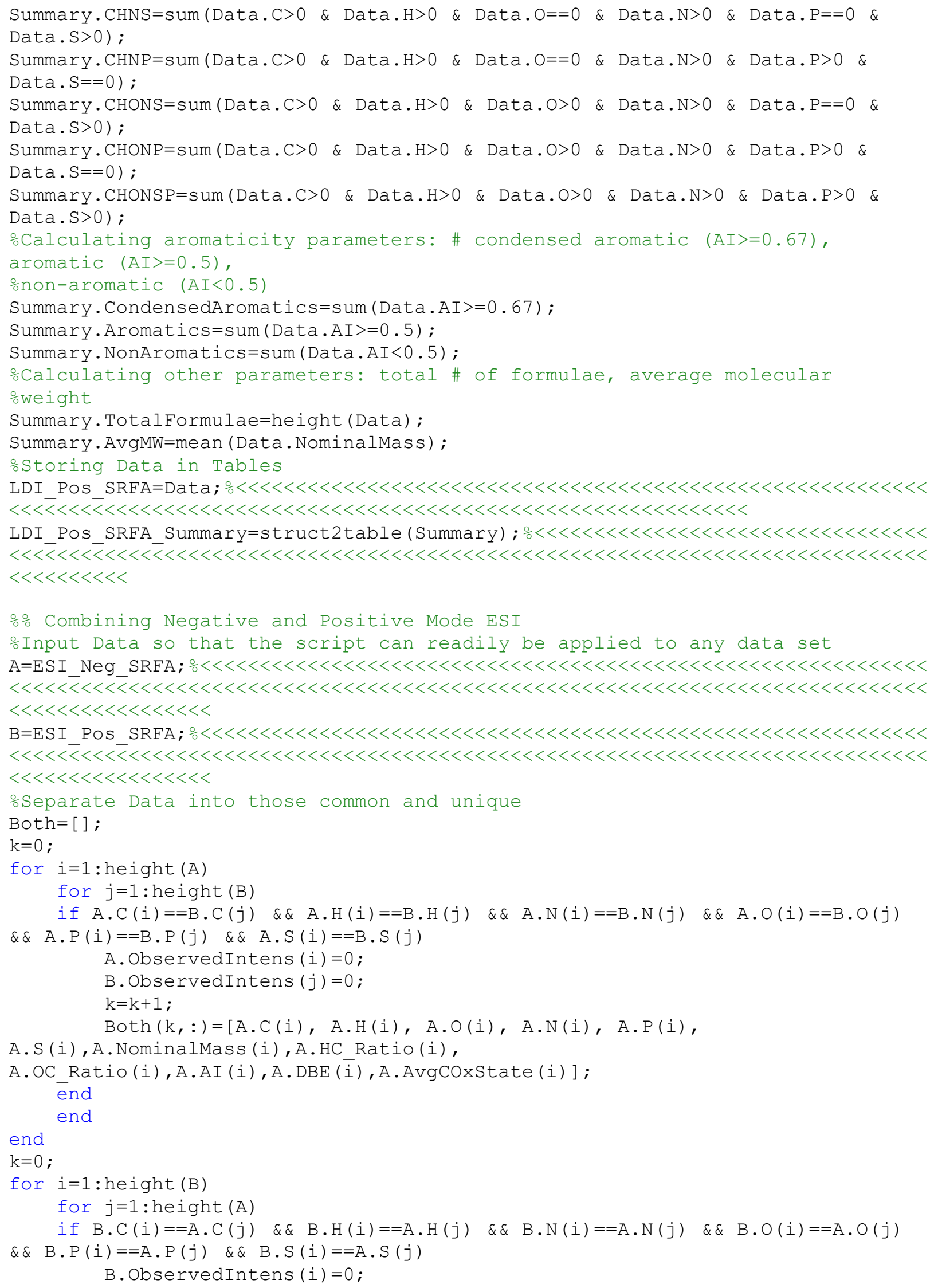


A. ObservedIntens $(j)=0$;

$\mathrm{k}=\mathrm{k}+1$;

Both $(k,:)=[B . C(i), B . H(i), B . O(i), B . N(i), B . P(i)$,

B.S (i), B.NominalMass (i), B.HC Ratio(i),

B.OC_Ratio(i),B.AI (i),B.DBE (i) , B.AvgCoxState (i) ] ;

end

end

end

oCombining Data Sets

$A(A$. Observedintens $==0,:)=[]$;

$A O n l_{Y}=[A . C, A . H, A . O, A . N, A . P, A . S$,

A. Nominalmass, A.HC Ratio,A.oC Ratio, A.AI, A.DBE, A.AvgCoxState];

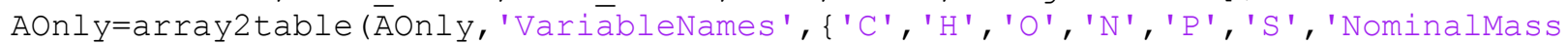
', 'HC_Ratio', 'OC_Ratio', 'AI', 'DBE', 'AvgCOxState'\}) ;

$\mathrm{B}(\mathrm{B} . \mathrm{Ob}$ servedinten $\mathrm{s}==0,:)=[]$;

$B O n l_{Y}=[B . C, B . H, B . O, B . N, B . P$,

B.S,B.NominalMass,B.HC_Ratio,B.OC_Ratio,B.AI, B.DBE, B.AvgCoxState] ;

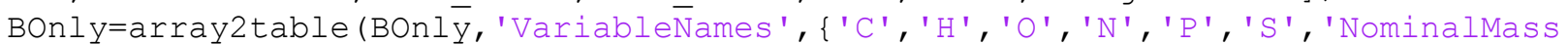
', 'HC_Ratio', 'OC_Ratio', 'AI', 'DBE', 'AvgCOxState'\}) ;

Both=array2table (Both, 'VariableNames', \{'C', 'H', 'O' , 'N', 'P' , 'S ', 'NominalMass', 'HC_Ratio', 'OC_Ratio', 'AI', 'DBE', 'AvgCOxState' \}) ;

Combined=[Both; AOnly; BOnly];

ostoring Data with distinct names

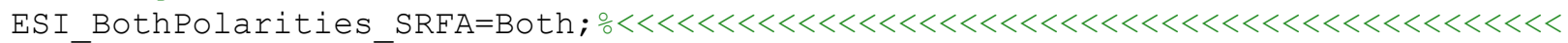
$<<\overline{<}<<<<<<<<<<<<<<\overline{<}<<<<<<<<<<<<<<<<<<<<<<<<<<<<<<<<<<<<<<<<<<<<<<<<<<<<<<<<<<~$ $<<<<<<<<<<<<<<<<<<<<<<<<<<<<<<<~$

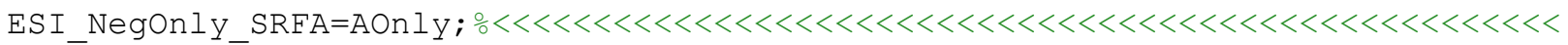
$<<\overline{<}<<<<<<<\bar{l}<<<<<<<<<<<<<<<<<<<<<<<<<<<<<<<<<<<<<<<<<<<<<<<<<<<<<<<<<<<<<<<<<$ $<<<<<<<<<<<<<<<<<<<<<<<<<~$

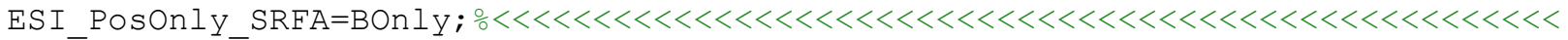

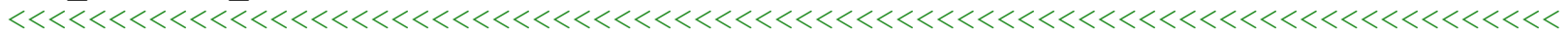
$<<<<<<<<<<<<<<<<<<<<<<<<<~$

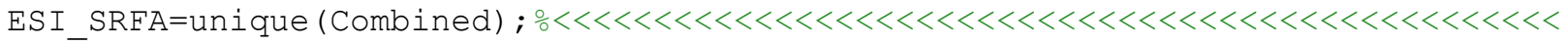

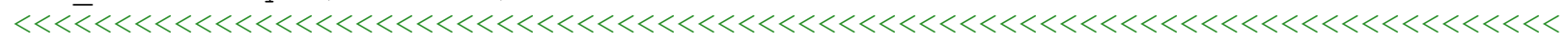
$<<<<<<<<<<<<<<<<<<<<<<<<<<<<~$

\% Summarizing Data

Data=AOnly;

oCalculating formulae type parameters: \# CHO, CHON, CHOS, CHOP, CHN, CHS, CHP, CHNS, CHONS formulae

Summary $\cdot \mathrm{CHO}=$ sum $($ Data. $\mathrm{C}>0$ \& Data. $\mathrm{H}>0$ \& Data. $\mathrm{O}>0$ \& Data. $\mathrm{N}==0$ \& Data. $\mathrm{P}==0$ \& Data. $\mathrm{S}==0$ );

Summary $\cdot \mathrm{CHON}=\operatorname{sum}($ Data. $\mathrm{C}>0$ \& Data.H $>0$ \& Data. $\mathrm{O}>0$ \& Data. $\mathrm{N}>0$ \& Data.P==0 \& Data. $\mathrm{S}==0$ );

Summary. $\mathrm{CHOS}=$ sum $($ Data. $\mathrm{C}>0$ \& Data.H $>0$ \& Data. $0>0$ \& Data. $\mathrm{N}==0$ \& Data. $\mathrm{P}==0$ \& Data. $\mathrm{S}>0$ );

Summary $\cdot \mathrm{CHOP}=\operatorname{sum}($ Data. $\mathrm{C}>0$ \& Data.H $>0$ \& Data. $\mathrm{O}>0$ \& Data. $\mathrm{N}==0$ \& Data.P $>0$ \& Data. $\mathrm{S}==0$ );

Summary. $\mathrm{CHN}=$ sum $($ Data. $\mathrm{C}>0$ \& Data. $\mathrm{H}>0$ \& Data. $\mathrm{O}==0$ \& Data. $\mathrm{N}>0$ \& Data.P==0 \& Data. $\mathrm{S}==0$ );

Summary. CHS $=$ sum $($ Data. $\mathrm{C}>0$ \& Data. $\mathrm{H}>0$ \& Data. $\mathrm{O}==0$ \& Data. $\mathrm{N}==0$ \& Data. $\mathrm{P}==0$ \&

Data. $\mathrm{S}>0$ );

Summary $\cdot \mathrm{CHNS}=\operatorname{sum}($ Data. $\mathrm{C}>0$ \& Data. $\mathrm{H}>0$ \& Data $\cdot \mathrm{O}==0$ \& Data $\cdot \mathrm{N}>0$ \& Data.P==0 \& Data. S>0);

Summary. $\mathrm{CHNP}=\operatorname{sum}($ Data. $\mathrm{C}>0$ \& Data. $\mathrm{H}>0$ \& Data. $\mathrm{O}==0$ \& Data. $\mathrm{N}>0$ \& Data.P $>0$ \&

Data. $\mathrm{S}=0$ ) ;

Summary. $\mathrm{CHONS}=\operatorname{sum}($ Data $. \mathrm{C}>0$ \& Data. $\mathrm{H}>0$ \& Data. $0>0$ \& Data. $>0$ \& Data.P==0 \&

Data. S>0); 
Summary. $\mathrm{CHONP}=$ sum $($ Data. $\mathrm{C}>0$ \& Data.H $>0$ \& Data. $0>0$ \& Data.N $>0$ \& Data.P $>0$ \&

Data. $\mathrm{S}==0$ );

Summary. $\mathrm{CHONSP}=$ sum $($ Data. $\mathrm{C}>0$ \& Data.H $>0$ \& Data.O $>0$ \& Data.N $>0$ \& Data.P>0 \& Data. S $>0$ );

ocalculating aromaticity parameters: \# condensed aromatic (AI>=0.67), aromatic $(A I>=0.5)$,

onon-aromatic $($ AI<0.5)

Summary. CondensedAromatics $=$ sum (Data. AI $>=0.67)$;

Summary. Aromatics=sum (Data.AI $>=0.5)$;

Summary. NonAromatics=sum (Data.AI<0.5);

ocalculating other parameters: total \# of formulae, average molecular 알ight

Summary. TotalFormulae=height (Data);

Summary. AvgMW=mean (Data. NominalMass);

ostoring Summary Data

ESI_NegOnly_SRFA_Summary=struct2table (Summary); $\circ<<<<<<<<<<<<<<<<<<<<<<<<<<<<<<<~$

$<<\overline{<}<<<<<<<\overline{<}<<<\overline{<}<<<<<<<<<<<<<<<<<<<<<<<<<<<<<<<<<<<<<<<<<<<<<<<<<<<<<<<<<<<<~$

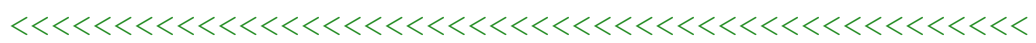

ㅇmmarizing Data

Data=BOnly;

oCalculating formulae type parameters: \# CHO, CHON, CHOS, CHOP, CHN, CHS, CHP, CHNS, CHONS formulae

Summary. $\mathrm{CHO}=\operatorname{sum}($ Data. $\mathrm{C}>0$ \& Data. $\mathrm{H}>0$ \& Data. $\mathrm{O}>0$ \& Data. $\mathrm{N}==0$ \& Data. $\mathrm{P}==0$ \&

Data. $\mathrm{S}==0$ );

Summary $\cdot \mathrm{CHON}=$ sum $($ Data. $\mathrm{C}>0$ \& Data.H $>0$ \& Data. $\mathrm{O}>0$ \& Data. $\mathrm{N}>0$ \& Data.P==0 \&

Data. $\mathrm{S}==0$ );

Summary $\cdot \mathrm{CHOS}=$ sum $($ Data. $\mathrm{C}>0$ \& Data. $\mathrm{H}>0$ \& Data. $\mathrm{O}>0$ \& Data. $\mathrm{N}==0$ \& Data. $\mathrm{P}==0$ \&

Data. $\mathrm{S}>0)$;

Summary. $\mathrm{CHOP}=\operatorname{sum}($ Data. $\mathrm{C}>0$ \& Data. $\mathrm{H}>0$ \& Data. $\mathrm{O}>0$ \& Data. $\mathrm{N}==0$ \& Data. $\mathrm{P}>0$ \&

Data. $\mathrm{S}==0$ );

Summary. $\mathrm{CHN}=$ sum $($ Data. $\mathrm{C}>0$ \& Data. $\mathrm{H}>0$ \& Data. $\mathrm{O}==0$ \& Data. $\mathrm{N}>0$ \& Data. $\mathrm{P}==0$ \&

Data. $\mathrm{S}==0$ );

Summary $\cdot \mathrm{CHS}=\operatorname{sum}($ Data $\cdot \mathrm{C}>0$ \& Data. $\mathrm{H}>0$ \& Data. $\mathrm{O}==0$ \& Data. $\mathrm{N}==0$ \& Data. $\mathrm{P}==0$ \&

Data. $\mathrm{S}>0$ );

Summary. $\mathrm{CHNS}=$ sum $($ Data. $\mathrm{C}>0$ \& Data. $\mathrm{H}>0$ \& Data. $\mathrm{O}==0$ \& Data. $\mathrm{N}>0$ \& Data. $\mathrm{P}==0$ \&

Data. S>0);

Summary $\cdot \mathrm{CHNP}=\operatorname{sum}($ Data. $\mathrm{C}>0$ \& Data. $\mathrm{H}>0$ \& Data. $\mathrm{O}==0$ \& Data. $\mathrm{N}>0$ \& Data.P $>0$ \&

Data. $\mathrm{S}==0$ ) ;

Summary. $\mathrm{CHONS}=\operatorname{sum}($ Data $. \mathrm{C}>0$ \& Data. $\mathrm{H}>0$ \& Data. $0>0$ \& Data. $\mathrm{N}>0$ \& Data. $\mathrm{P}==0$ \&

Data. $\mathrm{S}>0$ ) ;

Summary $\cdot \mathrm{CHONP}=$ sum $($ Data. $\mathrm{C}>0$ \& Data.H $>0$ \& Data. O $>0$ \& Data.N $>0$ \& Data.P $>0$ \&

Data. $\mathrm{S}==0$ ) ;

Summary. CHONSP $=$ sum $($ Data. $\mathrm{C}>0$ \& Data. $\mathrm{H}>0$ \& Data. $\mathrm{O}>0$ \& Data.N $>0$ \& Data.P $>0$ \&

Data. $\mathrm{S}>0)$;

oCalculating aromaticity parameters: \# condensed aromatic (AI>=0.67), aromatic $(\mathrm{AI}>=0.5)$,

onon-aromatic $($ AI $<0.5)$

Summary. CondensedAromatics $=$ sum (Data. AI $>=0.67)$;

Summary. Aromatics $=$ sum (Data.AI $>=0.5)$;

Summary. NonAromatics=sum (Data.AI<0.5);

ocalculating other parameters: total \# of formulae, average molecular

oweight

Summary. TotalFormulae=height (Data);

Summary. AvgMW=mean (Data. NominalMass);

ostoring Summary Data 


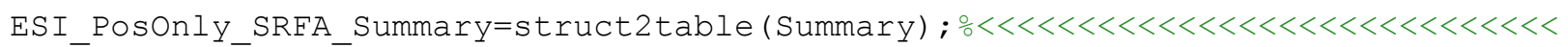
$<<\overline{<}<<<<<<<\overline{<}<<<\overline{<}<<<<<<<<<<<<<<<<<<<<<<<<<<<<<<<<<<<<<<<<<<<<<<<<<<<<<<<<<<<<<$

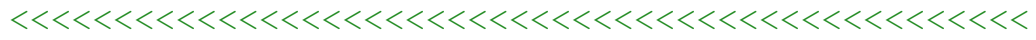

ㅇumarizing Data

Data=Both;

oCalculating formulae type parameters: \# CHO, CHON, CHOS, CHOP, CHN, CHS, CHP, CHNS, CHONS formulae

Summary $\cdot \mathrm{CHO}=$ sum $($ Data. $\mathrm{C}>0$ \& Data. $\mathrm{H}>0$ \& Data. $\mathrm{O}>0$ \& Data. $\mathrm{N}==0$ \& Data. $\mathrm{P}==0$ \&

Data. $\mathrm{S}==0$ );

Summary $\cdot \mathrm{CHON}=$ sum $($ Data. $\mathrm{C}>0$ \& Data.H $>0$ \& Data. $\mathrm{O}>0$ \& Data. $\mathrm{N}>0$ \& Data.P==0 \&

Data. $\mathrm{S}==0$ );

Summary. $\mathrm{CHOS}=$ sum $($ Data. $\mathrm{C}>0$ \& Data. $\mathrm{H}>0$ \& Data. $\mathrm{O}>0$ \& Data. $\mathrm{N}==0$ \& Data. $\mathrm{P}==0$ \&

Data. $\mathrm{S}>0$ );

Summary. $\mathrm{CHOP}=\mathrm{sum}($ Data. $\mathrm{C}>0$ \& Data.H $>0$ \& Data. $0>0$ \& Data. $\mathrm{N}==0$ \& Data.P $>0$ \&

Data. $\mathrm{S}==0$ );

Summary $\cdot \mathrm{CHN}=$ sum $($ Data. $\mathrm{C}>0$ \& Data. $\mathrm{H}>0$ \& Data. $\mathrm{O}==0$ \& Data. $\mathrm{N}>0$ \& Data. $\mathrm{P}==0$ \&

Data. $\mathrm{S}=0$ ) ;

Summary $\cdot \mathrm{CHS}=\operatorname{sum}($ Data. $\mathrm{C}>0$ \& Data. $\mathrm{H}>0$ \& Data. $\mathrm{O}==0$ \& Data. $\mathrm{N}==0$ \& Data. $\mathrm{P}==0$ \&

Data. $\mathrm{S}>0$ ) ;

Summary $\cdot \mathrm{CHNS}=\operatorname{sum}($ Data. $\mathrm{C}>0$ \& Data. $\mathrm{H}>0$ \& Data. $\mathrm{O}==0$ \& Data. $\mathrm{N}>0$ \& Data.P==0 \&

Data. S $>0$ );

Summary $\cdot \mathrm{CHNP}=\operatorname{sum}($ Data. $\mathrm{C}>0$ \& Data.H $>0$ \& Data. $\mathrm{O}==0$ \& Data. $\mathrm{N}>0$ \& Data.P $>0$ \&

Data. $\mathrm{S}==0$ );

Summary. $\mathrm{CHONS}=\operatorname{sum}($ Data. $\mathrm{C}>0$ \& Data. $\mathrm{H}>0$ \& Data. $0>0$ \& Data.N>0 \& Data.P==0 \&

Data. S>0);

Summary. $\mathrm{CHONP}=\operatorname{sum}($ Data. $\mathrm{C}>0$ \& Data.H $>0$ \& Data. O $>0$ \& Data.N $>0$ \& Data.P $>0$ \&

Data. $\mathrm{S}==0$ );

Summary. $\mathrm{CHONSP}=$ sum $($ Data. $\mathrm{C}>0$ \& Data.H $>0$ \& Data.O $>0$ \& Data.N $>0$ \& Data.P>0 \&

Data. $\mathrm{S}>0)$;

ocalculating aromaticity parameters: \# condensed aromatic (AI>=0.67),

aromatic $(\mathrm{AI}>=0.5)$,

onon-aromatic $(\mathrm{A} I<0.5)$

Summary. CondensedAromatics $=$ sum (Data. AI $>=0.67)$;

Summary. Aromatics $=$ sum (Data.AI $>=0.5)$;

Summary. NonAromatics=sum (Data.AI<0.5);

ocalculating other parameters: total \# of formulae, average molecular

oweight

Summary. TotalFormulae=height (Data);

Summary. AvgMW=mean (Data. NominalMass);

ostoring Summary Data

ESI_BothPolarities_SRFA_Summary=struct2table (Summary) ; $\circ<<<<<<<<<<<<<<<<<<<<<<~$

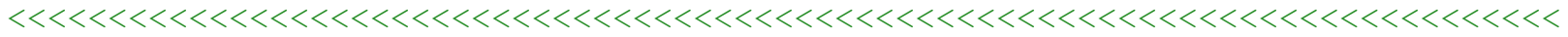

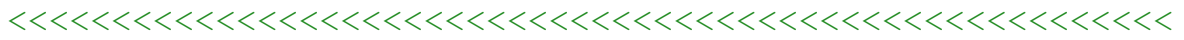

․ Summarizing Data

Data $=$ Combined;

oCalculating formulae type parameters: \# CHO, CHON, CHOS, CHOP, CHN, CHS, CHP, CHNS, CHONS formulae

Summary. $\mathrm{CHO}=\operatorname{sum}($ Data. $\mathrm{C}>0$ \& Data. $\mathrm{H}>0$ \& Data. $\mathrm{O}>0$ \& Data. $\mathrm{N}==0$ \& Data. $\mathrm{P}==0$ \& Data. $\mathrm{S}=0$ ) ;

Summary. $\mathrm{CHON}=\operatorname{sum}($ Data. $\mathrm{C}>0$ \& Data. $\mathrm{H}>0$ \& Data. $\mathrm{O}>0$ \& Data.N $>0$ \& Data.P==0 \&

Data. $\mathrm{S}==0$ ) ;

Summary $\cdot \mathrm{CHOS}=\operatorname{sum}($ Data. $\mathrm{C}>0$ \& Data. $\mathrm{H}>0$ \& Data. $\mathrm{O}>0$ \& Data. $\mathrm{N}==0$ \& Data. $\mathrm{P}==0$ \&

Data. $\mathrm{S}>0$ ) ;

Summary $\cdot \mathrm{CHOP}=\operatorname{sum}($ Data. $\mathrm{C}>0$ \& Data.H $>0$ \& Data. $\mathrm{O}>0$ \& Data. $\mathrm{N}==0$ \& Data.P $>0$ \&

Data. $\mathrm{S}=0$ ) ;

Summary. $\mathrm{CHN}=\operatorname{sum}($ Data. $\mathrm{C}>0$ \& Data. $\mathrm{H}>0$ \& Data. $\mathrm{O}==0$ \& Data. $\mathrm{N}>0$ \& Data. $\mathrm{P}==0$ \&

Data. $\mathrm{S}==0$ ); 


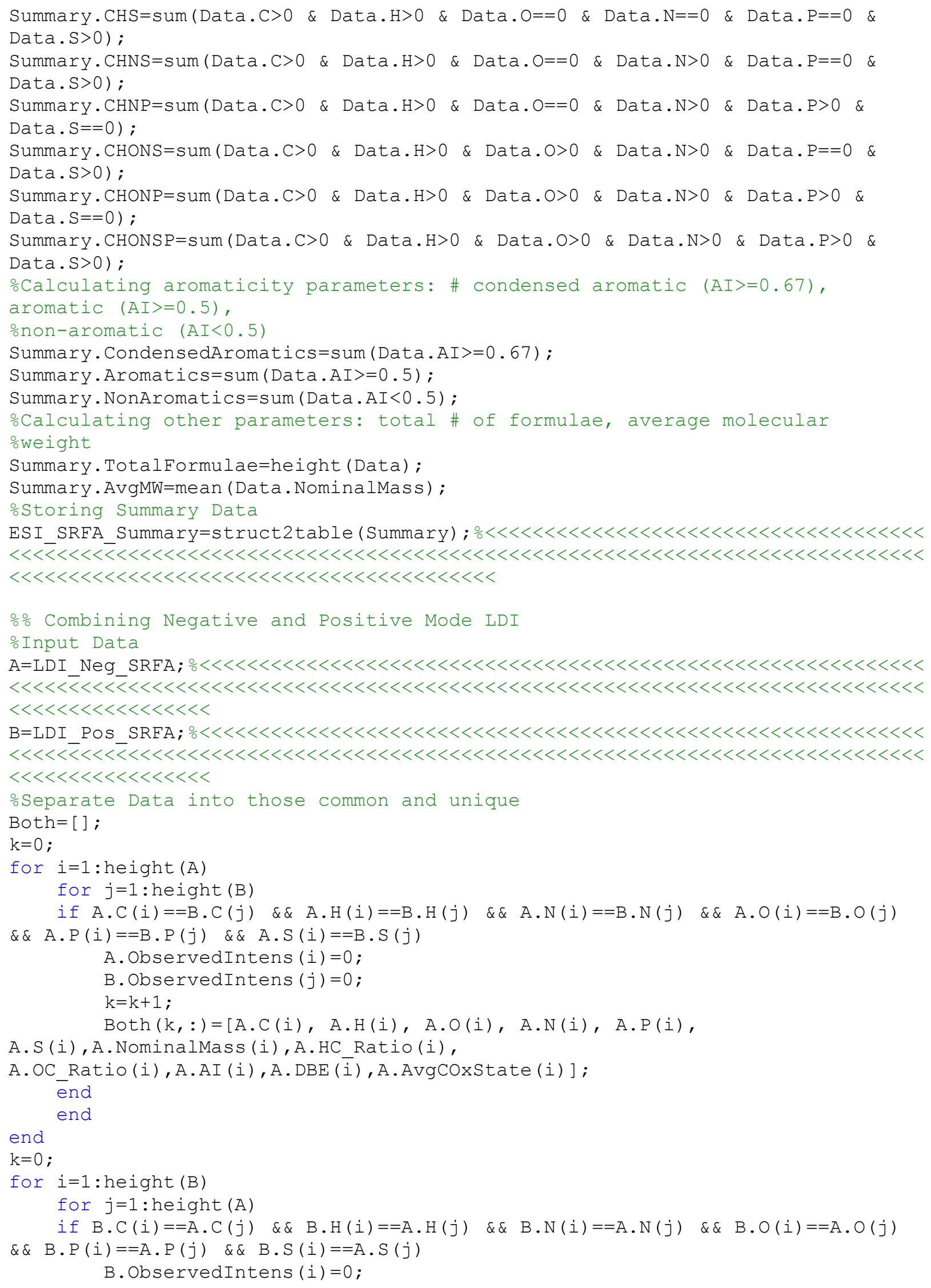


A. ObservedIntens $(j)=0$;

$\mathrm{k}=\mathrm{k}+1$;

Both $(k,:)=[B . C(i), B . H(i), B . O(i), B . N(i), B . P(i)$,

B.S(i),B.NominalMass (i), B.HC_Ratio(i),

B.OC_Ratio(i),B.AI (i),B.DBE (i) , B.AvgCoxState (i) ] ;

end

end

end

oCombining Data Sets

$A(A$. Observedintens $==0,:)=[]$;

$A O n l_{Y}=[A . C, A . H, A . O, A . N, A . P, A . S$,

A. Nominalmass, A.HC Ratio,A.oC Ratio, A.AI, A.DBE, A.AvgCoxState];

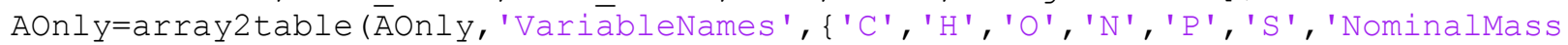
', 'HC_Ratio', 'OC_Ratio', 'AI', 'DBE', 'AvgCOxState'\}) ;

$\mathrm{B}(\mathrm{B} . \mathrm{Ob}$ servedinten $\mathrm{s}==0,:)=[]$;

$B O n l_{Y}=[B . C, B . H, B . O, B . N, B . P$,

B.S,B.NominalMass, B.HC_Ratio,B.oC_Ratio,B.AI,B.DBE,B.AvgCOxState] ;

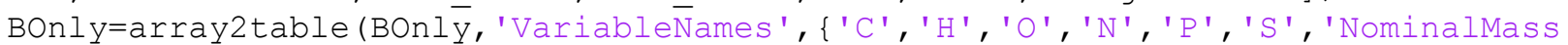
', 'HC_Ratio', 'OC_Ratio', 'AI', 'DBE', 'AvgCOxState'\}) ;

Both=array2table (Both, 'VariableNames', \{'C', 'H', 'O' , 'N', 'P' , 'S ', 'NominalMass', 'HC_Ratio', 'OC_Ratio', 'AI', 'DBE', 'AvgCOxState' \}) ;

Combined=[Both; AOnly; BOnly];

ostoring Data with distinct names

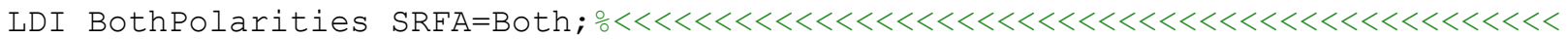
$<<\overline{<}<<<<<<<<<<<<<<\overline{<}<<<<<<<<<<<<<<<<<<<<<<<<<<<<<<<<<<<<<<<<<<<<<<<<<<<<<<<<<<~$ $<<<<<<<<<<<<<<<<<<<<<<<<<<<<<<<~$

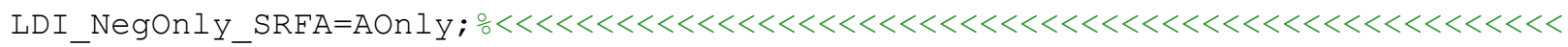
$<<\overline{<}<<<<<<<\overline{<}<<<<<<<<<<<<<<<<<<<<<<<<<<<<<<<<<<<<<<<<<<<<<<<<<<<<<<<<<<<<<<<<<~$ $<<<<<<<<<<<<<<<<<<<<<<<<<~$

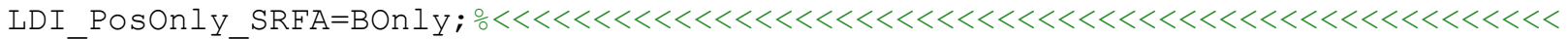

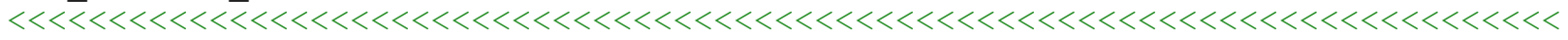
$<<<<<<<<<<<<<<<<<<<<<<<<<~$

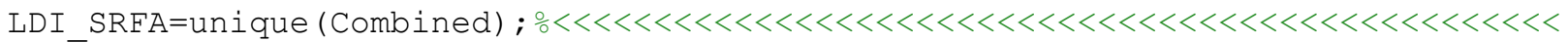

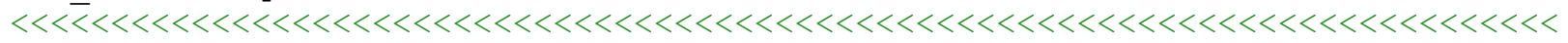
$<<<<<<<<<<<<<<<<<<<<<<<<<<<<~$

\% Summarizing Data

Data=AOnly;

oCalculating formulae type parameters: \# CHO, CHON, CHOS, CHOP, CHN, CHS, CHP, CHNS, CHONS formulae

Summary $\cdot \mathrm{CHO}=$ sum $($ Data. $\mathrm{C}>0$ \& Data. $\mathrm{H}>0$ \& Data. $\mathrm{O}>0$ \& Data. $\mathrm{N}==0$ \& Data. $\mathrm{P}==0$ \& Data. $\mathrm{S}==0$ );

Summary $\cdot \mathrm{CHON}=\operatorname{sum}($ Data. $\mathrm{C}>0$ \& Data.H $>0$ \& Data. $\mathrm{O}>0$ \& Data. $\mathrm{N}>0$ \& Data.P==0 \& Data. $\mathrm{S}==0$ );

Summary. $\mathrm{CHOS}=$ sum $($ Data. $\mathrm{C}>0$ \& Data.H $>0$ \& Data. $0>0$ \& Data. $\mathrm{N}==0$ \& Data. $\mathrm{P}==0$ \& Data. $\mathrm{S}>0$ );

Summary $\cdot \mathrm{CHOP}=\mathrm{sum}($ Data. $\mathrm{C}>0$ \& Data.H $>0$ \& Data. $\mathrm{O}>0$ \& Data. $\mathrm{N}==0$ \& Data.P $>0$ \& Data. $\mathrm{S}==0$ );

Summary. $\mathrm{CHN}=$ sum $($ Data. $\mathrm{C}>0$ \& Data. $\mathrm{H}>0$ \& Data. $\mathrm{O}==0$ \& Data. $\mathrm{N}>0$ \& Data.P==0 \& Data. $\mathrm{S}==0$ );

Summary $\cdot \mathrm{CHS}=\operatorname{sum}($ Data $\cdot \mathrm{C}>0$ \& Data. $\mathrm{H}>0$ \& Data. $\mathrm{O}==0$ \& Data. $\mathrm{N}==0$ \& Data. $\mathrm{P}==0$ \&

Data. $\mathrm{S}>0$ );

Summary $\cdot \mathrm{CHNS}=\operatorname{sum}($ Data. $\mathrm{C}>0$ \& Data. $\mathrm{H}>0$ \& Data $\cdot \mathrm{O}==0$ \& Data $\cdot \mathrm{N}>0$ \& Data.P==0 \& Data. $\mathrm{S}>0$ ) ;

Summary $\cdot \mathrm{CHNP}=\operatorname{sum}($ Data. $\mathrm{C}>0$ \& Data.H $>0$ \& Data. $\mathrm{O}==0$ \& Data.N $>0$ \& Data.P $>0$ \&

Data. $\mathrm{S}=0$ ) ;

Summary. $\mathrm{CHONS}=\operatorname{sum}($ Data $. \mathrm{C}>0$ \& Data. $\mathrm{H}>0$ \& Data. $0>0$ \& Data. $>0$ \& Data.P==0 \&

Data. S>0); 
Summary. $\mathrm{CHONP}=$ sum $($ Data. $\mathrm{C}>0$ \& Data.H $>0$ \& Data. $0>0$ \& Data.N $>0$ \& Data.P $>0$ \&

Data. $\mathrm{S}==0$ );

Summary. $\mathrm{CHONSP}=$ sum $($ Data. $\mathrm{C}>0$ \& Data.H $>0$ \& Data.O $>0$ \& Data.N $>0$ \& Data.P>0 \& Data. S $>0$ );

ocalculating aromaticity parameters: \# condensed aromatic (AI>=0.67), aromatic $(A I>=0.5)$,

onon-aromatic $($ A I $<0.5)$

Summary. CondensedAromatics $=$ sum (Data. AI $>=0.67)$;

Summary. Aromatics=sum (Data.AI $>=0.5)$;

Summary. NonAromatics=sum (Data.AI<0.5);

ocalculating other parameters: total \# of formulae, average molecular 알ight

Summary. TotalFormulae=height (Data);

Summary. AvgMW=mean (Data. NominalMass);

ostoring Summary Data

LDI_NegOnly_SRFA_Summary=struct2table (Summary); $\circ<<<<<<<<<<<<<<<<<<<<<<<<<<<<<<<~$

$<<\overline{<}<<<<<<<\overline{<}<<<\overline{<}<<<<<<<<<<<<<<<<<<<<<<<<<<<<<<<<<<<<<<<<<<<<<<<<<<<<<<<<<<<<~$ $<<<<<<<<<<<<<<<<<<<<<<<<<<<<<<<<<<<<<<<<<<<<<<<<<~$

ㅇmmarizing Data

Data=BOnly;

oCalculating formulae type parameters: \# CHO, CHON, CHOS, CHOP, CHN, CHS, CHP, CHNS, CHONS formulae

Summary. $\mathrm{CHO}=\operatorname{sum}($ Data. $\mathrm{C}>0$ \& Data. $\mathrm{H}>0$ \& Data. $\mathrm{O}>0$ \& Data. $\mathrm{N}==0$ \& Data. $\mathrm{P}==0$ \&

Data. $\mathrm{S}==0$ );

Summary $\cdot \mathrm{CHON}=$ sum $($ Data. $\mathrm{C}>0$ \& Data.H $>0$ \& Data. $\mathrm{O}>0$ \& Data. $\mathrm{N}>0$ \& Data.P==0 \&

Data. $\mathrm{S}==0$ );

Summary $\cdot \mathrm{CHOS}=$ sum $($ Data. $\mathrm{C}>0$ \& Data. $\mathrm{H}>0$ \& Data. $\mathrm{O}>0$ \& Data. $\mathrm{N}==0$ \& Data. $\mathrm{P}==0$ \&

Data. $\mathrm{S}>0)$;

Summary. $\mathrm{CHOP}=\operatorname{sum}($ Data. $\mathrm{C}>0$ \& Data. $\mathrm{H}>0$ \& Data. $\mathrm{O}>0$ \& Data. $\mathrm{N}==0$ \& Data. $\mathrm{P}>0$ \&

Data. $\mathrm{S}==0$ );

Summary. $\mathrm{CHN}=$ sum $($ Data. $\mathrm{C}>0$ \& Data. $\mathrm{H}>0$ \& Data. $\mathrm{O}==0$ \& Data. $\mathrm{N}>0$ \& Data. $\mathrm{P}==0$ \&

Data. $\mathrm{S}==0$ );

Summary $\cdot \mathrm{CHS}=\operatorname{sum}($ Data $\cdot \mathrm{C}>0$ \& Data. $\mathrm{H}>0$ \& Data. $\mathrm{O}==0$ \& Data. $\mathrm{N}==0$ \& Data. $\mathrm{P}==0$ \&

Data. $\mathrm{S}>0$ );

Summary. $\mathrm{CHNS}=$ sum $($ Data. $\mathrm{C}>0$ \& Data. $\mathrm{H}>0$ \& Data. $\mathrm{O}==0$ \& Data. $\mathrm{N}>0$ \& Data. $\mathrm{P}==0$ \&

Data. S>0);

Summary $\cdot \mathrm{CHNP}=\operatorname{sum}($ Data. $\mathrm{C}>0$ \& Data. $\mathrm{H}>0$ \& Data. $\mathrm{O}==0$ \& Data. $\mathrm{N}>0$ \& Data.P $>0$ \&

Data. $\mathrm{S}==0$ ) ;

Summary. $\mathrm{CHONS}=\operatorname{sum}($ Data $. \mathrm{C}>0$ \& Data. $\mathrm{H}>0$ \& Data. $0>0$ \& Data. $\mathrm{N}>0$ \& Data. $\mathrm{P}==0$ \&

Data. $\mathrm{S}>0$ ) ;

Summary $\cdot \mathrm{CHONP}=$ sum $($ Data. $\mathrm{C}>0$ \& Data.H $>0$ \& Data. O $>0$ \& Data.N $>0$ \& Data.P $>0$ \&

Data. $\mathrm{S}==0$ ) ;

Summary. CHONSP $=$ sum $($ Data. $\mathrm{C}>0$ \& Data. $\mathrm{H}>0$ \& Data. $\mathrm{O}>0$ \& Data.N $>0$ \& Data.P $>0$ \&

Data. $\mathrm{S}>0)$;

oCalculating aromaticity parameters: \# condensed aromatic (AI>=0.67), aromatic $(\mathrm{AI}>=0.5)$,

onon-aromatic $($ AI $<0.5)$

Summary. CondensedAromatics $=$ sum (Data. AI $>=0.67)$;

Summary. Aromatics $=$ sum (Data.AI $>=0.5)$;

Summary. NonAromatics=sum (Data.AI<0.5);

ocalculating other parameters: total \# of formulae, average molecular

oweight

Summary. TotalFormulae=height (Data);

Summary. AvgMW=mean (Data. NominalMass);

ostoring Summary Data 
LDI_PosOnly_SRFA_Summary=struct2table (Summary) ; $\circ<<<<<<<<<<<<<<<<<<<<<<<<<<<<<<<<<~$ $<<\overline{<}<<<<<<<\overline{<}<<<\overline{<}<<<<<<<<<<<<<<<<<<<<<<<<<<<<<<<<<<<<<<<<<<<<<<<<<<<<<<<<<<<<~$

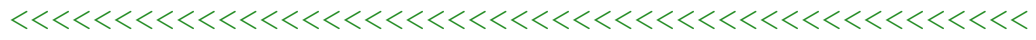

ㅇumarizing Data

Data=Both;

oCalculating formulae type parameters: \# CHO, CHON, CHOS, CHOP, CHN, CHS, CHP, CHNS, CHONS formulae

Summary $\cdot \mathrm{CHO}=$ sum $($ Data. $\mathrm{C}>0$ \& Data. $\mathrm{H}>0$ \& Data. $\mathrm{O}>0$ \& Data. $\mathrm{N}==0$ \& Data. $\mathrm{P}==0$ \&

Data. $\mathrm{S}==0$ );

Summary $\cdot \mathrm{CHON}=$ sum $($ Data. $\mathrm{C}>0$ \& Data.H $>0$ \& Data. $\mathrm{O}>0$ \& Data. $\mathrm{N}>0$ \& Data.P==0 \&

Data. $\mathrm{S}==0$ );

Summary. $\mathrm{CHOS}=$ sum $($ Data. $\mathrm{C}>0$ \& Data. $\mathrm{H}>0$ \& Data. $\mathrm{O}>0$ \& Data. $\mathrm{N}==0$ \& Data. $\mathrm{P}==0$ \&

Data. $\mathrm{S}>0$ );

Summary. $\mathrm{CHOP}=\mathrm{sum}($ Data. $\mathrm{C}>0$ \& Data.H $>0$ \& Data. $0>0$ \& Data. $\mathrm{N}==0$ \& Data.P $>0$ \&

Data. $\mathrm{S}==0$ );

Summary $\cdot \mathrm{CHN}=$ sum $($ Data. $\mathrm{C}>0$ \& Data. $\mathrm{H}>0$ \& Data. $\mathrm{O}==0$ \& Data. $\mathrm{N}>0$ \& Data. $\mathrm{P}==0$ \&

Data. $\mathrm{S}=0$ ) ;

Summary $\cdot \mathrm{CHS}=\operatorname{sum}($ Data. $\mathrm{C}>0$ \& Data. $\mathrm{H}>0$ \& Data. $\mathrm{O}==0$ \& Data. $\mathrm{N}==0$ \& Data. $\mathrm{P}==0$ \&

Data. $\mathrm{S}>0$ ) ;

Summary $\cdot \mathrm{CHNS}=\operatorname{sum}($ Data. $\mathrm{C}>0$ \& Data. $\mathrm{H}>0$ \& Data. $\mathrm{O}==0$ \& Data. $\mathrm{N}>0$ \& Data.P==0 \&

Data. S $>0$ );

Summary. $\mathrm{CHNP}=\operatorname{sum}($ Data. $\mathrm{C}>0$ \& Data.H $>0$ \& Data. $\mathrm{O}==0$ \& Data. $\mathrm{N}>0$ \& Data.P $>0$ \&

Data. $\mathrm{S}==0$ );

Summary. $\mathrm{CHONS}=\operatorname{sum}($ Data. $\mathrm{C}>0$ \& Data. $\mathrm{H}>0$ \& Data. $0>0$ \& Data.N>0 \& Data.P==0 \&

Data. S>0);

Summary. $\mathrm{CHONP}=\operatorname{sum}($ Data. $\mathrm{C}>0$ \& Data.H $>0$ \& Data. $\mathrm{O}>0$ \& Data.N $>0$ \& Data.P $>0$ \&

Data. $\mathrm{S}==0$ );

Summary. $\mathrm{CHONSP}=$ sum $($ Data. $\mathrm{C}>0$ \& Data.H $>0$ \& Data.O $>0$ \& Data.N $>0$ \& Data.P>0 \&

Data. $\mathrm{S}>0)$;

ocalculating aromaticity parameters: \# condensed aromatic (AI>=0.67),

aromatic $(\mathrm{AI}>=0.5)$,

onon-aromatic $(\mathrm{A} I<0.5)$

Summary. CondensedAromatics $=$ sum (Data. AI $>=0.67)$;

Summary. Aromatics $=$ sum (Data.AI $>=0.5)$;

Summary. NonAromatics=sum (Data.AI<0.5);

ocalculating other parameters: total \# of formulae, average molecular

oweight

Summary.TotalFormulae=height (Data);

Summary. AvgMW=mean (Data. NominalMass);

ostoring Summary Data

LDI_BothPolarities_SRFA_Summary=struct2table (Summary) ; $\circ<<<<<<<<<<<<<<<<<<<<<<$

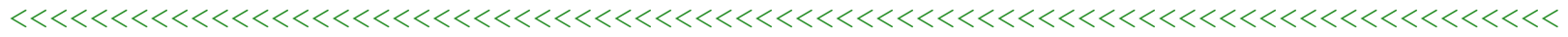

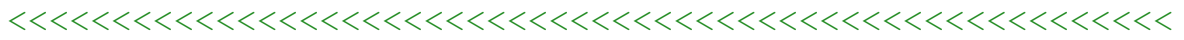

․ Summarizing Data

Data=Combined;

oCalculating formulae type parameters: \# CHO, CHON, CHOS, CHOP, CHN, CHS, CHP, CHNS, CHONS formulae

Summary. $\mathrm{CHO}=\operatorname{sum}($ Data. $\mathrm{C}>0$ \& Data. $\mathrm{H}>0$ \& Data. $\mathrm{O}>0$ \& Data. $\mathrm{N}==0$ \& Data. $\mathrm{P}==0$ \& Data. $\mathrm{S}=0$ ) ;

Summary. $\mathrm{CHON}=\operatorname{sum}($ Data. $\mathrm{C}>0$ \& Data. $\mathrm{H}>0$ \& Data. $\mathrm{O}>0$ \& Data.N $>0$ \& Data.P==0 \&

Data. $\mathrm{S}==0$ );

Summary $\cdot \mathrm{CHOS}=$ sum $($ Data. $\mathrm{C}>0$ \& Data.H $>0$ \& Data. $\mathrm{O}>0$ \& Data. $\mathrm{N}==0$ \& Data.P==0 \&

Data. $\mathrm{S}>0$ ) ;

Summary $\cdot \mathrm{CHOP}=\mathrm{sum}($ Data. $\mathrm{C}>0$ \& Data. $\mathrm{H}>0$ \& Data.O $>0$ \& Data. $\mathrm{N}==0$ \& Data.P $>0$ \&

Data. $\mathrm{S}=0$ ) ;

Summary. $\mathrm{CHN}=\operatorname{sum}($ Data. $\mathrm{C}>0$ \& Data.H $>0$ \& Data. $\mathrm{O}==0$ \& Data. $\mathrm{N}>0$ \& Data.P==0 \&

Data. $\mathrm{S}==0$ ); 


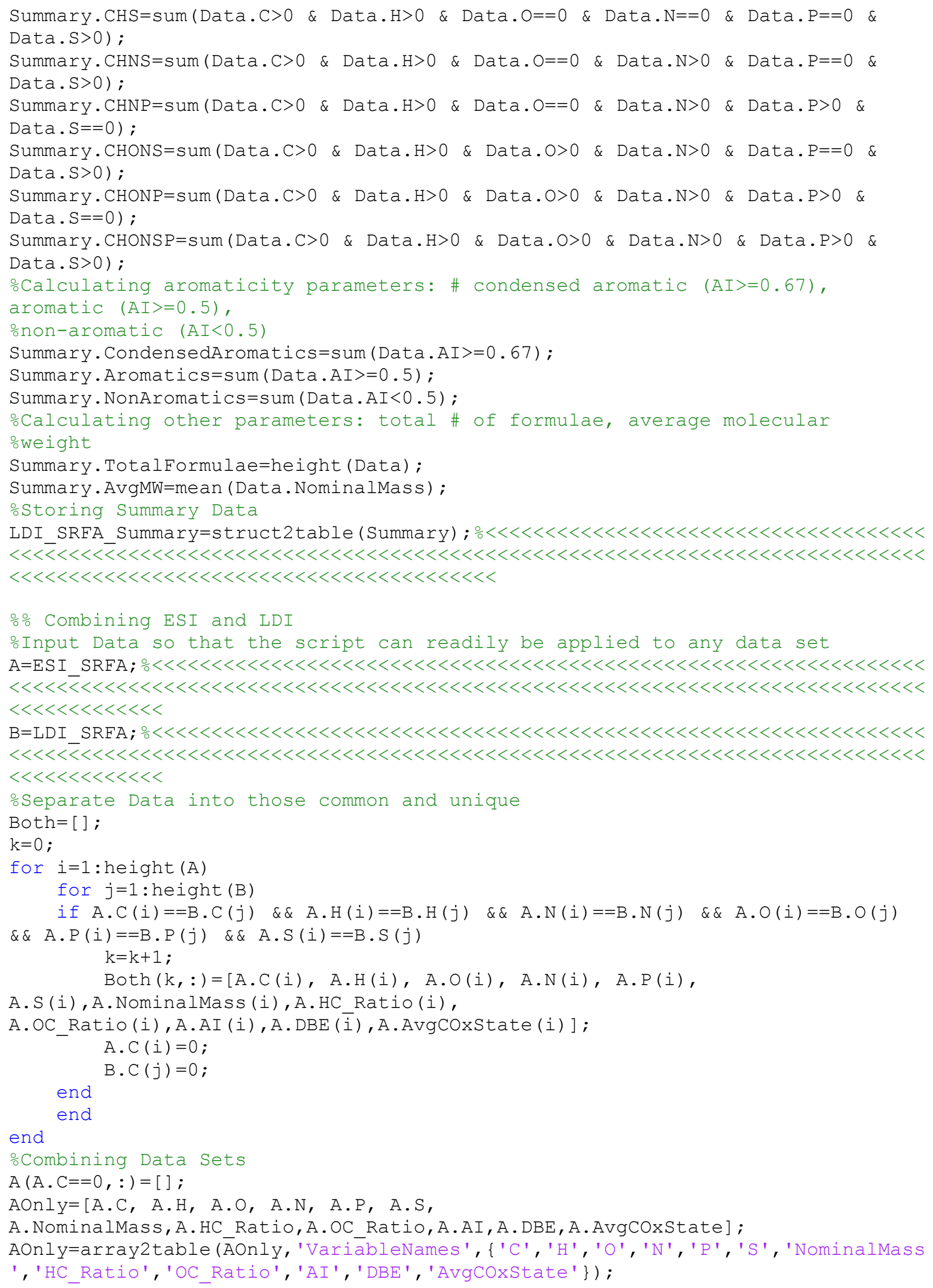


$\mathrm{B}(\mathrm{B} . \mathrm{C}==0,:)=[]$;

$\mathrm{BOnl} Y=[\mathrm{B} . \mathrm{C}, \mathrm{B} . \mathrm{H}, \mathrm{B} . \mathrm{O}, \mathrm{B} . \mathrm{N}, \mathrm{B} . \mathrm{P}$,

B.S,B.NominalMass,B.HC_Ratio,B.OC_Ratio, B.AI, B.DBE, B.AvgCoxState];

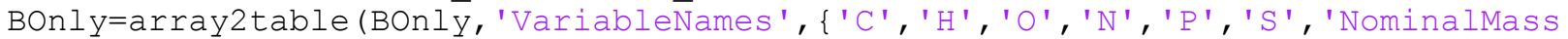

', 'HC_Ratio', 'OC_Ratio', 'AI ', 'DBE', 'AvgCoxstate' \}) ;

Both=äray2table (Both, 'VariableNames' , \{'C', 'H', 'O', 'N', 'P' , 'S ', 'NominalMass',

'HC_Ratio', 'oC_Ratio', 'AI', 'DBE', 'AvgCOxState'\});

Combined=[Both; AOnly; BOnly];

ostoring Data with distinct names

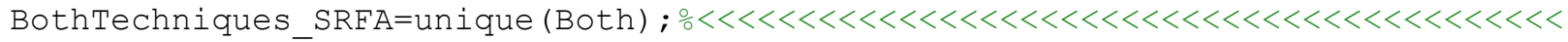

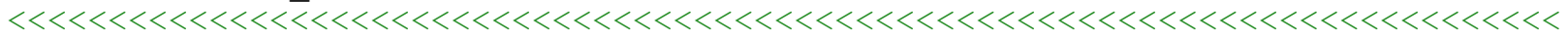

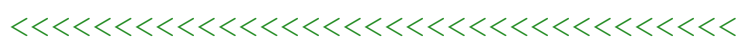

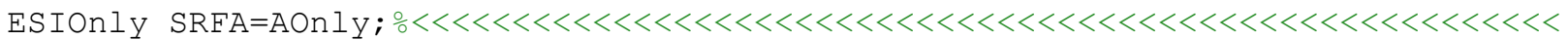

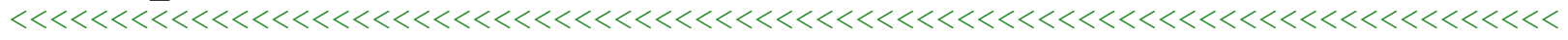
$<<<<<<<<<<<<<<<<<<<<<$

LDIOnIY_SRFA=BOnIY; $\circ \circ<<<<<<<<<<<<<<<<<<<<<<<<<<<<<<<<<<<<<<<<<<<<<<<<<<<<<<<<<~$

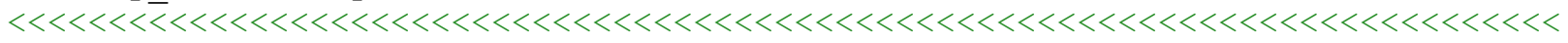
$<<<<<<<<<<<<<<<<<<<<<~$

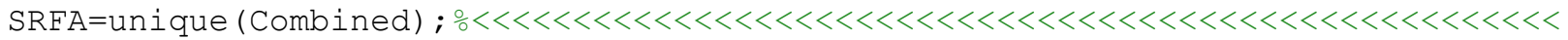

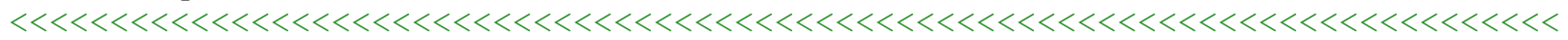
$<<<<<<<<<<<<<<<<<<<<<<<~$

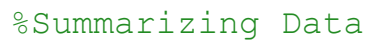

Data=AOnly;

oCalculating formulae type parameters: \# CHO, CHON, CHOS, CHOP, CHN, CHS, CHP, CHNS, CHONS formulae

Summary $\cdot \mathrm{CHO}=\operatorname{sum}($ Data. $\mathrm{C}>0$ \& Data. $\mathrm{H}>0$ \& Data.O $>0$ \& Data. $\mathrm{N}==0$ \& Data.P==0 \& Data. $\mathrm{S}==0$ );

Summary. $\mathrm{CHON}=$ sum $($ Data. $\mathrm{C}>0$ \& Data. $\mathrm{H}>0$ \& Data. O $>0$ \& Data.N $>0$ \& Data.P==0 \&

Data. $\mathrm{S}==0$ );

Summary. $\mathrm{CHOS}=$ sum $($ Data. $\mathrm{C}>0$ \& Data. $\mathrm{H}>0$ \& Data. $\mathrm{O}>0$ \& Data. $\mathrm{N}==0$ \& Data. $\mathrm{P}==0$ \& Data. $\mathrm{S}>0$ );

Summary. $\mathrm{CHOP}=\operatorname{sum}($ Data. $\mathrm{C}>0$ \& Data. $\mathrm{H}>0$ \& Data. $\mathrm{O}>0$ \& Data. $\mathrm{N}==0$ \& Data. $\mathrm{P}>0$ \&

Data. $\mathrm{S}=0$ ) ;

Summary. $\mathrm{CHN}=$ sum $($ Data. $\mathrm{C}>0$ \& Data. $\mathrm{H}>0$ \& Data. $\mathrm{O}==0$ \& Data. $\mathrm{N}>0$ \& Data. $\mathrm{P}==0$ \&

Data. $\mathrm{S}==0$ );

Summary $\cdot \mathrm{CHS}=\operatorname{sum}($ Data $\cdot \mathrm{C}>0$ \& Data. $\mathrm{H}>0$ \& Data $\cdot \mathrm{O}==0$ \& Data. $\mathrm{N}==0$ \& Data.P==0 \&

Data. S>0);

Summary $\cdot \mathrm{CHNS}=$ sum $($ Data. $\mathrm{C}>0$ \& Data. $\mathrm{H}>0$ \& Data. $\mathrm{O}==0$ \& Data. $\mathrm{N}>0$ \& Data. $\mathrm{P}==0$ \&

Data. $\mathrm{S}>0$ );

Summary. $\mathrm{CHNP}=\operatorname{sum}($ Data. $\mathrm{C}>0$ \& Data. $\mathrm{H}>0$ \& Data. $\mathrm{O}==0$ \& Data.N $>0$ \& Data.P $>0$ \&

Data. $\mathrm{S}==0$ );

Summary $\cdot \mathrm{CHONS}=\operatorname{sum}($ Data $\cdot \mathrm{C}>0$ \& Data. $\mathrm{H}>0$ \& Data. $\mathrm{O}>0$ \& Data.N $>0$ \& Data.P==0 \&

Data. $\mathrm{S}>0$ );

Summary. $\mathrm{CHONP}=$ sum (Data. $\mathrm{C}>0$ \& Data.H>0 \& Data.O $>0$ \& Data.N $>0$ \& Data.P $>0$ \&

Data. $\mathrm{S}==0$ );

Summary. $\mathrm{CHONSP}=$ sum $($ Data. $\mathrm{C}>0$ \& Data.H $>0$ \& Data.O $>0$ \& Data.N $>0$ \& Data.P>0 \&

Data. $\mathrm{S}>0$ );

ocalculating aromaticity parameters: \# condensed aromatic (AI>=0.67),

aromatic $(\mathrm{AI}>=0.5)$,

onon-aromatic $(\mathrm{AI}<0.5)$

Summary. CondensedAromatics $=$ sum (Data. AI $>=0.67)$;

Summary.Aromatics=sum (Data.AI>=0.5);

Summary. NonAromatics=sum (Data.AI<0.5);

ocalculating other parameters: total \# of formulae, average molecular oweight

Summary. TotalFormulae=height (Data) ;

Summary. AvgMW=mean (Data. NominalMass) ; 


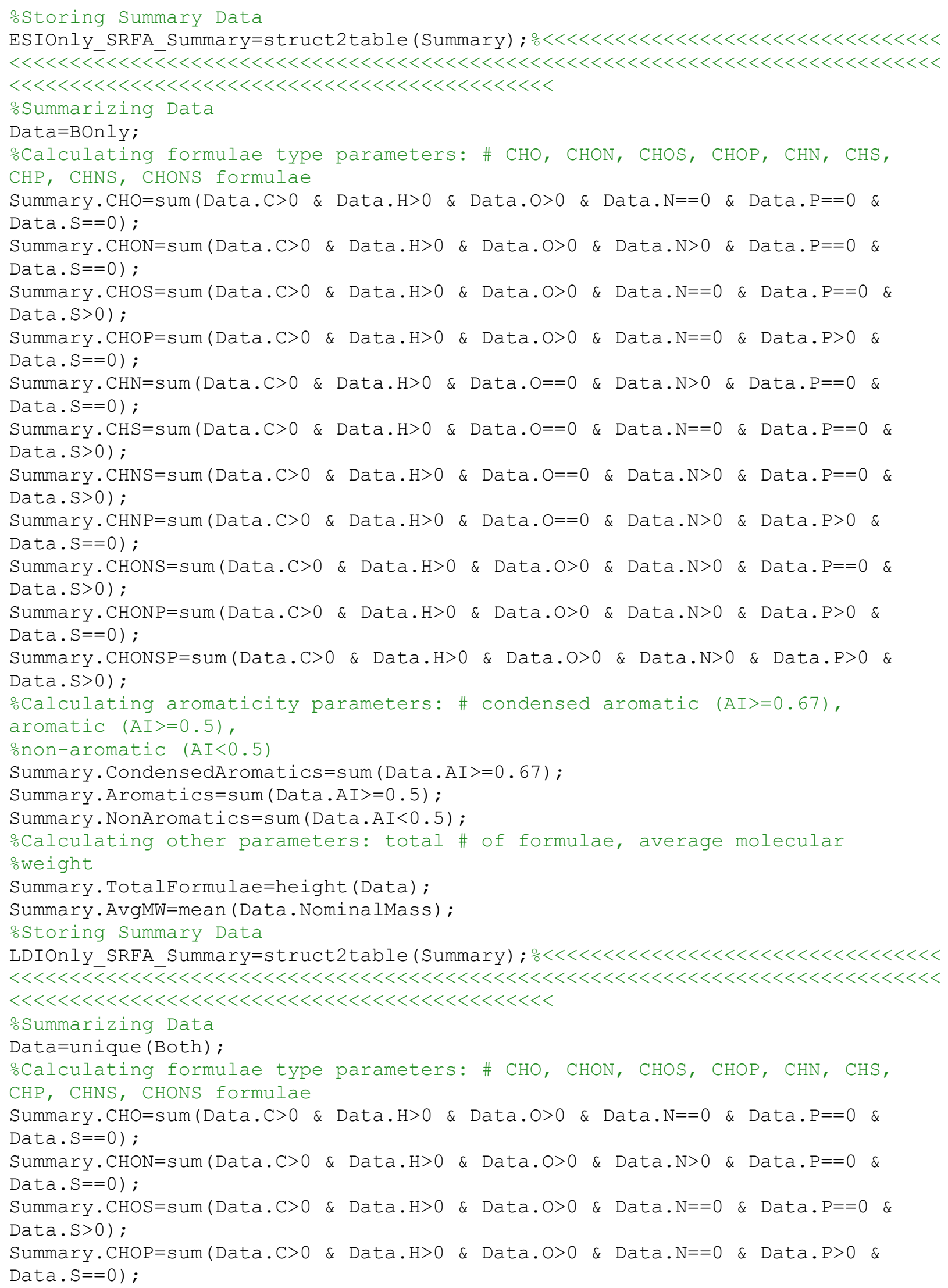


Summary. $\mathrm{CHN}=$ sum $($ Data. $\mathrm{C}>0$ \& Data.H $>0$ \& Data. $\mathrm{O}==0$ \& Data. $\mathrm{N}>0$ \& Data.P==0 \& Data. $\mathrm{S}==0$ );

Summary $\cdot \mathrm{CHS}=\operatorname{sum}($ Data $\cdot \mathrm{C}>0$ \& Data. $\mathrm{H}>0$ \& Data. $\mathrm{O}==0$ \& Data. $\mathrm{N}==0$ \& Data.P==0 \& Data. $\mathrm{S}>0$ );

Summary $\cdot \mathrm{CHNS}=\operatorname{sum}($ Data. $\mathrm{C}>0$ \& Data. $\mathrm{H}>0$ \& Data. $\mathrm{O}==0$ \& Data. $\mathrm{N}>0$ \& Data. $\mathrm{P}==0$ \& Data. S>0);

Summary. $\mathrm{CHNP}=\operatorname{sum}($ Data. $\mathrm{C}>0$ \& Data. $\mathrm{H}>0$ \& Data. $\mathrm{O}==0$ \& Data.N $>0$ \& Data.P $>0$ \&

Data. $\mathrm{S}==0$ );

Summary. $\mathrm{CHONS}=\operatorname{sum}($ Data. $\mathrm{C}>0$ \& Data. $\mathrm{H}>0$ \& Data.O $>0$ \& Data.N $>0$ \& Data.P==0 \&

Data. $\mathrm{S}>0$ );

Summary. $\mathrm{CHONP}=$ sum $($ Data. $\mathrm{C}>0$ \& Data.H $>0$ \& Data. O $>0$ \& Data.N $>0$ \& Data.P $>0$ \&

Data. $\mathrm{S}=0$ ) ;

Summary. $\mathrm{CHONSP}=$ sum $($ Data. $\mathrm{C}>0$ \& Data.H $>0$ \& Data.O $>0$ \& Data.N $>0$ \& Data.P>0 \&

Data. S>0);

ocalculating aromaticity parameters: \# condensed aromatic (AI>=0.67),

aromatic (AI>=0.5),

onon-aromatic $($ A I < 0.5$)$

Summary. CondensedAromatics $=$ sum (Data.AI $>=0.67)$;

Summary. Aromatics=sum (Data.AI $>=0.5)$;

Summary. NonAromatics $=$ sum (Data.AI<0.5);

ocalculating other parameters: total \# of formulae, average molecular

oweight

Summary. TotalFormulae=height (Data);

Summary. AvgMW=mean (Data. NominalMass);

ostoring Summary Data

BothTechniques_SRFA_Summary=struct2table (Summary); $\circ<<<<<<<<<<<<<<<<<<<<<<<<<<<<~$

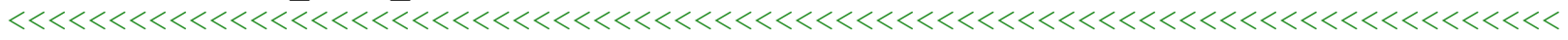

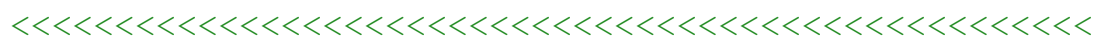

ㅇmmarizing Data

Data $=$ Combined;

oCalculating formulae type parameters: \# CHO, CHON, CHOS, CHOP, CHN, CHS, CHP, CHNS, CHONS formulae

Summary $\cdot \mathrm{CHO}=$ sum $($ Data. $\mathrm{C}>0$ \& Data. $\mathrm{H}>0$ \& Data. $\mathrm{O}>0$ \& Data. $\mathrm{N}==0$ \& Data. $\mathrm{P}==0$ \& Data. $\mathrm{S}==0$ );

Summary. $\mathrm{CHON}=\mathrm{sum}($ Data. $\mathrm{C}>0$ \& Data. $\mathrm{H}>0$ \& Data.O $>0$ \& Data.N $>0$ \& Data.P==0 \&

Data. $S==0$ ) ;

Summary. $\mathrm{CHOS}=\operatorname{sum}($ Data. $\mathrm{C}>0$ \& Data. $\mathrm{H}>0$ \& Data. $\mathrm{O}>0$ \& Data. $\mathrm{N}==0$ \& Data. $\mathrm{P}==0$ \&

Data. $\mathrm{S}>0$ );

Summary. $\mathrm{CHOP}=\operatorname{sum}($ Data. $\mathrm{C}>0$ \& Data. $\mathrm{H}>0$ \& Data. $\mathrm{O}>0$ \& Data. $\mathrm{N}==0$ \& Data.P>0 \&

Data. $\mathrm{S}==0$ );

Summary $\cdot \mathrm{CHN}=$ sum $($ Data. $\mathrm{C}>0$ \& Data. $\mathrm{H}>0$ \& Data. $\mathrm{O}==0$ \& Data. $\mathrm{N}>0$ \& Data. $\mathrm{P}==0$ \&

Data. $\mathrm{S}==0$ ) ;

Summary $\cdot \mathrm{CHS}=\operatorname{sum}($ Data $\cdot \mathrm{C}>0$ \& Data.H $>0$ \& Data $\cdot \mathrm{O}==0$ \& Data. $\mathrm{N}==0$ \& Data.P==0 \&

Data. $\mathrm{S}>0$ ) ;

Summary $\cdot \mathrm{CHNS}=$ sum $($ Data. $\mathrm{C}>0$ \& Data. $\mathrm{H}>0$ \& Data. $\mathrm{O}==0$ \& Data. $\mathrm{D}>0$ \& Data. $\mathrm{P}==0$ \&

Data. S>0);

Summary $\cdot \mathrm{CHNP}=\operatorname{sum}($ Data $\cdot \mathrm{C}>0$ \& Data. $\mathrm{H}>0$ \& Data. $\mathrm{O}==0$ \& Data.N $>0$ \& Data.P $>0$ \&

Data. $\mathrm{S}==0$ );

Summary $\cdot \mathrm{CHONS}=\operatorname{sum}($ Data. $\mathrm{C}>0$ \& Data. $\mathrm{H}>0$ \& Data. $\mathrm{O}>0$ \& Data. $\mathrm{N}>0$ \& Data. $\mathrm{P}==0$ \&

Data. $\mathrm{S}>0$ ) ;

Summary. $\mathrm{CHONP}=$ sum $($ Data. $\mathrm{C}>0$ \& Data.H $>0$ \& Data. $0>0$ \& Data.N $>0$ \& Data.P $>0$ \&

Data. $\mathrm{S}==0$ );

Summary. $\mathrm{CHONSP}=$ sum $($ Data. $\mathrm{C}>0$ \& Data. $\mathrm{H}>0$ \& Data.O $>0$ \& Data.N $>0$ \& Data.P $>0$ \&

Data. $\mathrm{S}>0$ );

ocalculating aromaticity parameters: \# condensed aromatic (AI>=0.67),

aromatic $(\mathrm{AI}>=0.5)$,

onon-aromatic $($ A I < 0.5$)$ 
Summary. CondensedAromatics $=$ sum (Data. AI $>=0.67)$;

Summary. Aromatics $=$ sum (Data.AI $>=0.5)$;

Summary. NonAromatics=sum (Data.AI<0.5);

ocalculating other parameters: total \# of formulae, average molecular

oweight

Summary. TotalFormulae=height (Data);

Summary. AvgMW=mean (Data. NominalMass);

oStoring Summary Data

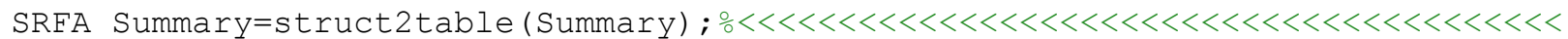
$<<<<\overline{<}<<<<<<<<<<<<<<<<<<<<<<<<<<<<<<<<<<<<<<<<<<<<<<<<<<<<<<<<<<<<<<<<<<<<<<<<~$

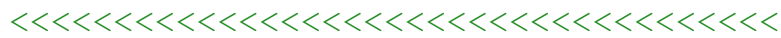

응 Repeating for post oxidation SRFA

응 Negative Mode ESI: Data Clean Up

\%Loading negative mode background impurities

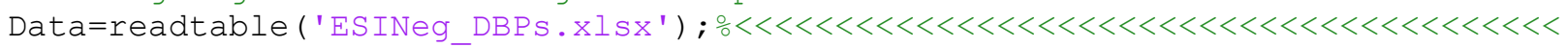
$<<<<<<<<<<<<<<<<<<<<<<\overline{<}<<<<<<<<<<<<<<<<<<<<<<<<<<<<<<<<<<<<<<<<<<<<<<<<<<<<<<~$ $<<<<<<<<<<<<<<<<<<<<<<<<<<<<<<<<<<~$

Data=unique (Data);

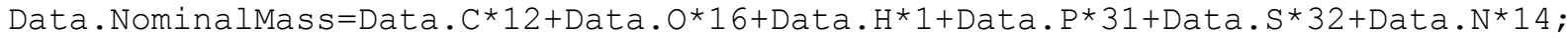

Data. KMD CH2=Data. NominalMass -

$0.998883 \overline{4} *\left(\right.$ Data. $C * 12+$ Data. $H^{*} 1.007825+$ Data. $N * 14.003074+$ Data. $0 * 15.994915+$ Data.P $\star 30.973763+$ Data. $S * 31.972072$ );

Data.KMD_COO=Data.NominalMass-

$1.000231^{\star}\left(\right.$ Data. C*12+Data.H*1.007825+Data. $N^{\star} 14.003074+$ Data. $0 * 15.994915+$ Data.P* $30.973763+$ Data. $S * 31.972072)$;

Data.KMD O2=Data.NominalMass-

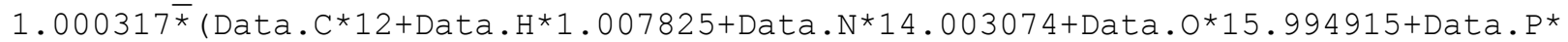
$30.973763+$ Data.. * 31.972072 );

Data.KMD_H2=Data. NominalMass-

$0.992236^{\star}($ Data. C*12+Data.H*1.007825+Data.N*14.003074+Data.0*15.994915+Data.P*

$30.973763+$ Data. $S * 31.972072)$;

Data. $\mathrm{H}=$ Data. $\mathrm{H}+1$;

Data.HC_Ratio = Data.H ./ Data.C;

Data.OC_Ratio = Data.O ./ Data.C;

Data.NC_Ratio = Data.N ./ Data.C;

Data.SC_Ratio = Data.S ./ Data.C;

Data.PC Ratio = Data.P ./ Data.C;

Data. $\mathrm{DBE}=1+0.5 *(2 *$ Data.C - Data.H + Data. $\mathrm{N}+$ Data.P $)$;

Data.AI $=(1+$ Data.C - Data.O - Data.S - 0.5* (Data.N + Data.P + Data.H) $) . /$

(Data.C - Data.O - Data.N - Data.S - Data.P);

Data.AvgCoxstate = 2*Data.OC_Ratio - Data.HC_Ratio;

Remove data points based on following criteria

ㄴ. Error (allowable: ppm $<=0.5$ )

Data (Data.errPpm $>0.5,:)=[]$;

Data (Data.errPpm<-0.5,:) = [] ;

\%2. Stubbins, 2010 :

$\therefore \mathrm{A} . \mathrm{DBE}=$ integer, greater than or equal to 0

Data (Data.DBE =round (Data.DBE), : ) = [];

Data $($ Data. $\mathrm{DBE}<0,:)=[]$;

$\because \mathrm{B} . \mathrm{C} \quad<=50$

Data (Data.C $>50,:)=[]$;

$\because \mathrm{C} . \mathrm{H}>=2 \&<=2 * \mathrm{C}+2$

Data (Data. $\mathrm{H}<2,:)=[]$;

Data (Data.H $>($ Data. $C * 2+2),:)=[]$;

D. $\mathrm{O}=<\mathrm{C}+2$

Data $($ Data. O $>($ Data $. C+2),:)=[]$; 


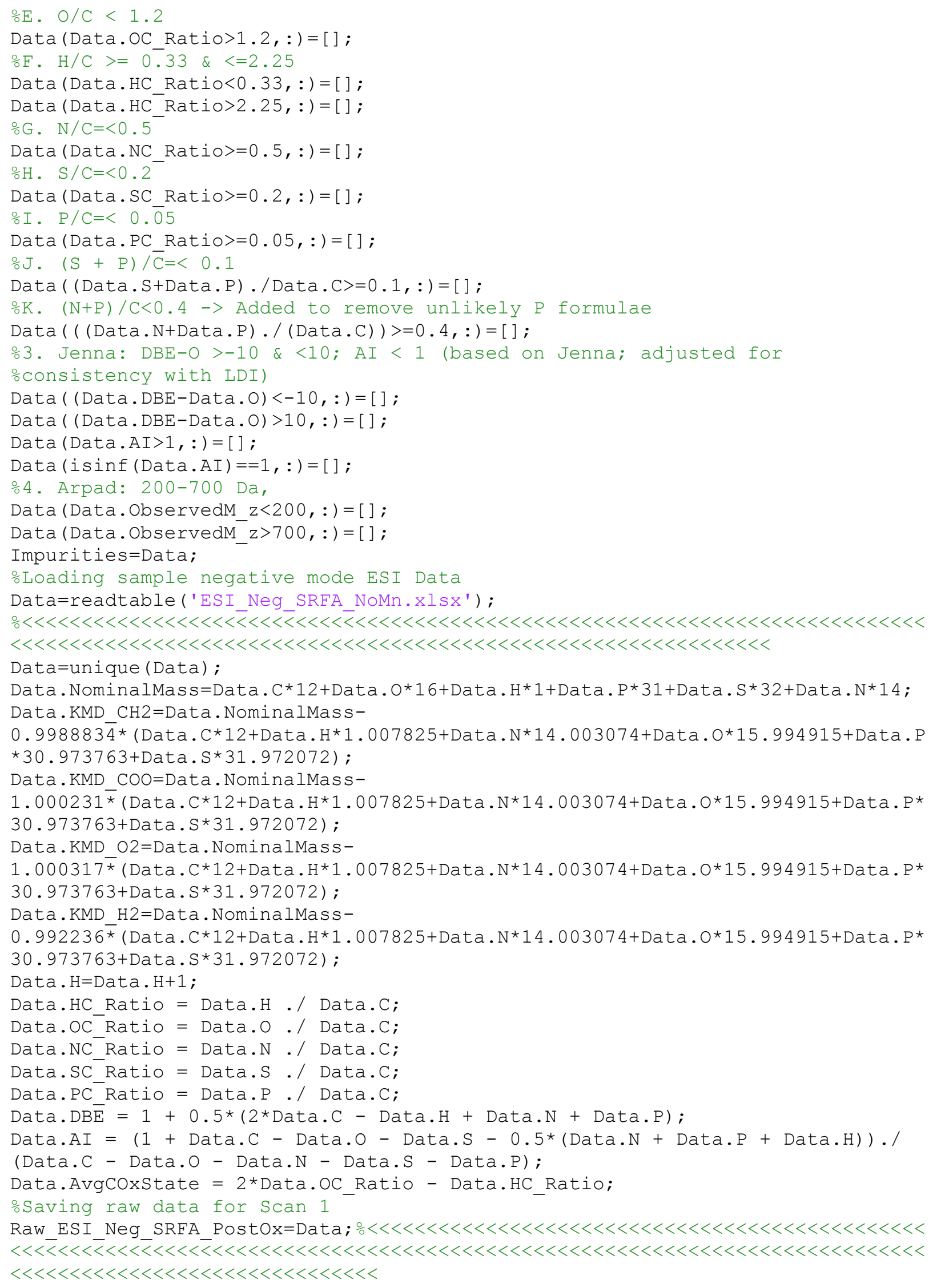




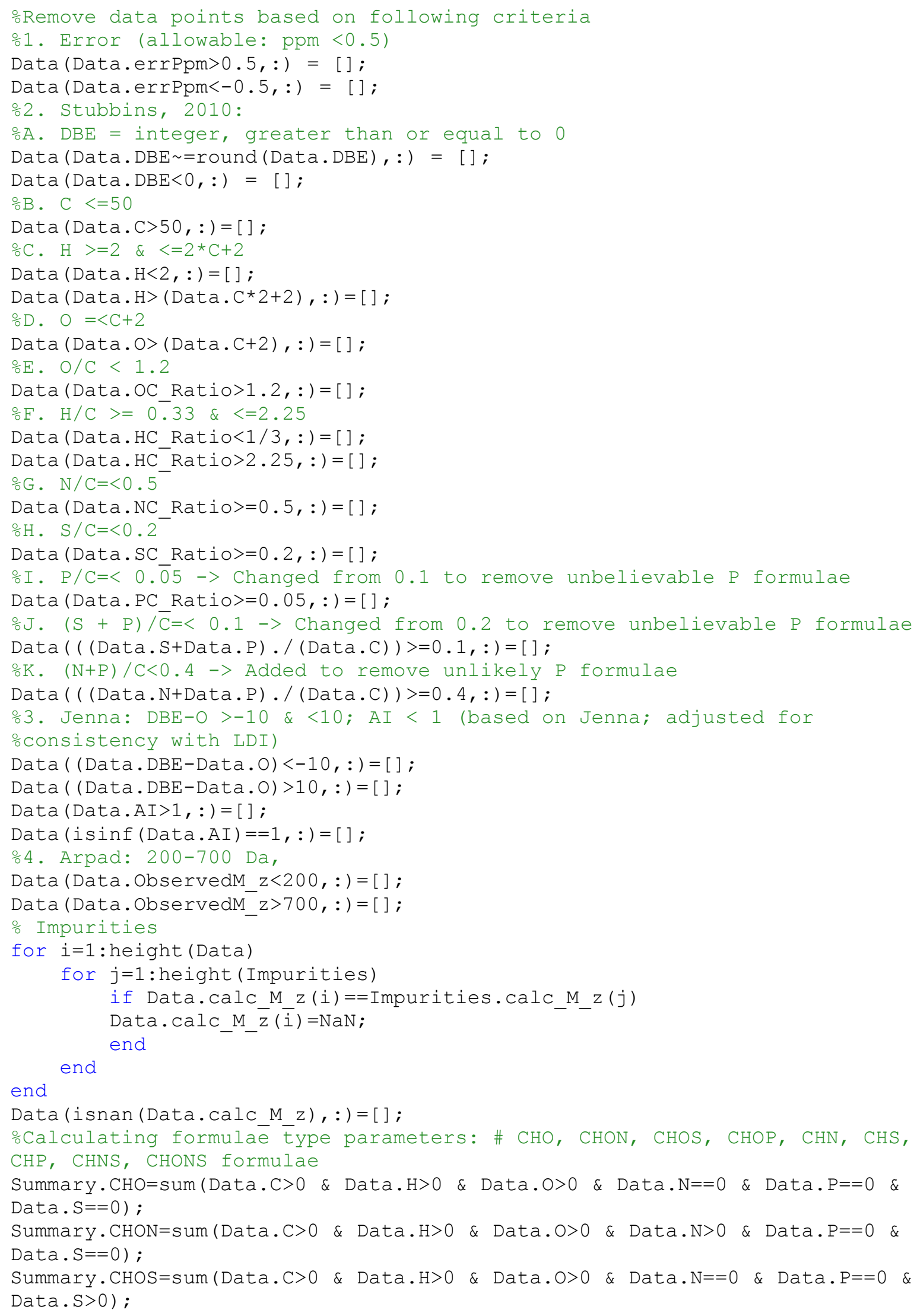


Summary. $\mathrm{CHOP}=\operatorname{sum}($ Data. $\mathrm{C}>0$ \& Data. $\mathrm{H}>0$ \& Data. $\mathrm{O}>0$ \& Data. $\mathrm{N}==0$ \& Data.P $>0$ \& Data. $\mathrm{S}==0$ );

Summary $\cdot \mathrm{CHN}=$ sum $($ Data. $\mathrm{C}>0$ \& Data. $\mathrm{H}>0$ \& Data. $\mathrm{O}==0$ \& Data. $\mathrm{N}>0$ \& Data.P==0 \& Data. $\mathrm{S}==0$ );

Summary $\cdot \mathrm{CHS}=\operatorname{sum}($ Data $\cdot \mathrm{C}>0$ \& Data. $\mathrm{H}>0$ \& Data. $\mathrm{O}==0$ \& Data. $\mathrm{N}==0$ \& Data. $\mathrm{P}==0$ \& Data. S>0);

Summary $\cdot \mathrm{CHNS}=$ sum $($ Data $\cdot \mathrm{C}>0$ \& Data. $\mathrm{H}>0$ \& Data. $\mathrm{O}==0$ \& Data. $\mathrm{N}>0$ \& Data. $\mathrm{P}==0$ \&

Data. $\mathrm{S}>0$ ) ;

Summary $\cdot \mathrm{CHNP}=\operatorname{sum}($ Data $\cdot \mathrm{C}>0$ \& Data. $\mathrm{H}>0$ \& Data. $\mathrm{O}==0$ \& Data.N $>0$ \& Data.P>0 \&

Data. $\mathrm{S}==0$ );

Summary $\cdot \mathrm{CHONS}=$ sum $($ Data $\cdot \mathrm{C}>0$ \& Data. $\mathrm{H}>0$ \& Data. $\mathrm{O}>0$ \& Data. $>0$ \& Data.P==0 \&

Data. $\mathrm{S}>0$ );

Summary. $\mathrm{CHONP}=$ sum $($ Data. $\mathrm{C}>0$ \& Data.H $>0$ \& Data.O>0 \& Data.N>0 \& Data.P>0 \&

Data. $\mathrm{S}==0$ );

Summary. $\mathrm{CHONSP}=$ sum $($ Data. $\mathrm{C}>0$ \& Data.H $>0$ \& Data.O $>0$ \& Data.N $>0$ \& Data.P $>0$ \&

Data. $\mathrm{S}>0$ ) ;

ocalculating aromaticity parameters: \# condensed aromatic (AI>=0.67),

aromatic $(\mathrm{AI}>=0.5)$,

onon-aromatic $(\mathrm{AI}<0.5)$

Summary. CondensedAromatics $=$ sum (Data. AI $>=0.67)$;

Summary.Aromatics=sum (Data.AI>=0.5);

Summary. NonAromatics=sum (Data.AI<0.5);

oCalculating other parameters: total \# of formulae, average molecular

oweight

Summary. TotalFormulae=height (Data);

Summary. AvgMW=mean (Data. NominalMass) ;

ostoring Data in Tables

ESI_Neg_SRFA_PostOx=Data $; \circ \circ<<<<<<<<<<<<<<<<<<<<<<<<<<<<<<<<<<<<<<<<<<<<<<<<<<<<<<~$ $<<\overline{<}<<\overline{<}<<<<\overline{<}<<<<<<<<<<<<<<<<<<<<<<<<<<<<<<<<<<<<<<<<<<<<<<<<<<<<<<<<<<<<<<<<<$ $<<<<<<<<<<<<<<<<<<<<<<<<<<<~$

ESI_Neg_SRFA_PostOx_Summary=struct2table (Summary) ; $\circ<<<<<<<<<<<<<<<<<<<<<<<<<<~$ $<<\overline{<}<<\overline{<}<<<<\overline{<}<<<<<<\overline{<}<<<<<<<<<<<<<<<<<<<<<<<<<<<<<<<<<<<<<<<<<<<<<<<<<<<<<<<<<~$

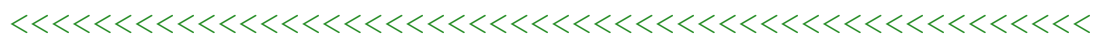

응 Positive Mode ESI: Data Clean Up

oLoading positive mode ESI impurities

Data $=$ readtable ('DBP_Control.xlsx') ;

Data. NominalMass=Data $\cdot \mathrm{C} * 12+$ Data $\cdot 0 * 16+$ Data $\cdot H * 1+$ Data $\cdot P * 31+$ Data $. S * 32+$ Data $\cdot N * 14+D$ ata. $\mathrm{Na} * 23$

Data.KMD_CH2=Data. NominalMass-

$0.998883 \overline{4} *\left(\right.$ Data. $C * 12+$ Data. $H^{*} 1.007825+$ Data. $N^{*} 14.003074+$ Data. $0 * 15.994915+$ Data. P *30.973763+Data.S*31.972072+Data.Na*22.98977);

Data.KMD COO=Data. NominalMass-

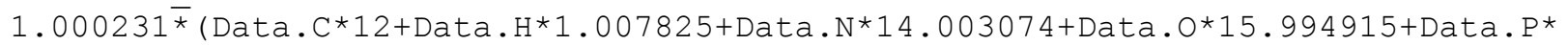
$30.973763+$ Data.. *31.972072+Data.Na*22.98977);

Data.KMD_O2=Data. NominalMass-

$1.000317^{\star}\left(\right.$ Data. $C * 12+$ Data. $H^{*} 1.007825+$ Data. $N^{\star} 14.003074+$ Data. $0 * 15.994915+$ Data. $P *$

$30.973763+$ Data.S*31.972072+Data.Na*22.98977);

Data.KMD_H2=Data. NominalMass-

$0.992236^{\bar{*}}$ (Data. C*12+Data.H*1.007825+Data.N*14.003074+Data.0*15.994915+Data.P* $30.973763+$ Data.S*31.972072+Data.Na*22.98977);

for $i=1$ :height (Data)

if Data.Na(i)==0

Data.H(i)=Data.H (i) -1 ;

end

end

Data.HC_Ratio = Data.H ./ Data.C; 


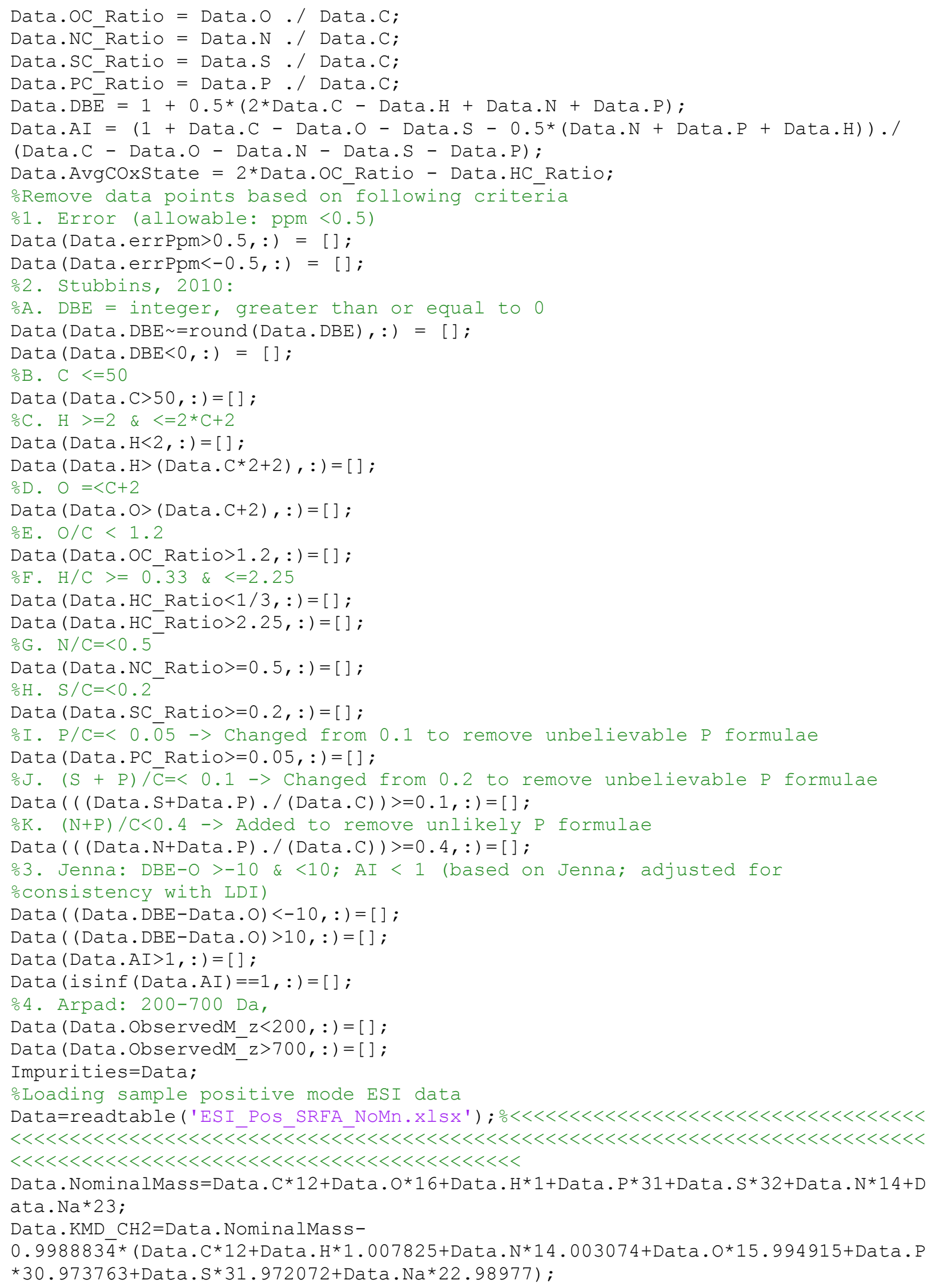




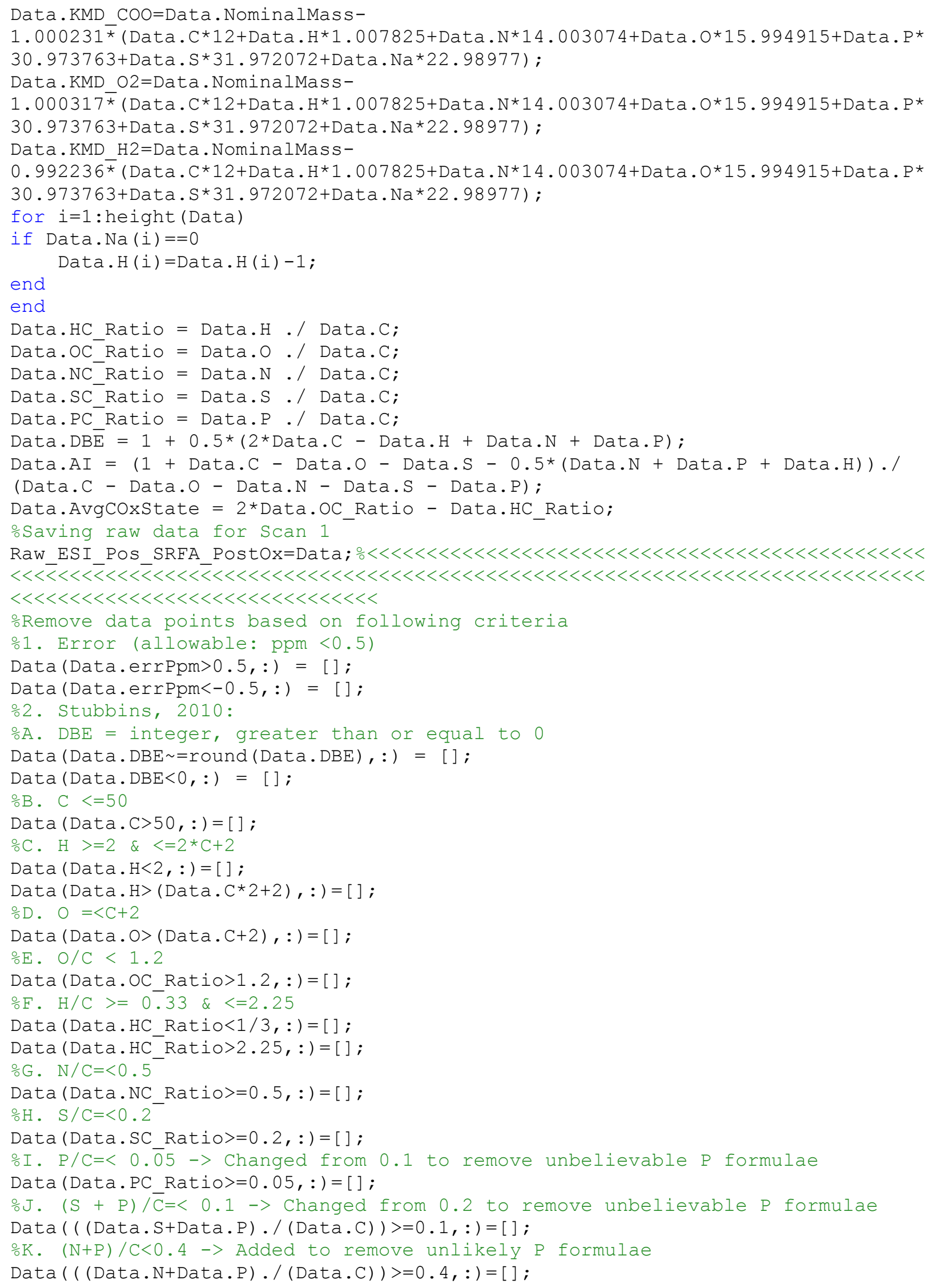




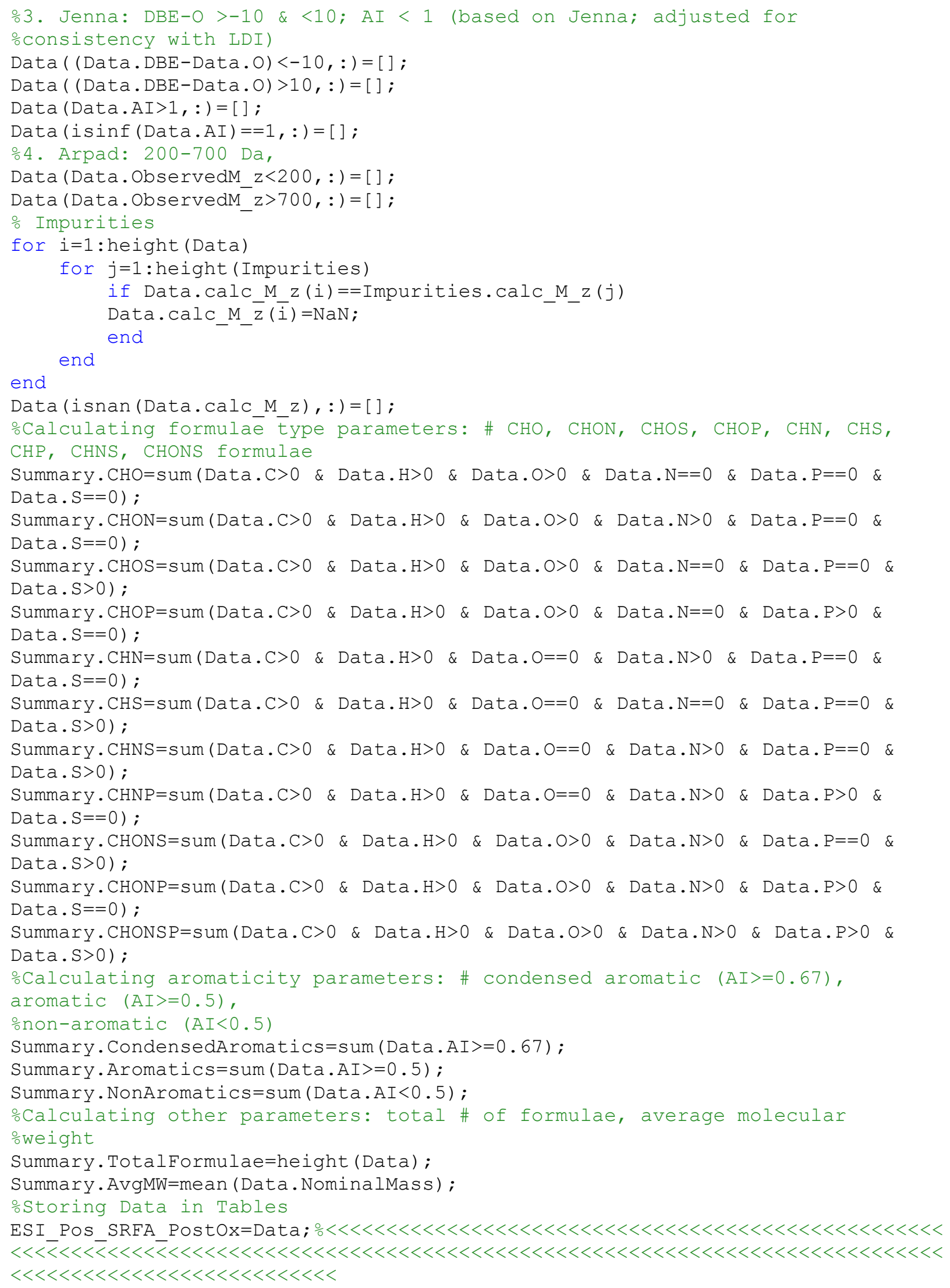


ESI_Pos_SRFA_PostOx_Summary=struct2table (Summary) ; $\circ<<<<<<<<<<<<<<<<<<<<<<<<<<<<<~$ $<<\overline{<}<<\overline{\bar{l}}<<<<\overline{<}<<<<<\overline{<} \overline{<}<<<<<<<<<<<<<<<<<<<<<<<<<<<<<<<<<<<<<<<<<<<<<<<<<<<<<<<<<$

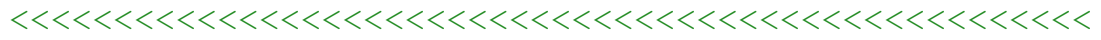

\% Negative Mode LDI: Data Clean Up

\%LDI assumed to have no background ions

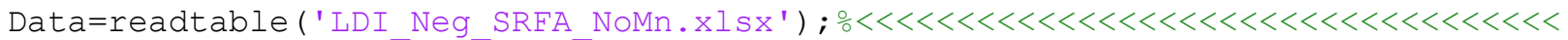
$<<<<<<<<<<<<<<<<<<<\overline{<}<<<\overline{<}<<<\overline{<}<<<<<<<<<<<<<<<<<<<<<<<<<<<<<<<<<<<<<<<<<<<<<<<<<~$ $<<<<<<<<<<<<<<<<<<<<<<<<<<<<<<<<<<<<<<<<<<<<<~$

Data=unique (Data);

Data. NominalMass=Data. $\mathrm{C} * 12+$ Data. $0 * 16+$ Data $\cdot H * 1+$ Data. $P * 31+$ Data $. S * 32+D a t a \cdot N * 14 ;$ Data. KMD CH2=Data. NominalMass-

$0.998883 \overline{4} *\left(\right.$ Data. $C * 12+$ Data. $H * 1.007825+$ Data. $N^{*} 14.003074+$ Data. $0 * 15.994915+$ Data. P *30.973763+Data.S*31.972072);

Data.KMD COO=Data. NominalMass -

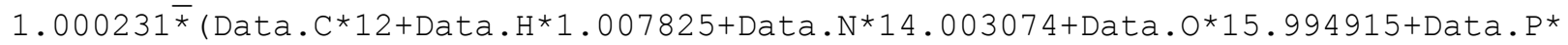
$30.973763+$ Data. $S * 31.972072)$;

Data.KMD O2=Data. NominalMass-

$1.000317^{\bar{*}}\left(\right.$ Data. $\mathrm{C}^{\star} 12+$ Data. $\mathrm{H}^{\star} 1.007825+$ Data. $\mathrm{N}^{\star} 14.003074+\mathrm{Data} .0 * 15.994915+\mathrm{Data} . \mathrm{P} *$ $30.973763+$ Data. S*31.972072);

Data.KMD H2=Data. NominalMass-

$0.992236^{\star}$ (Data.C*12+Data.H*1.007825+Data.N*14.003074+Data. $* 15.994915+$ Data.P* $30.973763+$ Data..$* 31.972072)$;

Data.DBE $=1+0.5 *(2 *$ Data.C - Data.H + Data.N + Data.P $)$;

for $i=1$ : height (Data)

if Data.DBE (i) =round (Data.DBE (i))

Data.H (i) =Data.H(i) + ;

Data. IonType $(i)=0 ; \% H-$ Adduct $s=0$

else

Data.IonType(i)=2; №lecularIons=2 \%Temporary fix until I figure out how

to enter names into a table end

end

Data.HC_Ratio = Data.H ./ Data.C;

Data.OC_Ratio = Data.O./ Data.C;

Data.NC Ratio = Data.N ./ Data.C;

Data.SC_Ratio = Data.S ./ Data.C;

Data.PC Ratio = Data.P ./ Data.C;

Data. $\mathrm{DBE}=1+0.5 *(2 *$ Data.C - Data.H + Data. $\mathrm{N}+$ Data.P $)$;

Data.AI $=(1+$ Data.C - Data.O - Data.S - 0.5* (Data.N + Data.P + Data.H) $) . /$

(Data.C - Data.O - Data.N - Data.S - Data.P);

Data.AvgCoxstate = 2*Data.OC_Ratio - Data.HC_Ratio;

osaving raw data

Raw LDI Neg SRFA PostOx =Data; $\stackrel{\circ}{\circ}<<<<<<<<<<<<<<<<<<<<<<<<<<<<<<<<<<<<<<<<<<<<<<<<<<~$

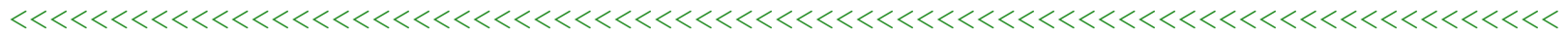
$<<<<<<<<<<<<<<<<<<<<<<<<<<<<<<<<<<~$

oRemove data points based on following criteria

\%1. Error (allowable: ppm <0.5)

Data (Data.errPpm>0.5,:) = [];

Data (Data.errPpm<-0.5,:) = [] ;

ㄴ. Stubbins, 2010 :

\%A. $\mathrm{DBE}=$ integer, greater than or equal to 0

Data (Data.DBE =round (Data.DBE), : ) = [];

Data (Data. $\mathrm{DBE}<0,:)=[]$;

ㅇ. $\mathrm{C}<=50$

Data (Data.C $>50,:)=[]$;

ㄷ. $\mathrm{H}>=2 \&<=2 * \mathrm{C}+2$ 


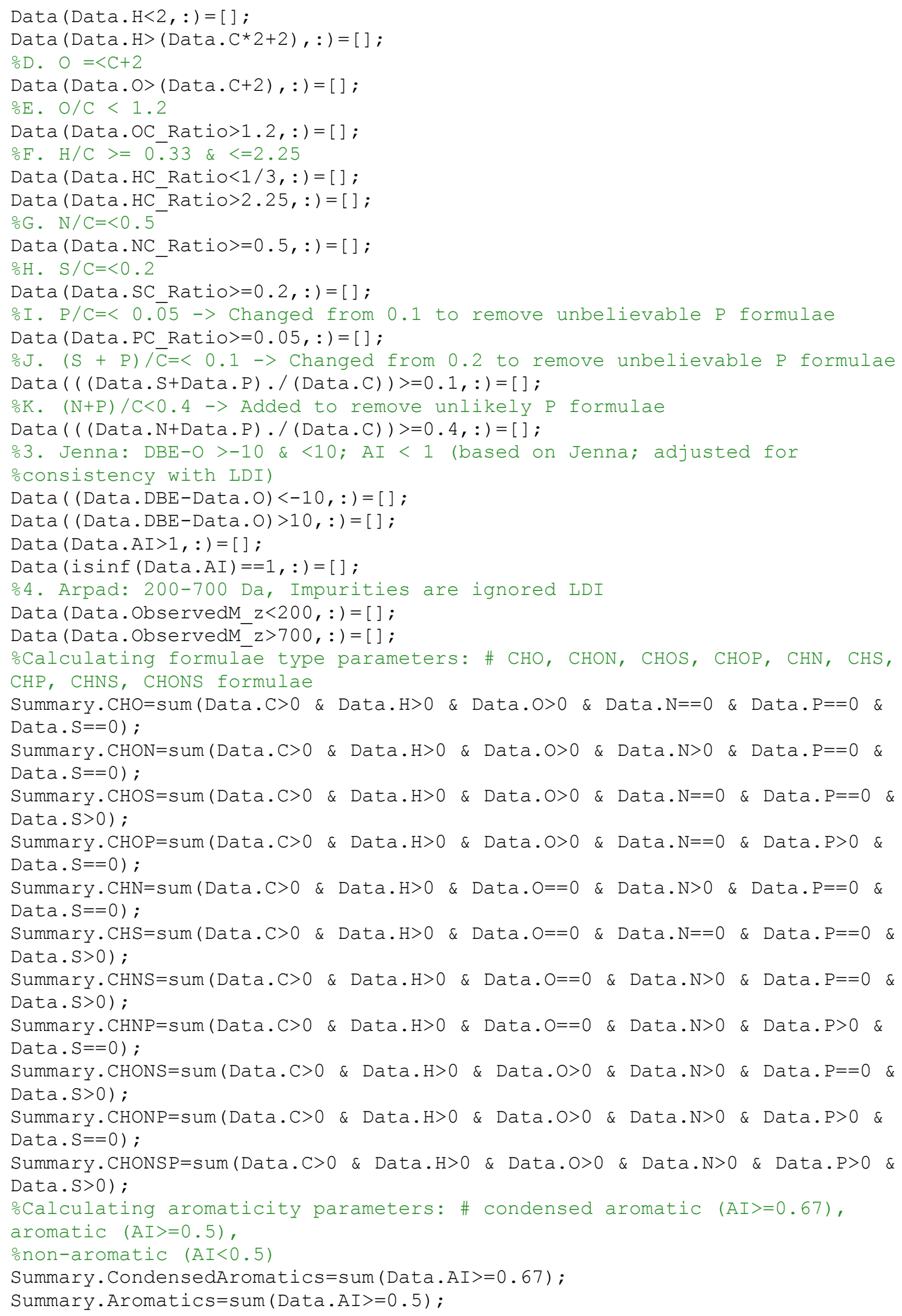


Summary. NonAromatics=sum (Data.AI<0.5);

ocalculating other parameters: total \# of formulae, average molecular

oweight

Summary. TotalFormulae=height (Data);

Summary. AvgMW=mean (Data. NominalMass);

ostoring Data in Tables

LDI Neg SRFA PostOx=Data $;$ 응 $<<<<<<<<<<<<<<<<<<<<<<<<<<<<<<<<<<<<<<<<<<<<<<<<<<<<<~$

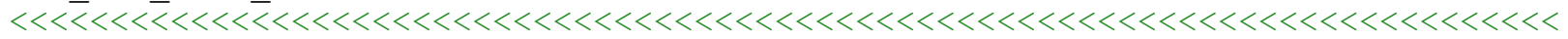
$<<<<<<<<<<<<<<<<<<<<<<<<<~$

LDI_Neg_SRFA_Postox_Summary=struct2table (Summary) ; $\circ<<<<<<<<<<<<<<<<<<<<<<<<<<<<~$

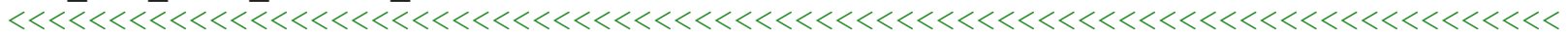

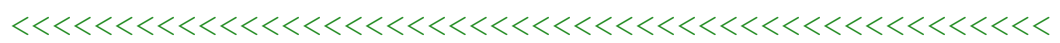

응 Positive Mode LDI: Data Clean Up

\%LDI assumed to have no background ions

oLoading sample positive mode LDI data

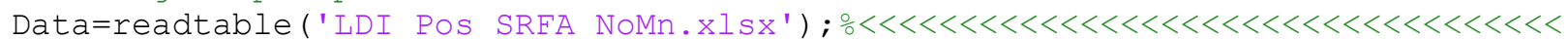
$<<<<<<<<<<<<<<<<<<<\overline{<}<<\overline{\bar{l}}<<<\overline{\bar{\alpha}}<<<<<<<<<<<<<<<<<<<<<<<<<<<<<<<<<<<<<<<<<<<<<<<<$

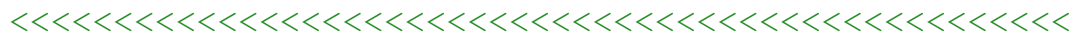

Data=unique (Data);

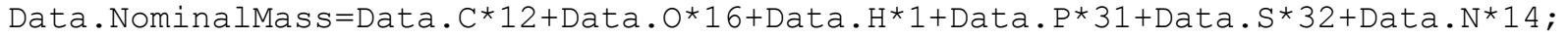

Data. KMD CH2=Data. NominalMass-

$0.998883 \overline{4} *\left(\right.$ Data. $C * 12+$ Data. $H^{*} 1.007825+$ Data. $N * 14.003074+$ Data. $0 * 15.994915+$ Data.P $\star 30.973763+$ Data. $\mathrm{S} * 31.972072$ );

Data.KMD_COO=Data.NominalMass-

$1.000231^{\star}\left(\right.$ Data. $C * 12+$ Data $. H * 1.007825+$ Data. $N^{*} 14.003074+$ Data. $0 * 15.994915+$ Data. $P *$ $30.973763+$ Data.. * 31.972072 );

Data.KMD $02=$ Data. NominalMass-

$1.000317^{\bar{*}}\left(\right.$ Data. $\mathrm{C} * 12+$ Data. $\mathrm{H}^{*} 1.007825+$ Data $. \mathrm{N}^{*} 14.003074+$ Data. $0 * 15.994915+\mathrm{Data} . \mathrm{P} *$ $30.973763+$ Data. $S * 31.972072)$;

Data.KMD_H2=Data.NominalMass-

$0.992236^{\star}\left(\right.$ Data. $C^{*} 12+$ Data $. H^{*} 1.007825+$ Data $. N^{*} 14.003074+$ Data $.0 * 15.994915+$ Data. $P^{*}$

$30.973763+$ Data. $S * 31.972072)$;

Data.DBE $=1+0.5 *(2 *$ Data.C - Data.H + Data.N + Data.P $)$;

for $i=1$ : height (Data)

if Data.DBE(i) =round (Data.DBE(i)) \&\& Data.Na(i)==0

Data.H $(i)=$ Data.H $(i)+1$;

Data. IonType $(i)=0 ; \circ H-$ Adduct $s=0$

elseif Data.DBE(i)==round (Data.DBE(i)) \&\& Data.Na(i) $>0$

Data.IonType $(i)=1 ; \circ \mathrm{Na}$-Adducts else

Data. IonType (i)=2; $\frac{\circ}{\circ}$ MolecularIons=2 \%Temporary fix until I figure out how

to enter names into a table end

end

Data.HC_Ratio = Data.H ./ Data.C;

Data.OC_Ratio = Data.O./ Data.C;

Data.NC_Ratio = Data.N ./ Data.C;

Data.SC Ratio = Data.S./ Data.C;

Data.PC_Ratio = Data.P./ Data.C;

Data.DBE $=1+0.5 *(2 *$ Data.C - Data.H + Data.N + Data.P $)$;

Data.AI $=(1+$ Data.C - Data.O - Data.S - 0.5* (Data.N + Data.P + Data.H) $) \cdot /$

(Data.C - Data.O - Data.N - Data.S - Data.P);

Data.AvgCoxstate = 2*Data.OC_Ratio - Data.HC_Ratio;

oSaving raw data for Scan 1 


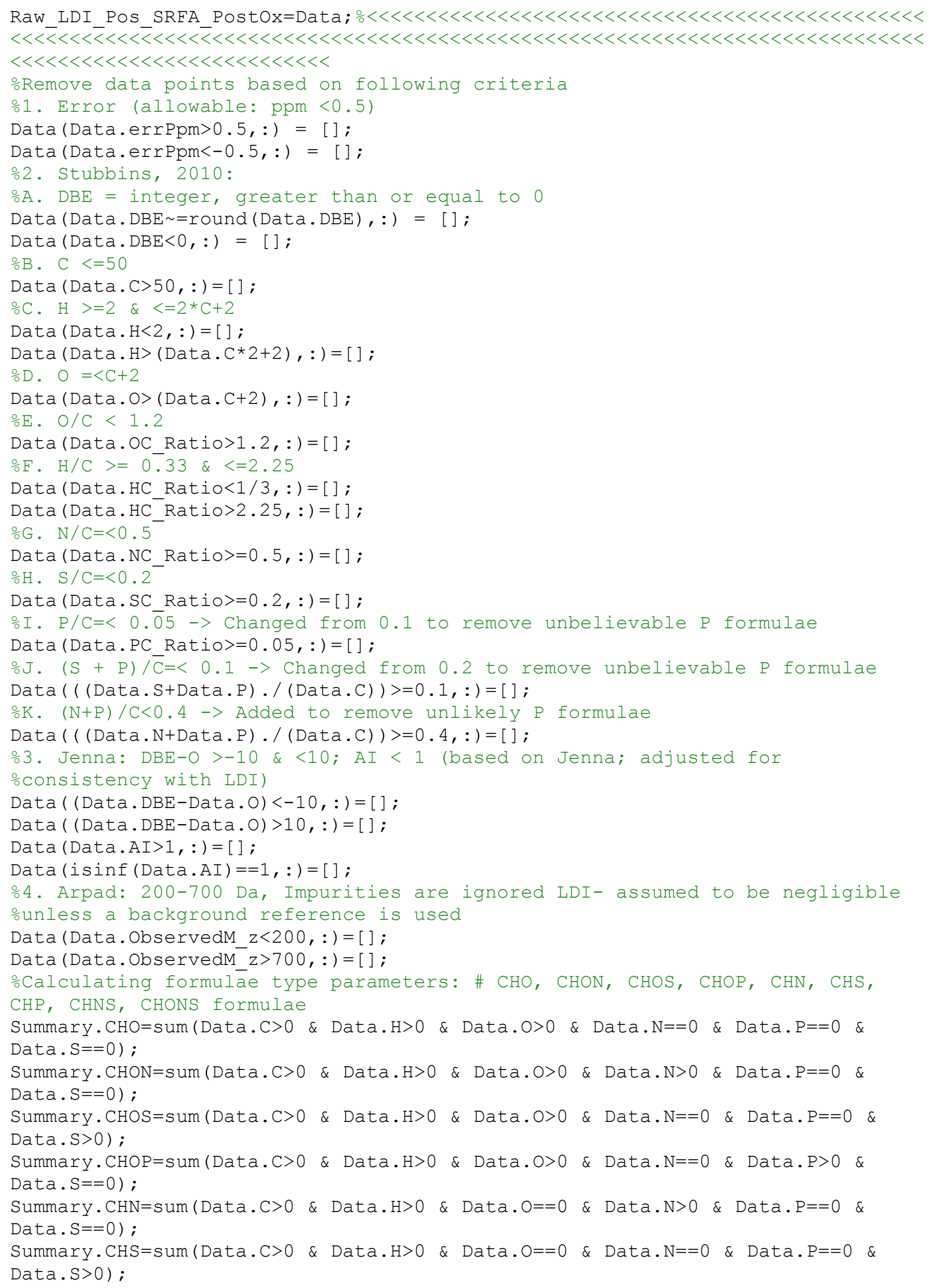




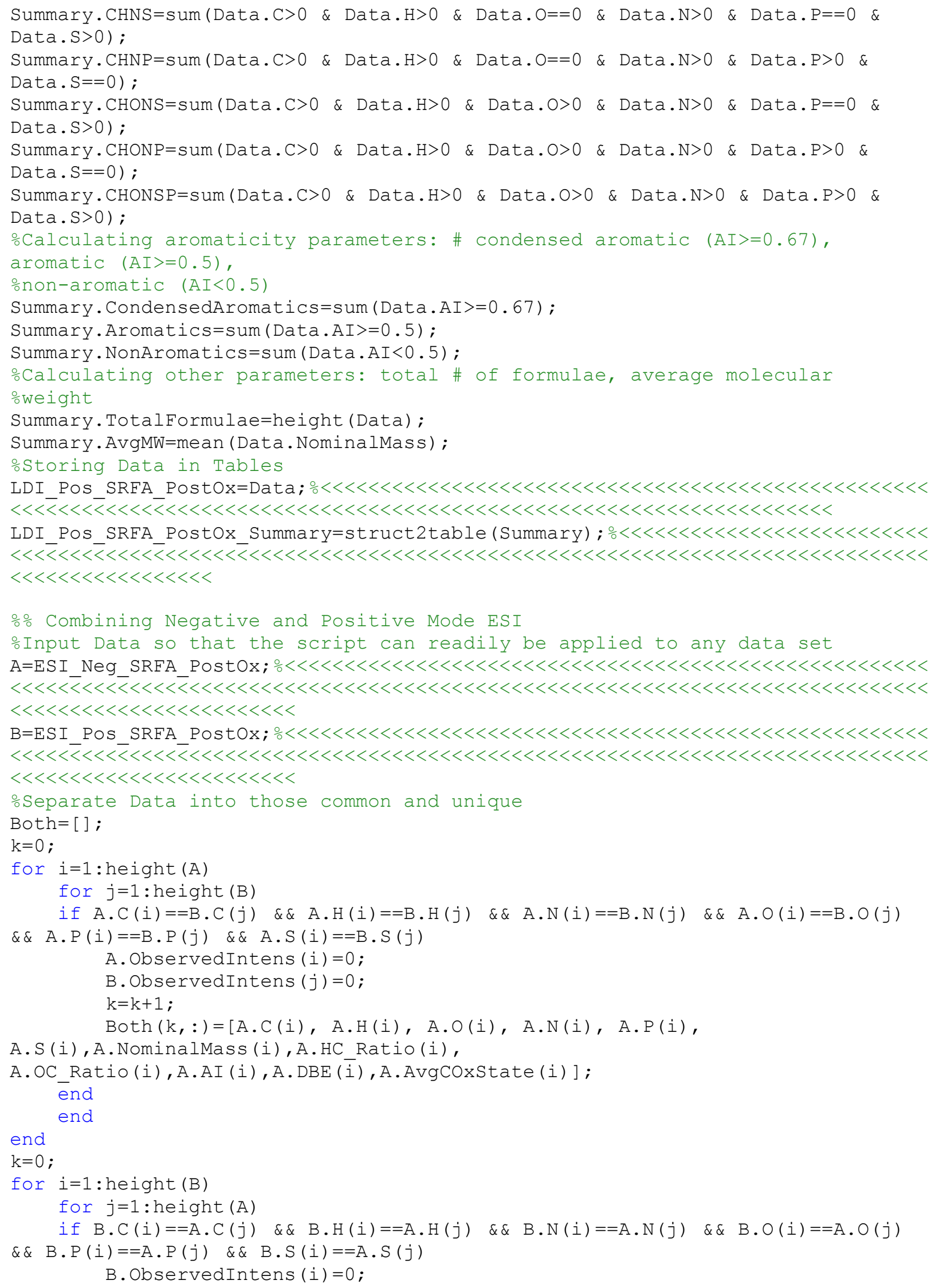


A. ObservedIntens $(j)=0$;

$\mathrm{k}=\mathrm{k}+1$;

Both $(k,:)=[B . C(i), B . H(i), B . O(i), B . N(i), B . P(i)$,

B.S (i), B.NominalMass (i), B.HC Ratio(i),

B.OC_Ratio(i),B.AI (i),B.DBE (i) , B.AvgCoxState (i) ] ;

end

end

end

oCombining Data Sets

$A(A$. Observedintens $==0,:)=[]$;

$A O n l_{Y}=[A . C, A . H, A . O, A . N, A . P, A . S$,

A. Nominalmass, A.HC Ratio,A.oC Ratio, A.AI, A.DBE, A.AvgCoxState];

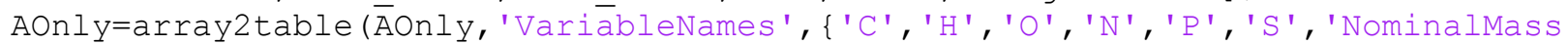
', 'HC_Ratio', 'OC_Ratio', 'AI', 'DBE', 'AvgCOxState'\}) ;

$\mathrm{B}(\mathrm{B} . \mathrm{Ob}$ servedinten $\mathrm{s}==0,:)=[]$;

$B O n l_{Y}=[B . C, B . H, B . O, B . N, B . P$,

B.S,B.NominalMass,B.HC_Ratio,B.OC_Ratio,B.AI, B.DBE, B.AvgCoxState] ;

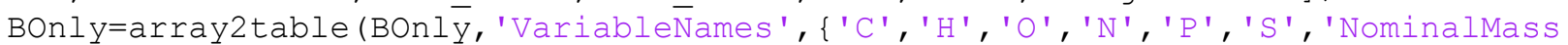
', 'HC_Ratio', 'OC_Ratio', 'AI', 'DBE', 'AvgCOxState'\}) ;

Both=array2table (Both, 'VariableNames', \{'C', 'H', 'O' , 'N', 'P' , 'S ', 'NominalMass', 'HC_Ratio', 'OC_Ratio', 'AI', 'DBE', 'AvgCOxState' \}) ;

Combined=[Both; AOnly; BOnly];

ostoring Data with distinct names

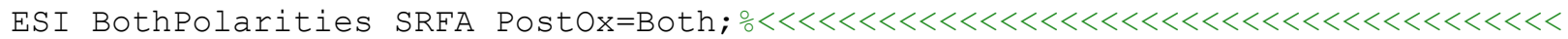
$<<<\overline{<}<<<<<<<<<<<<<<\overline{<}<<<<\overline{<}<<<<<<<<<<<<<<<<<<<<<<<<<<<<<<<<<<<<<<<<<<<<<<<<<<<<<~$ $<<<<<<<<<<<<<<<<<<<<<<<<<<<<<<<<<<<<<<~$

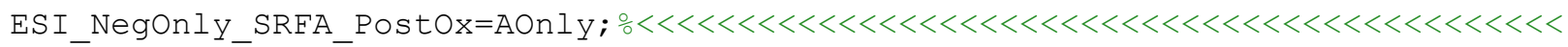
$<<\overline{\bar{l}}<<<<<<<\overline{<}<<<\overline{\bar{l}}<<<<<<<<<<<<<<<<<<<<<<<<<<<<<<<<<<<<<<<<<<<<<<<<<<<<<<<<<<<<$ $<<<<<<<<<<<<<<<<<<<<<<<<<<<<<<<<~$

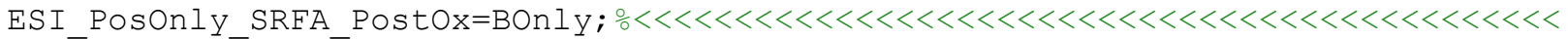

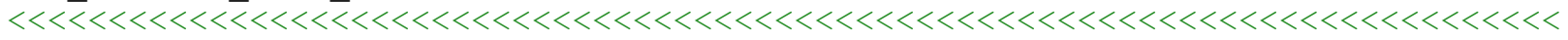
$<<<<<<<<<<<<<<<<<<<<<<<<<<<<<<<<~$

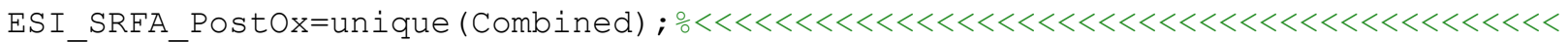
$<<\overline{<}<<<\overline{<}<<<<<<<<<<<<<<<<<<<<<<<<<<<<<<<<<<<<<<<<<<<<<<<<<<<<<<<<<<<<<<<<<<<<~$

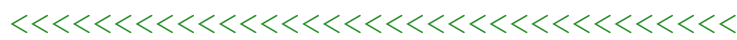

\% Summarizing Data

Data=AOnly;

oCalculating formulae type parameters: \# CHO, CHON, CHOS, CHOP, CHN, CHS, CHP, CHNS, CHONS formulae

Summary $\cdot \mathrm{CHO}=$ sum $($ Data. $\mathrm{C}>0$ \& Data. $\mathrm{H}>0$ \& Data. $\mathrm{O}>0$ \& Data. $\mathrm{N}==0$ \& Data. $\mathrm{P}==0$ \& Data. $\mathrm{S}==0$ );

Summary $\cdot \mathrm{CHON}=\operatorname{sum}($ Data. $\mathrm{C}>0$ \& Data.H $>0$ \& Data. $\mathrm{O}>0$ \& Data. $\mathrm{N}>0$ \& Data.P==0 \& Data. $\mathrm{S}==0$ );

Summary. $\mathrm{CHOS}=$ sum $($ Data. $\mathrm{C}>0$ \& Data.H $>0$ \& Data. $0>0$ \& Data. $\mathrm{N}==0$ \& Data. $\mathrm{P}==0$ \& Data. $\mathrm{S}>0$ );

Summary $\cdot \mathrm{CHOP}=\operatorname{sum}($ Data. $\mathrm{C}>0$ \& Data.H $>0$ \& Data. $\mathrm{O}>0$ \& Data. $\mathrm{N}==0$ \& Data.P $>0$ \& Data. $\mathrm{S}==0$ );

Summary. $\mathrm{CHN}=$ sum $($ Data. $\mathrm{C}>0$ \& Data. $\mathrm{H}>0$ \& Data. $\mathrm{O}==0$ \& Data. $\mathrm{N}>0$ \& Data. $\mathrm{P}==0$ \& Data. $\mathrm{S}==0$ );

Summary. CHS $=$ sum $($ Data. $\mathrm{C}>0$ \& Data. $\mathrm{H}>0$ \& Data. $\mathrm{O}==0$ \& Data. $\mathrm{N}==0$ \& Data. $\mathrm{P}==0$ \&

Data. $\mathrm{S}>0$ );

Summary $\cdot \mathrm{CHNS}=\operatorname{sum}($ Data. $\mathrm{C}>0$ \& Data. $\mathrm{H}>0$ \& Data $\cdot \mathrm{O}==0$ \& Data $\cdot \mathrm{N}>0$ \& Data.P==0 \& Data. S>0);

Summary. $\mathrm{CHNP}=\operatorname{sum}($ Data. $\mathrm{C}>0$ \& Data. $\mathrm{H}>0$ \& Data. $\mathrm{O}==0$ \& Data. $\mathrm{N}>0$ \& Data.P $>0$ \&

Data. $\mathrm{S}=0$ ) ;

Summary. $\mathrm{CHONS}=\operatorname{sum}($ Data. $\mathrm{C}>0$ \& Data. $\mathrm{H}>0$ \& Data. $0>0$ \& Data.N $>0$ \& Data.P==0 \&

Data. S>0); 
Summary. $\mathrm{CHONP}=$ sum $($ Data. $\mathrm{C}>0$ \& Data.H $>0$ \& Data. $0>0$ \& Data.N $>0$ \& Data.P $>0$ \&

Data. $\mathrm{S}==0$ );

Summary. $\mathrm{CHONSP}=$ sum $($ Data. $\mathrm{C}>0$ \& Data.H $>0$ \& Data.O $>0$ \& Data.N $>0$ \& Data.P>0 \& Data. S $>0$ );

ocalculating aromaticity parameters: \# condensed aromatic (AI>=0.67), aromatic $(A I>=0.5)$,

onon-aromatic $($ A I $<0.5)$

Summary. CondensedAromatics $=$ sum (Data. AI $>=0.67)$;

Summary. Aromatics=sum (Data.AI $>=0.5)$;

Summary. NonAromatics=sum (Data.AI<0.5);

ocalculating other parameters: total \# of formulae, average molecular 알ight

Summary. TotalFormulae=height (Data);

Summary. AvgMW=mean (Data. NominalMass);

ostoring Summary Data

ESI_NegOnly_SRFA_PostOx_Summary=struct2table (Summary) ; $\circ<<<<<<<<<<<<<<<<<<<<<<<<~$

$<<\overline{<}<<<<<<<\overline{<}<<<\overline{<}<<<<<\overline{<}<<<<<<<<<<<<<<<<<<<<<<<<<<<<<<<<<<<<<<<<<<<<<<<<<<<<<<~$

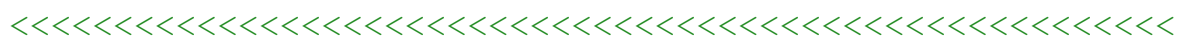

ㅇmmarizing Data

Data=BOnly;

oCalculating formulae type parameters: \# CHO, CHON, CHOS, CHOP, CHN, CHS, CHP, CHNS, CHONS formulae

Summary. $\mathrm{CHO}=\operatorname{sum}($ Data. $\mathrm{C}>0$ \& Data. $\mathrm{H}>0$ \& Data. $\mathrm{O}>0$ \& Data. $\mathrm{N}==0$ \& Data. $\mathrm{P}==0$ \&

Data. $\mathrm{S}==0$ );

Summary $\cdot \mathrm{CHON}=$ sum $($ Data. $\mathrm{C}>0$ \& Data.H $>0$ \& Data. $\mathrm{O}>0$ \& Data. $\mathrm{N}>0$ \& Data.P==0 \&

Data. $\mathrm{S}==0$ );

Summary $\cdot \mathrm{CHOS}=\operatorname{sum}($ Data. $\mathrm{C}>0$ \& Data. $\mathrm{H}>0$ \& Data. $\mathrm{O}>0$ \& Data. $\mathrm{N}==0$ \& Data. $\mathrm{P}==0$ \&

Data. $\mathrm{S}>0)$;

Summary. $\mathrm{CHOP}=\operatorname{sum}($ Data. $\mathrm{C}>0$ \& Data. $\mathrm{H}>0$ \& Data. $\mathrm{O}>0$ \& Data. $\mathrm{N}==0$ \& Data. $\mathrm{P}>0$ \&

Data. $\mathrm{S}==0$ );

Summary. $\mathrm{CHN}=$ sum $($ Data. $\mathrm{C}>0$ \& Data. $\mathrm{H}>0$ \& Data. $\mathrm{O}==0$ \& Data. $\mathrm{N}>0$ \& Data. $\mathrm{P}==0$ \&

Data. $\mathrm{S}==0$ );

Summary $\cdot \mathrm{CHS}=\operatorname{sum}($ Data $\cdot \mathrm{C}>0$ \& Data. $\mathrm{H}>0$ \& Data. $\mathrm{O}==0$ \& Data. $\mathrm{N}==0$ \& Data. $\mathrm{P}==0$ \&

Data. $\mathrm{S}>0$ );

Summary. $\mathrm{CHNS}=$ sum $($ Data. $\mathrm{C}>0$ \& Data. $\mathrm{H}>0$ \& Data. $\mathrm{O}==0$ \& Data. $\mathrm{N}>0$ \& Data. $\mathrm{P}==0$ \&

Data. S>0);

Summary $\cdot \mathrm{CHNP}=\operatorname{sum}($ Data. $\mathrm{C}>0$ \& Data. $\mathrm{H}>0$ \& Data. $\mathrm{O}==0$ \& Data. $\mathrm{N}>0$ \& Data.P $>0$ \&

Data. $\mathrm{S}==0$ ) ;

Summary. $\mathrm{CHONS}=\operatorname{sum}($ Data. $\mathrm{C}>0$ \& Data. $\mathrm{H}>0$ \& Data. $0>0$ \& Data.N $>0$ \& Data.P==0 \&

Data. $\mathrm{S}>0$ ) ;

Summary $\cdot \mathrm{CHONP}=$ sum $($ Data. $\mathrm{C}>0$ \& Data.H $>0$ \& Data. O $>0$ \& Data.N $>0$ \& Data.P $>0$ \&

Data. $\mathrm{S}==0$ ) ;

Summary. CHONSP $=$ sum $($ Data. $\mathrm{C}>0$ \& Data. $\mathrm{H}>0$ \& Data. $\mathrm{O}>0$ \& Data.N $>0$ \& Data.P $>0$ \&

Data. $\mathrm{S}>0)$;

oCalculating aromaticity parameters: \# condensed aromatic (AI>=0.67), aromatic $(\mathrm{AI}>=0.5)$,

onon-aromatic $(\mathrm{AI}<0.5)$

Summary. CondensedAromatics $=$ sum (Data. AI $>=0.67)$;

Summary. Aromatics $=$ sum (Data.AI $>=0.5)$;

Summary. NonAromatics=sum (Data.AI<0.5);

ocalculating other parameters: total \# of formulae, average molecular

oweight

Summary. TotalFormulae=height (Data);

Summary. AvgMW=mean (Data. NominalMass);

ostoring Summary Data 
ESI_PosOnIy_SRFA_PostOx_Summary=struct2table (Summary) ; $\circ<<<<<<<<<<<<<<<<<<<<<<<~$ $<<\overline{<}<<<<<<<\overline{<}<<<\overline{<}<<<<<<\overline{<}<<<<<<<<<<<<<<<<<<<<<<<<<<<<<<<<<<<<<<<<<<<<<<<<<<<<<<$

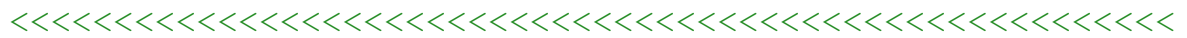

ㅇumarizing Data

Data=Both;

oCalculating formulae type parameters: \# CHO, CHON, CHOS, CHOP, CHN, CHS, CHP, CHNS, CHONS formulae

Summary $\cdot \mathrm{CHO}=$ sum $($ Data. $\mathrm{C}>0$ \& Data. $\mathrm{H}>0$ \& Data. $\mathrm{O}>0$ \& Data. $\mathrm{N}==0$ \& Data. $\mathrm{P}==0$ \&

Data. $\mathrm{S}==0$ );

Summary $\cdot \mathrm{CHON}=$ sum $($ Data. $\mathrm{C}>0$ \& Data.H $>0$ \& Data. $\mathrm{O}>0$ \& Data. $\mathrm{N}>0$ \& Data.P==0 \&

Data. $\mathrm{S}==0$ );

Summary. $\mathrm{CHOS}=$ sum $($ Data. $\mathrm{C}>0$ \& Data. $\mathrm{H}>0$ \& Data. $\mathrm{O}>0$ \& Data. $\mathrm{N}==0$ \& Data. $\mathrm{P}==0$ \&

Data. $\mathrm{S}>0$ );

Summary. $\mathrm{CHOP}=\mathrm{sum}($ Data. $\mathrm{C}>0$ \& Data.H $>0$ \& Data. $0>0$ \& Data. $\mathrm{N}==0$ \& Data.P $>0$ \&

Data. $\mathrm{S}==0$ );

Summary $\cdot \mathrm{CHN}=$ sum $($ Data. $\mathrm{C}>0$ \& Data. $\mathrm{H}>0$ \& Data. $\mathrm{O}==0$ \& Data. $\mathrm{N}>0$ \& Data. $\mathrm{P}==0$ \&

Data. $\mathrm{S}=0$ ) ;

Summary $\cdot \mathrm{CHS}=\operatorname{sum}($ Data $\cdot \mathrm{C}>0$ \& Data. $\mathrm{H}>0$ \& Data. $\mathrm{O}==0$ \& Data. $\mathrm{N}==0$ \& Data. $\mathrm{P}==0$ \&

Data. $\mathrm{S}>0$ ) ;

Summary. $\mathrm{CHNS}=$ sum $($ Data. $\mathrm{C}>0$ \& Data. $\mathrm{H}>0$ \& Data. $\mathrm{O}==0$ \& Data. $\mathrm{N}>0$ \& Data. $\mathrm{P}==0$ \&

Data. S $>0$ );

Summary $\cdot \mathrm{CHNP}=\mathrm{sum}($ Data. $\mathrm{C}>0$ \& Data.H $>0$ \& Data. $\mathrm{O}==0$ \& Data. $\mathrm{N}>0$ \& Data.P $>0$ \&

Data. $\mathrm{S}==0$ );

Summary. $\mathrm{CHONS}=\operatorname{sum}($ Data. $\mathrm{C}>0$ \& Data. $\mathrm{H}>0$ \& Data. $0>0$ \& Data.N>0 \& Data.P==0 \&

Data. S>0);

Summary. $\mathrm{CHONP}=\operatorname{sum}($ Data. $\mathrm{C}>0$ \& Data.H $>0$ \& Data. O $>0$ \& Data.N $>0$ \& Data.P $>0$ \&

Data. $\mathrm{S}==0$ );

Summary. $\mathrm{CHONSP}=$ sum $($ Data. $\mathrm{C}>0$ \& Data.H $>0$ \& Data.O $>0$ \& Data.N $>0$ \& Data.P>0 \&

Data. $\mathrm{S}>0)$;

ocalculating aromaticity parameters: \# condensed aromatic (AI>=0.67),

aromatic $(\mathrm{AI}>=0.5)$,

onon-aromatic $(\mathrm{AI}<0.5)$

Summary. CondensedAromatics $=$ sum (Data. AI $>=0.67)$;

Summary. Aromatics $=$ sum (Data.AI $>=0.5)$;

Summary. NonAromatics=sum (Data.AI<0.5);

ocalculating other parameters: total \# of formulae, average molecular

oweight

Summary.TotalFormulae=height (Data);

Summary. AvgMW=mean (Data. NominalMass);

ostoring Summary Data

ESI_BothPolarities_SRFA_PostOx_Summary=struct2table (Summary); $\circ 0<<<<<<<<<<<<<<<$

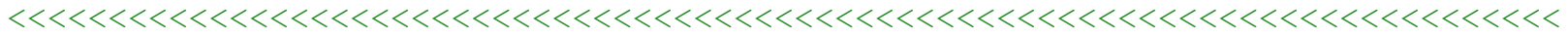

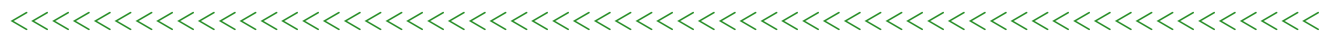

․ Summarizing Data

Data $=$ Combined;

oCalculating formulae type parameters: \# CHO, CHON, CHOS, CHOP, CHN, CHS, CHP, CHNS, CHONS formulae

Summary. $\mathrm{CHO}=\operatorname{sum}($ Data. $\mathrm{C}>0$ \& Data. $\mathrm{H}>0$ \& Data. $\mathrm{O}>0$ \& Data. $\mathrm{N}==0$ \& Data. $\mathrm{P}==0$ \& Data. $\mathrm{S}=0$ ) ;

Summary. $\mathrm{CHON}=\operatorname{sum}($ Data. $\mathrm{C}>0$ \& Data. $\mathrm{H}>0$ \& Data. $\mathrm{O}>0$ \& Data.N $>0$ \& Data.P==0 \&

Data. $\mathrm{S}==0$ );

Summary $\cdot \mathrm{CHOS}=\operatorname{sum}($ Data. $\mathrm{C}>0$ \& Data. $\mathrm{H}>0$ \& Data. $\mathrm{O}>0$ \& Data. $\mathrm{N}==0$ \& Data. $\mathrm{P}==0$ \&

Data. $\mathrm{S}>0$ ) ;

Summary $\cdot \mathrm{CHOP}=\operatorname{sum}($ Data. $\mathrm{C}>0$ \& Data.H $>0$ \& Data. $\mathrm{O}>0$ \& Data. $\mathrm{N}==0$ \& Data.P $>0$ \&

Data. $\mathrm{S}=0$ ) ;

Summary. $\mathrm{CHN}=\operatorname{sum}($ Data. $\mathrm{C}>0$ \& Data. $\mathrm{H}>0$ \& Data. $\mathrm{O}==0$ \& Data. $\mathrm{N}>0$ \& Data. $\mathrm{P}==0$ \&

Data. $\mathrm{S}==0$ ) ; 


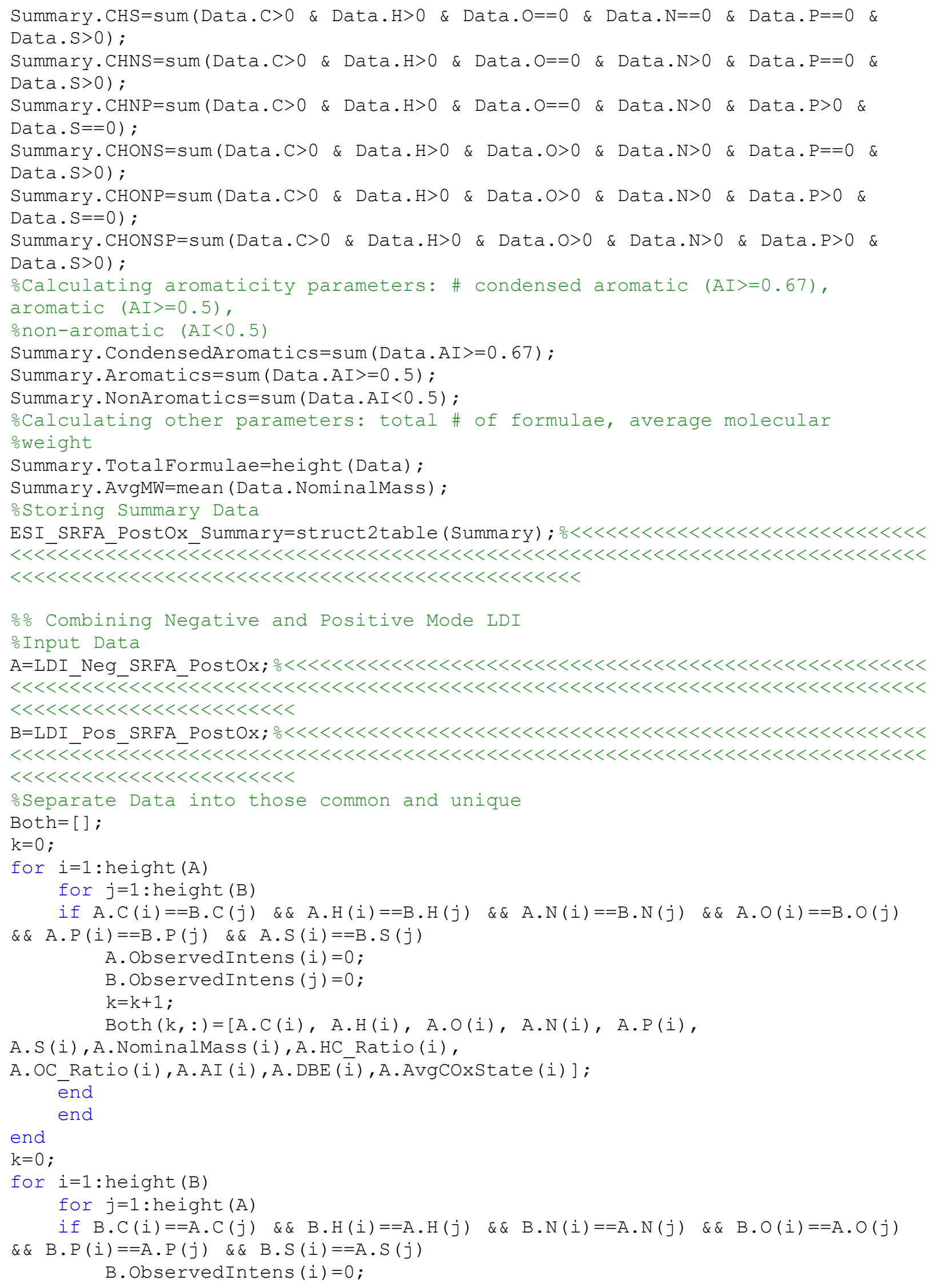


A. ObservedIntens $(j)=0$;

$\mathrm{k}=\mathrm{k}+1$;

Both $(k,:)=[B . C(i), B . H(i), B . O(i), B . N(i), B . P(i)$,

B.S (i), B.NominalMass (i), B.HC Ratio(i),

B.OC_Ratio(i),B.AI (i),B.DBE (i) , B.AvgCoxState (i) ] ;

end

end

end

oCombining Data Sets

$A(A$. Observedintens $==0,:)=[]$;

$A O n l_{Y}=[A . C, A . H, A . O, A . N, A . P, A . S$,

A. Nominalmass, A.HC Ratio,A.oC Ratio, A.AI, A.DBE, A.AvgCoxState];

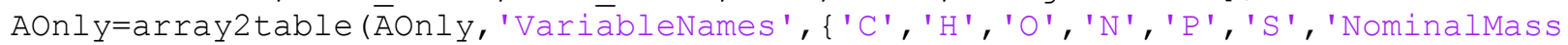
', 'HC_Ratio', 'OC_Ratio', 'AI', 'DBE', 'AvgCOxState'\}) ;

$\mathrm{B}(\mathrm{B} . \mathrm{Ob}$ servedinten $\mathrm{s}==0,:)=[]$;

$B O n l_{Y}=[B . C, B . H, B . O, B . N, B . P$,

B.S,B.NominalMass,B.HC_Ratio,B.OC_Ratio,B.AI, B.DBE, B.AvgCoxState] ;

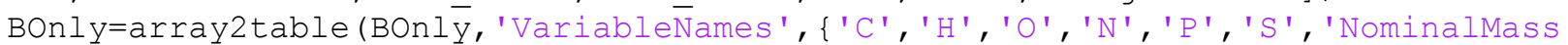
', 'HC_Ratio', 'OC_Ratio', 'AI', 'DBE', 'AvgCOxState'\}) ;

Both=array2table (Both, 'VariableNames', \{'C', 'H', 'O' , 'N', 'P' , 'S ', 'NominalMass', 'HC_Ratio', 'OC_Ratio', 'AI', 'DBE', 'AvgCOxState' \}) ;

Combined=[Both; AOnly; BOnly];

ostoring Data with distinct names

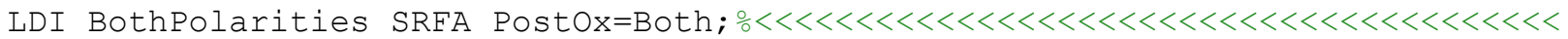
$<<\overline{<}<<<<<<<<<<<<<<<\overline{<}<<<<\overline{<}<<<<<<<<<<<<<<<<<<<<<<<<<<<<<<<<<<<<<<<<<<<<<<<<<<<<<~$

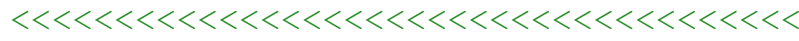

LDI_NegOnly_SRFA_PostOx=AOnIy; $\frac{\circ}{\circ}<<<<<<<<<<<<<<<<<<<<<<<<<<<<<<<<<<<<<<<<<<<<<<<~$ $<<\overline{\bar{l}}<<<<<<<\overline{<}<<<\overline{\bar{l}}<<<<<<<<<<<<<<<<<<<<<<<<<<<<<<<<<<<<<<<<<<<<<<<<<<<<<<<<<<<<$ $<<<<<<<<<<<<<<<<<<<<<<<<<<<<<<<<~$

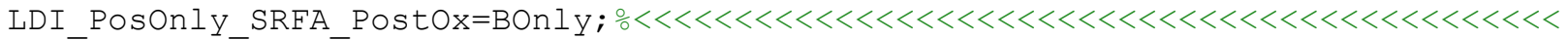
$<<\overline{\bar{l}}<<<<<<<\overline{<}<<<\overline{<}<<<<<<<<<<<<<<<<<<<<<<<<<<<<<<<<<<<<<<<<<<<<<<<<<<<<<<<<<<<<$ $<<<<<<<<<<<<<<<<<<<<<<<<<<<<<<<<~$

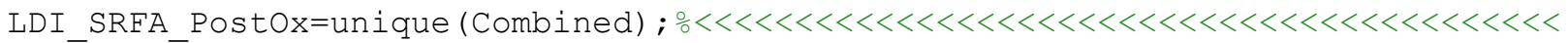
$<<\overline{<}<<<\overline{<}<<<<<<<<<<<<<<<<<<<<<<<<<<<<<<<<<<<<<<<<<<<<<<<<<<<<<<<<<<<<<<<<<<<<~$

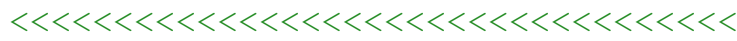

\% Summarizing Data

Data=AOnly;

oCalculating formulae type parameters: \# CHO, CHON, CHOS, CHOP, CHN, CHS, CHP, CHNS, CHONS formulae

Summary $\cdot \mathrm{CHO}=$ sum $($ Data. $\mathrm{C}>0$ \& Data. $\mathrm{H}>0$ \& Data. $\mathrm{O}>0$ \& Data. $\mathrm{N}==0$ \& Data. $\mathrm{P}==0$ \& Data. $\mathrm{S}==0$ );

Summary $\cdot \mathrm{CHON}=\operatorname{sum}($ Data. $\mathrm{C}>0$ \& Data.H $>0$ \& Data. $\mathrm{O}>0$ \& Data. $\mathrm{N}>0$ \& Data.P==0 \& Data. $\mathrm{S}==0$ );

Summary. $\mathrm{CHOS}=$ sum $($ Data. $\mathrm{C}>0$ \& Data.H $>0$ \& Data. $0>0$ \& Data. $\mathrm{N}==0$ \& Data. $\mathrm{P}==0$ \& Data. $\mathrm{S}>0$ );

Summary $\cdot \mathrm{CHOP}=\mathrm{sum}($ Data. $\mathrm{C}>0$ \& Data.H $>0$ \& Data. $\mathrm{O}>0$ \& Data. $\mathrm{N}==0$ \& Data.P $>0$ \& Data. $\mathrm{S}==0$ );

Summary. $\mathrm{CHN}=$ sum $($ Data. $\mathrm{C}>0$ \& Data. $\mathrm{H}>0$ \& Data. $\mathrm{O}==0$ \& Data. $\mathrm{N}>0$ \& Data. $\mathrm{P}==0$ \& Data. $\mathrm{S}==0$ );

Summary $\cdot \mathrm{CHS}=\operatorname{sum}($ Data $\cdot \mathrm{C}>0$ \& Data. $\mathrm{H}>0$ \& Data. $\mathrm{O}==0$ \& Data. $\mathrm{N}==0$ \& Data. $\mathrm{P}==0$ \&

Data. $\mathrm{S}>0$ );

Summary $\cdot \mathrm{CHNS}=\operatorname{sum}($ Data. $\mathrm{C}>0$ \& Data. $\mathrm{H}>0$ \& Data. $\mathrm{O}==0$ \& Data. $\mathrm{N}>0$ \& Data. $\mathrm{P}==0$ \& Data. S>0);

Summary. $\mathrm{CHNP}=\operatorname{sum}($ Data. $\mathrm{C}>0$ \& Data. $\mathrm{H}>0$ \& Data. $\mathrm{O}==0$ \& Data. $\mathrm{N}>0$ \& Data.P $>0$ \&

Data. $\mathrm{S}=0$ ) ;

Summary. $\mathrm{CHONS}=\operatorname{sum}($ Data $. \mathrm{C}>0$ \& Data. $\mathrm{H}>0$ \& Data. $0>0$ \& Data. $>0$ \& Data.P==0 \&

Data. S>0); 
Summary. $\mathrm{CHONP}=$ sum $($ Data. $\mathrm{C}>0$ \& Data.H $>0$ \& Data. $0>0$ \& Data.N $>0$ \& Data.P $>0$ \&

Data. $\mathrm{S}==0$ );

Summary. $\mathrm{CHONSP}=$ sum $($ Data. $\mathrm{C}>0$ \& Data.H $>0$ \& Data.O $>0$ \& Data.N $>0$ \& Data.P>0 \& Data. S $>0$ );

ocalculating aromaticity parameters: \# condensed aromatic (AI>=0.67), aromatic $(A I>=0.5)$,

onon-aromatic $($ A I $<0.5)$

Summary. CondensedAromatics $=$ sum (Data. AI $>=0.67)$;

Summary. Aromatics=sum (Data.AI $>=0.5)$;

Summary. NonAromatics=sum (Data.AI<0.5);

ocalculating other parameters: total \# of formulae, average molecular 알ight

Summary. TotalFormulae=height (Data);

Summary. AvgMW=mean (Data. NominalMass);

ostoring Summary Data

LDI_NegOnly_SRFA_PostOx_Summary=struct2table (Summary) ; $\circ<<<<<<<<<<<<<<<<<<<<<<<<~$

$<<\overline{<}<<<<<<<\overline{<}<<<\overline{<}<<<<<\overline{<}<<<<<<<<<<<<<<<<<<<<<<<<<<<<<<<<<<<<<<<<<<<<<<<<<<<<<<~$

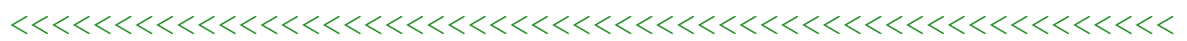

ㅇmmarizing Data

Data=BOnly;

oCalculating formulae type parameters: \# CHO, CHON, CHOS, CHOP, CHN, CHS, CHP, CHNS, CHONS formulae

Summary. $\mathrm{CHO}=\operatorname{sum}($ Data. $\mathrm{C}>0$ \& Data. $\mathrm{H}>0$ \& Data. $\mathrm{O}>0$ \& Data. $\mathrm{N}==0$ \& Data. $\mathrm{P}==0$ \&

Data. $\mathrm{S}==0$ );

Summary $\cdot \mathrm{CHON}=$ sum $($ Data. $\mathrm{C}>0$ \& Data.H $>0$ \& Data. $\mathrm{O}>0$ \& Data. $\mathrm{N}>0$ \& Data.P==0 \&

Data. $\mathrm{S}==0$ );

Summary $\cdot \mathrm{CHOS}=\operatorname{sum}($ Data. $\mathrm{C}>0$ \& Data. $\mathrm{H}>0$ \& Data. $\mathrm{O}>0$ \& Data. $\mathrm{N}==0$ \& Data. $\mathrm{P}==0$ \&

Data. $\mathrm{S}>0)$;

Summary. $\mathrm{CHOP}=\operatorname{sum}($ Data. $\mathrm{C}>0$ \& Data. $\mathrm{H}>0$ \& Data. $\mathrm{O}>0$ \& Data. $\mathrm{N}==0$ \& Data. $\mathrm{P}>0$ \&

Data. $\mathrm{S}==0$ );

Summary. $\mathrm{CHN}=$ sum $($ Data. $\mathrm{C}>0$ \& Data. $\mathrm{H}>0$ \& Data. $\mathrm{O}==0$ \& Data. $\mathrm{N}>0$ \& Data. $\mathrm{P}==0$ \&

Data. $\mathrm{S}==0$ );

Summary $\cdot \mathrm{CHS}=\operatorname{sum}($ Data $\cdot \mathrm{C}>0$ \& Data. $\mathrm{H}>0$ \& Data. $\mathrm{O}==0$ \& Data. $\mathrm{N}==0$ \& Data. $\mathrm{P}==0$ \&

Data. $\mathrm{S}>0$ );

Summary. $\mathrm{CHNS}=$ sum $($ Data. $\mathrm{C}>0$ \& Data. $\mathrm{H}>0$ \& Data. $\mathrm{O}==0$ \& Data. $\mathrm{N}>0$ \& Data. $\mathrm{P}==0$ \&

Data. S>0);

Summary $\cdot \mathrm{CHNP}=\operatorname{sum}($ Data. $\mathrm{C}>0$ \& Data. $\mathrm{H}>0$ \& Data. $\mathrm{O}==0$ \& Data. $\mathrm{N}>0$ \& Data.P $>0$ \&

Data. $\mathrm{S}==0$ ) ;

Summary. $\mathrm{CHONS}=\operatorname{sum}($ Data. $\mathrm{C}>0$ \& Data. $\mathrm{H}>0$ \& Data. $0>0$ \& Data.N $>0$ \& Data.P==0 \&

Data. $\mathrm{S}>0$ ) ;

Summary $\cdot \mathrm{CHONP}=$ sum $($ Data. $\mathrm{C}>0$ \& Data.H $>0$ \& Data. O $>0$ \& Data.N $>0$ \& Data.P $>0$ \&

Data. $\mathrm{S}==0$ ) ;

Summary. CHONSP $=$ sum $($ Data. $\mathrm{C}>0$ \& Data. $\mathrm{H}>0$ \& Data. $\mathrm{O}>0$ \& Data.N $>0$ \& Data.P $>0$ \&

Data. $\mathrm{S}>0)$;

oCalculating aromaticity parameters: \# condensed aromatic (AI>=0.67), aromatic $(\mathrm{AI}>=0.5)$,

onon-aromatic $(\mathrm{AI}<0.5)$

Summary. CondensedAromatics $=$ sum (Data. AI $>=0.67)$;

Summary. Aromatics $=$ sum (Data.AI $>=0.5)$;

Summary. NonAromatics=sum (Data.AI<0.5);

ocalculating other parameters: total \# of formulae, average molecular

oweight

Summary. TotalFormulae=height (Data);

Summary. AvgMW=mean (Data. NominalMass);

ostoring Summary Data 
LDI_PosOnIy_SRFA_PostOx_Summary=struct2table (Summary) ; $\circ<<<<<<<<<<<<<<<<<<<<<<<~$ $<<\overline{<}<<<<<<<\overline{<}<<<\overline{\bar{l}}<<<<<\overline{<} \overline{<}<<<<<<<<<<<<<<<<<<<<<<<<<<<<<<<<<<<<<<<<<<<<<<<<<<<<<$

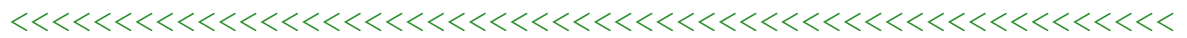

ㅇumarizing Data

Data=Both;

oCalculating formulae type parameters: \# CHO, CHON, CHOS, CHOP, CHN, CHS, CHP, CHNS, CHONS formulae

Summary $\cdot \mathrm{CHO}=$ sum $($ Data. $\mathrm{C}>0$ \& Data. $\mathrm{H}>0$ \& Data. $\mathrm{O}>0$ \& Data. $\mathrm{N}==0$ \& Data. $\mathrm{P}==0$ \&

Data. $\mathrm{S}==0$ );

Summary $\cdot \mathrm{CHON}=$ sum $($ Data. $\mathrm{C}>0$ \& Data.H $>0$ \& Data. $\mathrm{O}>0$ \& Data. $\mathrm{N}>0$ \& Data.P==0 \&

Data. $\mathrm{S}==0$ );

Summary. $\mathrm{CHOS}=$ sum $($ Data. $\mathrm{C}>0$ \& Data. $\mathrm{H}>0$ \& Data. $\mathrm{O}>0$ \& Data. $\mathrm{N}==0$ \& Data. $\mathrm{P}==0$ \&

Data. $\mathrm{S}>0$ );

Summary. $\mathrm{CHOP}=\mathrm{sum}($ Data. $\mathrm{C}>0$ \& Data.H $>0$ \& Data. $0>0$ \& Data. $\mathrm{N}==0$ \& Data.P $>0$ \&

Data. $\mathrm{S}==0$ );

Summary $\cdot \mathrm{CHN}=$ sum $($ Data. $\mathrm{C}>0$ \& Data. $\mathrm{H}>0$ \& Data. $\mathrm{O}==0$ \& Data. $\mathrm{N}>0$ \& Data. $\mathrm{P}==0$ \&

Data. $\mathrm{S}=0$ ) ;

Summary $\cdot \mathrm{CHS}=\operatorname{sum}($ Data $\cdot \mathrm{C}>0$ \& Data. $\mathrm{H}>0$ \& Data. $\mathrm{O}==0$ \& Data. $\mathrm{N}==0$ \& Data. $\mathrm{P}==0$ \&

Data. $\mathrm{S}>0$ ) ;

Summary. $\mathrm{CHNS}=$ sum $($ Data. $\mathrm{C}>0$ \& Data. $\mathrm{H}>0$ \& Data. $\mathrm{O}==0$ \& Data. $\mathrm{N}>0$ \& Data. $\mathrm{P}==0$ \&

Data. S $>0$ );

Summary $\cdot \mathrm{CHNP}=\operatorname{sum}($ Data. $\mathrm{C}>0$ \& Data.H $>0$ \& Data. $\mathrm{O}==0$ \& Data. $\mathrm{N}>0$ \& Data.P $>0$ \&

Data. $\mathrm{S}==0$ );

Summary. $\mathrm{CHONS}=\operatorname{sum}($ Data. $\mathrm{C}>0$ \& Data. $\mathrm{H}>0$ \& Data. $0>0$ \& Data.N>0 \& Data.P==0 \&

Data. S>0);

Summary. $\mathrm{CHONP}=\operatorname{sum}($ Data. $\mathrm{C}>0$ \& Data.H $>0$ \& Data. O $>0$ \& Data.N $>0$ \& Data.P $>0$ \&

Data. $\mathrm{S}==0$ );

Summary. $\mathrm{CHONSP}=$ sum $($ Data. $\mathrm{C}>0$ \& Data.H $>0$ \& Data.O $>0$ \& Data.N $>0$ \& Data.P>0 \&

Data. $\mathrm{S}>0)$;

ocalculating aromaticity parameters: \# condensed aromatic (AI>=0.67),

aromatic $(\mathrm{AI}>=0.5)$,

onon-aromatic $(\mathrm{A} I<0.5)$

Summary. CondensedAromatics $=$ sum (Data. AI $>=0.67)$;

Summary. Aromatics $=$ sum (Data.AI $>=0.5)$;

Summary. NonAromatics=sum (Data.AI<0.5);

ocalculating other parameters: total \# of formulae, average molecular

oweight

Summary.TotalFormulae=height (Data);

Summary. AvgMW=mean (Data. NominalMass);

ostoring Summary Data

LDI_BothPolarities_SRFA_Postox_Summary=struct2table (Summary); $\circ$ o $<<<<<<<<<<<<<<<$

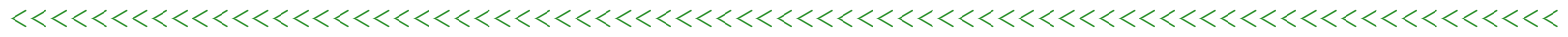

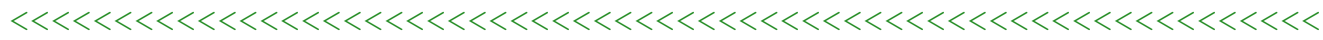

․ Summarizing Data

Data $=$ Combined;

oCalculating formulae type parameters: \# CHO, CHON, CHOS, CHOP, CHN, CHS, CHP, CHNS, CHONS formulae

Summary. $\mathrm{CHO}=\operatorname{sum}($ Data. $\mathrm{C}>0$ \& Data. $\mathrm{H}>0$ \& Data. $\mathrm{O}>0$ \& Data. $\mathrm{N}==0$ \& Data. $\mathrm{P}==0$ \& Data. $\mathrm{S}=0$ ) ;

Summary. $\mathrm{CHON}=\operatorname{sum}($ Data. $\mathrm{C}>0$ \& Data. $\mathrm{H}>0$ \& Data. $\mathrm{O}>0$ \& Data.N $>0$ \& Data.P==0 \&

Data. $\mathrm{S}==0$ );

Summary $\cdot \mathrm{CHOS}=$ sum $($ Data. $\mathrm{C}>0$ \& Data.H $>0$ \& Data. $\mathrm{O}>0$ \& Data. $\mathrm{N}==0$ \& Data.P==0 \& Data. $\mathrm{S}>0$ ) ;

Summary $\cdot \mathrm{CHOP}=\operatorname{sum}($ Data. $\mathrm{C}>0$ \& Data.H $>0$ \& Data. $\mathrm{O}>0$ \& Data. $\mathrm{N}==0$ \& Data.P $>0$ \&

Data. $\mathrm{S}=0$ ) ;

Summary. $\mathrm{CHN}=\operatorname{sum}($ Data. $\mathrm{C}>0$ \& Data. $\mathrm{H}>0$ \& Data. $\mathrm{O}==0$ \& Data. $\mathrm{N}>0$ \& Data. $\mathrm{P}==0$ \&

Data. $\mathrm{S}==0$ ); 


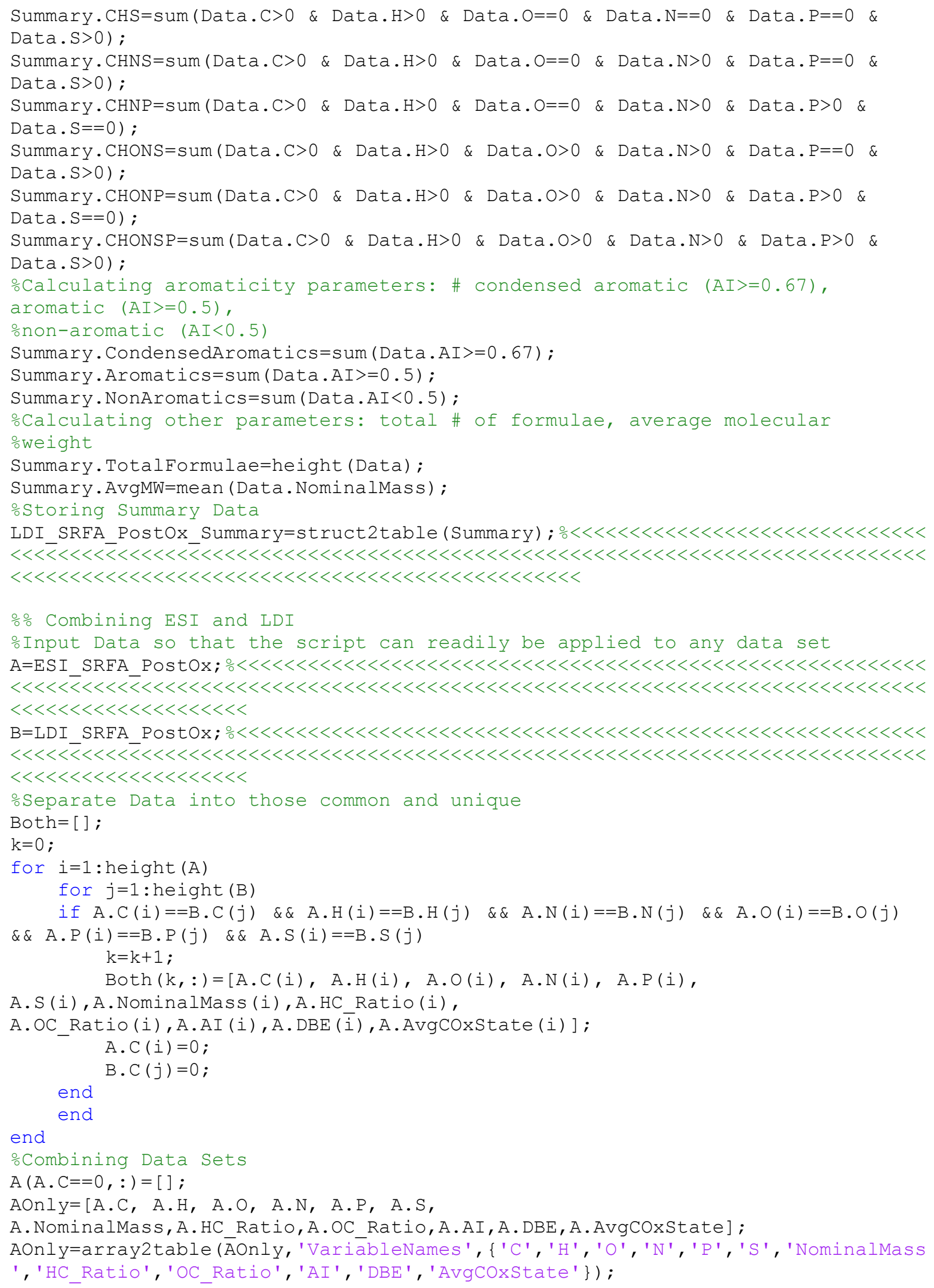


$\mathrm{B}(\mathrm{B} . \mathrm{C}==0,:)=[]$;

$\mathrm{BOnl} Y=[\mathrm{B} . \mathrm{C}, \mathrm{B} . \mathrm{H}, \mathrm{B} . \mathrm{O}, \mathrm{B} . \mathrm{N}, \mathrm{B} . \mathrm{P}$,

B.S,B.NominalMass,B.HC_Ratio,B.OC_Ratio, B.AI, B.DBE, B.AvgCoxState];

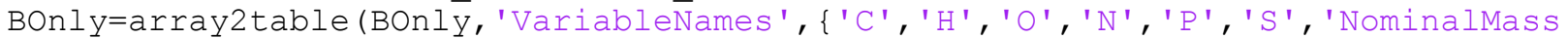

', 'HC_Ratio', 'OC_Ratio', 'AI ', 'DBE', 'AvgCoxstate' \}) ;

Both=äray2table (Both, 'VariableNames' , \{'C', 'H', 'O', 'N', 'P' , 'S ', 'NominalMass',

'HC_Ratio', 'oC_Ratio', 'AI', 'DBE', 'AvgCOxState'\});

Combined=[Both; AOnly; BOnly];

ostoring Data with distinct names

BothTechniques_SRFA_PostOx=unique (Both) ; 응 $<<<<<<<<<<<<<<<<<<<<<<<<<<<<<<<<<<<<<<~$

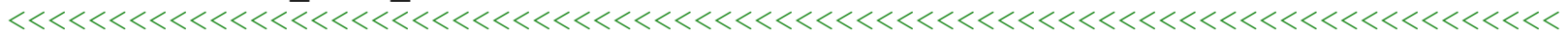

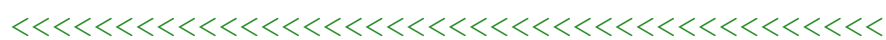

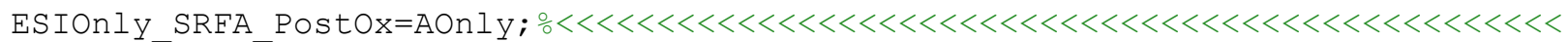

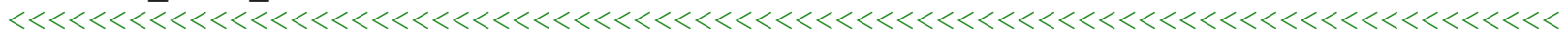
$<<<<<<<<<<<<<<<<<<<<<<<<<<<<~$

LDIOnIy_SRFA_PostOx=BOnIy; $\circ<<<<<<<<<<<<<<<<<<<<<<<<<<<<<<<<<<<<<<<<<<<<<<<<<<<<~$ $<<<<<<<\bar{l}<<<\overline{<}<<<<<<<<<<<<<<<<<<<<<<<<<<<<<<<<<<<<<<<<<<<<<<<<<<<<<<<<<<<<<<<<~$ $<<<<<<<<<<<<<<<<<<<<<<<<<<<<~$

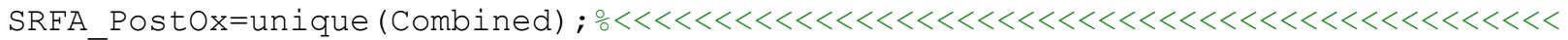

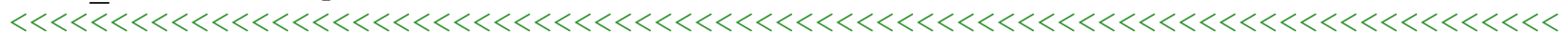
$<<<<<<<<<<<<<<<<<<<<<<<<<<<<<<~$

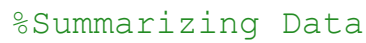

Data=AOnly;

oCalculating formulae type parameters: \# CHO, CHON, CHOS, CHOP, CHN, CHS, CHP, CHNS, CHONS formulae

Summary $\cdot \mathrm{CHO}=\operatorname{sum}($ Data. $\mathrm{C}>0$ \& Data. $\mathrm{H}>0$ \& Data.O $>0$ \& Data. $\mathrm{N}==0$ \& Data.P==0 \& Data. $\mathrm{S}==0$ );

Summary. $\mathrm{CHON}=$ sum $($ Data. $\mathrm{C}>0$ \& Data. $\mathrm{H}>0$ \& Data. O $>0$ \& Data.N $>0$ \& Data.P==0 \&

Data. $\mathrm{S}==0$ );

Summary. $\mathrm{CHOS}=$ sum $($ Data. $\mathrm{C}>0$ \& Data. $\mathrm{H}>0$ \& Data. $\mathrm{O}>0$ \& Data. $\mathrm{N}==0$ \& Data. $\mathrm{P}==0$ \& Data. $\mathrm{S}>0$ );

Summary. $\mathrm{CHOP}=\operatorname{sum}($ Data. $\mathrm{C}>0$ \& Data. $\mathrm{H}>0$ \& Data. $\mathrm{O}>0$ \& Data. $\mathrm{N}==0$ \& Data. $\mathrm{P}>0$ \&

Data. $\mathrm{S}=0$ ) ;

Summary. $\mathrm{CHN}=$ sum $($ Data. $\mathrm{C}>0$ \& Data. $\mathrm{H}>0$ \& Data. $\mathrm{O}==0$ \& Data. $\mathrm{N}>0$ \& Data. $\mathrm{P}==0$ \&

Data. $\mathrm{S}==0$ );

Summary $\cdot \mathrm{CHS}=\operatorname{sum}($ Data. $\mathrm{C}>0$ \& Data. $\mathrm{H}>0$ \& Data. $\mathrm{O}==0$ \& Data. $\mathrm{N}==0$ \& Data. $\mathrm{P}==0$ \&

Data. S>0);

Summary $\cdot \mathrm{CHNS}=\operatorname{sum}($ Data. $\mathrm{C}>0$ \& Data. $\mathrm{H}>0$ \& Data. $\mathrm{O}==0$ \& Data. $\mathrm{N}>0$ \& Data.P==0 \&

Data. $\mathrm{S}>0$ );

Summary. $\mathrm{CHNP}=\operatorname{sum}($ Data. $\mathrm{C}>0$ \& Data. $\mathrm{H}>0$ \& Data. $\mathrm{O}==0$ \& Data.N $>0$ \& Data.P $>0$ \&

Data. $\mathrm{S}==0$ );

Summary $\cdot \mathrm{CHONS}=\operatorname{sum}($ Data $\cdot \mathrm{C}>0$ \& Data. $\mathrm{H}>0$ \& Data. $\mathrm{O}>0$ \& Data.N $>0$ \& Data.P==0 \&

Data. $\mathrm{S}>0$ ) ;

Summary. $\mathrm{CHONP}=$ sum (Data. $\mathrm{C}>0$ \& Data.H>0 \& Data.O $>0$ \& Data.N $>0$ \& Data.P $>0$ \&

Data. $\mathrm{S}==0$ ) ;

Summary. $\mathrm{CHONSP}=$ sum $($ Data. $\mathrm{C}>0$ \& Data.H $>0$ \& Data.O $>0$ \& Data.N $>0$ \& Data.P>0 \&

Data. $\mathrm{S}>0$ );

ocalculating aromaticity parameters: \# condensed aromatic (AI>=0.67),

aromatic $(\mathrm{AI}>=0.5)$,

onon-aromatic $(\mathrm{AI}<0.5)$

Summary. CondensedAromatics $=$ sum (Data. AI $>=0.67)$;

Summary.Aromatics=sum (Data.AI>=0.5);

Summary. NonAromatics=sum (Data.AI<0.5);

ocalculating other parameters: total \# of formulae, average molecular \%weight

Summary. TotalFormulae=height (Data);

Summary. AvgMW=mean (Data. NominalMass) ; 


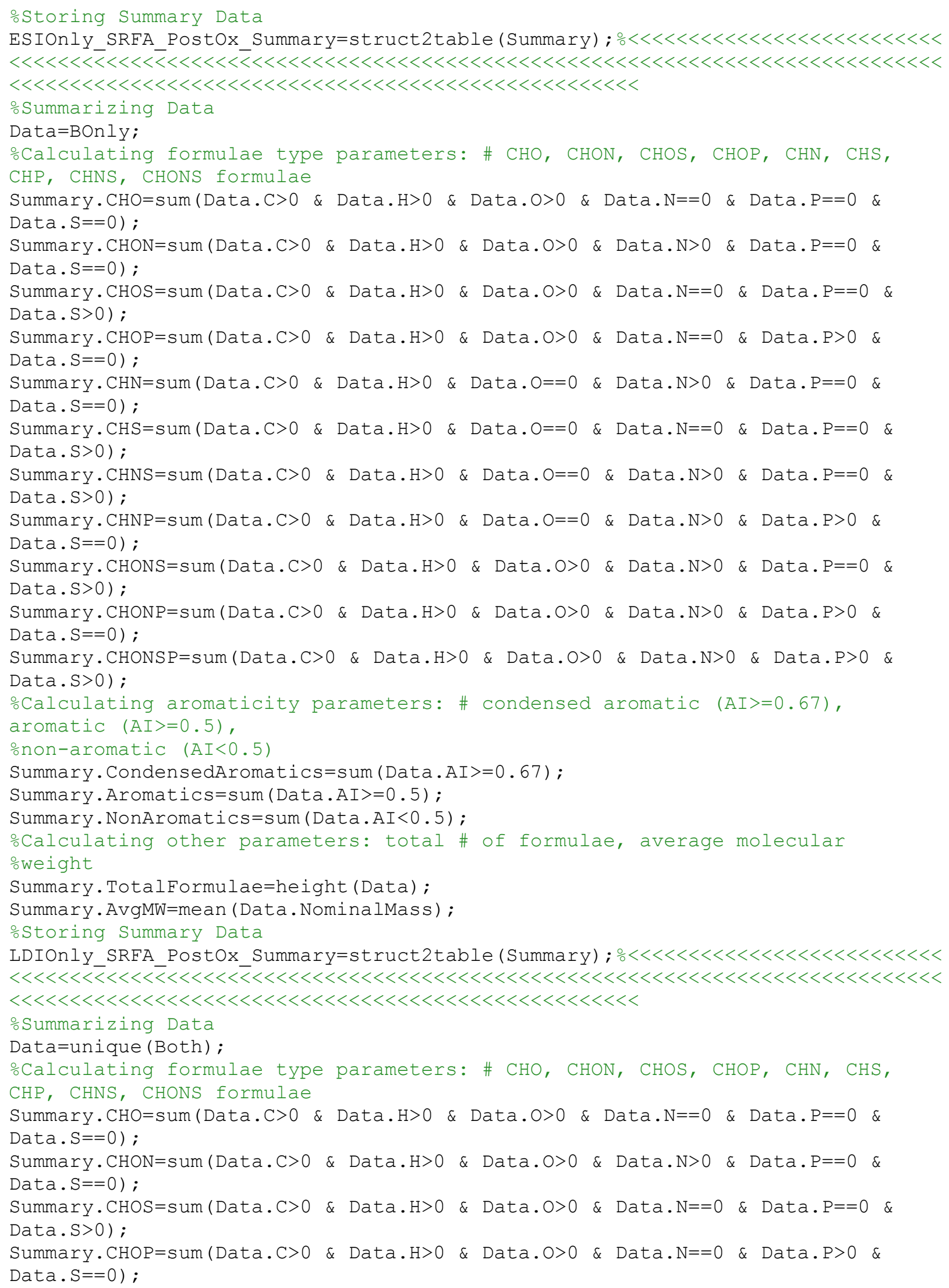


Summary. $\mathrm{CHN}=$ sum $($ Data. $\mathrm{C}>0$ \& Data.H $>0$ \& Data. $\mathrm{O}==0$ \& Data. $\mathrm{N}>0$ \& Data.P==0 \& Data. $\mathrm{S}==0$ );

Summary. CHS $=$ sum $($ Data. $\mathrm{C}>0$ \& Data.H $>0$ \& Data. $\mathrm{O}==0$ \& Data. $\mathrm{N}==0$ \& Data.P==0 \& Data. $\mathrm{S}>0$ );

Summary $\cdot \mathrm{CHNS}=\operatorname{sum}($ Data. $\mathrm{C}>0$ \& Data. $\mathrm{H}>0$ \& Data. $\mathrm{O}==0$ \& Data. $\mathrm{N}>0$ \& Data. $\mathrm{P}==0$ \& Data. S>0);

Summary. $\mathrm{CHNP}=\operatorname{sum}($ Data. $\mathrm{C}>0$ \& Data. $\mathrm{H}>0$ \& Data. $\mathrm{O}==0$ \& Data.N $>0$ \& Data.P $>0$ \&

Data. $\mathrm{S}=0$ ) ;

Summary $\cdot \mathrm{CHONS}=\operatorname{sum}($ Data $\cdot \mathrm{C}>0$ \& Data. $\mathrm{H}>0$ \& Data. $\mathrm{O}>0$ \& Data.N $>0$ \& Data.P==0 \&

Data. $\mathrm{S}>0$ );

Summary. $\mathrm{CHONP}=$ sum $($ Data. $\mathrm{C}>0$ \& Data.H $>0$ \& Data. O $>0$ \& Data.N $>0$ \& Data.P $>0$ \&

Data. $\mathrm{S}=0$ ) ;

Summary. $\mathrm{CHONSP}=$ sum $($ Data. $\mathrm{C}>0$ \& Data.H $>0$ \& Data.O $>0$ \& Data.N $>0$ \& Data.P>0 \&

Data. S>0);

ocalculating aromaticity parameters: \# condensed aromatic (AI>=0.67),

aromatic (AI>=0.5),

onon-aromatic $($ AI $<0.5)$

Summary. CondensedAromatics $=$ sum (Data. AI $>=0.67)$;

Summary. Aromatics=sum (Data.AI $>=0.5)$;

Summary. NonAromatics=sum (Data.AI<0.5);

ocalculating other parameters: total \# of formulae, average molecular

\%weight

Summary. TotalFormulae=height (Data);

Summary. AvgMW=mean (Data. NominalMass);

ostoring Summary Data

BothTechniques_SRFA_Postox_Summary=struct2table (Summary); $\circ<<<<<<<<<<<<<<<<<<<<~$

$<<<<<<<<<<<<<<\overline{<}<<<<\overline{<}<<<<<\overline{<}<<<<<<<<<<<<<<<<<<<<<<<<<<<<<<<<<<<<<<<<<<<<<<<<<<~$

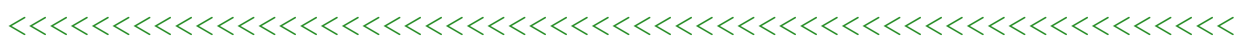

oSummarizing Data

Data $=$ Combined;

oCalculating formulae type parameters: \# CHO, CHON, CHOS, CHOP, CHN, CHS, CHP, CHNS, CHONS formulae

Summary $\cdot \mathrm{CHO}=$ sum $($ Data. $\mathrm{C}>0$ \& Data. $\mathrm{H}>0$ \& Data. $\mathrm{O}>0$ \& Data. $\mathrm{N}==0$ \& Data. $\mathrm{P}==0$ \&

Data. $\mathrm{S}==0$ );

Summary. $\mathrm{CHON}=\mathrm{sum}($ Data. $\mathrm{C}>0$ \& Data. $\mathrm{H}>0$ \& Data.O $>0$ \& Data.N $>0$ \& Data.P==0 \&

Data. $\mathrm{S}==0$ );

Summary. $\mathrm{CHOS}=$ sum $($ Data. $\mathrm{C}>0$ \& Data. $\mathrm{H}>0$ \& Data. $\mathrm{O}>0$ \& Data. $\mathrm{N}==0$ \& Data. $\mathrm{P}==0$ \&

Data. $\mathrm{S}>0$ );

Summary. $\mathrm{CHOP}=\operatorname{sum}($ Data. $\mathrm{C}>0$ \& Data. $\mathrm{H}>0$ \& Data. $\mathrm{O}>0$ \& Data. $\mathrm{N}==0$ \& Data.P>0 \&

Data. $\mathrm{S}==0$ );

Summary $\cdot \mathrm{CHN}=$ sum $($ Data. $\mathrm{C}>0$ \& Data. $\mathrm{H}>0$ \& Data. $\mathrm{O}==0$ \& Data. $\mathrm{N}>0$ \& Data. $\mathrm{P}==0$ \&

Data. $\mathrm{S}==0$ ) ;

Summary $\cdot \mathrm{CHS}=\operatorname{sum}($ Data $\cdot \mathrm{C}>0$ \& Data.H $>0$ \& Data $\cdot \mathrm{O}==0$ \& Data. $\mathrm{N}==0$ \& Data.P==0 \&

Data. $\mathrm{S}>0$ ) ;

Summary $\cdot \mathrm{CHNS}=\mathrm{sum}($ Data $\cdot \mathrm{C}>0$ \& Data. $\mathrm{H}>0$ \& Data. $\mathrm{O}==0$ \& Data. $>0$ \& Data.P==0 \&

Data. $\mathrm{S}>0$ );

Summary $\cdot \mathrm{CHNP}=\operatorname{sum}($ Data $\cdot \mathrm{C}>0$ \& Data. $\mathrm{H}>0$ \& Data. $\mathrm{O}==0$ \& Data.N $>0$ \& Data.P $>0$ \&

Data. $\mathrm{S}==0$ );

Summary. $\mathrm{CHONS}=\operatorname{sum}($ Data $. \mathrm{C}>0$ \& Data. $\mathrm{H}>0$ \& Data. $\mathrm{O}>0$ \& Data.N>0 \& Data.P==0 \&

Data. $\mathrm{S}>0$ ) ;

Summary. $\mathrm{CHONP}=$ sum $($ Data. $\mathrm{C}>0$ \& Data.H $>0$ \& Data. $0>0$ \& Data.N $>0$ \& Data.P $>0$ \&

Data. $\mathrm{S}==0$ );

Summary. $\mathrm{CHONSP}=$ sum $($ Data. $\mathrm{C}>0$ \& Data. $\mathrm{H}>0$ \& Data.O $>0$ \& Data.N $>0$ \& Data.P $>0$ \&

Data. $\mathrm{S}>0$ );

ocalculating aromaticity parameters: \# condensed aromatic (AI>=0.67),

aromatic (AI $>=0.5)$,

onon-aromatic $($ A I < 0.5$)$ 
Summary. CondensedAromatics $=$ sum (Data.AI $>=0.67)$;

Summary. Aromatics $=$ sum (Data.AI $>=0.5)$;

Summary. NonAromatics=sum (Data.AI<0.5);

ocalculating other parameters: total \# of formulae, average molecular

oweight

Summary. TotalFormulae=height (Data);

Summary. AvgMW=mean (Data. NominalMass);

ostoring Summary Data

SRFA Postox Summary=struct2table (Summary); $\circ<<<<<<<<<<<<<<<<<<<<<<<<<<<<<<<<<<<<~$ $<<<<\overline{<}<<<<<<\overline{<}<<<<<<<<<<<<<<<<<<<<<<<<<<<<<<<<<<<<<<<<<<<<<<<<<<<<<<<<<<<<<<<<<~$

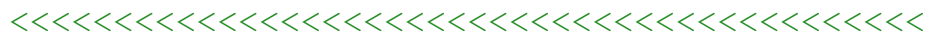

응 Generic code to combine negative and positive ESI and LDI FT-ICR-MS data for 1 sample

oThe following code is organized into sections as follows:

ㅇ. Clean up negative mode ESI data (remove impurities and chemical

oformulae that are unlikely to exist)

\%2. Clean up positive mode ESI data (remove impurities and chemical

oformulae that are unlikely to exist)

⒊ Clean up negative mode LDI data (remove chemical formulae that are

ounlikely to exist)

․ 4. Clean up positive mode LDI data (remove chemical formulae that are

ounlikely to exist)

⒌ Compare and combine negative and positive mode ESI data

⒍ Compare and combine negative and positive mode LDI data

\%7. Compare and combine LDI and ESI data to give final list of chemical

oformulae identified in DOM sample

oUser inputs:

\%1. Data files in excel format with chemical formulae assigned in Bruker

oCompass Data Analysis Software

$\div$ A. Background negative mode ESI scan

B. Background positive mode ESI scan

ㄷ. Negative mode ESI scan

D. Positive mode ESI scan

‥ Negative mode LDI scan

$\circ \mathrm{F}$. Positive mode ESI scan

⒉ Data file names as you chose

oll inputs denoted by

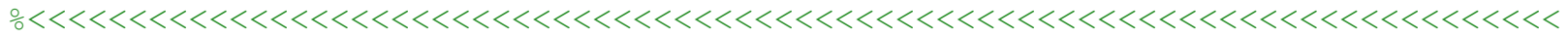

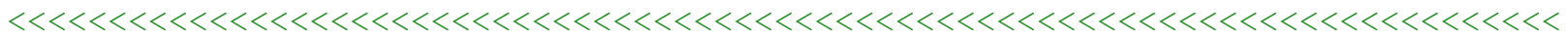
$<<<<<$

응 Negative Mode ESI: Data Clean Up

oLoading negative mode background impurities

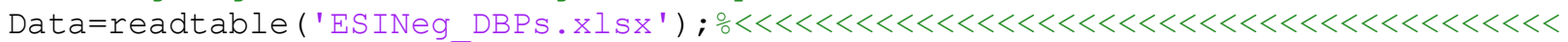
$<<<<<<<<<<<<<<<<<<<<<<\overline{<}<<<<<<<<<<<<<<<<<<<<<<<<<<<<<<<<<<<<<<<<<<<<<<<<<<<<<<~$ $<<<<<<<<<<<<<<<<<<<<<<<<<<<<<<<<<<~$

Data=unique (Data);

Data $\cdot$ NominalMass=Data $\cdot C * 12+$ Data $\cdot 0 * 16+$ Data $\cdot H * 1+$ Data $\cdot P * 31+$ Data $\cdot S * 32+$ Data $\cdot N * 14$;

Data. KMD CH2=Data. NominalMass-

$0.998883 \overline{4} *($ Data. $C * 12+$ Data. H*1.007825+Data. N*14.003074+Data. $0 * 15.994915+$ Data.P

$\star 30.973763+$ Data. $\mathrm{S} * 31.972072$ );

Data.KMD_COO=Data.NominalMass-

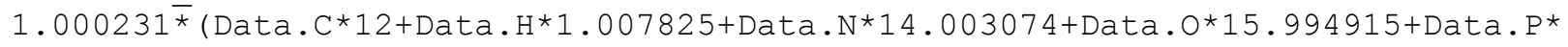
$30.973763+$ Data. S*31.972072); 


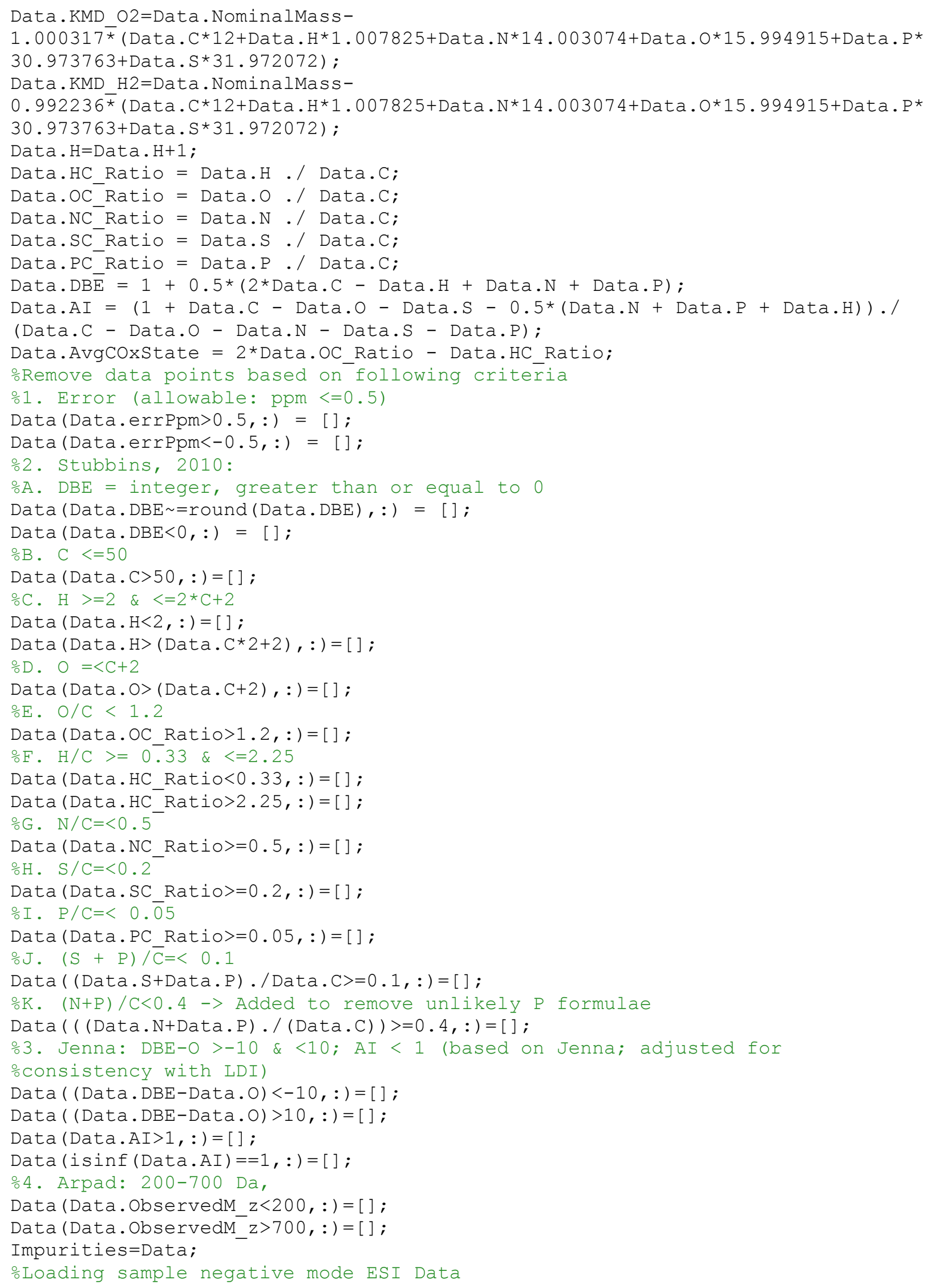




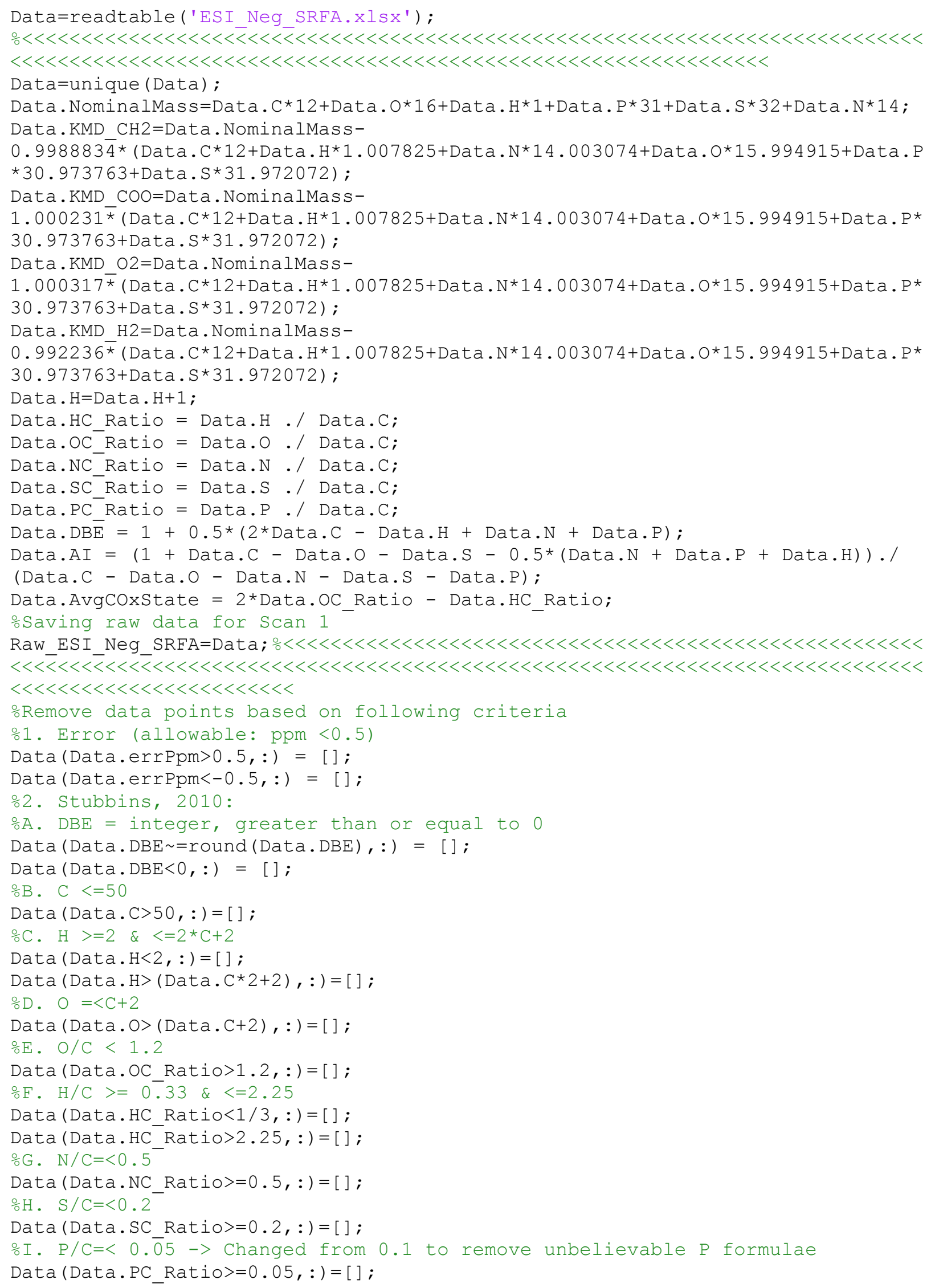


J. $(\mathrm{S}+\mathrm{P}) / \mathrm{C}=<0.1->$ Changed from 0.2 to remove unbelievable $\mathrm{P}$ formulae $\operatorname{Data}((($ Data.S+Data.P).$/($ Data. C $))>=0.1,:)=[]$;

\%K. $(\mathrm{N}+\mathrm{P}) / \mathrm{C}<0.4->$ Added to remove unlikely P formulae

$\operatorname{Data}((($ Data. N+Data.P $) . /($ Data.C $))>=0.4,:)=[]$;

ㅇ. Jenna: DBE-0 >-10 \& <10; AI < 1 (based on Jenna; adjusted for

oconsistency with LDI)

Data ( (Data.DBE-Data.0)<-10,: ) = [ ] ;

Data ( (Data.DBE-Data.O) $>10,:)=[$;

Data (Data.AI>1,:) = [ ] ;

Data (isinf (Data.AI) $==1,:)=[$;

\%4. Arpad: 200-700 Da,

Data (Data.ObservedM $z<200,:$ ) = [ ] ;

Data (Data.ObservedM_z $>700,:$ ) = [ ] ;

\% Impurities

for $i=1$ : height (Data)

for $j=1$ :height (Impurities)

if Data.calc_M_z(i)==Impurities.calc_M_z(j)

Data.calc_M $\bar{z}(\bar{i})=\mathrm{NaN}$;

end

end

end

Data (isnan (Data.calc_M_z), : ) = [ ] ;

ocalculating formulae type parameters: \# CHO, CHON, CHOS, CHOP, CHN, CHS, CHP, CHNS, CHONS formulae

Summary $\cdot \mathrm{CHO}=$ sum $($ Data. $\mathrm{C}>0$ \& Data. $\mathrm{H}>0$ \& Data. $\mathrm{O}>0$ \& Data. $\mathrm{N}==0$ \& Data. $\mathrm{P}==0$ \&

Data. $\mathrm{S}==0$ );

Summary $\cdot \mathrm{CHON}=\operatorname{sum}($ Data. $\mathrm{C}>0$ \& Data.H $>0$ \& Data. $\mathrm{O}>0$ \& Data. $\mathrm{N}>0$ \& Data.P==0 \&

Data. $\mathrm{S}==0$ );

Summary. $\mathrm{CHOS}=$ sum $($ Data. $\mathrm{C}>0$ \& Data. $\mathrm{H}>0$ \& Data. $\mathrm{O}>0$ \& Data. $\mathrm{N}==0$ \& Data. $\mathrm{P}==0$ \&

Data. $\mathrm{S}>0$ );

Summary $\cdot \mathrm{CHOP}=\operatorname{sum}($ Data. $\mathrm{C}>0$ \& Data.H $>0$ \& Data. $\mathrm{O}>0$ \& Data. $\mathrm{N}==0$ \& Data. P>0 \&

Data. $\mathrm{S}==0$ );

Summary $\cdot \mathrm{CHN}=$ sum $($ Data. $\mathrm{C}>0$ \& Data. $\mathrm{H}>0$ \& Data. $\mathrm{O}==0$ \& Data. $\mathrm{N}>0$ \& Data. $\mathrm{P}==0$ \&

Data. $\mathrm{S}==0$ );

Summary $\cdot \mathrm{CHS}=$ sum $($ Data $\cdot \mathrm{C}>0$ \& Data. $\mathrm{H}>0$ \& Data. $\mathrm{O}==0$ \& Data. $\mathrm{N}==0$ \& Data. $\mathrm{P}==0$ \&

Data. S>0);

Summary. $\mathrm{CHNS}=\operatorname{sum}($ Data. $\mathrm{C}>0$ \& Data. $\mathrm{H}>0$ \& Data. $\mathrm{O}==0$ \& Data. $\mathrm{N}>0$ \& Data. $\mathrm{P}==0$ \&

Data. S $>0$ );

Summary $\cdot \mathrm{CHNP}=\mathrm{Sum}($ Data. $\mathrm{C}>0$ \& Data.H $>0$ \& Data. $\mathrm{O}==0$ \& Data. $\mathrm{N}>0$ \& Data.P $>0$ \&

Data. $\mathrm{S}==0$ );

Summary $\cdot \mathrm{CHONS}=\operatorname{sum}($ Data $\cdot \mathrm{C}>0$ \& Data. $\mathrm{H}>0$ \& Data. $0>0$ \& Data.N>0 \& Data.P==0 \&

Data. S $>0$ );

Summary. $\mathrm{CHONP}=\operatorname{sum}($ Data. $\mathrm{C}>0$ \& Data.H $>0$ \& Data. $\mathrm{O}>0$ \& Data.N $>0$ \& Data.P $>0$ \&

Data. $\mathrm{S}==0$ );

Summary. $\mathrm{CHONSP}=$ sum $($ Data. $\mathrm{C}>0$ \& Data.H $>0$ \& Data.O $>0$ \& Data.N $>0$ \& Data.P>0 \&

Data. $\mathrm{S}>0$ ) ;

ocalculating aromaticity parameters: \# condensed aromatic (AI>=0.67),

aromatic $(A I>=0.5)$,

onon-aromatic $($ AI $<0.5)$

Summary. CondensedAromatics $=$ sum (Data. AI $>=0.67)$;

Summary. Aromatics $=$ sum (Data.AI $>=0.5)$;

Summary. NonAromatics=sum (Data.AI<0.5);

ocalculating other parameters: total \# of formulae, average molecular

oweight

Summary. TotalFormulae=height (Data) ;

Summary. AvgMW=mean (Data. NominalMass);

ostoring Data in Tables 


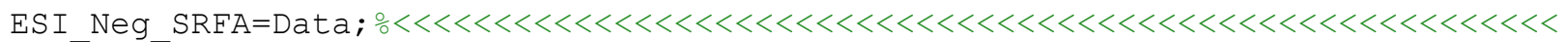
$<<\overline{<}<<\overline{<}<<<<<<<<<<<<<<<<<<<<<<<<<<<<<<<<<<<<<<<<<<<<<<<<<<<<<<<<<<<<<<<<<<<<<<~$ $<<<<<<<<<<<<<<<<<<<<~$

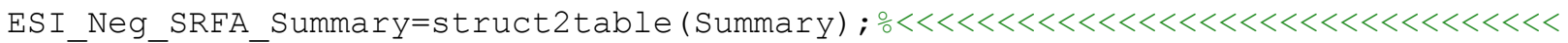
$<<\overline{<}<<\overline{\bar{l}}<<<<\overline{<}<<<<<<<<<<<<<<<<<<<<<<<<<<<<<<<<<<<<<<<<<<<<<<<<<<<<<<<<<<<<<<<<$

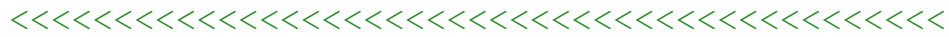

응 Positive Mode ESI: Data Clean Up

oLoading positive mode ESI impurities

Data $=$ readtable ('DBP_Control.xlsx') ;

Data. NominalMass=Data $\cdot \mathrm{C} * 12+$ Data $\cdot 0 * 16+$ Data $\cdot H * 1+$ Data $\cdot P * 31+$ Data $. S * 32+D a t a \cdot N * 14+D$ ata. $\mathrm{Na}$ 23;

Data.KMD_CH2=Data.NominalMass-

$0.998883 \overline{4} *($ Data. $C * 12+$ Data. $H * 1.007825+$ Data $. N * 14.003074+$ Data. $0 * 15.994915+$ Data.P *30.973763+Data.S*31.972072+Data.Na*22.98977);

Data.KMD COO=Data. NominalMass-

$1.000231^{\bar{*}}\left(\right.$ Data. $\mathrm{C} * 12+$ Data $. H^{*} 1.007825+$ Data $. N^{*} 14.003074+$ Data. $0 * 15.994915+$ Data. $P *$ $30.973763+$ Data.. *31.972072+Data.Na*22.98977);

Data.KMD 02=Data. NominalMass-

$1.000317^{\star}\left(\right.$ Data. $C * 12+$ Data $. H^{*} 1.007825+$ Data. $N^{*} 14.003074+$ Data. $0 * 15.994915+$ Data. $P *$ $30.973763+$ Data. $S * 31.972072+$ Data. Na*22.98977);

Data.KMD_H2=Data.NominalMass-

$0.992236^{\star}$ (Data. C*12+Data.H*1.007825+Data. $N^{\star} 14.003074+$ Data. $0 * 15.994915+$ Data.P* $30.973763+$ Data.S*31.972072+Data.Na*22.98977);

for $i=1$ : height (Data)

if $\operatorname{Data.Na}(i)==0$

Data.H(i)=Data.H (i) -1 ;

end

end

Data.HC Ratio = Data.H ./ Data.C;

Data.OC_Ratio = Data.O./ Data.C;

Data.NC_Ratio = Data.N./ Data.C;

Data.SC Ratio = Data.S./ Data.C;

Data.PC Ratio = Data.P ./ Data.C;

Data.DBE $=1+0.5 *(2 *$ Data.C - Data.H + Data. $N+$ Data.P $)$;

Data.AI = (1 + Data.C - Data.O - Data.S - 0.5* (Data.N + Data.P + Data.H))./

(Data.C - Data.O - Data.N - Data.S - Data.P);

Data.AvgCoxstate = 2*Data.oC_Ratio - Data.HC_Ratio;

Remove data points based on following criteria

\%1. Error (allowable: ppm <0.5)

Data (Data.errPpm $>0.5,:)=[]$;

Data (Data.errPpm<-0.5,:) = [] ;

⒉ Stubbins, 2010 :

○A. $\mathrm{DBE}=$ integer, greater than or equal to 0

Data (Data.DBE =round (Data.DBE), : ) = [];

Data (Data. $\mathrm{DBE}<0,:)=[]$;

ㅇ. $\mathrm{C}<=50$

Data (Data.C $>50,:)=[]$;

ㄷ. $\mathrm{H}>=2 \&<=2 * \mathrm{C}+2$

Data (Data. $\mathrm{H}<2,:)=[]$;

Data (Data.H $>($ Data. C*2+2), : ) = [ ] ;

$\div \mathrm{D} . \mathrm{O}=<\mathrm{C}+2$

Data $($ Data. O $>($ Data $. C+2),:)=[]$;

ㅌ. $O / C<1.2$

Data (Data.oC Ratio>1.2, : )=[] ;

ㄷ․ $\mathrm{H} / \mathrm{C}>=0.33 \&<=2.25$

Data (Data.HC_Ratio $<1 / 3,:)=[]$; 


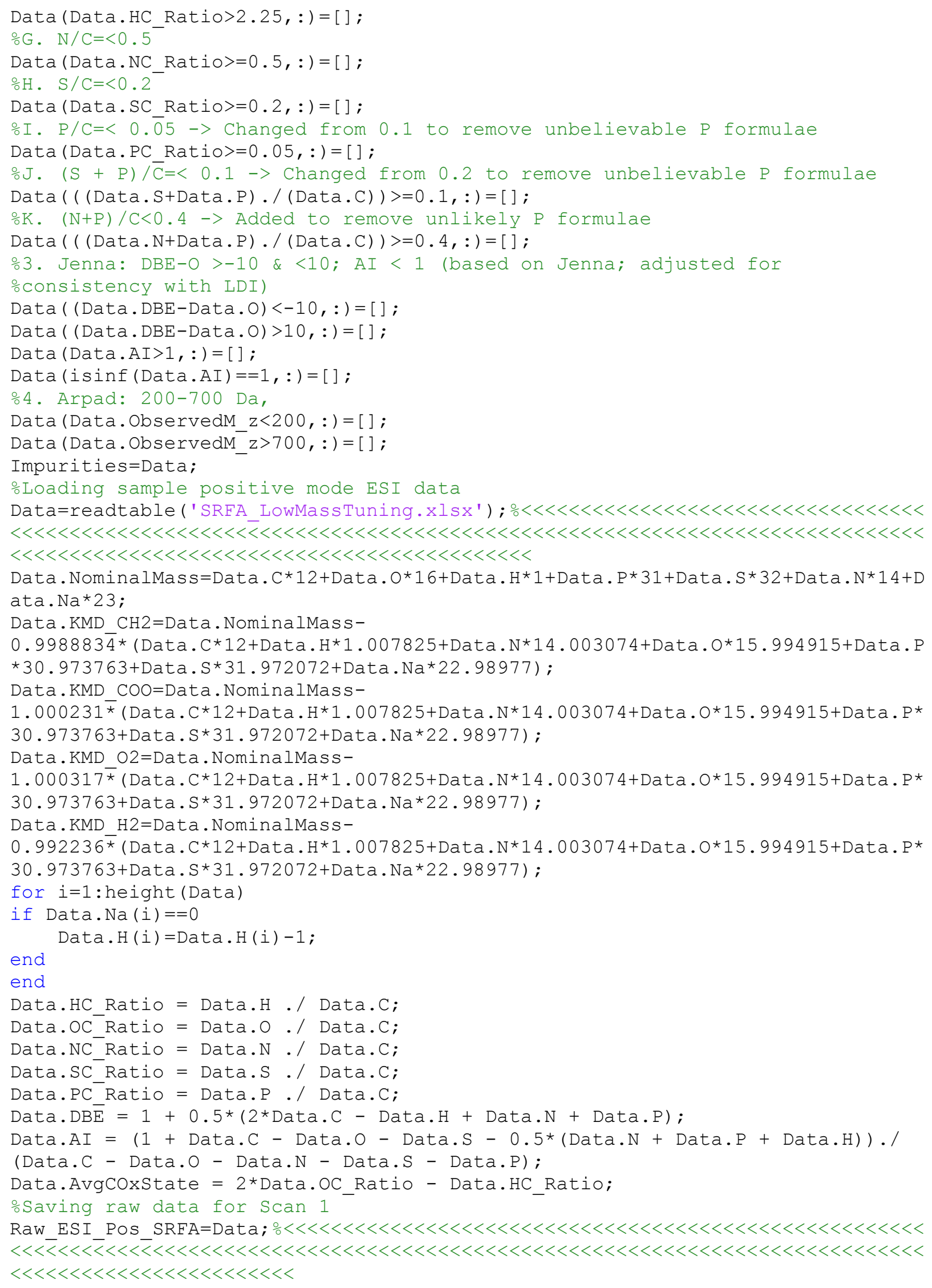




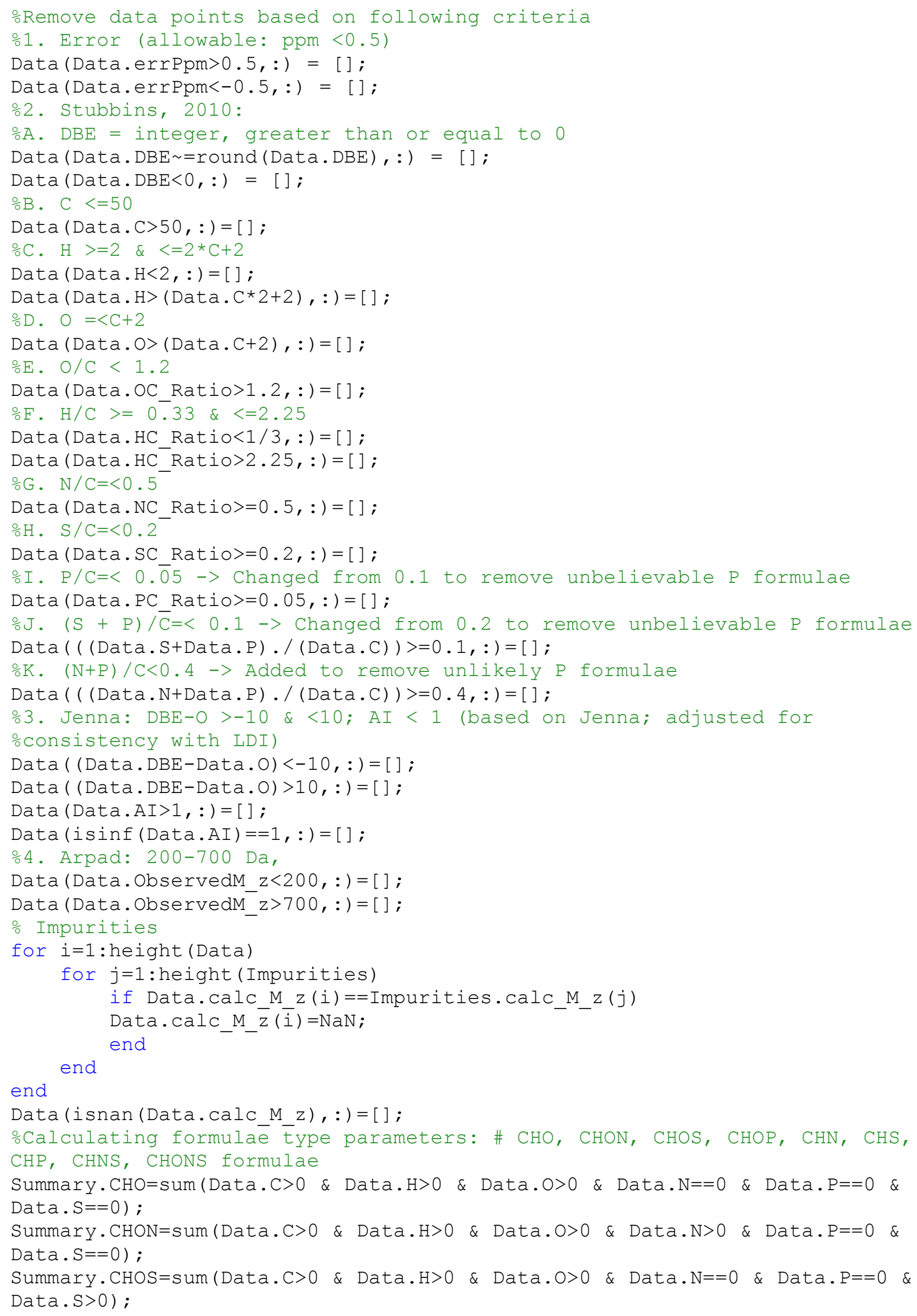


Summary. $\mathrm{CHOP}=\operatorname{sum}($ Data. $\mathrm{C}>0$ \& Data. $\mathrm{H}>0$ \& Data. $\mathrm{O}>0$ \& Data.N==0 \& Data.P $>0$ \& Data. $\mathrm{S}==0$ );

Summary $\cdot \mathrm{CHN}=$ sum $($ Data. $\mathrm{C}>0$ \& Data. $\mathrm{H}>0$ \& Data. $\mathrm{O}==0$ \& Data. $\mathrm{N}>0$ \& Data.P==0 \& Data. $\mathrm{S}==0$ );

Summary $\cdot \mathrm{CHS}=\operatorname{sum}($ Data $\cdot \mathrm{C}>0$ \& Data. $\mathrm{H}>0$ \& Data. $\mathrm{O}==0$ \& Data. $\mathrm{N}==0$ \& Data. $\mathrm{P}==0$ \& Data. S>0);

Summary $\cdot \mathrm{CHNS}=$ sum $($ Data $\cdot \mathrm{C}>0$ \& Data. $\mathrm{H}>0$ \& Data. $\mathrm{O}==0$ \& Data. $\mathrm{N}>0$ \& Data. $\mathrm{P}==0$ \&

Data. $\mathrm{S}>0$ ) ;

Summary $\cdot \mathrm{CHNP}=\operatorname{sum}($ Data $\cdot \mathrm{C}>0$ \& Data. $\mathrm{H}>0$ \& Data. $\mathrm{O}==0$ \& Data.N $>0$ \& Data.P>0 \&

Data. $\mathrm{S}==0$ );

Summary $\cdot \mathrm{CHONS}=$ sum $($ Data $\cdot \mathrm{C}>0$ \& Data. $\mathrm{H}>0$ \& Data. $\mathrm{O}>0$ \& Data. $>0$ \& Data.P==0 \&

Data. $\mathrm{S}>0$ );

Summary. $\mathrm{CHONP}=$ sum $($ Data. $\mathrm{C}>0$ \& Data.H $>0$ \& Data.O>0 \& Data.N>0 \& Data.P>0 \&

Data. $\mathrm{S}==0$ );

Summary. $\mathrm{CHONSP}=$ sum $($ Data. $\mathrm{C}>0$ \& Data.H $>0$ \& Data.O $>0$ \& Data.N $>0$ \& Data.P $>0$ \&

Data. $\mathrm{S}>0$ ) ;

ocalculating aromaticity parameters: \# condensed aromatic (AI>=0.67),

aromatic $(\mathrm{AI}>=0.5)$,

onon-aromatic $(\mathrm{AI}<0.5)$

Summary. CondensedAromatics $=$ sum (Data. AI $>=0.67)$;

Summary.Aromatics=sum (Data.AI>=0.5);

Summary. NonAromatics=sum (Data.AI<0.5);

oCalculating other parameters: total \# of formulae, average molecular

oweight

Summary. TotalFormulae=height (Data);

Summary. AvgMW=mean (Data. NominalMass);

ostoring Data in Tables

ESI_Pos_SRFA=Data; $\circ \circ<<<<<<<<<<<<<<<<<<<<<<<<<<<<<<<<<<<<<<<<<<<<<<<<<<<<<<<<<<<<<<~$

$<<\overline{<}<<\overline{<}<<<<<<<<<<<<<<<<<<<<<<<<<<<<<<<<<<<<<<<<<<<<<<<<<<<<<<<<<<<<<<<<<<<<<<$ $<<<<<<<<<<<<<<<<<<<<$

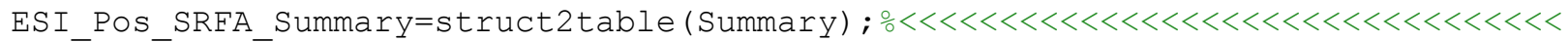
$<<\overline{<}<<\overline{<}<<<<\overline{<}<<<<<<<<<<<<<<<<<<<<<<<<<<<<<<<<<<<<<<<<<<<<<<<<<<<<<<<<<<<<<<<<~$

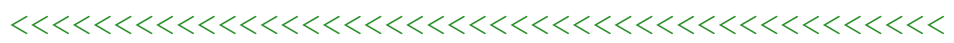

응 Negative Mode LDI: Data Clean Up

LDI assumed to have no background ions

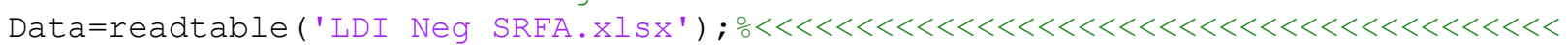
$<<<<<<<<<<<<<<<<<<\overline{<}<<\overline{<}<<<<<<<<<<<<<<<<<<<<<<<<<<<<<<<<<<<<<<<<<<<<<<<<<<<<<<$

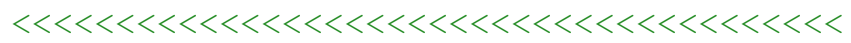

Data=unique (Data);

Data $\cdot$ NominalMass=Data $\cdot C * 12+$ Data $\cdot 0 * 16+$ Data $\cdot H * 1+$ Data $\cdot P * 31+$ Data $\cdot S * 32+D a t a \cdot N * 14 ;$

Data.KMD CH2=Data. NominalMass-

$0.998883 \overline{4} *($ Data. $C * 12+$ Data. H*1.007825+Data. $N * 14.003074+$ Data. $0 * 15.994915+$ Data.P

$\star 30.973763+$ Data. S*31.972072);

Data.KMD COO=Data. NominalMass-

$1.000231^{\star}\left(\right.$ Data. C*12+Data.H*1.007825+Data. $N^{\star 1} 14.003074+$ Data.0*15.994915+Data.P* $30.973763+$ Data. S*31.972072);

Data.KMD $02=$ Data. NominalMass-

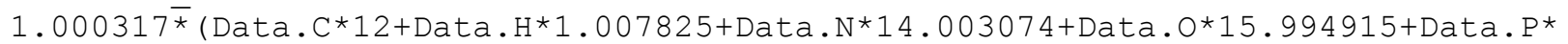
$30.973763+$ Data. $S * 31.972072)$;

Data.KMD_H2=Data. NominalMass-

$0.992236^{\star}\left(\right.$ Data. $C * 12+$ Data. $H^{*} 1.007825+$ Data $. N * 14.003074+$ Data. $0 * 15.994915+$ Data. $P *$

$30.973763+$ Data. $S * 31.972072) ;$

Data.DBE $=1+0.5 *(2 *$ Data.C - Data.H + Data.N + Data.P $)$;

for $i=1$ : height (Data)

if Data.DBE (i) round (Data.DBE (i))

Data.H (i) =Data.H (i) +1; 


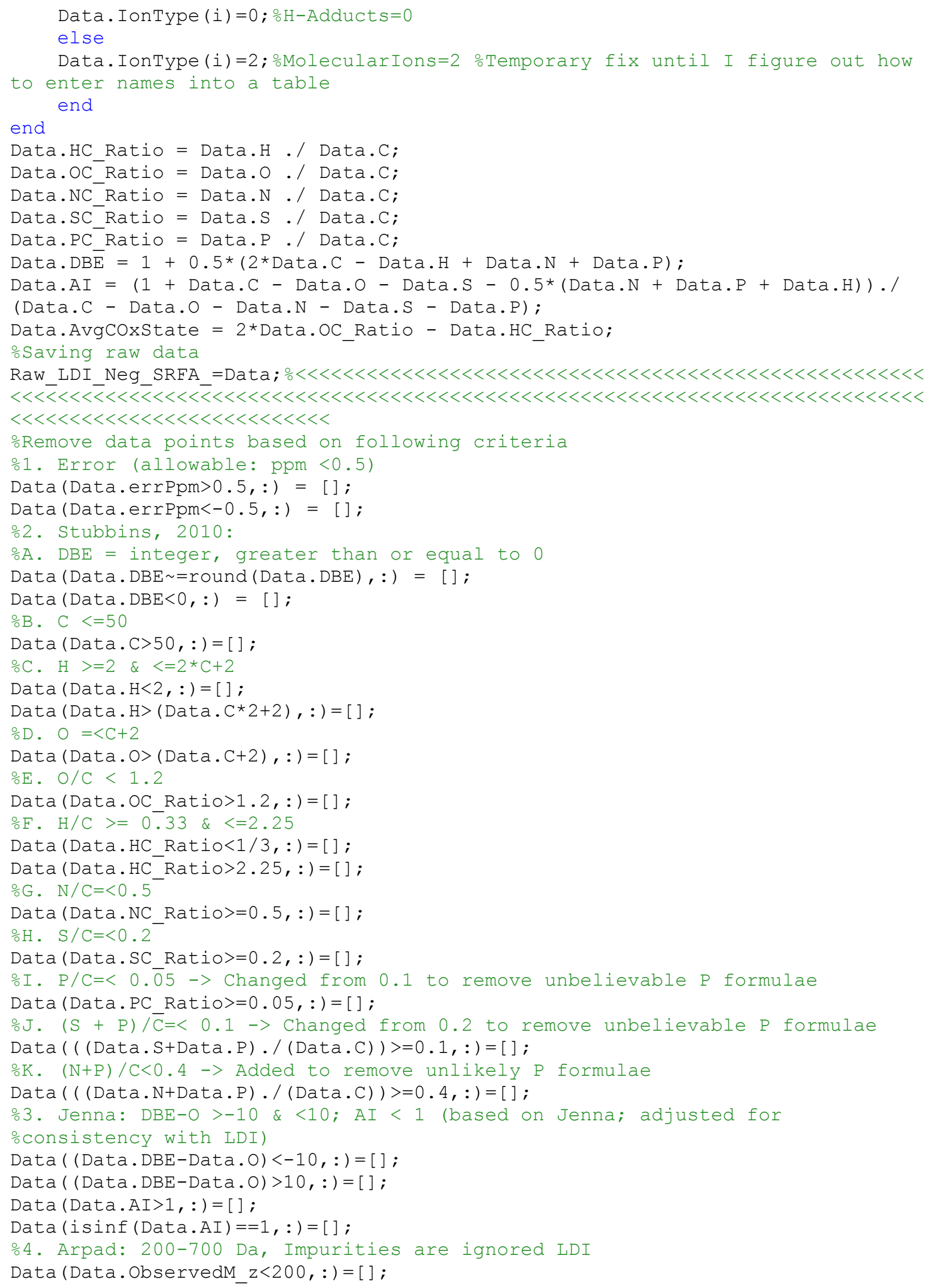


Data (Data. ObservedM_z>700,:) =[] ;

ocalculating formulāe type parameters: \# CHO, CHON, CHOS, CHOP, CHN, CHS, CHP, CHNS, CHONS formulae

Summary $\cdot \mathrm{CHO}=$ sum (Data. $\mathrm{C}>0$ \& Data. $\mathrm{H}>0$ \& Data. $\mathrm{O}>0$ \& Data. $\mathrm{N}==0$ \& Data. $\mathrm{P}==0$ \& $\operatorname{Data} . \mathrm{S}==0$ );

Summary $\cdot \mathrm{CHON}=\operatorname{sum}($ Data. $\mathrm{C}>0$ \& Data.H $>0$ \& Data. $0>0$ \& Data. $\mathrm{N}>0$ \& Data. $\mathrm{P}==0$ \& Data. $\mathrm{S}==0$ );

Summary $\cdot \mathrm{CHOS}=$ sum $($ Data. $\mathrm{C}>0$ \& Data.H $>0$ \& Data.O $>0$ \& Data. $\mathrm{N}==0$ \& Data. $\mathrm{P}==0$ \& Data. S>0);

Summary $\cdot \mathrm{CHOP}=\mathrm{sum}($ Data. $\mathrm{C}>0$ \& Data.H $>0$ \& Data. $\mathrm{O}>0$ \& Data. $\mathrm{N}==0$ \& Data. $\mathrm{P}>0$ \& Data. $\mathrm{S}==0$ );

Summary. $\mathrm{CHN}=$ sum $($ Data. $\mathrm{C}>0$ \& Data.H $>0$ \& Data. $\mathrm{O}==0$ \& Data. $>0$ \& Data.P==0 \&

Data. $\mathrm{S}=0$ ) ;

Summary $\cdot \mathrm{CHS}=\operatorname{sum}($ Data $\cdot \mathrm{C}>0$ \& Data. $\mathrm{H}>0$ \& Data $\cdot \mathrm{O}==0$ \& Data. $\mathrm{N}==0$ \& Data. $\mathrm{P}==0$ \& Data. $\mathrm{S}>0$ );

Summary $\cdot \mathrm{CHNS}=\operatorname{sum}($ Data. $\mathrm{C}>0$ \& Data. $\mathrm{H}>0$ \& Data. $\mathrm{O}==0$ \& Data. $\mathrm{N}>0$ \& Data.P==0 \& Data. $\mathrm{S}>0$ );

Summary. $\mathrm{CHNP}=\operatorname{sum}($ Data. $\mathrm{C}>0$ \& Data. $\mathrm{H}>0$ \& Data. $\mathrm{O}==0$ \& Data.N $>0$ \& Data.P $>0$ \&

Data. $\mathrm{S}==0)$;

Summary $\cdot \mathrm{CHONS}=\operatorname{sum}($ Data $\cdot \mathrm{C}>0$ \& Data. $\mathrm{H}>0$ \& Data. $\mathrm{O}>0$ \& Data. $\mathrm{N}>0$ \& Data.P==0 \&

Data. $\mathrm{S}>0$ );

Summary. $\mathrm{CHONP}=$ sum $($ Data. $\mathrm{C}>0$ \& Data.H $>0$ \& Data. O $>0$ \& Data.N $>0$ \& Data.P $>0$ \&

Data. $\mathrm{S}==0$ );

Summary. $\mathrm{CHONSP}=$ sum $($ Data. $\mathrm{C}>0$ \& Data.H $>0$ \& Data.O $>0$ \& Data.N $>0$ \& Data.P>0 \&

Data. S>0);

ocalculating aromaticity parameters: \# condensed aromatic (AI>=0.67),

aromatic $(\mathrm{AI}>=0.5)$,

onon-aromatic $($ AI $<0.5)$

Summary. CondensedAromatics $=$ sum (Data. AI $>=0.67)$;

Summary. Aromatics $=$ sum (Data.AI $>=0.5)$;

Summary. NonAromatics=sum (Data.AI<0.5);

ocalculating other parameters: total \# of formulae, average molecular

oweight

Summary. TotalFormulae=height (Data);

Summary. AvgMW=mean (Data. NominalMass);

\% Storing Data in Tables

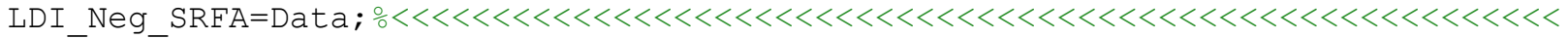

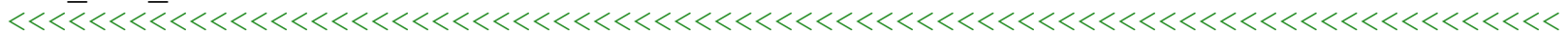

$<<<<<<<<<<<<<<<<<<~$

LDI Neg SRFA Summary=struct2table (Summary) ; 으 $<<<<<<<<<<<<<<<<<<<<<<<<<<<<<<<<<<<~$ $<<<\overline{<}<<<<<<<<\overline{<}<<<<<<<<<<<<<<<<<<<<<<<<<<<<<<<<<<<<<<<<<<<<<<<<<<<<<<<<<<<<<<<<~$

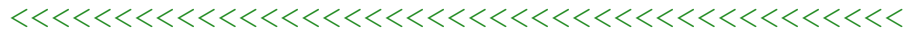

응 Positive Mode LDI: Data Clean Up

oLDI assumed to have no background ions

oLoading sample positive mode LDI data

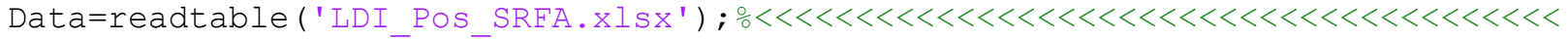
$<<<<<<<<<<<<<<<<<<<\overline{<}<<<\overline{<}<<<<<<<<<<<<<<<<<<<<<<<<<<<<<<<<<<<<<<<<<<<<<<<<<<<<<~$ $<<<<<<<<<<<<<<<<<<<<<<<<<<<<<<<<<<<<<<<<<<<<<<~$

Data=unique (Data);

Data. NominalMass=Data $\cdot \mathrm{C} * 12+$ Data $\cdot 0 * 16+$ Data $\cdot H * 1+$ Data. $P * 31+$ Data $\cdot S * 32+$ Data $\cdot N * 14$; Data.KMD_CH2=Data.NominalMass-

$0.998883 \overline{4} *\left(\right.$ Data. $C * 12+$ Data. $H^{*} 1.007825+$ Data. $N^{*} 14.003074+$ Data. $0 * 15.994915+$ Data. P $\star 30.973763+$ Data.. *31.972072);

Data.KMD COO=Data.NominalMass-

$1.000231^{\star}\left(\right.$ Data. $C^{\star} 12+$ Data.H*1.007825+Data. $N^{\star} 14.003074+$ Data. $0 * 15.994915+$ Data.P* $30.973763+$ Data. S*31.972072); 


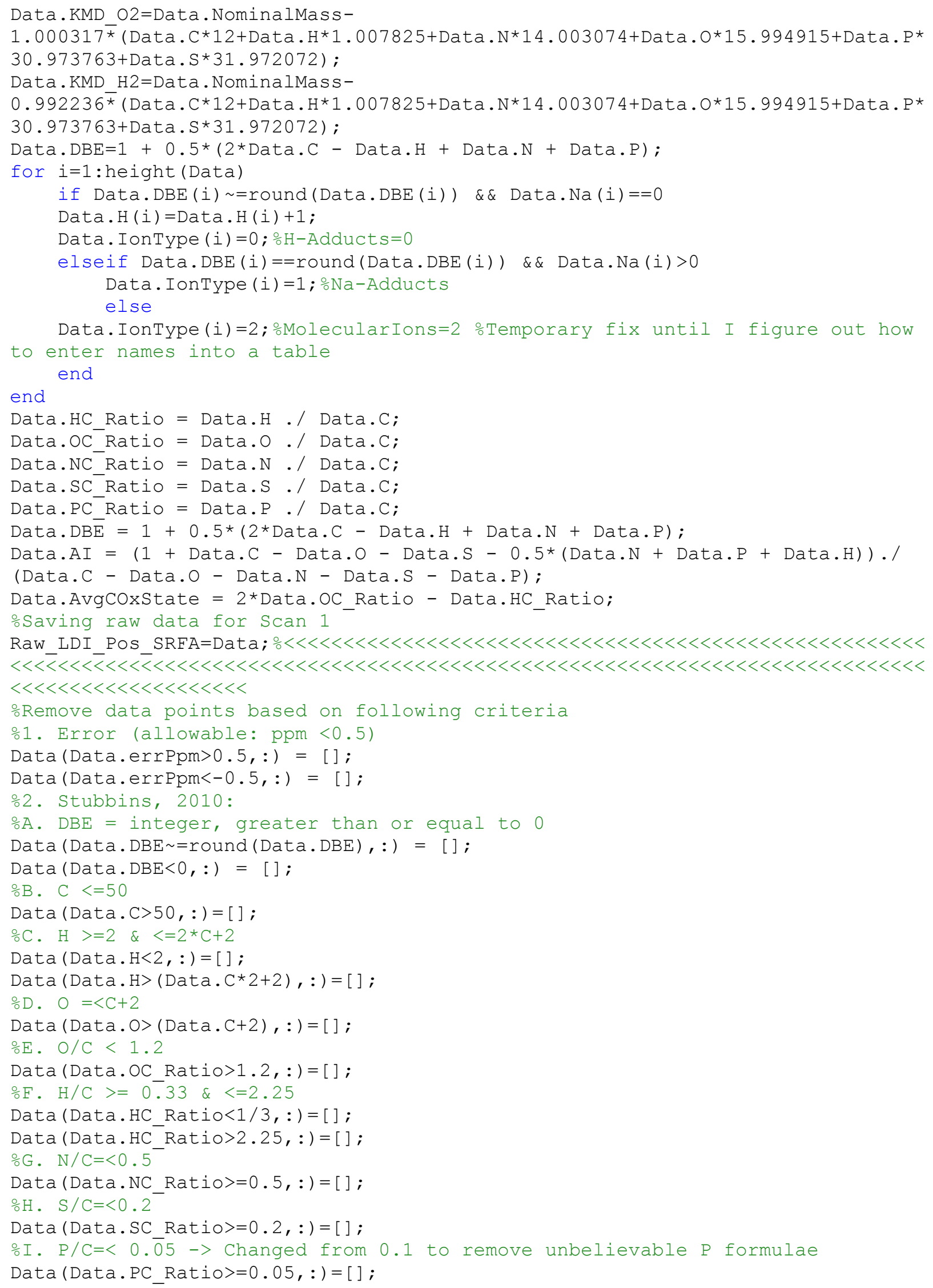


J. $(\mathrm{S}+\mathrm{P}) / \mathrm{C}=<0.1->$ Changed from 0.2 to remove unbelievable $\mathrm{P}$ formulae $\operatorname{Data}((($ Data.S+Data.P).$/($ Data. C $))>=0.1,:)=[]$;

\%K. $(\mathrm{N}+\mathrm{P}) / \mathrm{C}<0.4->$ Added to remove unlikely P formulae

$\operatorname{Data}((($ Data.N+Data.P).$/($ Data.C $))>=0.4,:)=[]$;

ㅇ. Jenna: DBE-0 >-10 \& <10; AI < 1 (based on Jenna; adjusted for

oconsistency with LDI)

Data ( (Data.DBE-Data.O) $<-10,:)=[$ ] ;

Data ( (Data.DBE-Data.O) $>10,:)=[$;

Data (Data.AI>1,:) = [ ] ;

Data $($ isinf $($ Data.AI $)==1,:)=[]$;

\%4. Arpad: 200-700 Da, Impurities are ignored LDI- assumed to be negligible ounless a background reference is used

Data (Data. ObservedM $z<200,:$ ) $=[]$;

Data (Data. ObservedM_z>700,: ) = [] ;

ocalculating formulāe type parameters: \# CHO, CHON, CHOS, CHOP, CHN, CHS, CHP, CHNS, CHONS formulae

Summary $\cdot \mathrm{CHO}=$ sum (Data. $\mathrm{C}>0$ \& Data. $\mathrm{H}>0$ \& Data. $\mathrm{O}>0$ \& Data. $\mathrm{N}==0$ \& Data. $\mathrm{P}==0$ \& Data. $\mathrm{S}==0$ );

Summary $\cdot \mathrm{CHON}=$ sum $($ Data. $\mathrm{C}>0$ \& Data.H $>0$ \& Data. $0>0$ \& Data. $\mathrm{N}>0$ \& Data.P==0 \& Data. $\mathrm{S}==0$ );

Summary. $\mathrm{CHOS}=$ sum $($ Data. $\mathrm{C}>0$ \& Data. $\mathrm{H}>0$ \& Data. $\mathrm{O}>0$ \& Data. $\mathrm{N}==0$ \& Data. $\mathrm{P}==0$ \& Data. S $>0$ );

Summary. $\mathrm{CHOP}=\mathrm{sum}($ Data. $\mathrm{C}>0$ \& Data.H $>0$ \& Data. $0>0$ \& Data. $\mathrm{N}==0$ \& Data.P $>0$ \& Data. $\mathrm{S}==0$ );

Summary $\cdot \mathrm{CHN}=$ sum $($ Data. $\mathrm{C}>0$ \& Data. $\mathrm{H}>0$ \& Data. $\mathrm{O}==0$ \& Data. $\mathrm{N}>0$ \& Data.P==0 \& Data. $\mathrm{S}==0$ );

Summary $\cdot \mathrm{CHS}=\operatorname{sum}($ Data $\cdot \mathrm{C}>0$ \& Data. $\mathrm{H}>0$ \& Data $\cdot \mathrm{O}==0$ \& Data. $\mathrm{N}==0$ \& Data. $\mathrm{P}==0$ \& Data. $\mathrm{S}>0$ );

Summary. $\mathrm{CHNS}=$ sum $($ Data. $\mathrm{C}>0$ \& Data. $\mathrm{H}>0$ \& Data. $\mathrm{O}==0$ \& Data. $\mathrm{D}>0$ \& Data. $\mathrm{P}==0$ \& Data. S>0);

Summary $\cdot \mathrm{CHNP}=\operatorname{sum}($ Data $\cdot \mathrm{C}>0$ \& Data. $\mathrm{H}>0$ \& Data. $\mathrm{O}==0$ \& Data.N $>0$ \& Data.P $>0$ \& Data. $\mathrm{S}==0$ );

Summary. $\mathrm{CHONS}=\operatorname{sum}($ Data $. \mathrm{C}>0$ \& Data. $\mathrm{H}>0$ \& Data. $\mathrm{O}>0$ \& Data. $\mathrm{N}>0$ \& Data.P==0 \& Data. $\mathrm{S}>0$ ) ;

Summary. $\mathrm{CHONP}=$ sum $($ Data. $\mathrm{C}>0$ \& Data.H $>0$ \& Data. O $>0$ \& Data.N $>0$ \& Data.P $>0$ \& Data. $\mathrm{S}=0$ ) ;

Summary. CHONSP $=$ sum $($ Data. $\mathrm{C}>0$ \& Data. $\mathrm{H}>0$ \& Data. $\mathrm{O}>0$ \& Data.N $>0$ \& Data.P $>0$ \& Data. $\mathrm{S}>0$ );

ocalculating aromaticity parameters: \# condensed aromatic (AI>=0.67), aromatic $(\mathrm{AI}>=0.5)$,

onon-aromatic $(\mathrm{AI}<0.5)$

Summary. CondensedAromatics $=$ sum (Data. AI $>=0.67)$;

Summary. Aromatics $=$ sum (Data.AI $>=0.5)$;

Summary. NonAromatics=sum (Data.AI<0.5);

ocalculating other parameters: total \# of formulae, average molecular

\%weight

Summary. TotalFormulae=height (Data);

Summary. AvgMW=mean (Data. NominalMass);

ostoring Data in Tables

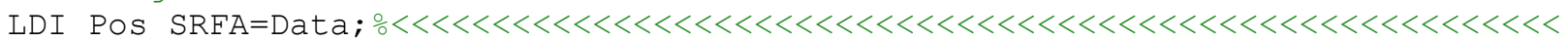
$<<<\overline{<}<<\overline{<}<<<<<<<<<<<<<<<<<<<<<<<<<<<<<<<<<<<<<<<<<<<<<<<<<<<<<<~$

LDI_Pos_SRFA_Summary=struct2table (Summary) ; $\circ<<<<<<<<<<<<<<<<<<<<<<<<<<<<<<<<<~$ $<<\overline{<}<<\overline{<} \overline{<}<<<\overline{<}<<<<<<<<<<<<<<<<<<<<<<<<<<<<<<<<<<<<<<<<<<<<<<<<<<<<<<<<<<<<<<<<~$ $<<<<<<<<<<$

\% Combining Negative and Positive Mode ESI

oInput Data so that the script can readily be applied to any data set 


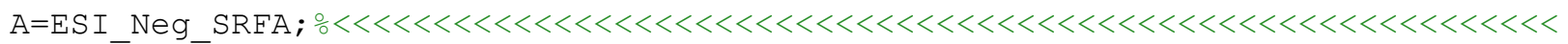
$<<<<\overline{<}<<<\overline{<}<<<<<<<<<<<<<<<<<<<<<<<<<<<<<<<<<<<<<<<<<<<<<<<<<<<<<<<<<<<<<<<<<<<~$ $<<<<<<<<<<<<<<<<<~$

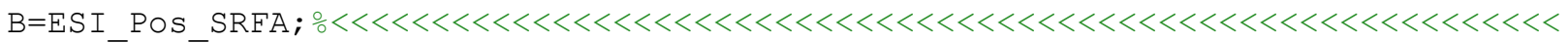

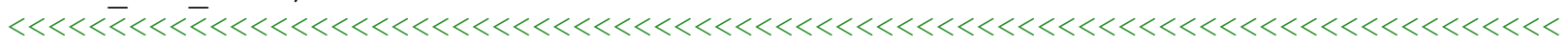
$<<<<<<<<<<<<<<<<<$

o Separate Data into those common and unique

Both=[ ]

$\mathrm{k}=0$;

for $i=1$ :height $(A)$

for $j=1$ :height $(B)$

if $A \cdot C(i)==B \cdot C(j) \quad \& \& A \cdot H(i)==B \cdot H(j) \quad \& \& A \cdot N(i)==B \cdot N(j) \quad \& \& A \cdot O(i)==B \cdot O(j)$

$\& \& A \cdot P(i)==B \cdot P(j) \quad \& \& A \cdot S(i)==B \cdot S(j)$

A. ObservedIntens $(i)=0$;

B. ObservedIntens $(j)=0$;

$\mathrm{k}=\mathrm{k}+1$;

Both $(k,:)=[A . C(i), A . H(i), A . O(i), A . N(i), A . P(i)$,

A.S(i), A.NominalMass (i), A.HC Ratio(i),

A.OC_Ratio(i),A.AI (i), A. DBE (i), A.AvgCoxState (i) ] ;

end

end

end

$\mathrm{k}=0$;

for $i=1:$ height (B)

for $j=1: \operatorname{height}(A)$

if $B \cdot C(i)==A \cdot C(j) \quad \& \& B \cdot H(i)==A \cdot H(j) \quad \& \& B \cdot N(i)==A \cdot N(j) \quad \& \& \quad B \cdot O(i)==A \cdot O(j)$

$\& \& B \cdot P(i)==A \cdot P(j) \quad \& \& \quad B \cdot S(i)==A \cdot S(j)$

B. ObservedIntens $(i)=0$;

A. Observedintens $(j)=0$;

$\mathrm{k}=\mathrm{k}+1$;

$\operatorname{Both}(k,:)=[B . C(i), B . H(i), B . O(i), B . N(i), B . P(i)$,

B.S (i), B.NominalMass (i), B.HC_Ratio(i),

B.OC Ratio(i), B.AI (i), B.DBE(i), B.AvgCOxState (i) ] ;

end

end

end

\%Combining Data Sets

A $(A$. ObservedIntens $==0,:)=[]$;

$A O n l_{Y}=[A . C, A . H, A . O, A . N, A . P, A . S$,

A.Nominalmass, A.HC_Ratio,A.oC_Ratio,A.AI, A.DBE, A.AvgCoxState];

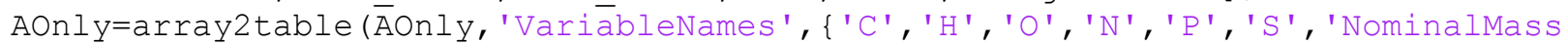
', 'HC Ratio', 'OC Ratio', 'AI ', 'DBE', 'AvgCoxstate' \});

$\mathrm{B}(\mathrm{B} . \mathrm{O} \overline{\mathrm{b}}$ servedinten $\mathrm{s}==0,:)=[]$;

$B O n l_{Y}=[B . C, B . H, B . O, B . N, B . P$,

B.S, B. NominalMass, B.HC Ratio,B.OC Ratio, B.AI, B.DBE, B. AvgCoxstate];

Bonly=array2table (BOnlȳy, 'Variableñames', \{'C', 'H', 'O', 'N', 'P' , 'S ', 'NominalMass ', 'HC_Ratio', 'OC_Ratio', 'AI', 'DBE', 'AvgCOxState'\});

Both=array2table (Both, 'VariableNames' , \{'C', 'H', 'O', 'N', 'P', 'S ', 'NominalMass', 'HC_Ratio', 'OC_Ratio', 'AI', 'DBE', 'AvgCOxState'\});

Combined=[Both; AOnly; BOnly];

ostoring Data with distinct names

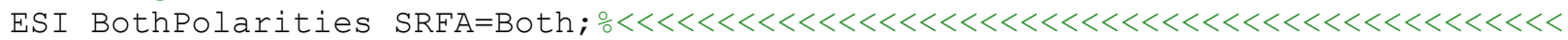
$<<\overline{<}<<<<<<<<<<<<<<\overline{<}<<<<<<<<<<<<<<<<<<<<<<<<<<<<<<<<<<<<<<<<<<<<<<<<<<<<<<<<<<~$ $<<<<<<<<<<<<<<<<<<<<<<<<<<<<<<<~$

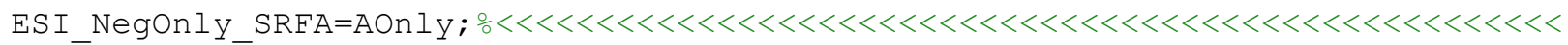
$<<\overline{<}<<<<<<<\overline{<}<<<<<<<<<<<<<<<<<<<<<<<<<<<<<<<<<<<<<<<<<<<<<<<<<<<<<<<<<<<<<<<<<~$ $<<<<<<<<<<<<<<<<<<<<<<<<<~$ 


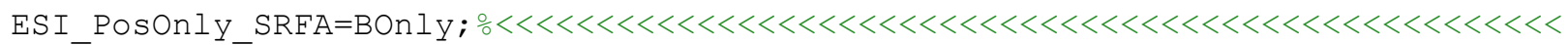
$<<\overline{<}<<<<<<<\overline{<}<<<<<<<<<<<<<<<<<<<<<<<<<<<<<<<<<<<<<<<<<<<<<<<<<<<<<<<<<<<<<<<<<~$ $<<<<<<<<<<<<<<<<<<<<<<<<<$

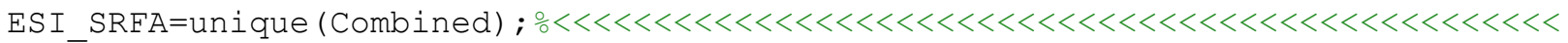

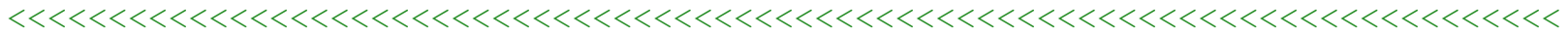
$<<<<<<<<<<<<<<<<<<<<<<<<<<<<~$

ㅇ Summarizing Data

Data=AOnly;

oCalculating formulae type parameters: \# CHO, CHON, CHOS, CHOP, CHN, CHS, CHP, CHNS, CHONS formulae

Summary $\cdot \mathrm{CHO}=$ sum (Data. $\mathrm{C}>0$ \& Data. $\mathrm{H}>0$ \& Data. $\mathrm{O}>0$ \& Data. $\mathrm{N}==0$ \& Data. $\mathrm{P}==0$ \& Data. $\mathrm{S}=0$ ) ;

Summary. $\mathrm{CHON}=$ sum $($ Data. $\mathrm{C}>0$ \& Data. $\mathrm{H}>0$ \& Data.O $>0$ \& Data.N $>0$ \& Data.P==0 \& Data. $\mathrm{S}==0$ );

Summary. $\mathrm{CHOS}=$ sum $($ Data. $\mathrm{C}>0$ \& Data. $\mathrm{H}>0$ \& Data. $\mathrm{O}>0$ \& Data. $\mathrm{N}==0$ \& Data. $\mathrm{P}==0$ \& Data. $\mathrm{S}>0$ );

Summary. $\mathrm{CHOP}=\operatorname{sum}($ Data. $\mathrm{C}>0$ \& Data.H $>0$ \& Data. $\mathrm{O}>0$ \& Data. $\mathrm{N}==0$ \& Data.P $>0$ \& Data. $\mathrm{S}==0$ );

Summary $\cdot \mathrm{CHN}=$ sum $($ Data. $\mathrm{C}>0$ \& Data. $\mathrm{H}>0$ \& Data. $\mathrm{O}==0$ \& Data. $\mathrm{N}>0$ \& Data. $\mathrm{P}==0$ \& Data. $\mathrm{S}==0$ );

Summary. $\mathrm{CHS}=\operatorname{sum}($ Data $\cdot \mathrm{C}>0$ \& Data. $\mathrm{H}>0$ \& Data $\cdot \mathrm{O}==0$ \& Data. $\mathrm{N}==0$ \& Data. $\mathrm{P}==0$ \& Data. S $>0$ );

Summary. $\mathrm{CHNS}=$ sum $($ Data. $\mathrm{C}>0$ \& Data. $\mathrm{H}>0$ \& Data. $\mathrm{O}==0$ \& Data. $\mathrm{N}>0$ \& Data. $\mathrm{P}==0$ \&

Data. $\mathrm{S}>0$ );

Summary $\cdot \mathrm{CHNP}=\mathrm{sum}($ Data. $\mathrm{C}>0$ \& Data.H $>0$ \& Data. $\mathrm{O}==0$ \& Data.N $>0$ \& Data.P $>0$ \&

Data. $\mathrm{S}==0$ );

Summary $\cdot \mathrm{CHONS}=\operatorname{sum}($ Data $\cdot \mathrm{C}>0$ \& Data. $\mathrm{H}>0$ \& Data. $\mathrm{O}>0$ \& Data. $\mathrm{N}>0$ \& Data. $\mathrm{P}==0$ \&

Data. $\mathrm{S}>0)$;

Summary $\cdot \mathrm{CHONP}=$ sum $($ Data. $\mathrm{C}>0$ \& Data.H $>0$ \& Data. O $>0$ \& Data.N $>0$ \& Data.P $>0$ \&

Data. $\mathrm{S}==0$ );

Summary. $\mathrm{CHONSP}=$ sum $($ Data. $\mathrm{C}>0$ \& Data. $\mathrm{H}>0$ \& Data. $\mathrm{O}>0$ \& Data.N $>0$ \& Data.P $>0$ \&

Data. S>0);

ocalculating aromaticity parameters: \# condensed aromatic (AI>=0.67),

aromatic $(\mathrm{AI}>=0.5)$,

onon-aromatic $($ AI<0.5)

Summary. CondensedAromatics $=$ sum (Data. AI $>=0.67)$;

Summary. Aromatics=sum (Data.AI>=0.5);

Summary. NonAromatics=sum (Data.AI<0.5);

ocalculating other parameters: total \# of formulae, average molecular

oweight

Summary. TotalFormulae=height (Data);

Summary. AvgMW=mean (Data. NominalMass);

ostoring Summary Data

ESI NegOnly_SRFA Summary=struct2table (Summary); $\circ<<<<<<<<<<<<<<<<<<<<<<<<<<<<<<<<~$ $<<\overline{\bar{l}}<<<<<<<\overline{<}<<<<\overline{<}<<<<<<<<<<<<<<<<<<<<<<<<<<<<<<<<<<<<<<<<<<<<<<<<<<<<<<<<<<<<~$

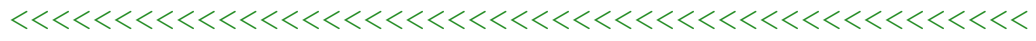

o Summarizing Data

Data=BOnly;

oCalculating formulae type parameters: \# CHO, CHON, CHOS, CHOP, CHN, CHS, CHP, CHNS, CHONS formulae

Summary $\cdot \mathrm{CHO}=$ sum $($ Data. $\mathrm{C}>0$ \& Data. $\mathrm{H}>0$ \& Data. $\mathrm{O}>0$ \& Data. $\mathrm{N}==0$ \& Data. $\mathrm{P}==0$ \&

Data. $\mathrm{S}==0$ );

Summary $\cdot \mathrm{CHON}=\operatorname{sum}($ Data. $\mathrm{C}>0$ \& Data.H $>0$ \& Data. $\mathrm{O}>0$ \& Data. $>0$ \& Data.P==0 \& Data. $\mathrm{S}==0$ );

Summary. $\mathrm{CHOS}=$ sum $($ Data. $\mathrm{C}>0$ \& Data. $\mathrm{H}>0$ \& Data. $\mathrm{O}>0$ \& Data. $\mathrm{N}==0$ \& Data. $\mathrm{P}==0$ \&

Data. $\mathrm{S}>0$ ) ; 
Summary. $\mathrm{CHOP}=\mathrm{sum}($ Data. $\mathrm{C}>0$ \& Data.H $>0$ \& Data.O $>0$ \& Data.N==0 \& Data.P>0 \& Data. $\mathrm{S}==0$ );

Summary $\cdot \mathrm{CHN}=$ sum $($ Data. $\mathrm{C}>0$ \& Data. $\mathrm{H}>0$ \& Data. $\mathrm{O}==0$ \& Data. $\mathrm{N}>0$ \& Data.P==0 \& Data. $\mathrm{S}==0$ ) ;

Summary $\cdot \mathrm{CHS}=\operatorname{sum}($ Data $\cdot \mathrm{C}>0$ \& Data. $\mathrm{H}>0$ \& Data. $\mathrm{O}==0$ \& Data. $\mathrm{N}==0$ \& Data. $\mathrm{P}==0$ \& Data. $\mathrm{S}>0$ );

Summary $\cdot \mathrm{CHNS}=$ sum $($ Data $\cdot \mathrm{C}>0$ \& Data. $\mathrm{H}>0$ \& Data. $\mathrm{O}==0$ \& Data. $\mathrm{N}>0$ \& Data. $\mathrm{P}==0$ \&

Data. $\mathrm{S}>0$ ) ;

Summary $\cdot \mathrm{CHNP}=\operatorname{sum}($ Data $\cdot \mathrm{C}>0$ \& Data. $\mathrm{H}>0$ \& Data. $\mathrm{O}==0$ \& Data.N $>0$ \& Data.P>0 \&

Data. $\mathrm{S}==0$ );

Summary. $\mathrm{CHONS}=$ sum $($ Data $. \mathrm{C}>0$ \& Data. $\mathrm{H}>0$ \& Data. $\mathrm{O}>0$ \& Data. $>0$ \& Data.P==0 \&

Data. $\mathrm{S}>0$ );

Summary. $\mathrm{CHONP}=$ sum $($ Data. $\mathrm{C}>0$ \& Data.H $>0$ \& Data.O>0 \& Data.N>0 \& Data.P>0 \&

Data. $\mathrm{S}==0$ );

Summary. $\mathrm{CHONSP}=$ sum $($ Data. $\mathrm{C}>0$ \& Data. $\mathrm{H}>0$ \& Data.O $>0$ \& Data.N $>0$ \& Data.P $>0$ \&

Data. $\mathrm{S}>0$ ) ;

ocalculating aromaticity parameters: \# condensed aromatic (AI>=0.67),

aromatic $(A I>=0.5)$,

onon-aromatic $(\mathrm{AI}<0.5)$

Summary. CondensedAromatics $=$ sum (Data. AI $>=0.67)$;

Summary. Aromatics $=$ sum (Data.AI $>=0.5)$;

Summary. NonAromatics=sum (Data.AI<0.5);

oCalculating other parameters: total \# of formulae, average molecular

oweight

Summary. TotalFormulae=height (Data);

Summary. AvgMW=mean (Data. NominalMass);

oStoring Summary Data

ESI_PosOnly_SRFA_Summary=struct2table (Summary) ; $\circ<<<<<<<<<<<<<<<<<<<<<<<<<<<<<<<~$

$<<\overline{<}<<<<<<<\overline{<}<<<\overline{<}<<<<<<<<<<<<<<<<<<<<<<<<<<<<<<<<<<<<<<<<<<<<<<<<<<<<<<<<<<<<<$

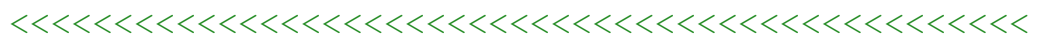

ㅇmmarizing Data

Data=Both;

oCalculating formulae type parameters: \# CHO, CHON, CHOS, CHOP, CHN, CHS, CHP, CHNS, CHONS formulae

Summary $\cdot \mathrm{CHO}=$ sum $($ Data. $\mathrm{C}>0$ \& Data. $\mathrm{H}>0$ \& Data. $\mathrm{O}>0$ \& Data. $\mathrm{N}==0$ \& Data. $\mathrm{P}==0$ \& Data. $\mathrm{S}==0$ );

Summary $\cdot \mathrm{CHON}=$ sum $($ Data $\cdot \mathrm{C}>0$ \& Data. $\mathrm{H}>0$ \& Data. $\mathrm{O}>0$ \& Data.N $>0$ \& Data.P==0 \&

Data. $\mathrm{S}==0$ );

Summary. CHOS $=$ sum $($ Data. $\mathrm{C}>0$ \& Data. $\mathrm{H}>0$ \& Data. $\mathrm{O}>0$ \& Data. $\mathrm{N}==0$ \& Data.P==0 \&

Data. $\mathrm{S}>0$ ) ;

Summary. $\mathrm{CHOP}=\mathrm{sum}($ Data. $\mathrm{C}>0$ \& Data.H $>0$ \& Data.O $>0$ \& Data.N==0 \& Data.P>0 \&

Data. $\mathrm{S}==0$ );

Summary. $\mathrm{CHN}=$ sum $($ Data. $\mathrm{C}>0$ \& Data. $\mathrm{H}>0$ \& Data. $\mathrm{O}==0$ \& Data. $\mathrm{N}>0$ \& Data.P==0 \&

Data. $\mathrm{S}==0$ );

Summary $\cdot \mathrm{CHS}=\operatorname{sum}($ Data $. \mathrm{C}>0$ \& Data. $\mathrm{H}>0$ \& Data. $\mathrm{O}==0$ \& Data. $\mathrm{N}==0$ \& Data. $\mathrm{P}==0$ \&

Data. $\mathrm{S}>0$ ) ;

Summary $\cdot \mathrm{CHNS}=$ sum $($ Data $\cdot \mathrm{C}>0$ \& Data. $\mathrm{H}>0$ \& Data $\cdot \mathrm{O}==0$ \& Data. $\mathrm{N}>0$ \& Data.P==0 \&

Data. $\mathrm{S}>0$ );

Summary. $\mathrm{CHNP}=$ sum $($ Data. $\mathrm{C}>0$ \& Data. $\mathrm{H}>0$ \& Data. $\mathrm{O}==0$ \& Data. $\mathrm{N}>0$ \& Data.P $>0$ \&

Data. $\mathrm{S}==0$ );

Summary $\cdot \mathrm{CHONS}=$ sum $($ Data $\cdot \mathrm{C}>0$ \& Data. $\mathrm{H}>0$ \& Data. $\mathrm{O}>0$ \& Data.N $>0$ \& Data.P==0 \&

Data. $\mathrm{S}>0$ ) ;

Summary. $\mathrm{CHONP}=$ sum $($ Data. $\mathrm{C}>0$ \& Data.H $>0$ \& Data. $\mathrm{O}>0$ \& Data.N $>0$ \& Data.P>0 \&

Data. $\mathrm{S}==0$ );

Summary. $\mathrm{CHONSP}=$ sum $($ Data. $\mathrm{C}>0$ \& Data. $\mathrm{H}>0$ \& Data. $\mathrm{O}>0$ \& Data.N $>0$ \& Data.P $>0$ \&

Data. $\mathrm{S}>0$ ) ; 
oCalculating aromaticity parameters: \# condensed aromatic (AI>=0.67), aromatic $(\mathrm{AI}>=0.5)$,

onon-aromatic $(\mathrm{AI}<0.5)$

Summary. CondensedAromatics $=$ sum (Data.AI $>=0.67)$;

Summary.Aromatics=sum (Data.AI $>=0.5)$;

Summary. NonAromatics=sum (Data.AI<0.5);

ocalculating other parameters: total \# of formulae, average molecular

oweight

Summary. TotalFormulae=height (Data);

Summary . AvgMW=mean (Data. NominalMass);

ostoring Summary Data

ESI_BothPolarities SRFA Summary=struct2table (Summary); $\circ<<<<<<<<<<<<<<<<<<<<<<~$ $<<\overline{<}<<<<<<<<<<<<<<\overline{<}<<<\overline{<}<<<<<<<<<<<<<<<<<<<<<<<<<<<<<<<<<<<<<<<<<<<<<<<<<<<<<<~$

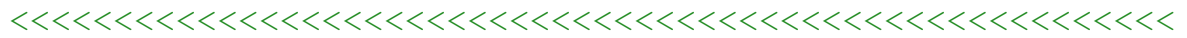

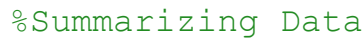

Data $=$ Combined;

oCalculating formulae type parameters: \# CHO, CHON, CHOS, CHOP, CHN, CHS, CHP, CHNS, CHONS formulae

Summary $\cdot \mathrm{CHO}=$ sum $($ Data. $\mathrm{C}>0$ \& Data. $\mathrm{H}>0$ \& Data. $\mathrm{O}>0$ \& Data. $\mathrm{N}==0$ \& Data. $\mathrm{P}==0$ \& Data. $\mathrm{S}==0$ );

Summary $\cdot \mathrm{CHON}=$ Sum $($ Data. $\mathrm{C}>0$ \& Data. $\mathrm{H}>0$ \& Data. $\mathrm{O}>0$ \& Data. $\mathrm{N}>0$ \& Data.P==0 \& Data. $\mathrm{S}==0$ );

Summary. $\mathrm{CHOS}=$ sum $($ Data. $\mathrm{C}>0$ \& Data. $\mathrm{H}>0$ \& Data. $\mathrm{O}>0$ \& Data. $\mathrm{N}==0$ \& Data. $\mathrm{P}==0$ \&

Data. $\mathrm{S}>0$ );

Summary $\cdot \mathrm{CHOP}=$ sum $($ Data. $\mathrm{C}>0$ \& Data.H $>0$ \& Data. $\mathrm{O}>0$ \& Data. $\mathrm{N}==0$ \& Data.P $>0$ \&

Data. $\mathrm{S}==0$ );

Summary $\cdot \mathrm{CHN}=$ sum $($ Data. $\mathrm{C}>0$ \& Data. $\mathrm{H}>0$ \& Data. $\mathrm{O}==0$ \& Data. $\mathrm{N}>0$ \& Data. $\mathrm{P}==0$ \&

Data. $\mathrm{S}==0$ );

Summary $\cdot \mathrm{CHS}=\operatorname{sum}($ Data $\cdot \mathrm{C}>0$ \& Data. $\mathrm{H}>0$ \& Data $\cdot \mathrm{O}==0$ \& Data. $\mathrm{N}==0$ \& Data. $\mathrm{P}==0$ \&

Data. $\mathrm{S}>0$ );

Summary. $\mathrm{CHNS}=$ sum $($ Data. $\mathrm{C}>0$ \& Data. $\mathrm{H}>0$ \& Data. $\mathrm{O}==0$ \& Data. $\mathrm{N}>0$ \& Data. $\mathrm{P}==0$ \&

Data. S>0);

Summary $\cdot \mathrm{CHNP}=\operatorname{sum}($ Data. $\mathrm{C}>0$ \& Data.H $>0$ \& Data. $\mathrm{O}==0$ \& Data. $\mathrm{N}>0$ \& Data.P $>0$ \&

Data. $\mathrm{S}==0$ );

Summary. $\mathrm{CHONS}=\operatorname{sum}($ Data $. \mathrm{C}>0$ \& Data. $\mathrm{H}>0$ \& Data. $0>0$ \& Data.N>0 \& Data.P==0 \&

Data. S>0);

Summary. $\mathrm{CHONP}=$ sum $($ Data. $\mathrm{C}>0$ \& Data. $\mathrm{H}>0$ \& Data. $\mathrm{O}>0$ \& Data.N $>0$ \& Data.P $>0$ \&

Data. $\mathrm{S}==0$ ) ;

Summary. $\mathrm{CHONSP}=$ sum $($ Data. $\mathrm{C}>0$ \& Data.H $>0$ \& Data.O $>0$ \& Data.N $>0$ \& Data.P>0 \&

Data. $\mathrm{S}>0$ );

ocalculating aromaticity parameters: \# condensed aromatic (AI>=0.67),

aromatic (AI>=0.5),

onon-aromatic $(\mathrm{A} I<0.5)$

Summary. CondensedAromatics $=$ sum (Data. AI $>=0.67)$;

Summary. Aromatics $=$ sum (Data.AI $>=0.5)$;

Summary. NonAromatics=sum (Data.AI<0.5);

ocalculating other parameters: total \# of formulae, average molecular

oweight

Summary. TotalFormulae=height (Data);

Summary. AvgMW=mean (Data. NominalMass) ;

ostoring Summary Data

ESI SRFA Summary=struct2table (Summary) ; 아 $<<<<<<<<<<<<<<<<<<<<<<<<<<<<<<<<<<<<<<<<~$

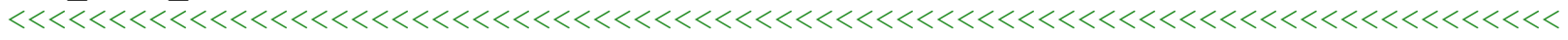

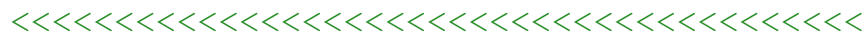

응 Combining Negative and Positive Mode LDI

oInput Data 


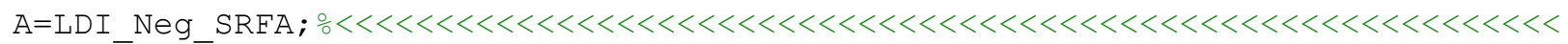
$<<<<\overline{<}<<<\overline{<}<<<<<<<<<<<<<<<<<<<<<<<<<<<<<<<<<<<<<<<<<<<<<<<<<<<<<<<<<<<<<<<<<<<~$ $<<<<<<<<<<<<<<<<<~$

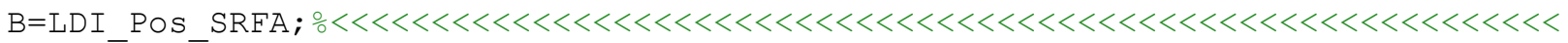

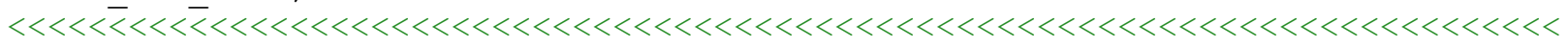
$<<<<<<<<<<<<<<<<<$

o Separate Data into those common and unique

Both=[ ]

$\mathrm{k}=0$;

for $i=1$ :height $(A)$

for $j=1$ :height $(B)$

if $A \cdot C(i)==B \cdot C(j) \quad \& \& A \cdot H(i)==B \cdot H(j) \quad \& \& A \cdot N(i)==B \cdot N(j) \quad \& \& A \cdot O(i)==B \cdot O(j)$

$\& \& A \cdot P(i)==B \cdot P(j) \quad \& \& A \cdot S(i)==B \cdot S(j)$

A. ObservedIntens $(i)=0$;

B. ObservedIntens $(j)=0$;

$\mathrm{k}=\mathrm{k}+1$;

$\operatorname{Both}(k,:)=[A . C(i), A . H(i), A . O(i), A . N(i), A . P(i)$,

A.S (i), A.NominalMass (i), A.HC Ratio(i),

A.OC_Ratio(i),A.AI (i), A. DBE (i), A.AvgCoxState (i) ] ;

end

end

end

$\mathrm{k}=0$;

for $i=1$ :height (B)

for $j=1$ :height $(A)$

if $B \cdot C(i)==A \cdot C(j) \quad \& \& B \cdot H(i)==A \cdot H(j) \quad \& \& B \cdot N(i)==A \cdot N(j) \quad \& \& \quad B \cdot O(i)==A \cdot O(j)$

$\& \& \quad B \cdot P(i)==A \cdot P(j) \quad \& \& \quad B . S(i)==A \cdot S(j)$

B. ObservedIntens $(i)=0$;

A. ObservedIntens $(j)=0$;

$\mathrm{k}=\mathrm{k}+1$;

$\operatorname{Both}(k,:)=[B . C(i), B . H(i), B . O(i), B . N(i), B . P(i)$,

B.S (i), B.NominalMass (i), B.HC_Ratio(i),

B.OC Ratio(i),B.AI (i), B.DBE(i), B.AvgCOxState (i) ] ;

end

end

end

\%Combining Data Sets

A $(A$. Observedintens $==0,:)=[]$;

$A O n l_{Y}=[A . C, A . H, A . O, A . N, A . P, A . S$,

A.NominalMass, A.HC Ratio,A.oC Ratio,A.AI, A.DBE, A.AvgCoxState];

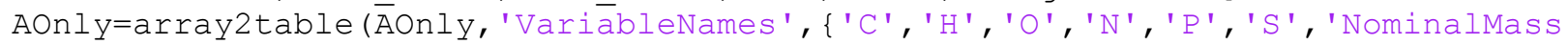
', 'HC Ratio', 'OC Ratio', 'AI ', 'DBE', 'AvgCoxState' \}) ;

$\mathrm{B}(\mathrm{B} . \mathrm{O} \overline{\mathrm{b}}$ servedinten $\mathrm{s}==0,:)=[]$;

$B O n l_{Y}=[B . C, B . H, B . O, B . N, B . P$,

B.S,B.NominalMass, B.HC Ratio,B.OC Ratio,B.AI, B.DBE, B. AvgCoxstate];

Bonly=array2table (BOnlȳ, 'Variableñames ', \{'C', 'H', 'O', 'N', ' P' , 'S ', 'NominalMass ', 'HC_Ratio', 'OC_Ratio', 'AI', 'DBE', 'AvgCoxstate' \}) ;

Both=array2table (Both, 'VariableNames' , \{'C', 'H', 'O', 'N', 'P', 'S ', 'NominalMass', 'HC Ratio', 'OC Ratio', 'AI', 'DBE', 'AvgCOxState'\});

Combined=[Both; AOnly; BOnly];

ostoring Data with distinct names

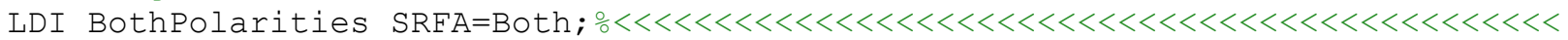
$<<\overline{<}<<<<<<<<<<<<<<\overline{<}<<<<<<<<<<<<<<<<<<<<<<<<<<<<<<<<<<<<<<<<<<<<<<<<<<<<<<<<<<~$ $<<<<<<<<<<<<<<<<<<<<<<<<<<<<<<<~$

LDI NegOnly SRFA=AOnly; $\circ \circ<<<<<<<<<<<<<<<<<<<<<<<<<<<<<<<<<<<<<<<<<<<<<<<<<<<<<<~$ $<<\overline{<}<<<<<<<\overline{<}<<<<<<<<<<<<<<<<<<<<<<<<<<<<<<<<<<<<<<<<<<<<<<<<<<<<<<<<<<<<<<<<<~$ $<<<<<<<<<<<<<<<<<<<<<<<<<~$ 
LDI_PosOnIY_SRFA=BOnIY; $\circ 2<<<<<<<<<<<<<<<<<<<<<<<<<<<<<<<<<<<<<<<<<<<<<<<<<<<<<<<~$ $<<\overline{<}<<<<<<<\overline{<}<<<<<<<<<<<<<<<<<<<<<<<<<<<<<<<<<<<<<<<<<<<<<<<<<<<<<<<<<<<<<<<<<~$ $<<<<<<<<<<<<<<<<<<<<<<<<<$

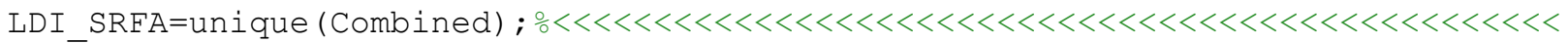
$<<\overline{<}<<<<<<<<<<<<<<<<<<<<<<<<<<<<<<<<<<<<<<<<<<<<<<<<<<<<<<<<<<<<<<<<<<<<<<<<<~$ $<<<<<<<<<<<<<<<<<<<<<<<<<<<<~$

ㅇ Summarizing Data

Data=AOnly;

oCalculating formulae type parameters: \# CHO, CHON, CHOS, CHOP, CHN, CHS, CHP, CHNS, CHONS formulae

Summary $\cdot \mathrm{CHO}=$ sum (Data. $\mathrm{C}>0$ \& Data. $\mathrm{H}>0$ \& Data. $\mathrm{O}>0$ \& Data. $\mathrm{N}==0$ \& Data. $\mathrm{P}==0$ \& Data. $\mathrm{S}=0$ ) );

Summary. $\mathrm{CHON}=$ sum $($ Data. $\mathrm{C}>0$ \& Data. $\mathrm{H}>0$ \& Data.O $>0$ \& Data.N $>0$ \& Data.P==0 \&

Data. $\mathrm{S}==0$ );

Summary $\cdot \mathrm{CHOS}=$ sum $($ Data. $\mathrm{C}>0$ \& Data. $\mathrm{H}>0$ \& Data. $\mathrm{O}>0$ \& Data. $\mathrm{N}==0$ \& Data. $\mathrm{P}==0$ \&

Data. S>0);

Summary $\cdot \mathrm{CHOP}=\mathrm{sum}($ Data. $\mathrm{C}>0$ \& Data.H $>0$ \& Data. $0>0$ \& Data. $\mathrm{N}==0$ \& Data.P $>0$ \&

Data. $\mathrm{S}==0$ );

Summary $\cdot \mathrm{CHN}=$ sum $($ Data. $\mathrm{C}>0$ \& Data. $\mathrm{H}>0$ \& Data. $\mathrm{O}==0$ \& Data. $\mathrm{N}>0$ \& Data. $\mathrm{P}==0$ \&

Data. $\mathrm{S}==0$ );

Summary. $\mathrm{CHS}=\operatorname{sum}($ Data $\cdot \mathrm{C}>0$ \& Data. $\mathrm{H}>0$ \& Data $\cdot \mathrm{O}==0$ \& Data. $\mathrm{N}==0$ \& Data. $\mathrm{P}==0$ \&

Data. S $>0$ );

Summary. $\mathrm{CHNS}=$ sum $($ Data $. \mathrm{C}>0$ \& Data. $\mathrm{H}>0$ \& Data. $\mathrm{O}==0$ \& Data. $\mathrm{N}>0$ \& Data. $\mathrm{P}==0$ \&

Data. $\mathrm{S}>0$ );

Summary $\cdot \mathrm{CHNP}=\mathrm{sum}($ Data. $\mathrm{C}>0$ \& Data.H $>0$ \& Data. $\mathrm{O}==0$ \& Data.N $>0$ \& Data.P $>0$ \&

Data. $\mathrm{S}==0$ );

Summary $\cdot \mathrm{CHONS}=\operatorname{sum}($ Data $\cdot \mathrm{C}>0$ \& Data. $\mathrm{H}>0$ \& Data. $\mathrm{O}>0$ \& Data. $\mathrm{N}>0$ \& Data. $\mathrm{P}==0$ \&

Data. $\mathrm{S}>0)$;

Summary. $\mathrm{CHONP}=$ sum $($ Data. $\mathrm{C}>0$ \& Data. $\mathrm{H}>0$ \& Data. $\mathrm{O}>0$ \& Data. $\mathrm{N}>0$ \& Data.P $>0$ \&

Data. $\mathrm{S}==0$ );

Summary. $\mathrm{CHONSP}=$ sum $($ Data. $\mathrm{C}>0$ \& Data. $\mathrm{H}>0$ \& Data. $\mathrm{O}>0$ \& Data.N $>0$ \& Data.P $>0$ \&

Data. S>0);

oCalculating aromaticity parameters: \# condensed aromatic (AI>=0.67),

aromatic $(\mathrm{AI}>=0.5)$,

onon-aromatic $($ AI<0.5)

Summary. CondensedAromatics $=$ sum (Data. AI $>=0.67)$;

Summary. Aromatics=sum (Data.AI>=0.5);

Summary. NonAromatics=sum (Data.AI<0.5);

ocalculating other parameters: total \# of formulae, average molecular

oweight

Summary. TotalFormulae=height (Data);

Summary. AvgMW=mean (Data. NominalMass);

ostoring Summary Data

LDI NegOnly_SRFA Summary=struct2table (Summary); $\circ<<<<<<<<<<<<<<<<<<<<<<<<<<<<<<<<~$ $<<\overline{\bar{l}}<<<<<<<\overline{<}<<<\overline{\bar{l}}<<<<<<<<<<<<<<<<<<<<<<<<<<<<<<<<<<<<<<<<<<<<<<<<<<<<<<<<<<<<<$

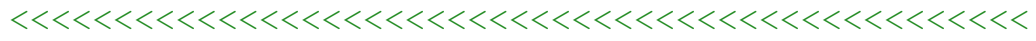

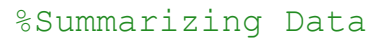

Data=BOnly;

oCalculating formulae type parameters: \# CHO, CHON, CHOS, CHOP, CHN, CHS, CHP, CHNS, CHONS formulae

Summary $\cdot \mathrm{CHO}=$ sum $($ Data. $\mathrm{C}>0$ \& Data. $\mathrm{H}>0$ \& Data. $\mathrm{O}>0$ \& Data. $\mathrm{N}==0$ \& Data. $\mathrm{P}==0$ \&

Data. $\mathrm{S}==0$ );

Summary $\cdot \mathrm{CHON}=\operatorname{sum}($ Data. $\mathrm{C}>0$ \& Data.H $>0$ \& Data. $\mathrm{O}>0$ \& Data. $>0$ \& Data.P==0 \& Data. $\mathrm{S}==0$ );

Summary. $\mathrm{CHOS}=$ sum $($ Data. $\mathrm{C}>0$ \& Data. $\mathrm{H}>0$ \& Data. $\mathrm{O}>0$ \& Data. $\mathrm{N}==0$ \& Data. $\mathrm{P}==0$ \&

Data. $\mathrm{S}>0$ ) ; 
Summary. $\mathrm{CHOP}=\mathrm{sum}($ Data. $\mathrm{C}>0$ \& Data.H $>0$ \& Data.O $>0$ \& Data.N==0 \& Data.P>0 \& Data. $\mathrm{S}==0$ );

Summary $\cdot \mathrm{CHN}=$ sum $($ Data. $\mathrm{C}>0$ \& Data. $\mathrm{H}>0$ \& Data. $\mathrm{O}==0$ \& Data. $\mathrm{N}>0$ \& Data.P==0 \& Data. $\mathrm{S}==0$ ) ;

Summary $\cdot \mathrm{CHS}=\operatorname{sum}($ Data $\cdot \mathrm{C}>0$ \& Data. $\mathrm{H}>0$ \& Data. $\mathrm{O}==0$ \& Data. $\mathrm{N}==0$ \& Data. $\mathrm{P}==0$ \& Data. $\mathrm{S}>0$ );

Summary $\cdot \mathrm{CHNS}=$ sum $($ Data $\cdot \mathrm{C}>0$ \& Data. $\mathrm{H}>0$ \& Data. $\mathrm{O}==0$ \& Data. $\mathrm{N}>0$ \& Data. $\mathrm{P}==0$ \&

Data. $\mathrm{S}>0$ ) ;

Summary $\cdot \mathrm{CHNP}=\operatorname{sum}($ Data $\cdot \mathrm{C}>0$ \& Data. $\mathrm{H}>0$ \& Data. $\mathrm{O}==0$ \& Data.N $>0$ \& Data.P>0 \&

Data. $\mathrm{S}==0$ );

Summary. $\mathrm{CHONS}=$ sum $($ Data $. \mathrm{C}>0$ \& Data. $\mathrm{H}>0$ \& Data. $\mathrm{O}>0$ \& Data. $>0$ \& Data.P==0 \&

Data. $\mathrm{S}>0$ );

Summary. $\mathrm{CHONP}=$ sum $($ Data. $\mathrm{C}>0$ \& Data.H $>0$ \& Data.O>0 \& Data.N>0 \& Data.P>0 \&

Data. $\mathrm{S}==0$ );

Summary. $\mathrm{CHONSP}=$ sum $($ Data. $\mathrm{C}>0$ \& Data. $\mathrm{H}>0$ \& Data.O $>0$ \& Data.N $>0$ \& Data.P $>0$ \&

Data. S>0);

ocalculating aromaticity parameters: \# condensed aromatic (AI>=0.67),

aromatic $(A I>=0.5)$,

onon-aromatic $(\mathrm{AI}<0.5)$

Summary. CondensedAromatics $=$ sum (Data. AI $>=0.67)$;

Summary. Aromatics $=$ sum (Data.AI $>=0.5)$;

Summary. NonAromatics=sum (Data.AI<0.5);

oCalculating other parameters: total \# of formulae, average molecular

oweight

Summary. TotalFormulae=height (Data);

Summary. AvgMW=mean (Data. NominalMass);

ostoring Summary Data

LDI_PosOnly_SREA_Summary=struct2table (Summary) ; $\circ<<<<<<<<<<<<<<<<<<<<<<<<<<<<<<~$

$<<\overline{<}<<<<<<<\overline{<}<<<\overline{<}<<<<<<<<<<<<<<<<<<<<<<<<<<<<<<<<<<<<<<<<<<<<<<<<<<<<<<<<<<<<<$

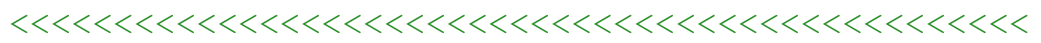

ㅇmmarizing Data

Data=Both;

oCalculating formulae type parameters: \# CHO, CHON, CHOS, CHOP, CHN, CHS, CHP, CHNS, CHONS formulae

Summary $\cdot \mathrm{CHO}=$ sum $($ Data. $\mathrm{C}>0$ \& Data. $\mathrm{H}>0$ \& Data. $\mathrm{O}>0$ \& Data. $\mathrm{N}==0$ \& Data. $\mathrm{P}==0$ \& Data. $\mathrm{S}==0$ );

Summary $\cdot \mathrm{CHON}=$ sum $($ Data $\cdot \mathrm{C}>0$ \& Data. $\mathrm{H}>0$ \& Data. $\mathrm{O}>0$ \& Data.N $>0$ \& Data.P==0 \&

Data. $\mathrm{S}==0$ );

Summary. CHOS $=$ sum $($ Data. $\mathrm{C}>0$ \& Data. $\mathrm{H}>0$ \& Data. $\mathrm{O}>0$ \& Data. $\mathrm{N}==0$ \& Data.P==0 \&

Data. $\mathrm{S}>0$ ) ;

Summary. $\mathrm{CHOP}=\mathrm{sum}($ Data. $\mathrm{C}>0$ \& Data.H $>0$ \& Data.O $>0$ \& Data.N==0 \& Data.P>0 \&

Data. $\mathrm{S}==0$ );

Summary. $\mathrm{CHN}=$ sum $($ Data. $\mathrm{C}>0$ \& Data. $\mathrm{H}>0$ \& Data. $\mathrm{O}==0$ \& Data. $\mathrm{N}>0$ \& Data.P==0 \&

Data. $\mathrm{S}==0$ );

Summary $\cdot \mathrm{CHS}=\operatorname{sum}($ Data $. \mathrm{C}>0$ \& Data. $\mathrm{H}>0$ \& Data. $\mathrm{O}==0$ \& Data. $\mathrm{N}==0$ \& Data. $\mathrm{P}==0$ \&

Data. $\mathrm{S}>0$ ) ;

Summary $\cdot \mathrm{CHNS}=$ sum $($ Data $\cdot \mathrm{C}>0$ \& Data. $\mathrm{H}>0$ \& Data $\cdot \mathrm{O}==0$ \& Data. $\mathrm{N}>0$ \& Data.P==0 \&

Data. $\mathrm{S}>0$ );

Summary. $\mathrm{CHNP}=$ sum $($ Data. $\mathrm{C}>0$ \& Data. $\mathrm{H}>0$ \& Data. $\mathrm{O}==0$ \& Data. $\mathrm{N}>0$ \& Data.P $>0$ \&

Data. $\mathrm{S}==0$ );

Summary $\cdot \mathrm{CHONS}=$ sum $($ Data $\cdot \mathrm{C}>0$ \& Data. $\mathrm{H}>0$ \& Data. $\mathrm{O}>0$ \& Data.N $>0$ \& Data.P==0 \&

Data. $\mathrm{S}>0$ ) ;

Summary. $\mathrm{CHONP}=$ sum $($ Data. $\mathrm{C}>0$ \& Data.H $>0$ \& Data. $\mathrm{O}>0$ \& Data.N $>0$ \& Data.P>0 \&

Data. $\mathrm{S}==0$ );

Summary. $\mathrm{CHONSP}=$ sum $($ Data. $\mathrm{C}>0$ \& Data. $\mathrm{H}>0$ \& Data. $\mathrm{O}>0$ \& Data.N $>0$ \& Data.P $>0$ \&

Data. $\mathrm{S}>0$ ) ; 
oCalculating aromaticity parameters: \# condensed aromatic (AI>=0.67), aromatic $(\mathrm{AI}>=0.5)$,

onon-aromatic $(\mathrm{AI}<0.5)$

Summary. CondensedAromatics $=$ sum (Data. AI $>=0.67)$;

Summary.Aromatics=sum (Data.AI $>=0.5)$;

Summary. NonAromatics=sum (Data.AI<0.5);

ocalculating other parameters: total \# of formulae, average molecular

oweight

Summary. TotalFormulae=height (Data);

Summary . AvgMW=mean (Data. NominalMass);

o.Storing Summary Data

LDI_BothPolarities_SRFA_Summary=struct2table (Summary); $\circ<<<<<<<<<<<<<<<<<<<<<<<$

$<<\overline{<}<<<<<<<<<<<<<<\overline{<}<<<\overline{<}<<<<<<<<<<<<<<<<<<<<<<<<<<<<<<<<<<<<<<<<<<<<<<<<<<<<<<~$

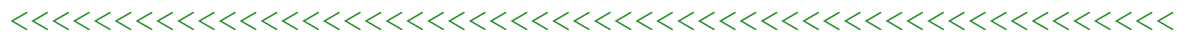

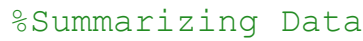

Data $=$ Combined;

oCalculating formulae type parameters: \# CHO, CHON, CHOS, CHOP, CHN, CHS, CHP, CHNS, CHONS formulae

Summary $\cdot \mathrm{CHO}=$ sum $($ Data. $\mathrm{C}>0$ \& Data. $\mathrm{H}>0$ \& Data. $\mathrm{O}>0$ \& Data. $\mathrm{N}==0$ \& Data. $\mathrm{P}==0$ \& Data. $\mathrm{S}==0$ );

Summary $\cdot \mathrm{CHON}=$ Sum $($ Data. $\mathrm{C}>0$ \& Data. $\mathrm{H}>0$ \& Data. $\mathrm{O}>0$ \& Data. $\mathrm{N}>0$ \& Data.P==0 \&

Data. $\mathrm{S}==0$ );

Summary. $\mathrm{CHOS}=$ sum $($ Data. $\mathrm{C}>0$ \& Data. $\mathrm{H}>0$ \& Data. $\mathrm{O}>0$ \& Data. $\mathrm{N}==0$ \& Data. $\mathrm{P}==0$ \&

Data. $\mathrm{S}>0$ );

Summary $\cdot \mathrm{CHOP}=$ sum $($ Data. $\mathrm{C}>0$ \& Data.H $>0$ \& Data. $\mathrm{O}>0$ \& Data. $\mathrm{N}==0$ \& Data.P $>0$ \&

Data. $\mathrm{S}==0$ );

Summary $\cdot \mathrm{CHN}=$ sum $($ Data. $\mathrm{C}>0$ \& Data. $\mathrm{H}>0$ \& Data. $\mathrm{O}==0$ \& Data. $\mathrm{N}>0$ \& Data. $\mathrm{P}==0$ \&

Data. $\mathrm{S}==0$ );

Summary $\cdot \mathrm{CHS}=\operatorname{sum}($ Data. $\mathrm{C}>0$ \& Data. $\mathrm{H}>0$ \& Data. $\mathrm{O}==0$ \& Data. $\mathrm{N}==0$ \& Data. $\mathrm{P}==0$ \&

Data. $\mathrm{S}>0$ ) ;

Summary. $\mathrm{CHNS}=$ sum $($ Data. $\mathrm{C}>0$ \& Data. $\mathrm{H}>0$ \& Data. $\mathrm{O}==0$ \& Data. $\mathrm{N}>0$ \& Data. $\mathrm{P}==0$ \&

Data. S>0);

Summary $\cdot \mathrm{CHNP}=\operatorname{sum}($ Data. $\mathrm{C}>0$ \& Data.H $>0$ \& Data. $\mathrm{O}==0$ \& Data. $\mathrm{N}>0$ \& Data.P $>0$ \&

Data. $\mathrm{S}==0$ );

Summary. $\mathrm{CHONS}=\operatorname{sum}($ Data $. \mathrm{C}>0$ \& Data. $\mathrm{H}>0$ \& Data. $0>0$ \& Data.N>0 \& Data.P==0 \&

Data. S>0);

Summary. $\mathrm{CHONP}=$ sum $($ Data. $\mathrm{C}>0$ \& Data. $\mathrm{H}>0$ \& Data. $\mathrm{O}>0$ \& Data.N $>0$ \& Data.P $>0$ \&

Data. $\mathrm{S}==0$ );

Summary. $\mathrm{CHONSP}=$ sum $($ Data. $\mathrm{C}>0$ \& Data.H $>0$ \& Data.O $>0$ \& Data.N $>0$ \& Data.P>0 \&

Data. $\mathrm{S}>0$ );

ocalculating aromaticity parameters: \# condensed aromatic (AI>=0.67),

aromatic (AI>=0.5),

onon-aromatic $(\mathrm{A} I<0.5)$

Summary. CondensedAromatics $=$ sum (Data. AI $>=0.67)$;

Summary. Aromatics $=$ sum (Data.AI>=0.5);

Summary. NonAromatics=sum (Data.AI<0.5);

ocalculating other parameters: total \# of formulae, average molecular

oweight

Summary. TotalFormulae=height (Data);

Summary. AvgMW=mean (Data. NominalMass);

ostoring Summary Data

LDI SRFA Summary=struct2table (Summary) ; 아 $<<<<<<<<<<<<<<<<<<<<<<<<<<<<<<<<<<<<<<<<~$ $<<\overline{\bar{l}}<<<<\overline{<}<<<<<<<<<<<<<<<<<<<<<<<<<<<<<<<<<<<<<<<<<<<<<<<<<<<<<<<<<<<<<<<<<<<<~$

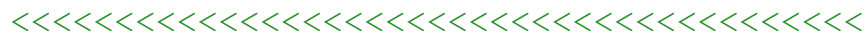

\% Combining ESI and LDI

\%Input Data so that the script can readily be applied to any data set 


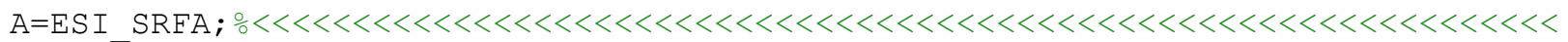
$<<<<\overline{<}<<<<<<<<<<<<<<<<<<<<<<<<<<<<<<<<<<<<<<<<<<<<<<<<<<<<<<<<<<<<<<<<<<<<<<<~$ $<<<<<<<<<<<<<$

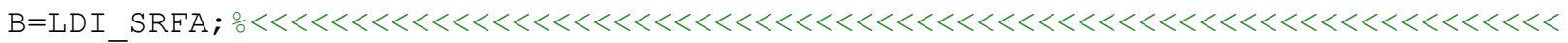
$<<<<\overline{<}<<<<<<<<<<<<<<<<<<<<<<<<<<<<<<<<<<<<<<<<<<<<<<<<<<<<<<<<<<<<<<<<<<<<<<<~$ $<<<<<<<<<<<<$

o Separate Data into those common and unique

Both $=[]$;

$\mathrm{k}=0$;

for $i=1$ :height $(A)$

for $j=1$ : height $(B)$

if $A \cdot C(i)==B \cdot C(j) \quad \& \& A \cdot H(i)==B \cdot H(j) \quad \& \& A \cdot N(i)==B \cdot N(j) \quad \& \& A \cdot O(i)==B \cdot O(j)$

$\& \& A \cdot P(i)==B \cdot P(j) \quad \& \& A \cdot S(i)==B \cdot S(j)$

$\mathrm{k}=\mathrm{k}+1$;

$\operatorname{Both}(k,:)=[A . C(i), A . H(i), A . O(i), A . N(i), A . P(i)$,

A.S (i), A.NominalMass (i), A.HC_Ratio(i),

A.OC_Ratio(i), A.AI (i), A.DBE(i), A.AvgCOxState (i)] ;

A. $C(i)=0$;

$B . C(j)=0$;

end

end

end

oCombining Data Sets

$\mathrm{A}(\mathrm{A} \cdot \mathrm{C}==0,:)=[]$;

$A O n l_{Y}=[A . C, A . H, A . O, A . N, A . P, A . S$,

A.NominalMass,A.HC_Ratio,A.OC_Ratio,A.AI,A.DBE,A.AvgCoxState] ;

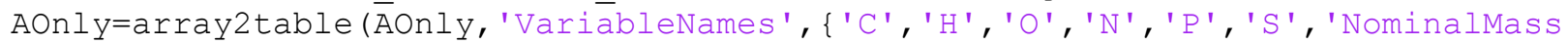
', 'HC Ratio', 'OC Ratio', 'AI', 'DBE', 'AvgCOxState' \}) ;

$\mathrm{B}(\mathrm{B} \cdot \mathrm{C}==0,:)=[]$;

$B O n l_{Y}=[B . C, B . H, B . O, B . N, B . P$,

B.S,B.NominalMass,B.HC Ratio,B.OC_Ratio,B.AI, B.DBE, B.AvgCoxstate];

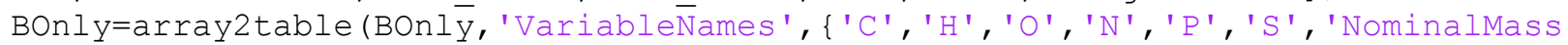
', 'HC Ratio',' 'OC Ratio', 'AI', 'DBE', 'AvgCOxState'\}) ;

Both $=\bar{a}$ rray2table (Both, 'VariableNames ', \{'C', 'H', 'O' , 'N', 'P' , 'S ', 'NominalMass ', 'HC Ratio', 'OC Ratio', 'AI', 'DBE', 'AvgCOxState'\});

Combined=[Both; AOnly; BOnly];

ostoring Data with distinct names

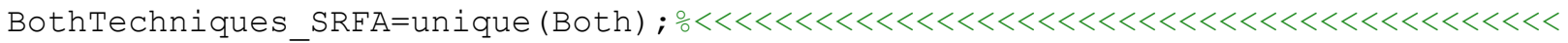
$<<<<<<<<<<<<<<\overline{<}<<<<<<<<<<<<<<<<<<<<<<<<<<<<<<<<<<<<<<<<<<<<<<<<<<<<<<<<<<<<<<~$ $<<<<<<<<<<<<<<<<<<<<<<<<<<<<<<<<<<<~$

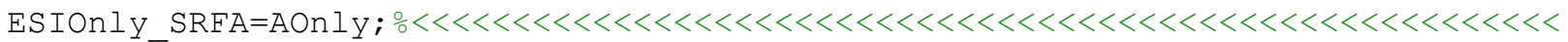

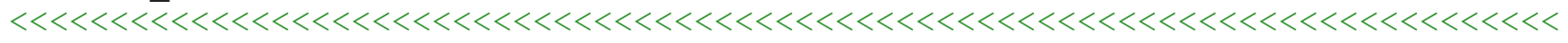
$<<<<<<<<<<<<<<<<<<<<<$

LDIOnly_SRFA=BOnly; $\circ<<<<<<<<<<<<<<<<<<<<<<<<<<<<<<<<<<<<<<<<<<<<<<<<<<<<<<<<<~$ $<<<<<<\overline{<}<<<<<<<<<<<<<<<<<<<<<<<<<<<<<<<<<<<<<<<<<<<<<<<<<<<<<<<<<<<<<<<<<<<<<~$ $<<<<<<<<<<<<<<<<<<<<<$

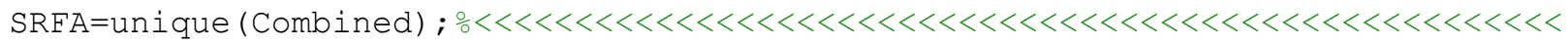

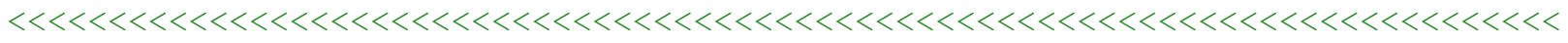
$<<<<<<<<<<<<<<<<<<<<<<<<~$

ㅇmmarizing Data

Data=AOnly;

oCalculating formulae type parameters: \# CHO, CHON, CHOS, CHOP, CHN, CHS, CHP, CHNS, CHONS formulae

Summary. $\mathrm{CHO}=\operatorname{sum}($ Data. $\mathrm{C}>0$ \& Data. $\mathrm{H}>0$ \& Data. $\mathrm{O}>0$ \& Data. $\mathrm{N}==0$ \& Data. $\mathrm{P}==0$ \& Data. $\mathrm{S}=0$ ) ;

Summary. $\mathrm{CHON}=\operatorname{sum}($ Data. $\mathrm{C}>0$ \& Data. $\mathrm{H}>0$ \& Data. $\mathrm{O}>0$ \& Data.N $>0$ \& Data.P==0 \&

Data. $\mathrm{S}==0$ ) ; 


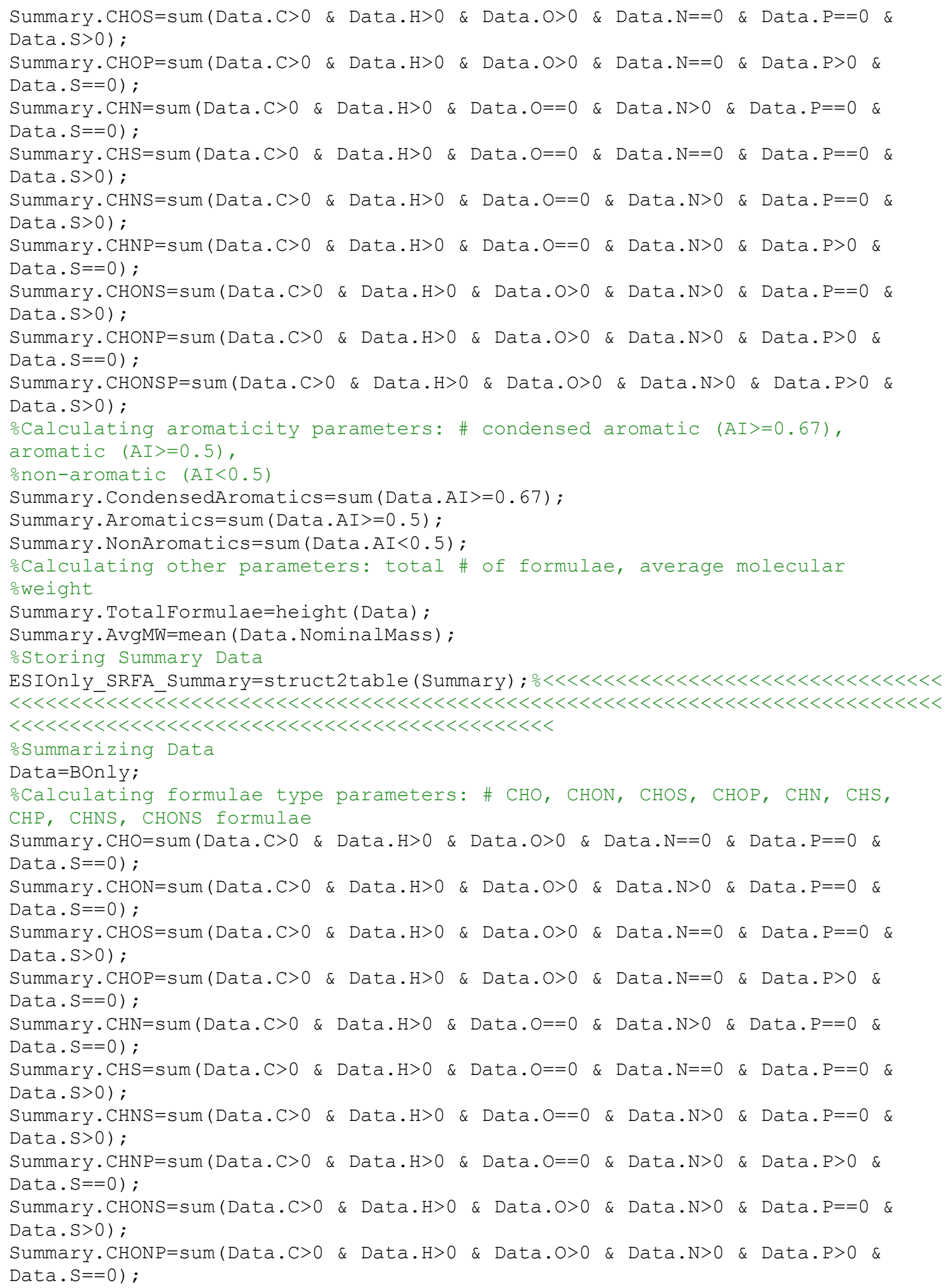


Summary. $\mathrm{CHONSP}=$ sum $($ Data. $\mathrm{C}>0$ \& Data. $\mathrm{H}>0$ \& Data. $\mathrm{O}>0$ \& Data.N $>0$ \& Data.P $>0$ \& Data. $\mathrm{S}>0$ );

ocalculating aromaticity parameters: \# condensed aromatic (AI>=0.67), aromatic $(A I>=0.5)$,

onon-aromatic $(\mathrm{AI}<0.5)$

Summary. CondensedAromatics $=\operatorname{sum}($ Data. $A I>=0.67)$;

Summary. Aromatics $=$ sum (Data.AI $>=0.5)$;

Summary. NonAromatics $=$ sum (Data.AI<0.5);

ocalculating other parameters: total \# of formulae, average molecular

\%weight

Summary. TotalFormulae=height (Data);

Summary. AvgMW=mean (Data. NominalMass);

ostoring Summary Data

LDIOnly_SRFA_Summary=struct2table (Summary) ; 은 $<<<<<<<<<<<<<<<<<<<<<<<<<<<<<<<<<<<~$

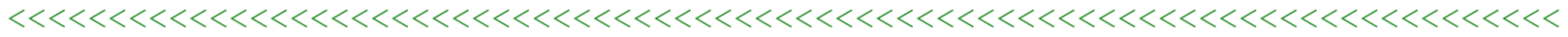

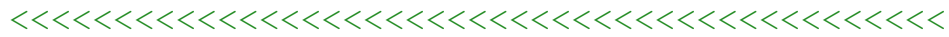

․ Summarizing Data

Data=unique (Both);

oCalculating formulae type parameters: \# CHO, CHON, CHOS, CHOP, CHN, CHS, CHP, CHNS, CHONS formulae

Summary. $\mathrm{CHO}=\operatorname{sum}($ Data. $\mathrm{C}>0$ \& Data.H $>0$ \& Data. $\mathrm{O}>0$ \& Data. $\mathrm{N}==0$ \& Data. $\mathrm{P}==0$ \& Data. $\mathrm{S}==0$ );

Summary $\cdot \mathrm{CHON}=$ sum $($ Data. $\mathrm{C}>0$ \& Data.H $>0$ \& Data. $0>0$ \& Data. $\mathrm{N}>0$ \& Data.P==0 \&

Data. $\mathrm{S}==0$ );

Summary $\cdot \mathrm{CHOS}=$ sum $($ Data. $\mathrm{C}>0$ \& Data.H $>0$ \& Data. $\mathrm{O}>0$ \& Data. $\mathrm{N}==0$ \& Data.P==0 \&

Data. S>0);

Summary $\cdot \mathrm{CHOP}=\operatorname{sum}($ Data. $\mathrm{C}>0$ \& Data.H $>0$ \& Data. $\mathrm{O}>0$ \& Data. $\mathrm{N}==0$ \& Data. $\mathrm{P}>0$ \&

Data. $\mathrm{S}==0$ );

Summary. $\mathrm{CHN}=\operatorname{sum}($ Data. $\mathrm{C}>0$ \& Data. $\mathrm{H}>0$ \& Data. $\mathrm{O}==0$ \& Data. $\mathrm{N}>0$ \& Data. $\mathrm{P}==0$ \&

Data. $\mathrm{S}==0$ );

Summary $\cdot \mathrm{CHS}=\operatorname{sum}($ Data $\cdot \mathrm{C}>0$ \& Data. $\mathrm{H}>0$ \& Data $\cdot \mathrm{O}==0$ \& Data. $\mathrm{N}==0$ \& Data. $\mathrm{P}==0$ \&

Data. S>0);

Summary. $\mathrm{CHNS}=$ sum $($ Data. $\mathrm{C}>0$ \& Data. $\mathrm{H}>0$ \& Data. $\mathrm{O}==0$ \& Data. $\mathrm{N}>0$ \& Data. $\mathrm{P}==0$ \&

Data. $\mathrm{S}>0$ );

Summary $\cdot \mathrm{CHNP}=\mathrm{sum}($ Data. $\mathrm{C}>0$ \& Data.H $>0$ \& Data. $\mathrm{O}==0$ \& Data. $\mathrm{N}>0$ \& Data.P $>0$ \&

Data. $\mathrm{S}==0$ );

Summary. CHONS $=$ sum $($ Data $\cdot \mathrm{C}>0$ \& Data. $\mathrm{H}>0$ \& Data. $\mathrm{O}>0$ \& Data. $\mathrm{N}>0$ \& Data. $\mathrm{P}==0$ \&

Data. $\mathrm{S}>0$ ) ;

Summary. $\mathrm{CHONP}=$ sum (Data. $\mathrm{C}>0$ \& Data.H>0 \& Data.O $>0$ \& Data.N $>0$ \& Data.P $>0$ \&

Data. $\mathrm{S}==0$ );

Summary. $\mathrm{CHONSP}=$ sum $($ Data. $\mathrm{C}>0$ \& Data.H $>0$ \& Data.O $>0$ \& Data.N $>0$ \& Data.P>0 \&

Data. S>0);

oCalculating aromaticity parameters: \# condensed aromatic (AI>=0.67),

aromatic $(A I>=0.5)$,

onon-aromatic $($ AI $<0.5)$

Summary. CondensedAromatics $=$ sum (Data. AI $>=0.67)$;

Summary. Aromatics=sum (Data.AI $>=0.5)$;

Summary. NonAromatics=sum (Data.AI<0.5);

ocalculating other parameters: total \# of formulae, average molecular

oweight

Summary. TotalFormulae=height (Data);

Summary. AvgMW=mean (Data. NominalMass) ;

ostoring Summary Data

BothTechniques_SRFA_Summary=struct2table (Summary) ; $\circ<<<<<<<<<<<<<<<<<<<<<<<<<<~$ $<<<<<<<<<<<<<\overline{<}<<<<\overline{<}<<<<<<<<<<<<<<<<<<<<<<<<<<<<<<<<<<<<<<<<<<<<<<<<<<<<<<<<<<$

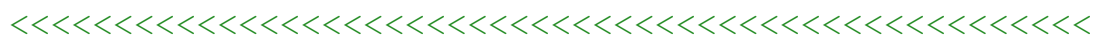

\% Summarizing Data 
Data=Combined;

oCalculating formulae type parameters: \# CHO, CHON, CHOS, CHOP, CHN, CHS, CHP, CHNS, CHONS formulae

Summary $\cdot \mathrm{CHO}=$ sum $($ Data. $\mathrm{C}>0$ \& Data. $\mathrm{H}>0$ \& Data. $\mathrm{O}>0$ \& Data. $\mathrm{N}==0$ \& Data. $\mathrm{P}==0$ \& Data. $\mathrm{S}==0$ );

Summary $\cdot \mathrm{CHON}=\operatorname{sum}($ Data. $\mathrm{C}>0$ \& Data.H $>0$ \& Data. $0>0$ \& Data. $\mathrm{N}>0$ \& Data. $\mathrm{P}==0$ \& Data. $\mathrm{S}==0$ );

Summary $\cdot \mathrm{CHOS}=$ sum $($ Data. $\mathrm{C}>0$ \& Data.H $>0$ \& Data.O $>0$ \& Data. $\mathrm{N}==0$ \& Data. $\mathrm{P}==0$ \& Data. S>0);

Summary $\cdot \mathrm{CHOP}=\operatorname{sum}($ Data. $\mathrm{C}>0$ \& Data. $\mathrm{H}>0$ \& Data. $\mathrm{O}>0$ \& Data. $\mathrm{N}==0$ \& Data.P $>0$ \& Data. $\mathrm{S}==0$ );

Summary. CHN $=$ sum $($ Data. $\mathrm{C}>0$ \& Data.H $>0$ \& Data. $\mathrm{O}==0$ \& Data. $>0$ \& Data.P==0 \&

Data. $\mathrm{S}==0$ );

Summary. $\mathrm{CHS}=\operatorname{sum}($ Data. $\mathrm{C}>0$ \& Data. $\mathrm{H}>0$ \& Data. $\mathrm{O}==0$ \& Data. $\mathrm{N}==0$ \& Data. $\mathrm{P}==0$ \& Data. $\mathrm{S}>0$ );

Summary $\cdot \mathrm{CHNS}=\operatorname{sum}($ Data. $\mathrm{C}>0$ \& Data. $\mathrm{H}>0$ \& Data. $\mathrm{O}==0$ \& Data. $\mathrm{N}>0$ \& Data.P==0 \& Data. $\mathrm{S}>0$ );

Summary. $\mathrm{CHNP}=\operatorname{sum}($ Data. $\mathrm{C}>0$ \& Data. $\mathrm{H}>0$ \& Data. $\mathrm{O}==0$ \& Data.N $>0$ \& Data.P $>0$ \&

Data. $\mathrm{S}==0$ );

Summary. $\mathrm{CHONS}=\operatorname{sum}($ Data $\cdot \mathrm{C}>0$ \& Data. $\mathrm{H}>0$ \& Data. $\mathrm{O}>0$ \& Data. $\mathrm{N}>0$ \& Data. $\mathrm{P}==0$ \&

Data. S $>0$ );

Summary. $\mathrm{CHONP}=$ sum $($ Data. $\mathrm{C}>0$ \& Data.H $>0$ \& Data. O $>0$ \& Data.N $>0$ \& Data.P $>0$ \&

Data. $\mathrm{S}==0$ );

Summary. $\mathrm{CHONSP}=$ sum $($ Data. $\mathrm{C}>0$ \& Data.H $>0$ \& Data.O $>0$ \& Data.N $>0$ \& Data.P>0 \&

Data. S>0);

ocalculating aromaticity parameters: \# condensed aromatic (AI>=0.67),

aromatic $(A I>=0.5)$,

onon-aromatic $($ AI $<0.5)$

Summary. CondensedAromatics $=$ sum (Data. AI $>=0.67)$;

Summary. Aromatics $=$ sum (Data.AI $>=0.5)$;

Summary. NonAromatics=sum (Data.AI<0.5);

ocalculating other parameters: total \# of formulae, average molecular

oweight

Summary. TotalFormulae=height (Data);

Summary. AvgMW=mean (Data. NominalMass);

ostoring Summary Data

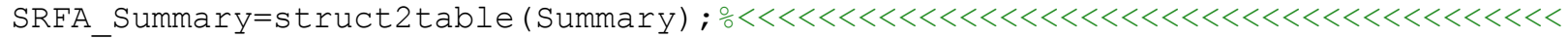
$<<<<\overline{<}<<<<<<<<<<<<<<<<<<<<<<<<<<<<<<<<<<<<<<<<<<<<<<<<<<<<<<<<<<<<<<<<<<<<<<<<~$

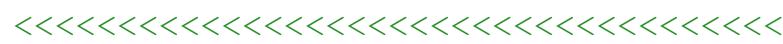

응 Repeating for post oxidation SRFA

\% Negative Mode ESI: Data Clean Up

\%Loading negative mode background impurities

Data=readtable ('ESINeg DBPS.XIsX') ; $\circ<<<<<<<<<<<<<<<<<<<<<<<<<<<<<<<<<<<<<<<<<<<~$

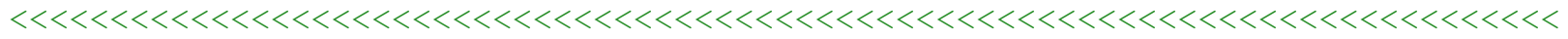
$<<<<<<<<<<<<<<<<<<<<<<<<<<<<<<<<<<~$

Data=unique (Data);

Data. NominalMass=Data $\cdot \mathbf{C} * 12+$ Data $\cdot 0 * 16+$ Data $\cdot H * 1+$ Data $\cdot P * 31+$ Data $\cdot S * 32+D a t a \cdot N * 14$;

Data. KMD CH2=Data. NominalMass-

$0.998883 \overline{4} *\left(\right.$ Data. $C * 12+$ Data. $H * 1.007825+$ Data. $N^{*} 14.003074+$ Data. $0 * 15.994915+$ Data.P $* 30.973763+$ Data.S*31.972072);

Data.KMD_COO=Data.NominalMass-

$1.000231^{\star}($ Data. C*12+Data.H*1.007825+Data. N*14.003074+Data.0*15.994915+Data.P*

$30.973763+$ Data. $S * 31.972072)$;

Data.KMD 02=Data. NominalMass-

$1.000317^{\bar{*}}\left(\right.$ Data. $\mathrm{C}^{\star} 12+$ Data. $\mathrm{H}^{\star} 1.007825+$ Data $. \mathrm{N}^{\star} 14.003074+\mathrm{Data} .0 * 15.994915+\mathrm{Data} . \mathrm{P} *$ $30.973763+$ Data. $\mathrm{S} * 31.972072)$; 


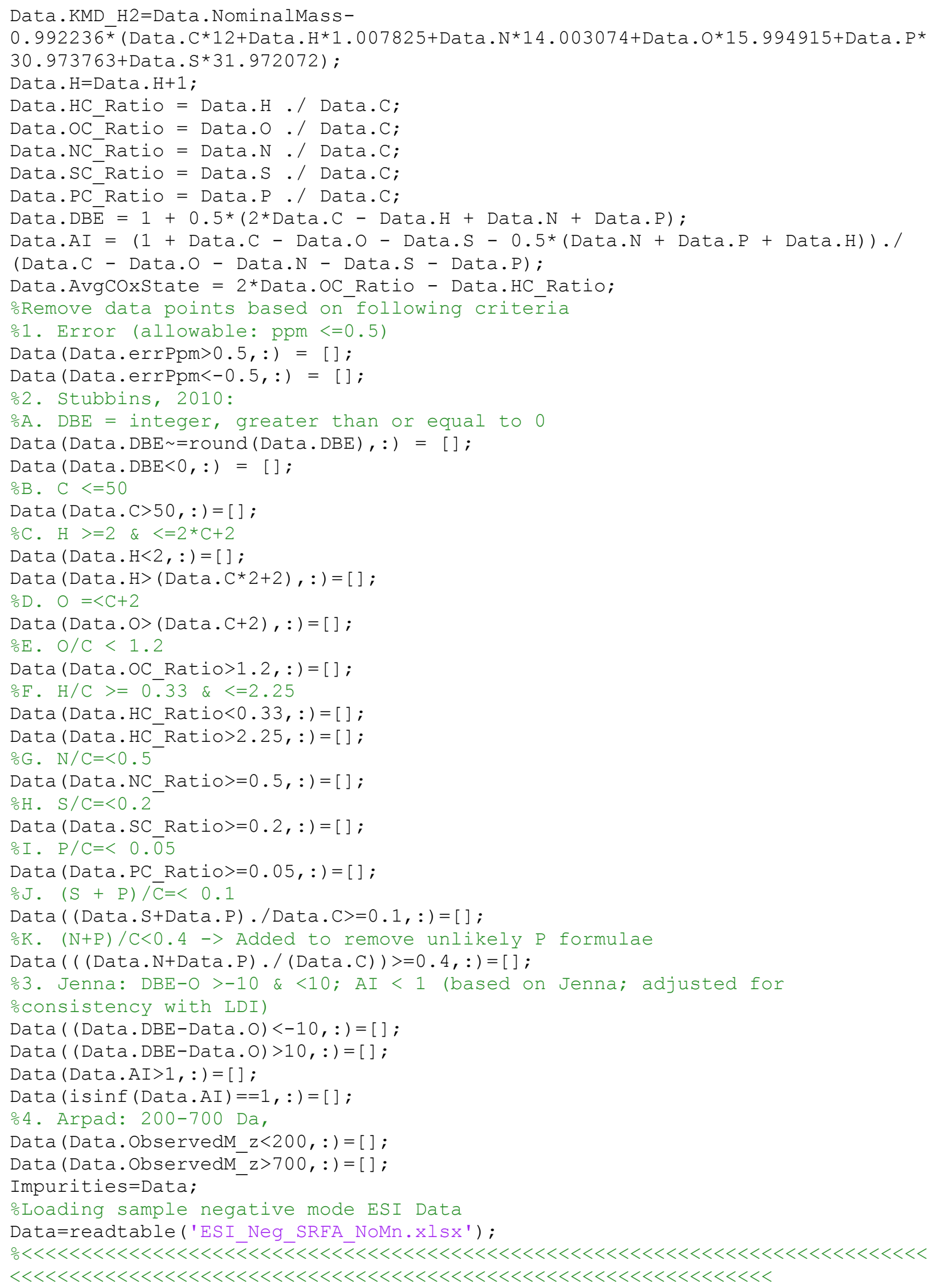




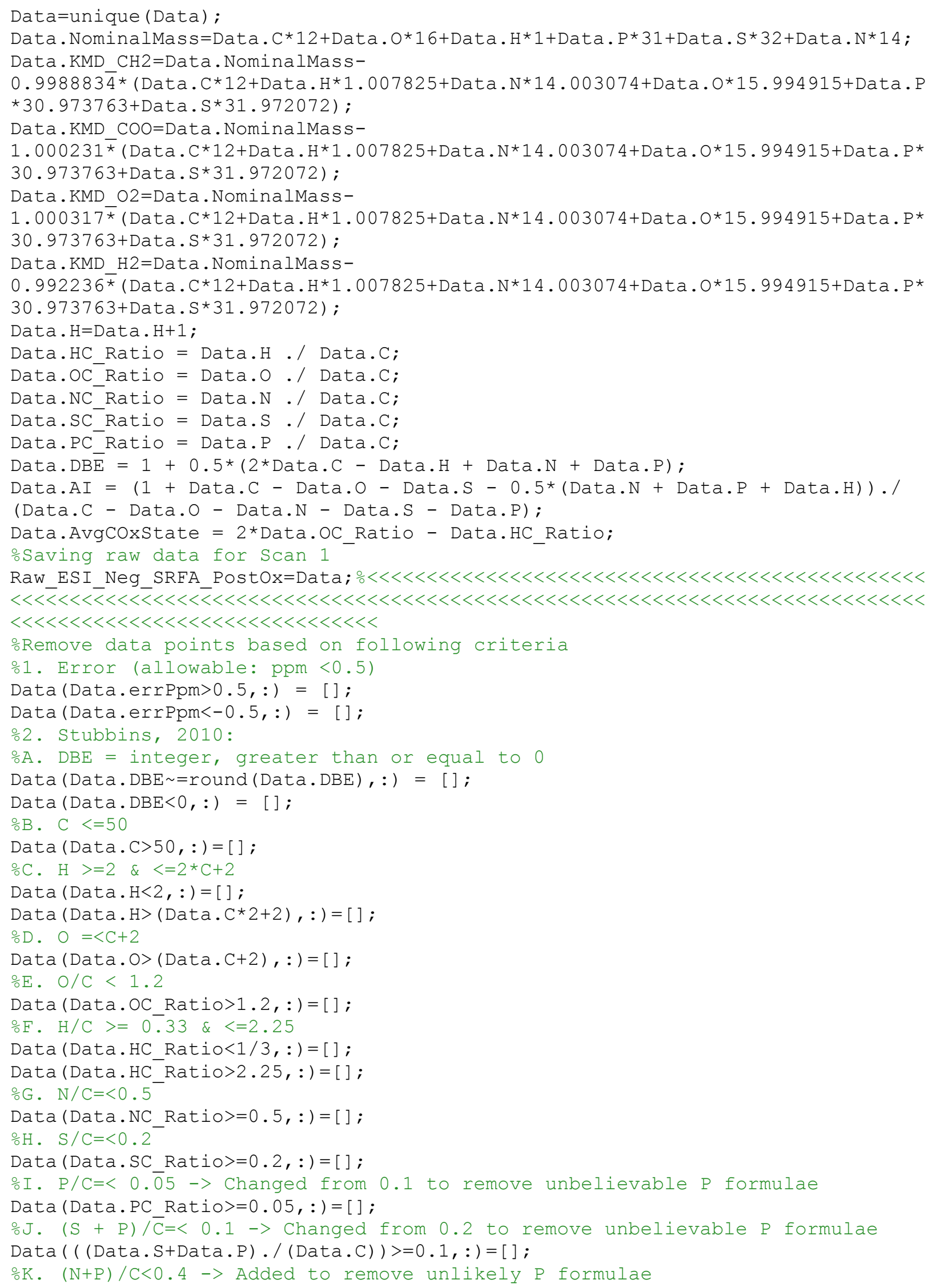


$\operatorname{Data}((($ Data.N+Data.P).$/($ Data.C $))>=0.4,:)=[]$;

\%3. Jenna: DBE-O >-10 \& <10; AI < 1 (based on Jenna; adjusted for

oconsistency with LDI)

Data ( (Data.DBE-Data.O) $<-10,:)=[]$;

Data ( (Data.DBE-Data.O) $>10,:)=[]$;

$\operatorname{Data}($ Data.AI>1, : ) $=[]$;

Data $($ isinf $($ Data.AI $)==1,:)=[]$;

\%4. Arpad: 200-700 Da,

Data (Data.ObservedM $z<200,:$ ) =[] ;

Data (Data. ObservedM_z>700,: ) =[] ;

\% Impurities

for $i=1$ : height (Data)

for $j=1$ : height (Impurities)

if Data.calc_M_z(i)==Impurities.calc_M_z(j)

Data.calc_M z $\bar{z}(\bar{i})=\mathrm{NaN}$;

end

end

end

Data (isnan (Data.calc_M_z), : ) =[] ;

oCalculating formulae type parameters: \# CHO, CHON, CHOS, CHOP, CHN, CHS, CHP, CHNS, CHONS formulae

Summary $\cdot \mathrm{CHO}=$ sum $($ Data. $\mathrm{C}>0$ \& Data. $\mathrm{H}>0$ \& Data. $\mathrm{O}>0$ \& Data. $\mathrm{N}==0$ \& Data. $\mathrm{P}==0$ \& Data. $\mathrm{S}==0$ );

Summary. $\mathrm{CHON}=$ sum $($ Data. $\mathrm{C}>0$ \& Data.H $>0$ \& Data.O $>0$ \& Data.N $>0$ \& Data.P==0 \&

Data. $S==0$ );

Summary $\cdot \mathrm{CHOS}=\operatorname{sum}($ Data. $\mathrm{C}>0$ \& Data. $\mathrm{H}>0$ \& Data. $\mathrm{O}>0$ \& Data. $\mathrm{N}==0$ \& Data. $\mathrm{P}==0$ \&

Data. $\mathrm{S}>0$ );

Summary. $\mathrm{CHOP}=\operatorname{sum}($ Data. $\mathrm{C}>0$ \& Data.H $>0$ \& Data. $\mathrm{O}>0$ \& Data. $\mathrm{N}==0$ \& Data.P>0 \&

Data. $\mathrm{S}==0$ ) ;

Summary $\cdot \mathrm{CHN}=$ sum $($ Data. $\mathrm{C}>0$ \& Data.H $>0$ \& Data. $\mathrm{O}==0$ \& Data. $\mathrm{N}>0$ \& Data. $\mathrm{P}==0$ \&

Data. $\mathrm{S}==0$ );

Summary $\cdot \mathrm{CHS}=\operatorname{sum}($ Data $\cdot \mathrm{C}>0$ \& Data. $\mathrm{H}>0$ \& Data $\cdot \mathrm{O}==0$ \& Data. $\mathrm{N}==0$ \& Data. $\mathrm{P}==0$ \&

Data. $\mathrm{S}>0$ );

Summary. $\mathrm{CHNS}=$ sum $($ Data. $\mathrm{C}>0$ \& Data. $\mathrm{H}>0$ \& Data. $\mathrm{O}==0$ \& Data. $\mathrm{N}>0$ \& Data. $\mathrm{P}==0$ \&

Data. $\mathrm{S}>0$ );

Summary $\cdot \mathrm{CHNP}=\operatorname{sum}($ Data. $\mathrm{C}>0$ \& Data.H $>0$ \& Data. $\mathrm{O}==0$ \& Data.N $>0$ \& Data.P $>0$ \&

Data. $\mathrm{S}==0$ ) ;

Summary $\cdot \mathrm{CHONS}=\operatorname{sum}($ Data $\cdot \mathrm{C}>0$ \& Data. $\mathrm{H}>0$ \& Data. $\mathrm{O}>0$ \& Data.N $>0$ \& Data.P==0 \&

Data. $\mathrm{S}>0$ );

Summary. $\mathrm{CHONP}=$ sum $($ Data. $\mathrm{C}>0$ \& Data.H $>0$ \& Data. O $>0$ \& Data.N $>0$ \& Data.P $>0$ \&

Data. $\mathrm{S}==0$ ) ;

Summary. CHONSP $=$ sum $($ Data. $\mathrm{C}>0$ \& Data.H $>0$ \& Data.O $>0$ \& Data.N $>0$ \& Data.P $>0$ \&

Data. S $>0$ );

ocalculating aromaticity parameters: \# condensed aromatic (AI>=0.67),

aromatic $(A I>=0.5)$,

onon-aromatic $(\mathrm{AI}<0.5)$

Summary CondensedAromatics $=\operatorname{sum}($ Data. $A I>=0.67)$;

Summary.Aromatics=sum (Data.AI $>=0.5)$;

Summary. NonAromatics=sum (Data.AI<0.5);

ocalculating other parameters: total \# of formulae, average molecular

알ight

Summary. TotalFormulae=height (Data);

Summary. AvgMW=mean (Data. NominalMass);

ostoring Data in Tables

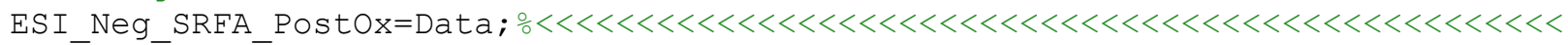
$<<\overline{<}<<\overline{<}<<<<\overline{<}<<<<<<<<<<<<<<<<<<<<<<<<<<<<<<<<<<<<<<<<<<<<<<<<<<<<<<<<<<<<<<<<~$ $<<<<<<<<<<<<<<<<<<<<<<<<<<<~$ 
ESI_Neg_SRFA_PostOx_Summary=struct2table (Summary) ; $\circ<<<<<<<<<<<<<<<<<<<<<<<<<<<<<~$ $<<\overline{<}<<<\overline{<}<<<\overline{<} \overline{<}<<<<<\overline{<} \overline{<}<<<<<<<<<<<<<<<<<<<<<<<<<<<<<<<<<<<<<<<<<<<<<<<<<<<<<<<<<$

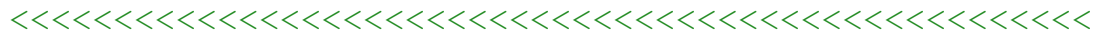

\%

oLoading positive mode ESI impurities

Data $=$ readtable ('DBP Control.xlsx');

Data $\cdot$ NominalMass=Data $\cdot C * 12+$ Data $\cdot 0 * 16+$ Data $\cdot H * 1+$ Data $\cdot P * 31+$ Data $\cdot S * 32+D a t a \cdot N * 14+D$ ata. $\mathrm{Na} * 23$;

Data. KMD CH2=Data. NominalMass-

$0.998883 \overline{4} *($ Data. $C * 12+$ Data. H*1.007825+Data. $N * 14.003074+$ Data. $0 * 15.994915+$ Data.P $\star 30.973763+$ Data.. *31.972072+Data.Na*22.98977);

Data.KMD COO=Data. NominalMass-

1.000231 ( Data. C*12+Data.H*1.007825+Data.N*14.003074+Data.0*15.994915+Data.P* $30.973763+$ Data.S*31.972072+Data.Na*22.98977);

Data.KMD 02=Data. NominalMass-

$1.000317^{\bar{*}}\left(\right.$ Data. $\mathrm{C} * 12+$ Data $. H^{*} 1.007825+$ Data $. N^{*} 14.003074+$ Data. $0 * 15.994915+$ Data. $P *$ $30.973763+$ Data. $\mathrm{S} * 31.972072+$ Data. $N a * 22.98977)$;

Data.KMD H2=Data. NominalMass-

$0.992236^{\star}\left(\right.$ Data. $C * 12+$ Data $. H * 1.007825+$ Data. $N^{*} 14.003074+$ Data. $0 * 15.994915+$ Data. $P *$

$30.973763+$ Data. $S * 31.972072+$ Data. Na*22.98977);

for $i=1$ :height (Data)

if $\mathrm{Data} . \mathrm{Na}(\mathrm{i})==0$

Data.H(i)=Data.H(i) -1 ;

end

end

Data.HC Ratio = Data.H ./ Data.C;

Data.oc Ratio = Data.O./ Data.C;

Data.NC_Ratio = Data.N./ Data.C;

Data.SCRatio = Data.S ./ Data.C;

Data.PC_Ratio = Data.P ./ Data.C;

Data. $\mathrm{DBE}=1+0.5 *(2 *$ Data. $\mathrm{C}-\operatorname{Data} \cdot \mathrm{H}+\operatorname{Data} \cdot \mathrm{N}+\operatorname{Data} \cdot \mathrm{P})$;

Data.AI $=(1+$ Data.C - Data.O - Data.S - 0.5*(Data.N + Data.P + Data.H) $)$.

(Data.C - Data.O - Data.N - Data.S - Data.P);

Data.AvgCoxstate = 2*Data.OC_Ratio - Data.HC_Ratio;

oRemove data points based on following criteria

\%1. Error (allowable: ppm <0.5)

Data (Data.errPpm>0.5,:) = [];

Data (Data.errPpm<-0.5,:) = [ ] ;

\%2. Stubbins, 2010 :

\%A. $\mathrm{DBE}=$ integer, greater than or equal to 0

Data (Data.DBE =round (Data.DBE), : ) = [];

Data (Data. $\mathrm{DBE}<0,:)=[]$;

B. $\mathrm{C}<=50$

Data (Data.C>50,: ) = [ ]

ㄷ. $\mathrm{H}>=2 \&<=2 * \mathrm{C}+2$

Data (Data. $\mathrm{H}<2,:)=[]$;

Data $($ Data. $\mathrm{H}>($ Data. $\mathrm{C} * 2+2),:)=[]$

$\therefore \mathrm{D} . \mathrm{O}=<\mathrm{C}+2$

Data (Data. O $>($ Data. $C+2),:)=[]$;

․ $\mathrm{O} / \mathrm{C}<1.2$

Data (Data.OC_Ratio>1.2, :) =[ ] ;

으. H/C $>=0.33 \&<=2.25$

Data (Data.HC Ratio<1/3,:) = [] ;

Data (Data.HC Ratio>2.25, : ) = [ ] ;

$\because \mathrm{G} . \mathrm{N} / \mathrm{C}=<0.5$

Data (Data.NC_Ratio $>=0.5,:)=[]$; 
$\circ \mathrm{H} \cdot \mathrm{S} / \mathrm{C}=<\mathrm{O} \cdot 2$

Data (Data.SC Ratio $>=0.2,:$ ) $=[]$;

ㄷ. $\mathrm{P} / \mathrm{C}=<0 . \overline{0} 5->$ Changed from 0.1 to remove unbelievable P formulae

Data (Data.PC_Ratio>=0.05, :) =[ ] ;

ㄱ. $(\mathrm{S}+\mathrm{P}) / \overline{\mathrm{C}}=<0.1->$ Changed from 0.2 to remove unbelievable $\mathrm{P}$ formulae

$\operatorname{Data}((($ Data.S+Data.P).$/($ Data.C $))>=0.1,:)=[]$;

$\circ \mathrm{K} . \quad(\mathrm{N}+\mathrm{P}) / \mathrm{C}<0.4->$ Added to remove unlikely P formulae

$\operatorname{Data}((($ Data.N+Data.P).$/($ Data. C $))>=0.4,:)=[]$;

⒊ Jenna: DBE-O >-10 \& <10; AI < 1 (based on Jenna; adjusted for

oconsistency with LDI)

Data ( (Data.DBE-Data.O) $<-10,:)=[]$;

Data $(($ Data.DBE-Data.O) $>10,:)=[]$;

Data (Data.AI $>1,:)=[]$;

Data $($ isinf $($ Data.AI $)==1,:)=[]$;

\%4. Arpad: 200-700 Da,

Data (Data. ObservedM $z<200,:$ ) = [] ;

Data (Data.ObservedM_z $>700,:)=[$ ] ;

Impurities=Data ;

oLoading sample positive mode ESI data

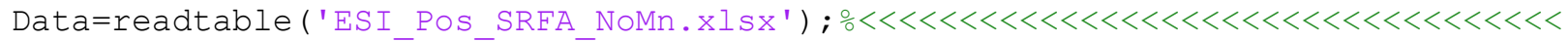
$<<<<<<<<<<<<<<<<<<<\overline{<}<<<\overline{<}<<<\overline{<}<<<<<<<<<<<<<<<<<<<<<<<<<<<<<<<<<<<<<<<<<<<<<<<<<~$ $<<<<<<<<<<<<<<<<<<<<<<<<<<<<<<<<<<<<<<<<<<<~$

Data $\cdot$ NominalMass $=$ Data $\cdot C * 12+$ Data $\cdot 0 * 16+$ Data $\cdot H * 1+$ Data $\cdot P * 31+$ Data $\cdot S * 32+D a t a \cdot N * 14+D$ ata. $\mathrm{Na} 23$;

Data. KMD CH2=Data. NominalMass-

$0.998883 \overline{4} *($ Data. $C * 12+$ Data. $H * 1.007825+$ Data. $N * 14.003074+$ Data. $0 * 15.994915+$ Data.P *30.973763+Data.S*31.972072+Data.Na*22.98977);

Data.KMD COO=Data.NominalMass-

$1.000231^{\star}\left(\right.$ Data. $C * 12+$ Data $. H^{*} 1.007825+$ Data. $N^{*} 14.003074+$ Data. $0 * 15.994915+$ Data. $P *$ $30.973763+$ Data. $\mathrm{S} * 31.972072+$ Data. $\mathrm{Na} * 22.98977$ );

Data.KMD_O2=Data.NominalMass-

$1.0003177^{\bar{*}}\left(\right.$ Data. $C * 12+$ Data. $H^{*} 1.007825+$ Data. $N^{*} 14.003074+$ Data. $0 * 15.994915+$ Data. $P *$ $30.973763+$ Data. $\mathrm{S} * 31.972072+$ Data. Na*22.98977);

Data.KMD H2=Data. NominalMass-

$0.992236^{\star}$ (Data. C*12+Data.H*1.007825+Data.N*14.003074+Data.0*15.994915+Data.P* $30.973763+$ Data.. *31.972072+Data.Na*22.98977);

for $i=1$ : height (Data)

if Data.Na(i)==0

Data.H $($ i $)=$ Data.H $($ i $)-1$;

end

end

Data.HC_Ratio = Data.H ./ Data.C;

Data.oC Ratio = Data.O ./ Data.C;

Data.NC Ratio = Data.N ./ Data.C;

Data.SC Ratio = Data.S./ Data.C;

Data.PC_Ratio = Data.P./ Data.C;

Data.DBE $=1+0.5 *(2 *$ Data.C - Data.H + Data. $N+$ Data.P $)$;

Data.AI $=(1+$ Data.C - Data.O - Data.S - 0.5* (Data.N + Data.P + Data.H) $) \cdot /$

(Data.C - Data.O - Data.N - Data.S - Data.P);

Data.AvgCoxstate = 2*Data.oC_Ratio - Data.HC_Ratio;

o Saving raw data for Scan 1

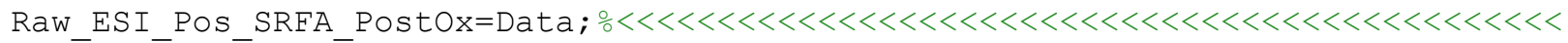
$<<\overline{<}<<<\overline{<}<<\overline{<} \overline{<}<<<\overline{<}<<<<<<<<<<<<<<<<<<<<<<<<<<<<<<<<<<<<<<<<<<<<<<<<<<<<<<<<<<<<$

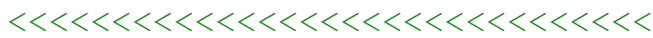

Remove data points based on following criteria

\%1. Error (allowable: ppm <0.5)

Data (Data.errPpm>0.5,:) = []; 


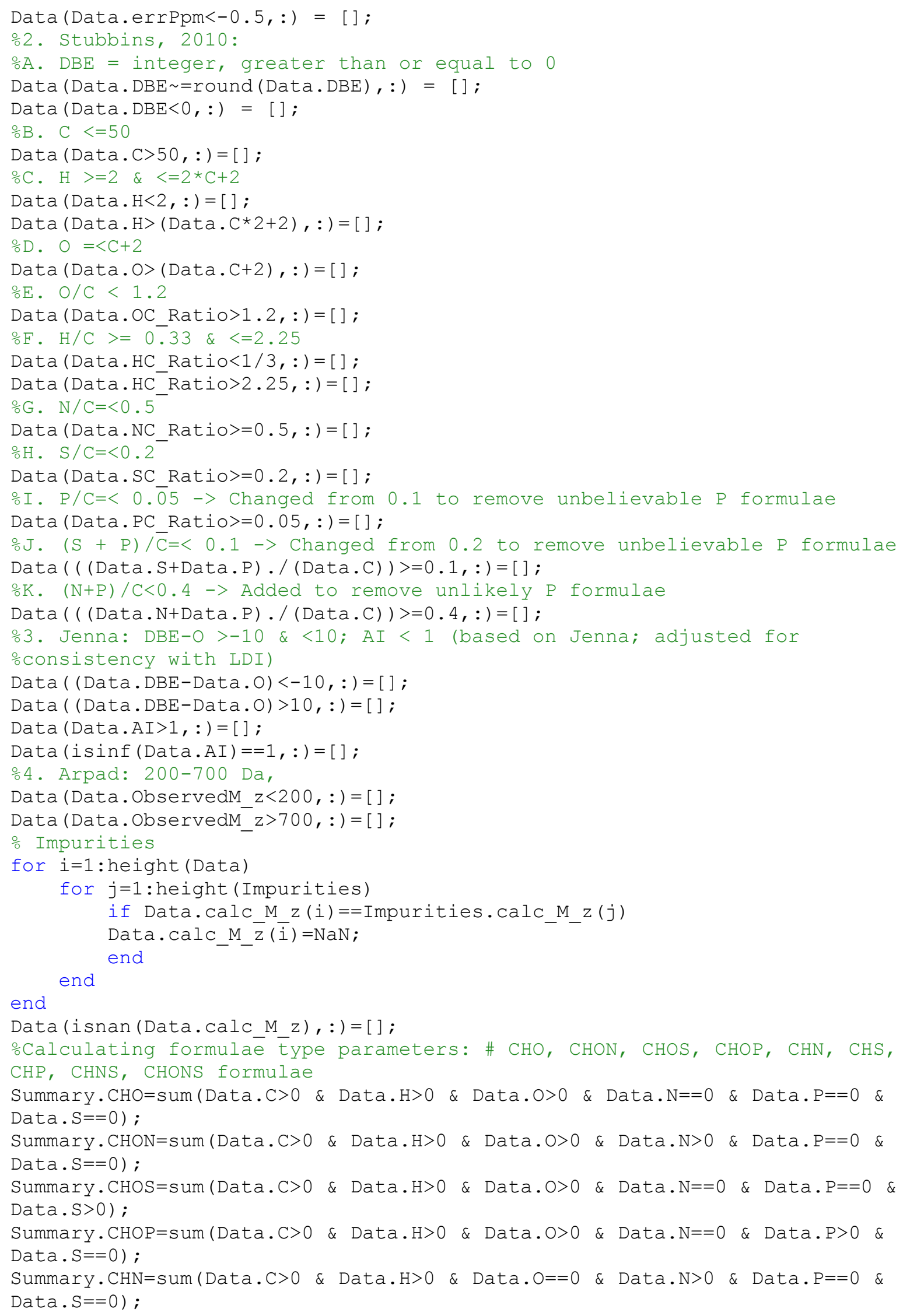


Summary $\cdot \mathrm{CHS}=\operatorname{sum}($ Data $\cdot \mathrm{C}>0$ \& Data. $\mathrm{H}>0$ \& Data. $\mathrm{O}==0$ \& Data. $\mathrm{N}==0$ \& Data. $\mathrm{P}==0$ \& Data. $\mathrm{S}>0$ );

Summary. CHNS $=$ sum $($ Data. $\mathrm{C}>0$ \& Data. $\mathrm{H}>0$ \& Data. $\mathrm{O}==0$ \& Data. $\mathrm{N}>0$ \& Data.P==0 \& Data. S $>0$ );

Summary. $\mathrm{CHNP}=\operatorname{sum}($ Data. $\mathrm{C}>0$ \& Data. $\mathrm{H}>0$ \& Data. $\mathrm{O}==0$ \& Data. $\mathrm{N}>0$ \& Data.P $>0$ \& Data. $\mathrm{S}==0$ );

Summary. CHONS $=$ sum $($ Data. $\mathrm{C}>0$ \& Data. $\mathrm{H}>0$ \& Data.O $>0$ \& Data.N $>0$ \& Data.P==0 \&

Data. $\mathrm{S}>0$ ) ;

Summary. $\mathrm{CHONP}=$ sum $($ Data. $\mathrm{C}>0$ \& Data. $\mathrm{H}>0$ \& Data. $\mathrm{O}>0$ \& Data.N $>0$ \& Data.P $>0$ \&

Data. $\mathrm{S}==0$ );

Summary. $\mathrm{CHONSP}=$ sum $($ Data. $\mathrm{C}>0$ \& Data. $\mathrm{H}>0$ \& Data. $\mathrm{O}>0$ \& Data.N $>0$ \& Data.P $>0$ \&

Data. S>0);

ocalculating aromaticity parameters: \# condensed aromatic (AI>=0.67), aromatic $(\mathrm{AI}>=0.5)$,

onon-aromatic $(\mathrm{A} I<0.5)$

Summary. CondensedAromatics $=$ sum (Data. AI $>=0.67)$;

Summary. Aromatics=sum (Data.AI $>=0.5)$;

Summary. NonAromatics=sum (Data.AI<0.5);

ocalculating other parameters: total \# of formulae, average molecular

oweight

Summary. TotalFormulae=height (Data);

Summary. AvgMW=mean (Data. NominalMass);

ostoring Data in Tables

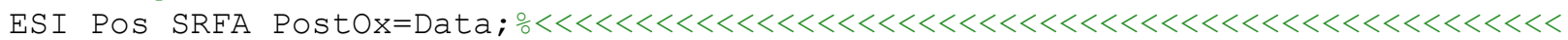
$<<\overline{<}<<<\overline{<}<<<<\overline{<}<<<<<<<<<<<<<<<<<<<<<<<<<<<<<<<<<<<<<<<<<<<<<<<<<<<<<<<<<<<<<<<<~$ $<<<<<<<<<<<<<<<<<<<<<<<<<<<~$

ESI_Pos_SRFA_PostOx_Summary=struct2table (Summary) ; $\circ<<<<<<<<<<<<<<<<<<<<<<<<<<<~$ $<<\overline{\bar{l}}<<\overline{\bar{l}}<<<<\overline{<}<<<<<\overline{<} \overline{<}<<<<<<<<<<<<<<<<<<<<<<<<<<<<<<<<<<<<<<<<<<<<<<<<<<<<<<<<<$ 난

응 Negative Mode LDI: Data Clean Up

oLDI assumed to have no background ions

Data=readtable ('LDI Neg SRFA NoMn.xlsX') ; $\circ<<<<<<<<<<<<<<<<<<<<<<<<<<<<<<<<<<<<<<<~$ $<<<<<<<<<<<<<<<<<<<\overline{<}<<<\overline{<}<<<\overline{<}<<<<<<<<<<<<<<<<<<<<<<<<<<<<<<<<<<<<<<<<<<<<<<<<<~$

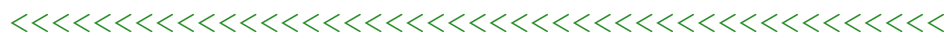

Data=unique (Data);

Data $\cdot$ NominalMass=Data $\cdot C * 12+$ Data $\cdot 0 * 16+$ Data $\cdot H * 1+$ Data $\cdot P * 31+$ Data $\cdot S * 32+$ Data $\cdot N * 14$;

Data. KMD CH2=Data. NominalMass-

$0.998883 \overline{4} *\left(\right.$ Data. $C * 12+$ Data. $H * 1.007825+$ Data. $N^{*} 14.003074+$ Data. $0 * 15.994915+$ Data. P $\star 30.973763+$ Data.. *31.972072);

Data.KMD_COO=Data. NominalMass-

$1.000231^{\star}($ Data. C*12+Data.H*1.007825+Data. N*14.003074+Data. O*15.994915+Data.P* $30.973763+$ Data..$* 31.972072)$;

Data.KMD 02=Data. NominalMass-

$1.000317^{\star}\left(\right.$ Data. $C * 12+$ Data.H*1.007825+Data. $\mathrm{N}^{\star} 14.003074+$ Data. $0 * 15.994915+$ Data. $\mathrm{P} *$ $30.973763+$ Data. $S * 31.972072)$;

Data. KMD H2=Data. NominalMass-

$0.992236^{\star}\left(\right.$ Data. C*12+Data.H*1.007825+Data. $N^{*} 14.003074+$ Data. $0 * 15.994915+$ Data.P*

$30.973763+$ Data. $S * 31.972072)$;

Data. DBE $=1+0.5 *(2 *$ Data. C - Data.H + Data.N + Data.P $)$;

for $i=1$ : height (Data)

if Data.DBE (i) =round (Data.DBE (i))

Data.H $($ i $)=$ Data.H $(i)+1$;

Data. IonType $(i)=0 ; \circ H-$ Adduct $s=0$

else

Data.IonType $(i)=2 ; \%$ MolecularIons=2 \%Temporary fix until I figure out how to enter names into a table 


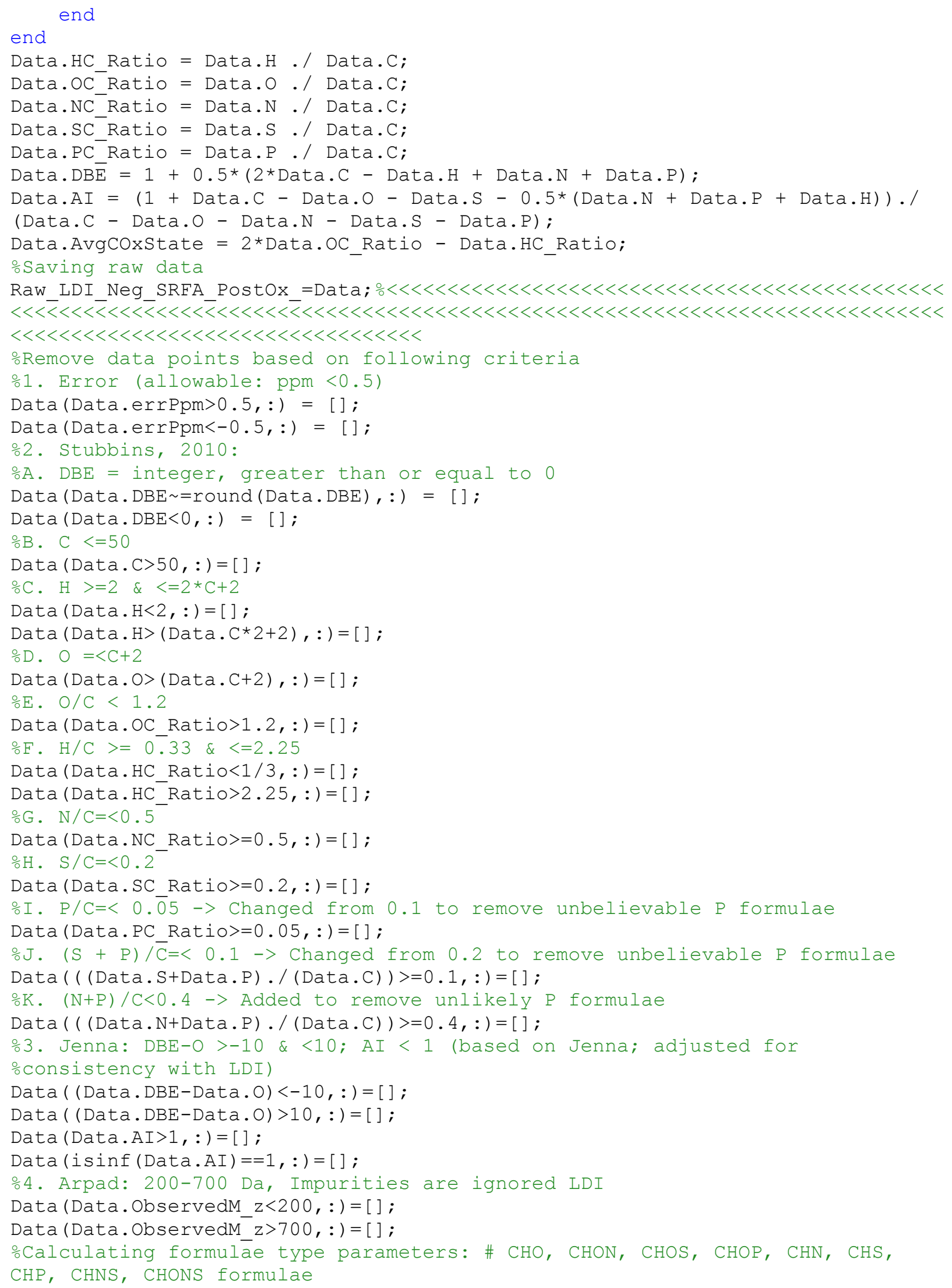


Summary. $\mathrm{CHO}=\operatorname{sum}($ Data. $\mathrm{C}>0$ \& Data.H $>0$ \& Data. $\mathrm{O}>0$ \& Data. $\mathrm{N}==0$ \& Data.P==0 \& Data. $\mathrm{S}==0$ ); Summary. $\mathrm{CHON}=\operatorname{sum}($ Data. $\mathrm{C}>0$ \& Data.H $>0$ \& Data. $0>0$ \& Data. $\mathrm{N}>0$ \& Data.P==0 \& Data. $\mathrm{S}==0$ );

Summary $\cdot \mathrm{CHOS}=$ sum $($ Data. $\mathrm{C}>0$ \& Data. $\mathrm{H}>0$ \& Data. $\mathrm{O}>0$ \& Data. $\mathrm{N}==0$ \& Data. $\mathrm{P}==0$ \& Data. $\mathrm{S}>0$ ); Summary. $\mathrm{CHOP}=\operatorname{sum}($ Data. $\mathrm{C}>0$ \& Data. $\mathrm{H}>0$ \& Data.O $>0$ \& Data. $\mathrm{N}==0$ \& Data.P $>0$ \& Data. $\mathrm{S}==0$ ); Summary $\cdot \mathrm{CHN}=$ sum $($ Data. $\mathrm{C}>0$ \& Data. $\mathrm{H}>0$ \& Data. $\mathrm{O}==0$ \& Data. $\mathrm{N}>0$ \& Data. $\mathrm{P}==0$ \& Data. $\mathrm{S}==0$ );

Summary $\cdot \mathrm{CHS}=\operatorname{sum}($ Data $\cdot \mathrm{C}>0$ \& Data. $\mathrm{H}>0$ \& Data $\cdot \mathrm{O}==0$ \& Data. $\mathrm{N}==0$ \& Data. $\mathrm{P}==0$ \& Data. $\mathrm{S}>0$ ) ; Summary $\cdot \mathrm{CHNS}=$ sum $($ Data $\cdot \mathrm{C}>0$ \& Data. $\mathrm{H}>0$ \& Data. $\mathrm{O}==0$ \& Data. $\mathrm{D}>0$ \& Data. $\mathrm{P}==0$ \& Data. S>0); Summary. $\mathrm{CHNP}=\operatorname{sum}($ Data $\cdot \mathrm{C}>0$ \& Data. $\mathrm{H}>0$ \& Data. $\mathrm{O}==0$ \& Data.N $>0$ \& Data.P>0 \& Data. $\mathrm{S}=0$ ) ; Summary. $\mathrm{CHONS}=\operatorname{sum}($ Data. $\mathrm{C}>0$ \& Data. $\mathrm{H}>0$ \& Data. $\mathrm{O}>0$ \& Data. $\mathrm{N}>0$ \& Data.P==0 \& Data. $\mathrm{S}>0$ ); Summary. $\mathrm{CHONP}=$ sum (Data. $\mathrm{C}>0$ \& Data.H>0 \& Data. O $>0$ \& Data.N $>0$ \& Data.P $>0$ \& Data. $\mathrm{S}==0$ ); Summary. $\mathrm{CHONSP}=$ sum $($ Data. $\mathrm{C}>0$ \& Data. $\mathrm{H}>0$ \& Data. $\mathrm{O}>0$ \& Data.N $>0$ \& Data.P $>0$ \& Data. S $>0)$;

ocalculating aromaticity parameters: \# condensed aromatic (AI>=0.67), aromatic $(A I>=0.5)$,

onon-aromatic $(\mathrm{AI}<0.5)$

Summary. CondensedAromatics $=$ sum (Data. AI $>=0.67)$;

Summary. Aromatics=sum (Data.AI>=0.5);

Summary. NonAromatics $=$ sum (Data. AI $<0.5)$;

ocalculating other parameters: total \# of formulae, average molecular oweight

Summary. TotalFormulae=height (Data);

Summary.AvgMW=mean (Data. NominalMass);

ostoring Data in Tables

LDI Neg SRFA PostOx=Data $;$ 응 $<<<<<<<<<<<<<<<<<<<<<<<<<<<<<<<<<<<<<<<<<<<<<<<<<<<<<~$

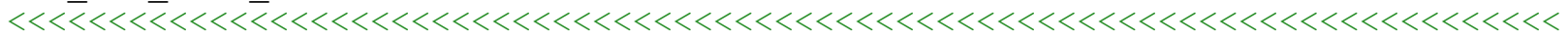
$<<<<<<<<<<<<<<<<<<<<<<<<<~$

LDI_Neg_SRFA_PostOx_Summary=struct2table (Summary) ; $\circ<<<<<<<<<<<<<<<<<<<<<<<<<<<~$ $<<<\overline{<}<<<\overline{<}<<<<\overline{<}<<<<<<\overline{<}<<<<<<<<<<<<<<<<<<<<<<<<<<<<<<<<<<<<<<<<<<<<<<<<<<<<<<<<<~$

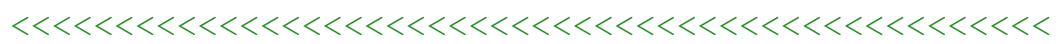

응 Positive Mode LDI: Data Clean Up

oLDI assumed to have no background ions

oLoding sample positive mode LDI data

Data=readtable('LDI Pos SRFA NoMn.xlsx') ; $\circ<<<<<<<<<<<<<<<<<<<<<<<<<<<<<<<<<<<<<<~$

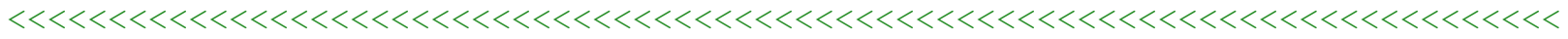

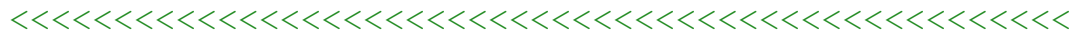

Data=unique (Data);

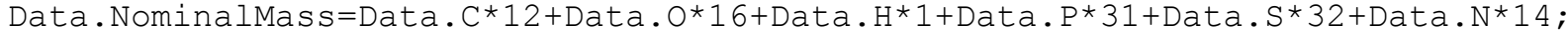

Data. $\mathrm{KMD} \mathrm{CH} 2=$ Data. NominalMass-

$0.998883 \overline{4} *\left(\right.$ Data. $C * 12+$ Data. $H^{*} 1.007825+$ Data. $N^{*} 14.003074+$ Data. $0 * 15.994915+$ Data.$P$ $* 30.973763+$ Data.S*31.972072);

Data.KMD COO=Data. NominalMass-

$1.000231^{\star}($ Data. C*12+Data.H*1.007825+Data. N*14.003074+Data.0*15.994915+Data.P* $30.973763+$ Data. $S * 31.972072)$;

Data.KMD O2=Data. NominalMass-

$1.000317^{\bar{*}}\left(\right.$ Data. $\mathrm{C}^{\star} 12+$ Data. $\mathrm{H}^{\star} 1.007825+$ Data $. \mathrm{N}^{\star} 14.003074+\mathrm{Data} .0 * 15.994915+\mathrm{Data} . \mathrm{P} *$ $30.973763+$ Data.. *31.972072); 


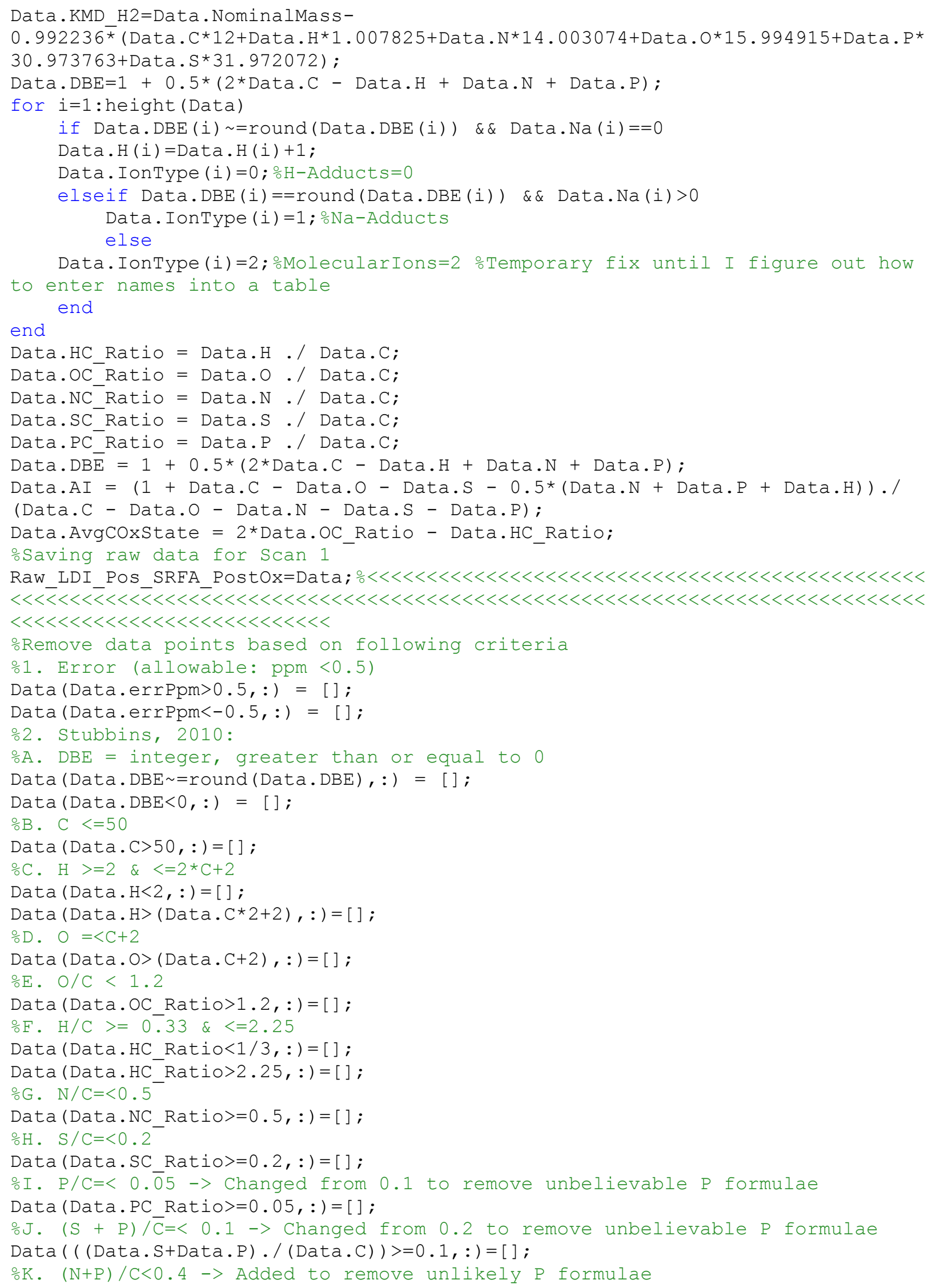

\section{S105}


$\operatorname{Data}((($ Data.N+Data.P).$/($ Data.C $))>=0.4,:)=[]$;

⒊ Jenna: DBE-0 >-10 \& <10; AI < 1 (based on Jenna; adjusted for

oconsistency with LDI)

Data ( (Data.DBE-Data.O) $<-10,:)=[]$;

Data ( (Data.DBE-Data.O) $>10,:)=[]$;

Data (Data.AI>1, : ) $=[]$;

$\operatorname{Data}($ isinf $($ Data. AI $)==1,:)=[]$;

\%4. Arpad: 200-700 Da, Impurities are ignored LDI- assumed to be negligible ounless a background reference is used

Data (Data. ObservedM_z<200,: ) = [] ;

Data (Data. ObservedM_z>700,: ) = [] ;

oCalculating formulāe type parameters: \# CHO, CHON, CHOS, CHOP, CHN, CHS, CHP, CHNS, CHONS formulae

Summary $\cdot \mathrm{CHO}=$ sum $($ Data. $\mathrm{C}>0$ \& Data. $\mathrm{H}>0$ \& Data. $\mathrm{O}>0$ \& Data. $\mathrm{N}==0$ \& Data. $\mathrm{P}==0$ \&

Data. $\mathrm{S}==0$ );

Summary $\cdot \mathrm{CHON}=$ sum $($ Data. $\mathrm{C}>0$ \& Data.H $>0$ \& Data. $\mathrm{O}>0$ \& Data. $\mathrm{N}>0$ \& Data.P==0 \&

Data. $\mathrm{S}=0$ ) ;

Summary $\cdot \mathrm{CHOS}=$ sum $($ Data. $\mathrm{C}>0$ \& Data.H $>0$ \& Data. O $>0$ \& Data. $\mathrm{N}==0$ \& Data. $\mathrm{P}==0$ \&

Data. $\mathrm{S}>0$ ) ;

Summary $\cdot \mathrm{CHOP}=\operatorname{sum}($ Data. $\mathrm{C}>0$ \& Data.H $>0$ \& Data. $\mathrm{O}>0$ \& Data. $\mathrm{N}==0$ \& Data.P $>0$ \&

Data. $\mathrm{S}==0$ );

Summary $\cdot \mathrm{CHN}=$ sum $($ Data. $\mathrm{C}>0$ \& Data. $\mathrm{H}>0$ \& Data. $\mathrm{O}==0$ \& Data. $\mathrm{N}>0$ \& Data. $\mathrm{P}==0$ \&

Data. $\mathrm{S}==0$ );

Summary. $\mathrm{CHS}=\operatorname{sum}($ Data. $\mathrm{C}>0$ \& Data. $\mathrm{H}>0$ \& Data. $\mathrm{O}==0$ \& Data. $\mathrm{N}==0$ \& Data. $\mathrm{P}==0$ \&

Data. S>0);

Summary $\cdot \mathrm{CHNS}=\operatorname{sum}($ Data. $\mathrm{C}>0$ \& Data. $\mathrm{H}>0$ \& Data. $\mathrm{O}==0$ \& Data. $\mathrm{N}>0$ \& Data.P==0 \&

Data. $\mathrm{S}>0$ );

Summary $\cdot \mathrm{CHNP}=\mathrm{sum}($ Data. $\mathrm{C}>0$ \& Data.H $>0$ \& Data. $\mathrm{O}==0$ \& Data.N $>0$ \& Data.P $>0$ \&

Data. $\mathrm{S}==0$ );

Summary $\cdot \mathrm{CHONS}=\operatorname{sum}($ Data. $\mathrm{C}>0$ \& Data. $\mathrm{H}>0$ \& Data. $0>0$ \& Data.N $>0$ \& Data.P==0 \&

Data. S>0);

Summary. $\mathrm{CHONP}=$ sum $($ Data. $\mathrm{C}>0$ \& Data. $\mathrm{H}>0$ \& Data. $\mathrm{O}>0$ \& Data. $\mathrm{N}>0$ \& Data.P $>0$ \&

Data. $\mathrm{S}=0$ ) ;

Summary. $\mathrm{CHONSP}=$ sum $($ Data. $\mathrm{C}>0$ \& Data.H $>0$ \& Data.O $>0$ \& Data.N $>0$ \& Data.P>0 \&

Data. $\mathrm{S}>0$ );

ocalculating aromaticity parameters: \# condensed aromatic (AI>=0.67),

aromatic $(A I>=0.5)$,

onon-aromatic $(\mathrm{AI}<0.5)$

Summary. CondensedAromatics $=$ sum (Data.AI $>=0.67)$;

Summary. Aromatics $=$ sum (Data.AI $>=0.5)$;

Summary. NonAromatics=sum (Data.AI<0.5);

ocalculating other parameters: total \# of formulae, average molecular

oweight

Summary.TotalFormulae=height (Data);

Summary. AvgMW=mean (Data. NominalMass);

ostoring Data in Tables

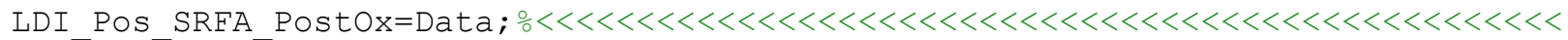
$<<<\overline{<}<<\overline{\bar{l}}<<<<\overline{<}<<<<<<<<<<<<<<<<<<<<<<<<<<<<<<<<<<<<<<<<<<<<<<<<<<<<<<<<~$

LDI_Pos_SRFA_Postox_Summary=struct2table (Summary) ; $\circ<<<<<<<<<<<<<<<<<<<<<<<<<<<~$ $<<\overline{<}<<\overline{\bar{l}}<<<<\overline{<}<<<<<<\overline{<}<<<<<<<<<<<<<<<<<<<<<<<<<<<<<<<<<<<<<<<<<<<<<<<<<<<<<<<<<$ $<<<<<<<<<<<<<<<<<~$

응 Combining Negative and Positive Mode ESI

oInput Data so that the script can readily be applied to any data set

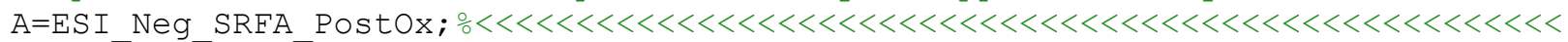
$<<<<\overline{<}<<<\overline{<}<<<\overline{<}<<<<<<<<<<<<<<<<<<<<<<<<<<<<<<<<<<<<<<<<<<<<<<<<<<<<<<<<<<<<<<~$

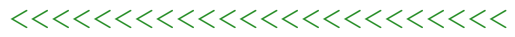




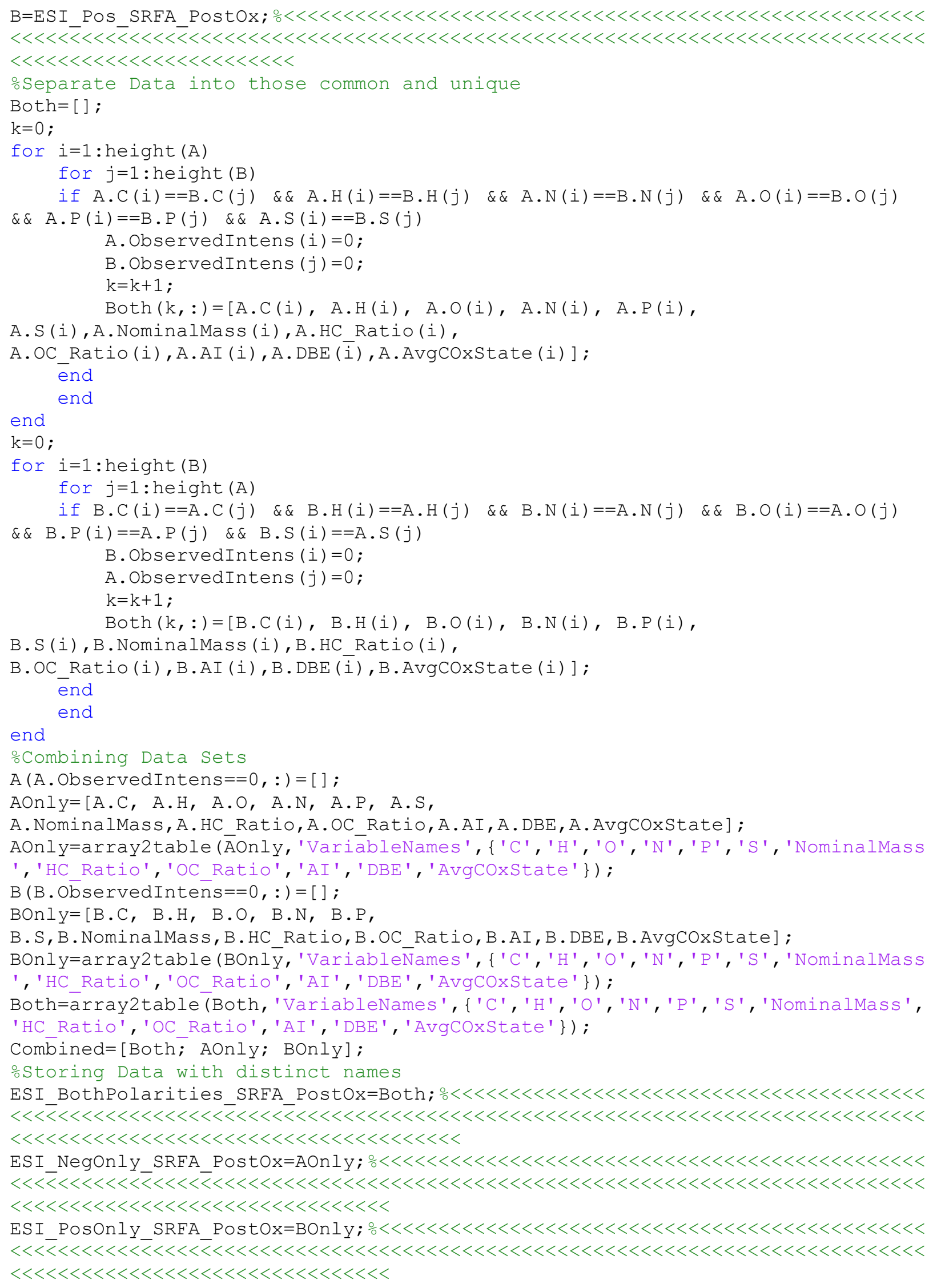




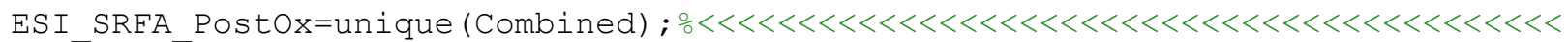
$<<\overline{<}<<<\overline{<} \bar{l}<<<<<<<<<<<<<<<<<<<<<<<<<<<<<<<<<<<<<<<<<<<<<<<<<<<<<<<<<<<<<<<<<<<<$

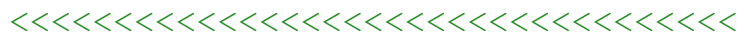

ㅇumarizing Data

Data=AOnly;

oCalculating formulae type parameters: \# CHO, CHON, CHOS, CHOP, CHN, CHS, CHP, CHNS, CHONS formulae

Summary $\cdot \mathrm{CHO}=$ sum $($ Data. $\mathrm{C}>0$ \& Data. $\mathrm{H}>0$ \& Data. $\mathrm{O}>0$ \& Data. $\mathrm{N}==0$ \& Data. $\mathrm{P}==0$ \&

Data. $\mathrm{S}==0$ );

Summary $\cdot \mathrm{CHON}=$ sum $($ Data. $\mathrm{C}>0$ \& Data.H $>0$ \& Data. $\mathrm{O}>0$ \& Data. $\mathrm{N}>0$ \& Data.P==0 \&

Data. $\mathrm{S}==0$ );

Summary. $\mathrm{CHOS}=$ sum $($ Data. $\mathrm{C}>0$ \& Data. $\mathrm{H}>0$ \& Data. $\mathrm{O}>0$ \& Data. $\mathrm{N}==0$ \& Data. $\mathrm{P}==0$ \&

Data. $\mathrm{S}>0$ );

Summary. $\mathrm{CHOP}=\mathrm{sum}($ Data. $\mathrm{C}>0$ \& Data.H $>0$ \& Data. $0>0$ \& Data. $\mathrm{N}==0$ \& Data.P $>0$ \&

Data. $\mathrm{S}==0$ );

Summary $\cdot \mathrm{CHN}=$ sum $($ Data. $\mathrm{C}>0$ \& Data. $\mathrm{H}>0$ \& Data. $\mathrm{O}==0$ \& Data. $\mathrm{N}>0$ \& Data. $\mathrm{P}==0$ \&

Data. $\mathrm{S}=0$ ) ;

Summary $\cdot \mathrm{CHS}=\operatorname{sum}($ Data $\cdot \mathrm{C}>0$ \& Data. $\mathrm{H}>0$ \& Data. $\mathrm{O}==0$ \& Data. $\mathrm{N}==0$ \& Data. $\mathrm{P}==0$ \&

Data. $\mathrm{S}>0$ ) ;

Summary. $\mathrm{CHNS}=$ sum $($ Data. $\mathrm{C}>0$ \& Data. $\mathrm{H}>0$ \& Data. $\mathrm{O}==0$ \& Data. $\mathrm{N}>0$ \& Data. $\mathrm{P}==0$ \&

Data. S $>0$ );

Summary $\cdot \mathrm{CHNP}=\operatorname{sum}($ Data. $\mathrm{C}>0$ \& Data.H $>0$ \& Data. $\mathrm{O}==0$ \& Data. $\mathrm{N}>0$ \& Data.P $>0$ \&

Data. $\mathrm{S}==0$ );

Summary. $\mathrm{CHONS}=\operatorname{sum}($ Data. $\mathrm{C}>0$ \& Data. $\mathrm{H}>0$ \& Data. $0>0$ \& Data.N>0 \& Data.P==0 \&

Data. S>0);

Summary. $\mathrm{CHONP}=\operatorname{sum}($ Data. $\mathrm{C}>0$ \& Data.H $>0$ \& Data. O $>0$ \& Data.N $>0$ \& Data.P $>0$ \&

Data. $\mathrm{S}==0$ );

Summary. $\mathrm{CHONSP}=$ sum $($ Data. $\mathrm{C}>0$ \& Data.H $>0$ \& Data.O $>0$ \& Data.N $>0$ \& Data.P>0 \&

Data. $\mathrm{S}>0)$;

ocalculating aromaticity parameters: \# condensed aromatic (AI>=0.67),

aromatic $(\mathrm{AI}>=0.5)$,

onon-aromatic $(\mathrm{A} I<0.5)$

Summary. CondensedAromatics $=$ sum (Data. AI $>=0.67)$;

Summary. Aromatics $=$ sum (Data.AI $>=0.5)$;

Summary. NonAromatics=sum (Data.AI<0.5);

ocalculating other parameters: total \# of formulae, average molecular

oweight

Summary.TotalFormulae=height (Data);

Summary. AvgMW=mean (Data. NominalMass);

ostoring Summary Data

ESI_NegOnly_SRFA_PostOx_Summary=struct2table (Summary) ; $\circ<<<<<<<<<<<<<<<<<<<<<<<~$

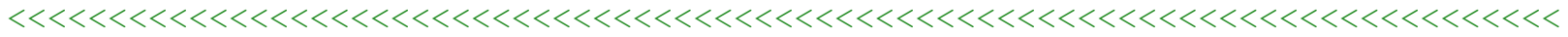

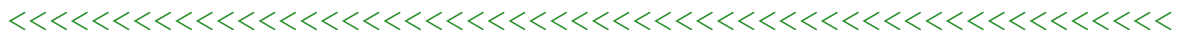

․ Summarizing Data

Data=BOnly;

oCalculating formulae type parameters: \# CHO, CHON, CHOS, CHOP, CHN, CHS, CHP, CHNS, CHONS formulae

Summary. $\mathrm{CHO}=\operatorname{sum}($ Data. $\mathrm{C}>0$ \& Data. $\mathrm{H}>0$ \& Data. $\mathrm{O}>0$ \& Data. $\mathrm{N}==0$ \& Data. $\mathrm{P}==0$ \& Data. $\mathrm{S}==0$ );

Summary. $\mathrm{CHON}=\operatorname{sum}($ Data. $\mathrm{C}>0$ \& Data. $\mathrm{H}>0$ \& Data. $\mathrm{O}>0$ \& Data.N $>0$ \& Data.P==0 \&

Data. $\mathrm{S}==0$ ) ;

Summary $\cdot \mathrm{CHOS}=$ sum $($ Data. $\mathrm{C}>0$ \& Data.H $>0$ \& Data. $\mathrm{O}>0$ \& Data. $\mathrm{N}==0$ \& Data.P==0 \&

Data. $\mathrm{S}>0$ ) ;

Summary $\cdot \mathrm{CHOP}=\mathrm{sum}($ Data. $\mathrm{C}>0$ \& Data. $\mathrm{H}>0$ \& Data. $\mathrm{O}>0$ \& Data. $\mathrm{N}==0$ \& Data.P $>0$ \&

Data. $\mathrm{S}=0$ ) ;

Summary. $\mathrm{CHN}=\operatorname{sum}($ Data. $\mathrm{C}>0$ \& Data. $\mathrm{H}>0$ \& Data. $\mathrm{O}==0$ \& Data. $\mathrm{N}>0$ \& Data. $\mathrm{P}==0$ \&

Data. $\mathrm{S}==0$ ) ; 
Summary $\cdot \mathrm{CHS}=\operatorname{sum}($ Data $\cdot \mathrm{C}>0$ \& Data. $\mathrm{H}>0$ \& Data. $\mathrm{O}==0$ \& Data. $\mathrm{N}==0$ \& Data. $\mathrm{P}==0$ \& Data. $\mathrm{S}>0$ ) ;

Summary. CHNS $=$ sum $($ Data. $\mathrm{C}>0$ \& Data. $\mathrm{H}>0$ \& Data. $\mathrm{O}==0$ \& Data. $\mathrm{N}>0$ \& Data.P==0 \& Data. S $>0$ );

Summary. $\mathrm{CHNP}=\operatorname{sum}($ Data. $\mathrm{C}>0$ \& Data. $\mathrm{H}>0$ \& Data. $\mathrm{O}==0$ \& Data. $\mathrm{N}>0$ \& Data.P $>0$ \& Data. $\mathrm{S}==0$ );

Summary. CHONS $=$ sum $($ Data. $\mathrm{C}>0$ \& Data. $\mathrm{H}>0$ \& Data.O $>0$ \& Data.N $>0$ \& Data.P==0 \& Data. $\mathrm{S}>0$ ) ;

Summary. $\mathrm{CHONP}=$ sum $($ Data. $\mathrm{C}>0$ \& Data. $\mathrm{H}>0$ \& Data. $\mathrm{O}>0$ \& Data.N $>0$ \& Data.P $>0$ \& Data. $\mathrm{S}==0$ );

Summary. $\mathrm{CHONSP}=$ sum $($ Data. $\mathrm{C}>0$ \& Data.H $>0$ \& Data. O $>0$ \& Data.N $>0$ \& Data.P $>0$ \& Data. S>0);

ocalculating aromaticity parameters: \# condensed aromatic (AI>=0.67), aromatic $(\mathrm{AI}>=0.5)$,

onon-aromatic $(\mathrm{A} I<0.5)$

Summary. CondensedAromatics $=$ sum (Data. AI $>=0.67)$;

Summary. Aromatics=sum (Data.AI $>=0.5)$;

Summary. NonAromatics=sum (Data.AI<0.5);

ocalculating other parameters: total \# of formulae, average molecular

oweight

Summary.TotalFormulae=height (Data);

Summary. AvgMW=mean (Data. NominalMass);

o.Storing Summary Data

ESI PosOnly SRFA PostOx Summary=struct2table (Summary); $\circ<<<<<<<<<<<<<<<<<<<<<<<<~$

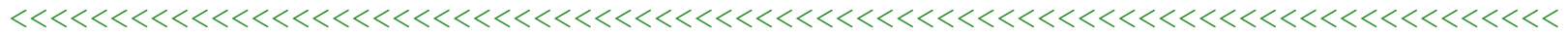

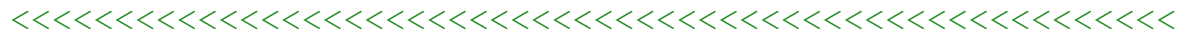

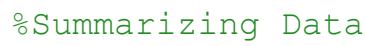

Data=Both;

oCalculating formulae type parameters: \# CHO, CHON, CHOS, CHOP, CHN, CHS, CHP, CHNS, CHONS formulae

Summary $\cdot \mathrm{CHO}=\operatorname{sum}($ Data. $\mathrm{C}>0$ \& Data. $\mathrm{H}>0$ \& Data. $\mathrm{O}>0$ \& Data. $\mathrm{N}==0$ \& Data. $\mathrm{P}==0$ \&

Data. $\mathrm{S}==0$ );

Summary $\cdot \mathrm{CHON}=\operatorname{sum}($ Data. $\mathrm{C}>0$ \& Data.H $>0$ \& Data. $0>0$ \& Data. $\mathrm{N}>0$ \& Data. $\mathrm{P}==0$ \&

Data. $\mathrm{S}==0$ );

Summary. $\mathrm{CHOS}=$ sum $($ Data. $\mathrm{C}>0$ \& Data. $\mathrm{H}>0$ \& Data.O $>0$ \& Data. $\mathrm{N}==0$ \& Data. $\mathrm{P}==0$ \&

Data. S>0);

Summary $\cdot \mathrm{CHOP}=$ sum $($ Data. $\mathrm{C}>0$ \& Data. $\mathrm{H}>0$ \& Data. $\mathrm{O}>0$ \& Data. $\mathrm{N}==0$ \& Data. P $>0$ \&

Data. $\mathrm{S}==0$ ) ;

Summary. $\mathrm{CHN}=$ sum $($ Data. $\mathrm{C}>0$ \& Data. $\mathrm{H}>0$ \& Data. $\mathrm{O}==0$ \& Data. $\mathrm{N}>0$ \& Data. $\mathrm{P}==0$ \&

Data. $\mathrm{S}==0$ );

Summary $\cdot \mathrm{CHS}=\operatorname{sum}($ Data. $\mathrm{C}>0$ \& Data. $\mathrm{H}>0$ \& Data. $\mathrm{O}==0$ \& Data. $\mathrm{N}==0$ \& Data. $\mathrm{P}==0$ \&

Data. S $>0$ );

Summary $\cdot \mathrm{CHNS}=\operatorname{sum}($ Data. $\mathrm{C}>0$ \& Data. $\mathrm{H}>0$ \& Data. $\mathrm{O}==0$ \& Data. $\mathrm{N}>0$ \& Data.P==0 \&

Data. $\mathrm{S}>0$ );

Summary. $\mathrm{CHNP}=\operatorname{sum}($ Data. $\mathrm{C}>0$ \& Data. $\mathrm{H}>0$ \& Data. $\mathrm{O}==0$ \& Data. $\mathrm{N}>0$ \& Data.P $>0$ \&

Data. $\mathrm{S}=0$ ) ;

Summary. $\mathrm{CHONS}=\operatorname{sum}($ Data $\cdot \mathrm{C}>0$ \& Data. $\mathrm{H}>0$ \& Data. $\mathrm{O}>0$ \& Data. $\mathrm{N}>0$ \& Data. $\mathrm{P}==0$ \&

Data. S>0);

Summary. $\mathrm{CHONP}=$ sum $($ Data. $\mathrm{C}>0$ \& Data. $\mathrm{H}>0$ \& Data. O $>0$ \& Data.N $>0$ \& Data.P $>0$ \&

Data. $\mathrm{S}==0$ );

Summary. $\mathrm{CHONSP}=$ sum $($ Data. $\mathrm{C}>0$ \& Data.H $>0$ \& Data.O $>0$ \& Data.N $>0$ \& Data.P>0 \&

Data. S>0);

oCalculating aromaticity parameters: \# condensed aromatic (AI>=0.67),

aromatic $(A I>=0.5)$,

onon-aromatic $($ AI $<0.5)$

Summary. CondensedAromatics $=$ sum (Data. AI $>=0.67)$;

Summary.Aromatics $=$ sum (Data.AI>=0.5); 
Summary. NonAromatics=sum (Data.AI<0.5);

ocalculating other parameters: total \# of formulae, average molecular

oweight

Summary. TotalFormulae=height (Data);

Summary. AvgMW=mean (Data. NominalMass);

ostoring Summary Data

ESI_BothPolarities_SRFA_Postox_Summary=struct2table (Summary); $\circ<<<<<<<<<<<<<<<<$

$<<<\overline{<}<<<<<<<<<<<<<<\overline{<}<<<<\overline{<}<<<<<<\overline{<}<<<<<<<<<<<<<<<<<<<<<<<<<<<<<<<<<<<<<<<<<<<<<<~$

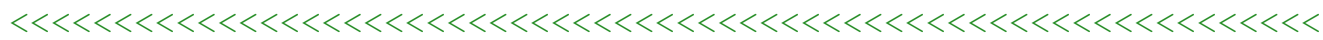

o Summarizing Data

Data $=$ Combined;

oCalculating formulae type parameters: \# CHO, CHON, CHOS, CHOP, CHN, CHS, CHP, CHNS, CHONS formulae

Summary $\cdot \mathrm{CHO}=$ sum $($ Data. $\mathrm{C}>0$ \& Data. $\mathrm{H}>0$ \& Data. $\mathrm{O}>0$ \& Data. $\mathrm{N}==0$ \& Data. $\mathrm{P}==0$ \&

Data. $\mathrm{S}==0$ );

Summary $\cdot \mathrm{CHON}=\operatorname{sum}($ Data. $\mathrm{C}>0$ \& Data.H $>0$ \& Data. $\mathrm{O}>0$ \& Data. $>0$ \& Data.P==0 \&

Data. $\mathrm{S}=0$ ) ;

Summary $\cdot \mathrm{CHOS}=$ sum $($ Data. $\mathrm{C}>0$ \& Data.H $>0$ \& Data. O $>0$ \& Data. $\mathrm{N}==0$ \& Data. $\mathrm{P}==0$ \&

Data. $\mathrm{S}>0$ );

Summary $\cdot \mathrm{CHOP}=\operatorname{sum}($ Data. $\mathrm{C}>0$ \& Data.H $>0$ \& Data. $\mathrm{O}>0$ \& Data. $\mathrm{N}==0$ \& Data.P $>0$ \&

Data. $\mathrm{S}==0$ );

Summary $\cdot \mathrm{CHN}=$ sum $($ Data. $\mathrm{C}>0$ \& Data. $\mathrm{H}>0$ \& Data. $\mathrm{O}==0$ \& Data. $\mathrm{N}>0$ \& Data. $\mathrm{P}==0$ \&

Data. $\mathrm{S}==0$ );

Summary $\cdot \mathrm{CHS}=\operatorname{sum}($ Data $\cdot \mathrm{C}>0$ \& Data. $\mathrm{H}>0$ \& Data. $\mathrm{O}==0$ \& Data. $\mathrm{N}==0$ \& Data. $\mathrm{P}==0$ \&

Data. S>0);

Summary. $\mathrm{CHNS}=$ sum $($ Data. $\mathrm{C}>0$ \& Data. $\mathrm{H}>0$ \& Data. $\mathrm{O}==0$ \& Data. $\mathrm{N}>0$ \& Data. $\mathrm{P}==0$ \&

Data. $\mathrm{S}>0$ );

Summary. $\mathrm{CHNP}=\mathrm{sum}($ Data. $\mathrm{C}>0$ \& Data. $\mathrm{H}>0$ \& Data. $\mathrm{O}==0$ \& Data.N $>0$ \& Data.P $>0$ \&

Data. $\mathrm{S}==0$ );

Summary $\cdot \mathrm{CHONS}=\operatorname{sum}($ Data. $\mathrm{C}>0$ \& Data. $\mathrm{H}>0$ \& Data. $0>0$ \& Data.N $>0$ \& Data.P==0 \&

Data. S>0);

Summary. $\mathrm{CHONP}=$ sum $($ Data. $\mathrm{C}>0$ \& Data. $\mathrm{H}>0$ \& Data. $\mathrm{O}>0$ \& Data. $\mathrm{N}>0$ \& Data.P $>0$ \&

Data. $\mathrm{S}==0$ );

Summary. $\mathrm{CHONSP}=$ sum $($ Data. $\mathrm{C}>0$ \& Data.H $>0$ \& Data.O $>0$ \& Data.N $>0$ \& Data.P>0 \&

Data. $\mathrm{S}>0$ );

ocalculating aromaticity parameters: \# condensed aromatic (AI>=0.67),

aromatic $(A I>=0.5)$,

onon-aromatic $(\mathrm{AI}<0.5)$

Summary. CondensedAromatics $=$ sum (Data. AI $>=0.67)$;

Summary. Aromatics $=$ sum (Data.AI $>=0.5)$;

Summary. NonAromatics=sum (Data.AI<0.5);

ocalculating other parameters: total \# of formulae, average molecular

oweight

Summary. TotalFormulae=height (Data);

Summary. AvgMW=mean (Data. NominalMass);

ostoring Summary Data

ESI SRFA Postox Summary=struct2table (Summary); $\circ<<<<<<<<<<<<<<<<<<<<<<<<<<<<<<<<~$ $<<<\overline{<}<<<<\overline{<}<<<<<<\overline{<}<<<<<<<<<<<<<<<<<<<<<<<<<<<<<<<<<<<<<<<<<<<<<<<<<<<<<<<<<<<<<~$

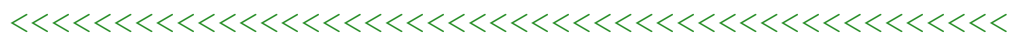

응 Combining Negative and Positive Mode LDI

oInput Data

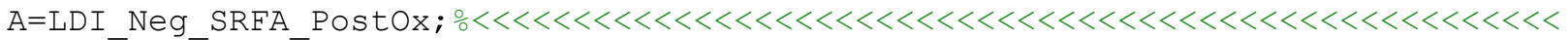

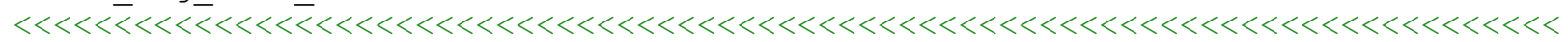
$<<<<<<<<<<<<<<<<<<<<<<<<~$ 


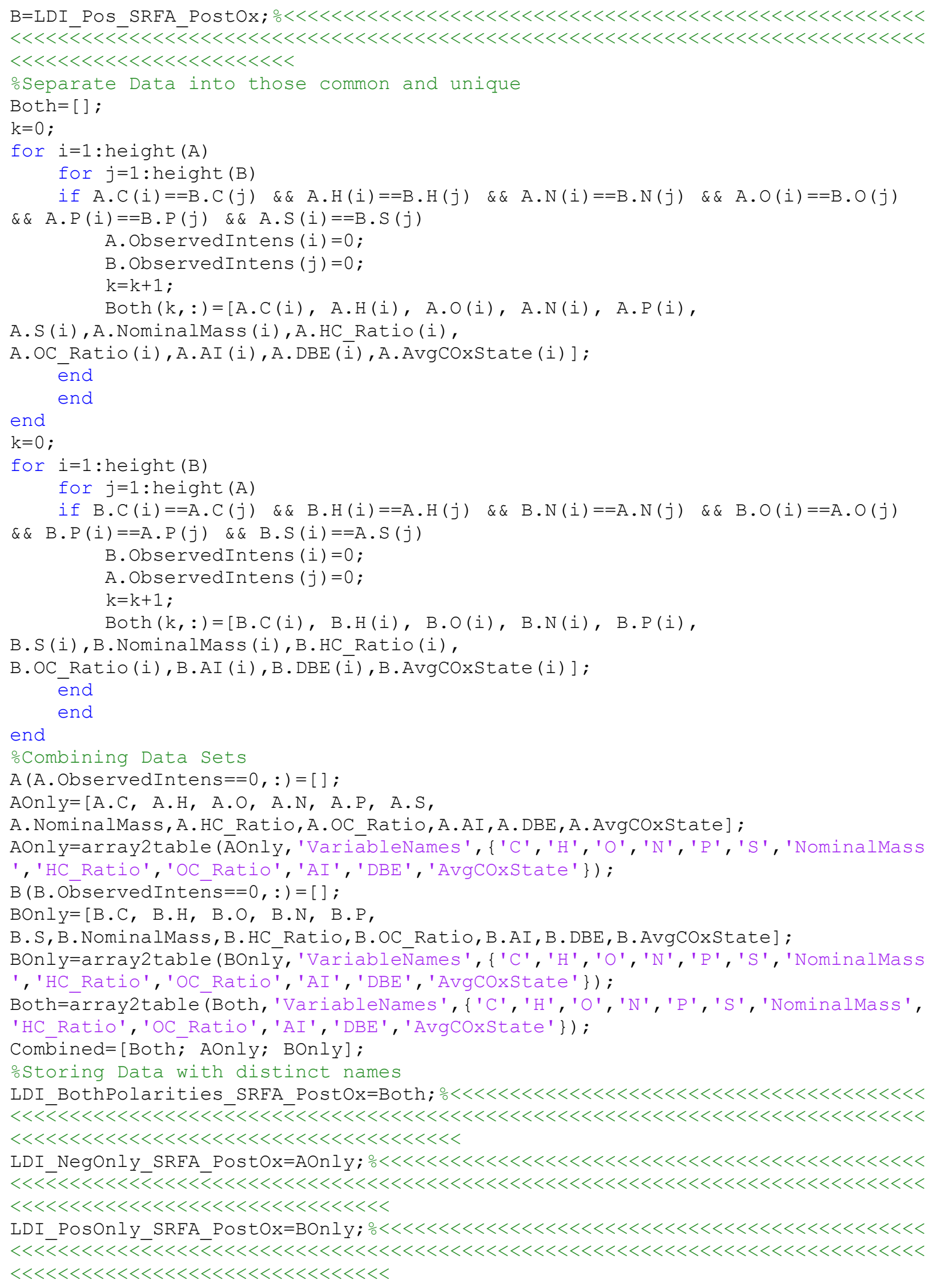




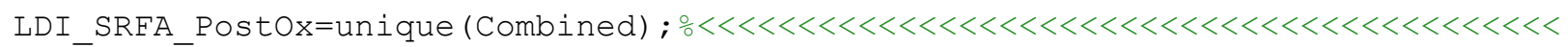
$<<\overline{<}<<<\overline{<} \overline{<}<<<<<<<<<<<<<<<<<<<<<<<<<<<<<<<<<<<<<<<<<<<<<<<<<<<<<<<<<<<<<<<<<<<<$

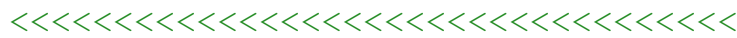

ㅇumarizing Data

Data=AOnly;

oCalculating formulae type parameters: \# CHO, CHON, CHOS, CHOP, CHN, CHS, CHP, CHNS, CHONS formulae

Summary $\cdot \mathrm{CHO}=$ sum $($ Data. $\mathrm{C}>0$ \& Data. $\mathrm{H}>0$ \& Data. $\mathrm{O}>0$ \& Data. $\mathrm{N}==0$ \& Data. $\mathrm{P}==0$ \&

Data. $\mathrm{S}==0$ );

Summary $\cdot \mathrm{CHON}=$ sum $($ Data. $\mathrm{C}>0$ \& Data.H $>0$ \& Data. $\mathrm{O}>0$ \& Data. $\mathrm{N}>0$ \& Data.P==0 \&

Data. $\mathrm{S}==0$ );

Summary. $\mathrm{CHOS}=$ sum $($ Data. $\mathrm{C}>0$ \& Data. $\mathrm{H}>0$ \& Data. $\mathrm{O}>0$ \& Data. $\mathrm{N}==0$ \& Data. $\mathrm{P}==0$ \&

Data. $\mathrm{S}>0$ );

Summary. $\mathrm{CHOP}=\mathrm{sum}($ Data. $\mathrm{C}>0$ \& Data.H $>0$ \& Data. $0>0$ \& Data. $\mathrm{N}==0$ \& Data.P $>0$ \&

Data. $\mathrm{S}==0$ );

Summary $\cdot \mathrm{CHN}=$ sum $($ Data. $\mathrm{C}>0$ \& Data. $\mathrm{H}>0$ \& Data. $\mathrm{O}==0$ \& Data. $\mathrm{N}>0$ \& Data. $\mathrm{P}==0$ \&

Data. $\mathrm{S}=0$ ) ;

Summary. CHS $=$ sum $($ Data. $\mathrm{C}>0$ \& Data.H $>0$ \& Data. $\mathrm{O}==0$ \& Data. $\mathrm{N}==0$ \& Data.P==0 \&

Data. $\mathrm{S}>0$ ) ;

Summary $\cdot \mathrm{CHNS}=\operatorname{sum}($ Data. $\mathrm{C}>0$ \& Data. $\mathrm{H}>0$ \& Data. $\mathrm{O}==0$ \& Data. $\mathrm{N}>0$ \& Data.P==0 \&

Data. S $>0$ );

Summary $\cdot \mathrm{CHNP}=\mathrm{sum}($ Data. $\mathrm{C}>0$ \& Data.H $>0$ \& Data. $\mathrm{O}==0$ \& Data. $\mathrm{N}>0$ \& Data.P $>0$ \&

Data. $\mathrm{S}==0$ );

Summary. $\mathrm{CHONS}=\operatorname{sum}($ Data. $\mathrm{C}>0$ \& Data. $\mathrm{H}>0$ \& Data. $0>0$ \& Data.N>0 \& Data.P==0 \&

Data. S>0);

Summary. $\mathrm{CHONP}=$ sum $($ Data. $\mathrm{C}>0$ \& Data.H $>0$ \& Data.O $>0$ \& Data.N $>0$ \& Data.P>0\&

Data. $\mathrm{S}==0$ );

Summary. $\mathrm{CHONSP}=$ sum $($ Data. $\mathrm{C}>0$ \& Data.H $>0$ \& Data.O $>0$ \& Data.N $>0$ \& Data.P>0 \&

Data. $\mathrm{S}>0)$;

ocalculating aromaticity parameters: \# condensed aromatic (AI>=0.67),

aromatic $(\mathrm{AI}>=0.5)$,

onon-aromatic $(\mathrm{A} I<0.5)$

Summary. CondensedAromatics $=$ sum (Data. AI $>=0.67)$;

Summary. Aromatics $=$ sum (Data.AI $>=0.5)$;

Summary. NonAromatics=sum (Data.AI<0.5);

ocalculating other parameters: total \# of formulae, average molecular

oweight

Summary.TotalFormulae=height (Data);

Summary. AvgMW=mean (Data. NominalMass);

ostoring Summary Data

LDI_NegOnIy_SREA_PostOx_Summary=struct2table (Summary) ; $\circ<<<<<<<<<<<<<<<<<<<<<<~$

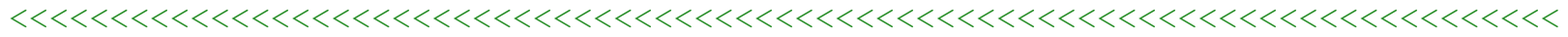

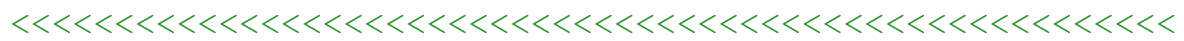

․ Summarizing Data

Data $=$ BOnly;

oCalculating formulae type parameters: \# CHO, CHON, CHOS, CHOP, CHN, CHS, CHP, CHNS, CHONS formulae

Summary. $\mathrm{CHO}=\operatorname{sum}($ Data. $\mathrm{C}>0$ \& Data. $\mathrm{H}>0$ \& Data. $\mathrm{O}>0$ \& Data. $\mathrm{N}==0$ \& Data. $\mathrm{P}==0$ \& Data. $\mathrm{S}=0$ ) ;

Summary. $\mathrm{CHON}=\operatorname{sum}($ Data. $\mathrm{C}>0$ \& Data. $\mathrm{H}>0$ \& Data. $\mathrm{O}>0$ \& Data.N $>0$ \& Data.P==0 \&

Data. $\mathrm{S}==0$ ) ;

Summary $\cdot \mathrm{CHOS}=$ sum $($ Data. $\mathrm{C}>0$ \& Data.H $>0$ \& Data. $\mathrm{O}>0$ \& Data. $\mathrm{N}==0$ \& Data.P==0 \&

Data. $\mathrm{S}>0$ ) ;

Summary $\cdot \mathrm{CHOP}=\operatorname{sum}($ Data. $\mathrm{C}>0$ \& Data.H $>0$ \& Data. $\mathrm{O}>0$ \& Data. $\mathrm{N}==0$ \& Data.P $>0$ \&

Data. $\mathrm{S}=0$ ) ;

Summary. $\mathrm{CHN}=\operatorname{sum}($ Data. $\mathrm{C}>0$ \& Data. $\mathrm{H}>0$ \& Data. $\mathrm{O}==0$ \& Data. $\mathrm{N}>0$ \& Data. $\mathrm{P}==0$ \&

Data. $\mathrm{S}==0$ ) ; 
Summary $\cdot \mathrm{CHS}=\operatorname{sum}($ Data $\cdot \mathrm{C}>0$ \& Data. $\mathrm{H}>0$ \& Data. $\mathrm{O}==0$ \& Data. $\mathrm{N}==0$ \& Data. $\mathrm{P}==0$ \& Data. $\mathrm{S}>0$ ) ;

Summary. CHNS $=$ sum $($ Data. $\mathrm{C}>0$ \& Data. $\mathrm{H}>0$ \& Data. $\mathrm{O}==0$ \& Data. $\mathrm{N}>0$ \& Data.P==0 \& Data. S $>0$ );

Summary. $\mathrm{CHNP}=\operatorname{sum}($ Data. $\mathrm{C}>0$ \& Data. $\mathrm{H}>0$ \& Data. $\mathrm{O}==0$ \& Data. $\mathrm{N}>0$ \& Data.P $>0$ \& Data. $\mathrm{S}==0$ );

Summary. CHONS $=$ sum $($ Data. $\mathrm{C}>0$ \& Data. $\mathrm{H}>0$ \& Data.O $>0$ \& Data.N $>0$ \& Data.P==0 \& Data. $\mathrm{S}>0$ ) ;

Summary. $\mathrm{CHONP}=$ sum $($ Data. $\mathrm{C}>0$ \& Data. $\mathrm{H}>0$ \& Data. $\mathrm{O}>0$ \& Data.N $>0$ \& Data.P $>0$ \& Data. $\mathrm{S}==0$ );

Summary. $\mathrm{CHONSP}=$ sum $($ Data. $\mathrm{C}>0$ \& Data.H $>0$ \& Data. O $>0$ \& Data.N $>0$ \& Data.P $>0$ \& Data. S>0);

ocalculating aromaticity parameters: \# condensed aromatic (AI>=0.67), aromatic $(\mathrm{AI}>=0.5)$,

onon-aromatic $(\mathrm{A} I<0.5)$

Summary. CondensedAromatics $=$ sum (Data. AI $>=0.67)$;

Summary. Aromatics $=$ sum (Data. AI $>=0.5)$;

Summary. NonAromatics=sum (Data.AI<0.5);

ocalculating other parameters: total \# of formulae, average molecular

oweight

Summary.TotalFormulae=height (Data);

Summary . AvgMW=mean (Data. NominalMass);

o.Storing Summary Data

LDI PosOnly SRFA Postox Summary=struct2table (Summary); $\circ<<<<<<<<<<<<<<<<<<<<<<<<~$ $<<\overline{<}<<<<<<<\overline{<}<<<\overline{<}<<<<<\overline{<}<<<<<<<<<<<<<<<<<<<<<<<<<<<<<<<<<<<<<<<<<<<<<<<<<<<<<$

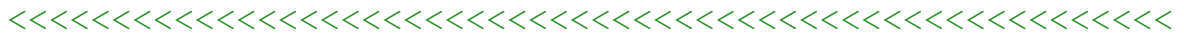

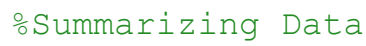

Data=Both;

oCalculating formulae type parameters: \# CHO, CHON, CHOS, CHOP, CHN, CHS, CHP, CHNS, CHONS formulae

Summary $\cdot \mathrm{CHO}=\operatorname{sum}($ Data. $\mathrm{C}>0$ \& Data. $\mathrm{H}>0$ \& Data. $\mathrm{O}>0$ \& Data. $\mathrm{N}==0$ \& Data. $\mathrm{P}==0$ \&

Data. $\mathrm{S}==0$ );

Summary $\cdot \mathrm{CHON}=\operatorname{sum}($ Data. $\mathrm{C}>0$ \& Data.H $>0$ \& Data. $0>0$ \& Data. $\mathrm{N}>0$ \& Data. $\mathrm{P}==0$ \&

Data. $\mathrm{S}==0$ );

Summary. $\mathrm{CHOS}=$ sum $($ Data. $\mathrm{C}>0$ \& Data. $\mathrm{H}>0$ \& Data.O $>0$ \& Data. $\mathrm{N}==0$ \& Data. $\mathrm{P}==0$ \&

Data. S>0);

Summary $\cdot \mathrm{CHOP}=$ sum $($ Data. $\mathrm{C}>0$ \& Data. $\mathrm{H}>0$ \& Data. $\mathrm{O}>0$ \& Data. $\mathrm{N}==0$ \& Data. P $>0$ \&

Data. $\mathrm{S}==0$ ) ;

Summary. $\mathrm{CHN}=$ sum $($ Data. $\mathrm{C}>0$ \& Data. $\mathrm{H}>0$ \& Data. $\mathrm{O}==0$ \& Data. $\mathrm{N}>0$ \& Data. $\mathrm{P}==0$ \&

Data. $\mathrm{S}==0$ );

Summary $\cdot \mathrm{CHS}=\operatorname{sum}($ Data. $\mathrm{C}>0$ \& Data. $\mathrm{H}>0$ \& Data. $\mathrm{O}==0$ \& Data. $\mathrm{N}==0$ \& Data. $\mathrm{P}==0$ \&

Data. S $>0$ );

Summary $\cdot \mathrm{CHNS}=\operatorname{sum}($ Data. $\mathrm{C}>0$ \& Data. $\mathrm{H}>0$ \& Data. $\mathrm{O}==0$ \& Data. $\mathrm{N}>0$ \& Data.P==0 \&

Data. $\mathrm{S}>0$ );

Summary. $\mathrm{CHNP}=\operatorname{sum}($ Data. $\mathrm{C}>0$ \& Data. $\mathrm{H}>0$ \& Data. $\mathrm{O}==0$ \& Data. $\mathrm{N}>0$ \& Data.P $>0$ \&

Data. $\mathrm{S}=0$ ) ;

Summary. $\mathrm{CHONS}=\operatorname{sum}($ Data $\cdot \mathrm{C}>0$ \& Data. $\mathrm{H}>0$ \& Data. $\mathrm{O}>0$ \& Data. $\mathrm{N}>0$ \& Data. $\mathrm{P}==0$ \&

Data. S>0);

Summary. $\mathrm{CHONP}=$ sum $($ Data. $\mathrm{C}>0$ \& Data. $\mathrm{H}>0$ \& Data. O $>0$ \& Data.N $>0$ \& Data.P $>0$ \&

Data. $\mathrm{S}==0$ );

Summary. $\mathrm{CHONSP}=$ sum $($ Data. $\mathrm{C}>0$ \& Data.H $>0$ \& Data.O $>0$ \& Data.N $>0$ \& Data.P>0 \&

Data. S>0);

oCalculating aromaticity parameters: \# condensed aromatic (AI>=0.67),

aromatic $(A I>=0.5)$,

onon-aromatic $($ AI $<0.5)$

Summary. CondensedAromatics $=$ sum (Data. AI $>=0.67)$;

Summary.Aromatics=sum (Data.AI>=0.5); 
Summary. NonAromatics=sum (Data.AI<0.5);

ocalculating other parameters: total \# of formulae, average molecular

oweight

Summary. TotalFormulae=height (Data);

Summary. AvgMW=mean (Data. NominalMass) ;

ostoring Summary Data

LDI BothPolarities_SRFA Postox_Summary=struct2table (Summary); $\circ<<<<<<<<<<<<<<<<$

$<<<\overline{<}<<<<<<<<<<<<<<\overline{<}<<<<\overline{<}<<<<<<\overline{<}<<<<<<<<<<<<<<<<<<<<<<<<<<<<<<<<<<<<<<<<<<<<<<~$

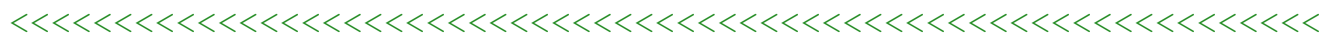

o Summarizing Data

Data $=$ Combined;

oCalculating formulae type parameters: \# CHO, CHON, CHOS, CHOP, CHN, CHS, CHP, CHNS, CHONS formulae

Summary $\cdot \mathrm{CHO}=$ sum $($ Data. $\mathrm{C}>0$ \& Data. $\mathrm{H}>0$ \& Data. $\mathrm{O}>0$ \& Data. $\mathrm{N}==0$ \& Data. $\mathrm{P}==0$ \&

Data. $\mathrm{S}==0$ );

Summary $\cdot \mathrm{CHON}=\operatorname{sum}($ Data. $\mathrm{C}>0$ \& Data.H $>0$ \& Data. $\mathrm{O}>0$ \& Data. $>0$ \& Data.P==0 \&

Data. $\mathrm{S}=0$ ) ;

Summary. CHOS $=$ sum $($ Data. $\mathrm{C}>0$ \& Data.H>0 \& Data.O>0 \& Data.N==0 \& Data.P==0 \&

Data. $\mathrm{S}>0$ );

Summary $\cdot \mathrm{CHOP}=\operatorname{sum}($ Data. $\mathrm{C}>0$ \& Data.H $>0$ \& Data. $\mathrm{O}>0$ \& Data. $\mathrm{N}==0$ \& Data.P $>0$ \&

Data. $\mathrm{S}==0$ );

Summary $\cdot \mathrm{CHN}=$ sum $($ Data. $\mathrm{C}>0$ \& Data. $\mathrm{H}>0$ \& Data. $\mathrm{O}==0$ \& Data. $\mathrm{N}>0$ \& Data. $\mathrm{P}==0$ \&

Data. $\mathrm{S}==0$ );

Summary. CHS $=$ sum $($ Data. $\mathrm{C}>0$ \& Data.H $>0$ \& Data. $\mathrm{O}==0$ \& Data. $\mathrm{N}==0$ \& Data.P==0 \&

Data. S>0);

Summary. $\mathrm{CHNS}=$ sum $($ Data. $\mathrm{C}>0$ \& Data. $\mathrm{H}>0$ \& Data. $\mathrm{O}==0$ \& Data. $\mathrm{N}>0$ \& Data. $\mathrm{P}==0$ \&

Data. $\mathrm{S}>0$ );

Summary. $\mathrm{CHNP}=\operatorname{sum}($ Data. $\mathrm{C}>0$ \& Data. $\mathrm{H}>0$ \& Data. $\mathrm{O}==0$ \& Data. $\mathrm{N}>0$ \& Data.P $>0$ \&

Data. $\mathrm{S}==0$ );

Summary $\cdot \mathrm{CHONS}=\operatorname{sum}($ Data. $\mathrm{C}>0$ \& Data. $\mathrm{H}>0$ \& Data. $0>0$ \& Data.N $>0$ \& Data.P==0 \&

Data. S>0);

Summary. $\mathrm{CHONP}=$ sum $($ Data. $\mathrm{C}>0$ \& Data. $\mathrm{H}>0$ \& Data. $\mathrm{O}>0$ \& Data. $\mathrm{N}>0$ \& Data.P $>0$ \&

Data. $\mathrm{S}==0$ );

Summary. $\mathrm{CHONSP}=$ sum $($ Data. $\mathrm{C}>0$ \& Data.H $>0$ \& Data.O $>0$ \& Data.N $>0$ \& Data.P>0 \&

Data. $\mathrm{S}>0$ );

ocalculating aromaticity parameters: \# condensed aromatic (AI>=0.67),

aromatic $(A I>=0.5)$,

onon-aromatic $(\mathrm{AI}<0.5)$

Summary. CondensedAromatics $=$ sum (Data. AI $>=0.67)$;

Summary. Aromatics $=$ sum (Data.AI $>=0.5)$;

Summary. NonAromatics=sum (Data.AI<0.5);

ocalculating other parameters: total \# of formulae, average molecular

\%weight

Summary. TotalFormulae=height (Data);

Summary. AvgMW=mean (Data. NominalMass);

ostoring Summary Data

LDI SRFA Postox Summary=struct2table (Summary); $\circ<<<<<<<<<<<<<<<<<<<<<<<<<<<<<<<<~$ $<<<\overline{<}<<<<\overline{<}<<<<<<\overline{<}<<<<<<<<<<<<<<<<<<<<<<<<<<<<<<<<<<<<<<<<<<<<<<<<<<<<<<<<<<<<<~$

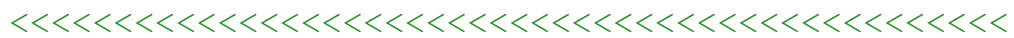

응 Combining ESI and LDI

onput Data so that the script can readily be applied to any data set

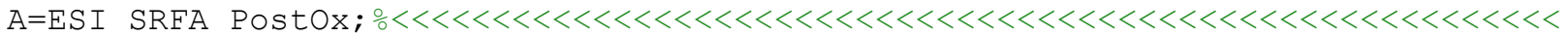
$<<<<<\overline{<}<<<\overline{<} \overline{<}<<<<<<<<<<<<<<<<<<<<<<<<<<<<<<<<<<<<<<<<<<<<<<<<<<<<<<<<<<<<<<<<<<~$ $<<<<<<<<<<<<<<<<<<<<~$ 


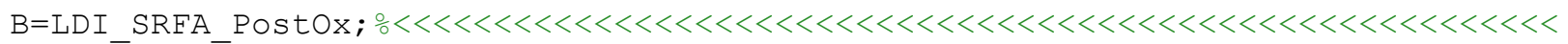
$<<<<\overline{\bar{l}}<<<\overline{<} \overline{<}<<<<<<<<<<<<<<<<<<<<<<<<<<<<<<<<<<<<<<<<<<<<<<<<<<<<<<<<<<<<<<<<<<~$ $<<<<<<<<<<<<<<<<<<<<~$

o Separate Data into those common and unique

Both=[ ] ;

$\mathrm{k}=0$;

for $i=1$ :height $(A)$ for $j=1$ :height $(B)$

if $A \cdot C(i)==B \cdot C(j) \quad \& \& A \cdot H(i)==B \cdot H(j) \quad \& \& A \cdot N(i)==B \cdot N(j) \quad \& \& A \cdot O(i)==B \cdot O(j)$

$\& \& A \cdot P(i)==B \cdot P(j) \quad \& \& A \cdot S(i)==B \cdot S(j)$

$\mathrm{k}=\mathrm{k}+1$;

$\operatorname{Both}(k,:)=[A . C(i), A . H(i), A . O(i), A . N(i), A . P(i)$,

A.S (i), A.NominalMass (i), A.HC Ratio(i),

A. OC Ratio(i), A.AI (i), A. DBE (i), A.AvgCoxState (i) ] ;

$$
\begin{aligned}
& \text { A. } C(i)=0 ; \\
& \text { B. C }(j)=0 ;
\end{aligned}
$$

end

end

Combining Data Sets

$A(A . C==0,:)=[]$;

$A O n l y=[A . C, A . H, A . O, A . N, A . P, A . S$,

A.NominalMass, A.HC Ratio,A.oC Ratio,A.AI, A.DBE, A.AvgCoxState];

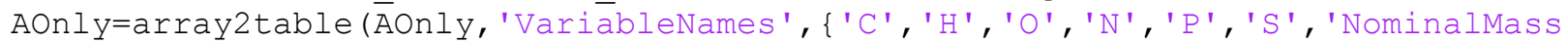
', 'HC_Ratio', 'OC_Ratio', 'AI', 'DBE', 'AvgCOxState' \});

$\mathrm{B}(\mathrm{B} \cdot \mathrm{C}==0,:)=[]$;

$\mathrm{BOnl} Y=[B . \mathrm{C}, \mathrm{B} . \mathrm{H}, \mathrm{B} . \mathrm{O}, \mathrm{B} . \mathrm{N}, \mathrm{B} . \mathrm{P}$,

B.S,B.NominalMass, B.HC Ratio,B.OC Ratio, B.AI, B.DBE, B. AvgCoxState];

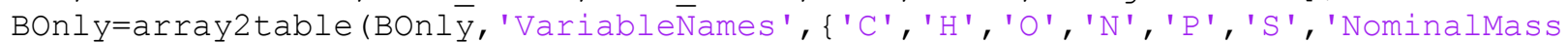
', 'HC Ratio', 'OC Ratio', 'AI', 'DBE', 'AvgCoxstate'\}) ;

Both=array2table (Both, 'VariableNames', \{'C', 'H', 'O', 'N', 'P' , 'S ', 'NominalMass', 'HC_Ratio', 'OC_Ratio', 'AI', 'DBE', 'AvgCoxstate' \}) ;

Combined=[Both; AOnly; BOnly];

ostoring Data with distinct names

BothTechniques SRFA PostOx=unique (Both); $\frac{\circ}{\circ}<<<<<<<<<<<<<<<<<<<<<<<<<<<<<<<<<<<<<~$ $<<<<<<<<<<<<<<\overline{<}<<<\overline{<}<<<<<<<<<<<<<<<<<<<<<<<<<<<<<<<<<<<<<<<<<<<<<<<<<<<<<<<<<~$

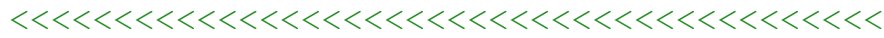

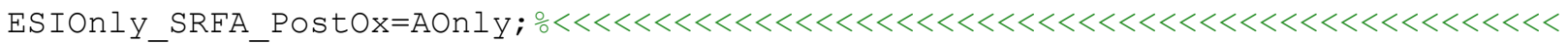
$<<<<<<<\bar{l}<<<\overline{\bar{l}}<<<<<<<<<<<<<<<<<<<<<<<<<<<<<<<<<<<<<<<<<<<<<<<<<<<<<<<<<<<<<<<<$ $<<<<<<<<<<<<<<<<<<<<<<<<<<<<~$

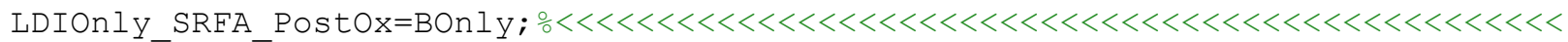

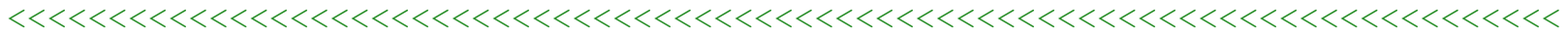
$<<<<<<<<<<<<<<<<<<<<<<<<<<<<~$

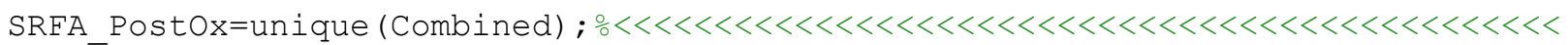
$<<<\overline{<}<<<<<<<<<<<<<<<<<<<<<<<<<<<<<<<<<<<<<<<<<<<<<<<<<<<<<<<<<<<<<<<<<<<<<<<<~$

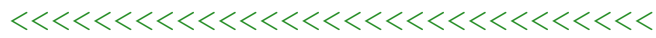

o Summarizing Data

Data=AOnly;

oCalculating formulae type parameters: \# CHO, CHON, CHOS, CHOP, CHN, CHS, CHP, CHNS, CHONS formulae

Summary $\cdot \mathrm{CHO}=$ sum $($ Data. $\mathrm{C}>0$ \& Data. $\mathrm{H}>0$ \& Data. $\mathrm{O}>0$ \& Data. $\mathrm{N}==0$ \& Data. $\mathrm{P}==0$ \&

Data. $\mathrm{S}=0$ ) ;

Summary $\cdot \mathrm{CHON}=\operatorname{sum}($ Data. $\mathrm{C}>0$ \& Data.H $>0$ \& Data. $\mathrm{O}>0$ \& Data. $>0$ \& Data.P==0 \& Data. $\mathrm{S}==0$ );

Summary. $\mathrm{CHOS}=$ sum $($ Data. $\mathrm{C}>0$ \& Data. $\mathrm{H}>0$ \& Data. $\mathrm{O}>0$ \& Data. $\mathrm{N}==0$ \& Data. $\mathrm{P}==0$ \&

Data. $\mathrm{S}>0$ ) ; 
Summary. $\mathrm{CHOP}=\mathrm{sum}($ Data. $\mathrm{C}>0$ \& Data.H $>0$ \& Data.O $>0$ \& Data.N==0 \& Data.P>0 \& Data. $\mathrm{S}==0$ );

Summary $\cdot \mathrm{CHN}=$ sum $($ Data. $\mathrm{C}>0$ \& Data. $\mathrm{H}>0$ \& Data. $\mathrm{O}==0$ \& Data. $\mathrm{N}>0$ \& Data.P==0 \& Data. $\mathrm{S}==0$ ) ;

Summary $\cdot \mathrm{CHS}=\operatorname{sum}($ Data $\cdot \mathrm{C}>0$ \& Data. $\mathrm{H}>0$ \& Data. $\mathrm{O}==0$ \& Data. $\mathrm{N}==0$ \& Data. $\mathrm{P}==0$ \& Data. $\mathrm{S}>0$ );

Summary $\cdot \mathrm{CHNS}=$ sum $($ Data $\cdot \mathrm{C}>0$ \& Data. $\mathrm{H}>0$ \& Data. $\mathrm{O}==0$ \& Data. $\mathrm{N}>0$ \& Data. $\mathrm{P}==0$ \&

Data. $\mathrm{S}>0$ ) ;

Summary $\cdot \mathrm{CHNP}=\operatorname{sum}($ Data $\cdot \mathrm{C}>0$ \& Data. $\mathrm{H}>0$ \& Data. $\mathrm{O}==0$ \& Data.N $>0$ \& Data.P>0 \&

Data. $\mathrm{S}==0$ );

Summary. $\mathrm{CHONS}=$ sum $($ Data $. \mathrm{C}>0$ \& Data. $\mathrm{H}>0$ \& Data. $\mathrm{O}>0$ \& Data. $>0$ \& Data.P==0 \&

Data. $\mathrm{S}>0$ );

Summary. $\mathrm{CHONP}=$ sum $($ Data. $\mathrm{C}>0$ \& Data.H $>0$ \& Data.O>0 \& Data.N>0 \& Data.P>0 \&

Data. $\mathrm{S}==0$ );

Summary. $\mathrm{CHONSP}=$ sum $($ Data. $\mathrm{C}>0$ \& Data. $\mathrm{H}>0$ \& Data.O $>0$ \& Data.N $>0$ \& Data.P $>0$ \&

Data. S>0);

ocalculating aromaticity parameters: \# condensed aromatic (AI>=0.67),

aromatic $(\mathrm{AI}>=0.5)$,

onon-aromatic $(\mathrm{AI}<0.5)$

Summary. CondensedAromatics $=$ sum (Data. AI $>=0.67)$;

Summary. Aromatics $=$ sum (Data.AI $>=0.5)$;

Summary. NonAromatics=sum (Data.AI<0.5);

oCalculating other parameters: total \# of formulae, average molecular

oweight

Summary. TotalFormulae=height (Data);

Summary. AvgMW=mean (Data. NominalMass);

ostoring Summary Data

ESIOnly_SRFA_Postox_Summary=struct2table (Summary) ; $\circ<<<<<<<<<<<<<<<<<<<<<<<<<<<<$

$<<<<<<<\overline{<}<<<\overline{<} \overline{<}<<<<<\overline{<}<<<<<<<<<<<<<<<<<<<<<<<<<<<<<<<<<<<<<<<<<<<<<<<<<<<<<<<<<<$

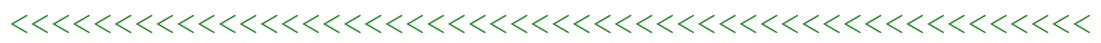

ㅇmmarizing Data

Data=BOnly;

oCalculating formulae type parameters: \# CHO, CHON, CHOS, CHOP, CHN, CHS, CHP, CHNS, CHONS formulae

Summary $\cdot \mathrm{CHO}=$ sum $($ Data. $\mathrm{C}>0$ \& Data. $\mathrm{H}>0$ \& Data. $\mathrm{O}>0$ \& Data. $\mathrm{N}==0$ \& Data. $\mathrm{P}==0$ \& Data. $\mathrm{S}==0$ );

Summary $\cdot \mathrm{CHON}=$ sum $($ Data $\cdot \mathrm{C}>0$ \& Data. $\mathrm{H}>0$ \& Data. $\mathrm{O}>0$ \& Data.N $>0$ \& Data.P==0 \&

Data. $\mathrm{S}==0$ );

Summary. $\mathrm{CHOS}=$ sum $($ Data. $\mathrm{C}>0$ \& Data. $\mathrm{H}>0$ \& Data. $\mathrm{O}>0$ \& Data. $\mathrm{N}==0$ \& Data. $\mathrm{P}==0$ \&

Data. $\mathrm{S}>0$ ) ;

Summary $\cdot \mathrm{CHOP}=\operatorname{sum}($ Data. $\mathrm{C}>0$ \& Data.H $>0$ \& Data.O $>0$ \& Data.N==0 \& Data.P>0 \&

Data. $\mathrm{S}==0$ );

Summary. $\mathrm{CHN}=$ sum $($ Data. $\mathrm{C}>0$ \& Data. $\mathrm{H}>0$ \& Data. $\mathrm{O}==0$ \& Data. $\mathrm{N}>0$ \& Data.P==0 \&

Data. $\mathrm{S}==0$ );

Summary $\cdot \mathrm{CHS}=\operatorname{sum}($ Data $. \mathrm{C}>0$ \& Data. $\mathrm{H}>0$ \& Data. $\mathrm{O}==0$ \& Data. $\mathrm{N}==0$ \& Data. $\mathrm{P}==0$ \&

Data. $\mathrm{S}>0$ ) ;

Summary. CHNS $=$ sum $($ Data. $\mathrm{C}>0$ \& Data. $\mathrm{H}>0$ \& Data. $\mathrm{O}==0$ \& Data. $>0$ \& Data.P==0 \&

Data. $\mathrm{S}>0$ );

Summary. $\mathrm{CHNP}=$ sum $($ Data. $\mathrm{C}>0$ \& Data. $\mathrm{H}>0$ \& Data. $\mathrm{O}==0$ \& Data. $\mathrm{N}>0$ \& Data.P $>0$ \&

Data. $\mathrm{S}==0$ );

Summary $\cdot \mathrm{CHONS}=$ sum $($ Data. $\mathrm{C}>0$ \& Data. $\mathrm{H}>0$ \& Data. $0>0$ \& Data. $>0$ \& Data.P==0 \&

Data. $\mathrm{S}>0$ ) ;

Summary. $\mathrm{CHONP}=$ sum $($ Data. $\mathrm{C}>0$ \& Data.H $>0$ \& Data. $\mathrm{O}>0$ \& Data.N $>0$ \& Data.P>0 \&

Data. $\mathrm{S}==0$ );

Summary. $\mathrm{CHONSP}=$ sum $($ Data. $\mathrm{C}>0$ \& Data. $\mathrm{H}>0$ \& Data. $\mathrm{O}>0$ \& Data.N $>0$ \& Data.P $>0$ \&

Data. $\mathrm{S}>0$ ) ; 
oCalculating aromaticity parameters: \# condensed aromatic (AI>=0.67), aromatic $(\mathrm{AI}>=0.5)$,

onon-aromatic (AI<0.5)

Summary. CondensedAromatics $=$ sum (Data.AI $>=0.67)$;

Summary.Aromatics=sum (Data.AI $>=0.5)$;

Summary. NonAromatics=sum (Data.AI<0.5);

ocalculating other parameters: total \# of formulae, average molecular

oweight

Summary. TotalFormulae=height (Data);

Summary . AvgMW=mean (Data. NominalMass);

ostoring Summary Data

LDIOnly_SRFA Postox_Summary=struct2table (Summary); $\circ<<<<<<<<<<<<<<<<<<<<<<<<<<<$ $<<<<<<<\overline{<}<<<\overline{<}<<<<<<\overline{<}<<<<<<<<<<<<<<<<<<<<<<<<<<<<<<<<<<<<<<<<<<<<<<<<<<<<<<<<<~$

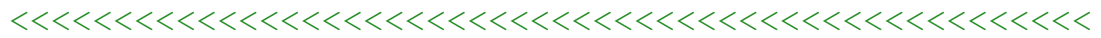

․ Summarizing Data

Data=unique (Both);

oCalculating formulae type parameters: \# CHO, CHON, CHOS, CHOP, CHN, CHS, CHP, CHNS, CHONS formulae

Summary $\cdot \mathrm{CHO}=$ sum $($ Data. $\mathrm{C}>0$ \& Data. $\mathrm{H}>0$ \& Data. $\mathrm{O}>0$ \& Data. $\mathrm{N}==0$ \& Data. $\mathrm{P}==0$ \& Data. $\mathrm{S}==0$ );

Summary $\cdot \mathrm{CHON}=$ Sum $($ Data. $\mathrm{C}>0$ \& Data. $\mathrm{H}>0$ \& Data. $\mathrm{O}>0$ \& Data. $\mathrm{N}>0$ \& Data.P==0 \& Data. $\mathrm{S}==0$ );

Summary. $\mathrm{CHOS}=$ sum $($ Data. $\mathrm{C}>0$ \& Data. $\mathrm{H}>0$ \& Data. $\mathrm{O}>0$ \& Data. $\mathrm{N}==0$ \& Data. $\mathrm{P}==0$ \&

Data. $\mathrm{S}>0$ );

Summary $\cdot \mathrm{CHOP}=$ sum $($ Data. $\mathrm{C}>0$ \& Data.H $>0$ \& Data. $\mathrm{O}>0$ \& Data. $\mathrm{N}==0$ \& Data.P $>0$ \&

Data. $\mathrm{S}==0$ );

Summary $\cdot \mathrm{CHN}=$ sum $($ Data. $\mathrm{C}>0$ \& Data. $\mathrm{H}>0$ \& Data. $\mathrm{O}==0$ \& Data. $\mathrm{N}>0$ \& Data. $\mathrm{P}==0$ \&

Data. $\mathrm{S}==0$ );

Summary $\cdot \mathrm{CHS}=\operatorname{sum}($ Data $\cdot \mathrm{C}>0$ \& Data. $\mathrm{H}>0$ \& Data $\cdot \mathrm{O}==0$ \& Data. $\mathrm{N}==0$ \& Data. $\mathrm{P}==0$ \&

Data. $\mathrm{S}>0$ );

Summary. $\mathrm{CHNS}=$ sum $($ Data. $\mathrm{C}>0$ \& Data. $\mathrm{H}>0$ \& Data. $\mathrm{O}==0$ \& Data. $\mathrm{N}>0$ \& Data. $\mathrm{P}==0$ \&

Data. S>0);

Summary $\cdot \mathrm{CHNP}=\operatorname{sum}($ Data. $\mathrm{C}>0$ \& Data.H $>0$ \& Data. $\mathrm{O}==0$ \& Data. $\mathrm{N}>0$ \& Data.P $>0$ \&

Data. $\mathrm{S}==0$ );

Summary. $\mathrm{CHONS}=\operatorname{sum}($ Data $. \mathrm{C}>0$ \& Data. $\mathrm{H}>0$ \& Data. $0>0$ \& Data.N>0 \& Data.P==0 \&

Data. S>0);

Summary. $\mathrm{CHONP}=$ sum $($ Data. $\mathrm{C}>0$ \& Data. $\mathrm{H}>0$ \& Data. $\mathrm{O}>0$ \& Data.N $>0$ \& Data.P $>0$ \&

Data. $\mathrm{S}==0$ ) ;

Summary. $\mathrm{CHONSP}=$ sum $($ Data. $\mathrm{C}>0$ \& Data.H $>0$ \& Data.O $>0$ \& Data.N $>0$ \& Data.P>0 \&

Data. $\mathrm{S}>0$ );

ocalculating aromaticity parameters: \# condensed aromatic (AI>=0.67),

aromatic (AI>=0.5),

onon-aromatic $(\mathrm{A} I<0.5)$

Summary. CondensedAromatics $=$ sum (Data. AI $>=0.67)$;

Summary. Aromatics $=$ sum (Data.AI $>=0.5)$;

Summary. NonAromatics=sum (Data.AI<0.5);

ocalculating other parameters: total \# of formulae, average molecular

oweight

Summary. TotalFormulae=height (Data);

Summary. AvgMW=mean (Data. NominalMass);

ostoring Summary Data

BothTechniques_SRFA_PostOx_Summary=struct2table (Summary); $\circ<<<<<<<<<<<<<<<<<<<~$ $<<<<<<<<<<<<<<\overline{<}<<<<<<<<<<<<<<<<<<<<<<<<<<<<<<<<<<<<<<<<<<<<<<<<<<<<<<<<<<<<<<<~$

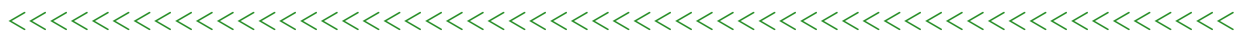

ㅇmmarizing Data

Data $=$ Combined; 
oCalculating formulae type parameters: \# CHO, CHON, CHOS, CHOP, CHN, CHS, CHP, CHNS, CHONS formulae

Summary $\cdot \mathrm{CHO}=$ sum (Data. $\mathrm{C}>0$ \& Data. $\mathrm{H}>0$ \& Data. $\mathrm{O}>0$ \& Data. $\mathrm{N}==0$ \& Data. $\mathrm{P}==0$ \& Data. $\mathrm{S}==0$ );

Summary. $\mathrm{CHON}=\operatorname{sum}($ Data. $\mathrm{C}>0$ \& Data. $\mathrm{H}>0$ \& Data. $\mathrm{O}>0$ \& Data. $\mathrm{N}>0$ \& Data.P==0 \& Data. $\mathrm{S}=0$ ) ) ;

Summary. CHOS $=$ sum $($ Data. $\mathrm{C}>0$ \& Data.H $>0$ \& Data.O>0 \& Data.N==0 \& Data.P==0 \& Data. $\mathrm{S}>0$ );

Summary $\cdot \mathrm{CHOP}=\operatorname{sum}($ Data. $\mathrm{C}>0$ \& Data.H $>0$ \& Data. $\mathrm{O}>0$ \& Data. $\mathrm{N}==0$ \& Data.P $>0$ \& Data. $\mathrm{S}==0$ );

Summary $\cdot \mathrm{CHN}=$ sum $($ Data. $\mathrm{C}>0$ \& Data. $\mathrm{H}>0$ \& Data. $\mathrm{O}==0$ \& Data. $\mathrm{N}>0$ \& Data. $\mathrm{P}==0$ \&

Data. $\mathrm{S}=0$ ) ;

Summary $\cdot \mathrm{CHS}=\operatorname{sum}($ Data $\cdot \mathrm{C}>0$ \& Data. $\mathrm{H}>0$ \& Data. $\mathrm{O}==0$ \& Data. $\mathrm{N}==0$ \& Data. $\mathrm{P}==0$ \&

Data. S>0);

Summary. $\mathrm{CHNS}=$ sum (Data. $\mathrm{C}>0$ \& Data. $\mathrm{H}>0$ \& Data. $\mathrm{O}==0$ \& Data. $\mathrm{N}>0$ \& Data. $\mathrm{P}==0$ \&

Data. S>0);

Summary $\cdot \mathrm{CHNP}=\operatorname{sum}($ Data. $\mathrm{C}>0$ \& Data.H $>0$ \& Data. $\mathrm{O}==0$ \& Data.N $>0$ \& Data.P $>0$ \&

Data. $\mathrm{S}==0$ );

Summary $\cdot \mathrm{CHONS}=\operatorname{sum}($ Data $\cdot \mathrm{C}>0$ \& Data. $\mathrm{H}>0$ \& Data. $\mathrm{O}>0$ \& Data.N $>0$ \& Data.P==0 \&

Data. S>0);

Summary. $\mathrm{CHONP}=\operatorname{sum}($ Data. $\mathrm{C}>0$ \& Data. $\mathrm{H}>0$ \& Data. $\mathrm{O}>0$ \& Data.N $>0$ \& Data.P $>0$ \&

Data. $\mathrm{S}==0$ );

Summary. $\mathrm{CHONSP}=$ sum $($ Data. $\mathrm{C}>0$ \& Data. $\mathrm{H}>0$ \& Data. $\mathrm{O}>0$ \& Data.N $>0$ \& Data.P $>0$ \&

Data. $\mathrm{S}>0$ );

ocalculating aromaticity parameters: \# condensed aromatic (AI>=0.67),

aromatic $(\mathrm{AI}>=0.5)$,

onon-aromatic $(\mathrm{A} I<0.5)$

Summary. CondensedAromatics $=$ sum (Data.AI $>=0.67)$;

Summary.Aromatics $=$ sum (Data.AI $>=0.5)$;

Summary. NonAromatics=sum (Data.AI<0.5);

ocalculating other parameters: total \# of formulae, average molecular

oweight

Summary. TotalFormulae=height (Data);

Summary. AvgMW=mean (Data. NominalMass);

ostoring Summary Data

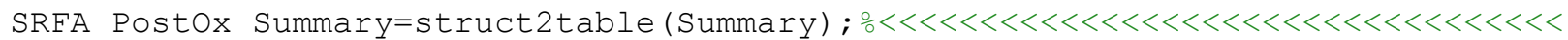
$<<<\overline{\bar{l}}<<<<<\overline{\bar{l}}<<<<<<<<<<<<<<<<<<<<<<<<<<<<<<<<<<<<<<<<<<<<<<<<<<<<<<<<<<<<<<<<<~$

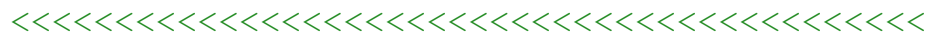

응 Repeating for GLSM DOM

응 Negative Mode ESI: Data Clean Up

oLoading negative mode background impurities

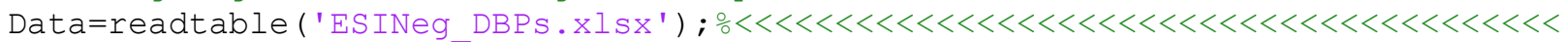
$<<<<<<<<<<<<<<<<<<<<<<\overline{<}<<<<<<<<<<<<<<<<<<<<<<<<<<<<<<<<<<<<<<<<<<<<<<<<<<<<<<~$ $<<<<<<<<<<<<<<<<<<<<<<<<<<<<<<<<<<~$

Data=unique (Data);

Data. NominalMass=Data. C*12+Data.0*16+Data.H*1+Data.P*31+Data.S*32+Data.N*14;

Data. KMD CH2=Data. NominalMass-

$0.998883 \overline{4} *\left(\right.$ Data. $C * 12+$ Data. $H^{*} 1.007825+$ Data. $N^{*} 14.003074+$ Data. $0 * 15.994915+$ Data.$P$

$\star 30.973763+$ Data.. *31.972072);

Data.KMD COO=Data. NominalMass-

$1.000231^{\star}\left(\right.$ Data. C*12+Data.H*1.007825+Data. $N^{*} 14.003074+$ Data. $0 * 15.994915+$ Data.P*

$30.973763+$ Data..$* 31.972072)$;

Data.KMD 02=Data. NominalMass-

$1.000317^{\bar{*}}\left(\right.$ Data. $\mathrm{C}^{\star} 12+$ Data. $\mathrm{H}^{\star} 1.007825+$ Data $. \mathrm{N}^{\star} 14.003074+\mathrm{Data} .0 * 15.994915+\mathrm{Data} . \mathrm{P} *$

$30.973763+$ Data. S*31.972072); 
Data.KMD H2=Data. NominalMass-

$0.992236^{\star}\left(\right.$ Data. $C^{*} 12+$ Data $. H^{*} 1.007825+$ Data $. N^{*} 14.003074+$ Data. $0 * 15.994915+$ Data. $P *$ $30.973763+$ Data.S*31.972072);

Data. H=Data.H+1;

Data.HC_Ratio = Data.H ./ Data.C;

Data.oC Ratio = Data.O ./ Data.C;

Data.NC Ratio = Data.N./ Data.C;

Data.SC Ratio = Data.S./ Data.C;

Data.PC Ratio = Data.P ./ Data.C;

Data. $\mathrm{DB} \overline{\mathrm{E}}=1+0.5 *(2 *$ Data. C - Data.H + Data. $\mathrm{N}+$ Data.P $)$;

Data.AI = (1 + Data.C - Data.O - Data.S - 0.5* (Data.N + Data.P + Data.H) $). /$

(Data.C - Data.O - Data.N - Data.S - Data.P);

Data.AvgCoxstate = 2*Data.oC Ratio - Data.HC Ratio;

\%Remove data points based on following criteria

ㄴ. Error (allowable: ppm $<=0.5$ )

Data (Data. errPpm>0.5,:) = [];

Data (Data.errPpm<-0.5,:) = [ ] ;

⒉ Stubbins, 2010 :

\%A. $\mathrm{DBE}=$ integer, greater than or equal to 0

Data (Data.DBE =round (Data.DBE), : ) = [];

Data $($ Data. $\mathrm{DBE}<0,:)=[]$;

ㅇ. $\mathrm{C}<=50$

Data (Data.C $>50,:$ ) $=[]$;

ㄷ. $\mathrm{H}>=2 \&<=2 * \mathrm{C}+2$

Data (Data. $\mathrm{H}<2,:$ ) $=[]$;

Data $($ Data.H $>($ Data. $C * 2+2),:)=[]$;

$\because \mathrm{D} \cdot \mathrm{O}=<\mathrm{C}+2$

Data (Data.o $>($ Data.C+2), : ) $=[]$;

E. $\mathrm{O} / \mathrm{C}<1.2$

Data (Data.oC Ratio>1.2,:) =[] ;

ㄷ․ $\mathrm{H} / \mathrm{C}>=0.33 \&<=2.25$

Data (Data.HC_Ratio<0.33,:) = [] ;

Data (Data.HC Ratio>2.25,:) =[ ] ;

$\div \mathrm{G} . \mathrm{N} / \mathrm{C}=<0.5$

Data (Data.NC Ratio $>=0.5,:)=[]$;

$\div \mathrm{H} . \mathrm{S} / \mathrm{C}=<0.2$

Data (Data.SC_Ratio>=0.2, :) $=[]$;

\%I. $\mathrm{P} / \mathrm{C}=<0 . \overline{0} 5$

Data (Data.PC Ratio $>=0.05$, :) $=[$ ] ;

ㅇ. $(\mathrm{S}+\mathrm{P}) / \overline{\mathrm{C}}=<0.05$

Data ( (Data.S+Data.P) . /Data.C $>=0.05,:)=[]$;

ㅇ. Jenna: DBE-O >-10 \& <10; AI < 1 (based on Jenna; adjusted for

oconsistency with LDI)

Data ( (Data.DBE-Data.0)<-10, : )= [] ;

Data $(($ Data.DBE-Data.O) $>10,:)=[]$;

Data (Data.AI $>1,:$ ) $=[]$;

Data $($ isinf $($ Data.AI $)==1,:)=[]$;

\%4. Arpad: 200-700 Da,

Data (Data. ObservedM_z<200,:) = [] ;

Data (Data.ObservedM ${ }^{-} \mathrm{z}>700,:$ ) $=[$ ] ;

Impurities=Data;

oLoding sample negative mode ESI Data

Data $=$ readtable ('ESI_Neg_GLSM.xlsx') ;

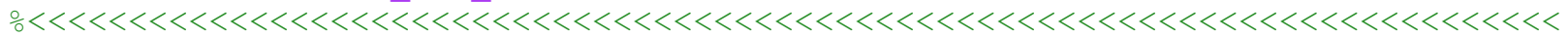
$<<<<<<<<<<<<<<<<<<<<<<<<<<<<<<<<<<<<<<<<<<<<<<<<<<<<<<<<<<<<<<<<~$

Data=unique (Data);

Data. NominalMass=Data. $\mathrm{C} * 12+$ Data $\cdot 0 * 16+$ Data $\cdot H * 1+$ Data. $P * 31+$ Data. $S * 32+D a t a \cdot N * 14$; 
Data.KMD CH2=Data. NominalMass-

$0.998883 \overline{4} *\left(\right.$ Data. $C * 12+$ Data. $H^{*} 1.007825+$ Data. $N^{*} 14.003074+$ Data. $0 * 15.994915+$ Data.$P$ $\star 30.973763+$ Data.S*31.972072);

Data.KMD_COO=Data.NominalMass-

1.000231 * $($ Data. C*12+Data.H*1.007825+Data. N*14.003074+Data.0*15.994915+Data.P*

$30.973763+$ Data. $S * 31.972072)$;

Data.KMD_O2=Data.NominalMass -

$1.000317^{\star}$ (Data. C*12+Data.H*1.007825+Data.N*14.003074+Data.0*15.994915+Data.P* $30.973763+$ Data.S*31.972072);

Data.KMD_H2=Data.NominalMass-

$0.992236^{\star}($ Data. C*12+Data.H*1.007825+Data. N*14.003074+Data.0*15.994915+Data.P*

$30.973763+$ Data. $S * 31.972072)$;

Data. $\mathrm{H}=$ Data. $\mathrm{H}+1$;

Data.HC Ratio = Data.H ./ Data.C;

Data.oC-Ratio = Data.O ./ Data.C;

Data.NC Ratio = Data.N./ Data.C;

Data.SC_Ratio = Data.S./ Data.C;

Data.PC Ratio = Data.P./ Data.C;

Data.DBE $=1+0.5 *(2 *$ Data.C - Data.H + Data. $N+$ Data.P $)$;

Data.AI = (1 + Data.C - Data.O - Data.S - 0.5* (Data.N + Data.P + Data.H) $). /$

(Data.C - Data.O - Data.N - Data.S - Data.P);

Data.AvgCoxstate = 2*Data.OC_Ratio - Data.HC_Ratio;

oSaving raw data for Scan 1

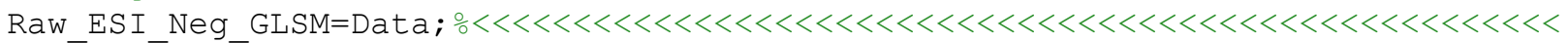
$<<\overline{<}<<<\overline{<}<<<\overline{<}<<<<<<<<<<<<<<<<<<<<<<<<<<<<<<<<<<<<<<<<<<<<<<<<<<<<<<<<<<<<<<<<<~$ $<<<<<<<<<<<<<<<<<<<<<<<<~$

Remove data points based on following criteria

ㄴ. Error (allowable: ppm <0.5)

Data (Data.errPpm $>0.5,:)=[]$;

Data (Data.errPpm<-0.5,:) = [] ;

\%2. Stubbins, 2010 :

$\div \mathrm{A} . \mathrm{DBE}=$ integer, greater than or equal to 0

Data (Data. DBE =round (Data.DBE), : ) = [ ] ;

Data (Data. $\mathrm{DBE}<0,:)=[]$;

ㅇ. $\mathrm{C}<=50$

Data (Data.C $>50,:)=[]$;

$\div \mathrm{C} \cdot \mathrm{H}>=2 \&<=2 * \mathrm{C}+2$

Data (Data. $\mathrm{H}<2,:)=[]$;

Data (Data.H $>($ Data. $C * 2+2),:)=[]$;

D. $\mathrm{O}=<\mathrm{C}+2$

Data $($ Data. O $>($ Data. $C+2),:)=[]$;

ㅌ. $O / C<1.2$

Data (Data.oC_Ratio>1.2,:) =[ ] ;

F. $\mathrm{H} / \mathrm{C}>=0.33 \&<=2.25$

Data (Data.HC Ratio<1/3, :) =[ ] ;

Data (Data.HC_Ratio $>2.25,:)=[]$;

$\div \mathrm{G} . \mathrm{N} / \mathrm{C}=<0.5$

Data (Data.NC_Ratio $>=0.5,:)=[]$;

$\div \mathrm{H} . \mathrm{S} / \mathrm{C}=<0.2$

Data (Data.SC Ratio>=0.2, :) =[] ;

ㅇ. $\mathrm{P} / \mathrm{C}=<0 . \overline{0} 5->$ Changed from 0.1 to remove unbelievable P formulae

Data (Data.PC Ratio $>=0.05,:$ ) $=[$ ] ;

J. $(\mathrm{S}+\mathrm{P}) / \overline{\mathrm{C}}=<0.1->$ Changed from 0.2 to remove unbelievable $\mathrm{P}$ formulae

$\operatorname{Data}((($ Data.S+Data.P).$/($ Data. C $))>=0.1,:)=[]$;

K. $(\mathrm{N}+\mathrm{P}) / \mathrm{C}<0.4->$ Added to remove unlikely P formulae

$\operatorname{Data}((($ Data.N+Data.P).$/($ Data. C $))>=0.4,:)=[]$;

⒊ Jenna: DBE-O >-10\&<10; AI < 1 (based on Jenna; adjusted for

\section{S120}




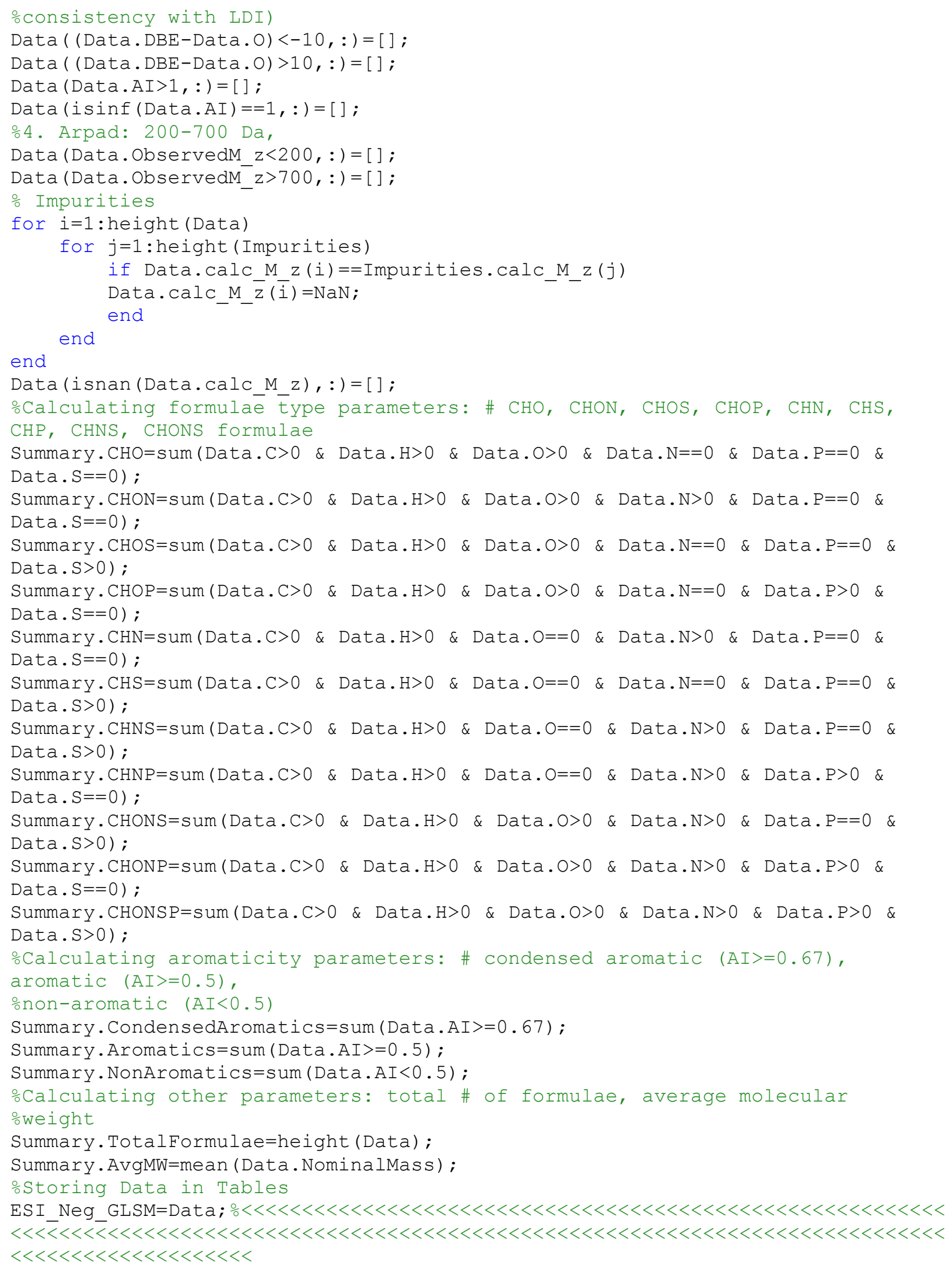




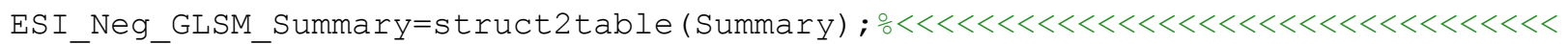

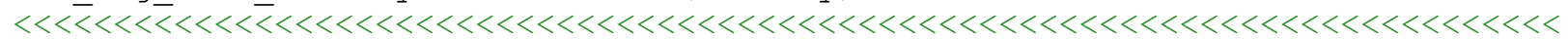

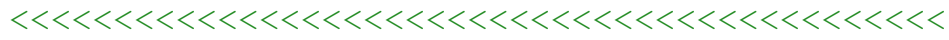

\%

oLoading positive mode ESI impurities

Data $=$ readtable ('DBP Control.xlsx');

Data $\cdot$ NominalMass=Data $\cdot C * 12+$ Data $\cdot 0 * 16+$ Data $\cdot H * 1+$ Data $\cdot P * 31+$ Data $\cdot S * 32+D a t a \cdot N * 14+D$ ata. $\mathrm{Na} * 23$;

Data. KMD CH2=Data. NominalMass-

$0.998883 \overline{4} *($ Data. $C * 12+$ Data. H*1.007825+Data. $N * 14.003074+$ Data. $0 * 15.994915+$ Data.P $\star 30.973763+$ Data.. *31.972072+Data.Na*22.98977);

Data.KMD COO=Data. NominalMass-

1.000231 ॠ (Data. C*12+Data.H*1.007825+Data. $N^{\star} 14.003074+$ Data.0*15.994915+Data.P* 30.973763+Data.S*31.972072+Data.Na*22.98977);

Data.KMD 02=Data. NominalMass-

$1.000317^{\bar{*}}\left(\right.$ Data. $\mathrm{C} * 12+$ Data $. H^{*} 1.007825+$ Data $. N^{*} 14.003074+$ Data. $0 * 15.994915+$ Data. $P *$ $30.973763+$ Data. $\mathrm{S} * 31.972072+$ Data.Na*22.98977);

Data.KMD H2=Data. NominalMass-

$0.992236^{\star}\left(\right.$ Data. $C * 12+$ Data $. H * 1.007825+$ Data. $N^{*} 14.003074+$ Data. $0 * 15.994915+$ Data. $P *$

$30.973763+$ Data. $S * 31.972072+$ Data. Na*22.98977);

for $i=1$ : height (Data)

if $\mathrm{Data} . \mathrm{Na}(\mathrm{i})==0$

Data.H(i)=Data.H(i) -1 ;

end

end

Data.HC Ratio = Data.H ./ Data.C;

Data.oc Ratio = Data.O./ Data.C;

Data.NC_Ratio = Data.N./ Data.C;

Data.SC-Ratio = Data.S ./ Data.C;

Data.PC_Ratio = Data.P ./ Data.C;

Data. $\mathrm{DBE}=1+0.5 *(2 *$ Data. $\mathrm{C}-$ Data.H + Data. $\mathrm{N}+$ Data.P $)$;

Data.AI $=(1+$ Data.C - Data.O - Data.S - 0.5*(Data.N + Data.P + Data.H) $)$.

(Data.C - Data.O - Data.N - Data.S - Data.P);

Data.AvgCoxstate = 2*Data.OC_Ratio - Data.HC_Ratio;

Remove data points based on following criteria

\%1. Error (allowable: ppm <0.5)

Data (Data.errPpm $>0.5,:)=[]$;

Data (Data.errPpm<-0.5,:) = [ ] ;

\%2. Stubbins, 2010 :

$\div \mathrm{A} . \mathrm{DBE}=$ integer, greater than or equal to 0

Data (Data.DBE =round (Data.DBE), : ) = [];

Data (Data. $\mathrm{DBE}<0,:)=[]$;

B. $\mathrm{C}<=50$

Data (Data.C>50,: ) = [ ]

ㄷ. $\mathrm{H}>=2 \&<=2 * \mathrm{C}+2$

Data (Data. $\mathrm{H}<2,:)=[]$;

Data $($ Data. $\mathrm{H}>($ Data. $\mathrm{C} * 2+2),:)=[]$

$\therefore \mathrm{D} . \mathrm{O}=<\mathrm{C}+2$

Data (Data. O $>($ Data. $C+2),:$ ) $=[]$;

․ $\mathrm{O} / \mathrm{C}<1.2$

Data (Data.OC_Ratio>1.2, :) =[ ] ;

으. H/C $>=0.33 \&<=2.25$

Data (Data.HC Ratio<1/3,:) = [] ;

Data (Data.HC Ratio>2.25, : ) = [ ] ;

$\because \mathrm{G} . \mathrm{N} / \mathrm{C}=<0.5$

Data (Data.NC_Ratio $>=0.5,:)=[]$; 
$\circ \mathrm{H} \cdot \mathrm{S} / \mathrm{C}=<\mathrm{O} \cdot 2$

Data (Data.SC Ratio $>=0.2,:$ ) $=[]$;

ㄷ. $\mathrm{P} / \mathrm{C}=<0 . \overline{0} 5->$ Changed from 0.1 to remove unbelievable P formulae

Data (Data.PC_Ratio>=0.05, :) =[ ] ;

ㄱ. $(\mathrm{S}+\mathrm{P}) / \overline{\mathrm{C}}=<0.1->$ Changed from 0.2 to remove unbelievable $\mathrm{P}$ formulae

$\operatorname{Data}((($ Data.S+Data.P).$/($ Data.C $))>=0.1,:)=[]$;

$\circ \mathrm{K} . \quad(\mathrm{N}+\mathrm{P}) / \mathrm{C}<0.4->$ Added to remove unlikely P formulae

$\operatorname{Data}((($ Data.N+Data.P).$/($ Data. C $))>=0.4,:)=[]$;

⒊ Jenna: DBE-O >-10 \& <10; AI < 1 (based on Jenna; adjusted for

oconsistency with LDI)

Data ( (Data.DBE-Data.O) $<-10,:)=[]$;

Data $(($ Data.DBE-Data.O) $>10,:)=[]$;

Data (Data.AI $>1,:)=[]$;

Data $($ isinf $($ Data.AI $)==1,:)=[]$;

\%4. Arpad: 200-700 Da,

Data (Data. ObservedM $z<200,:$ ) = [] ;

Data (Data.ObservedM_z $>700,:)=[$ ] ;

Impurities=Data ;

oLoading sample positive mode ESI data

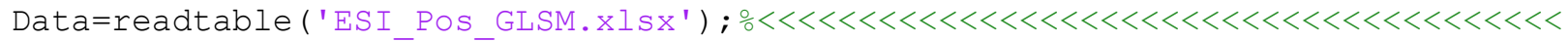

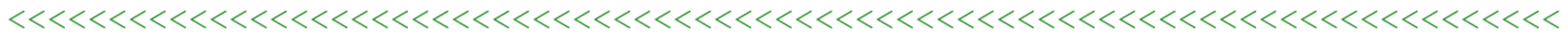
$<<<<<<<<<<<<<<<<<<<<<<<<<<<<<<<<<<<<<<~$

Data $\cdot$ NominalMass $=$ Data $\cdot C * 12+$ Data $\cdot 0 * 16+$ Data $\cdot H * 1+$ Data $\cdot P * 31+$ Data $\cdot S * 32+D a t a \cdot N * 14+D$ ata. $\mathrm{Na} 23$;

Data. KMD CH2=Data. NominalMass-

$0.998883 \overline{4} *\left(\right.$ Data. $C * 12+$ Data. $H * 1.007825+$ Data. $N^{*} 14.003074+$ Data. $0 * 15.994915+$ Data.P $\star 30.973763+$ Data.S*31.972072+Data.Na*22.98977);

Data.KMD COO=Data.NominalMass-

$1.000231^{\star}\left(\right.$ Data. $C * 12+$ Data $. H^{*} 1.007825+$ Data. $N^{*} 14.003074+$ Data. $0 * 15.994915+$ Data. $P *$ $30.973763+$ Data. $\mathrm{S} * 31.972072+$ Data. $\mathrm{Na} * 22.98977$ );

Data.KMD O2=Data.NominalMass-

$1.0003177^{\bar{*}}\left(\right.$ Data. $C * 12+$ Data. $H^{*} 1.007825+$ Data. $N^{*} 14.003074+$ Data. $0 * 15.994915+$ Data. $P *$ $30.973763+$ Data. S*31.972072+Data.Na*22.98977);

Data.KMD H2=Data. NominalMass-

$0.992236^{\star}$ (Data. C*12+Data.H*1.007825+Data.N*14.003074+Data.0*15.994915+Data.P* $30.973763+$ Data.. *31.972072+Data.Na*22.98977);

for $i=1$ : height (Data)

if $\operatorname{Data.Na}(i)==0$

Data.H $($ i $)=$ Data.H $($ i $)-1$;

end

end

Data.HC_Ratio = Data.H ./ Data.C;

Data.oC Ratio = Data.O ./ Data.C;

Data.NC Ratio = Data.N ./ Data.C;

Data.SC Ratio = Data.S./ Data.C;

Data.PC_Ratio = Data.P./ Data.C;

Data.DBE $=1+0.5 *(2 *$ Data.C - Data.H + Data. $N+$ Data.P $)$;

Data.AI $=(1+$ Data.C - Data.O - Data.S - 0.5* (Data.N + Data.P + Data.H) $) \cdot /$

(Data.C - Data.O - Data.N - Data.S - Data.P);

Data.AvgCoxstate = 2*Data.oC_Ratio - Data.HC_Ratio;

oSaving raw data for Scan 1

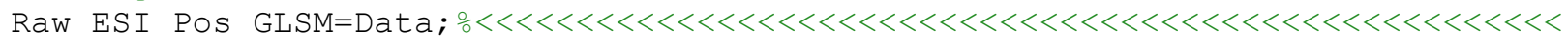

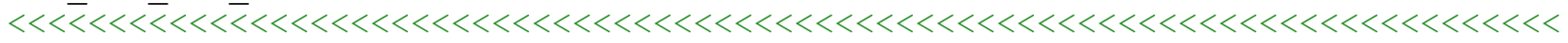
$<<<<<<<<<<<<<<<<<<<<<<<<~$

oRemove data points based on following criteria

⒈ Error (allowable: ppm <0.5)

Data (Data.errPpm>0.5,:) = []; 


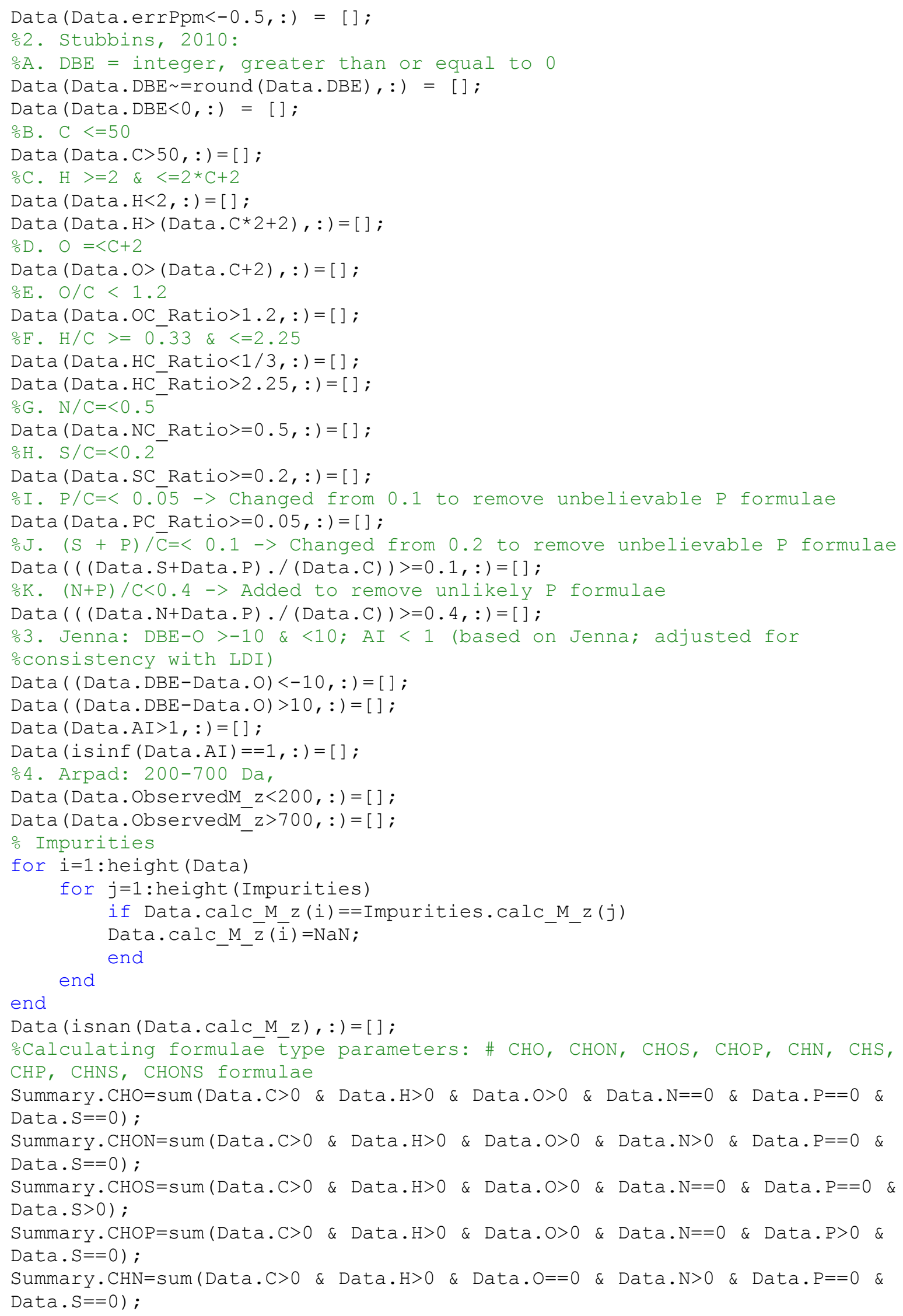


Summary $\cdot \mathrm{CHS}=\operatorname{sum}($ Data $\cdot \mathrm{C}>0$ \& Data. $\mathrm{H}>0$ \& Data. $\mathrm{O}==0$ \& Data. $\mathrm{N}==0$ \& Data. $\mathrm{P}==0$ \& Data. $\mathrm{S}>0$ );

Summary. CHNS $=$ sum $($ Data. $\mathrm{C}>0$ \& Data. $\mathrm{H}>0$ \& Data. $\mathrm{O}==0$ \& Data. $\mathrm{N}>0$ \& Data.P==0 \& Data. S $>0$ );

Summary. $\mathrm{CHNP}=\operatorname{sum}($ Data. $\mathrm{C}>0$ \& Data. $\mathrm{H}>0$ \& Data. $\mathrm{O}==0$ \& Data. $\mathrm{N}>0$ \& Data.P $>0$ \& Data. $\mathrm{S}==0$ );

Summary. CHONS $=$ sum $($ Data. $\mathrm{C}>0$ \& Data. $\mathrm{H}>0$ \& Data.O $>0$ \& Data.N $>0$ \& Data.P==0 \&

Data. $\mathrm{S}>0$ ) ;

Summary. $\mathrm{CHONP}=$ sum $($ Data. $\mathrm{C}>0$ \& Data. $\mathrm{H}>0$ \& Data. $\mathrm{O}>0$ \& Data.N $>0$ \& Data.P $>0$ \&

Data. $\mathrm{S}==0$ );

Summary. $\mathrm{CHONSP}=$ sum $($ Data. $\mathrm{C}>0$ \& Data. $\mathrm{H}>0$ \& Data. $\mathrm{O}>0$ \& Data.N $>0$ \& Data.P $>0$ \&

Data. S>0);

ocalculating aromaticity parameters: \# condensed aromatic (AI>=0.67), aromatic $(\mathrm{AI}>=0.5)$,

onon-aromatic $(\mathrm{A} I<0.5)$

Summary. CondensedAromatics $=$ sum (Data. AI $>=0.67)$;

Summary. Aromatics=sum (Data.AI $>=0.5)$;

Summary. NonAromatics=sum (Data.AI<0.5);

ocalculating other parameters: total \# of formulae, average molecular

oweight

Summary. TotalFormulae=height (Data);

Summary. AvgMW=mean (Data. NominalMass);

ostoring Data in Tables

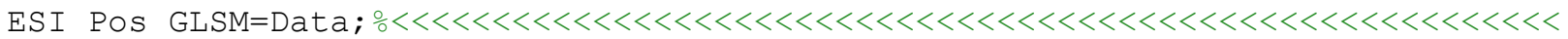

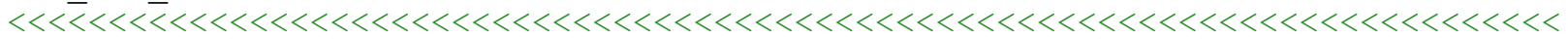
$<<<<<<<<<<<<<<<<<<<<~$

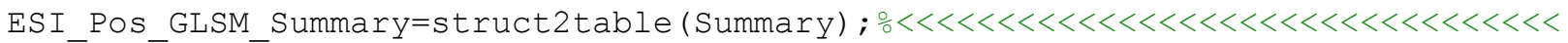
$<<\overline{<}<<\overline{\bar{l}}<<<\overline{<} \overline{<}<<<<<<<<<<<<<<<<<<<<<<<<<<<<<<<<<<<<<<<<<<<<<<<<<<<<<<<<<<<<<<<<$

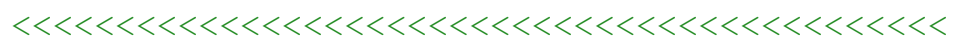

응 Negative Mode LDI: Data Clean Up

oLDI assumed to have no background ions

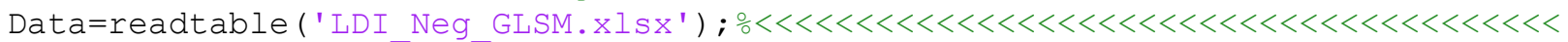

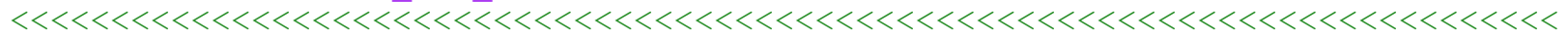

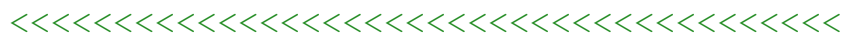

Data=unique (Data);

Data $\cdot$ NominalMass=Data $\cdot C * 12+$ Data $\cdot 0 * 16+$ Data $\cdot H * 1+$ Data $\cdot P * 31+$ Data $\cdot S * 32+$ Data $\cdot N * 14$;

Data. KMD CH2=Data. NominalMass-

$0.998883 \overline{4} *\left(\right.$ Data. $C * 12+$ Data. $H * 1.007825+$ Data. $N^{*} 14.003074+$ Data. $0 * 15.994915+$ Data. P $\star 30.973763+$ Data.. *31.972072);

Data.KMD_COO=Data.NominalMass-

$1.000231^{\star}($ Data. C*12+Data.H*1.007825+Data. N*14.003074+Data. O*15.994915+Data.P* $30.973763+$ Data..$* 31.972072)$;

Data.KMD 02=Data. NominalMass-

$1.000317^{\star}\left(\right.$ Data. $C * 12+$ Data.H*1.007825+Data. $\mathrm{N}^{\star} 14.003074+$ Data. $0 * 15.994915+$ Data. $\mathrm{P} *$ $30.973763+$ Data.S*31.972072);

Data. KMD H2=Data. NominalMass-

$0.992236^{\star}$ (Data. C*12+Data.H*1.007825+Data. $N^{*} 14.003074+$ Data. $0 * 15.994915+$ Data. P*

$30.973763+$ Data. $S * 31.972072)$;

Data.DBE $=1+0.5 *(2 *$ Data.C - Data.H + Data.N + Data.P $)$;

for $i=1$ : height (Data)

if Data.DBE (i) =round (Data.DBE (i))

Data.H $($ i $)=$ Data.H $(i)+1$;

Data. IonType $(i)=0 ; \circ H-$ Adduct $s=0$

else

Data.IonType $(i)=2 ; \%$ MolecularIons=2 \%Temporary fix until I figure out how to enter names into a table 


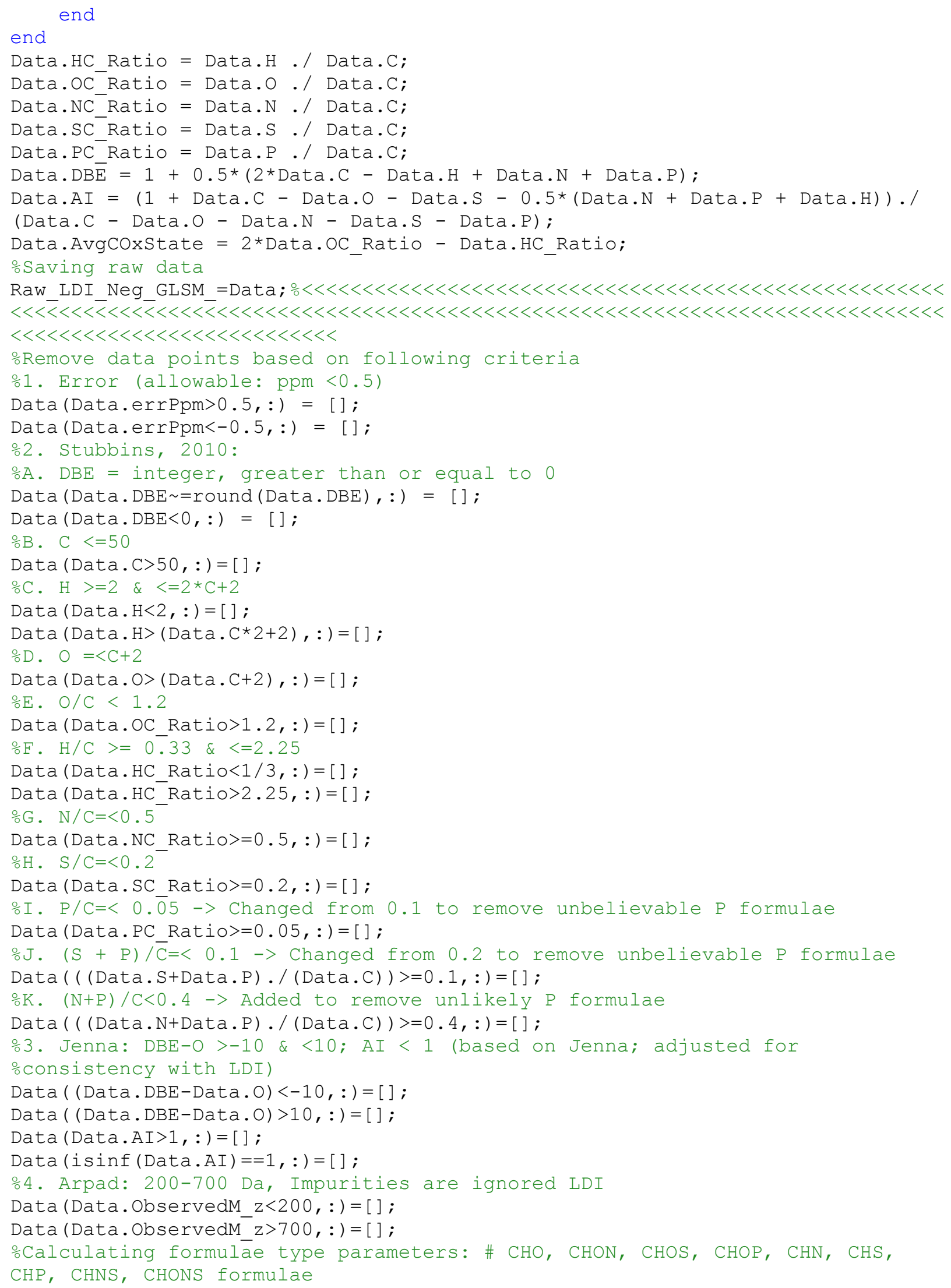


Summary. $\mathrm{CHO}=\operatorname{sum}($ Data. $\mathrm{C}>0$ \& Data.H $>0$ \& Data. $\mathrm{O}>0$ \& Data. $\mathrm{N}==0$ \& Data.P==0 \& Data. $\mathrm{S}==0$ ); Summary. $\mathrm{CHON}=\operatorname{sum}($ Data. $\mathrm{C}>0$ \& Data.H $>0$ \& Data. $0>0$ \& Data. $\mathrm{N}>0$ \& Data.P==0 \& Data. $\mathrm{S}==0$ );

Summary $\cdot \mathrm{CHOS}=$ sum $($ Data. $\mathrm{C}>0$ \& Data. $\mathrm{H}>0$ \& Data. $\mathrm{O}>0$ \& Data. $\mathrm{N}==0$ \& Data. $\mathrm{P}==0$ \& Data. $\mathrm{S}>0$ ); Summary. $\mathrm{CHOP}=\operatorname{sum}($ Data. $\mathrm{C}>0$ \& Data. $\mathrm{H}>0$ \& Data.O $>0$ \& Data. $\mathrm{N}==0$ \& Data.P $>0$ \& Data. $\mathrm{S}=0$ ) ; Summary $\cdot \mathrm{CHN}=$ sum $($ Data. $\mathrm{C}>0$ \& Data. $\mathrm{H}>0$ \& Data. $\mathrm{O}==0$ \& Data. $\mathrm{N}>0$ \& Data. $\mathrm{P}==0$ \& Data. $\mathrm{S}==0$ );

Summary $\cdot \mathrm{CHS}=\operatorname{sum}($ Data $\cdot \mathrm{C}>0$ \& Data. $\mathrm{H}>0$ \& Data $\cdot \mathrm{O}==0$ \& Data. $\mathrm{N}==0$ \& Data. $\mathrm{P}==0$ \& Data. $\mathrm{S}>0$ ) ; Summary $\cdot \mathrm{CHNS}=$ sum $($ Data $\cdot \mathrm{C}>0$ \& Data. $\mathrm{H}>0$ \& Data. $\mathrm{O}==0$ \& Data. $\mathrm{D}>0$ \& Data. $\mathrm{P}==0$ \& Data. S>0); Summary. $\mathrm{CHNP}=\operatorname{sum}($ Data $\cdot \mathrm{C}>0$ \& Data. $\mathrm{H}>0$ \& Data. $\mathrm{O}==0$ \& Data.N $>0$ \& Data.P>0 \& Data. $\mathrm{S}=0$ ) ; Summary. $\mathrm{CHONS}=\operatorname{sum}($ Data. $\mathrm{C}>0$ \& Data. $\mathrm{H}>0$ \& Data. $\mathrm{O}>0$ \& Data. $\mathrm{N}>0$ \& Data.P==0 \& Data. $\mathrm{S}>0$ ); Summary. $\mathrm{CHONP}=$ sum (Data. $\mathrm{C}>0$ \& Data.H>0 \& Data. O $>0$ \& Data.N $>0$ \& Data.P $>0$ \& Data. $\mathrm{S}==0$ ); Summary. $\mathrm{CHONSP}=$ sum $($ Data. $\mathrm{C}>0$ \& Data. $\mathrm{H}>0$ \& Data. $\mathrm{O}>0$ \& Data.N $>0$ \& Data.P $>0$ \& Data. S $>0)$;

ocalculating aromaticity parameters: \# condensed aromatic (AI>=0.67), aromatic $(A I>=0.5)$, onon-aromatic $(\mathrm{AI}<0.5)$

Summary. CondensedAromatics $=$ sum (Data. AI $>=0.67)$;

Summary. Aromatics $=$ sum (Data.AI $>=0.5)$;

Summary. NonAromatics $=$ sum $($ Data. AI $<0.5)$;

ocalculating other parameters: total \# of formulae, average molecular oweight

Summary. TotalFormulae=height (Data);

Summary.AvgMW=mean (Data. NominalMass);

ostoring Data in Tables

LDI Neg GLSM=Data; $\circ 2<<<<<<<<<<<<<<<<<<<<<<<<<<<<<<<<<<<<<<<<<<<<<<<<<<<<<<<<<<<<~$

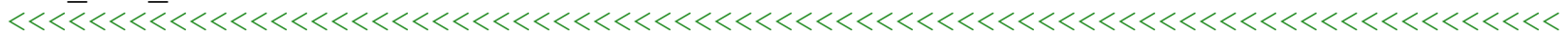
$<<<<<<<<<<<<<<<<<<$

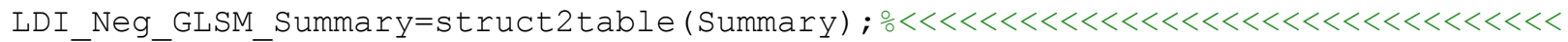
$<<<\overline{<}<<<\bar{L}<<<<\overline{<}<<<<<<<<<<<<<<<<<<<<<<<<<<<<<<<<<<<<<<<<<<<<<<<<<<<<<<<<<<<<<<<<~$

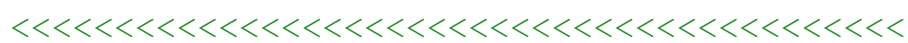

응 Positive Mode LDI: Data Clean Up

oLDI assumed to have no background ions

oLoading sample positive mode LDI data

Data=readtable ('LDI POS GLSM.xlsx') ; 은 $<<<<<<<<<<<<<<<<<<<<<<<<<<<<<<<<<<<<<<<<<<~$

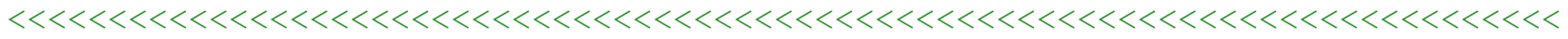

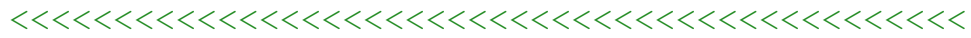

Data=unique (Data);

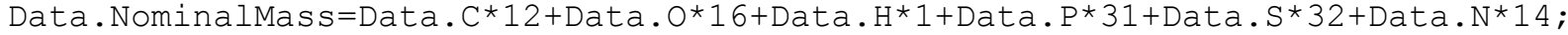

Data. $\mathrm{KMD} \mathrm{CH} 2=$ Data. NominalMass-

$0.998883 \overline{4} *($ Data $. C * 12+$ Data $. H * 1.007825+$ Data $. N * 14.003074+$ Data. $0 * 15.994915+$ Data.$P$ *30.973763+Data.S*31.972072);

Data.KMD COO=Data. NominalMass-

$1.000231^{\star}\left(\right.$ Data. C*12+Data.H*1.007825+Data. $N^{*} 14.003074+$ Data. $0 * 15.994915+$ Data. $P *$ $30.973763+$ Data..$* 31.972072)$;

Data.KMD $02=$ Data. NominalMass-

$1.000317^{\bar{*}}\left(\right.$ Data. $\mathrm{C}^{\star} 12+$ Data. $\mathrm{H}^{\star} 1.007825+$ Data $. \mathrm{N}^{\star} 14.003074+\mathrm{Data} .0 * 15.994915+\mathrm{Data} . \mathrm{P} *$ $30.973763+$ Data.. *31.972072); 


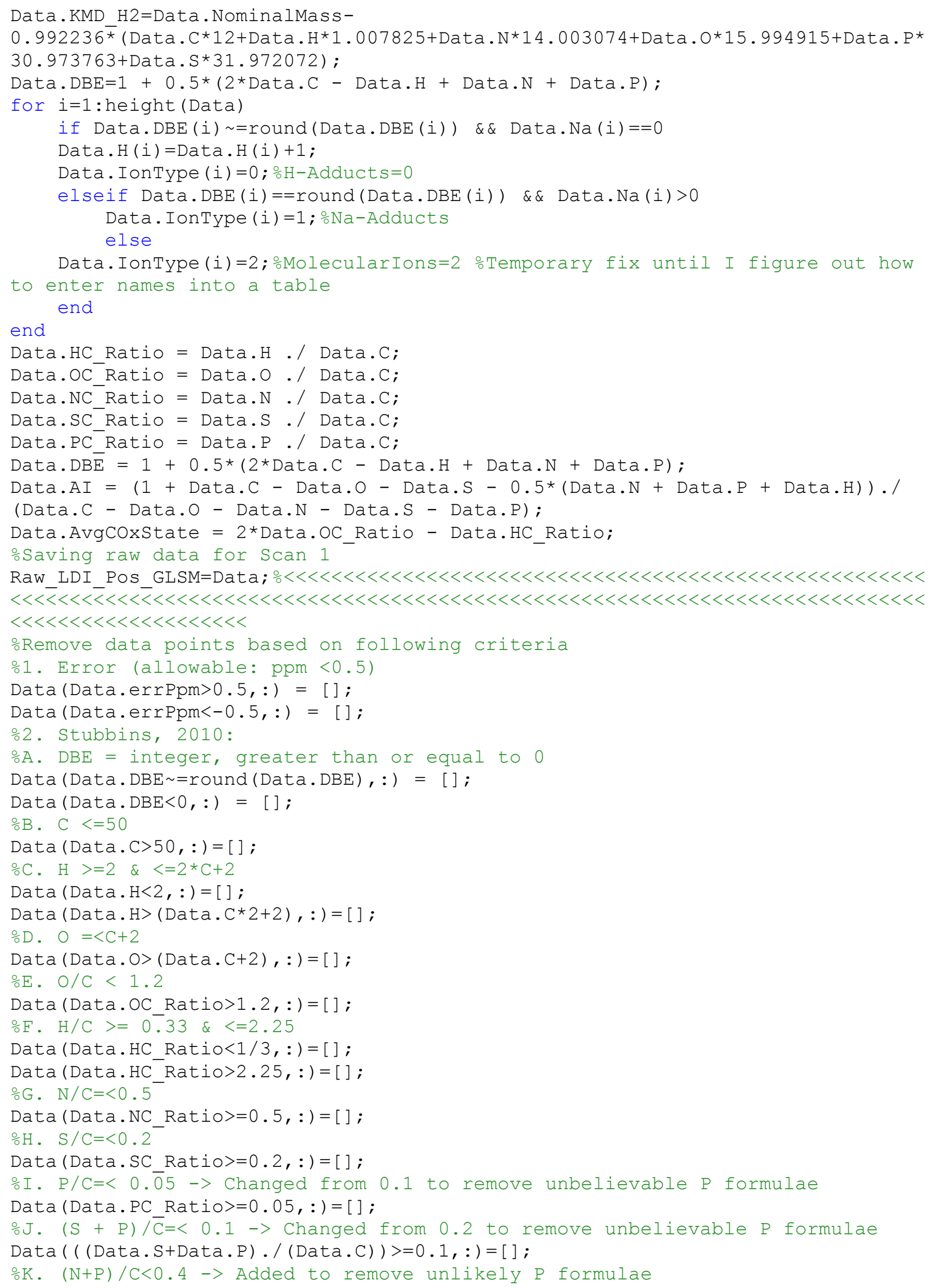


$\operatorname{Data}((($ Data.N+Data.P).$/($ Data.C $))>=0.4,:)=[]$;

⒊ Jenna: DBE-0 >-10 \& <10; AI < 1 (based on Jenna; adjusted for

oconsistency with LDI)

Data ( (Data.DBE-Data.O) $<-10,:)=[]$;

Data ( (Data.DBE-Data.O) $>10,:)=[]$;

Data (Data.AI>1, : ) $=[]$;

$\operatorname{Data}($ isinf $($ Data. AI $)==1,:)=[]$;

\%4. Arpad: 200-700 Da, Impurities are ignored LDI- assumed to be negligible ounless a background reference is used

Data (Data. ObservedM_z<200,: ) = [] ;

Data (Data. ObservedM_z>700,: ) = [] ;

oCalculating formulāe type parameters: \# CHO, CHON, CHOS, CHOP, CHN, CHS, CHP, CHNS, CHONS formulae

Summary $\cdot \mathrm{CHO}=$ sum $($ Data. $\mathrm{C}>0$ \& Data. $\mathrm{H}>0$ \& Data. $\mathrm{O}>0$ \& Data. $\mathrm{N}==0$ \& Data. $\mathrm{P}==0$ \&

Data. $\mathrm{S}==0$ );

Summary $\cdot \mathrm{CHON}=$ sum $($ Data. $\mathrm{C}>0$ \& Data.H $>0$ \& Data. $\mathrm{O}>0$ \& Data. $\mathrm{N}>0$ \& Data.P==0 \&

Data. $\mathrm{S}=0$ ) ;

Summary $\cdot \mathrm{CHOS}=$ sum $($ Data. $\mathrm{C}>0$ \& Data.H $>0$ \& Data. O $>0$ \& Data. $\mathrm{N}==0$ \& Data. $\mathrm{P}==0$ \&

Data. $\mathrm{S}>0$ ) ;

Summary $\cdot \mathrm{CHOP}=\operatorname{sum}($ Data. $\mathrm{C}>0$ \& Data.H $>0$ \& Data. $\mathrm{O}>0$ \& Data. $\mathrm{N}==0$ \& Data.P $>0$ \&

Data. $\mathrm{S}==0$ );

Summary $\cdot \mathrm{CHN}=$ sum $($ Data. $\mathrm{C}>0$ \& Data. $\mathrm{H}>0$ \& Data. $\mathrm{O}==0$ \& Data. $\mathrm{N}>0$ \& Data. $\mathrm{P}==0$ \&

Data. $\mathrm{S}==0$ );

Summary. $\mathrm{CHS}=\operatorname{sum}($ Data. $\mathrm{C}>0$ \& Data. $\mathrm{H}>0$ \& Data. $\mathrm{O}==0$ \& Data. $\mathrm{N}==0$ \& Data. $\mathrm{P}==0$ \&

Data. S>0);

Summary $\cdot \mathrm{CHNS}=\operatorname{sum}($ Data. $\mathrm{C}>0$ \& Data. $\mathrm{H}>0$ \& Data. $\mathrm{O}==0$ \& Data. $\mathrm{N}>0$ \& Data.P==0 \&

Data. $\mathrm{S}>0$ );

Summary $\cdot \mathrm{CHNP}=\mathrm{sum}($ Data. $\mathrm{C}>0$ \& Data.H $>0$ \& Data. $\mathrm{O}==0$ \& Data.N $>0$ \& Data.P $>0$ \&

Data. $\mathrm{S}==0$ );

Summary $\cdot \mathrm{CHONS}=\operatorname{sum}($ Data. $\mathrm{C}>0$ \& Data. $\mathrm{H}>0$ \& Data. $0>0$ \& Data.N $>0$ \& Data.P==0 \&

Data. S>0);

Summary. $\mathrm{CHONP}=$ sum $($ Data. $\mathrm{C}>0$ \& Data. $\mathrm{H}>0$ \& Data. $\mathrm{O}>0$ \& Data. $\mathrm{N}>0$ \& Data.P $>0$ \&

Data. $\mathrm{S}=0$ ) ;

Summary. $\mathrm{CHONSP}=$ sum $($ Data. $\mathrm{C}>0$ \& Data.H $>0$ \& Data.O $>0$ \& Data.N $>0$ \& Data.P>0 \&

Data. $\mathrm{S}>0$ );

ocalculating aromaticity parameters: \# condensed aromatic (AI>=0.67),

aromatic $(A I>=0.5)$,

onon-aromatic $(\mathrm{AI}<0.5)$

Summary. CondensedAromatics $=$ sum (Data.AI $>=0.67)$;

Summary. Aromatics $=$ sum (Data.AI $>=0.5)$;

Summary. NonAromatics=sum (Data.AI<0.5);

ocalculating other parameters: total \# of formulae, average molecular

oweight

Summary.TotalFormulae=height (Data);

Summary. AvgMW=mean (Data. NominalMass);

ostoring Data in Tables

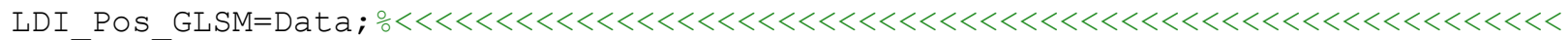
$<<<\overline{<}<<<\overline{\bar{l}}<<<<<<<<<<<<<<<<<<<<<<<<<<<<<<<<<<<<<<<<<<<<<<<<<<<<<<~$

LDI_Pos_GLSM_Summary=struct2table (Summary) ; $\circ<<<<<<<<<<<<<<<<<<<<<<<<<<<<<<<<<<~$ $<<\overline{<}<<\overline{<}<<<<\overline{<}<<<<<<<<<<<<<<<<<<<<<<<<<<<<<<<<<<<<<<<<<<<<<<<<<<<<<<<<<<<<<<<<~$ $<<<<<<<<<$

응 Combining Negative and Positive Mode ESI

oInput Data so that the script can readily be applied to any data set

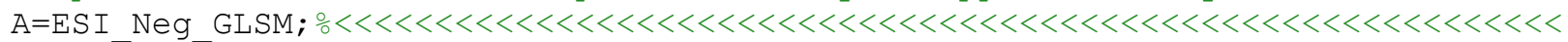

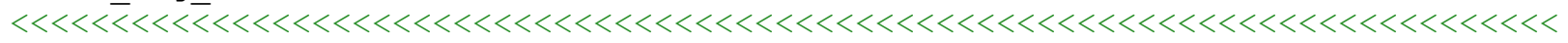
$<<<<<<<<<<<<<<<<<~$ 


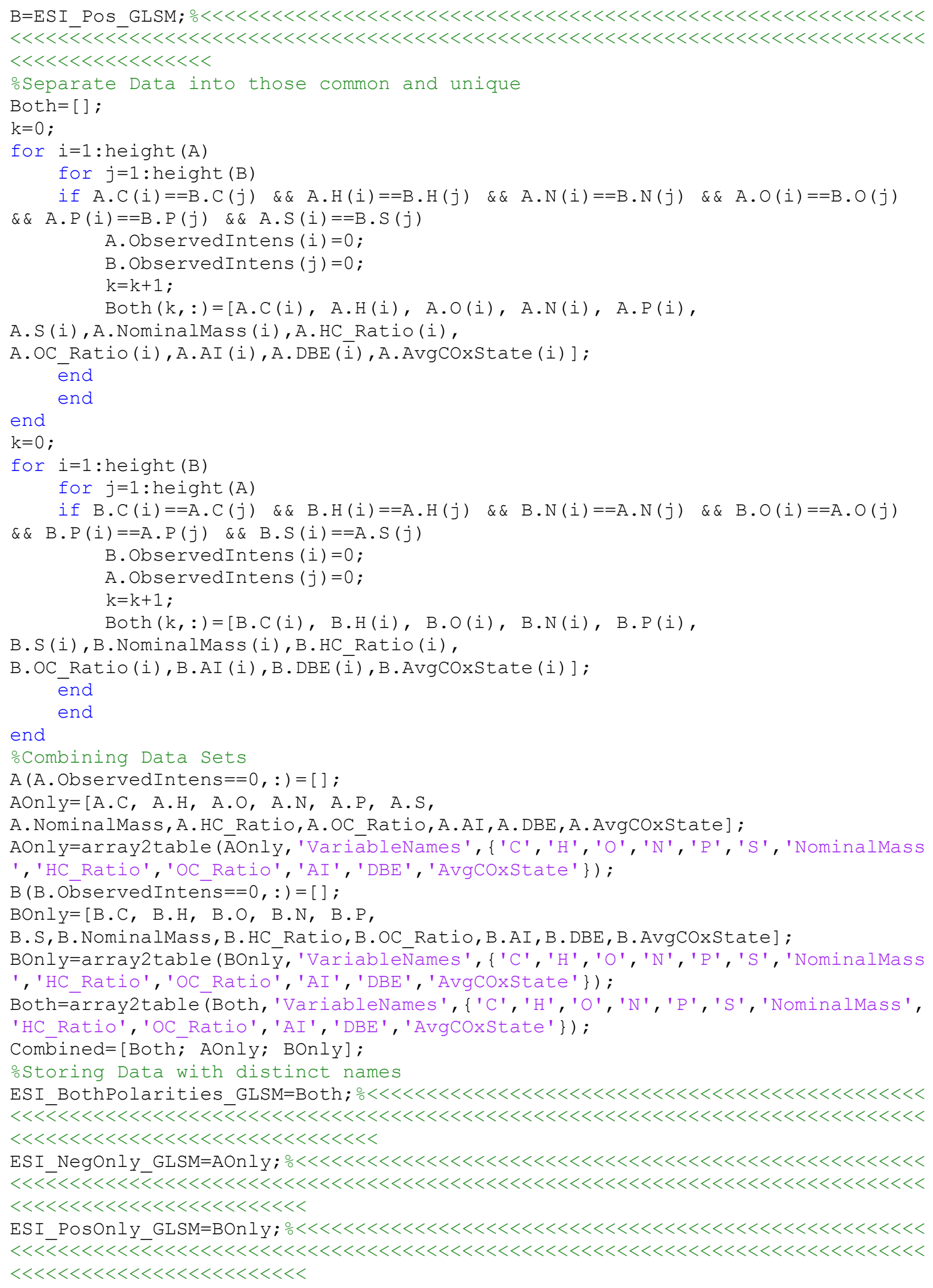




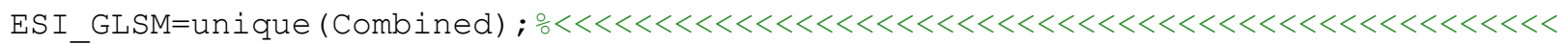
$<<\overline{<}<<<<<<<<<<<<<<<<<<<<<<<<<<<<<<<<<<<<<<<<<<<<<<<<<<<<<<<<<<<<<<<<<<<<<<<<<~$ $<<<<<<<<<<<<<<<<<<<<<<<<<<<<~$

ㅇumarizing Data

Data=AOnly;

oCalculating formulae type parameters: \# CHO, CHON, CHOS, CHOP, CHN, CHS, CHP, CHNS, CHONS formulae

Summary $\cdot \mathrm{CHO}=$ sum $($ Data. $\mathrm{C}>0$ \& Data. $\mathrm{H}>0$ \& Data. $\mathrm{O}>0$ \& Data. $\mathrm{N}==0$ \& Data. $\mathrm{P}==0$ \&

Data. $\mathrm{S}==0$ );

Summary $\cdot \mathrm{CHON}=$ sum $($ Data. $\mathrm{C}>0$ \& Data.H $>0$ \& Data. $\mathrm{O}>0$ \& Data. $\mathrm{N}>0$ \& Data.P==0 \&

Data. $\mathrm{S}==0$ );

Summary. $\mathrm{CHOS}=$ sum $($ Data. $\mathrm{C}>0$ \& Data. $\mathrm{H}>0$ \& Data. $\mathrm{O}>0$ \& Data. $\mathrm{N}==0$ \& Data. $\mathrm{P}==0$ \&

Data. $\mathrm{S}>0$ );

Summary. $\mathrm{CHOP}=\mathrm{sum}($ Data. $\mathrm{C}>0$ \& Data.H $>0$ \& Data. $0>0$ \& Data. $\mathrm{N}==0$ \& Data.P $>0$ \&

Data. $\mathrm{S}==0$ );

Summary $\cdot \mathrm{CHN}=$ sum $($ Data. $\mathrm{C}>0$ \& Data. $\mathrm{H}>0$ \& Data. $\mathrm{O}==0$ \& Data. $\mathrm{N}>0$ \& Data. $\mathrm{P}==0$ \&

Data. $\mathrm{S}=0$ ) ;

Summary $\cdot \mathrm{CHS}=\operatorname{sum}($ Data. $\mathrm{C}>0$ \& Data. $\mathrm{H}>0$ \& Data. $\mathrm{O}==0$ \& Data. $\mathrm{N}==0$ \& Data. $\mathrm{P}==0$ \&

Data. $\mathrm{S}>0$ ) ;

Summary. $\mathrm{CHNS}=$ sum $($ Data. $\mathrm{C}>0$ \& Data. $\mathrm{H}>0$ \& Data. $\mathrm{O}==0$ \& Data. $\mathrm{N}>0$ \& Data. $\mathrm{P}==0$ \&

Data. S $>0$ );

Summary $\cdot \mathrm{CHNP}=\operatorname{sum}($ Data. $\mathrm{C}>0$ \& Data.H $>0$ \& Data. $\mathrm{O}==0$ \& Data. $\mathrm{N}>0$ \& Data.P $>0$ \&

Data. $\mathrm{S}==0$ );

Summary. $\mathrm{CHONS}=\operatorname{sum}($ Data. $\mathrm{C}>0$ \& Data. $\mathrm{H}>0$ \& Data. $0>0$ \& Data.N>0 \& Data.P==0 \&

Data. S>0);

Summary. $\mathrm{CHONP}=\operatorname{sum}($ Data. $\mathrm{C}>0$ \& Data.H $>0$ \& Data. O $>0$ \& Data.N $>0$ \& Data.P $>0$ \&

Data. $\mathrm{S}==0$ );

Summary. $\mathrm{CHONSP}=$ sum $($ Data. $\mathrm{C}>0$ \& Data.H $>0$ \& Data.O $>0$ \& Data.N $>0$ \& Data.P>0 \&

Data. $\mathrm{S}>0)$;

ocalculating aromaticity parameters: \# condensed aromatic (AI>=0.67),

aromatic $(\mathrm{AI}>=0.5)$,

onon-aromatic $(\mathrm{A} I<0.5)$

Summary. CondensedAromatics $=$ sum (Data. AI $>=0.67)$;

Summary. Aromatics $=$ sum (Data.AI $>=0.5)$;

Summary. NonAromatics=sum (Data.AI<0.5);

ocalculating other parameters: total \# of formulae, average molecular

oweight

Summary.TotalFormulae=height (Data);

Summary. AvgMW=mean (Data. NominalMass);

ostoring Summary Data

ESI_NegOnIy_GLSM_Summary=struct2table (Summary) ; $\circ<<<<<<<<<<<<<<<<<<<<<<<<<<<<<<<<~$

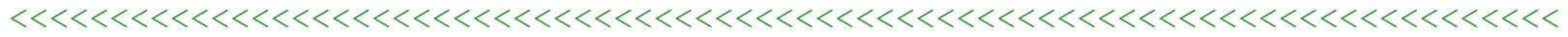

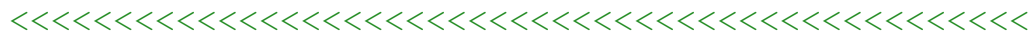

․ Summarizing Data

Data=BOnly;

oCalculating formulae type parameters: \# CHO, CHON, CHOS, CHOP, CHN, CHS, CHP, CHNS, CHONS formulae

Summary. $\mathrm{CHO}=\operatorname{sum}($ Data. $\mathrm{C}>0$ \& Data. $\mathrm{H}>0$ \& Data. $\mathrm{O}>0$ \& Data. $\mathrm{N}==0$ \& Data. $\mathrm{P}==0$ \& Data. $\mathrm{S}==0$ );

Summary. $\mathrm{CHON}=\operatorname{sum}($ Data. $\mathrm{C}>0$ \& Data. $\mathrm{H}>0$ \& Data. $\mathrm{O}>0$ \& Data.N $>0$ \& Data.P==0 \&

Data. $\mathrm{S}==0$ ) ;

Summary $\cdot \mathrm{CHOS}=$ sum $($ Data. $\mathrm{C}>0$ \& Data. $\mathrm{H}>0$ \& Data. $\mathrm{O}>0$ \& Data. $\mathrm{N}==0$ \& Data.P==0 \& Data. $\mathrm{S}>0$ ) ;

Summary. $\mathrm{CHOP}=\operatorname{sum}($ Data. $\mathrm{C}>0$ \& Data. $\mathrm{H}>0$ \& Data. $\mathrm{O}>0$ \& Data. $\mathrm{N}==0$ \& Data. $\mathrm{P}>0$ \&

Data. $\mathrm{S}=0$ ) ;

Summary. $\mathrm{CHN}=\operatorname{sum}($ Data. $\mathrm{C}>0$ \& Data. $\mathrm{H}>0$ \& Data. $\mathrm{O}==0$ \& Data. $\mathrm{N}>0$ \& Data. $\mathrm{P}==0$ \&

Data. $\mathrm{S}==0$ ) ; 
Summary $\cdot \mathrm{CHS}=\operatorname{sum}($ Data $\cdot \mathrm{C}>0$ \& Data. $\mathrm{H}>0$ \& Data. $\mathrm{O}==0$ \& Data. $\mathrm{N}==0$ \& Data. $\mathrm{P}==0$ \& Data. $\mathrm{S}>0$ ) ;

Summary. CHNS $=$ sum $($ Data. $\mathrm{C}>0$ \& Data. $\mathrm{H}>0$ \& Data. $\mathrm{O}==0$ \& Data. $\mathrm{N}>0$ \& Data.P==0 \& Data. S $>0$ );

Summary. $\mathrm{CHNP}=\operatorname{sum}($ Data. $\mathrm{C}>0$ \& Data. $\mathrm{H}>0$ \& Data. $\mathrm{O}==0$ \& Data. $\mathrm{N}>0$ \& Data.P $>0$ \& Data. $\mathrm{S}==0$ );

Summary. CHONS $=$ sum $($ Data. $\mathrm{C}>0$ \& Data. $\mathrm{H}>0$ \& Data.O $>0$ \& Data.N $>0$ \& Data.P==0 \& Data. $\mathrm{S}>0$ ) ;

Summary. $\mathrm{CHONP}=$ sum $($ Data. $\mathrm{C}>0$ \& Data. $\mathrm{H}>0$ \& Data. $\mathrm{O}>0$ \& Data.N $>0$ \& Data.P $>0$ \& Data. $\mathrm{S}==0$ );

Summary. $\mathrm{CHONSP}=$ sum $($ Data. $\mathrm{C}>0$ \& Data.H $>0$ \& Data. O $>0$ \& Data.N $>0$ \& Data.P $>0$ \& Data. S>0);

ocalculating aromaticity parameters: \# condensed aromatic (AI>=0.67), aromatic $(\mathrm{AI}>=0.5)$,

onon-aromatic $(\mathrm{A} I<0.5)$

Summary. CondensedAromatics $=$ sum (Data. AI $>=0.67)$;

Summary. Aromatics=sum (Data.AI $>=0.5)$;

Summary. NonAromatics=sum (Data.AI<0.5);

ocalculating other parameters: total \# of formulae, average molecular

oweight

Summary.TotalFormulae=height (Data);

Summary. AvgMW=mean (Data. NominalMass);

o.Storing Summary Data

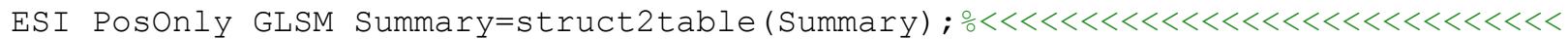

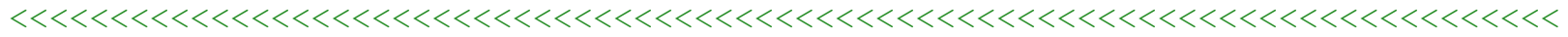

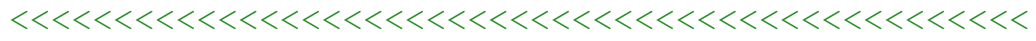

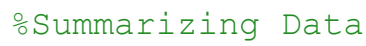

Data=Both;

oCalculating formulae type parameters: \# CHO, CHON, CHOS, CHOP, CHN, CHS, CHP, CHNS, CHONS formulae

Summary $\cdot \mathrm{CHO}=\operatorname{sum}($ Data. $\mathrm{C}>0$ \& Data. $\mathrm{H}>0$ \& Data. $\mathrm{O}>0$ \& Data. $\mathrm{N}==0$ \& Data. $\mathrm{P}==0$ \&

Data. $\mathrm{S}==0$ );

Summary $\cdot \mathrm{CHON}=\operatorname{sum}($ Data. $\mathrm{C}>0$ \& Data.H $>0$ \& Data. $0>0$ \& Data. $\mathrm{N}>0$ \& Data. $\mathrm{P}==0$ \&

Data. $\mathrm{S}==0$ );

Summary. $\mathrm{CHOS}=$ sum $($ Data. $\mathrm{C}>0$ \& Data. $\mathrm{H}>0$ \& Data.O $>0$ \& Data. $\mathrm{N}==0$ \& Data. $\mathrm{P}==0$ \&

Data. S>0);

Summary $\cdot \mathrm{CHOP}=$ sum $($ Data. $\mathrm{C}>0$ \& Data. $\mathrm{H}>0$ \& Data. $\mathrm{O}>0$ \& Data. $\mathrm{N}==0$ \& Data. P $>0$ \&

Data. $\mathrm{S}==0$ ) ;

Summary. $\mathrm{CHN}=$ sum $($ Data. $\mathrm{C}>0$ \& Data. $\mathrm{H}>0$ \& Data. $\mathrm{O}==0$ \& Data. $\mathrm{N}>0$ \& Data. $\mathrm{P}==0$ \&

Data. $\mathrm{S}==0$ );

Summary $\cdot \mathrm{CHS}=\operatorname{sum}($ Data. $\mathrm{C}>0$ \& Data. $\mathrm{H}>0$ \& Data. $\mathrm{O}==0$ \& Data. $\mathrm{N}==0$ \& Data. $\mathrm{P}==0$ \&

Data. S $>0$ );

Summary $\cdot \mathrm{CHNS}=\operatorname{sum}($ Data. $\mathrm{C}>0$ \& Data. $\mathrm{H}>0$ \& Data. $\mathrm{O}==0$ \& Data. $\mathrm{N}>0$ \& Data.P==0 \&

Data. $\mathrm{S}>0$ );

Summary. $\mathrm{CHNP}=\operatorname{sum}($ Data. $\mathrm{C}>0$ \& Data. $\mathrm{H}>0$ \& Data. $\mathrm{O}==0$ \& Data. $\mathrm{N}>0$ \& Data.P $>0$ \&

Data. $\mathrm{S}=0$ ) ;

Summary. $\mathrm{CHONS}=\operatorname{sum}($ Data $\cdot \mathrm{C}>0$ \& Data. $\mathrm{H}>0$ \& Data. $\mathrm{O}>0$ \& Data. $\mathrm{N}>0$ \& Data. $\mathrm{P}==0$ \&

Data. S>0);

Summary. $\mathrm{CHONP}=$ sum $($ Data. $\mathrm{C}>0$ \& Data. $\mathrm{H}>0$ \& Data. O $>0$ \& Data.N $>0$ \& Data.P $>0$ \&

Data. $\mathrm{S}==0$ );

Summary. $\mathrm{CHONSP}=$ sum $($ Data. $\mathrm{C}>0$ \& Data.H $>0$ \& Data.O $>0$ \& Data.N $>0$ \& Data.P>0 \&

Data. S>0);

oCalculating aromaticity parameters: \# condensed aromatic (AI>=0.67),

aromatic $(A I>=0.5)$,

onon-aromatic $($ AI $<0.5)$

Summary. CondensedAromatics $=$ sum (Data. AI $>=0.67)$;

Summary.Aromatics $=$ sum (Data.AI>=0.5); 
Summary. NonAromatics=sum (Data.AI<0.5);

ocalculating other parameters: total \# of formulae, average molecular

oweight

Summary. TotalFormulae=height (Data);

Summary. AvgMW=mean (Data. NominalMass);

oStoring Summary Data

ESI BothPolarities_GLSM Summary=struct2table (Summary) ; $\circ<<<<<<<<<<<<<<<<<<<<<<<~$

$<<<\overline{<}<<<<<<<<<<<<<<\overline{<}<<<<\overline{<}<<<<<<<<<<<<<<<<<<<<<<<<<<<<<<<<<<<<<<<<<<<<<<<<<<<<<~$

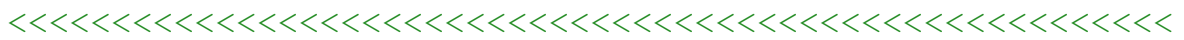

o Summarizing Data

Data $=$ Combined;

oCalculating formulae type parameters: \# CHO, CHON, CHOS, CHOP, CHN, CHS, CHP, CHNS, CHONS formulae

Summary $\cdot \mathrm{CHO}=$ sum $($ Data. $\mathrm{C}>0$ \& Data. $\mathrm{H}>0$ \& Data. $\mathrm{O}>0$ \& Data. $\mathrm{N}==0$ \& Data. $\mathrm{P}==0$ \&

Data. $\mathrm{S}==0$ );

Summary $\cdot \mathrm{CHON}=\operatorname{sum}($ Data. $\mathrm{C}>0$ \& Data.H $>0$ \& Data. $\mathrm{O}>0$ \& Data. $>0$ \& Data.P==0 \&

Data. $\mathrm{S}=0$ ) ;

Summary $\cdot \mathrm{CHOS}=$ sum $($ Data. $\mathrm{C}>0$ \& Data.H $>0$ \& Data. O $>0$ \& Data. $\mathrm{N}==0$ \& Data. $\mathrm{P}==0$ \&

Data. $\mathrm{S}>0$ );

Summary $\cdot \mathrm{CHOP}=\operatorname{sum}($ Data. $\mathrm{C}>0$ \& Data.H $>0$ \& Data. $\mathrm{O}>0$ \& Data. $\mathrm{N}==0$ \& Data.P $>0$ \&

Data. $\mathrm{S}==0$ );

Summary $\cdot \mathrm{CHN}=$ sum $($ Data. $\mathrm{C}>0$ \& Data. $\mathrm{H}>0$ \& Data. $\mathrm{O}==0$ \& Data. $\mathrm{N}>0$ \& Data. $\mathrm{P}==0$ \&

Data. $\mathrm{S}==0$ );

Summary $\cdot \mathrm{CHS}=\operatorname{sum}($ Data $\cdot \mathrm{C}>0$ \& Data. $\mathrm{H}>0$ \& Data. $\mathrm{O}==0$ \& Data. $\mathrm{N}==0$ \& Data. $\mathrm{P}==0$ \&

Data. S>0);

Summary. $\mathrm{CHNS}=$ sum $($ Data. $\mathrm{C}>0$ \& Data. $\mathrm{H}>0$ \& Data. $\mathrm{O}==0$ \& Data. $\mathrm{N}>0$ \& Data. $\mathrm{P}==0$ \&

Data. $\mathrm{S}>0$ );

Summary. $\mathrm{CHNP}=\mathrm{sum}($ Data. $\mathrm{C}>0$ \& Data. $\mathrm{H}>0$ \& Data. $\mathrm{O}==0$ \& Data.N $>0$ \& Data.P $>0$ \&

Data. $\mathrm{S}==0$ );

Summary $\cdot \mathrm{CHONS}=\operatorname{sum}($ Data. $\mathrm{C}>0$ \& Data. $\mathrm{H}>0$ \& Data. $0>0$ \& Data.N $>0$ \& Data.P==0 \&

Data. S>0);

Summary. $\mathrm{CHONP}=$ sum $($ Data. $\mathrm{C}>0$ \& Data. $\mathrm{H}>0$ \& Data. $\mathrm{O}>0$ \& Data. $\mathrm{N}>0$ \& Data.P $>0$ \&

Data. $\mathrm{S}==0$ );

Summary. $\mathrm{CHONSP}=$ sum $($ Data. $\mathrm{C}>0$ \& Data.H $>0$ \& Data.O $>0$ \& Data.N $>0$ \& Data.P>0 \&

Data. $\mathrm{S}>0$ );

ocalculating aromaticity parameters: \# condensed aromatic (AI>=0.67),

aromatic $(A I>=0.5)$,

onon-aromatic $(\mathrm{AI}<0.5)$

Summary. CondensedAromatics $=$ sum (Data. AI $>=0.67)$;

Summary. Aromatics $=$ sum (Data.AI $>=0.5)$;

Summary. NonAromatics=sum (Data.AI<0.5);

ocalculating other parameters: total \# of formulae, average molecular

\%weight

Summary. TotalFormulae=height (Data);

Summary. AvgMW=mean (Data. NominalMass) ;

ostoring Summary Data

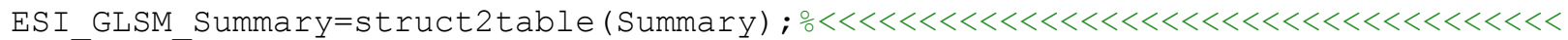
$<<\overline{<}<<<\overline{\bar{l}}<<<<<<<<<<<<<<<<<<<<<<<<<<<<<<<<<<<<<<<<<<<<<<<<<<<<<<<<<<<<<<<<<<<<$

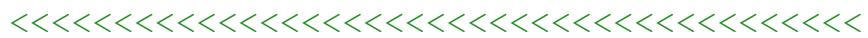

응 Combining Negative and Positive Mode LDI

oInput Data

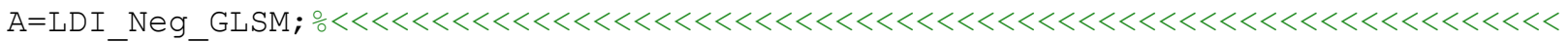
$<<<<\overline{<}<<\overline{<}<<<<<<<<<<<<<<<<<<<<<<<<<<<<<<<<<<<<<<<<<<<<<<<<<<<<<<<<<<<<<<<<<<<<~$ $<<<<<<<<<<<<<<<<<~$ 


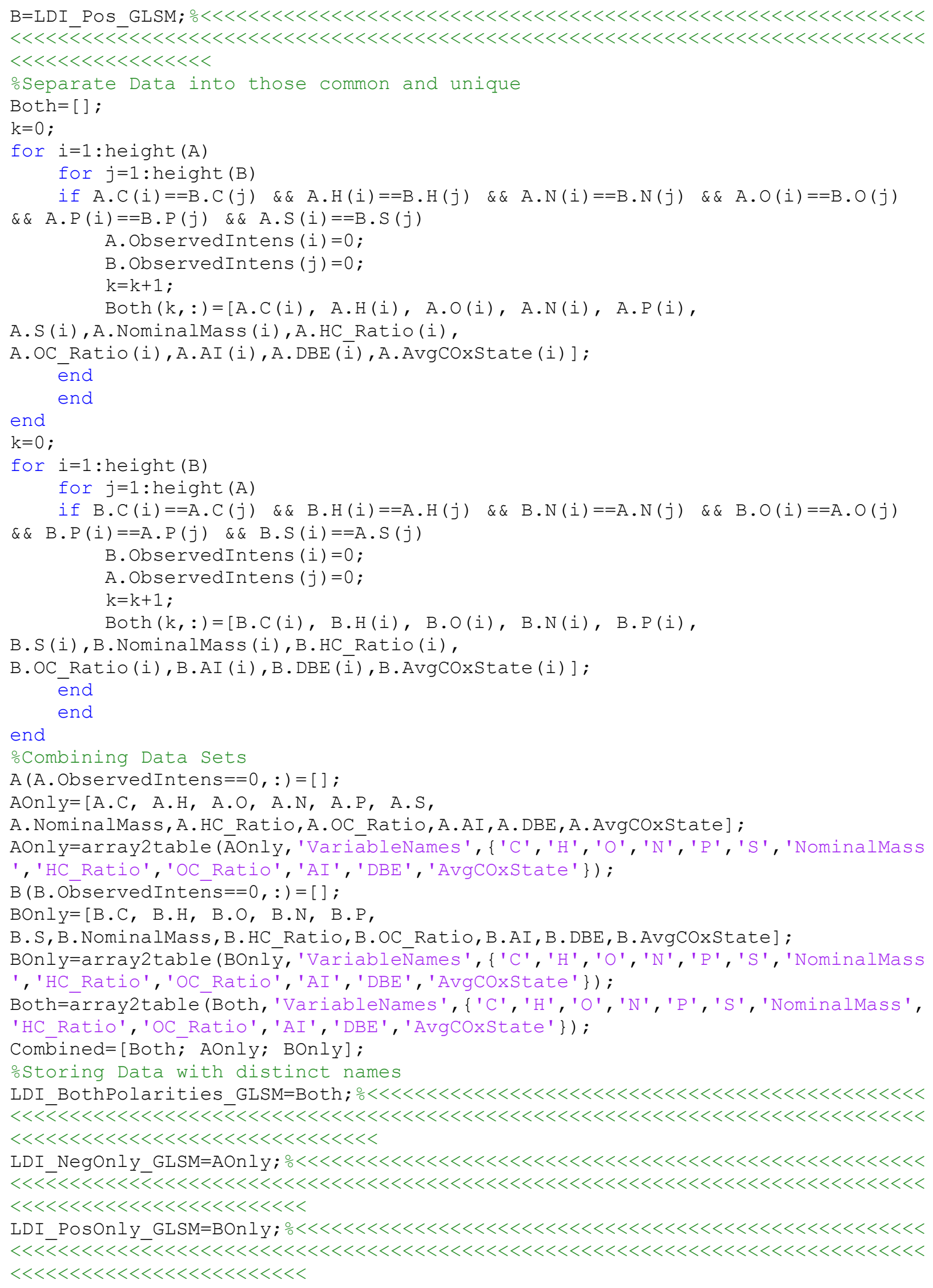




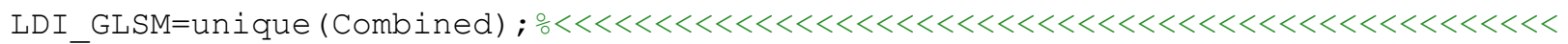
$<<\overline{<}<<<<<<<<<<<<<<<<<<<<<<<<<<<<<<<<<<<<<<<<<<<<<<<<<<<<<<<<<<<<<<<<<<<<<<<<<~$ $<<<<<<<<<<<<<<<<<<<<<<<<<<<<~$

ㅇumarizing Data

Data=AOnly;

oCalculating formulae type parameters: \# CHO, CHON, CHOS, CHOP, CHN, CHS, CHP, CHNS, CHONS formulae

Summary $\cdot \mathrm{CHO}=$ sum $($ Data. $\mathrm{C}>0$ \& Data. $\mathrm{H}>0$ \& Data. $\mathrm{O}>0$ \& Data. $\mathrm{N}==0$ \& Data. $\mathrm{P}==0$ \&

Data. $\mathrm{S}==0$ );

Summary $\cdot \mathrm{CHON}=$ sum $($ Data. $\mathrm{C}>0$ \& Data.H $>0$ \& Data. $\mathrm{O}>0$ \& Data. $\mathrm{N}>0$ \& Data.P==0 \&

Data. $\mathrm{S}==0$ );

Summary. $\mathrm{CHOS}=$ sum $($ Data. $\mathrm{C}>0$ \& Data. $\mathrm{H}>0$ \& Data. $\mathrm{O}>0$ \& Data. $\mathrm{N}==0$ \& Data. $\mathrm{P}==0$ \&

Data. $\mathrm{S}>0$ );

Summary. $\mathrm{CHOP}=\mathrm{sum}($ Data. $\mathrm{C}>0$ \& Data.H $>0$ \& Data. $0>0$ \& Data. $\mathrm{N}==0$ \& Data.P $>0$ \&

Data. $\mathrm{S}==0$ );

Summary $\cdot \mathrm{CHN}=$ sum $($ Data. $\mathrm{C}>0$ \& Data. $\mathrm{H}>0$ \& Data. $\mathrm{O}==0$ \& Data. $\mathrm{N}>0$ \& Data. $\mathrm{P}==0$ \&

Data. $\mathrm{S}=0$ ) ;

Summary $\cdot \mathrm{CHS}=\operatorname{sum}($ Data. $\mathrm{C}>0$ \& Data. $\mathrm{H}>0$ \& Data. $\mathrm{O}==0$ \& Data. $\mathrm{N}==0$ \& Data. $\mathrm{P}==0$ \&

Data. $\mathrm{S}>0$ ) ;

Summary $\cdot \mathrm{CHNS}=\operatorname{sum}($ Data. $\mathrm{C}>0$ \& Data. $\mathrm{H}>0$ \& Data. $\mathrm{O}==0$ \& Data. $\mathrm{N}>0$ \& Data.P==0 \&

Data. S $>0$ );

Summary $\cdot \mathrm{CHNP}=\operatorname{sum}($ Data. $\mathrm{C}>0$ \& Data.H $>0$ \& Data. $\mathrm{O}==0$ \& Data. $\mathrm{N}>0$ \& Data.P $>0$ \&

Data. $\mathrm{S}==0$ );

Summary. $\mathrm{CHONS}=\operatorname{sum}($ Data. $\mathrm{C}>0$ \& Data. $\mathrm{H}>0$ \& Data. $0>0$ \& Data.N>0 \& Data.P==0 \&

Data. S>0);

Summary. $\mathrm{CHONP}=\operatorname{sum}($ Data. $\mathrm{C}>0$ \& Data.H $>0$ \& Data. O $>0$ \& Data.N $>0$ \& Data.P $>0$ \&

Data. $\mathrm{S}==0$ );

Summary. $\mathrm{CHONSP}=$ sum $($ Data. $\mathrm{C}>0$ \& Data.H $>0$ \& Data.O $>0$ \& Data.N $>0$ \& Data.P>0 \&

Data. $\mathrm{S}>0)$;

ocalculating aromaticity parameters: \# condensed aromatic (AI>=0.67),

aromatic $(\mathrm{AI}>=0.5)$,

onon-aromatic $(\mathrm{A} I<0.5)$

Summary. CondensedAromatics $=$ sum (Data. AI $>=0.67)$;

Summary. Aromatics $=$ sum (Data.AI $>=0.5)$;

Summary. NonAromatics=sum (Data.AI<0.5);

ocalculating other parameters: total \# of formulae, average molecular

oweight

Summary.TotalFormulae=height (Data);

Summary. AvgMW=mean (Data. NominalMass);

ostoring Summary Data

LDI_NegOnly_GLSM_Summary=struct2table (Summary); $\circ<<<<<<<<<<<<<<<<<<<<<<<<<<<<<<<~$

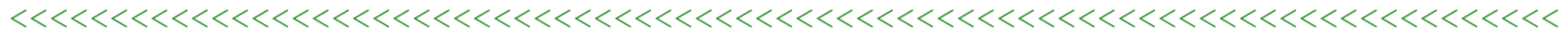

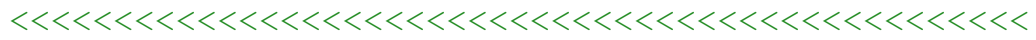

․ Summarizing Data

Data=BOnly;

oCalculating formulae type parameters: \# CHO, CHON, CHOS, CHOP, CHN, CHS, CHP, CHNS, CHONS formulae

Summary. $\mathrm{CHO}=\operatorname{sum}($ Data. $\mathrm{C}>0$ \& Data. $\mathrm{H}>0$ \& Data. $\mathrm{O}>0$ \& Data. $\mathrm{N}==0$ \& Data. $\mathrm{P}==0$ \& Data. $\mathrm{S}=0$ ) ;

Summary. $\mathrm{CHON}=\operatorname{sum}($ Data. $\mathrm{C}>0$ \& Data. $\mathrm{H}>0$ \& Data. $\mathrm{O}>0$ \& Data.N $>0$ \& Data.P==0 \&

Data. $\mathrm{S}==0$ ) ;

Summary $\cdot \mathrm{CHOS}=$ sum $($ Data. $\mathrm{C}>0$ \& Data. $\mathrm{H}>0$ \& Data. $\mathrm{O}>0$ \& Data. $\mathrm{N}==0$ \& Data.P==0 \& Data. $\mathrm{S}>0$ ) ;

Summary $\cdot \mathrm{CHOP}=\mathrm{sum}($ Data. $\mathrm{C}>0$ \& Data. $\mathrm{H}>0$ \& Data. $\mathrm{O}>0$ \& Data. $\mathrm{N}==0$ \& Data.P $>0$ \&

Data. $\mathrm{S}=0$ ) ;

Summary. $\mathrm{CHN}=\operatorname{sum}($ Data. $\mathrm{C}>0$ \& Data. $\mathrm{H}>0$ \& Data. $\mathrm{O}==0$ \& Data. $\mathrm{N}>0$ \& Data. $\mathrm{P}==0$ \&

Data. $\mathrm{S}==0$ ) ; 
Summary $\cdot \mathrm{CHS}=\operatorname{sum}($ Data $\cdot \mathrm{C}>0$ \& Data. $\mathrm{H}>0$ \& Data. $\mathrm{O}==0$ \& Data. $\mathrm{N}==0$ \& Data. $\mathrm{P}==0$ \& Data. $\mathrm{S}>0$ ) ;

Summary. CHNS $=$ sum $($ Data. $\mathrm{C}>0$ \& Data. $\mathrm{H}>0$ \& Data. $\mathrm{O}==0$ \& Data. $\mathrm{N}>0$ \& Data.P==0 \& Data. S $>0$ );

Summary. $\mathrm{CHNP}=\operatorname{sum}($ Data. $\mathrm{C}>0$ \& Data. $\mathrm{H}>0$ \& Data. $\mathrm{O}==0$ \& Data. $\mathrm{N}>0$ \& Data.P $>0$ \& Data. $\mathrm{S}==0$ );

Summary. CHONS $=$ sum $($ Data. $\mathrm{C}>0$ \& Data. $\mathrm{H}>0$ \& Data.O $>0$ \& Data.N $>0$ \& Data.P==0 \& Data. $\mathrm{S}>0$ ) ;

Summary. $\mathrm{CHONP}=$ sum $($ Data. $\mathrm{C}>0$ \& Data. $\mathrm{H}>0$ \& Data. $\mathrm{O}>0$ \& Data.N $>0$ \& Data.P $>0$ \& Data. $\mathrm{S}==0$ );

Summary. $\mathrm{CHONSP}=$ sum $($ Data. $\mathrm{C}>0$ \& Data.H $>0$ \& Data. O $>0$ \& Data.N $>0$ \& Data.P $>0$ \& Data. S>0);

ocalculating aromaticity parameters: \# condensed aromatic (AI>=0.67), aromatic $(\mathrm{AI}>=0.5)$,

onon-aromatic $(\mathrm{A} I<0.5)$

Summary. CondensedAromatics $=$ sum (Data. AI $>=0.67)$;

Summary. Aromatics=sum (Data.AI $>=0.5)$;

Summary. NonAromatics=sum (Data.AI<0.5);

ocalculating other parameters: total \# of formulae, average molecular

oweight

Summary.TotalFormulae=height (Data);

Summary. AvgMW=mean (Data. NominalMass);

o.Storing Summary Data

LDI PosOnly GLSM Summary=struct2table (Summary); $\circ<<<<<<<<<<<<<<<<<<<<<<<<<<<<<<<<<~$

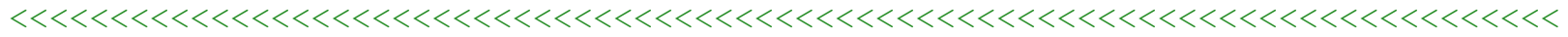

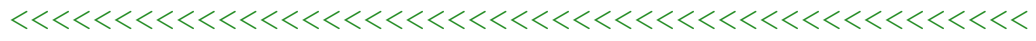

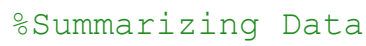

Data=Both;

oCalculating formulae type parameters: \# CHO, CHON, CHOS, CHOP, CHN, CHS, CHP, CHNS, CHONS formulae

Summary $\cdot \mathrm{CHO}=\operatorname{sum}($ Data. $\mathrm{C}>0$ \& Data. $\mathrm{H}>0$ \& Data. $\mathrm{O}>0$ \& Data. $\mathrm{N}==0$ \& Data. $\mathrm{P}==0$ \&

Data. $\mathrm{S}==0$ );

Summary $\cdot \mathrm{CHON}=\operatorname{sum}($ Data. $\mathrm{C}>0$ \& Data.H $>0$ \& Data. $0>0$ \& Data. $\mathrm{N}>0$ \& Data. $\mathrm{P}==0$ \&

Data. $\mathrm{S}==0$ );

Summary. $\mathrm{CHOS}=$ sum $($ Data. $\mathrm{C}>0$ \& Data. $\mathrm{H}>0$ \& Data.O $>0$ \& Data. $\mathrm{N}==0$ \& Data. $\mathrm{P}==0$ \&

Data. S>0);

Summary $\cdot \mathrm{CHOP}=$ sum $($ Data. $\mathrm{C}>0$ \& Data. $\mathrm{H}>0$ \& Data. $\mathrm{O}>0$ \& Data. $\mathrm{N}==0$ \& Data. P $>0$ \&

Data. $\mathrm{S}==0$ ) ;

Summary. $\mathrm{CHN}=$ sum $($ Data. $\mathrm{C}>0$ \& Data. $\mathrm{H}>0$ \& Data. $\mathrm{O}==0$ \& Data. $\mathrm{N}>0$ \& Data. $\mathrm{P}==0$ \&

Data. $\mathrm{S}==0$ );

Summary $\cdot \mathrm{CHS}=\operatorname{sum}($ Data. $\mathrm{C}>0$ \& Data. $\mathrm{H}>0$ \& Data. $\mathrm{O}==0$ \& Data. $\mathrm{N}==0$ \& Data. $\mathrm{P}==0$ \&

Data. S $>0$ );

Summary $\cdot \mathrm{CHNS}=\operatorname{sum}($ Data. $\mathrm{C}>0$ \& Data. $\mathrm{H}>0$ \& Data. $\mathrm{O}==0$ \& Data. $\mathrm{N}>0$ \& Data.P==0 \&

Data. $\mathrm{S}>0$ );

Summary. $\mathrm{CHNP}=\operatorname{sum}($ Data. $\mathrm{C}>0$ \& Data. $\mathrm{H}>0$ \& Data. $\mathrm{O}==0$ \& Data. $\mathrm{N}>0$ \& Data.P $>0$ \&

Data. $\mathrm{S}=0$ ) ;

Summary. $\mathrm{CHONS}=\operatorname{sum}($ Data $\cdot \mathrm{C}>0$ \& Data. $\mathrm{H}>0$ \& Data. $\mathrm{O}>0$ \& Data. $\mathrm{N}>0$ \& Data. $\mathrm{P}==0$ \&

Data. S>0);

Summary. $\mathrm{CHONP}=$ sum $($ Data. $\mathrm{C}>0$ \& Data. $\mathrm{H}>0$ \& Data. O $>0$ \& Data.N $>0$ \& Data.P $>0$ \&

Data. $\mathrm{S}==0$ );

Summary. $\mathrm{CHONSP}=$ sum $($ Data. $\mathrm{C}>0$ \& Data.H $>0$ \& Data.O $>0$ \& Data.N $>0$ \& Data.P>0 \&

Data. S>0);

oCalculating aromaticity parameters: \# condensed aromatic (AI>=0.67),

aromatic $(A I>=0.5)$,

onon-aromatic $($ AI $<0.5)$

Summary. CondensedAromatics $=$ sum (Data. AI $>=0.67)$;

Summary.Aromatics $=$ sum (Data.AI>=0.5); 
Summary. NonAromatics=sum (Data.AI<0.5);

ocalculating other parameters: total \# of formulae, average molecular

oweight

Summary. TotalFormulae=height (Data);

Summary. AvgMW=mean (Data. NominalMass) ;

ostoring Summary Data

LDI BothPolarities GLSM Summary=struct2table (Summary); $\circ<<<<<<<<<<<<<<<<<<<<<<<~$

$<<<\overline{<}<<<<<<<<<<<<<<\overline{<}<<<<\overline{<}<<<<<<<<<<<<<<<<<<<<<<<<<<<<<<<<<<<<<<<<<<<<<<<<<<<<<~$

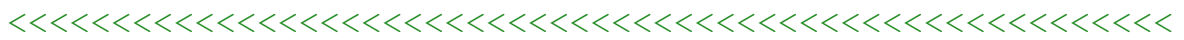

o Summarizing Data

Data $=$ Combined;

oCalculating formulae type parameters: \# CHO, CHON, CHOS, CHOP, CHN, CHS, CHP, CHNS, CHONS formulae

Summary $\cdot \mathrm{CHO}=$ sum $($ Data. $\mathrm{C}>0$ \& Data. $\mathrm{H}>0$ \& Data. $\mathrm{O}>0$ \& Data. $\mathrm{N}==0$ \& Data. $\mathrm{P}==0$ \&

Data. $\mathrm{S}==0$ );

Summary $\cdot \mathrm{CHON}=$ sum $($ Data. $\mathrm{C}>0$ \& Data.H $>0$ \& Data. $\mathrm{O}>0$ \& Data. $\mathrm{N}>0$ \& Data.P==0 \&

Data. $\mathrm{S}==0$ );

Summary $\cdot \mathrm{CHOS}=$ sum $($ Data. $\mathrm{C}>0$ \& Data.H $>0$ \& Data. O $>0$ \& Data. $\mathrm{N}==0$ \& Data. $\mathrm{P}==0$ \&

Data. $\mathrm{S}>0$ ) ;

Summary $\cdot \mathrm{CHOP}=\operatorname{sum}($ Data. $\mathrm{C}>0$ \& Data.H $>0$ \& Data. $\mathrm{O}>0$ \& Data. $\mathrm{N}==0$ \& Data.P $>0$ \&

Data. $\mathrm{S}==0$ );

Summary $\cdot \mathrm{CHN}=$ sum $($ Data. $\mathrm{C}>0$ \& Data. $\mathrm{H}>0$ \& Data. $\mathrm{O}==0$ \& Data. $\mathrm{N}>0$ \& Data. $\mathrm{P}==0$ \&

Data. $\mathrm{S}==0$ );

Summary $\cdot \mathrm{CHS}=\operatorname{sum}($ Data $\cdot \mathrm{C}>0$ \& Data. $\mathrm{H}>0$ \& Data. $\mathrm{O}==0$ \& Data. $\mathrm{N}==0$ \& Data. $\mathrm{P}==0$ \&

Data. S>0);

Summary. $\mathrm{CHNS}=$ sum $($ Data. $\mathrm{C}>0$ \& Data. $\mathrm{H}>0$ \& Data. $\mathrm{O}==0$ \& Data. $\mathrm{N}>0$ \& Data. $\mathrm{P}==0$ \&

Data. $\mathrm{S}>0$ );

Summary. $\mathrm{CHNP}=\mathrm{sum}($ Data. $\mathrm{C}>0$ \& Data. $\mathrm{H}>0$ \& Data. $\mathrm{O}==0$ \& Data.N $>0$ \& Data.P $>0$ \&

Data. $\mathrm{S}==0$ );

Summary $\cdot \mathrm{CHONS}=\operatorname{sum}($ Data. $\mathrm{C}>0$ \& Data. $\mathrm{H}>0$ \& Data. $0>0$ \& Data.N $>0$ \& Data.P==0 \&

Data. $\mathrm{S}>0$ );

Summary. $\mathrm{CHONP}=$ sum $($ Data. $\mathrm{C}>0$ \& Data. $\mathrm{H}>0$ \& Data. $\mathrm{O}>0$ \& Data. $\mathrm{N}>0$ \& Data.P $>0$ \&

Data. $\mathrm{S}==0$ );

Summary. $\mathrm{CHONSP}=$ sum $($ Data. $\mathrm{C}>0$ \& Data.H $>0$ \& Data.O $>0$ \& Data.N $>0$ \& Data.P>0 \&

Data. $\mathrm{S}>0$ );

ocalculating aromaticity parameters: \# condensed aromatic (AI>=0.67),

aromatic $(A I>=0.5)$,

onon-aromatic $(\mathrm{AI}<0.5)$

Summary. CondensedAromatics $=$ sum (Data. AI $>=0.67)$;

Summary. Aromatics $=$ sum (Data.AI $>=0.5)$;

Summary. NonAromatics=sum (Data.AI<0.5);

ocalculating other parameters: total \# of formulae, average molecular

oweight

Summary. TotalFormulae=height (Data);

Summary. AvgMW=mean (Data. NominalMass);

ostoring Summary Data

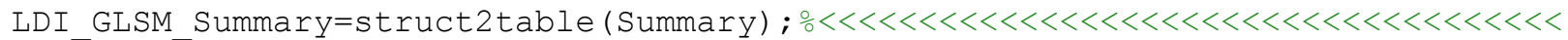
$<<\overline{\bar{l}}<<<\overline{\bar{l}}<<<<<<<<<<<<<<<<<<<<<<<<<<<<<<<<<<<<<<<<<<<<<<<<<<<<<<<<<<<<<<<<<<<<~$

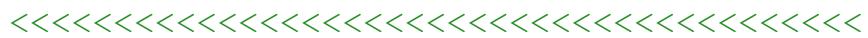

응 Combining ESI and LDI

oInput Data so that the script can readily be applied to any data set

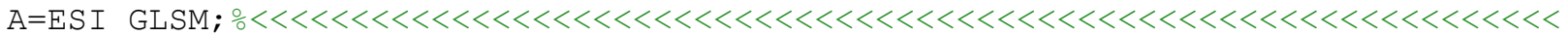
$<<<<\overline{<}<<<<<<<<<<<<<<<<<<<<<<<<<<<<<<<<<<<<<<<<<<<<<<<<<<<<<<<<<<<<<<<<<<<<<<<~$ $<<<<<<<<<<<<<$ 


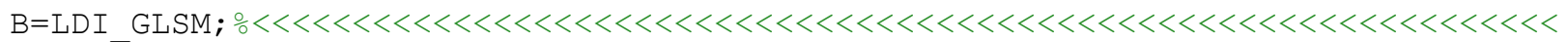
$<<<<\overline{<}<<<<<<<<<<<<<<<<<<<<<<<<<<<<<<<<<<<<<<<<<<<<<<<<<<<<<<<<<<<<<<<<<<<<<<<~$ $<<<<<<<<<<<<$

o Separate Data into those common and unique

Both=[ ] ;

$\mathrm{k}=0$;

for $i=1$ :height $(\mathrm{A})$

for $j=1$ :height $(B)$

if $A \cdot C(i)==B \cdot C(j) \quad \& \& A \cdot H(i)==B \cdot H(j) \quad \& \& A \cdot N(i)==B \cdot N(j) \quad \& \& A \cdot O(i)==B \cdot O(j)$

$\& \& A \cdot P(i)==B \cdot P(j) \quad \& \& A \cdot S(i)==B \cdot S(j)$

$\mathrm{k}=\mathrm{k}+1$;

$\operatorname{Both}(k,:)=[A . C(i), A . H(i), A . O(i), A . N(i), A . P(i)$,

A.S (i), A.NominalMass (i), A.HC Ratio(i),

A. OC Ratio(i), A.AI (i), A.DBE(i), A.AvgCOxState (i) ] ;

$$
\begin{aligned}
& \text { A. } C(i)=0 ; \\
& \text { B. C }(j)=0 ;
\end{aligned}
$$

end

end

Combining Data Sets

$A(A . C==0,:)=[]$;

$A O n l_{Y}=[A . C, A . H, A . O, A . N, A . P, A . S$,

A.Nominalmass,A.HC_Ratio,A.oC_Ratio,A.AI, A.DBE,A.AvgCoxstate];

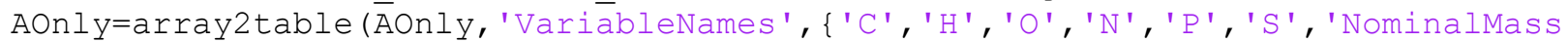
', 'HC_Ratio', 'OC_Ratio', 'AI', 'DBE', 'AvgCOxState' \});

$\mathrm{B}(\mathrm{B} \cdot \mathrm{C}==0,:)=[]$;

$\mathrm{BOnl} Y=[B . \mathrm{C}, \mathrm{B} . \mathrm{H}, \mathrm{B} . \mathrm{O}, \mathrm{B} . \mathrm{N}, \mathrm{B} . \mathrm{P}$,

B.S,B.NominalMass, B.HC Ratio,B.OC Ratio, B.AI, B.DBE, B. AvgCoxState];

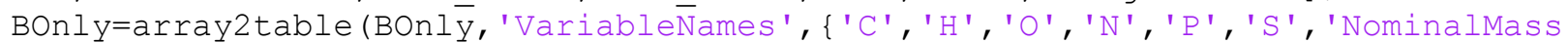
', 'HC Ratio', 'OC Ratio', 'AI', 'DBE', 'AvgCoxstate'\}) ;

Both=array2table (Both, 'VariableNames', \{'C', 'H', 'O', 'N', 'P' , 'S ', 'NominalMass', 'HC_Ratio', 'OC_Ratio', 'AI', 'DBE', 'AvgCoxstate' \}) ;

Combined=[Both; AOnly; BOnly];

ostoring Data with distinct names

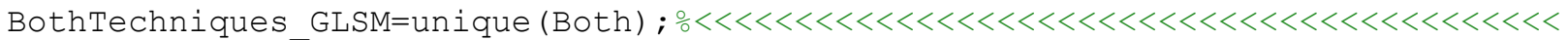

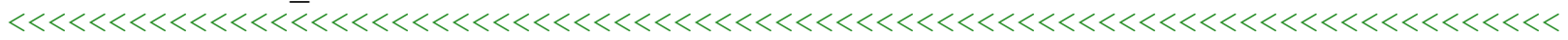

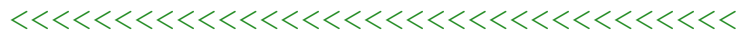

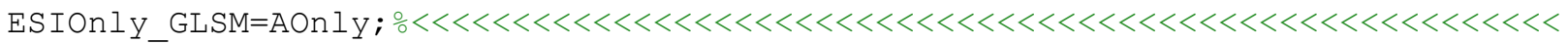

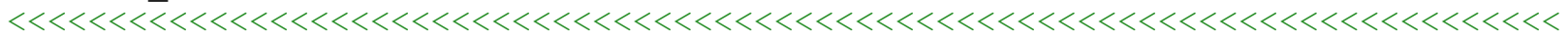
$<<<<<<<<<<<<<<<<<<<<<$

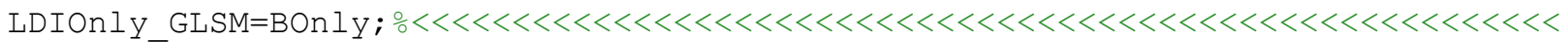

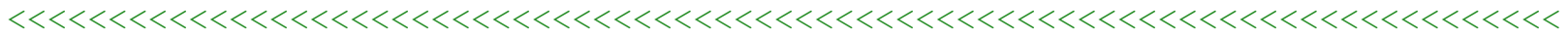
$<<<<<<<<<<<<<<<<<<<<<~$

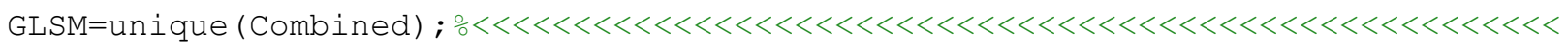

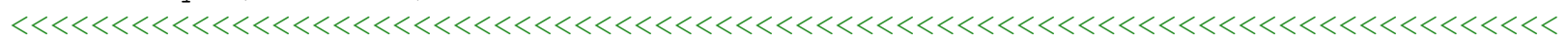
$<<<<<<<<<<<<<<<<<<<<<<<~$

o Summarizing Data

Data=AOnly;

oCalculating formulae type parameters: \# CHO, CHON, CHOS, CHOP, CHN, CHS, CHP, CHNS, CHONS formulae

Summary $\cdot \mathrm{CHO}=$ sum $($ Data. $\mathrm{C}>0$ \& Data. $\mathrm{H}>0$ \& Data. $\mathrm{O}>0$ \& Data. $\mathrm{N}==0$ \& Data. $\mathrm{P}==0$ \&

Data. $\mathrm{S}==0$ );

Summary $\cdot \mathrm{CHON}=\operatorname{sum}($ Data. $\mathrm{C}>0$ \& Data.H $>0$ \& Data. $\mathrm{O}>0$ \& Data. $>0$ \& Data.P==0 \& Data. $\mathrm{S}==0$ );

Summary. $\mathrm{CHOS}=$ sum $($ Data. $\mathrm{C}>0$ \& Data. $\mathrm{H}>0$ \& Data. $\mathrm{O}>0$ \& Data. $\mathrm{N}==0$ \& Data. $\mathrm{P}==0$ \&

Data. S>0); 
Summary. $\mathrm{CHOP}=\mathrm{sum}($ Data. $\mathrm{C}>0$ \& Data.H $>0$ \& Data.O $>0$ \& Data.N==0 \& Data.P>0 \& Data. $\mathrm{S}==0$ );

Summary $\cdot \mathrm{CHN}=$ sum $($ Data. $\mathrm{C}>0$ \& Data. $\mathrm{H}>0$ \& Data. $\mathrm{O}==0$ \& Data. $\mathrm{N}>0$ \& Data.P==0 \& Data. $\mathrm{S}==0$ ) ;

Summary $\cdot \mathrm{CHS}=\operatorname{sum}($ Data $\cdot \mathrm{C}>0$ \& Data. $\mathrm{H}>0$ \& Data. $\mathrm{O}==0$ \& Data. $\mathrm{N}==0$ \& Data. $\mathrm{P}==0$ \& Data. $\mathrm{S}>0$ );

Summary $\cdot \mathrm{CHNS}=$ sum $($ Data $\cdot \mathrm{C}>0$ \& Data. $\mathrm{H}>0$ \& Data. $\mathrm{O}==0$ \& Data. $\mathrm{N}>0$ \& Data. $\mathrm{P}==0$ \&

Data. $\mathrm{S}>0$ ) ;

Summary $\cdot \mathrm{CHNP}=\operatorname{sum}($ Data $\cdot \mathrm{C}>0$ \& Data. $\mathrm{H}>0$ \& Data. $\mathrm{O}==0$ \& Data.N $>0$ \& Data.P>0 \&

Data. $\mathrm{S}==0$ );

Summary. $\mathrm{CHONS}=\operatorname{sum}($ Data. $\mathrm{C}>0$ \& Data. $\mathrm{H}>0$ \& Data. $\mathrm{O}>0$ \& Data. $>0$ \& Data.P==0 \&

Data. $\mathrm{S}>0$ );

Summary. $\mathrm{CHONP}=$ sum $($ Data. $\mathrm{C}>0$ \& Data.H $>0$ \& Data.O>0 \& Data.N>0 \& Data.P>0 \&

Data. $\mathrm{S}==0$ );

Summary. $\mathrm{CHONSP}=$ sum $($ Data. $\mathrm{C}>0$ \& Data. $\mathrm{H}>0$ \& Data.O $>0$ \& Data.N $>0$ \& Data.P $>0$ \&

Data. S>0);

ocalculating aromaticity parameters: \# condensed aromatic (AI>=0.67),

aromatic $(\mathrm{AI}>=0.5)$,

onon-aromatic $(\mathrm{AI}<0.5)$

Summary. CondensedAromatics $=$ sum (Data. AI $>=0.67)$;

Summary. Aromatics $=$ sum (Data.AI $>=0.5)$;

Summary. NonAromatics=sum (Data.AI<0.5);

oCalculating other parameters: total \# of formulae, average molecular

oweight

Summary. TotalFormulae=height (Data);

Summary. AvgMW=mean (Data. NominalMass);

ostoring Summary Data

ESIOnly_GLSM_Summary=struct2table (Summary) ; $\circ<<<<<<<<<<<<<<<<<<<<<<<<<<<<<<<<<<~$

$<<<<<<<\overline{<}<<<\overline{<} \overline{<}<<<<<<<<<<<<<<<<<<<<<<<<<<<<<<<<<<<<<<<<<<<<<<<<<<<<<<<<<<<<<<<<$

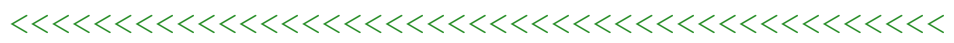

ㅇmmarizing Data

Data=BOnly;

oCalculating formulae type parameters: \# CHO, CHON, CHOS, CHOP, CHN, CHS, CHP, CHNS, CHONS formulae

Summary $\cdot \mathrm{CHO}=$ sum $($ Data. $\mathrm{C}>0$ \& Data. $\mathrm{H}>0$ \& Data. $\mathrm{O}>0$ \& Data. $\mathrm{N}==0$ \& Data. $\mathrm{P}==0$ \& Data. $\mathrm{S}==0$ );

Summary $\cdot \mathrm{CHON}=$ sum $($ Data $\cdot \mathrm{C}>0$ \& Data. $\mathrm{H}>0$ \& Data. $\mathrm{O}>0$ \& Data.N $>0$ \& Data.P==0 \&

Data. $\mathrm{S}==0$ );

Summary. $\mathrm{CHOS}=$ sum $($ Data. $\mathrm{C}>0$ \& Data. $\mathrm{H}>0$ \& Data. $\mathrm{O}>0$ \& Data. $\mathrm{N}==0$ \& Data. $\mathrm{P}==0$ \&

Data. $\mathrm{S}>0$ ) ;

Summary. $\mathrm{CHOP}=\mathrm{sum}($ Data. $\mathrm{C}>0$ \& Data.H $>0$ \& Data.O $>0$ \& Data.N==0 \& Data.P>0 \&

Data. $\mathrm{S}==0$ );

Summary. $\mathrm{CHN}=$ sum $($ Data. $\mathrm{C}>0$ \& Data. $\mathrm{H}>0$ \& Data. $\mathrm{O}==0$ \& Data. $\mathrm{N}>0$ \& Data.P==0 \&

Data. $\mathrm{S}==0$ );

Summary $\cdot \mathrm{CHS}=\operatorname{sum}($ Data $. \mathrm{C}>0$ \& Data. $\mathrm{H}>0$ \& Data. $\mathrm{O}==0$ \& Data. $\mathrm{N}==0$ \& Data. $\mathrm{P}==0$ \&

Data. $\mathrm{S}>0$ ) ;

Summary. CHNS $=$ sum $($ Data. $\mathrm{C}>0$ \& Data. $\mathrm{H}>0$ \& Data. $\mathrm{O}==0$ \& Data. $>0$ \& Data.P==0 \&

Data. $\mathrm{S}>0$ );

Summary. $\mathrm{CHNP}=$ sum $($ Data. $\mathrm{C}>0$ \& Data. $\mathrm{H}>0$ \& Data. $\mathrm{O}==0$ \& Data. $\mathrm{N}>0$ \& Data.P $>0$ \&

Data. $\mathrm{S}==0$ );

Summary $\cdot \mathrm{CHONS}=$ sum $($ Data. $\mathrm{C}>0$ \& Data. $\mathrm{H}>0$ \& Data. $0>0$ \& Data. $>0$ \& Data.P==0 \&

Data. $\mathrm{S}>0$ ) ;

Summary. $\mathrm{CHONP}=$ sum $($ Data. $\mathrm{C}>0$ \& Data.H $>0$ \& Data. $\mathrm{O}>0$ \& Data.N $>0$ \& Data.P>0 \&

Data. $\mathrm{S}==0$ );

Summary. $\mathrm{CHONSP}=$ sum $($ Data. $\mathrm{C}>0$ \& Data. $\mathrm{H}>0$ \& Data. $\mathrm{O}>0$ \& Data.N $>0$ \& Data.P $>0$ \&

Data. $\mathrm{S}>0$ ) ; 
oCalculating aromaticity parameters: \# condensed aromatic (AI>=0.67), aromatic $(\mathrm{AI}>=0.5)$,

onon-aromatic $(\mathrm{AI}<0.5)$

Summary. CondensedAromatics $=$ sum (Data.AI $>=0.67)$;

Summary.Aromatics=sum (Data.AI $>=0.5)$;

Summary. NonAromatics=sum (Data.AI<0.5);

ocalculating other parameters: total \# of formulae, average molecular

oweight

Summary. TotalFormulae=height (Data);

Summary. AvgMW=mean (Data. NominalMass);

ostoring Summary Data

LDIOnIy_GLSM_Summary=struct2table (Summary) ; $\circ<<<<<<<<<<<<<<<<<<<<<<<<<<<<<<<<<<~$

$<<<<<<<\overline{<}<<<\overline{<}<<<<<<<<<<<<<<<<<<<<<<<<<<<<<<<<<<<<<<<<<<<<<<<<<<<<<<<<<<<<<<<<~$

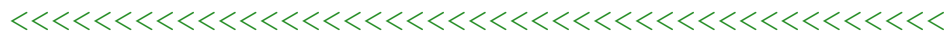

․ Summarizing Data

Data=unique (Both);

oCalculating formulae type parameters: \# CHO, CHON, CHOS, CHOP, CHN, CHS, CHP, CHNS, CHONS formulae

Summary $\cdot \mathrm{CHO}=$ sum (Data. $\mathrm{C}>0$ \& Data. $\mathrm{H}>0$ \& Data. $\mathrm{O}>0$ \& Data. $\mathrm{N}==0$ \& Data. $\mathrm{P}==0$ \&

Data. $\mathrm{S}==0$ );

Summary $\cdot \mathrm{CHON}=$ Sum $($ Data. $\mathrm{C}>0$ \& Data. $\mathrm{H}>0$ \& Data. $\mathrm{O}>0$ \& Data. $\mathrm{N}>0$ \& Data.P==0 \&

Data. $\mathrm{S}==0$ );

Summary. $\mathrm{CHOS}=$ sum $($ Data. $\mathrm{C}>0$ \& Data. $\mathrm{H}>0$ \& Data. $\mathrm{O}>0$ \& Data. $\mathrm{N}==0$ \& Data. $\mathrm{P}==0$ \&

Data. $\mathrm{S}>0$ );

Summary $\cdot \mathrm{CHOP}=$ sum $($ Data. $\mathrm{C}>0$ \& Data.H $>0$ \& Data. $\mathrm{O}>0$ \& Data. $\mathrm{N}==0$ \& Data.P $>0$ \&

Data. $\mathrm{S}==0$ );

Summary $\cdot \mathrm{CHN}=$ sum $($ Data. $\mathrm{C}>0$ \& Data. $\mathrm{H}>0$ \& Data. $\mathrm{O}==0$ \& Data. $\mathrm{N}>0$ \& Data.P==0 \&

Data. $\mathrm{S}==0$ );

Summary $\cdot \mathrm{CHS}=\operatorname{sum}($ Data $\cdot \mathrm{C}>0$ \& Data. $\mathrm{H}>0$ \& Data. $\mathrm{O}==0$ \& Data. $\mathrm{N}==0$ \& Data. $\mathrm{P}==0$ \&

Data. $\mathrm{S}>0$ );

Summary. $\mathrm{CHNS}=\operatorname{sum}($ Data. $\mathrm{C}>0$ \& Data. $\mathrm{H}>0$ \& Data. $\mathrm{O}==0$ \& Data. $\mathrm{N}>0$ \& Data. $\mathrm{P}==0$ \&

Data. S>0);

Summary $\cdot \mathrm{CHNP}=\operatorname{sum}($ Data. $\mathrm{C}>0$ \& Data.H $>0$ \& Data. $\mathrm{O}==0$ \& Data. $\mathrm{N}>0$ \& Data.P $>0$ \&

Data. $\mathrm{S}==0$ );

Summary. $\mathrm{CHONS}=\operatorname{sum}($ Data $. \mathrm{C}>0$ \& Data. $\mathrm{H}>0$ \& Data. $0>0$ \& Data.N>0 \& Data.P==0 \&

Data. S>0);

Summary. $\mathrm{CHONP}=$ sum $($ Data. $\mathrm{C}>0$ \& Data. $\mathrm{H}>0$ \& Data. $\mathrm{O}>0$ \& Data.N $>0$ \& Data.P $>0$ \&

Data. $\mathrm{S}==0$ ) ;

Summary. $\mathrm{CHONSP}=$ sum $($ Data. $\mathrm{C}>0$ \& Data.H $>0$ \& Data.O $>0$ \& Data.N $>0$ \& Data.P>0 \&

Data. $\mathrm{S}>0$ );

oCalculating aromaticity parameters: \# condensed aromatic (AI>=0.67),

aromatic $(A I>=0.5)$,

onon-aromatic $($ AI $<0.5)$

Summary. CondensedAromatics $=$ sum (Data. AI $>=0.67)$;

Summary. Aromatics $=$ sum (Data.AI $>=0.5)$;

Summary. NonAromatics=sum (Data.AI<0.5);

ocalculating other parameters: total \# of formulae, average molecular

oweight

Summary. TotalFormulae=height (Data);

Summary. AvgMW=mean (Data. NominalMass);

ostoring Summary Data

BothTechniques_GLSM_Summary=struct2table (Summary) ; $\circ<<<<<<<<<<<<<<<<<<<<<<<<<<~$

$<<<<<<<<<<<<<\overline{<}<<<\overline{<}<<<<<<<<<<<<<<<<<<<<<<<<<<<<<<<<<<<<<<<<<<<<<<<<<<<<<<<<<~$

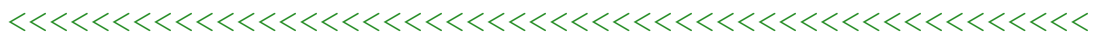

oSummarizing Data

Data $=$ Combined; 
oCalculating formulae type parameters: \# CHO, CHON, CHOS, CHOP, CHN, CHS, CHP, CHNS, CHONS formulae

Summary $\cdot \mathrm{CHO}=$ sum (Data. $\mathrm{C}>0$ \& Data. $\mathrm{H}>0$ \& Data. $\mathrm{O}>0$ \& Data. $\mathrm{N}==0$ \& Data. $\mathrm{P}==0$ \& Data. $\mathrm{S}==0$ );

Summary. $\mathrm{CHON}=\operatorname{sum}($ Data. $\mathrm{C}>0$ \& Data. $\mathrm{H}>0$ \& Data. $\mathrm{O}>0$ \& Data. $\mathrm{N}>0$ \& Data.P==0 \& Data. $\mathrm{S}=0$ ) ) ;

Summary. CHOS $=$ sum $($ Data. $\mathrm{C}>0$ \& Data.H $>0$ \& Data.O>0 \& Data.N==0 \& Data.P==0 \& Data. $\mathrm{S}>0$ );

Summary $\cdot \mathrm{CHOP}=\operatorname{sum}($ Data. $\mathrm{C}>0$ \& Data.H $>0$ \& Data. $\mathrm{O}>0$ \& Data. $\mathrm{N}==0$ \& Data.P $>0$ \& Data. $\mathrm{S}==0$ );

Summary $\cdot \mathrm{CHN}=$ sum $($ Data. $\mathrm{C}>0$ \& Data. $\mathrm{H}>0$ \& Data. $\mathrm{O}==0$ \& Data. $\mathrm{N}>0$ \& Data. $\mathrm{P}==0$ \&

Data. $\mathrm{S}=0$ ) ;

Summary $\cdot \mathrm{CHS}=\operatorname{sum}($ Data $\cdot \mathrm{C}>0$ \& Data. $\mathrm{H}>0$ \& Data. $\mathrm{O}==0$ \& Data. $\mathrm{N}==0$ \& Data. $\mathrm{P}==0$ \&

Data. S>0);

Summary. $\mathrm{CHNS}=$ sum (Data. $\mathrm{C}>0$ \& Data. $\mathrm{H}>0$ \& Data. $\mathrm{O}==0$ \& Data. $\mathrm{N}>0$ \& Data. $\mathrm{P}==0$ \&

Data. S>0);

Summary $\cdot \mathrm{CHNP}=\operatorname{sum}($ Data. $\mathrm{C}>0$ \& Data.H $>0$ \& Data. $\mathrm{O}==0$ \& Data.N $>0$ \& Data.P $>0$ \&

Data. $\mathrm{S}==0$ );

Summary $\cdot \mathrm{CHONS}=\operatorname{sum}($ Data $\cdot \mathrm{C}>0$ \& Data. $\mathrm{H}>0$ \& Data. $\mathrm{O}>0$ \& Data.N $>0$ \& Data.P==0 \&

Data. S>0);

Summary. $\mathrm{CHONP}=\operatorname{sum}($ Data. $\mathrm{C}>0$ \& Data. $\mathrm{H}>0$ \& Data. $\mathrm{O}>0$ \& Data.N $>0$ \& Data.P $>0$ \&

Data. $\mathrm{S}==0$ );

Summary. $\mathrm{CHONSP}=$ sum $($ Data. $\mathrm{C}>0$ \& Data.H $>0$ \& Data.O $>0$ \& Data.N $>0$ \& Data.P>0 \&

Data. $\mathrm{S}>0$ );

ocalculating aromaticity parameters: \# condensed aromatic (AI>=0.67),

aromatic $(\mathrm{AI}>=0.5)$,

onon-aromatic $(\mathrm{A} I<0.5)$

Summary. CondensedAromatics $=$ sum (Data.AI $>=0.67)$;

Summary.Aromatics $=$ sum (Data.AI $>=0.5)$;

Summary. NonAromatics=sum (Data.AI<0.5);

ocalculating other parameters: total \# of formulae, average molecular

oweight

Summary. TotalFormulae=height (Data);

Summary. AvgMW=mean (Data. NominalMass);

ostoring Summary Data

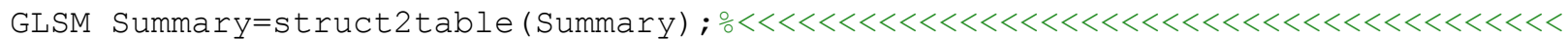
$<<<\overline{\bar{l}}<<<<<<<<<<<<<<<<<<<<<<<<<<<<<<<<<<<<<<<<<<<<<<<<<<<<<<<<<<<<<<<<<<<<<<<<~$

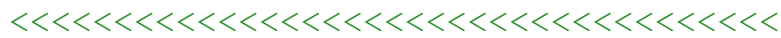

응 Repeating for post oxidation GLSM

응 Negative Mode ESI: Data Clean Up

oLoading negative mode background impurities

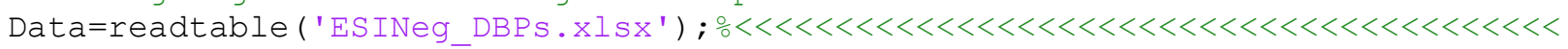
$<<<<<<<<<<<<<<<<<<<<<<\overline{<}<<<<<<<<<<<<<<<<<<<<<<<<<<<<<<<<<<<<<<<<<<<<<<<<<<<<<<~$ $<<<<<<<<<<<<<<<<<<<<<<<<<<<<<<<<<<~$

Data=unique (Data);

Data. NominalMass=Data. C*12+Data.0*16+Data.H*1+Data.P*31+Data.S*32+Data.N*14;

Data. KMD CH2=Data. NominalMass -

$0.998883 \overline{4} *\left(\right.$ Data. $C * 12+$ Data. $H^{*} 1.007825+$ Data. $N^{*} 14.003074+$ Data. $0 * 15.994915+$ Data.$P$

$\star 30.973763+$ Data.. *31.972072);

Data.KMD_COO=Data.NominalMass-

$1.000231^{\star}\left(\right.$ Data. $C * 12+$ Data $. H * 1.007825+$ Data. $N^{*} 14.003074+$ Data. $0 * 15.994915+$ Data. $P *$

$30.973763+$ Data..$* 31.972072)$;

Data.KMD 02=Data. NominalMass-

$1.000317^{\bar{*}}\left(\right.$ Data. $\mathrm{C}^{\star} 12+$ Data. $\mathrm{H}^{\star} 1.007825+$ Data $. \mathrm{N}^{\star} 14.003074+\mathrm{Data} .0 * 15.994915+\mathrm{Data} . \mathrm{P} *$

$30.973763+$ Data.S*31.972072); 


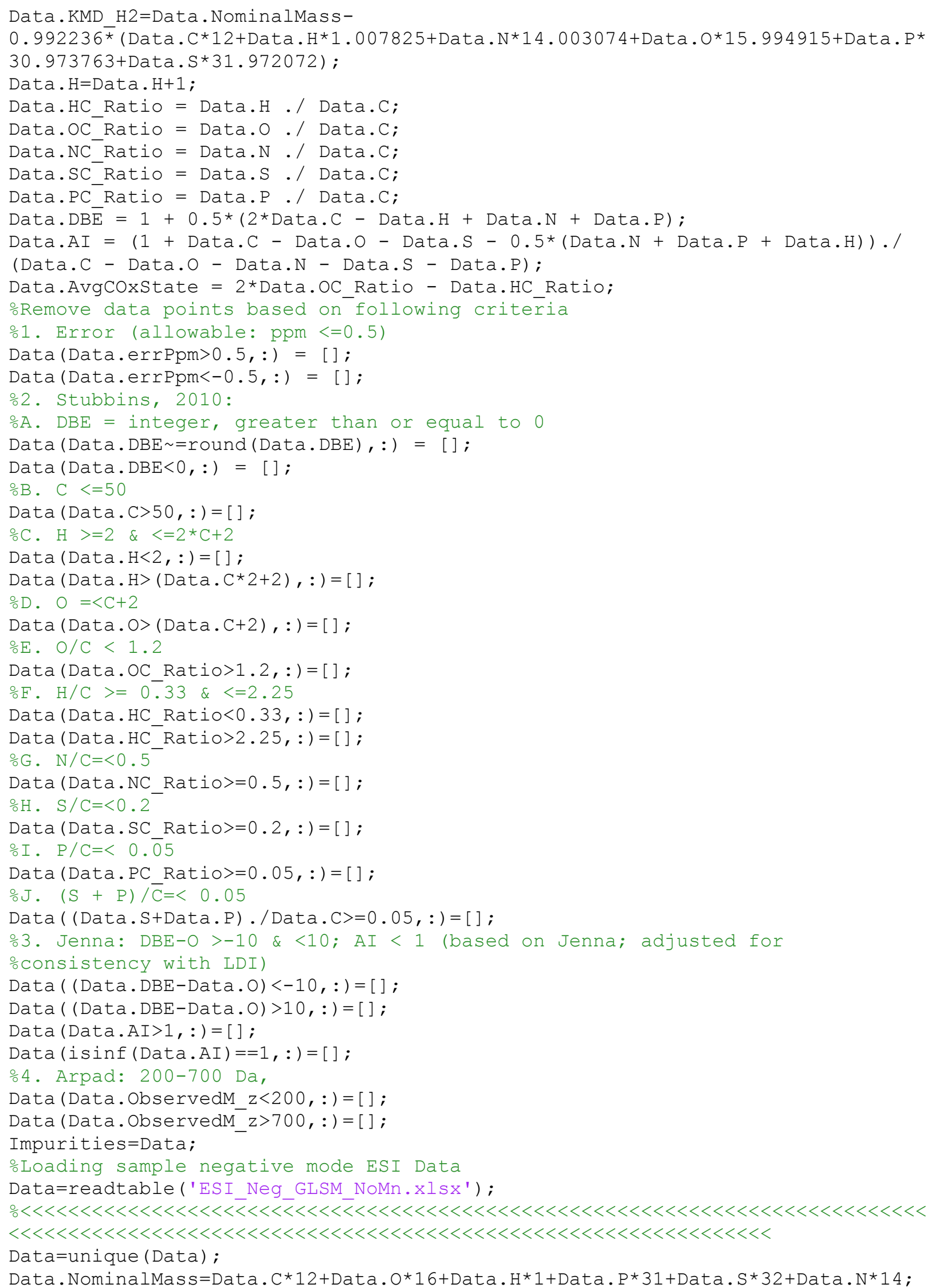


Data.KMD CH2=Data. NominalMass-

$0.998883 \overline{4} *\left(\right.$ Data. $C * 12+$ Data. $H^{*} 1.007825+$ Data. $N^{*} 14.003074+$ Data. $0 * 15.994915+$ Data.$P$ *30.973763+Data.S*31.972072);

Data.KMD_COO=Data.NominalMass-

1.000231 * $($ Data. C*12+Data.H*1.007825+Data. N*14.003074+Data.0*15.994915+Data.P*

$30.973763+$ Data. $S * 31.972072)$;

Data.KMD_O2=Data.NominalMass -

$1.000317^{\star}$ (Data. C*12+Data.H*1.007825+Data.N*14.003074+Data.0*15.994915+Data.P* $30.973763+$ Data.S*31.972072);

Data.KMD_H2=Data.NominalMass-

$0.992236^{\star}($ Data. C*12+Data.H*1.007825+Data. N*14.003074+Data.0*15.994915+Data.P*

$30.973763+$ Data. $S * 31.972072)$;

Data. $\mathrm{H}=$ Data. $\mathrm{H}+1$;

Data.HC Ratio = Data.H ./ Data.C;

Data.oC-Ratio = Data.O ./ Data.C;

Data.NC Ratio = Data.N./ Data.C;

Data.SC_Ratio = Data.S./ Data.C;

Data.PC Ratio = Data.P./ Data.C;

Data.DBE $=1+0.5 *(2 *$ Data.C - Data.H + Data. $N+$ Data.P $)$;

Data.AI = (1 + Data.C - Data.O - Data.S - 0.5* (Data.N + Data.P + Data.H) $). /$

(Data.C - Data.O - Data.N - Data.S - Data.P);

Data.AvgCoxstate = 2*Data.OC_Ratio - Data.HC_Ratio;

oSaving raw data for Scan 1

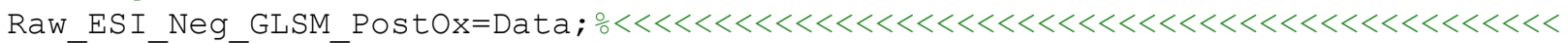

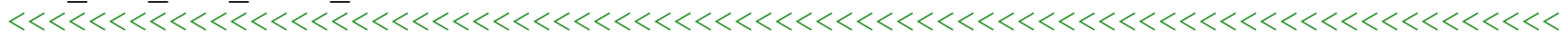
$<<<<<<<<<<<<<<<<<<<<<<<<<<<<<<<~$

Remove data points based on following criteria

ㄴ. Error (allowable: ppm <0.5)

Data (Data.errPpm $>0.5,:)=[]$;

Data (Data.errPpm<-0.5,:) = [] ;

\%2. Stubbins, 2010 :

$\div \mathrm{A} . \mathrm{DBE}=$ integer, greater than or equal to 0

Data (Data. DBE =round (Data.DBE), : ) = [ ] ;

Data $($ Data. $\mathrm{DBE}<0,:)=[]$;

ㅇ. $\mathrm{C}<=50$

Data (Data.C $>50,:)=[]$;

$\div \mathrm{C} \cdot \mathrm{H}>=2 \&<=2 * \mathrm{C}+2$

Data (Data. $\mathrm{H}<2,:)=[]$;

Data (Data.H $>($ Data. $C * 2+2),:)=[]$;

D. $\mathrm{O}=<\mathrm{C}+2$

Data $($ Data. O $>($ Data. $C+2),:)=[]$;

ㅌ. $O / C<1.2$

Data (Data.oC_Ratio>1.2,:) =[ ] ;

F. $\mathrm{H} / \mathrm{C}>=0.33 \&<=2.25$

Data (Data.HC Ratio<1/3, :) =[ ] ;

Data (Data.HC_Ratio $>2.25,:)=[]$;

$\div \mathrm{G} . \mathrm{N} / \mathrm{C}=<0.5$

Data (Data.NC_Ratio $>=0.5,:)=[]$;

$\div \mathrm{H} . \mathrm{S} / \mathrm{C}=<0.2$

Data (Data.SC_Ratio $>=0.2,:)=[]$;

ㅇ. $\mathrm{P} / \mathrm{C}=<0 . \overline{0} 5->$ Changed from 0.1 to remove unbelievable P formulae

Data (Data.PC Ratio $>=0.05,:$ ) $=[]$;

J. $(\mathrm{S}+\mathrm{P}) / \overline{\mathrm{C}}=<0.1->$ Changed from 0.2 to remove unbelievable $\mathrm{P}$ formulae

$\operatorname{Data}((($ Data.S+Data.P).$/($ Data. C $))>=0.1,:)=[]$;

K. $(\mathrm{N}+\mathrm{P}) / \mathrm{C}<0.4->$ Added to remove unlikely P formulae

$\operatorname{Data}((($ Data.N+Data.P).$/($ Data. C $))>=0.4,:)=[]$;

⒊ Jenna: DBE-O >-10\&<10; AI < 1 (based on Jenna; adjusted for

\section{S143}




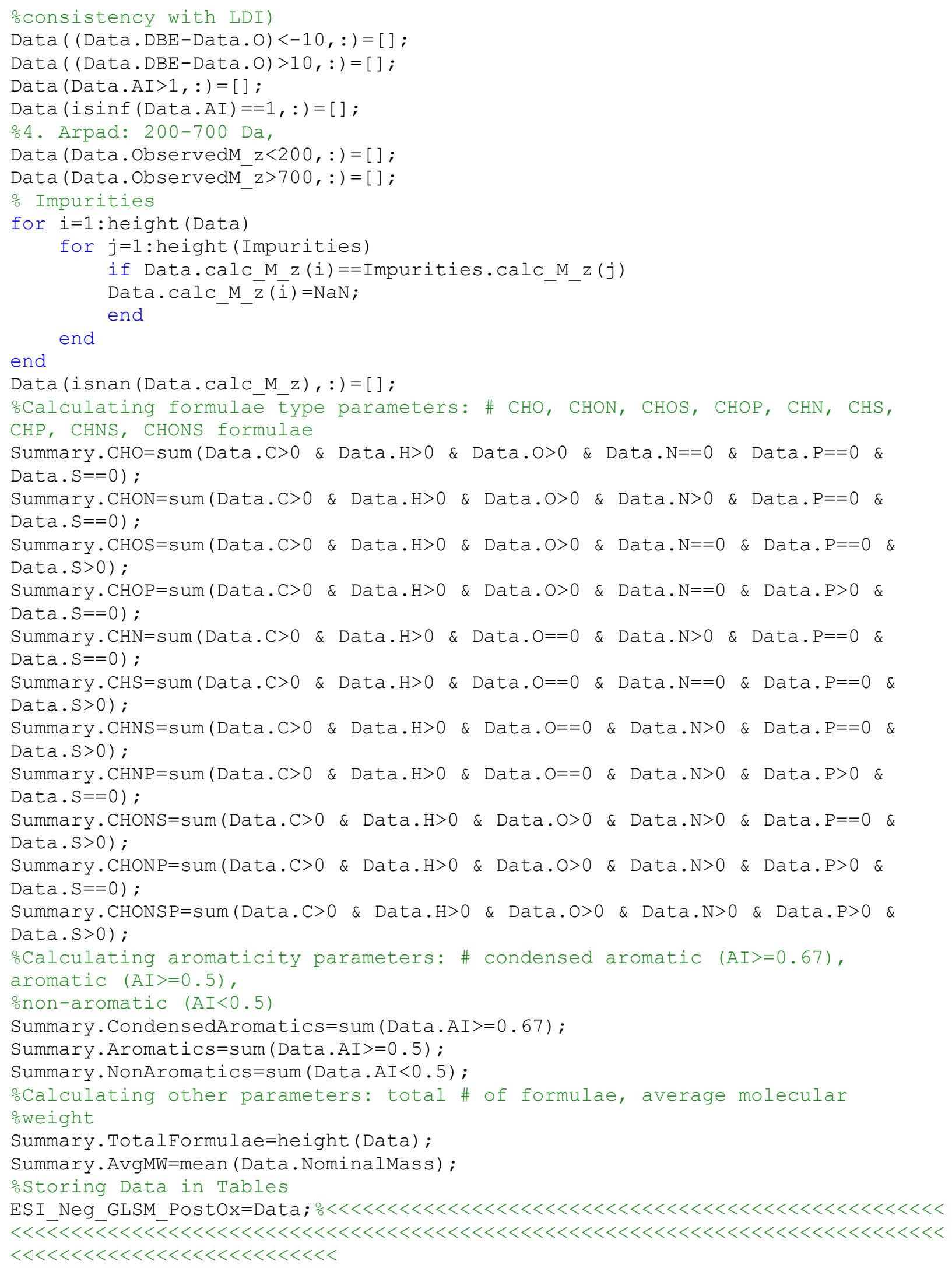


ESI_Neg_GLSM_PostOx_Summary=struct2table (Summary) ; $\circ<<<<<<<<<<<<<<<<<<<<<<<<<<<<<~$ $<<\overline{<}<<\overline{<}<<<<\overline{<}<<<<<<\overline{<}<<<<<<<<<<<<<<<<<<<<<<<<<<<<<<<<<<<<<<<<<<<<<<<<<<<<<<<<<<$

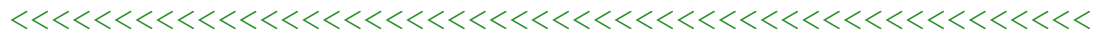

\%

oLoading positive mode ESI impurities

Data $=$ readtable ('DBP Control.xlsx');

Data $\cdot$ NominalMass=Data $\cdot C * 12+$ Data $\cdot 0 * 16+$ Data $\cdot H * 1+$ Data $\cdot P * 31+$ Data $\cdot S * 32+D a t a \cdot N * 14+D$ ata. $\mathrm{Na} * 23$;

Data. KMD CH2=Data. NominalMass-

$0.998883 \overline{4} *($ Data. $C * 12+$ Data. H*1.007825+Data. $N * 14.003074+$ Data. $0 * 15.994915+$ Data.P $\star 30.973763+$ Data.. *31.972072+Data.Na*22.98977);

Data.KMD COO=Data. NominalMass-

1.000231 ( Data. C*12+Data.H*1.007825+Data.N*14.003074+Data.0*15.994915+Data.P* $30.973763+$ Data.S*31.972072+Data.Na*22.98977);

Data.KMD 02=Data. NominalMass-

$1.000317^{\bar{*}}\left(\right.$ Data. $\mathrm{C} * 12+$ Data $. H^{*} 1.007825+$ Data $. N^{*} 14.003074+$ Data. $0 * 15.994915+$ Data. $P *$ $30.973763+$ Data. $\mathrm{S} * 31.972072+$ Data. $N a * 22.98977)$;

Data.KMD H2=Data. NominalMass-

$0.992236^{\star}\left(\right.$ Data. $C * 12+$ Data $. H * 1.007825+$ Data. $N^{*} 14.003074+$ Data. $0 * 15.994915+$ Data. $P *$

$30.973763+$ Data. $S * 31.972072+$ Data. Na*22.98977);

for $i=1$ :height (Data)

if $\mathrm{Data} . \mathrm{Na}(\mathrm{i})==0$

Data.H(i)=Data.H(i) -1 ;

end

end

Data.HC Ratio = Data.H ./ Data.C;

Data.oc Ratio = Data.O./ Data.C;

Data.NC_Ratio = Data.N./ Data.C;

Data.SCRatio = Data.S ./ Data.C;

Data.PC_Ratio = Data.P ./ Data.C;

Data. $\mathrm{DBE}=1+0.5 *(2 *$ Data. $\mathrm{C}-$ Data.H + Data. $\mathrm{N}+$ Data.P $)$;

Data.AI $=(1+$ Data.C - Data.O - Data.S - 0.5*(Data.N + Data.P + Data.H) $)$.

(Data.C - Data.O - Data.N - Data.S - Data.P);

Data.AvgCoxstate = 2*Data.OC_Ratio - Data.HC_Ratio;

Remove data points based on following criteria

\%1. Error (allowable: ppm <0.5)

Data (Data.errPpm $>0.5,:)=[]$;

Data (Data.errPpm<-0.5,:) = [ ] ;

\%2. Stubbins, 2010 :

\%A. $\mathrm{DBE}=$ integer, greater than or equal to 0

Data (Data.DBE =round (Data.DBE), : ) = [];

Data (Data. $\mathrm{DBE}<0,:)=[]$;

B. $\mathrm{C}<=50$

Data (Data.C>50,: ) = [ ]

ㄷ. $\mathrm{H}>=2 \&<=2 * \mathrm{C}+2$

Data (Data. $\mathrm{H}<2,:)=[]$;

Data $($ Data. $\mathrm{H}>($ Data. $\mathrm{C} * 2+2),:)=[]$

$\therefore \mathrm{D} . \mathrm{O}=<\mathrm{C}+2$

Data (Data. O $>($ Data. $C+2),:$ ) $=[]$;

․ $\mathrm{O} / \mathrm{C}<1.2$

Data (Data.OC_Ratio>1.2, :) =[ ] ;

으. H/C $>=0.33 \&<=2.25$

Data (Data.HC Ratio<1/3,:) = [] ;

Data (Data.HC Ratio>2.25, : ) = [ ] ;

$\because \mathrm{G} . \mathrm{N} / \mathrm{C}=<0.5$

Data (Data.NC_Ratio $>=0.5,:)=[]$; 
$\circ \mathrm{H} \cdot \mathrm{S} / \mathrm{C}=<\mathrm{O} \cdot 2$

Data (Data.SC Ratio $>=0.2,:$ ) $=[]$;

ㄷ. $\mathrm{P} / \mathrm{C}=<0 . \overline{0} 5->$ Changed from 0.1 to remove unbelievable P formulae

Data (Data.PC_Ratio>=0.05, :) =[ ] ;

ㄱ. $(\mathrm{S}+\mathrm{P}) / \overline{\mathrm{C}}=<0.1->$ Changed from 0.2 to remove unbelievable $\mathrm{P}$ formulae

$\operatorname{Data}((($ Data.S+Data.P).$/($ Data.C $))>=0.1,:)=[]$;

$\circ \mathrm{K} . \quad(\mathrm{N}+\mathrm{P}) / \mathrm{C}<0.4->$ Added to remove unlikely P formulae

$\operatorname{Data}((($ Data.N+Data.P).$/($ Data. C $))>=0.4,:)=[]$;

⒊ Jenna: DBE-O >-10 \& <10; AI < 1 (based on Jenna; adjusted for

oconsistency with LDI)

Data ( (Data.DBE-Data.O) $<-10,:)=[]$;

Data $(($ Data.DBE-Data.O) $>10,:)=[]$;

Data (Data.AI $>1,:)=[]$;

Data $($ isinf $($ Data.AI $)==1,:)=[]$;

\%4. Arpad: 200-700 Da,

Data (Data. ObservedM $z<200,:$ ) = [] ;

Data (Data.ObservedM_z $>700,:)=[$ ] ;

Impurities=Data ;

oLoading sample positive mode ESI data

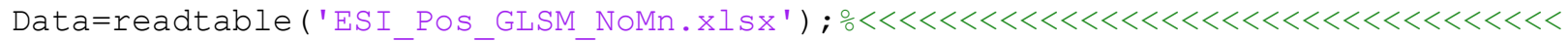
$<<<<<<<<<<<<<<<<<<<\overline{<}<<<\overline{<}<<<\overline{<}<<<<<<<<<<<<<<<<<<<<<<<<<<<<<<<<<<<<<<<<<<<<<<<<~$ $<<<<<<<<<<<<<<<<<<<<<<<<<<<<<<<<<<<<<<<<<<<~$

Data $\cdot$ NominalMass $=$ Data $\cdot C * 12+$ Data $\cdot 0 * 16+$ Data $\cdot H * 1+$ Data $\cdot P * 31+$ Data $\cdot S * 32+D a t a \cdot N * 14+D$ ata. $\mathrm{Na} 23$;

Data. KMD CH2=Data. NominalMass-

$0.998883 \overline{4} *($ Data. $C * 12+$ Data. $H * 1.007825+$ Data. $N * 14.003074+$ Data. $0 * 15.994915+$ Data.P *30.973763+Data.S*31.972072+Data.Na*22.98977);

Data.KMD COO=Data.NominalMass-

$1.000231^{\star}\left(\right.$ Data. $C * 12+$ Data $. H^{*} 1.007825+$ Data. $N^{*} 14.003074+$ Data. $0 * 15.994915+$ Data. $P *$ $30.973763+$ Data. $\mathrm{S} * 31.972072+$ Data. $\mathrm{Na} * 22.98977$ );

Data.KMD_O2=Data.NominalMass-

$1.0003177^{\bar{*}}\left(\right.$ Data. $C * 12+$ Data. $H^{*} 1.007825+$ Data. $N^{*} 14.003074+$ Data. $0 * 15.994915+$ Data. $P *$ $30.973763+$ Data. $\mathrm{S} * 31.972072+$ Data. Na*22.98977);

Data.KMD H2=Data. NominalMass-

$0.992236^{\star}$ (Data. C*12+Data.H*1.007825+Data.N*14.003074+Data.0*15.994915+Data.P* $30.973763+$ Data.. *31.972072+Data.Na*22.98977);

for $i=1$ : height (Data)

if Data.Na(i)==0

Data.H $($ i $)=$ Data.H $($ i $)-1$;

end

end

Data.HC_Ratio = Data.H ./ Data.C;

Data.oC Ratio = Data.O ./ Data.C;

Data.NC Ratio = Data.N ./ Data.C;

Data.SC Ratio = Data.S./ Data.C;

Data.PC_Ratio = Data.P./ Data.C;

Data.DBE $=1+0.5 *(2 *$ Data.C - Data.H + Data. $N+$ Data.P $)$;

Data.AI $=(1+$ Data.C - Data.O - Data.S - 0.5* (Data.N + Data.P + Data.H) $) \cdot /$

(Data.C - Data.O - Data.N - Data.S - Data.P);

Data.AvgCoxstate = 2*Data.OC_Ratio - Data.HC_Ratio;

o Saving raw data for Scan 1

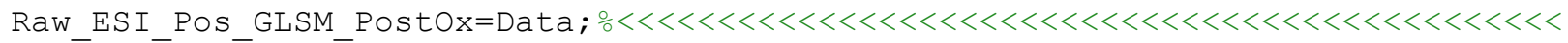
$<<\overline{<}<<<\overline{<}<<\overline{<}<<<\overline{<} \overline{<}<<<<<<<<<<<<<<<<<<<<<<<<<<<<<<<<<<<<<<<<<<<<<<<<<<<<<<<<<<<<$

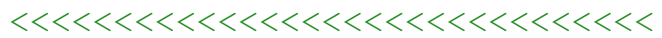

Remove data points based on following criteria

ㄴ. Error (allowable: ppm <0.5)

Data (Data.errPpm>0.5,:) = []; 


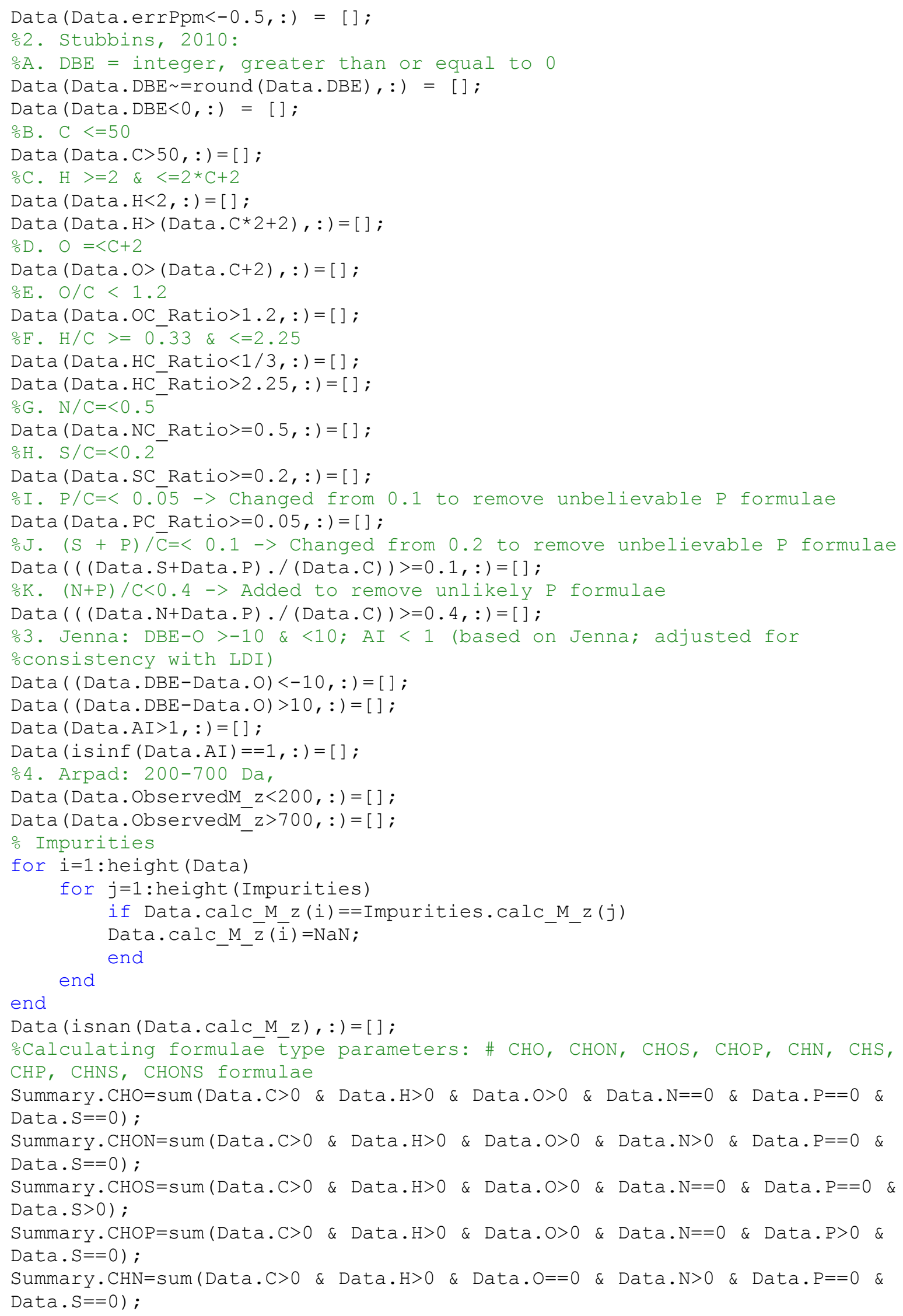


Summary $\cdot \mathrm{CHS}=\operatorname{sum}($ Data $\cdot \mathrm{C}>0$ \& Data. $\mathrm{H}>0$ \& Data. $\mathrm{O}==0$ \& Data. $\mathrm{N}==0$ \& Data. $\mathrm{P}==0$ \& Data. $\mathrm{S}>0$ );

Summary. CHNS $=$ sum $($ Data. $\mathrm{C}>0$ \& Data. $\mathrm{H}>0$ \& Data. $\mathrm{O}==0$ \& Data. $\mathrm{N}>0$ \& Data.P==0 \& Data. S $>0$ );

Summary. $\mathrm{CHNP}=\operatorname{sum}($ Data. $\mathrm{C}>0$ \& Data. $\mathrm{H}>0$ \& Data. $\mathrm{O}==0$ \& Data. $\mathrm{N}>0$ \& Data.P $>0$ \& Data. $\mathrm{S}==0$ );

Summary. CHONS $=$ sum $($ Data. $\mathrm{C}>0$ \& Data. $\mathrm{H}>0$ \& Data.O $>0$ \& Data.N $>0$ \& Data.P==0 \&

Data. $\mathrm{S}>0$ ) ;

Summary. $\mathrm{CHONP}=$ sum $($ Data. $\mathrm{C}>0$ \& Data. $\mathrm{H}>0$ \& Data. $\mathrm{O}>0$ \& Data.N $>0$ \& Data.P $>0$ \&

Data. $\mathrm{S}==0$ );

Summary. $\mathrm{CHONSP}=$ sum $($ Data. $\mathrm{C}>0$ \& Data. $\mathrm{H}>0$ \& Data. $\mathrm{O}>0$ \& Data.N $>0$ \& Data.P $>0$ \&

Data. S>0);

ocalculating aromaticity parameters: \# condensed aromatic (AI>=0.67), aromatic $(\mathrm{AI}>=0.5)$,

onon-aromatic $(\mathrm{A} I<0.5)$

Summary. CondensedAromatics $=$ sum (Data. AI $>=0.67)$;

Summary. Aromatics=sum (Data.AI $>=0.5)$;

Summary. NonAromatics=sum (Data.AI<0.5);

ocalculating other parameters: total \# of formulae, average molecular

oweight

Summary.TotalFormulae=height (Data);

Summary. AvgMW=mean (Data. NominalMass);

ostoring Data in Tables

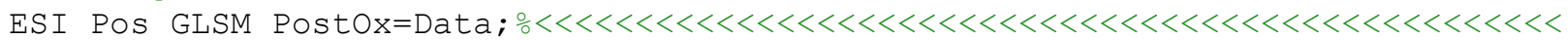
$<<\overline{<}<<\overline{<}<<<<<\overline{<}<<<<<<<<<<<<<<<<<<<<<<<<<<<<<<<<<<<<<<<<<<<<<<<<<<<<<<<<<<<<<<<<~$ $<<<<<<<<<<<<<<<<<<<<<<<<<<<~$

ESI_Pos_GLSM_PostOx_Summary=struct2table (Summary) ; $\circ<<<<<<<<<<<<<<<<<<<<<<<<<<<~$ $<<\overline{\bar{l}}<<\overline{\bar{l}}<<<\overline{<} \overline{<}<<<<<\overline{<} \overline{<}<<<<<<<<<<<<<<<<<<<<<<<<<<<<<<<<<<<<<<<<<<<<<<<<<<<<<<<<<$

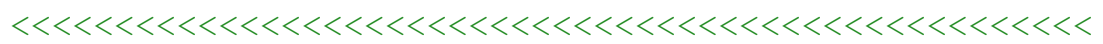

응 Negative Mode LDI: Data Clean Up

oLDI assumed to have no background ions

Data=readtable ('LDI Neg GLSM NoMn.xlsX') ; 은 $<<<<<<<<<<<<<<<<<<<<<<<<<<<<<<<<<<<<<~$

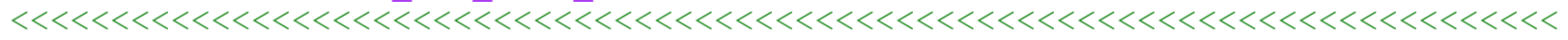

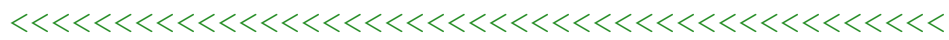

Data=unique (Data);

Data $\cdot$ NominalMass=Data $\cdot C * 12+$ Data $\cdot 0 * 16+$ Data $\cdot H * 1+$ Data $\cdot P * 31+$ Data $\cdot S * 32+$ Data $\cdot N * 14$;

Data. KMD CH2=Data. NominalMass-

$0.998883 \overline{4} *\left(\right.$ Data. $C * 12+$ Data. $H * 1.007825+$ Data. $N^{*} 14.003074+$ Data. $0 * 15.994915+$ Data. P $\star 30.973763+$ Data.. *31.972072);

Data.KMD_COO=Data. NominalMass-

$1.000231^{\star}($ Data. C*12+Data.H*1.007825+Data. N*14.003074+Data. O*15.994915+Data.P* $30.973763+$ Data. $S * 31.972072$ );

Data.KMD 02=Data. NominalMass-

$1.000317^{\star}\left(\right.$ Data. $C * 12+$ Data.H*1.007825+Data. $\mathrm{N}^{\star} 14.003074+$ Data. $0 * 15.994915+$ Data. $\mathrm{P} *$ $30.973763+$ Data.S*31.972072);

Data.KMD H2=Data. NominalMass-

$0.992236^{\star}\left(\right.$ Data. $C * 12+$ Data $. H * 1.007825+$ Data. $N^{*} 14.003074+$ Data. $0 * 15.994915+$ Data. $P *$

$30.973763+$ Data. $S * 31.972072)$;

Data. DBE $=1+0.5 *(2 *$ Data. C - Data.H + Data.N + Data.P $)$;

for $i=1$ : height (Data)

if Data.DBE (i) =round (Data.DBE (i))

Data.H $($ i $)=$ Data.H $(i)+1$;

Data. IonType $(i)=0 ; \circ H-$ Adduct $s=0$

else

Data. IonType $(i)=2 ; \%$ MolecularIons=2 \%Temporary fix until I figure out how to enter names into a table 


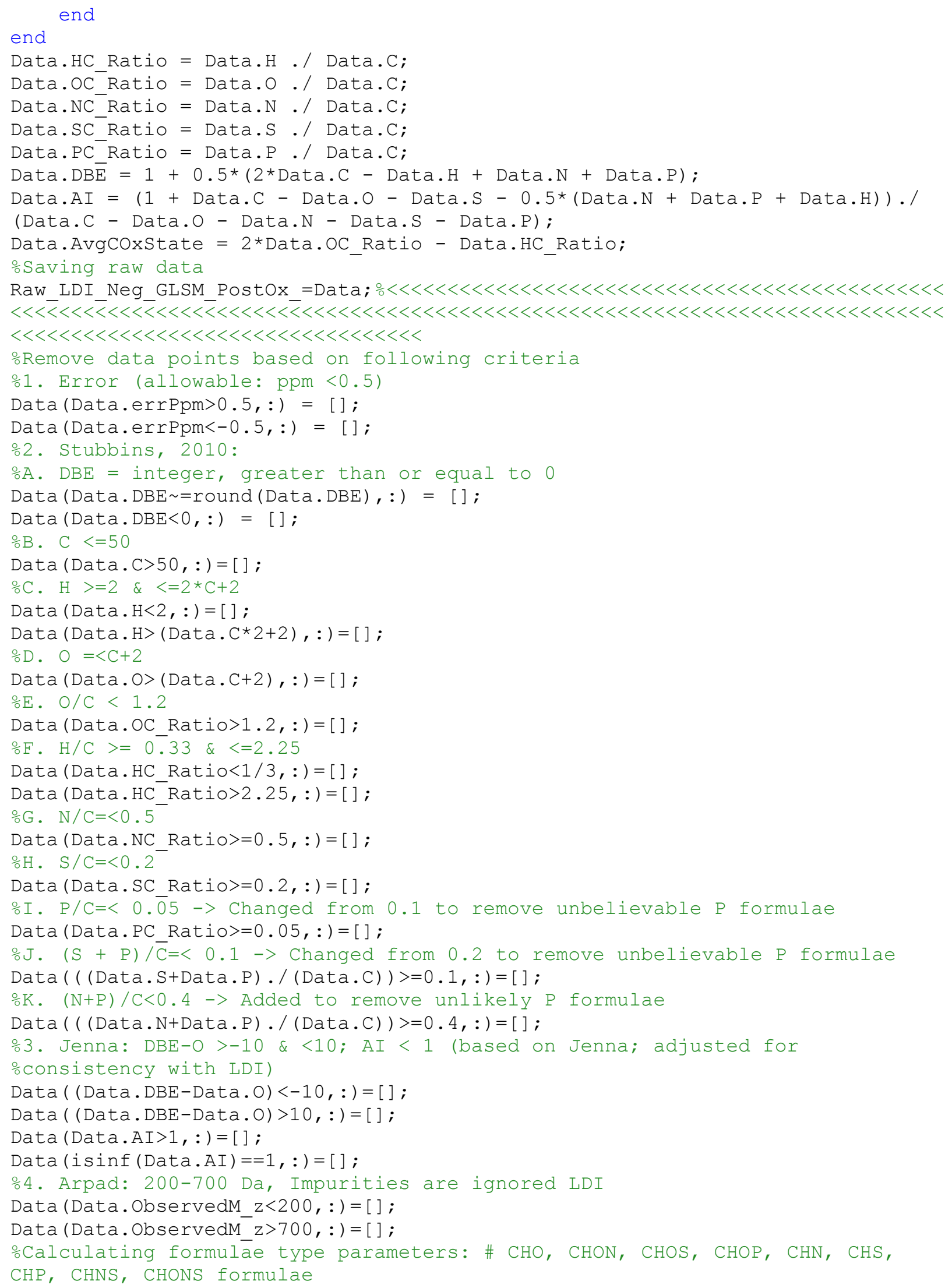


Summary. $\mathrm{CHO}=\operatorname{sum}($ Data. $\mathrm{C}>0$ \& Data.H $>0$ \& Data. $\mathrm{O}>0$ \& Data. $\mathrm{N}==0$ \& Data.P==0 \& Data. $\mathrm{S}==0$ ); Summary. $\mathrm{CHON}=\operatorname{sum}($ Data. $\mathrm{C}>0$ \& Data.H $>0$ \& Data. $0>0$ \& Data. $\mathrm{N}>0$ \& Data.P==0 \& Data. $\mathrm{S}==0$ );

Summary $\cdot \mathrm{CHOS}=$ sum $($ Data. $\mathrm{C}>0$ \& Data. $\mathrm{H}>0$ \& Data. $\mathrm{O}>0$ \& Data. $\mathrm{N}==0$ \& Data. $\mathrm{P}==0$ \& Data. $\mathrm{S}>0$ ); Summary. $\mathrm{CHOP}=\operatorname{sum}($ Data. $\mathrm{C}>0$ \& Data. $\mathrm{H}>0$ \& Data.O $>0$ \& Data. $\mathrm{N}==0$ \& Data.P $>0$ \& Data. $\mathrm{S}==0$ ); Summary $\cdot \mathrm{CHN}=$ sum $($ Data. $\mathrm{C}>0$ \& Data. $\mathrm{H}>0$ \& Data. $\mathrm{O}==0$ \& Data. $\mathrm{N}>0$ \& Data. $\mathrm{P}==0$ \& Data. $\mathrm{S}==0$ );

Summary $\cdot \mathrm{CHS}=\operatorname{sum}($ Data $\cdot \mathrm{C}>0$ \& Data. $\mathrm{H}>0$ \& Data $\cdot \mathrm{O}==0$ \& Data. $\mathrm{N}==0$ \& Data. $\mathrm{P}==0$ \& Data. $\mathrm{S}>0$ ) ; Summary $\cdot \mathrm{CHNS}=$ sum $($ Data $\cdot \mathrm{C}>0$ \& Data. $\mathrm{H}>0$ \& Data. $\mathrm{O}==0$ \& Data. $\mathrm{D}>0$ \& Data. $\mathrm{P}==0$ \& Data. S>0); Summary $\cdot \mathrm{CHNP}=\operatorname{sum}($ Data $\cdot \mathrm{C}>0$ \& Data. $\mathrm{H}>0$ \& Data. $\mathrm{O}==0$ \& Data.N $>0$ \& Data.P $>0$ \& Data. $\mathrm{S}=0$ ) ; Summary. $\mathrm{CHONS}=\operatorname{sum}($ Data. $\mathrm{C}>0$ \& Data. $\mathrm{H}>0$ \& Data. $\mathrm{O}>0$ \& Data. $\mathrm{N}>0$ \& Data.P==0 \& Data. $\mathrm{S}>0$ ); Summary. $\mathrm{CHONP}=$ sum (Data. $\mathrm{C}>0$ \& Data.H>0 \& Data. O $>0$ \& Data.N $>0$ \& Data.P $>0$ \& Data. $\mathrm{S}==0$ ); Summary. $\mathrm{CHONSP}=$ sum $($ Data. $\mathrm{C}>0$ \& Data. $\mathrm{H}>0$ \& Data. $\mathrm{O}>0$ \& Data.N $>0$ \& Data.P $>0$ \& Data. S $>0)$;

ocalculating aromaticity parameters: \# condensed aromatic (AI>=0.67), aromatic $(A I>=0.5)$,

onon-aromatic $(\mathrm{AI}<0.5)$

Summary. CondensedAromatics $=$ sum (Data. AI $>=0.67)$;

Summary. Aromatics=sum (Data.AI>=0.5);

Summary. NonAromatics $=$ sum (Data. AI $<0.5)$;

ocalculating other parameters: total \# of formulae, average molecular oweight

Summary. TotalFormulae=height (Data);

Summary.AvgMW=mean (Data. NominalMass);

ostoring Data in Tables

LDI Neg GLSM PostOx=Data $;$ 응 $<<<<<<<<<<<<<<<<<<<<<<<<<<<<<<<<<<<<<<<<<<<<<<<<<<<<<<~$

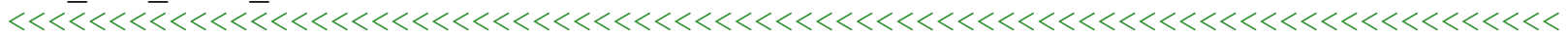
$<<<<<<<<<<<<<<<<<<<<<<<<<~$

LDI_Neg_GLSM_PostOx_Summary=struct2table (Summary) ; $\circ<<<<<<<<<<<<<<<<<<<<<<<<<<<~$ $<<<\overline{<}<<<\overline{<}<<<<\overline{<}<<<<<<\overline{<}<<<<<<<<<<<<<<<<<<<<<<<<<<<<<<<<<<<<<<<<<<<<<<<<<<<<<<<<<~$

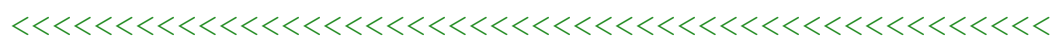

응 Positive Mode LDI: Data Clean Up

oLDI assumed to have no background ions

oLoding sample positive mode LDI data

Data=readtable ('LDI Pos GLSM NoMn.xlsx') ; $\circ<<<<<<<<<<<<<<<<<<<<<<<<<<<<<<<<<<<<<~$

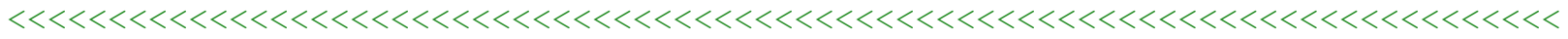

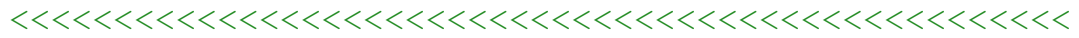

Data=unique (Data);

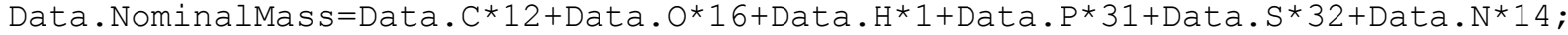

Data. $\mathrm{KMD} \mathrm{CH} 2=$ Data. NominalMass-

$0.998883 \overline{4} *\left(\right.$ Data. $C * 12+$ Data. $H^{*} 1.007825+$ Data. $N^{*} 14.003074+$ Data. $0 * 15.994915+$ Data.$P$ $* 30.973763+$ Data.S*31.972072);

Data.KMD COO=Data. NominalMass-

$1.000231^{\star}($ Data. C*12+Data.H*1.007825+Data. N*14.003074+Data.0*15.994915+Data.P* $30.973763+$ Data. $S * 31.972072)$;

Data.KMD O2=Data. NominalMass-

$1.000317^{\bar{*}}\left(\right.$ Data. $\mathrm{C}^{\star} 12+$ Data. $\mathrm{H}^{\star} 1.007825+$ Data $. \mathrm{N}^{\star} 14.003074+\mathrm{Data} .0 * 15.994915+\mathrm{Data} . \mathrm{P} *$ $30.973763+$ Data.. *31.972072); 


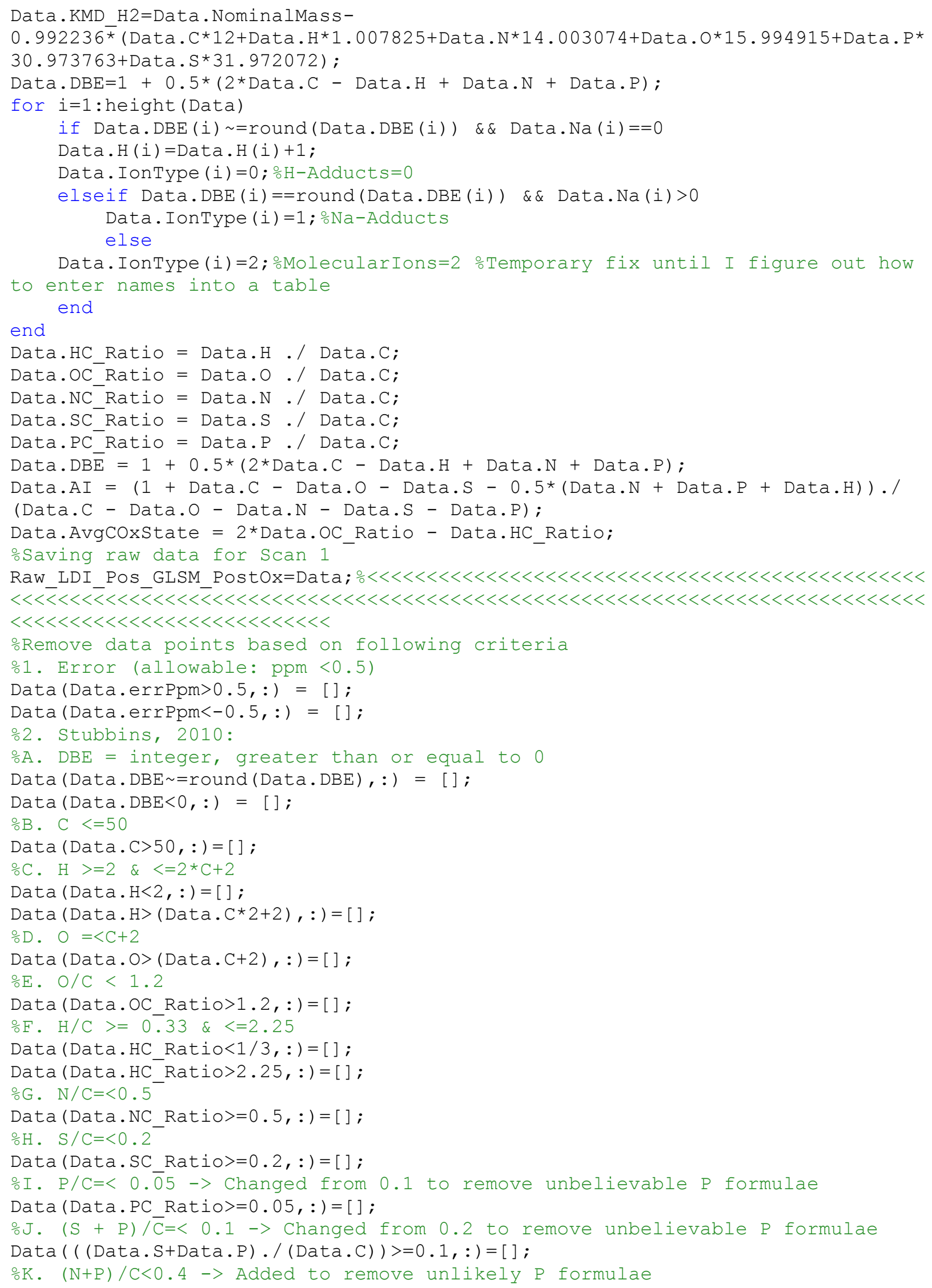

\section{S151}


$\operatorname{Data}((($ Data.N+Data.P).$/($ Data.C $))>=0.4,:)=[]$;

⒊ Jenna: DBE-0 >-10 \& <10; AI < 1 (based on Jenna; adjusted for

oconsistency with LDI)

Data ( (Data.DBE-Data.O) $<-10,:)=[]$;

Data ( (Data.DBE-Data.O) $>10,:)=[]$;

Data (Data.AI>1, : ) $=[]$;

$\operatorname{Data}($ isinf $($ Data. AI $)==1,:)=[]$;

\%4. Arpad: 200-700 Da, Impurities are ignored LDI- assumed to be negligible ounless a background reference is used

Data (Data. ObservedM_z<200,: ) = [] ;

Data (Data. ObservedM_z>700,: ) = [] ;

oCalculating formulāe type parameters: \# CHO, CHON, CHOS, CHOP, CHN, CHS, CHP, CHNS, CHONS formulae

Summary $\cdot \mathrm{CHO}=$ sum $($ Data. $\mathrm{C}>0$ \& Data. $\mathrm{H}>0$ \& Data. $\mathrm{O}>0$ \& Data. $\mathrm{N}==0$ \& Data. $\mathrm{P}==0$ \&

Data. $\mathrm{S}==0$ );

Summary $\cdot \mathrm{CHON}=$ sum $($ Data. $\mathrm{C}>0$ \& Data.H $>0$ \& Data. $\mathrm{O}>0$ \& Data. $\mathrm{N}>0$ \& Data.P==0 \&

Data. $\mathrm{S}=0$ ) ;

Summary $\cdot \mathrm{CHOS}=$ sum $($ Data. $\mathrm{C}>0$ \& Data.H $>0$ \& Data. O $>0$ \& Data. $\mathrm{N}==0$ \& Data. $\mathrm{P}==0$ \&

Data. $\mathrm{S}>0$ ) ;

Summary $\cdot \mathrm{CHOP}=\operatorname{sum}($ Data. $\mathrm{C}>0$ \& Data.H $>0$ \& Data. $\mathrm{O}>0$ \& Data. $\mathrm{N}==0$ \& Data.P $>0$ \&

Data. $\mathrm{S}==0$ );

Summary $\cdot \mathrm{CHN}=$ sum $($ Data. $\mathrm{C}>0$ \& Data. $\mathrm{H}>0$ \& Data. $\mathrm{O}==0$ \& Data. $\mathrm{N}>0$ \& Data. $\mathrm{P}==0$ \&

Data. $\mathrm{S}==0$ );

Summary. $\mathrm{CHS}=\operatorname{sum}($ Data. $\mathrm{C}>0$ \& Data. $\mathrm{H}>0$ \& Data. $\mathrm{O}==0$ \& Data. $\mathrm{N}==0$ \& Data. $\mathrm{P}==0$ \&

Data. S>0);

Summary $\cdot \mathrm{CHNS}=\operatorname{sum}($ Data. $\mathrm{C}>0$ \& Data. $\mathrm{H}>0$ \& Data. $\mathrm{O}==0$ \& Data. $\mathrm{N}>0$ \& Data.P==0 \&

Data. $\mathrm{S}>0$ );

Summary $\cdot \mathrm{CHNP}=\mathrm{sum}($ Data. $\mathrm{C}>0$ \& Data.H $>0$ \& Data. $\mathrm{O}==0$ \& Data.N $>0$ \& Data.P $>0$ \&

Data. $\mathrm{S}==0$ );

Summary $\cdot \mathrm{CHONS}=\operatorname{sum}($ Data. $\mathrm{C}>0$ \& Data. $\mathrm{H}>0$ \& Data. $0>0$ \& Data.N $>0$ \& Data.P==0 \&

Data. S>0);

Summary. $\mathrm{CHONP}=$ sum $($ Data. $\mathrm{C}>0$ \& Data. $\mathrm{H}>0$ \& Data. $\mathrm{O}>0$ \& Data. $\mathrm{N}>0$ \& Data.P $>0$ \&

Data. $\mathrm{S}=0$ ) ;

Summary. $\mathrm{CHONSP}=$ sum $($ Data. $\mathrm{C}>0$ \& Data.H $>0$ \& Data.O $>0$ \& Data.N $>0$ \& Data.P>0 \&

Data. $\mathrm{S}>0$ );

oCalculating aromaticity parameters: \# condensed aromatic (AI>=0.67),

aromatic $(A I>=0.5)$,

onon-aromatic $(\mathrm{AI}<0.5)$

Summary. CondensedAromatics $=$ sum (Data.AI $>=0.67)$;

Summary. Aromatics $=$ sum (Data.AI $>=0.5)$;

Summary. NonAromatics=sum (Data.AI<0.5);

ocalculating other parameters: total \# of formulae, average molecular

oweight

Summary.TotalFormulae=height (Data);

Summary. AvgMW=mean (Data. NominalMass);

ostoring Data in Tables

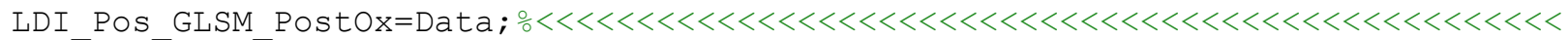
$<<<\overline{<}<<\overline{<}<<<<\overline{<}<<<<<<<<<<<<<<<<<<<<<<<<<<<<<<<<<<<<<<<<<<<<<<<<<<<<<<<<~$

LDI_Pos_GLSM_PostOx_Summary=struct2table (Summary) ; $\circ<<<<<<<<<<<<<<<<<<<<<<<<<<<~$ $<<\overline{<}<<\overline{\bar{l}}<<<<\overline{<}<<<<<\overline{<}<<<<<<<<<<<<<<<<<<<<<<<<<<<<<<<<<<<<<<<<<<<<<<<<<<<<<<<<<<$ $<<<<<<<<<<<<<<<<<~$

응 Combining Negative and Positive Mode ESI

oInput Data so that the script can readily be applied to any data set

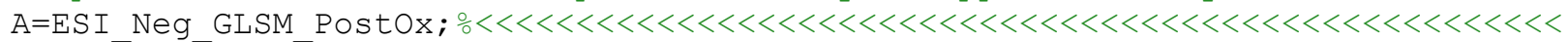
$<<<<\overline{<}<<<\overline{<}<<<\overline{<}<<<<<<<<<<<<<<<<<<<<<<<<<<<<<<<<<<<<<<<<<<<<<<<<<<<<<<<<<<<<<<~$

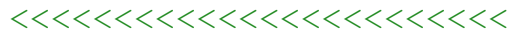




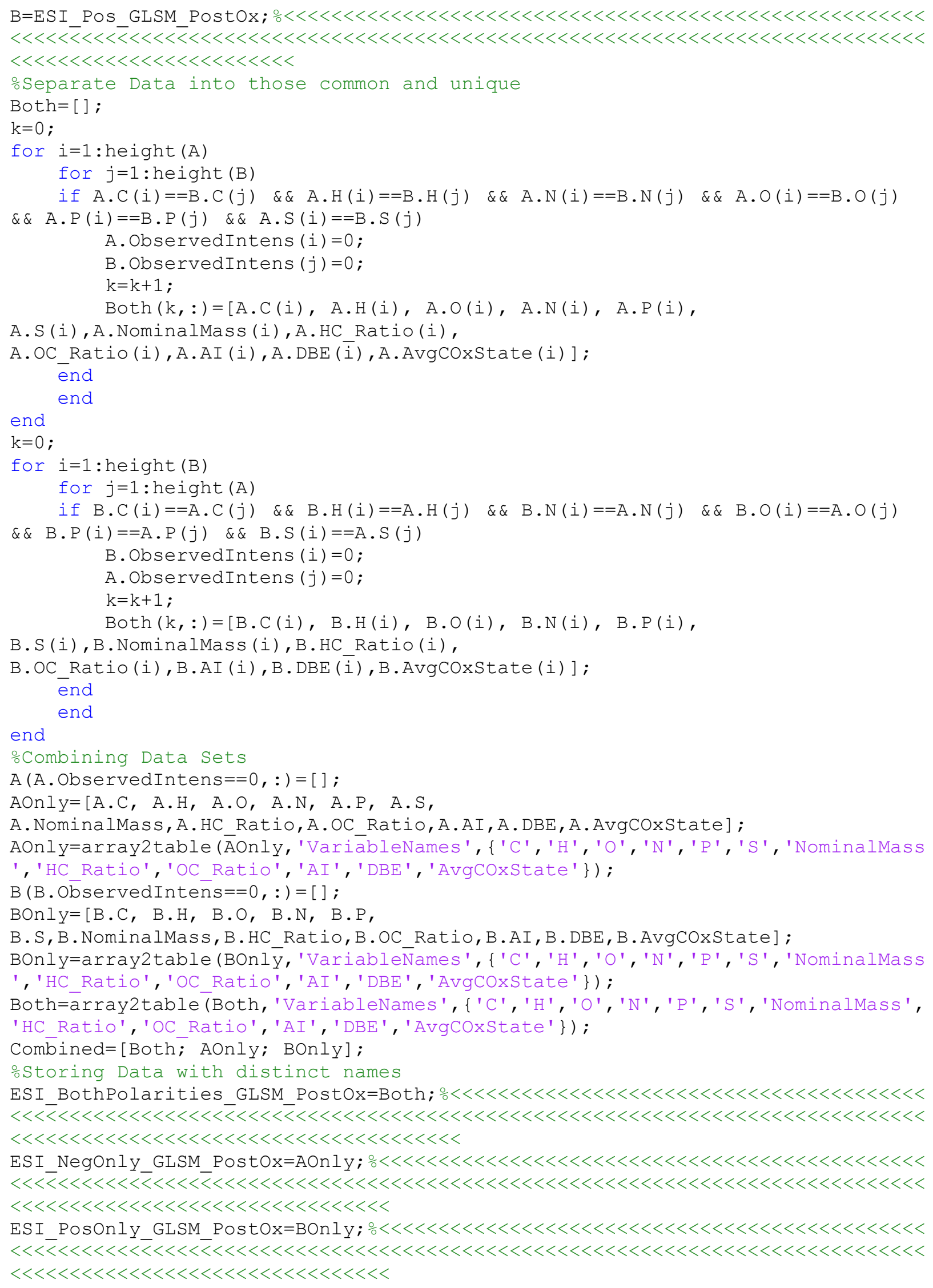




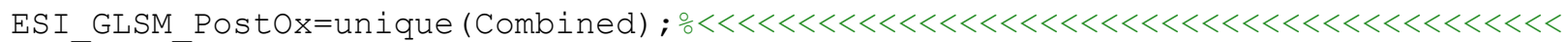
$<<\overline{<}<<<\overline{<} \bar{l}<<<<<<<<<<<<<<<<<<<<<<<<<<<<<<<<<<<<<<<<<<<<<<<<<<<<<<<<<<<<<<<<<<<<~$

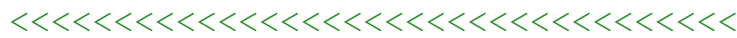

ㅇumarizing Data

Data=AOnly;

oCalculating formulae type parameters: \# CHO, CHON, CHOS, CHOP, CHN, CHS, CHP, CHNS, CHONS formulae

Summary $\cdot \mathrm{CHO}=$ sum $($ Data. $\mathrm{C}>0$ \& Data. $\mathrm{H}>0$ \& Data. $\mathrm{O}>0$ \& Data. $\mathrm{N}==0$ \& Data. $\mathrm{P}==0$ \&

Data. $\mathrm{S}=0$ ) ;

Summary $\cdot \mathrm{CHON}=$ sum $($ Data. $\mathrm{C}>0$ \& Data.H $>0$ \& Data. $\mathrm{O}>0$ \& Data. $\mathrm{N}>0$ \& Data.P==0 \&

Data. $\mathrm{S}==0$ );

Summary. $\mathrm{CHOS}=$ sum $($ Data. $\mathrm{C}>0$ \& Data. $\mathrm{H}>0$ \& Data. $\mathrm{O}>0$ \& Data. $\mathrm{N}==0$ \& Data. $\mathrm{P}==0$ \&

Data. $\mathrm{S}>0$ );

Summary. $\mathrm{CHOP}=\mathrm{sum}($ Data. $\mathrm{C}>0$ \& Data.H $>0$ \& Data. $0>0$ \& Data. $\mathrm{N}==0$ \& Data.P $>0$ \&

Data. $\mathrm{S}==0$ );

Summary $\cdot \mathrm{CHN}=$ sum $($ Data. $\mathrm{C}>0$ \& Data. $\mathrm{H}>0$ \& Data. $\mathrm{O}==0$ \& Data. $\mathrm{N}>0$ \& Data. $\mathrm{P}==0$ \&

Data. $\mathrm{S}=0$ ) ;

Summary $\cdot \mathrm{CHS}=\operatorname{sum}($ Data. $\mathrm{C}>0$ \& Data. $\mathrm{H}>0$ \& Data. $\mathrm{O}==0$ \& Data. $\mathrm{N}==0$ \& Data. $\mathrm{P}==0$ \&

Data. $\mathrm{S}>0$ ) ;

Summary $\cdot \mathrm{CHNS}=\operatorname{sum}($ Data. $\mathrm{C}>0$ \& Data. $\mathrm{H}>0$ \& Data. $\mathrm{O}==0$ \& Data. $\mathrm{N}>0$ \& Data.P==0 \&

Data. S $>0$ );

Summary $\cdot \mathrm{CHNP}=\operatorname{sum}($ Data. $\mathrm{C}>0$ \& Data.H $>0$ \& Data. $\mathrm{O}==0$ \& Data. $\mathrm{N}>0$ \& Data.P $>0$ \&

Data. $\mathrm{S}==0$ );

Summary. $\mathrm{CHONS}=\operatorname{sum}($ Data. $\mathrm{C}>0$ \& Data. $\mathrm{H}>0$ \& Data. $0>0$ \& Data.N>0 \& Data.P==0 \&

Data. S>0);

Summary. $\mathrm{CHONP}=$ sum $($ Data. $\mathrm{C}>0$ \& Data.H $>0$ \& Data.O $>0$ \& Data.N $>0$ \& Data.P>0\&

Data. $\mathrm{S}==0$ );

Summary. $\mathrm{CHONSP}=$ sum $($ Data. $\mathrm{C}>0$ \& Data.H $>0$ \& Data.O $>0$ \& Data.N $>0$ \& Data.P>0 \&

Data. $\mathrm{S}>0)$;

ocalculating aromaticity parameters: \# condensed aromatic (AI>=0.67),

aromatic $(\mathrm{AI}>=0.5)$,

onon-aromatic $(\mathrm{A} I<0.5)$

Summary. CondensedAromatics $=$ sum (Data. AI $>=0.67)$;

Summary. Aromatics $=$ sum (Data.AI $>=0.5)$;

Summary. NonAromatics=sum (Data.AI<0.5);

ocalculating other parameters: total \# of formulae, average molecular

oweight

Summary.TotalFormulae=height (Data);

Summary. AvgMW=mean (Data. NominalMass);

ostoring Summary Data

ESI_NegOnly_GLSM_PostOx_Summary=struct2table (Summary) ; $\circ<<<<<<<<<<<<<<<<<<<<<<<$

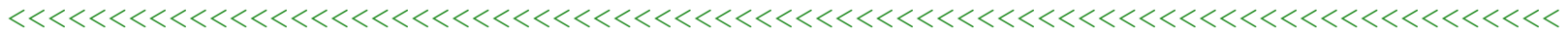

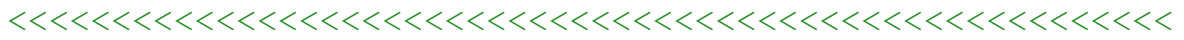

․ Summarizing Data

Data=BOnly;

oCalculating formulae type parameters: \# CHO, CHON, CHOS, CHOP, CHN, CHS, CHP, CHNS, CHONS formulae

Summary. $\mathrm{CHO}=\operatorname{sum}($ Data. $\mathrm{C}>0$ \& Data. $\mathrm{H}>0$ \& Data. $\mathrm{O}>0$ \& Data. $\mathrm{N}==0$ \& Data. $\mathrm{P}==0$ \& Data. $\mathrm{S}==0$ );

Summary. $\mathrm{CHON}=\operatorname{sum}($ Data. $\mathrm{C}>0$ \& Data. $\mathrm{H}>0$ \& Data. $\mathrm{O}>0$ \& Data.N $>0$ \& Data.P==0 \&

Data. $\mathrm{S}==0$ ) ;

Summary $\cdot \mathrm{CHOS}=$ sum $($ Data. $\mathrm{C}>0$ \& Data.H $>0$ \& Data. $\mathrm{O}>0$ \& Data. $\mathrm{N}==0$ \& Data.P==0 \&

Data. S>0);

Summary $\cdot \mathrm{CHOP}=\mathrm{sum}($ Data. $\mathrm{C}>0$ \& Data. $\mathrm{H}>0$ \& Data. $\mathrm{O}>0$ \& Data. $\mathrm{N}==0$ \& Data.P $>0$ \&

Data. $\mathrm{S}=0$ ) ;

Summary. $\mathrm{CHN}=\operatorname{sum}($ Data. $\mathrm{C}>0$ \& Data. $\mathrm{H}>0$ \& Data. $\mathrm{O}==0$ \& Data. $\mathrm{N}>0$ \& Data. $\mathrm{P}==0$ \&

Data. $\mathrm{S}==0$ ) ; 
Summary $\cdot \mathrm{CHS}=\operatorname{sum}($ Data $\cdot \mathrm{C}>0$ \& Data. $\mathrm{H}>0$ \& Data. $\mathrm{O}==0$ \& Data. $\mathrm{N}==0$ \& Data. $\mathrm{P}==0$ \& Data. $\mathrm{S}>0$ ) ;

Summary. CHNS $=$ sum $($ Data. $\mathrm{C}>0$ \& Data. $\mathrm{H}>0$ \& Data. $\mathrm{O}==0$ \& Data. $\mathrm{N}>0$ \& Data.P==0 \& Data. S $>0$ );

Summary. $\mathrm{CHNP}=\operatorname{sum}($ Data. $\mathrm{C}>0$ \& Data. $\mathrm{H}>0$ \& Data. $\mathrm{O}==0$ \& Data. $\mathrm{N}>0$ \& Data.P $>0$ \& Data. $\mathrm{S}==0$ );

Summary. CHONS $=$ sum $($ Data. $\mathrm{C}>0$ \& Data. $\mathrm{H}>0$ \& Data.O $>0$ \& Data.N $>0$ \& Data.P==0 \& Data. $\mathrm{S}>0$ ) ;

Summary. $\mathrm{CHONP}=$ sum $($ Data. $\mathrm{C}>0$ \& Data. $\mathrm{H}>0$ \& Data. $\mathrm{O}>0$ \& Data.N $>0$ \& Data.P $>0$ \& Data. $\mathrm{S}==0$ );

Summary. $\mathrm{CHONSP}=$ sum $($ Data. $\mathrm{C}>0$ \& Data.H $>0$ \& Data. O $>0$ \& Data.N $>0$ \& Data.P $>0$ \& Data. S>0);

ocalculating aromaticity parameters: \# condensed aromatic (AI>=0.67), aromatic $(\mathrm{AI}>=0.5)$,

onon-aromatic $(\mathrm{A} I<0.5)$

Summary. CondensedAromatics $=$ sum (Data. AI $>=0.67)$;

Summary. Aromatics $=$ sum (Data. AI $>=0.5)$;

Summary. NonAromatics=sum (Data.AI<0.5);

ocalculating other parameters: total \# of formulae, average molecular

oweight

Summary.TotalFormulae=height (Data);

Summary. AvgMW=mean (Data. NominalMass);

o.Storing Summary Data

ESI PosOnly GLSM PostOx Summary=struct2table (Summary); $\circ<<<<<<<<<<<<<<<<<<<<<<<<<~$

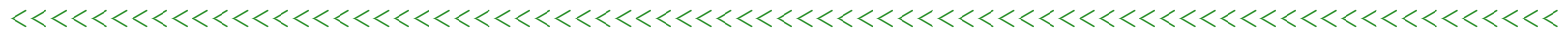

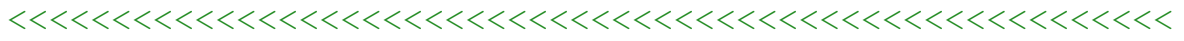

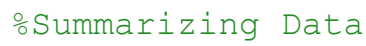

Data=Both;

oCalculating formulae type parameters: \# CHO, CHON, CHOS, CHOP, CHN, CHS, CHP, CHNS, CHONS formulae

Summary $\cdot \mathrm{CHO}=\operatorname{sum}($ Data. $\mathrm{C}>0$ \& Data. $\mathrm{H}>0$ \& Data. $\mathrm{O}>0$ \& Data. $\mathrm{N}==0$ \& Data. $\mathrm{P}==0$ \&

Data. $\mathrm{S}==0$ );

Summary $\cdot \mathrm{CHON}=\operatorname{sum}($ Data. $\mathrm{C}>0$ \& Data.H $>0$ \& Data. $0>0$ \& Data. $\mathrm{N}>0$ \& Data. $\mathrm{P}==0$ \&

Data. $\mathrm{S}==0$ );

Summary. $\mathrm{CHOS}=$ sum $($ Data. $\mathrm{C}>0$ \& Data. $\mathrm{H}>0$ \& Data.O $>0$ \& Data. $\mathrm{N}==0$ \& Data. $\mathrm{P}==0$ \&

Data. S>0);

Summary $\cdot \mathrm{CHOP}=$ sum $($ Data. $\mathrm{C}>0$ \& Data. $\mathrm{H}>0$ \& Data. $\mathrm{O}>0$ \& Data. $\mathrm{N}==0$ \& Data. P $>0$ \&

Data. $\mathrm{S}==0$ ) ;

Summary. $\mathrm{CHN}=$ sum $($ Data. $\mathrm{C}>0$ \& Data. $\mathrm{H}>0$ \& Data. $\mathrm{O}==0$ \& Data. $\mathrm{N}>0$ \& Data. $\mathrm{P}==0$ \&

Data. $\mathrm{S}==0$ );

Summary $\cdot \mathrm{CHS}=\operatorname{sum}($ Data. $\mathrm{C}>0$ \& Data. $\mathrm{H}>0$ \& Data. $\mathrm{O}==0$ \& Data. $\mathrm{N}==0$ \& Data. $\mathrm{P}==0$ \&

Data. S $>0$ );

Summary $\cdot \mathrm{CHNS}=\operatorname{sum}($ Data. $\mathrm{C}>0$ \& Data. $\mathrm{H}>0$ \& Data. $\mathrm{O}==0$ \& Data. $\mathrm{N}>0$ \& Data.P==0 \&

Data. $\mathrm{S}>0$ );

Summary. $\mathrm{CHNP}=\operatorname{sum}($ Data. $\mathrm{C}>0$ \& Data. $\mathrm{H}>0$ \& Data. $\mathrm{O}==0$ \& Data. $\mathrm{N}>0$ \& Data.P $>0$ \&

Data. $\mathrm{S}=0$ ) ;

Summary. $\mathrm{CHONS}=\operatorname{sum}($ Data $\cdot \mathrm{C}>0$ \& Data. $\mathrm{H}>0$ \& Data. $\mathrm{O}>0$ \& Data. $\mathrm{N}>0$ \& Data. $\mathrm{P}==0$ \&

Data. S>0);

Summary. $\mathrm{CHONP}=$ sum $($ Data. $\mathrm{C}>0$ \& Data. $\mathrm{H}>0$ \& Data. O $>0$ \& Data.N $>0$ \& Data.P $>0$ \&

Data. $\mathrm{S}==0$ );

Summary. $\mathrm{CHONSP}=$ sum $($ Data. $\mathrm{C}>0$ \& Data.H $>0$ \& Data.O $>0$ \& Data.N $>0$ \& Data.P>0 \&

Data. S>0);

oCalculating aromaticity parameters: \# condensed aromatic (AI>=0.67),

aromatic $(A I>=0.5)$,

onon-aromatic $($ AI $<0.5)$

Summary. CondensedAromatics $=$ sum (Data. AI $>=0.67)$;

Summary.Aromatics $=$ sum (Data.AI>=0.5); 
Summary. NonAromatics=sum (Data.AI<0.5);

ocalculating other parameters: total \# of formulae, average molecular

oweight

Summary. TotalFormulae=height (Data);

Summary. AvgMW=mean (Data. NominalMass);

oStoring Summary Data

ESI BothPolarities_GLSM Postox Summary=struct2table (Summary); $\circ<<<<<<<<<<<<<<<<$

$<<<\overline{<}<<<<<<<<<<<<<<\overline{<}<<<<\overline{<}<<<<<<\overline{<}<<<<<<<<<<<<<<<<<<<<<<<<<<<<<<<<<<<<<<<<<<<<<<~$

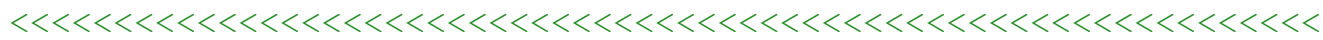

o Summarizing Data

Data $=$ Combined;

oCalculating formulae type parameters: \# CHO, CHON, CHOS, CHOP, CHN, CHS, CHP, CHNS, CHONS formulae

Summary $\cdot \mathrm{CHO}=$ sum $($ Data. $\mathrm{C}>0$ \& Data. $\mathrm{H}>0$ \& Data. $\mathrm{O}>0$ \& Data. $\mathrm{N}==0$ \& Data. $\mathrm{P}==0$ \&

Data. $\mathrm{S}==0$ );

Summary $\cdot \mathrm{CHON}=$ sum $($ Data. $\mathrm{C}>0$ \& Data.H $>0$ \& Data. $\mathrm{O}>0$ \& Data. $\mathrm{N}>0$ \& Data.P==0 \&

Data. $\mathrm{S}==0$ );

Summary $\cdot \mathrm{CHOS}=$ sum $($ Data. $\mathrm{C}>0$ \& Data.H $>0$ \& Data. O $>0$ \& Data. $\mathrm{N}==0$ \& Data. $\mathrm{P}==0$ \&

Data. $\mathrm{S}>0$ ) ;

Summary $\cdot \mathrm{CHOP}=\operatorname{sum}($ Data. $\mathrm{C}>0$ \& Data.H $>0$ \& Data. $\mathrm{O}>0$ \& Data. $\mathrm{N}==0$ \& Data.P $>0$ \&

Data. $\mathrm{S}==0$ );

Summary $\cdot \mathrm{CHN}=$ sum $($ Data. $\mathrm{C}>0$ \& Data. $\mathrm{H}>0$ \& Data. $\mathrm{O}==0$ \& Data. $\mathrm{N}>0$ \& Data. $\mathrm{P}==0$ \&

Data. $\mathrm{S}==0$ );

Summary $\cdot \mathrm{CHS}=\operatorname{sum}($ Data $\cdot \mathrm{C}>0$ \& Data. $\mathrm{H}>0$ \& Data. $\mathrm{O}==0$ \& Data. $\mathrm{N}==0$ \& Data. $\mathrm{P}==0$ \&

Data. S $>0$ );

Summary. $\mathrm{CHNS}=$ sum $($ Data. $\mathrm{C}>0$ \& Data. $\mathrm{H}>0$ \& Data. $\mathrm{O}==0$ \& Data. $\mathrm{N}>0$ \& Data. $\mathrm{P}==0$ \&

Data. $\mathrm{S}>0$ );

Summary. $\mathrm{CHNP}=\operatorname{sum}($ Data. $\mathrm{C}>0$ \& Data. $\mathrm{H}>0$ \& Data. $\mathrm{O}==0$ \& Data.N $>0$ \& Data.P>0 \&

Data. $\mathrm{S}==0$ );

Summary $\cdot \mathrm{CHONS}=\operatorname{sum}($ Data. $\mathrm{C}>0$ \& Data. $\mathrm{H}>0$ \& Data. $0>0$ \& Data.N $>0$ \& Data.P==0 \&

Data. $\mathrm{S}>0$ );

Summary. $\mathrm{CHONP}=$ sum $($ Data. $\mathrm{C}>0$ \& Data. $\mathrm{H}>0$ \& Data. $\mathrm{O}>0$ \& Data. $\mathrm{N}>0$ \& Data.P $>0$ \&

Data. $\mathrm{S}==0$ );

Summary. $\mathrm{CHONSP}=$ sum $($ Data. $\mathrm{C}>0$ \& Data.H $>0$ \& Data.O $>0$ \& Data.N $>0$ \& Data.P>0 \&

Data. $\mathrm{S}>0$ );

ocalculating aromaticity parameters: \# condensed aromatic (AI>=0.67),

aromatic $(A I>=0.5)$,

onon-aromatic $($ AI $<0.5)$

Summary. CondensedAromatics $=$ sum (Data. AI $>=0.67)$;

Summary. Aromatics $=$ sum (Data.AI $>=0.5)$;

Summary. NonAromatics=sum (Data.AI<0.5);

ocalculating other parameters: total \# of formulae, average molecular

oweight

Summary. TotalFormulae=height (Data);

Summary. AvgMW=mean (Data. NominalMass) ;

ostoring Summary Data

ESI GLSM PostOx Summary=struct2table (Summary); $\circ<<<<<<<<<<<<<<<<<<<<<<<<<<<<<<<<~$ $<<\overline{<}<<<\overline{\bar{l}}<<<<<<\overline{<}<<<<<<<<<<<<<<<<<<<<<<<<<<<<<<<<<<<<<<<<<<<<<<<<<<<<<<<<<<<<<$

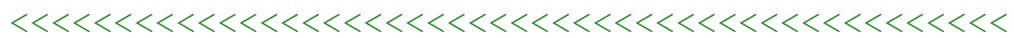

응 Combining Negative and Positive Mode LDI

oInput Data

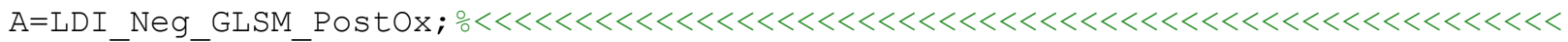
$<<<<\overline{\bar{l}}<<\overline{<} \overline{<}<<<\overline{\bar{l}}<<<<<<<<<<<<<<<<<<<<<<<<<<<<<<<<<<<<<<<<<<<<<<<<<<<<<<<<<<<<<<$ $<<<<<<<<<<<<<<<<<<<<<<<<~$ 


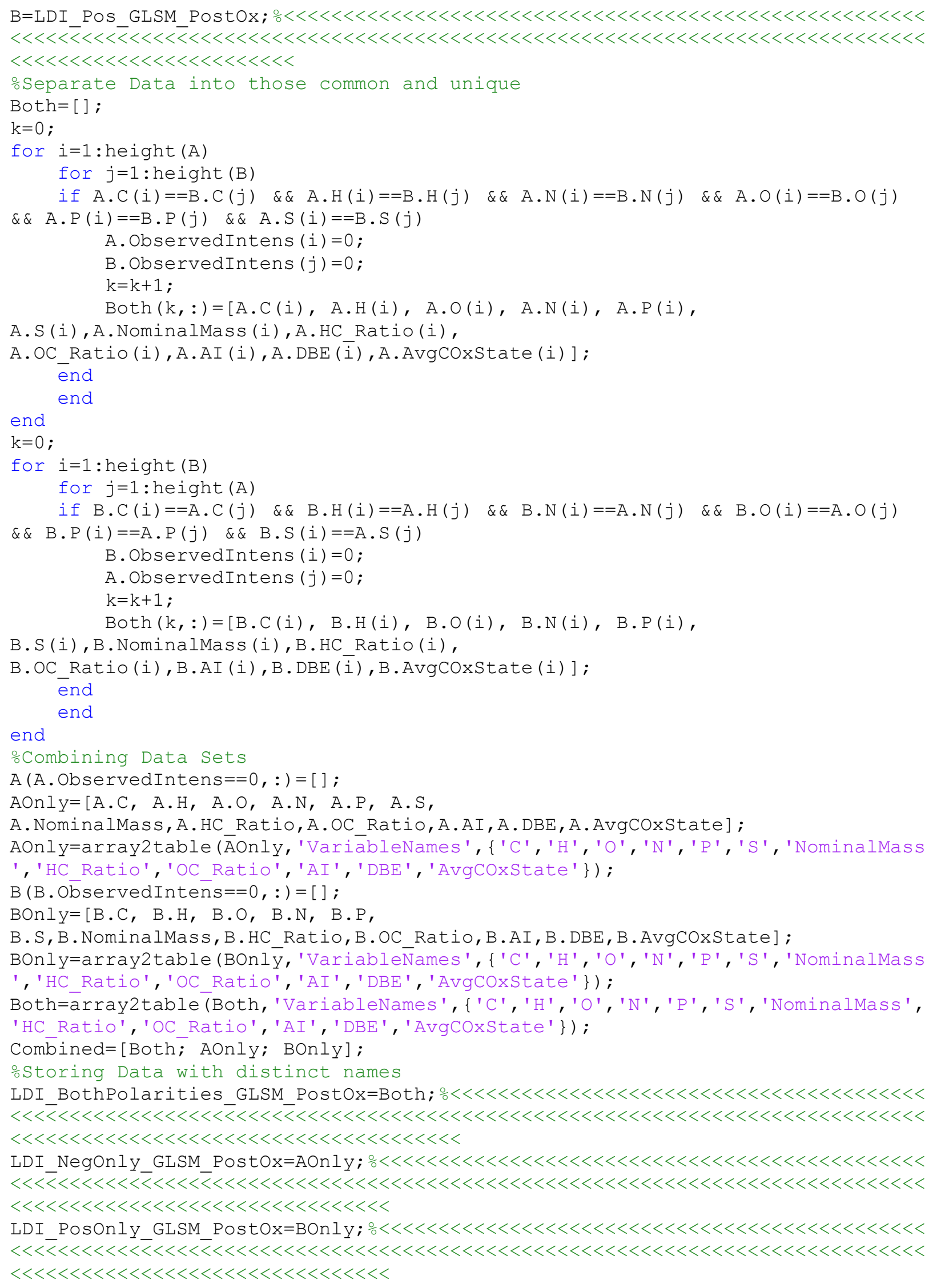




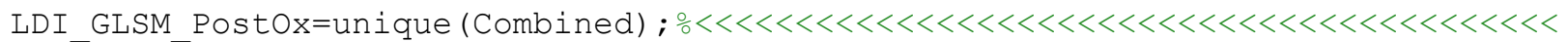
$<<\overline{<}<<<\overline{\bar{l}}<<<<<<<<<<<<<<<<<<<<<<<<<<<<<<<<<<<<<<<<<<<<<<<<<<<<<<<<<<<<<<<<<<<<$

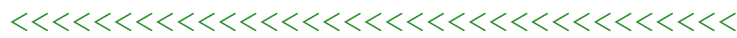

ㅇumarizing Data

Data=AOnly;

oCalculating formulae type parameters: \# CHO, CHON, CHOS, CHOP, CHN, CHS, CHP, CHNS, CHONS formulae

Summary $\cdot \mathrm{CHO}=$ sum $($ Data. $\mathrm{C}>0$ \& Data. $\mathrm{H}>0$ \& Data. $\mathrm{O}>0$ \& Data. $\mathrm{N}==0$ \& Data. $\mathrm{P}==0$ \&

Data. $\mathrm{S}==0$ );

Summary $\cdot \mathrm{CHON}=$ sum $($ Data. $\mathrm{C}>0$ \& Data.H $>0$ \& Data. $\mathrm{O}>0$ \& Data. $\mathrm{N}>0$ \& Data.P==0 \&

Data. $\mathrm{S}==0$ );

Summary. $\mathrm{CHOS}=$ sum $($ Data. $\mathrm{C}>0$ \& Data. $\mathrm{H}>0$ \& Data. $\mathrm{O}>0$ \& Data. $\mathrm{N}==0$ \& Data. $\mathrm{P}==0$ \&

Data. $\mathrm{S}>0$ );

Summary. $\mathrm{CHOP}=\mathrm{sum}($ Data. $\mathrm{C}>0$ \& Data.H $>0$ \& Data. $0>0$ \& Data. $\mathrm{N}==0$ \& Data.P $>0$ \&

Data. $\mathrm{S}==0$ );

Summary $\cdot \mathrm{CHN}=$ sum $($ Data. $\mathrm{C}>0$ \& Data. $\mathrm{H}>0$ \& Data. $\mathrm{O}==0$ \& Data. $\mathrm{N}>0$ \& Data. $\mathrm{P}==0$ \&

Data. $\mathrm{S}=0$ ) ;

Summary $\cdot \mathrm{CHS}=\operatorname{sum}($ Data. $\mathrm{C}>0$ \& Data. $\mathrm{H}>0$ \& Data. $\mathrm{O}==0$ \& Data. $\mathrm{N}==0$ \& Data. $\mathrm{P}==0$ \&

Data. $\mathrm{S}>0$ ) ;

Summary $\cdot \mathrm{CHNS}=\operatorname{sum}($ Data. $\mathrm{C}>0$ \& Data. $\mathrm{H}>0$ \& Data. $\mathrm{O}==0$ \& Data. $\mathrm{N}>0$ \& Data.P==0 \&

Data. S $>0$ );

Summary $\cdot \mathrm{CHNP}=\operatorname{sum}($ Data. $\mathrm{C}>0$ \& Data.H $>0$ \& Data. $\mathrm{O}==0$ \& Data. $\mathrm{N}>0$ \& Data.P $>0$ \&

Data. $\mathrm{S}==0$ );

Summary. $\mathrm{CHONS}=\operatorname{sum}($ Data. $\mathrm{C}>0$ \& Data. $\mathrm{H}>0$ \& Data. $0>0$ \& Data.N>0 \& Data.P==0 \&

Data. S>0);

Summary. $\mathrm{CHONP}=$ sum $($ Data. $\mathrm{C}>0$ \& Data.H $>0$ \& Data.O $>0$ \& Data.N $>0$ \& Data.P>0\&

Data. $\mathrm{S}==0$ );

Summary. $\mathrm{CHONSP}=$ sum $($ Data. $\mathrm{C}>0$ \& Data.H $>0$ \& Data.O $>0$ \& Data.N $>0$ \& Data.P>0 \&

Data. $\mathrm{S}>0)$;

ocalculating aromaticity parameters: \# condensed aromatic (AI>=0.67),

aromatic $(\mathrm{AI}>=0.5)$,

onon-aromatic $(\mathrm{A} I<0.5)$

Summary. CondensedAromatics $=$ sum (Data. AI $>=0.67)$;

Summary. Aromatics $=$ sum (Data.AI $>=0.5)$;

Summary. NonAromatics=sum (Data.AI<0.5);

ocalculating other parameters: total \# of formulae, average molecular

oweight

Summary.TotalFormulae=height (Data);

Summary. AvgMW=mean (Data. NominalMass);

ostoring Summary Data

LDI_NegOnly_GLSM_PostOx_Summary=struct2table (Summary) ; $\circ<<<<<<<<<<<<<<<<<<<<<<<$

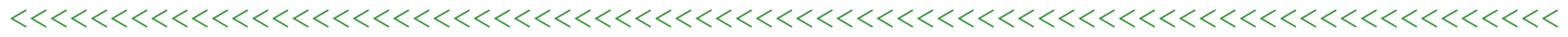

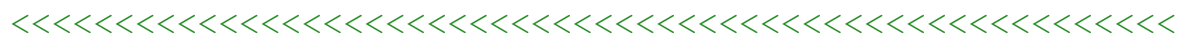

․ Summarizing Data

Data=BOnly ;

oCalculating formulae type parameters: \# CHO, CHON, CHOS, CHOP, CHN, CHS, CHP, CHNS, CHONS formulae

Summary. $\mathrm{CHO}=\operatorname{sum}($ Data. $\mathrm{C}>0$ \& Data. $\mathrm{H}>0$ \& Data. $\mathrm{O}>0$ \& Data. $\mathrm{N}==0$ \& Data. $\mathrm{P}==0$ \& Data. $\mathrm{S}==0$ );

Summary. $\mathrm{CHON}=\operatorname{sum}($ Data. $\mathrm{C}>0$ \& Data. $\mathrm{H}>0$ \& Data. $\mathrm{O}>0$ \& Data.N $>0$ \& Data.P==0 \&

Data. $\mathrm{S}==0$ ) ;

Summary $\cdot \mathrm{CHOS}=$ sum $($ Data. $\mathrm{C}>0$ \& Data.H $>0$ \& Data. $\mathrm{O}>0$ \& Data. $\mathrm{N}==0$ \& Data.P==0 \&

Data. $\mathrm{S}>0$ ) ;

Summary. $\mathrm{CHOP}=\operatorname{sum}($ Data. $\mathrm{C}>0$ \& Data. $\mathrm{H}>0$ \& Data. $\mathrm{O}>0$ \& Data. $\mathrm{N}==0$ \& Data. $\mathrm{P}>0$ \&

Data. $\mathrm{S}=0$ ) ;

Summary. $\mathrm{CHN}=\operatorname{sum}($ Data. $\mathrm{C}>0$ \& Data. $\mathrm{H}>0$ \& Data. $\mathrm{O}==0$ \& Data. $\mathrm{N}>0$ \& Data. $\mathrm{P}==0$ \&

Data. $\mathrm{S}==0$ ); 
Summary $\cdot \mathrm{CHS}=\operatorname{sum}($ Data $\cdot \mathrm{C}>0$ \& Data. $\mathrm{H}>0$ \& Data. $\mathrm{O}==0$ \& Data. $\mathrm{N}==0$ \& Data. $\mathrm{P}==0$ \& Data. $\mathrm{S}>0$ ) ;

Summary. CHNS $=$ sum $($ Data. $\mathrm{C}>0$ \& Data. $\mathrm{H}>0$ \& Data. $\mathrm{O}==0$ \& Data. $\mathrm{N}>0$ \& Data.P==0 \& Data. S $>0$ );

Summary. $\mathrm{CHNP}=\operatorname{sum}($ Data. $\mathrm{C}>0$ \& Data. $\mathrm{H}>0$ \& Data. $\mathrm{O}==0$ \& Data. $\mathrm{N}>0$ \& Data.P $>0$ \& Data. $\mathrm{S}==0$ );

Summary. CHONS $=$ sum $($ Data. $\mathrm{C}>0$ \& Data. $\mathrm{H}>0$ \& Data.O $>0$ \& Data.N $>0$ \& Data.P==0 \& Data. $\mathrm{S}>0$ ) ;

Summary. $\mathrm{CHONP}=$ sum $($ Data. $\mathrm{C}>0$ \& Data. $\mathrm{H}>0$ \& Data. $\mathrm{O}>0$ \& Data.N $>0$ \& Data.P $>0$ \& Data. $\mathrm{S}==0$ );

Summary. $\mathrm{CHONSP}=$ sum $($ Data. $\mathrm{C}>0$ \& Data.H $>0$ \& Data. O $>0$ \& Data.N $>0$ \& Data.P $>0$ \& Data. S>0);

ocalculating aromaticity parameters: \# condensed aromatic (AI>=0.67), aromatic $(\mathrm{AI}>=0.5)$,

onon-aromatic $(\mathrm{A} I<0.5)$

Summary. CondensedAromatics $=$ sum (Data. AI $>=0.67)$;

Summary. Aromatics $=$ sum (Data. AI $>=0.5)$;

Summary. NonAromatics=sum (Data.AI<0.5);

ocalculating other parameters: total \# of formulae, average molecular

oweight

Summary.TotalFormulae=height (Data);

Summary . AvgMW=mean (Data. NominalMass);

o.Storing Summary Data

LDI PosOnly GLSM PostOx Summary=struct2table (Summary); $\circ<<<<<<<<<<<<<<<<<<<<<<<<~$

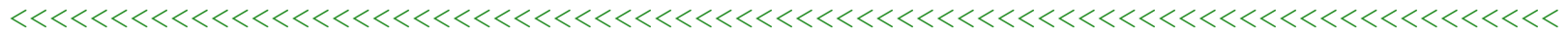

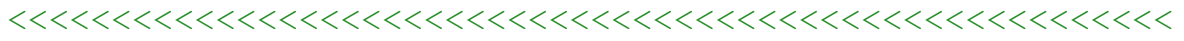

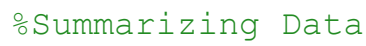

Data=Both;

oCalculating formulae type parameters: \# CHO, CHON, CHOS, CHOP, CHN, CHS, CHP, CHNS, CHONS formulae

Summary $\cdot \mathrm{CHO}=\operatorname{sum}($ Data. $\mathrm{C}>0$ \& Data. $\mathrm{H}>0$ \& Data. $\mathrm{O}>0$ \& Data. $\mathrm{N}==0$ \& Data. $\mathrm{P}==0$ \&

Data. $\mathrm{S}==0$ );

Summary $\cdot \mathrm{CHON}=\operatorname{sum}($ Data. $\mathrm{C}>0$ \& Data.H $>0$ \& Data. $0>0$ \& Data. $\mathrm{N}>0$ \& Data. $\mathrm{P}==0$ \&

Data. $\mathrm{S}==0$ );

Summary. $\mathrm{CHOS}=$ sum $($ Data. $\mathrm{C}>0$ \& Data. $\mathrm{H}>0$ \& Data.O $>0$ \& Data. $\mathrm{N}==0$ \& Data. $\mathrm{P}==0$ \&

Data. S>0);

Summary $\cdot \mathrm{CHOP}=$ sum $($ Data. $\mathrm{C}>0$ \& Data. $\mathrm{H}>0$ \& Data. $\mathrm{O}>0$ \& Data. $\mathrm{N}==0$ \& Data. P $>0$ \&

Data. $\mathrm{S}==0$ ) ;

Summary. $\mathrm{CHN}=$ sum $($ Data. $\mathrm{C}>0$ \& Data. $\mathrm{H}>0$ \& Data. $\mathrm{O}==0$ \& Data. $\mathrm{N}>0$ \& Data. $\mathrm{P}==0$ \&

Data. $\mathrm{S}==0$ );

Summary $\cdot \mathrm{CHS}=\operatorname{sum}($ Data. $\mathrm{C}>0$ \& Data. $\mathrm{H}>0$ \& Data. $\mathrm{O}==0$ \& Data. $\mathrm{N}==0$ \& Data. $\mathrm{P}==0$ \&

Data. S $>0$ );

Summary $\cdot \mathrm{CHNS}=\operatorname{sum}($ Data. $\mathrm{C}>0$ \& Data. $\mathrm{H}>0$ \& Data. $\mathrm{O}==0$ \& Data. $\mathrm{N}>0$ \& Data.P==0 \&

Data. $\mathrm{S}>0$ );

Summary. $\mathrm{CHNP}=\operatorname{sum}($ Data. $\mathrm{C}>0$ \& Data. $\mathrm{H}>0$ \& Data. $\mathrm{O}==0$ \& Data. $\mathrm{N}>0$ \& Data.P $>0$ \&

Data. $\mathrm{S}=0$ ) ;

Summary. $\mathrm{CHONS}=\operatorname{sum}($ Data $\cdot \mathrm{C}>0$ \& Data. $\mathrm{H}>0$ \& Data. $\mathrm{O}>0$ \& Data. $\mathrm{N}>0$ \& Data. $\mathrm{P}==0$ \&

Data. S>0);

Summary. $\mathrm{CHONP}=$ sum $($ Data. $\mathrm{C}>0$ \& Data. $\mathrm{H}>0$ \& Data. O $>0$ \& Data.N $>0$ \& Data.P $>0$ \&

Data. $\mathrm{S}==0$ );

Summary. $\mathrm{CHONSP}=$ sum $($ Data. $\mathrm{C}>0$ \& Data.H $>0$ \& Data.O $>0$ \& Data.N $>0$ \& Data.P>0 \&

Data. S>0);

oCalculating aromaticity parameters: \# condensed aromatic (AI>=0.67),

aromatic $(A I>=0.5)$,

onon-aromatic $($ AI $<0.5)$

Summary. CondensedAromatics $=$ sum (Data. AI $>=0.67)$;

Summary.Aromatics $=$ sum (Data.AI>=0.5); 
Summary. NonAromatics=sum (Data.AI<0.5);

ocalculating other parameters: total \# of formulae, average molecular

oweight

Summary. TotalFormulae=height (Data);

Summary. AvgMW=mean (Data. NominalMass) ;

ostoring Summary Data

LDI_BothPolarities GLSM Postox Summary=struct2table (Summary); $\circ<<<<<<<<<<<<<<<<$

$<<<\overline{<}<<<<<<<<<<<<<<\overline{<}<<<<\overline{<}<<<<<<\overline{<}<<<<<<<<<<<<<<<<<<<<<<<<<<<<<<<<<<<<<<<<<<<<<<~$

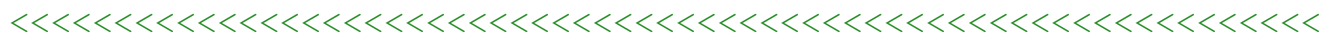

o Summarizing Data

Data $=$ Combined;

oCalculating formulae type parameters: \# CHO, CHON, CHOS, CHOP, CHN, CHS, CHP, CHNS, CHONS formulae

Summary $\cdot \mathrm{CHO}=$ sum $($ Data. $\mathrm{C}>0$ \& Data. $\mathrm{H}>0$ \& Data. $\mathrm{O}>0$ \& Data. $\mathrm{N}==0$ \& Data. $\mathrm{P}==0$ \&

Data. $\mathrm{S}==0$ );

Summary $\cdot \mathrm{CHON}=\operatorname{sum}($ Data. $\mathrm{C}>0$ \& Data.H $>0$ \& Data. $\mathrm{O}>0$ \& Data. $>0$ \& Data.P==0 \&

Data. $\mathrm{S}=0$ ) ;

Summary $\cdot \mathrm{CHOS}=$ sum $($ Data. $\mathrm{C}>0$ \& Data.H $>0$ \& Data. O $>0$ \& Data. $\mathrm{N}==0$ \& Data. $\mathrm{P}==0$ \&

Data. $\mathrm{S}>0$ );

Summary $\cdot \mathrm{CHOP}=\operatorname{sum}($ Data. $\mathrm{C}>0$ \& Data.H $>0$ \& Data. $\mathrm{O}>0$ \& Data. $\mathrm{N}==0$ \& Data.P $>0$ \&

Data. $\mathrm{S}==0$ );

Summary $\cdot \mathrm{CHN}=$ sum $($ Data. $\mathrm{C}>0$ \& Data. $\mathrm{H}>0$ \& Data. $\mathrm{O}==0$ \& Data. $\mathrm{N}>0$ \& Data. $\mathrm{P}==0$ \&

Data. $\mathrm{S}==0$ );

Summary. CHS $=$ sum $($ Data. $\mathrm{C}>0$ \& Data.H $>0$ \& Data. $\mathrm{O}==0$ \& Data. $\mathrm{N}==0$ \& Data.P==0 \&

Data. S>0);

Summary. $\mathrm{CHNS}=$ sum $($ Data. $\mathrm{C}>0$ \& Data. $\mathrm{H}>0$ \& Data. $\mathrm{O}==0$ \& Data. $\mathrm{N}>0$ \& Data. $\mathrm{P}==0$ \&

Data. $\mathrm{S}>0$ );

Summary. $\mathrm{CHNP}=\mathrm{sum}($ Data. $\mathrm{C}>0$ \& Data. $\mathrm{H}>0$ \& Data. $\mathrm{O}==0$ \& Data.N $>0$ \& Data.P $>0$ \&

Data. $\mathrm{S}==0$ );

Summary $\cdot \mathrm{CHONS}=\operatorname{sum}($ Data. $\mathrm{C}>0$ \& Data. $\mathrm{H}>0$ \& Data. $0>0$ \& Data.N $>0$ \& Data.P==0 \&

Data. S>0);

Summary. $\mathrm{CHONP}=$ sum $($ Data. $\mathrm{C}>0$ \& Data. $\mathrm{H}>0$ \& Data. $\mathrm{O}>0$ \& Data. $\mathrm{N}>0$ \& Data.P $>0$ \&

Data. $\mathrm{S}==0$ );

Summary. $\mathrm{CHONSP}=$ sum $($ Data. $\mathrm{C}>0$ \& Data.H $>0$ \& Data.O $>0$ \& Data.N $>0$ \& Data.P>0 \&

Data. $\mathrm{S}>0$ );

ocalculating aromaticity parameters: \# condensed aromatic (AI>=0.67),

aromatic $(A I>=0.5)$,

onon-aromatic $($ AI $<0.5)$

Summary. CondensedAromatics $=$ sum (Data. AI $>=0.67)$;

Summary. Aromatics $=$ sum (Data.AI $>=0.5)$;

Summary. NonAromatics=sum (Data.AI<0.5);

ocalculating other parameters: total \# of formulae, average molecular

oweight

Summary. TotalFormulae=height (Data);

Summary. AvgMW=mean (Data. NominalMass);

ostoring Summary Data

LDI GLSM Postox Summary=struct2table (Summary); $\circ<<<<<<<<<<<<<<<<<<<<<<<<<<<<<<<<~$ $<<\overline{<}<<<\overline{\bar{l}}<<<<<<\overline{<}<<<<<<<<<<<<<<<<<<<<<<<<<<<<<<<<<<<<<<<<<<<<<<<<<<<<<<<<<<<<<$

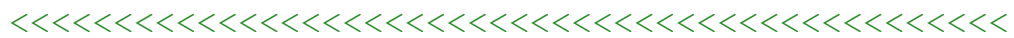

응 Combining ESI and LDI

onput Data so that the script can readily be applied to any data set

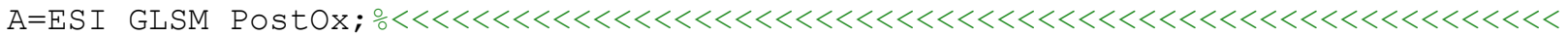
$<<<<\overline{<}<<<\overline{<} \overline{<}<<<<<<<<<<<<<<<<<<<<<<<<<<<<<<<<<<<<<<<<<<<<<<<<<<<<<<<<<<<<<<<<<<~$ $<<<<<<<<<<<<<<<<<<<<~$ 


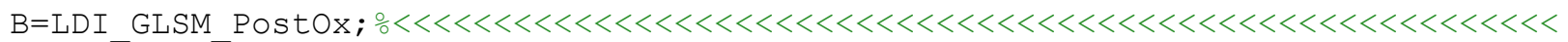

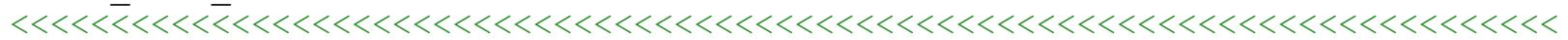
$<<<<<<<<<<<<<<<<<<<<~$

o Separate Data into those common and unique

Both=[ ] ;

$\mathrm{k}=0$;

for $i=1$ :height $(\mathrm{A})$ for $j=1$ :height $(B)$

if $A \cdot C(i)==B \cdot C(j) \quad \& \& A \cdot H(i)==B \cdot H(j) \quad \& \& A \cdot N(i)==B \cdot N(j) \quad \& \& A \cdot O(i)==B \cdot O(j)$

$\& \& \quad A \cdot P(i)==B \cdot P(j) \quad \& \& \quad A \cdot S(i)==B \cdot S(j)$

$\mathrm{k}=\mathrm{k}+1$;

$\operatorname{Both}(k,:)=[A . C(i), A . H(i), A . O(i), A . N(i), A . P(i)$,

A.S (i), A.NominalMass (i), A.HC Ratio(i),

A. OC Ratio(i), A.AI (i), A. DBE (i), A.AvgCoxState (i) ] ;

$$
\begin{aligned}
& \text { A. } C(i)=0 ; \\
& \text { B. C }(j)=0 ;
\end{aligned}
$$

end

end

Combining Data Sets

$A(A . C==0,:)=[]$;

$A O n l_{y}=[A . C, A . H, A . O, A . N, A . P, A . S$,

A.NominalMass, A.HC Ratio,A.oC Ratio,A.AI, A.DBE, A.AvgCoxState];

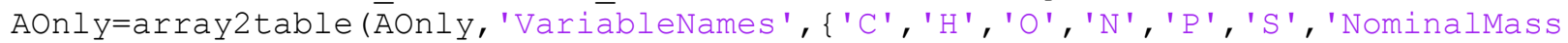
', 'HC_Ratio', 'OC_Ratio', 'AI', 'DBE', 'AvgCOxState' \});

$\mathrm{B}(\mathrm{B} \cdot \mathrm{C}==0,:)=[]$;

$\mathrm{BOnl} Y=[B . \mathrm{C}, \mathrm{B} . \mathrm{H}, \mathrm{B} . \mathrm{O}, \mathrm{B} . \mathrm{N}, \mathrm{B} . \mathrm{P}$,

B.S,B.NominalMass, B.HC Ratio,B.OC Ratio, B.AI, B.DBE, B. AvgCoxState];

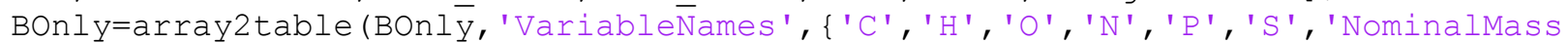
', 'HC Ratio', 'OC Ratio', 'AI', 'DBE', 'AvgCoxstate'\}) ;

Both=array2table (Both, 'VariableNames', \{'C', 'H', 'O', 'N', 'P' , 'S ', 'NominalMass', 'HC_Ratio', 'OC_Ratio', 'AI', 'DBE', 'AvgCoxstate' \}) ;

Combined=[Both; AOnly; BOnly];

ostoring Data with distinct names

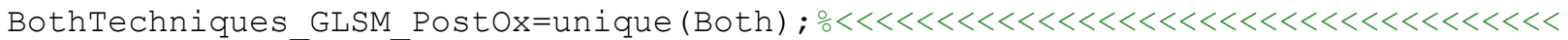
$<<<<<<<<<<<<<\overline{<}<<<\overline{<}<<<<<<<<<<<<<<<<<<<<<<<<<<<<<<<<<<<<<<<<<<<<<<<<<<<<<<<<<~$

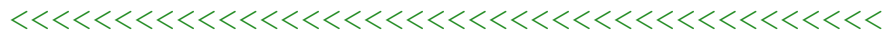

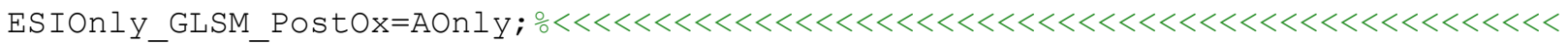
$<<<<<<<\overline{<}<<<\overline{\bar{l}}<<<<<<<<<<<<<<<<<<<<<<<<<<<<<<<<<<<<<<<<<<<<<<<<<<<<<<<<<<<<<<<<$ $<<<<<<<<<<<<<<<<<<<<<<<<<<<<~$

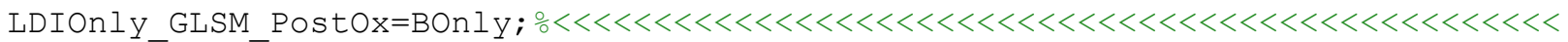

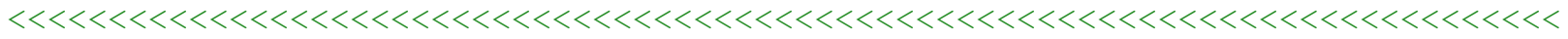
$<<<<<<<<<<<<<<<<<<<<<<<<<<<<~$

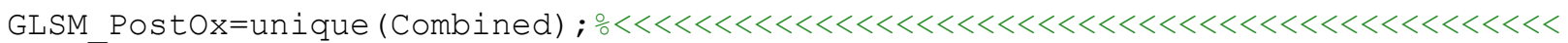
$<<<\overline{<}<<<<<<<<<<<<<<<<<<<<<<<<<<<<<<<<<<<<<<<<<<<<<<<<<<<<<<<<<<<<<<<<<<<<<<<<~$

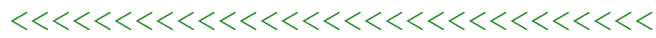

o Summarizing Data

Data=AOnly;

oCalculating formulae type parameters: \# CHO, CHON, CHOS, CHOP, CHN, CHS, CHP, CHNS, CHONS formulae

Summary $\cdot \mathrm{CHO}=$ sum $($ Data. $\mathrm{C}>0$ \& Data. $\mathrm{H}>0$ \& Data. $\mathrm{O}>0$ \& Data. $\mathrm{N}==0$ \& Data. $\mathrm{P}==0$ \&

Data. $\mathrm{S}=0$ ) ;

Summary $\cdot \mathrm{CHON}=\operatorname{sum}($ Data. $\mathrm{C}>0$ \& Data.H $>0$ \& Data. $\mathrm{O}>0$ \& Data. $>0$ \& Data.P==0 \& Data. $\mathrm{S}==0$ );

Summary. $\mathrm{CHOS}=$ sum $($ Data. $\mathrm{C}>0$ \& Data. $\mathrm{H}>0$ \& Data. $\mathrm{O}>0$ \& Data. $\mathrm{N}==0$ \& Data. $\mathrm{P}==0$ \&

Data. $\mathrm{S}>0$ ); 
Summary. $\mathrm{CHOP}=\mathrm{sum}($ Data. $\mathrm{C}>0$ \& Data.H $>0$ \& Data.O $>0$ \& Data.N==0 \& Data.P>0 \& Data. $\mathrm{S}==0$ );

Summary $\cdot \mathrm{CHN}=$ sum $($ Data. $\mathrm{C}>0$ \& Data. $\mathrm{H}>0$ \& Data. $\mathrm{O}==0$ \& Data. $\mathrm{N}>0$ \& Data.P==0 \& Data. $\mathrm{S}==0$ ) ;

Summary $\cdot \mathrm{CHS}=\operatorname{sum}($ Data $\cdot \mathrm{C}>0$ \& Data. $\mathrm{H}>0$ \& Data. $\mathrm{O}==0$ \& Data. $\mathrm{N}==0$ \& Data. $\mathrm{P}==0$ \& Data. $\mathrm{S}>0$ );

Summary $\cdot \mathrm{CHNS}=$ sum $($ Data $\cdot \mathrm{C}>0$ \& Data. $\mathrm{H}>0$ \& Data. $\mathrm{O}==0$ \& Data. $\mathrm{N}>0$ \& Data. $\mathrm{P}==0$ \&

Data. $\mathrm{S}>0$ ) ;

Summary $\cdot \mathrm{CHNP}=\operatorname{sum}($ Data $\cdot \mathrm{C}>0$ \& Data. $\mathrm{H}>0$ \& Data. $\mathrm{O}==0$ \& Data.N $>0$ \& Data.P>0 \&

Data. $\mathrm{S}==0$ );

Summary. $\mathrm{CHONS}=$ sum $($ Data $. \mathrm{C}>0$ \& Data. $\mathrm{H}>0$ \& Data. $\mathrm{O}>0$ \& Data. $>0$ \& Data.P==0 \&

Data. $\mathrm{S}>0$ );

Summary. $\mathrm{CHONP}=$ sum $($ Data. $\mathrm{C}>0$ \& Data.H $>0$ \& Data.O>0 \& Data.N>0 \& Data.P>0 \&

Data. $\mathrm{S}==0$ );

Summary. $\mathrm{CHONSP}=$ sum $($ Data. $\mathrm{C}>0$ \& Data. $\mathrm{H}>0$ \& Data.O $>0$ \& Data.N $>0$ \& Data.P $>0$ \&

Data. S>0);

ocalculating aromaticity parameters: \# condensed aromatic (AI>=0.67),

aromatic $(\mathrm{AI}>=0.5)$,

onon-aromatic $(\mathrm{AI}<0.5)$

Summary. CondensedAromatics $=$ sum (Data. AI $>=0.67)$;

Summary. Aromatics $=$ sum (Data.AI $>=0.5)$;

Summary. NonAromatics=sum (Data.AI<0.5);

oCalculating other parameters: total \# of formulae, average molecular

oweight

Summary. TotalFormulae=height (Data);

Summary. AvgMW=mean (Data. NominalMass);

ostoring Summary Data

ESIOnly_GLSM Postox_Summary=struct2table (Summary) ; $\circ<<<<<<<<<<<<<<<<<<<<<<<<<<<<$

$<<<<<<<\overline{<}<<<\overline{<} \overline{<}<<<<<\overline{<}<<<<<<<<<<<<<<<<<<<<<<<<<<<<<<<<<<<<<<<<<<<<<<<<<<<<<<<<<<$

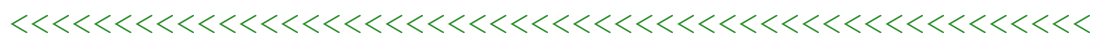

ㅇmmarizing Data

Data=BOnly;

oCalculating formulae type parameters: \# CHO, CHON, CHOS, CHOP, CHN, CHS, CHP, CHNS, CHONS formulae

Summary $\cdot \mathrm{CHO}=$ sum $($ Data. $\mathrm{C}>0$ \& Data. $\mathrm{H}>0$ \& Data. $\mathrm{O}>0$ \& Data. $\mathrm{N}==0$ \& Data. $\mathrm{P}==0$ \& Data. $\mathrm{S}==0$ );

Summary $\cdot \mathrm{CHON}=$ sum $($ Data $\cdot \mathrm{C}>0$ \& Data. $\mathrm{H}>0$ \& Data. $\mathrm{O}>0$ \& Data.N $>0$ \& Data.P==0 \&

Data. $\mathrm{S}==0$ );

Summary. $\mathrm{CHOS}=$ sum $($ Data. $\mathrm{C}>0$ \& Data. $\mathrm{H}>0$ \& Data. $\mathrm{O}>0$ \& Data. $\mathrm{N}==0$ \& Data. $\mathrm{P}==0$ \&

Data. $\mathrm{S}>0$ ) ;

Summary $\cdot \mathrm{CHOP}=\operatorname{sum}($ Data. $\mathrm{C}>0$ \& Data.H $>0$ \& Data.O $>0$ \& Data.N==0 \& Data.P>0 \&

Data. $\mathrm{S}==0$ );

Summary. $\mathrm{CHN}=$ sum $($ Data. $\mathrm{C}>0$ \& Data. $\mathrm{H}>0$ \& Data. $\mathrm{O}==0$ \& Data. $\mathrm{N}>0$ \& Data.P==0 \&

Data. $\mathrm{S}==0$ );

Summary $\cdot \mathrm{CHS}=\operatorname{sum}($ Data $. \mathrm{C}>0$ \& Data. $\mathrm{H}>0$ \& Data. $\mathrm{O}==0$ \& Data. $\mathrm{N}==0$ \& Data. $\mathrm{P}==0$ \&

Data. $\mathrm{S}>0$ ) ;

Summary. CHNS $=$ sum $($ Data. $\mathrm{C}>0$ \& Data. $\mathrm{H}>0$ \& Data. $\mathrm{O}==0$ \& Data. $>0$ \& Data.P==0 \&

Data. $\mathrm{S}>0$ );

Summary. $\mathrm{CHNP}=$ sum $($ Data. $\mathrm{C}>0$ \& Data. $\mathrm{H}>0$ \& Data. $\mathrm{O}==0$ \& Data. $\mathrm{N}>0$ \& Data.P $>0$ \&

Data. $\mathrm{S}==0$ );

Summary $\cdot \mathrm{CHONS}=$ sum $($ Data. $\mathrm{C}>0$ \& Data. $\mathrm{H}>0$ \& Data. $0>0$ \& Data. $>0$ \& Data.P==0 \&

Data. $\mathrm{S}>0$ ) ;

Summary. $\mathrm{CHONP}=$ sum $($ Data. $\mathrm{C}>0$ \& Data.H $>0$ \& Data. $\mathrm{O}>0$ \& Data.N $>0$ \& Data.P>0 \&

Data. $\mathrm{S}==0$ );

Summary. $\mathrm{CHONSP}=$ sum $($ Data. $\mathrm{C}>0$ \& Data. $\mathrm{H}>0$ \& Data. $\mathrm{O}>0$ \& Data.N $>0$ \& Data.P $>0$ \&

Data. $\mathrm{S}>0$ ) ; 
oCalculating aromaticity parameters: \# condensed aromatic (AI>=0.67), aromatic $(\mathrm{AI}>=0.5)$,

onon-aromatic (AI<0.5)

Summary. CondensedAromatics $=$ sum (Data.AI $>=0.67)$;

Summary.Aromatics=sum (Data.AI $>=0.5)$;

Summary. NonAromatics=sum (Data.AI<0.5);

ocalculating other parameters: total \# of formulae, average molecular

oweight

Summary. TotalFormulae=height (Data);

Summary . AvgMW=mean (Data. NominalMass);

ostoring Summary Data

LDIOnly_GLSM Postox Summary=struct2table (Summary); $\circ<<<<<<<<<<<<<<<<<<<<<<<<<<<<~$ $<<<<<<<\overline{<}<<<\overline{<}<<<<<\overline{<}<<<<<<<<<<<<<<<<<<<<<<<<<<<<<<<<<<<<<<<<<<<<<<<<<<<<<<<<<<~$

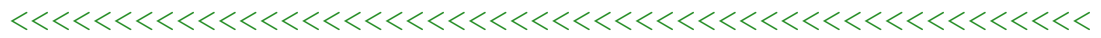

․ Summarizing Data

Data=unique (Both);

oCalculating formulae type parameters: \# CHO, CHON, CHOS, CHOP, CHN, CHS, CHP, CHNS, CHONS formulae

Summary $\cdot \mathrm{CHO}=$ sum (Data. $\mathrm{C}>0$ \& Data. $\mathrm{H}>0$ \& Data. $\mathrm{O}>0$ \& Data. $\mathrm{N}==0$ \& Data. $\mathrm{P}==0$ \& Data. $\mathrm{S}==0$ );

Summary $\cdot \mathrm{CHON}=$ Sum $($ Data. $\mathrm{C}>0$ \& Data. $\mathrm{H}>0$ \& Data. $\mathrm{O}>0$ \& Data. $\mathrm{N}>0$ \& Data.P==0 \& Data. $\mathrm{S}==0$ );

Summary. $\mathrm{CHOS}=$ sum $($ Data. $\mathrm{C}>0$ \& Data. $\mathrm{H}>0$ \& Data. $\mathrm{O}>0$ \& Data. $\mathrm{N}==0$ \& Data. $\mathrm{P}==0$ \&

Data. $\mathrm{S}>0$ ) ;

Summary $\cdot \mathrm{CHOP}=\mathrm{sum}($ Data. $\mathrm{C}>0$ \& Data.H $>0$ \& Data. $\mathrm{O}>0$ \& Data. $\mathrm{N}==0$ \& Data.P $>0$ \&

Data. $\mathrm{S}==0$ );

Summary $\cdot \mathrm{CHN}=$ sum $($ Data. $\mathrm{C}>0$ \& Data. $\mathrm{H}>0$ \& Data. $\mathrm{O}==0$ \& Data. $\mathrm{N}>0$ \& Data.P==0 \&

Data. $\mathrm{S}==0$ );

Summary $\cdot \mathrm{CHS}=\operatorname{sum}($ Data $\cdot \mathrm{C}>0$ \& Data. $\mathrm{H}>0$ \& Data. $\mathrm{O}==0$ \& Data. $\mathrm{N}==0$ \& Data. $\mathrm{P}==0$ \&

Data. $\mathrm{S}>0$ );

Summary. $\mathrm{CHNS}=\operatorname{sum}($ Data. $\mathrm{C}>0$ \& Data. $\mathrm{H}>0$ \& Data. $\mathrm{O}==0$ \& Data. $\mathrm{N}>0$ \& Data. $\mathrm{P}==0$ \&

Data. S>0);

Summary $\cdot \mathrm{CHNP}=\mathrm{sum}($ Data. $\mathrm{C}>0$ \& Data.H $>0$ \& Data. $\mathrm{O}==0$ \& Data. $\mathrm{N}>0$ \& Data.P $>0$ \&

Data. $\mathrm{S}==0$ );

Summary. $\mathrm{CHONS}=\operatorname{sum}($ Data $. \mathrm{C}>0$ \& Data. $\mathrm{H}>0$ \& Data. $0>0$ \& Data.N>0 \& Data.P==0 \&

Data. S>0);

Summary. $\mathrm{CHONP}=$ sum $($ Data. $\mathrm{C}>0$ \& Data. $\mathrm{H}>0$ \& Data. $\mathrm{O}>0$ \& Data.N $>0$ \& Data.P $>0$ \&

Data. $\mathrm{S}==0$ ) ;

Summary. $\mathrm{CHONSP}=$ sum $($ Data. $\mathrm{C}>0$ \& Data.H $>0$ \& Data.O $>0$ \& Data.N $>0$ \& Data.P>0 \&

Data. $\mathrm{S}>0$ );

oCalculating aromaticity parameters: \# condensed aromatic (AI>=0.67),

aromatic $(A I>=0.5)$,

onon-aromatic $($ AI $<0.5)$

Summary. CondensedAromatics $=$ sum (Data. AI $>=0.67)$;

Summary. Aromatics $=$ sum (Data.AI $>=0.5)$;

Summary. NonAromatics $=$ sum (Data.AI<0.5);

ocalculating other parameters: total \# of formulae, average molecular

oweight

Summary. TotalFormulae=height (Data);

Summary. AvgMW=mean (Data. NominalMass);

ostoring Summary Data

BothTechniques GLSM PostOx Summary=struct2table (Summary); $\circ<<<<<<<<<<<<<<<<<<<<<$ $<<<<<<<<<<<<<\overline{<}<<<\overline{<}<<<<<<<\overline{<}<<<<<<<<<<<<<<<<<<<<<<<<<<<<<<<<<<<<<<<<<<<<<<<<<<~$

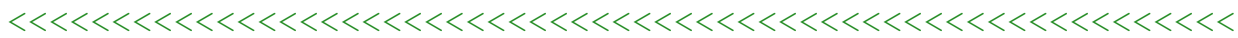

ㅇmmarizing Data

Data $=$ Combined; 
oCalculating formulae type parameters: \# CHO, CHON, CHOS, CHOP, CHN, CHS, CHP, CHNS, CHONS formulae

Summary $\cdot \mathrm{CHO}=$ sum $($ Data. $\mathrm{C}>0$ \& Data. $\mathrm{H}>0$ \& Data. $\mathrm{O}>0$ \& Data. $\mathrm{N}==0$ \& Data. $\mathrm{P}==0$ \& Data. $\mathrm{S}==0$ );

Summary. $\mathrm{CHON}=\operatorname{sum}($ Data. $\mathrm{C}>0$ \& Data. $\mathrm{H}>0$ \& Data. $\mathrm{O}>0$ \& Data. $\mathrm{N}>0$ \& Data.P==0 \& Data. $\mathrm{S}=0$ ) ) ;

Summary. $\mathrm{CHOS}=$ sum $($ Data. $\mathrm{C}>0$ \& Data. $\mathrm{H}>0$ \& Data. $0>0$ \& Data. $\mathrm{N}==0$ \& Data. $\mathrm{P}==0$ \& Data. $\mathrm{S}>0$ );

Summary $\cdot \mathrm{CHOP}=\operatorname{sum}($ Data. $\mathrm{C}>0$ \& Data.H $>0$ \& Data. $\mathrm{O}>0$ \& Data. $\mathrm{N}==0$ \& Data.P $>0$ \& Data. $\mathrm{S}==0$ );

Summary $\cdot \mathrm{CHN}=$ sum $($ Data. $\mathrm{C}>0$ \& Data. $\mathrm{H}>0$ \& Data. $\mathrm{O}==0$ \& Data. $\mathrm{N}>0$ \& Data. $\mathrm{P}==0$ \&

Data. $\mathrm{S}=0$ ) ;

Summary $\cdot \mathrm{CHS}=\operatorname{sum}($ Data. $\mathrm{C}>0$ \& Data. $\mathrm{H}>0$ \& Data. $\mathrm{O}==0$ \& Data. $\mathrm{N}==0$ \& Data. $\mathrm{P}==0$ \&

Data. S>0);

Summary. $\mathrm{CHNS}=$ sum (Data. $\mathrm{C}>0$ \& Data. $\mathrm{H}>0$ \& Data. $\mathrm{O}==0$ \& Data. $\mathrm{N}>0$ \& Data. $\mathrm{P}==0$ \&

Data. S>0);

Summary $\cdot \mathrm{CHNP}=\operatorname{sum}($ Data. $\mathrm{C}>0$ \& Data.H $>0$ \& Data. $\mathrm{O}==0$ \& Data.N $>0$ \& Data.P $>0$ \&

Data. $\mathrm{S}==0$ );

Summary $\cdot \mathrm{CHONS}=\operatorname{sum}($ Data $\cdot \mathrm{C}>0$ \& Data. $\mathrm{H}>0$ \& Data. $\mathrm{O}>0$ \& Data.N $>0$ \& Data.P==0 \&

Data. S>0);

Summary. $\mathrm{CHONP}=\operatorname{sum}($ Data. $\mathrm{C}>0$ \& Data. $\mathrm{H}>0$ \& Data. $\mathrm{O}>0$ \& Data.N $>0$ \& Data.P $>0$ \&

Data. $\mathrm{S}==0$ );

Summary. $\mathrm{CHONSP}=$ sum $($ Data. $\mathrm{C}>0$ \& Data.H $>0$ \& Data.O $>0$ \& Data.N $>0$ \& Data.P>0 \&

Data. $\mathrm{S}>0$ );

ocalculating aromaticity parameters: \# condensed aromatic (AI>=0.67),

aromatic $(\mathrm{AI}>=0.5)$,

onon-aromatic $(\mathrm{A} I<0.5)$

Summary. CondensedAromatics $=$ sum (Data.AI $>=0.67)$;

Summary. Aromatics $=$ sum (Data.AI $>=0.5)$;

Summary. NonAromatics=sum (Data.AI<0.5);

ocalculating other parameters: total \# of formulae, average molecular

oweight

Summary. TotalFormulae=height (Data);

Summary. AvgMW=mean (Data. NominalMass);

oStoring Summary Data

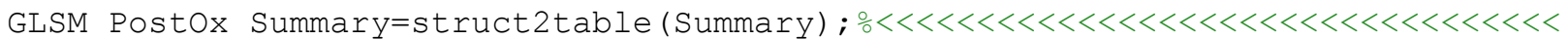
$<<<\overline{\bar{l}}<<<<<\overline{\bar{l}}<<<<<<<<<<<<<<<<<<<<<<<<<<<<<<<<<<<<<<<<<<<<<<<<<<<<<<<<<<<<<<<<<~$

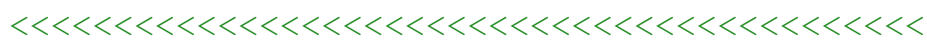

응 Comparing Pre- and Post-Oxidation SRFA

onput Data so that the script can readily be applied to any data set

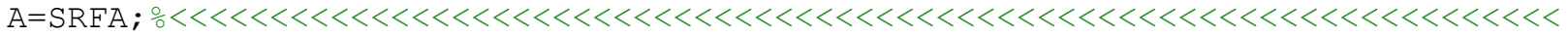

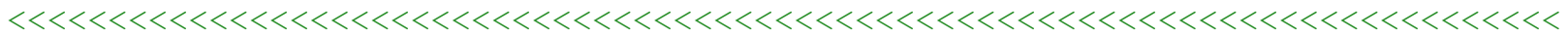
$<<<<<<<<<$

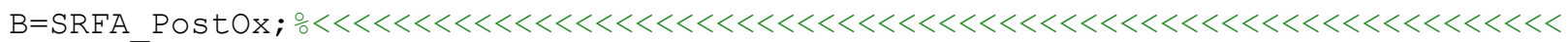

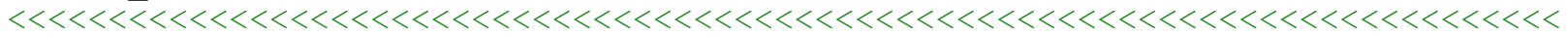
$<<<<<<<<<<<<<<<$

o Separate Data into those common and unique

Both $=[]$;

$\mathrm{k}=0$;

for $i=1$ :height $(A)$

for $j=1$ :height $(B)$

if $A \cdot C(i)==B \cdot C(j) \quad \& \& A \cdot H(i)==B \cdot H(j) \quad \& \& A \cdot N(i)==B \cdot N(j) \quad \& \& A \cdot O(i)==B \cdot O(j)$

$\& \& A \cdot P(i)==B \cdot P(j) \quad \& \& A \cdot S(i)==B \cdot S(j)$

$\mathrm{k}=\mathrm{k}+1$; 
$\operatorname{Both}(k,:)=[A . C(i), A . H(i), A . O(i), A . N(i), A . P(i)$,

A.S (i), A.NominalMass (i), A.HC_Ratio(i),

A.OC_Ratio(i),A.AI(i),A.DBE(i),A.AvgCOxState (i)] ;

$$
\text { A. C }(i)=0 \text {; }
$$

B. C $(j)=0$;

end

end

oCombining Data Sets

$A(A \cdot C==0,:)=[]$;

$A O n l_{Y}=[A . C, A . H, A . O, A . N, A . P, A . S$,

A. Nominalmass, A.HC Ratio,A.oC Ratio, A.AI, A.DBE, A.AvgCoxState];

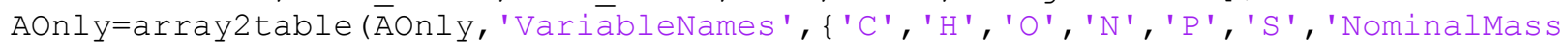
', 'HC_Ratio', 'OC_Ratio', 'AI', 'DBE', 'AvgCoxState'\});

$\mathrm{B}(\mathrm{B} \cdot \mathrm{C}==0,:)=[]$;

$\mathrm{BOnl} \mathrm{Y}_{\mathrm{B}}=[\mathrm{B} . \mathrm{C}, \mathrm{B} . \mathrm{H}, \mathrm{B} . \mathrm{O}, \mathrm{B} . \mathrm{N}, \mathrm{B} . \mathrm{P}$,

B.S,B.NominalMass,B.HC Ratio,B.oC Ratio, B.AI, B.DBE, B.AvgCoxState] ;

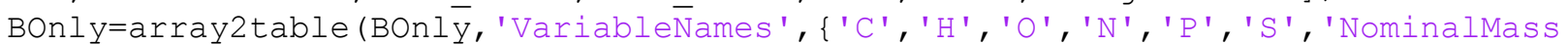
', 'HC_Ratio', 'OC_Ratio', 'AI', 'DBE', 'AvgCOxState'\}) ;

Both=array2table (Both, 'VariableNames', \{'C', 'H', 'O' , 'N', 'P' , 'S ', 'NominalMass', 'HC_Ratio', 'OC_Ratio', 'AI', 'DBE', 'AvgCOxState' \}) ;

ostoring Data with distinct names

Common PreandPost SRFA=unique (Both) ; $\circ<<<<<<<<<<<<<<<<<<<<<<<<<<<<<<<<<<<<<<<<<~$

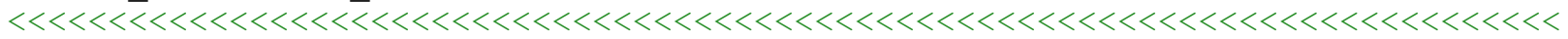

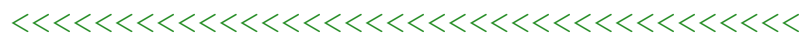

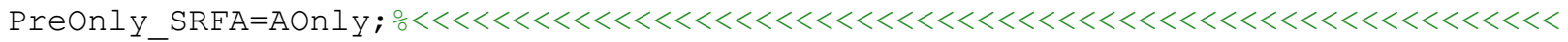

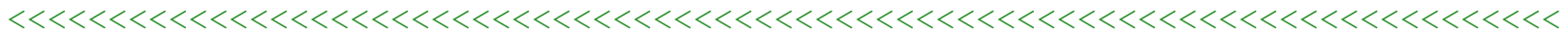
$<<<<<<<<<<<<<<<<<<<<<~$

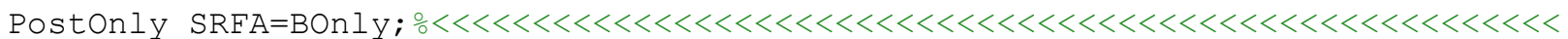

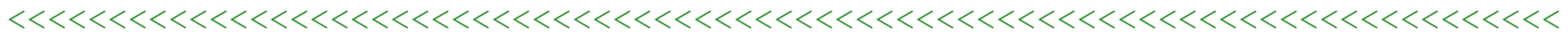
$<<<<<<<<<<<<<<<<<<<<<<~$

\% Summarizing Data

Data=AOnly;

oCalculating formulae type parameters: \# CHO, CHON, CHOS, CHOP, CHN, CHS, CHP, CHNS, CHONS formulae

Summary. $\mathrm{CHO}=\operatorname{sum}($ Data. $\mathrm{C}>0$ \& Data. $\mathrm{H}>0$ \& Data. $\mathrm{O}>0$ \& Data. $\mathrm{N}==0$ \& Data. $\mathrm{P}==0$ \& Data. $\mathrm{S}==0$ );

Summary. $\mathrm{CHON}=$ sum $($ Data. $\mathrm{C}>0$ \& Data. $\mathrm{H}>0$ \& Data. $\mathrm{O}>0$ \& Data. $\mathrm{N}>0$ \& Data.P==0 \&

Data. $\mathrm{S}=0$ ) ;

Summary $\cdot \mathrm{CHOS}=$ sum $($ Data. $\mathrm{C}>0$ \& Data.H $>0$ \& Data. O $>0$ \& Data. $\mathrm{N}==0$ \& Data. $\mathrm{P}==0$ \&

Data. S>0);

Summary. $\mathrm{CHOP}=\operatorname{sum}($ Data. $\mathrm{C}>0$ \& Data. $\mathrm{H}>0$ \& Data. $\mathrm{O}>0$ \& Data. $\mathrm{N}==0$ \& Data. $\mathrm{P}>0$ \&

Data. $\mathrm{S}==0$ );

Summary $\cdot \mathrm{CHN}=$ sum $($ Data. $\mathrm{C}>0$ \& Data. $\mathrm{H}>0$ \& Data. $\mathrm{O}==0$ \& Data. $\mathrm{N}>0$ \& Data. $\mathrm{P}==0$ \&

Data. $\mathrm{S}==0$ );

Summary $\cdot \mathrm{CHS}=\operatorname{sum}($ Data $\cdot \mathrm{C}>0$ \& Data. $\mathrm{H}>0$ \& Data $\cdot \mathrm{O}==0$ \& Data. $\mathrm{N}==0$ \& Data. $\mathrm{P}==0$ \&

Data.s>0);

Summary. $\mathrm{CHNS}=\operatorname{sum}($ Data. $\mathrm{C}>0$ \& Data. $\mathrm{H}>0$ \& Data. $\mathrm{O}==0$ \& Data. $\mathrm{N}>0$ \& Data. $\mathrm{P}==0$ \&

Data. S>0) ;

Summary. $\mathrm{CHNP}=\mathrm{sum}($ Data. $\mathrm{C}>0$ \& Data.H $>0$ \& Data. $\mathrm{O}==0$ \& Data. $\mathrm{N}>0$ \& Data.P $>0$ \&

Data. $\mathrm{S}=0$ ) ;

Summary $\cdot \mathrm{CHONS}=\operatorname{sum}($ Data $\cdot \mathrm{C}>0$ \& Data. $\mathrm{H}>0$ \& Data. $\mathrm{O}>0$ \& Data. $\mathrm{N}>0$ \& Data.P==0 \&

Data. $\mathrm{S}>0$ );

Summary. $\mathrm{CHONP}=\operatorname{sum}($ Data. $\mathrm{C}>0$ \& Data. $\mathrm{H}>0$ \& Data. $\mathrm{O}>0$ \& Data.N $>0$ \& Data.P>0\&

Data. $\mathrm{S}=0$ ) ;

Summary. $\mathrm{CHONSP}=$ sum $($ Data. $\mathrm{C}>0$ \& Data.H $>0$ \& Data.O $>0$ \& Data.N $>0$ \& Data.P $>0$ \&

Data.S $>0$ ); 
oCalculating aromaticity parameters: \# condensed aromatic (AI>=0.67), aromatic $(\mathrm{AI}>=0.5)$,

onon-aromatic $(\mathrm{AI}<0.5)$

Summary. CondensedAromatics $=$ sum (Data.AI $>=0.67)$;

Summary.Aromatics=sum (Data.AI $>=0.5)$;

Summary. NonAromatics=sum (Data.AI<0.5);

ocalculating other parameters: total \# of formulae, average molecular

oweight

Summary. TotalFormulae=height (Data);

Summary. AvgMW=mean (Data. NominalMass);

ostoring Summary Data

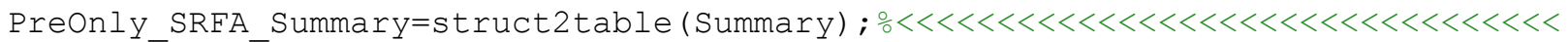
$<<<<<<<\overline{<}<<<\overline{<}<<<<<<<<<<<<<<<<<<<<<<<<<<<<<<<<<<<<<<<<<<<<<<<<<<<<<<<<<<<<<<<<~$

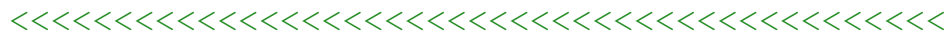

ㅇmmarizing Data

Data=BOnly;

oCalculating formulae type parameters: \# CHO, CHON, CHOS, CHOP, CHN, CHS, CHP, CHNS, CHONS formulae

Summary $\cdot \mathrm{CHO}=$ sum (Data. $\mathrm{C}>0$ \& Data. $\mathrm{H}>0$ \& Data. $\mathrm{O}>0$ \& Data. $\mathrm{N}==0$ \& Data. $\mathrm{P}==0$ \& Data. $\mathrm{S}==0$ );

Summary $\cdot \mathrm{CHON}=$ Sum $($ Data. $\mathrm{C}>0$ \& Data. $\mathrm{H}>0$ \& Data. $\mathrm{O}>0$ \& Data. $\mathrm{N}>0$ \& Data.P==0 \& Data. $\mathrm{S}==0$ );

Summary. $\mathrm{CHOS}=$ sum $($ Data. $\mathrm{C}>0$ \& Data. $\mathrm{H}>0$ \& Data. $\mathrm{O}>0$ \& Data. $\mathrm{N}==0$ \& Data. $\mathrm{P}==0$ \&

Data. $\mathrm{S}>0$ ) ;

Summary $\cdot \mathrm{CHOP}=$ sum $($ Data. $\mathrm{C}>0$ \& Data.H $>0$ \& Data. $\mathrm{O}>0$ \& Data. $\mathrm{N}==0$ \& Data.P $>0$ \&

Data. $\mathrm{S}==0$ );

Summary $\cdot \mathrm{CHN}=$ sum $($ Data. $\mathrm{C}>0$ \& Data. $\mathrm{H}>0$ \& Data. $\mathrm{O}==0$ \& Data. $\mathrm{N}>0$ \& Data.P==0 \&

Data. $\mathrm{S}==0$ );

Summary. $\mathrm{CHS}=\mathrm{sum}($ Data. $\mathrm{C}>0$ \& Data. $\mathrm{H}>0$ \& Data. $\mathrm{O}==0$ \& Data. $\mathrm{N}==0$ \& Data. $\mathrm{P}==0$ \&

Data. $\mathrm{S}>0$ );

Summary. $\mathrm{CHNS}=\operatorname{sum}($ Data. $\mathrm{C}>0$ \& Data. $\mathrm{H}>0$ \& Data. $\mathrm{O}==0$ \& Data. $\mathrm{N}>0$ \& Data. $\mathrm{P}==0$ \&

Data. S>0);

Summary $\cdot \mathrm{CHNP}=\mathrm{sum}($ Data. $\mathrm{C}>0$ \& Data.H $>0$ \& Data. $\mathrm{O}==0$ \& Data. $\mathrm{N}>0$ \& Data.P $>0$ \&

Data. $\mathrm{S}==0$ );

Summary. $\mathrm{CHONS}=\operatorname{sum}($ Data. $\mathrm{C}>0$ \& Data. $\mathrm{H}>0$ \& Data. $0>0$ \& Data.N>0 \& Data.P==0 \&

Data. S>0);

Summary. $\mathrm{CHONP}=$ sum $($ Data. $\mathrm{C}>0$ \& Data. $\mathrm{H}>0$ \& Data. $\mathrm{O}>0$ \& Data.N $>0$ \& Data.P $>0$ \&

Data. $\mathrm{S}==0$ ) ;

Summary. $\mathrm{CHONSP}=$ sum $($ Data. $\mathrm{C}>0$ \& Data.H $>0$ \& Data.O $>0$ \& Data.N $>0$ \& Data.P>0 \&

Data. $\mathrm{S}>0$ );

oCalculating aromaticity parameters: \# condensed aromatic (AI>=0.67),

aromatic $(A I>=0.5)$,

onon-aromatic $($ AI $<0.5)$

Summary. CondensedAromatics $=$ sum (Data. AI $>=0.67)$;

Summary. Aromatics $=$ sum (Data.AI $>=0.5)$;

Summary. NonAromatics=sum (Data.AI<0.5);

ocalculating other parameters: total \# of formulae, average molecular

oweight

Summary. TotalFormulae=height (Data);

Summary. AvgMW=mean (Data. NominalMass);

ostoring Summary Data

Postonly SRFA Summary=struct2table (Summary); $\circ<<<<<<<<<<<<<<<<<<<<<<<<<<<<<<<<<<<<~$ $<<<<<<<<\bar{L}<<<<<<<<<<<<<<<<<<<<<<<<<<<<<<<<<<<<<<<<<<<<<<<<<<<<<<<<<<<<<<<<<<<<~$

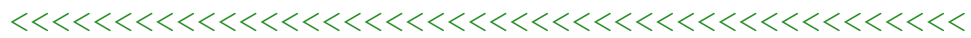

o Summarizing Data

Data=unique (Both); 
oCalculating formulae type parameters: \# CHO, CHON, CHOS, CHOP, CHN, CHS, CHP, CHNS, CHONS formulae

Summary $\cdot \mathrm{CHO}=$ sum $($ Data. $\mathrm{C}>0$ \& Data. $\mathrm{H}>0$ \& Data. $\mathrm{O}>0$ \& Data. $\mathrm{N}==0$ \& Data. $\mathrm{P}==0$ \& Data. $\mathrm{S}==0$ );

Summary. $\mathrm{CHON}=\operatorname{sum}($ Data. $\mathrm{C}>0$ \& Data. $\mathrm{H}>0$ \& Data. $\mathrm{O}>0$ \& Data. $\mathrm{N}>0$ \& Data.P==0 \& Data. $\mathrm{S}=0$ ) ) ;

Summary. $\mathrm{CHOS}=$ sum $($ Data. $\mathrm{C}>0$ \& Data. $\mathrm{H}>0$ \& Data. $0>0$ \& Data. $\mathrm{N}==0$ \& Data. $\mathrm{P}==0$ \& Data. $\mathrm{S}>0$ );

Summary $\cdot \mathrm{CHOP}=\operatorname{sum}($ Data. $\mathrm{C}>0$ \& Data.H $>0$ \& Data. $\mathrm{O}>0$ \& Data. $\mathrm{N}==0$ \& Data.P $>0$ \& Data. $\mathrm{S}==0$ );

Summary $\cdot \mathrm{CHN}=$ sum $($ Data. $\mathrm{C}>0$ \& Data. $\mathrm{H}>0$ \& Data. $\mathrm{O}==0$ \& Data. $\mathrm{N}>0$ \& Data. $\mathrm{P}==0$ \&

Data. $\mathrm{S}=0$ ) ;

Summary $\cdot \mathrm{CHS}=\operatorname{sum}($ Data. $\mathrm{C}>0$ \& Data. $\mathrm{H}>0$ \& Data. $\mathrm{O}==0$ \& Data. $\mathrm{N}==0$ \& Data. $\mathrm{P}==0$ \&

Data. S>0);

Summary. $\mathrm{CHNS}=$ sum (Data. $\mathrm{C}>0$ \& Data. $\mathrm{H}>0$ \& Data. $\mathrm{O}==0$ \& Data. $\mathrm{N}>0$ \& Data. $\mathrm{P}==0$ \&

Data. S>0);

Summary $\cdot \mathrm{CHNP}=\operatorname{sum}($ Data. $\mathrm{C}>0$ \& Data.H $>0$ \& Data. $\mathrm{O}==0$ \& Data.N $>0$ \& Data.P $>0$ \&

Data. $\mathrm{S}==0$ );

Summary $\cdot \mathrm{CHONS}=\operatorname{sum}($ Data $\cdot \mathrm{C}>0$ \& Data. $\mathrm{H}>0$ \& Data. $\mathrm{O}>0$ \& Data.N $>0$ \& Data.P==0 \&

Data. S>0);

Summary. $\mathrm{CHONP}=\operatorname{sum}($ Data. $\mathrm{C}>0$ \& Data. $\mathrm{H}>0$ \& Data. $\mathrm{O}>0$ \& Data.N $>0$ \& Data.P $>0$ \&

Data. $\mathrm{S}==0$ );

Summary. $\mathrm{CHONSP}=$ sum $($ Data. $\mathrm{C}>0$ \& Data. $\mathrm{H}>0$ \& Data. $\mathrm{O}>0$ \& Data.N $>0$ \& Data.P $>0$ \&

Data. $\mathrm{S}>0$ );

oCalculating aromaticity parameters: \# condensed aromatic (AI>=0.67),

aromatic $(\mathrm{AI}>=0.5)$,

onon-aromatic $(\mathrm{A} I<0.5)$

Summary. CondensedAromatics $=$ sum (Data.AI $>=0.67)$;

Summary. Aromatics $=$ sum (Data.AI $>=0.5)$;

Summary. NonAromatics=sum (Data.AI<0.5);

ocalculating other parameters: total \# of formulae, average molecular

oweight

Summary. TotalFormulae=height (Data);

Summary. AvgMW=mean (Data. NominalMass);

ostoring Summary Data

Common PreandPost SRFA Summary=struct2table (Summary); $\circ$ o $<<<<<<<<<<<<<<<<<<<<<<<<~$ $<<<<<<\overline{<}<<<<<<<<<\overline{\bar{l}}<<<\overline{<} \overline{<}<<<<<<<<<<<<<<<<<<<<<<<<<<<<<<<<<<<<<<<<<<<<<<<<<<<<<<$

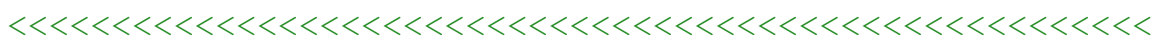

응 Comparing Pre- and Post-Oxidation GLSM

onput Data so that the script can readily be applied to any data set

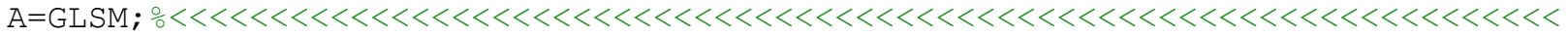

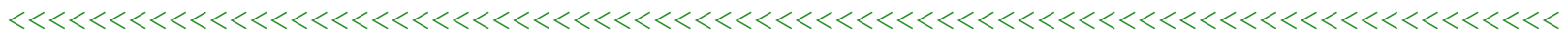

$<<<<<<<<<$

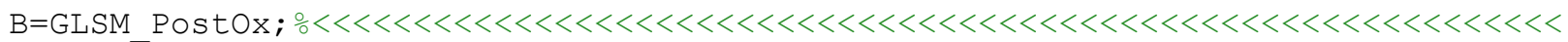

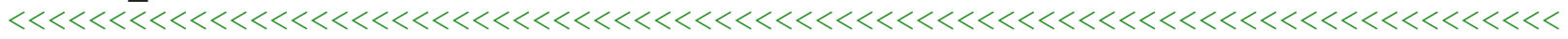
$<<<<<<<<<<<<<<<$

o Separate Data into those common and unique

Both $=[]$;

$\mathrm{k}=0$;

for $i=1$ : height $(A)$

for $j=1:$ height $(B)$

if $A \cdot C(i)==B \cdot C(j) \quad \& \& A \cdot H(i)==B \cdot H(j) \quad \& \& A \cdot N(i)==B \cdot N(j) \quad \& \& A \cdot O(i)==B \cdot O(j)$

$\& \& A \cdot P(i)==B \cdot P(j) \quad \& \& A \cdot S(i)==B \cdot S(j)$

$\mathrm{k}=\mathrm{k}+1$; 
$\operatorname{Both}(k,:)=[A . C(i), A . H(i), A . O(i), A . N(i), A . P(i)$,

A.S (i), A.NominalMass (i), A.HC_Ratio(i),

A.OC_Ratio(i),A.AI(i),A.DBE(i),A.AvgCOxState (i)] ;

$$
\text { A. C }(i)=0 \text {; }
$$

B. C $(j)=0$;

end

end

oCombining Data Sets

$A(A \cdot C==0,:)=[]$;

$A O n l_{Y}=[A . C, A . H, A . O, A . N, A . P, A . S$,

A. Nominalmass, A.HC Ratio,A.oC Ratio, A.AI, A.DBE, A.AvgCoxState];

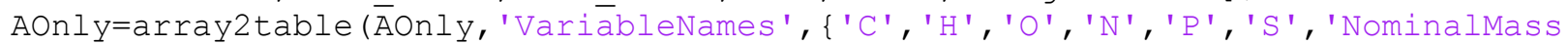
', 'HC_Ratio', 'OC_Ratio', 'AI', 'DBE', 'AvgCoxState'\});

$\mathrm{B}(\mathrm{B} \cdot \mathrm{C}==0,:)=[]$;

$\mathrm{BOnl} \mathrm{Y}_{\mathrm{B}}=[\mathrm{B} . \mathrm{C}, \mathrm{B} . \mathrm{H}, \mathrm{B} . \mathrm{O}, \mathrm{B} . \mathrm{N}, \mathrm{B} . \mathrm{P}$,

B.S,B.Nominalmass, B.HC_Ratio,B.OC_Ratio,B.AI, B.DBE, B.AvgCoxState] ;

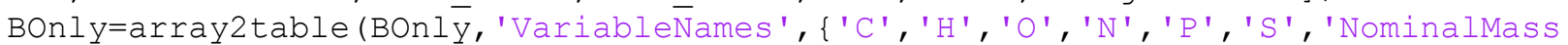
', 'HC_Ratio', 'OC_Ratio', 'AI', 'DBE', 'AvgCOxState'\}) ;

Both=array2table (Both, 'VariableNames', \{'C', 'H', 'O' , 'N', 'P' , 'S ', 'NominalMass', 'HC_Ratio', 'OC_Ratio', 'AI', 'DBE', 'AvgCOxState' \}) ;

ostoring Data with distinct names

Common PreandPost GLSM=unique (Both) ; $\circ<<<<<<<<<<<<<<<<<<<<<<<<<<<<<<<<<<<<<<<<<~$

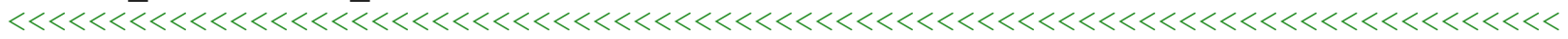

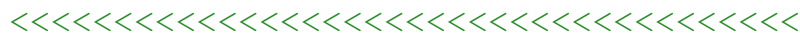

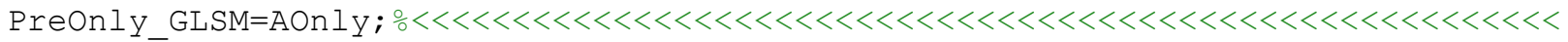

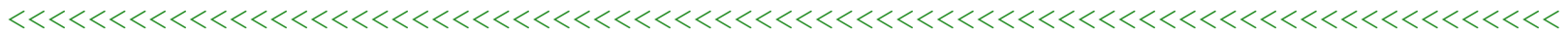
$<<<<<<<<<<<<<<<<<<<<<~$

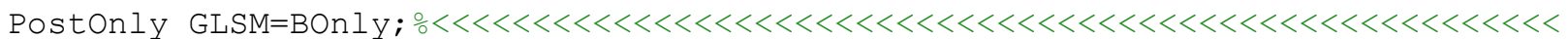

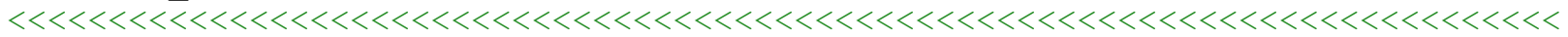
$<<<<<<<<<<<<<<<<<<<<<<~$

\% Summarizing Data

Data=AOnly;

oCalculating formulae type parameters: \# CHO, CHON, CHOS, CHOP, CHN, CHS, CHP, CHNS, CHONS formulae

Summary. $\mathrm{CHO}=\operatorname{sum}($ Data. $\mathrm{C}>0$ \& Data. $\mathrm{H}>0$ \& Data. $\mathrm{O}>0$ \& Data. $\mathrm{N}==0$ \& Data. $\mathrm{P}==0$ \& Data. $\mathrm{S}==0$ );

Summary. $\mathrm{CHON}=$ sum $($ Data. $\mathrm{C}>0$ \& Data. $\mathrm{H}>0$ \& Data. $\mathrm{O}>0$ \& Data. $\mathrm{N}>0$ \& Data.P==0 \&

Data. $\mathrm{S}=0$ ) ;

Summary $\cdot \mathrm{CHOS}=$ sum $($ Data. $\mathrm{C}>0$ \& Data.H $>0$ \& Data. O $>0$ \& Data. $\mathrm{N}==0$ \& Data. $\mathrm{P}==0$ \&

Data. S>0);

Summary. $\mathrm{CHOP}=\operatorname{sum}($ Data. $\mathrm{C}>0$ \& Data. $\mathrm{H}>0$ \& Data. $\mathrm{O}>0$ \& Data. $\mathrm{N}==0$ \& Data. $\mathrm{P}>0$ \&

Data. $\mathrm{S}==0$ );

Summary $\cdot \mathrm{CHN}=$ sum $($ Data. $\mathrm{C}>0$ \& Data. $\mathrm{H}>0$ \& Data. $\mathrm{O}==0$ \& Data. $\mathrm{N}>0$ \& Data. $\mathrm{P}==0$ \&

Data. $\mathrm{S}==0$ );

Summary $\cdot \mathrm{CHS}=\operatorname{sum}($ Data $\cdot \mathrm{C}>0$ \& Data. $\mathrm{H}>0$ \& Data $\cdot \mathrm{O}==0$ \& Data. $\mathrm{N}==0$ \& Data. $\mathrm{P}==0$ \&

Data.s>0);

Summary. $\mathrm{CHNS}=\operatorname{sum}($ Data. $\mathrm{C}>0$ \& Data. $\mathrm{H}>0$ \& Data. $\mathrm{O}==0$ \& Data. $\mathrm{N}>0$ \& Data. $\mathrm{P}==0$ \&

Data.S>0);

Summary $\cdot \mathrm{CHNP}=\mathrm{sum}($ Data. $\mathrm{C}>0$ \& Data.H $>0$ \& Data. $\mathrm{O}==0$ \& Data.N $>0$ \& Data.P $>0$ \&

Data. $\mathrm{S}=0$ ) ;

Summary $\cdot \mathrm{CHONS}=\operatorname{sum}($ Data $\cdot \mathrm{C}>0$ \& Data. $\mathrm{H}>0$ \& Data. $\mathrm{O}>0$ \& Data. $\mathrm{N}>0$ \& Data.P==0 \&

Data. $\mathrm{S}>0$ );

Summary. $\mathrm{CHONP}=\operatorname{sum}($ Data. $\mathrm{C}>0$ \& Data. $\mathrm{H}>0$ \& Data. $\mathrm{O}>0$ \& Data.N $>0$ \& Data.P>0\&

Data. $\mathrm{S}=0$ ) ;

Summary. $\mathrm{CHONSP}=$ sum $($ Data. $\mathrm{C}>0$ \& Data.H $>0$ \& Data.O $>0$ \& Data.N $>0$ \& Data.P $>0$ \&

Data. S>0); 
oCalculating aromaticity parameters: \# condensed aromatic (AI>=0.67), aromatic $(\mathrm{AI}>=0.5)$,

onon-aromatic $(\mathrm{AI}<0.5)$

Summary. CondensedAromatics $=$ sum (Data.AI $>=0.67)$;

Summary.Aromatics=sum (Data.AI $>=0.5)$;

Summary. NonAromatics=sum (Data.AI<0.5);

ocalculating other parameters: total \# of formulae, average molecular

oweight

Summary. TotalFormulae=height (Data);

Summary. AvgMW=mean (Data. NominalMass);

ostoring Summary Data

PreOnIy_GLSM_Summary=struct2table (Summary) ; $\circ<<<<<<<<<<<<<<<<<<<<<<<<<<<<<<<<<<~$ $<<<<<<<\overline{<}<<<\overline{<}<<<<<<<<<<<<<<<<<<<<<<<<<<<<<<<<<<<<<<<<<<<<<<<<<<<<<<<<<<<<<<<<~$

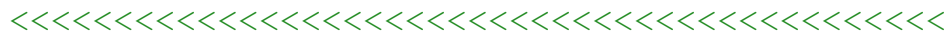

ㅇmmarizing Data

Data=BOnly;

oCalculating formulae type parameters: \# CHO, CHON, CHOS, CHOP, CHN, CHS, CHP, CHNS, CHONS formulae

Summary $\cdot \mathrm{CHO}=$ sum (Data. $\mathrm{C}>0$ \& Data. $\mathrm{H}>0$ \& Data. $\mathrm{O}>0$ \& Data. $\mathrm{N}==0$ \& Data. $\mathrm{P}==0$ \& Data. $\mathrm{S}==0$ );

Summary $\cdot \mathrm{CHON}=$ Sum $($ Data. $\mathrm{C}>0$ \& Data. $\mathrm{H}>0$ \& Data. $\mathrm{O}>0$ \& Data. $\mathrm{N}>0$ \& Data.P==0 \& Data. $\mathrm{S}==0$ );

Summary. $\mathrm{CHOS}=$ sum $($ Data. $\mathrm{C}>0$ \& Data. $\mathrm{H}>0$ \& Data. $\mathrm{O}>0$ \& Data. $\mathrm{N}==0$ \& Data. $\mathrm{P}==0$ \&

Data. $\mathrm{S}>0$ ) ;

Summary $\cdot \mathrm{CHOP}=$ sum $($ Data. $\mathrm{C}>0$ \& Data.H $>0$ \& Data. $\mathrm{O}>0$ \& Data. $\mathrm{N}==0$ \& Data.P $>0$ \&

Data. $\mathrm{S}==0$ );

Summary $\cdot \mathrm{CHN}=$ sum $($ Data. $\mathrm{C}>0$ \& Data. $\mathrm{H}>0$ \& Data. $\mathrm{O}==0$ \& Data. $\mathrm{N}>0$ \& Data.P==0 \&

Data. $\mathrm{S}==0$ );

Summary. $\mathrm{CHS}=\mathrm{sum}($ Data. $\mathrm{C}>0$ \& Data. $\mathrm{H}>0$ \& Data. $\mathrm{O}==0$ \& Data. $\mathrm{N}==0$ \& Data. $\mathrm{P}==0$ \&

Data. $\mathrm{S}>0$ );

Summary. $\mathrm{CHNS}=\operatorname{sum}($ Data. $\mathrm{C}>0$ \& Data. $\mathrm{H}>0$ \& Data. $\mathrm{O}==0$ \& Data. $\mathrm{N}>0$ \& Data. $\mathrm{P}==0$ \&

Data. S>0);

Summary $\cdot \mathrm{CHNP}=\mathrm{sum}($ Data. $\mathrm{C}>0$ \& Data.H $>0$ \& Data. $\mathrm{O}==0$ \& Data. $\mathrm{N}>0$ \& Data.P $>0$ \&

Data. $\mathrm{S}==0$ );

Summary. $\mathrm{CHONS}=\operatorname{sum}($ Data $. \mathrm{C}>0$ \& Data. $\mathrm{H}>0$ \& Data. $0>0$ \& Data.N>0 \& Data.P==0 \&

Data. S>0);

Summary. $\mathrm{CHONP}=$ sum $($ Data. $\mathrm{C}>0$ \& Data. $\mathrm{H}>0$ \& Data. $\mathrm{O}>0$ \& Data.N $>0$ \& Data.P $>0$ \&

Data. $\mathrm{S}==0$ ) ;

Summary. $\mathrm{CHONSP}=$ sum $($ Data. $\mathrm{C}>0$ \& Data.H $>0$ \& Data.O $>0$ \& Data.N $>0$ \& Data.P>0 \&

Data. $\mathrm{S}>0$ );

oCalculating aromaticity parameters: \# condensed aromatic (AI>=0.67),

aromatic $(A I>=0.5)$,

onon-aromatic $($ AI $<0.5)$

Summary. CondensedAromatics $=$ sum (Data.AI $>=0.67)$;

Summary. Aromatics $=$ sum (Data.AI $>=0.5)$;

Summary. NonAromatics=sum (Data.AI<0.5);

ocalculating other parameters: total \# of formulae, average molecular

oweight

Summary. TotalFormulae=height (Data);

Summary. AvgMW=mean (Data. NominalMass);

ostoring Summary Data

Postonly GLSM Summary=struct2table (Summary); $\circ<<<<<<<<<<<<<<<<<<<<<<<<<<<<<<<<<<<<~$

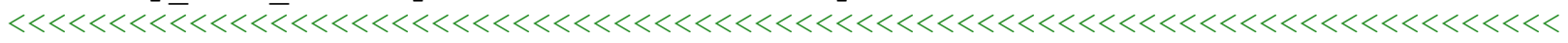

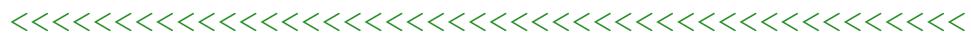

o Summarizing Data

Data=unique (Both); 
oCalculating formulae type parameters: \# CHO, CHON, CHOS, CHOP, CHN, CHS, CHP, CHNS, CHONS formulae

Summary $\cdot \mathrm{CHO}=$ sum $($ Data. $\mathrm{C}>0$ \& Data. $\mathrm{H}>0$ \& Data. $\mathrm{O}>0$ \& Data. $\mathrm{N}==0$ \& Data. $\mathrm{P}==0$ \& Data. $\mathrm{S}==0$ );

Summary. $\mathrm{CHON}=\operatorname{sum}($ Data. $\mathrm{C}>0$ \& Data. $\mathrm{H}>0$ \& Data. $\mathrm{O}>0$ \& Data. $\mathrm{N}>0$ \& Data.P==0 \& Data. $\mathrm{S}=0$ ) ) ;

Summary. $\mathrm{CHOS}=$ sum $($ Data. $\mathrm{C}>0$ \& Data. $\mathrm{H}>0$ \& Data. $0>0$ \& Data. $\mathrm{N}==0$ \& Data. $\mathrm{P}==0$ \& Data. $\mathrm{S}>0$ );

Summary $\cdot \mathrm{CHOP}=\operatorname{sum}($ Data. $\mathrm{C}>0$ \& Data.H $>0$ \& Data. $\mathrm{O}>0$ \& Data. $\mathrm{N}==0$ \& Data.P $>0$ \& Data. $\mathrm{S}==0$ );

Summary $\cdot \mathrm{CHN}=$ sum $($ Data. $\mathrm{C}>0$ \& Data. $\mathrm{H}>0$ \& Data. $\mathrm{O}==0$ \& Data. $\mathrm{N}>0$ \& Data. $\mathrm{P}==0$ \&

Data. $\mathrm{S}=0$ ) ;

Summary $\cdot \mathrm{CHS}=\operatorname{sum}($ Data. $\mathrm{C}>0$ \& Data. $\mathrm{H}>0$ \& Data. $\mathrm{O}==0$ \& Data. $\mathrm{N}==0$ \& Data. $\mathrm{P}==0$ \&

Data. S>0);

Summary. $\mathrm{CHNS}=$ sum (Data. $\mathrm{C}>0$ \& Data. $\mathrm{H}>0$ \& Data. $\mathrm{O}==0$ \& Data. $\mathrm{N}>0$ \& Data. $\mathrm{P}==0$ \&

Data. S>0);

Summary $\cdot \mathrm{CHNP}=\operatorname{sum}($ Data. $\mathrm{C}>0$ \& Data.H $>0$ \& Data. $\mathrm{O}==0$ \& Data.N $>0$ \& Data.P $>0$ \&

Data. $\mathrm{S}==0$ );

Summary $\cdot \mathrm{CHONS}=\operatorname{sum}($ Data $\cdot \mathrm{C}>0$ \& Data. $\mathrm{H}>0$ \& Data. $\mathrm{O}>0$ \& Data.N $>0$ \& Data.P==0 \&

Data. S>0);

Summary. $\mathrm{CHONP}=\operatorname{sum}($ Data. $\mathrm{C}>0$ \& Data. $\mathrm{H}>0$ \& Data. $\mathrm{O}>0$ \& Data.N $>0$ \& Data.P $>0$ \&

Data. $\mathrm{S}==0$ );

Summary. $\mathrm{CHONSP}=$ sum $($ Data. $\mathrm{C}>0$ \& Data. $\mathrm{H}>0$ \& Data. $\mathrm{O}>0$ \& Data.N $>0$ \& Data.P $>0$ \&

Data. S>0);

ocalculating aromaticity parameters: \# condensed aromatic (AI>=0.67),

aromatic $(\mathrm{AI}>=0.5)$,

onon-aromatic $(\mathrm{A} I<0.5)$

Summary. CondensedAromatics $=$ sum (Data.AI $>=0.67)$;

Summary. Aromatics $=$ sum (Data.AI $>=0.5)$;

Summary. NonAromatics=sum (Data.AI<0.5);

ocalculating other parameters: total \# of formulae, average molecular

oweight

Summary. TotalFormulae=height (Data);

Summary. AvgMW=mean (Data. NominalMass);

ostoring Summary Data

Common PreandPost GLSM Summary=struct2table (Summary); 응 $<<<<<<<<<<<<<<<<<<<<<<<<~$ $<<<<<<\overline{<}<<<<<<<<<\overline{\bar{l}}<<<\overline{<} \overline{<}<<<<<<<<<<<<<<<<<<<<<<<<<<<<<<<<<<<<<<<<<<<<<<<<<<<<<<$

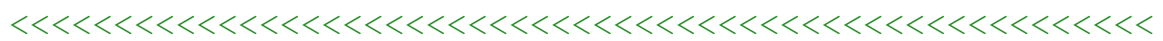

응 Comparing Pre- and Post-Oxidation SRFA for negative mode ESI data oInput Data so that the script can readily be applied to any data set

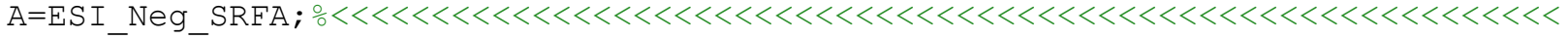

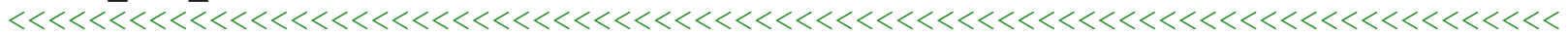
$<<<<<<<<<<<<<<<<<$

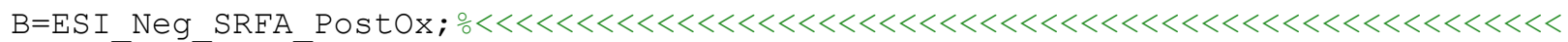

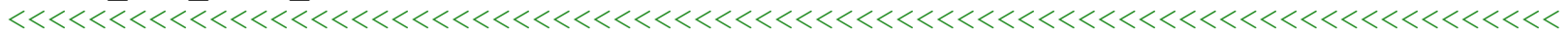
$<<<<<<<<<<<<<<<<<<<<<<<<~$

o Separate Data into those common and unique

Both $=[]$;

$\mathrm{k}=0$;

for $i=1$ :height $(A)$

for $j=1$ :height $(B)$

if $A \cdot C(i)==B \cdot C(j) \quad \& \& A \cdot H(i)==B \cdot H(j) \quad \& \& A \cdot N(i)==B \cdot N(j) \quad \& \& A \cdot O(i)==B \cdot O(j)$

$\& \& A \cdot P(i)==B \cdot P(j) \quad \& \& A \cdot S(i)==B \cdot S(j)$

$\mathrm{k}=\mathrm{k}+1$; 
$\operatorname{Both}(k,:)=[A . C(i), A . H(i), A . O(i), A . N(i), A . P(i)$,

A.S (i), A.NominalMass (i), A.HC_Ratio(i),

A.OC_Ratio(i),A.AI(i),A.DBE(i),A.AvgCOxState (i)] ;

$$
\text { A. C }(i)=0 \text {; }
$$

B. C $(j)=0$;

end

end

oCombining Data Sets

$A(A \cdot C==0,:)=[]$;

$A O n l_{Y}=[A . C, A . H, A . O, A . N, A . P, A . S$,

A. Nominalmass, A.HC Ratio,A.oC Ratio, A.AI, A.DBE, A.AvgCoxState];

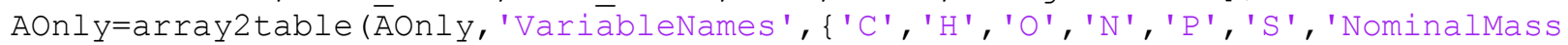
', 'HC_Ratio', 'OC_Ratio', 'AI', 'DBE', 'AvgCoxState'\});

$\mathrm{B}(\mathrm{B} \cdot \mathrm{C}==0,:)=[]$;

$\mathrm{BOnl} \mathrm{Y}_{\mathrm{B}}=[\mathrm{B} . \mathrm{C}, \mathrm{B} . \mathrm{H}, \mathrm{B} . \mathrm{O}, \mathrm{B} . \mathrm{N}, \mathrm{B} . \mathrm{P}$,

B.S,B.Nominalmass, B.HC_Ratio,B.OC_Ratio,B.AI, B.DBE, B.AvgCoxState] ;

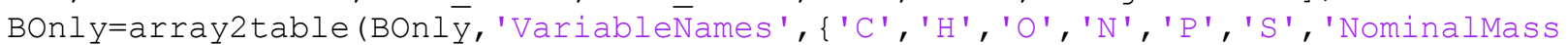
', 'HC_Ratio', 'OC_Ratio', 'AI', 'DBE', 'AvgCOxState'\}) ;

Both=array2table (Both, 'VariableNames', \{'C', 'H', 'O' , 'N', 'P' , 'S ', 'NominalMass', 'HC_Ratio', 'OC_Ratio', 'AI', 'DBE', 'AvgCOxState' \}) ;

ostoring Data with distinct names

Common PreandPost ESI Neg SRFA=unique (Both) ; $\circ<<<<<<<<<<<<<<<<<<<<<<<<<<<<<<<<<<~$ $<<<<<<\overline{<}<<<<<<<<<<\overline{<}<<<\overline{<}<<<\overline{<}<<<<<<<<<<<<<<<<<<<<<<<<<<<<<<<<<<<<<<<<<<<<<<<<<<<~$

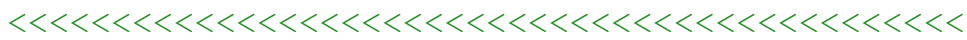

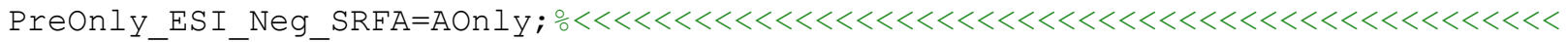
$<<<<<<\overline{<}<<<\overline{<}<<<\overline{<}<<<<<<<<<<<<<<<<<<<<<<<<<<<<<<<<<<<<<<<<<<<<<<<<<<<<<<<<<<<<<~$ $<<<<<<<<<<<<<<<<<<<<<<<<<<<<<~$

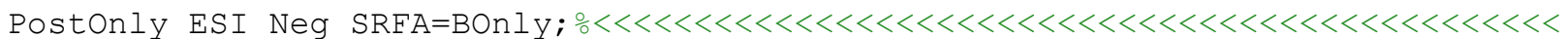

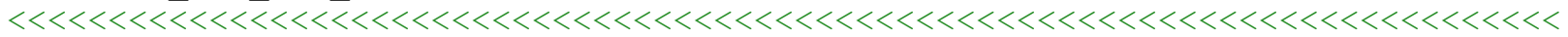
$<<<<<<<<<<<<<<<<<<<<<<<<<<<<<<~$

\% Summarizing Data

Data=AOnly;

oCalculating formulae type parameters: \# CHO, CHON, CHOS, CHOP, CHN, CHS, CHP, CHNS, CHONS formulae

Summary. $\mathrm{CHO}=\operatorname{sum}($ Data. $\mathrm{C}>0$ \& Data. $\mathrm{H}>0$ \& Data. $\mathrm{O}>0$ \& Data. $\mathrm{N}==0$ \& Data. $\mathrm{P}==0$ \& Data. $\mathrm{S}==0$ );

Summary. $\mathrm{CHON}=$ sum $($ Data. $\mathrm{C}>0$ \& Data. $\mathrm{H}>0$ \& Data. $\mathrm{O}>0$ \& Data. $\mathrm{N}>0$ \& Data.P==0 \&

Data. $\mathrm{S}=0$ ) ;

Summary $\cdot \mathrm{CHOS}=$ sum $($ Data. $\mathrm{C}>0$ \& Data.H $>0$ \& Data. O $>0$ \& Data. $\mathrm{N}==0$ \& Data. $\mathrm{P}==0$ \&

Data. S>0);

Summary. $\mathrm{CHOP}=\operatorname{sum}($ Data. $\mathrm{C}>0$ \& Data. $\mathrm{H}>0$ \& Data. $\mathrm{O}>0$ \& Data. $\mathrm{N}==0$ \& Data. $\mathrm{P}>0$ \&

Data. $\mathrm{S}==0$ ) ;

Summary. $\mathrm{CHN}=\operatorname{sum}($ Data. $\mathrm{C}>0$ \& Data. $\mathrm{H}>0$ \& Data. $\mathrm{O}==0$ \& Data. $\mathrm{N}>0$ \& Data. $\mathrm{P}==0$ \&

Data. $\mathrm{S}==0$ ) ;

Summary $\cdot \mathrm{CHS}=\operatorname{sum}($ Data $\cdot \mathrm{C}>0$ \& Data. $\mathrm{H}>0$ \& Data $\cdot \mathrm{O}==0$ \& Data. $\mathrm{N}==0$ \& Data. $\mathrm{P}==0$ \&

Data.s>0);

Summary. $\mathrm{CHNS}=\operatorname{sum}($ Data. $\mathrm{C}>0$ \& Data. $\mathrm{H}>0$ \& Data. $\mathrm{O}==0$ \& Data. $\mathrm{N}>0$ \& Data. $\mathrm{P}==0$ \&

Data. $\mathrm{S}>0$ );

Summary. $\mathrm{CHNP}=\mathrm{sum}($ Data. $\mathrm{C}>0$ \& Data.H $>0$ \& Data. $\mathrm{O}==0$ \& Data. $\mathrm{N}>0$ \& Data.P $>0$ \&

Data. $\mathrm{S}=0$ ) ;

Summary $\cdot \mathrm{CHONS}=\operatorname{sum}($ Data $\cdot \mathrm{C}>0$ \& Data. $\mathrm{H}>0$ \& Data. $\mathrm{O}>0$ \& Data. $\mathrm{N}>0$ \& Data.P==0 \&

Data. $\mathrm{S}>0$ );

Summary. $\mathrm{CHONP}=\operatorname{sum}($ Data. $\mathrm{C}>0$ \& Data. $\mathrm{H}>0$ \& Data. $\mathrm{O}>0$ \& Data.N $>0$ \& Data.P>0\&

Data. $\mathrm{S}=0$ ) ;

Summary. $\mathrm{CHONSP}=$ sum $($ Data. $\mathrm{C}>0$ \& Data.H $>0$ \& Data.O $>0$ \& Data.N $>0$ \& Data.P $>0$ \&

Data.S $>0$ ); 
oCalculating aromaticity parameters: \# condensed aromatic (AI>=0.67), aromatic $(\mathrm{AI}>=0.5)$,

onon-aromatic $($ AI $<0.5)$

Summary. CondensedAromatics $=$ sum (Data.AI $>=0.67)$;

Summary.Aromatics=sum (Data.AI $>=0.5)$;

Summary. NonAromatics=sum (Data.AI<0.5);

ocalculating other parameters: total \# of formulae, average molecular

oweight

Summary. TotalFormulae=height (Data);

Summary. AvgMW=mean (Data. NominalMass);

ostoring Summary Data

PreOnIy_ESI_Neg_SRFA_Summary=struct2table (Summary) $;$ 응 $<<<<<<<<<<<<<<<<<<<<<<<<<<~$ $<<<<<<<\overline{<}<<<\overline{<}<<<\overline{<}<<<<\overline{<}<<<<<<<<<<<<<<<<<<<<<<<<<<<<<<<<<<<<<<<<<<<<<<<<<<<<<<<<~$

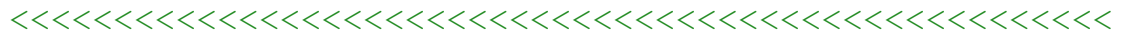

ㅇmmarizing Data

Data=BOnly;

oCalculating formulae type parameters: \# CHO, CHON, CHOS, CHOP, CHN, CHS, CHP, CHNS, CHONS formulae

Summary $\cdot \mathrm{CHO}=$ sum $($ Data. $\mathrm{C}>0$ \& Data. $\mathrm{H}>0$ \& Data. $\mathrm{O}>0$ \& Data. $\mathrm{N}==0$ \& Data. $\mathrm{P}==0$ \& Data. $\mathrm{S}=0$ ) ;

Summary $\cdot \mathrm{CHON}=$ sum $($ Data. $\mathrm{C}>0$ \& Data. $\mathrm{H}>0$ \& Data. $\mathrm{O}>0$ \& Data. $\mathrm{N}>0$ \& Data. $\mathrm{P}==0$ \& Data. $\mathrm{S}==0$ );

Summary. $\mathrm{CHOS}=$ sum $($ Data. $\mathrm{C}>0$ \& Data.H $>0$ \& Data. $\mathrm{O}>0$ \& Data. $\mathrm{N}==0$ \& Data. $\mathrm{P}==0$ \&

Data. $\mathrm{S}>0$ );

Summary $\cdot \mathrm{CHOP}=$ sum $($ Data. $\mathrm{C}>0$ \& Data. $\mathrm{H}>0$ \& Data. $\mathrm{O}>0$ \& Data. $\mathrm{N}==0$ \& Data. $\mathrm{P}>0$ \&

Data. $\mathrm{S}==0$ );

Summary $\cdot \mathrm{CHN}=$ sum $($ Data. $\mathrm{C}>0$ \& Data. $\mathrm{H}>0$ \& Data. $\mathrm{O}==0$ \& Data. $\mathrm{N}>0$ \& Data.P==0 \&

Data. $\mathrm{S}==0$ );

Summary. $\mathrm{CHS}=\mathrm{sum}($ Data. $\mathrm{C}>0$ \& Data. $\mathrm{H}>0$ \& Data. $\mathrm{O}==0$ \& Data. $\mathrm{N}==0$ \& Data. $\mathrm{P}==0$ \&

Data. $\mathrm{S}>0)$;

Summary. $\mathrm{CHNS}=\operatorname{sum}($ Data. $\mathrm{C}>0$ \& Data. $\mathrm{H}>0$ \& Data. $\mathrm{O}==0$ \& Data. $\mathrm{N}>0$ \& Data. $\mathrm{P}==0$ \&

Data. $\mathrm{S}>0$ );

Summary $\cdot \mathrm{CHNP}=\mathrm{sum}($ Data. $\mathrm{C}>0$ \& Data.H $>0$ \& Data. $\mathrm{O}==0$ \& Data. $\mathrm{N}>0$ \& Data. $\mathrm{P}>0$ \&

Data. $\mathrm{S}==0$ );

Summary. $\mathrm{CHONS}=\operatorname{sum}($ Data. $\mathrm{C}>0$ \& Data. $\mathrm{H}>0$ \& Data. $0>0$ \& Data.N>0 \& Data.P==0 \&

Data. S>0);

Summary. $\mathrm{CHONP}=$ sum $($ Data. $\mathrm{C}>0$ \& Data. $\mathrm{H}>0$ \& Data. $\mathrm{O}>0$ \& Data.N $>0$ \& Data.P $>0$ \&

Data. $\mathrm{S}==0$ ) ;

Summary. $\mathrm{CHONSP}=$ sum $($ Data. $\mathrm{C}>0$ \& Data. $\mathrm{H}>0$ \& Data. $\mathrm{O}>0$ \& Data.N $>0$ \& Data.P>0 \&

Data. $\mathrm{S}>0$ );

oCalculating aromaticity parameters: \# condensed aromatic (AI>=0.67),

aromatic $(A I>=0.5)$,

onon-aromatic $($ AI $<0.5)$

Summary. CondensedAromatics $=$ sum (Data.AI $>=0.67)$;

Summary. Aromatics $=$ sum (Data.AI $>=0.5)$;

Summary. NonAromatics=sum (Data.AI<0.5);

ocalculating other parameters: total \# of formulae, average molecular

oweight

Summary. TotalFormulae=height (Data);

Summary. AvgMW=mean (Data. NominalMass);

ostoring Summary Data

Postonly ESI Neg SRFA Summary=struct2table (Summary) ; $\circ<<<<<<<<<<<<<<<<<<<<<<<<<~$ $<<<<<<<<\overline{<}<<<\overline{<}<<<\overline{<}<<<<\overline{<}<<<<<<<<<<<<<<<<<<<<<<<<<<<<<<<<<<<<<<<<<<<<<<<<<<<<<<<~$

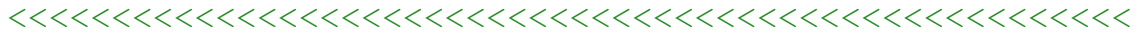

o Summarizing Data

Data=unique (Both) ; 
oCalculating formulae type parameters: \# CHO, CHON, CHOS, CHOP, CHN, CHS, CHP, CHNS, CHONS formulae

Summary $\cdot \mathrm{CHO}=$ sum $($ Data. $\mathrm{C}>0$ \& Data. $\mathrm{H}>0$ \& Data. $\mathrm{O}>0$ \& Data. $\mathrm{N}==0$ \& Data. $\mathrm{P}==0$ \& Data. $\mathrm{S}==0$ );

Summary. $\mathrm{CHON}=\operatorname{sum}($ Data. $\mathrm{C}>0$ \& Data. $\mathrm{H}>0$ \& Data. $\mathrm{O}>0$ \& Data. $\mathrm{N}>0$ \& Data.P==0 \& Data. $\mathrm{S}=0$ ) ) ;

Summary. $\mathrm{CHOS}=$ sum $($ Data. $\mathrm{C}>0$ \& Data. $\mathrm{H}>0$ \& Data. $0>0$ \& Data. $\mathrm{N}==0$ \& Data. $\mathrm{P}==0$ \& Data. $\mathrm{S}>0$ );

Summary $\cdot \mathrm{CHOP}=\operatorname{sum}($ Data. $\mathrm{C}>0$ \& Data.H $>0$ \& Data. $\mathrm{O}>0$ \& Data. $\mathrm{N}==0$ \& Data.P $>0$ \& Data. $\mathrm{S}==0$ );

Summary $\cdot \mathrm{CHN}=$ sum $($ Data. $\mathrm{C}>0$ \& Data. $\mathrm{H}>0$ \& Data. $\mathrm{O}==0$ \& Data. $\mathrm{N}>0$ \& Data. $\mathrm{P}==0$ \&

Data. $\mathrm{S}=0$ ) ;

Summary $\cdot \mathrm{CHS}=\operatorname{sum}($ Data. $\mathrm{C}>0$ \& Data. $\mathrm{H}>0$ \& Data. $\mathrm{O}==0$ \& Data. $\mathrm{N}==0$ \& Data. $\mathrm{P}==0$ \&

Data. S>0);

Summary. $\mathrm{CHNS}=$ sum (Data. $\mathrm{C}>0$ \& Data. $\mathrm{H}>0$ \& Data. $\mathrm{O}==0$ \& Data. $\mathrm{N}>0$ \& Data. $\mathrm{P}==0$ \&

Data. S>0);

Summary $\cdot \mathrm{CHNP}=\operatorname{sum}($ Data. $\mathrm{C}>0$ \& Data.H $>0$ \& Data. $\mathrm{O}==0$ \& Data.N $>0$ \& Data.P $>0$ \&

Data. $\mathrm{S}==0$ );

Summary $\cdot \mathrm{CHONS}=\operatorname{sum}($ Data $\cdot \mathrm{C}>0$ \& Data. $\mathrm{H}>0$ \& Data. $\mathrm{O}>0$ \& Data.N $>0$ \& Data.P==0 \&

Data. S>0);

Summary. $\mathrm{CHONP}=\operatorname{sum}($ Data. $\mathrm{C}>0$ \& Data. $\mathrm{H}>0$ \& Data. $\mathrm{O}>0$ \& Data.N $>0$ \& Data.P $>0$ \&

Data. $\mathrm{S}==0$ );

Summary. $\mathrm{CHONSP}=$ sum $($ Data. $\mathrm{C}>0$ \& Data.H $>0$ \& Data.O $>0$ \& Data.N $>0$ \& Data.P>0 \&

Data. $\mathrm{S}>0$ );

oCalculating aromaticity parameters: \# condensed aromatic (AI>=0.67),

aromatic $(\mathrm{AI}>=0.5)$,

onon-aromatic $(\mathrm{A} I<0.5)$

Summary. CondensedAromatics $=$ sum (Data.AI $>=0.67)$;

Summary.Aromatics $=$ sum (Data.AI $>=0.5)$;

Summary. NonAromatics=sum (Data.AI<0.5);

ocalculating other parameters: total \# of formulae, average molecular

oweight

Summary. TotalFormulae=height (Data);

Summary. AvgMW=mean (Data. NominalMass);

ostoring Summary Data

Common PreandPost ESI Neg SRFA Summary=struct2table (Summary); $\circ$ o $<<<<<<<<<<<<<<<<$

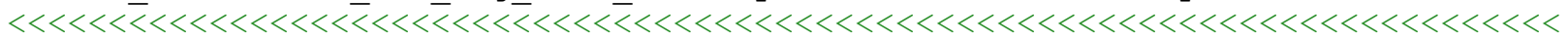

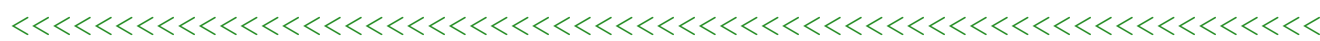

응 Comparing Pre- and Post-Oxidation GLSM for negative mode ESI

onput Data so that the script can readily be applied to any data set

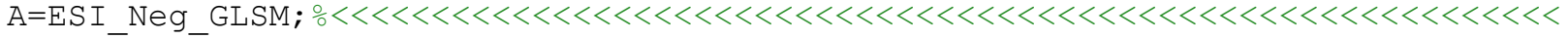

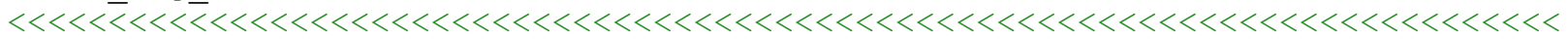
$<<<<<<<<<<<<<<<<<$

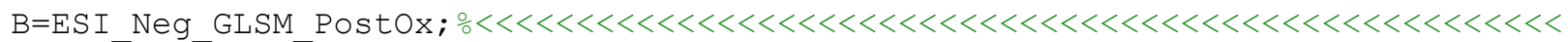

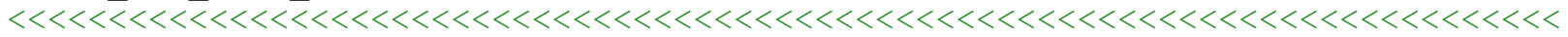
$<<<<<<<<<<<<<<<<<<<<<<<<~$

oseparate Data into those common and unique

Both $=[]$;

$\mathrm{k}=0$;

for $i=1$ :height $(A)$

for $j=1:$ height $(B)$

if $A \cdot C(i)==B \cdot C(j) \quad \& \& A \cdot H(i)==B \cdot H(j) \quad \& \& A \cdot N(i)==B \cdot N(j) \quad \& \& A \cdot O(i)==B \cdot O(j)$

$\& \& A \cdot P(i)==B \cdot P(j) \quad \& \& A \cdot S(i)==B \cdot S(j)$

$\mathrm{k}=\mathrm{k}+1$; 
$\operatorname{Both}(k,:)=[A . C(i), A . H(i), A . O(i), A . N(i), A . P(i)$,

A.S (i), A.NominalMass (i), A.HC_Ratio(i),

A.OC_Ratio(i),A.AI(i),A.DBE(i),A.AvgCOxState (i)] ;

$$
\text { A. C }(i)=0 \text {; }
$$

B. C $(j)=0$;

end

end

oCombining Data Sets

$A(A \cdot C==0,:)=[]$;

$A O n l_{Y}=[A . C, A . H, A . O, A . N, A . P, A . S$,

A. Nominalmass, A.HC Ratio,A.oC Ratio, A.AI, A.DBE, A.AvgCoxState];

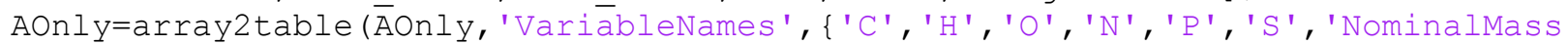
', 'HC_Ratio', 'OC_Ratio', 'AI', 'DBE', 'AvgCoxState'\});

$\mathrm{B}(\mathrm{B} \cdot \mathrm{C}==0,:)=[]$;

$\mathrm{BOnl} \mathrm{Y}_{\mathrm{B}}=[\mathrm{B} . \mathrm{C}, \mathrm{B} . \mathrm{H}, \mathrm{B} . \mathrm{O}, \mathrm{B} . \mathrm{N}, \mathrm{B} . \mathrm{P}$,

B.S,B.Nominalmass, B.HC_Ratio,B.OC_Ratio,B.AI, B.DBE, B.AvgCoxState] ;

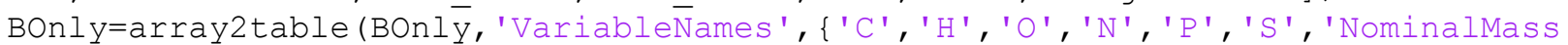
', 'HC_Ratio', 'OC_Ratio', 'AI', 'DBE', 'AvgCOxState'\}) ;

Both=array2table (Both, 'VariableNames', \{'C', 'H', 'O' , 'N', 'P' , 'S ', 'NominalMass', 'HC_Ratio', 'OC_Ratio', 'AI', 'DBE', 'AvgCOxState' \}) ;

ostoring Data with distinct names

Common PreandPost ESI Neg GLSM=unique (Both) ; $\circ<<<<<<<<<<<<<<<<<<<<<<<<<<<<<<<<<<<~$ $<<<<<<\overline{<}<<<<<<<<<<\overline{<}<<<\overline{<}<<\overline{<}<<<<<<<<<<<<<<<<<<<<<<<<<<<<<<<<<<<<<<<<<<<<<<<<<<<<~$

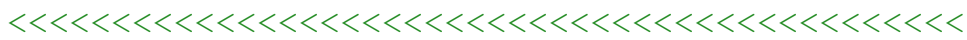

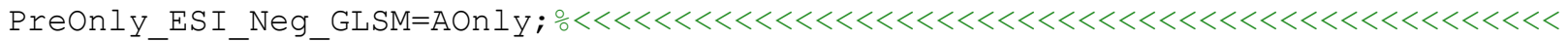
$<<<<<<\overline{<}<<<\overline{<}<<<\overline{<}<<<<<<<<<<<<<<<<<<<<<<<<<<<<<<<<<<<<<<<<<<<<<<<<<<<<<<<<<<<<<~$ $<<<<<<<<<<<<<<<<<<<<<<<<<<<<<~$

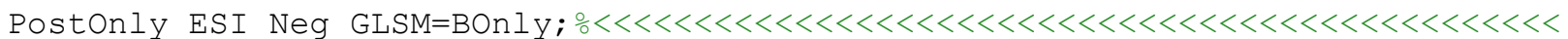

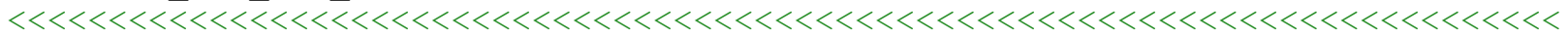
$<<<<<<<<<<<<<<<<<<<<<<<<<<<<<<~$

\% Summarizing Data

Data=AOnly;

oCalculating formulae type parameters: \# CHO, CHON, CHOS, CHOP, CHN, CHS, CHP, CHNS, CHONS formulae

Summary. $\mathrm{CHO}=\operatorname{sum}($ Data. $\mathrm{C}>0$ \& Data. $\mathrm{H}>0$ \& Data. $\mathrm{O}>0$ \& Data. $\mathrm{N}==0$ \& Data. $\mathrm{P}==0$ \& Data. $\mathrm{S}==0$ );

Summary. $\mathrm{CHON}=$ sum $($ Data. $\mathrm{C}>0$ \& Data. $\mathrm{H}>0$ \& Data. $\mathrm{O}>0$ \& Data. $\mathrm{N}>0$ \& Data.P==0 \&

Data. $\mathrm{S}=0$ ) ;

Summary $\cdot \mathrm{CHOS}=$ sum $($ Data. $\mathrm{C}>0$ \& Data.H $>0$ \& Data. O $>0$ \& Data. $\mathrm{N}==0$ \& Data. $\mathrm{P}==0$ \&

Data. S>0);

Summary. $\mathrm{CHOP}=\operatorname{sum}($ Data. $\mathrm{C}>0$ \& Data. $\mathrm{H}>0$ \& Data. $\mathrm{O}>0$ \& Data. $\mathrm{N}==0$ \& Data. $\mathrm{P}>0$ \&

Data. $\mathrm{S}==0$ ) ;

Summary $\cdot \mathrm{CHN}=$ sum $($ Data. $\mathrm{C}>0$ \& Data. $\mathrm{H}>0$ \& Data. $\mathrm{O}==0$ \& Data. $\mathrm{N}>0$ \& Data. $\mathrm{P}==0$ \&

Data. $\mathrm{S}==0$ ) ;

Summary $\cdot \mathrm{CHS}=\operatorname{sum}($ Data $\cdot \mathrm{C}>0$ \& Data. $\mathrm{H}>0$ \& Data $\cdot \mathrm{O}==0$ \& Data. $\mathrm{N}==0$ \& Data. $\mathrm{P}==0$ \&

Data.s>0);

Summary. $\mathrm{CHNS}=\operatorname{sum}($ Data. $\mathrm{C}>0$ \& Data. $\mathrm{H}>0$ \& Data. $\mathrm{O}==0$ \& Data. $\mathrm{N}>0$ \& Data. $\mathrm{P}==0$ \&

Data. $\mathrm{S}>0$ );

Summary. $\mathrm{CHNP}=\mathrm{sum}($ Data. $\mathrm{C}>0$ \& Data.H $>0$ \& Data. $\mathrm{O}==0$ \& Data. $\mathrm{N}>0$ \& Data.P $>0$ \&

Data. $\mathrm{S}=0$ ) ;

Summary $\cdot \mathrm{CHONS}=\operatorname{sum}($ Data $\cdot \mathrm{C}>0$ \& Data. $\mathrm{H}>0$ \& Data. $\mathrm{O}>0$ \& Data. $\mathrm{N}>0$ \& Data.P==0 \&

Data. $\mathrm{S}>0$ );

Summary. $\mathrm{CHONP}=\operatorname{sum}($ Data. $\mathrm{C}>0$ \& Data. $\mathrm{H}>0$ \& Data. $\mathrm{O}>0$ \& Data.N $>0$ \& Data.P>0\&

Data. $\mathrm{S}=0$ ) ;

Summary. $\mathrm{CHONSP}=$ sum $($ Data. $\mathrm{C}>0$ \& Data.H $>0$ \& Data.O $>0$ \& Data.N $>0$ \& Data.P $>0$ \&

Data. S>0); 
oCalculating aromaticity parameters: \# condensed aromatic (AI>=0.67), aromatic $(\mathrm{AI}>=0.5)$,

onon-aromatic (AI<0.5)

Summary. CondensedAromatics $=$ sum (Data.AI $>=0.67)$;

Summary.Aromatics=sum (Data.AI $>=0.5)$;

Summary. NonAromatics=sum (Data.AI<0.5);

ocalculating other parameters: total \# of formulae, average molecular

oweight

Summary. TotalFormulae=height (Data);

Summary. AvgMW=mean (Data. NominalMass);

ostoring Summary Data

PreOnIy_ESI_Neg_GLSM_Summary=struct2table (Summary) ; 응 $<<<<<<<<<<<<<<<<<<<<<<<<<<~$ $<<<<<<<\overline{<}<<<\overline{<}<<<\overline{<}<<<\overline{<}<<<<<<<<<<<<<<<<<<<<<<<<<<<<<<<<<<<<<<<<<<<<<<<<<<<<<<<<<~$

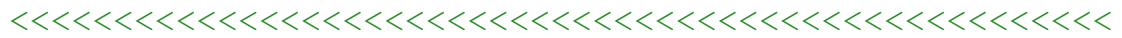

ㅇmmarizing Data

Data=BOnly;

oCalculating formulae type parameters: \# CHO, CHON, CHOS, CHOP, CHN, CHS, CHP, CHNS, CHONS formulae

Summary $\cdot \mathrm{CHO}=$ sum (Data. $\mathrm{C}>0$ \& Data. $\mathrm{H}>0$ \& Data. $\mathrm{O}>0$ \& Data. $\mathrm{N}==0$ \& Data. $\mathrm{P}==0$ \& Data. $\mathrm{S}==0$ );

Summary $\cdot \mathrm{CHON}=$ Sum $($ Data. $\mathrm{C}>0$ \& Data. $\mathrm{H}>0$ \& Data. $\mathrm{O}>0$ \& Data. $\mathrm{N}>0$ \& Data.P==0 \& Data. $\mathrm{S}==0$ );

Summary. $\mathrm{CHOS}=$ sum $($ Data. $\mathrm{C}>0$ \& Data. $\mathrm{H}>0$ \& Data. $\mathrm{O}>0$ \& Data. $\mathrm{N}==0$ \& Data. $\mathrm{P}==0$ \&

Data. $\mathrm{S}>0$ ) ;

Summary $\cdot \mathrm{CHOP}=\mathrm{sum}($ Data. $\mathrm{C}>0$ \& Data.H $>0$ \& Data. $\mathrm{O}>0$ \& Data. $\mathrm{N}==0$ \& Data.P $>0$ \&

Data. $\mathrm{S}==0$ );

Summary $\cdot \mathrm{CHN}=$ sum $($ Data. $\mathrm{C}>0$ \& Data. $\mathrm{H}>0$ \& Data. $\mathrm{O}==0$ \& Data. $\mathrm{N}>0$ \& Data.P==0 \&

Data. $\mathrm{S}==0$ );

Summary. $\mathrm{CHS}=\mathrm{sum}($ Data. $\mathrm{C}>0$ \& Data. $\mathrm{H}>0$ \& Data. $\mathrm{O}==0$ \& Data. $\mathrm{N}==0$ \& Data. $\mathrm{P}==0$ \&

Data. $\mathrm{S}>0$ );

Summary. $\mathrm{CHNS}=\operatorname{sum}($ Data. $\mathrm{C}>0$ \& Data. $\mathrm{H}>0$ \& Data. $\mathrm{O}==0$ \& Data. $\mathrm{N}>0$ \& Data. $\mathrm{P}==0$ \&

Data. S>0);

Summary $\cdot \mathrm{CHNP}=\mathrm{sum}($ Data. $\mathrm{C}>0$ \& Data.H $>0$ \& Data. $\mathrm{O}==0$ \& Data. $\mathrm{N}>0$ \& Data.P $>0$ \&

Data. $\mathrm{S}==0$ );

Summary. $\mathrm{CHONS}=\operatorname{sum}($ Data. $\mathrm{C}>0$ \& Data. $\mathrm{H}>0$ \& Data. $0>0$ \& Data.N>0 \& Data.P==0 \&

Data. S>0);

Summary. $\mathrm{CHONP}=$ sum $($ Data. $\mathrm{C}>0$ \& Data. $\mathrm{H}>0$ \& Data. $\mathrm{O}>0$ \& Data.N $>0$ \& Data.P $>0$ \&

Data. $\mathrm{S}==0$ ) ;

Summary. $\mathrm{CHONSP}=$ sum $($ Data. $\mathrm{C}>0$ \& Data.H $>0$ \& Data.O $>0$ \& Data.N $>0$ \& Data.P>0 \&

Data. $\mathrm{S}>0$ );

oCalculating aromaticity parameters: \# condensed aromatic (AI>=0.67),

aromatic $(A I>=0.5)$,

onon-aromatic $($ AI $<0.5)$

Summary. CondensedAromatics $=$ sum (Data. AI $>=0.67)$;

Summary. Aromatics $=$ sum (Data.AI $>=0.5)$;

Summary. NonAromatics=sum (Data.AI<0.5);

ocalculating other parameters: total \# of formulae, average molecular

oweight

Summary. TotalFormulae=height (Data);

Summary. AvgMW=mean (Data. NominalMass);

ostoring Summary Data

Postonly ESI Neg GLSM Summary=struct2table (Summary) ; $\circ<<<<<<<<<<<<<<<<<<<<<<<<<<$ $<<<<<<<<\overline{<}<<<\overline{<}<<<\overline{<}<<<<\overline{<}<<<<<<<<<<<<<<<<<<<<<<<<<<<<<<<<<<<<<<<<<<<<<<<<<<<<<<<~$

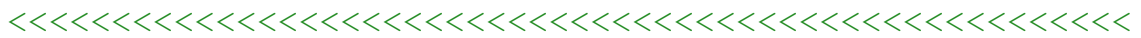

o Summarizing Data

Data=unique (Both); 
oCalculating formulae type parameters: \# CHO, CHON, CHOS, CHOP, CHN, CHS, CHP, CHNS, CHONS formulae

Summary $\cdot \mathrm{CHO}=$ sum $($ Data. $\mathrm{C}>0$ \& Data. $\mathrm{H}>0$ \& Data. $\mathrm{O}>0$ \& Data. $\mathrm{N}==0$ \& Data. $\mathrm{P}==0$ \& Data. $\mathrm{S}==0$ );

Summary. $\mathrm{CHON}=\operatorname{sum}($ Data. $\mathrm{C}>0$ \& Data. $\mathrm{H}>0$ \& Data. $\mathrm{O}>0$ \& Data. $\mathrm{N}>0$ \& Data.P==0 \& Data. $\mathrm{S}=0$ ) ) ;

Summary. $\mathrm{CHOS}=$ sum $($ Data. $\mathrm{C}>0$ \& Data. $\mathrm{H}>0$ \& Data. $0>0$ \& Data. $\mathrm{N}==0$ \& Data. $\mathrm{P}==0$ \& Data. $\mathrm{S}>0$ );

Summary $\cdot \mathrm{CHOP}=\operatorname{sum}($ Data. $\mathrm{C}>0$ \& Data.H $>0$ \& Data. $\mathrm{O}>0$ \& Data. $\mathrm{N}==0$ \& Data.P $>0$ \& Data. $\mathrm{S}==0$ );

Summary $\cdot \mathrm{CHN}=$ sum $($ Data. $\mathrm{C}>0$ \& Data. $\mathrm{H}>0$ \& Data. $\mathrm{O}==0$ \& Data. $\mathrm{N}>0$ \& Data. $\mathrm{P}==0$ \&

Data. $\mathrm{S}=0$ ) ;

Summary $\cdot \mathrm{CHS}=\operatorname{sum}($ Data. $\mathrm{C}>0$ \& Data. $\mathrm{H}>0$ \& Data. $\mathrm{O}==0$ \& Data. $\mathrm{N}==0$ \& Data. $\mathrm{P}==0$ \&

Data. S>0);

Summary. $\mathrm{CHNS}=$ sum (Data. $\mathrm{C}>0$ \& Data. $\mathrm{H}>0$ \& Data. $\mathrm{O}==0$ \& Data. $\mathrm{N}>0$ \& Data. $\mathrm{P}==0$ \&

Data. S>0);

Summary $\cdot \mathrm{CHNP}=\operatorname{sum}($ Data. $\mathrm{C}>0$ \& Data.H $>0$ \& Data. $\mathrm{O}==0$ \& Data.N $>0$ \& Data.P $>0$ \&

Data. $\mathrm{S}==0$ );

Summary $\cdot \mathrm{CHONS}=\operatorname{sum}($ Data $\cdot \mathrm{C}>0$ \& Data. $\mathrm{H}>0$ \& Data. $\mathrm{O}>0$ \& Data.N $>0$ \& Data.P==0 \&

Data. S>0);

Summary. $\mathrm{CHONP}=\operatorname{sum}($ Data. $\mathrm{C}>0$ \& Data. $\mathrm{H}>0$ \& Data. $\mathrm{O}>0$ \& Data.N $>0$ \& Data.P $>0$ \&

Data. $\mathrm{S}==0$ );

Summary. $\mathrm{CHONSP}=$ sum $($ Data. $\mathrm{C}>0$ \& Data. $\mathrm{H}>0$ \& Data. $\mathrm{O}>0$ \& Data.N $>0$ \& Data.P $>0$ \&

Data. $\mathrm{S}>0$ );

ocalculating aromaticity parameters: \# condensed aromatic (AI>=0.67),

aromatic $(\mathrm{AI}>=0.5)$,

onon-aromatic $(\mathrm{A} I<0.5)$

Summary. CondensedAromatics $=$ sum (Data.AI $>=0.67)$;

Summary. Aromatics $=$ sum (Data.AI $>=0.5)$;

Summary. NonAromatics=sum (Data.AI<0.5);

ocalculating other parameters: total \# of formulae, average molecular

oweight

Summary. TotalFormulae=height (Data);

Summary. AvgMW=mean (Data. NominalMass);

ostoring Summary Data

Common PreandPost ESI Neg GLSM Summary=struct2table (Summary); $\circ$ o $<<<<<<<<<<<<<<<<$ $<<<<<<\overline{<}<<<<<<<<<\overline{\bar{l}}<<<\overline{\bar{l}}<<\overline{<} \overline{<}<<<<\overline{<}<<<<<<<<<<<<<<<<<<<<<<<<<<<<<<<<<<<<<<<<<<<<<<$

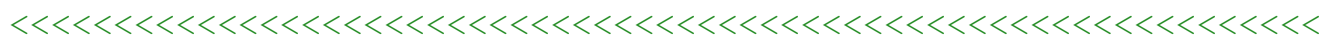

응 Comparing Pre- and Post-Oxidation SRFA for Positive mode ESI data oInput Data so that the script can readily be applied to any data set

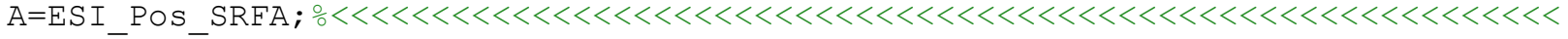

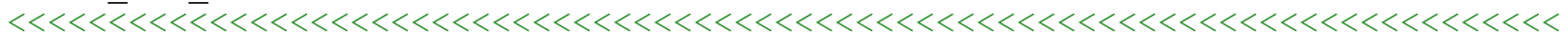
$<<<<<<<<<<<<<<<<<$

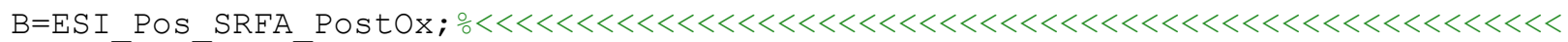
$<<<<<\overline{<}<<<\overline{<}<<<<\overline{<}<<<<<<<<<<<<<<<<<<<<<<<<<<<<<<<<<<<<<<<<<<<<<<<<<<<<<<<<<<<<<<~$ $<<<<<<<<<<<<<<<<<<<<<<<<~$

o Separate Data into those common and unique

Both $=[]$;

$\mathrm{k}=0$;

for $i=1$ :height $(A)$

for $j=1:$ height $(B)$

if $A \cdot C(i)==B \cdot C(j) \quad \& \& A \cdot H(i)==B \cdot H(j) \quad \& \& A \cdot N(i)==B \cdot N(j) \quad \& \& A \cdot O(i)==B \cdot O(j)$

$\& \& A \cdot P(i)==B \cdot P(j) \quad \& \& A \cdot S(i)==B \cdot S(j)$

$\mathrm{k}=\mathrm{k}+1$; 
$\operatorname{Both}(k,:)=[A . C(i), A . H(i), A . O(i), A . N(i), A . P(i)$,

A.S (i), A.NominalMass (i), A.HC_Ratio(i),

A.OC_Ratio(i),A.AI(i),A.DBE(i),A.AvgCOxState (i)] ;

$$
\text { A. C }(i)=0 \text {; }
$$

B. C $(j)=0$;

end

end

oCombining Data Sets

$A(A \cdot C==0,:)=[]$;

$A O n l_{Y}=[A . C, A . H, A . O, A . N, A . P, A . S$,

A. Nominalmass, A.HC Ratio,A.oC Ratio, A.AI, A.DBE, A.AvgCoxState];

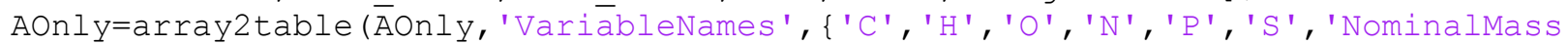
', 'HC_Ratio', 'OC_Ratio', 'AI', 'DBE', 'AvgCoxState'\});

$\mathrm{B}(\mathrm{B} \cdot \mathrm{C}==0,:)=[]$;

$\mathrm{BOnl} \mathrm{Y}_{\mathrm{B}}=[\mathrm{B} . \mathrm{C}, \mathrm{B} . \mathrm{H}, \mathrm{B} . \mathrm{O}, \mathrm{B} . \mathrm{N}, \mathrm{B} . \mathrm{P}$,

B.S,B.Nominalmass, B.HC_Ratio,B.OC_Ratio,B.AI, B.DBE, B.AvgCoxState] ;

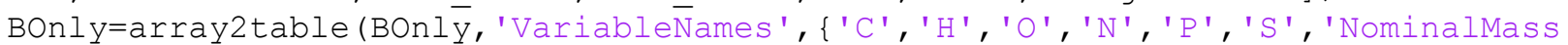
', 'HC_Ratio', 'OC_Ratio', 'AI', 'DBE', 'AvgCOxState'\}) ;

Both=array2table (Both, 'VariableNames', \{'C', 'H', 'O' , 'N', 'P' , 'S ', 'NominalMass', 'HC_Ratio', 'OC_Ratio', 'AI', 'DBE', 'AvgCOxState' \}) ;

ostoring Data with distinct names

Common PreandPost ESI Pos SRFA=unique (Both) ; $\circ<<<<<<<<<<<<<<<<<<<<<<<<<<<<<<<<<<<~$ $<<<<<<\overline{<}<<<<<<<<<<\overline{<}<<<\overline{<}<<<\overline{<}<<<<<<<<<<<<<<<<<<<<<<<<<<<<<<<<<<<<<<<<<<<<<<<<<<<~$

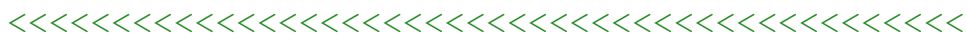

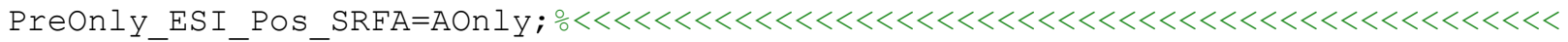
$<<<<<<\overline{<}<<<\overline{<}<<<\overline{<}<<<<<<<<<<<<<<<<<<<<<<<<<<<<<<<<<<<<<<<<<<<<<<<<<<<<<<<<<<<<<~$ $<<<<<<<<<<<<<<<<<<<<<<<<<<<<<~$

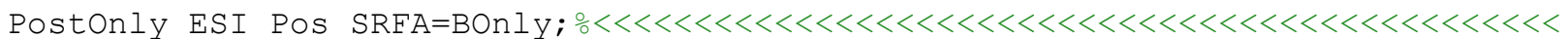

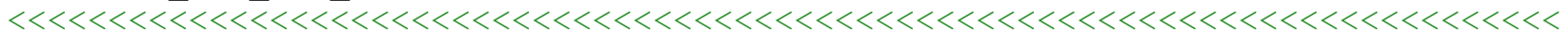
$<<<<<<<<<<<<<<<<<<<<<<<<<<<<<<~$

\% Summarizing Data

Data=AOnly;

oCalculating formulae type parameters: \# CHO, CHON, CHOS, CHOP, CHN, CHS, CHP, CHNS, CHONS formulae

Summary. $\mathrm{CHO}=\operatorname{sum}($ Data. $\mathrm{C}>0$ \& Data. $\mathrm{H}>0$ \& Data. $\mathrm{O}>0$ \& Data. $\mathrm{N}==0$ \& Data. $\mathrm{P}==0$ \& Data. $\mathrm{S}==0$ );

Summary. $\mathrm{CHON}=$ sum $($ Data. $\mathrm{C}>0$ \& Data. $\mathrm{H}>0$ \& Data. $\mathrm{O}>0$ \& Data. $\mathrm{N}>0$ \& Data.P==0 \&

Data. $\mathrm{S}=0$ ) ;

Summary $\cdot \mathrm{CHOS}=$ sum $($ Data. $\mathrm{C}>0$ \& Data.H $>0$ \& Data. O $>0$ \& Data. $\mathrm{N}==0$ \& Data. $\mathrm{P}==0$ \&

Data. S>0);

Summary. $\mathrm{CHOP}=\operatorname{sum}($ Data. $\mathrm{C}>0$ \& Data. $\mathrm{H}>0$ \& Data. $\mathrm{O}>0$ \& Data. $\mathrm{N}==0$ \& Data. $\mathrm{P}>0$ \&

Data. $\mathrm{S}==0$ ) ;

Summary $\cdot \mathrm{CHN}=$ sum $($ Data. $\mathrm{C}>0$ \& Data. $\mathrm{H}>0$ \& Data. $\mathrm{O}==0$ \& Data. $\mathrm{N}>0$ \& Data. $\mathrm{P}==0$ \&

Data. $\mathrm{S}==0$ ) ;

Summary $\cdot \mathrm{CHS}=\operatorname{sum}($ Data $\cdot \mathrm{C}>0$ \& Data. $\mathrm{H}>0$ \& Data $\cdot \mathrm{O}==0$ \& Data. $\mathrm{N}==0$ \& Data. $\mathrm{P}==0$ \&

Data.s>0);

Summary. $\mathrm{CHNS}=\operatorname{sum}($ Data. $\mathrm{C}>0$ \& Data. $\mathrm{H}>0$ \& Data. $\mathrm{O}==0$ \& Data. $\mathrm{N}>0$ \& Data. $\mathrm{P}==0$ \&

Data. $\mathrm{S}>0$ );

Summary. $\mathrm{CHNP}=\mathrm{sum}($ Data. $\mathrm{C}>0$ \& Data.H $>0$ \& Data. $\mathrm{O}==0$ \& Data. $\mathrm{N}>0$ \& Data.P $>0$ \&

Data. $\mathrm{S}=0$ ) ;

Summary $\cdot \mathrm{CHONS}=\operatorname{sum}($ Data $\cdot \mathrm{C}>0$ \& Data. $\mathrm{H}>0$ \& Data. $\mathrm{O}>0$ \& Data. $\mathrm{N}>0$ \& Data.P==0 \&

Data. $\mathrm{S}>0$ );

Summary. $\mathrm{CHONP}=\operatorname{sum}($ Data. $\mathrm{C}>0$ \& Data. $\mathrm{H}>0$ \& Data. $\mathrm{O}>0$ \& Data.N $>0$ \& Data.P>0\&

Data. $\mathrm{S}=0$ ) ;

Summary. $\mathrm{CHONSP}=$ sum $($ Data. $\mathrm{C}>0$ \& Data.H $>0$ \& Data.O $>0$ \& Data.N $>0$ \& Data.P $>0$ \&

Data.S $>0$ ); 
oCalculating aromaticity parameters: \# condensed aromatic (AI>=0.67), aromatic $(\mathrm{AI}>=0.5)$,

onon-aromatic $($ AI $<0.5)$

Summary. CondensedAromatics $=$ sum (Data.AI $>=0.67)$;

Summary.Aromatics=sum (Data.AI $>=0.5)$;

Summary. NonAromatics=sum (Data.AI<0.5);

ocalculating other parameters: total \# of formulae, average molecular

oweight

Summary. TotalFormulae=height (Data);

Summary. AvgMW=mean (Data. NominalMass);

ostoring Summary Data

PreOnly_ESI_Pos_SRFA_Summary=struct2table (Summary) ; 응 $<<<<<<<<<<<<<<<<<<<<<<<<<<~$ $<<<<<<<\overline{<}<<<\overline{<}<<<\overline{<}<<<<\overline{<}<<<<<<<<<<<<<<<<<<<<<<<<<<<<<<<<<<<<<<<<<<<<<<<<<<<<<<<<~$

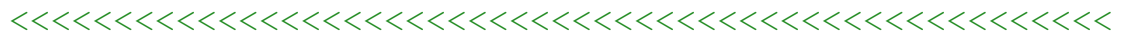

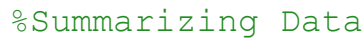

Data=BOnly;

oCalculating formulae type parameters: \# CHO, CHON, CHOS, CHOP, CHN, CHS, CHP, CHNS, CHONS formulae

Summary $\cdot \mathrm{CHO}=$ sum $($ Data. $\mathrm{C}>0$ \& Data. $\mathrm{H}>0$ \& Data. $\mathrm{O}>0$ \& Data. $\mathrm{N}==0$ \& Data. $\mathrm{P}==0$ \& Data. $\mathrm{S}=0$ ) ;

Summary $\cdot \mathrm{CHON}=$ sum $($ Data. $\mathrm{C}>0$ \& Data. $\mathrm{H}>0$ \& Data. $\mathrm{O}>0$ \& Data. $\mathrm{N}>0$ \& Data. $\mathrm{P}==0$ \& Data. $\mathrm{S}==0$ );

Summary. $\mathrm{CHOS}=$ sum $($ Data. $\mathrm{C}>0$ \& Data.H $>0$ \& Data. $\mathrm{O}>0$ \& Data. $\mathrm{N}==0$ \& Data. $\mathrm{P}==0$ \&

Data. $\mathrm{S}>0$ );

Summary $\cdot \mathrm{CHOP}=$ sum $($ Data. $\mathrm{C}>0$ \& Data. $\mathrm{H}>0$ \& Data. $\mathrm{O}>0$ \& Data. $\mathrm{N}==0$ \& Data. $\mathrm{P}>0$ \&

Data. $\mathrm{S}==0$ );

Summary $\cdot \mathrm{CHN}=$ sum $($ Data. $\mathrm{C}>0$ \& Data. $\mathrm{H}>0$ \& Data. $\mathrm{O}==0$ \& Data. $\mathrm{N}>0$ \& Data.P==0 \&

Data. $\mathrm{S}==0$ );

Summary. $\mathrm{CHS}=\mathrm{sum}($ Data. $\mathrm{C}>0$ \& Data. $\mathrm{H}>0$ \& Data. $\mathrm{O}==0$ \& Data. $\mathrm{N}==0$ \& Data. $\mathrm{P}==0$ \&

Data. $\mathrm{S}>0)$;

Summary. $\mathrm{CHNS}=\operatorname{sum}($ Data. $\mathrm{C}>0$ \& Data. $\mathrm{H}>0$ \& Data. $\mathrm{O}==0$ \& Data. $\mathrm{N}>0$ \& Data. $\mathrm{P}==0$ \&

Data. $\mathrm{S}>0$ );

Summary $\cdot \mathrm{CHNP}=\mathrm{sum}($ Data. $\mathrm{C}>0$ \& Data.H $>0$ \& Data. $\mathrm{O}==0$ \& Data. $\mathrm{N}>0$ \& Data. $\mathrm{P}>0$ \&

Data. $\mathrm{S}==0$ );

Summary. $\mathrm{CHONS}=\operatorname{sum}($ Data. $\mathrm{C}>0$ \& Data. $\mathrm{H}>0$ \& Data. $0>0$ \& Data.N>0 \& Data.P==0 \&

Data. S>0);

Summary. $\mathrm{CHONP}=$ sum $($ Data. $\mathrm{C}>0$ \& Data. $\mathrm{H}>0$ \& Data. $\mathrm{O}>0$ \& Data.N $>0$ \& Data.P $>0$ \&

Data. $\mathrm{S}==0$ ) ;

Summary. $\mathrm{CHONSP}=$ sum $($ Data. $\mathrm{C}>0$ \& Data. $\mathrm{H}>0$ \& Data. $\mathrm{O}>0$ \& Data.N $>0$ \& Data.P>0 \&

Data. $\mathrm{S}>0$ );

ocalculating aromaticity parameters: \# condensed aromatic (AI>=0.67),

aromatic $(A I>=0.5)$,

onon-aromatic $($ AI $<0.5)$

Summary. CondensedAromatics $=$ sum (Data.AI $>=0.67)$;

Summary. Aromatics $=$ sum (Data.AI $>=0.5)$;

Summary. NonAromatics=sum (Data.AI<0.5);

ocalculating other parameters: total \# of formulae, average molecular

oweight

Summary. TotalFormulae=height (Data);

Summary. AvgMW=mean (Data. NominalMass);

o.storing Summary Data

Postonly ESI POS SRFA Summary=struct2table (Summary) ; $\circ<<<<<<<<<<<<<<<<<<<<<<<<<<~$ $<<<<<<<<\overline{<}<<<\overline{<}<<<\overline{<}<<<<\overline{<}<<<<<<<<<<<<<<<<<<<<<<<<<<<<<<<<<<<<<<<<<<<<<<<<<<<<<<<~$

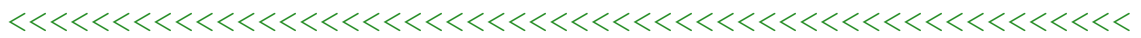

o Summarizing Data

Data=unique (Both); 
oCalculating formulae type parameters: \# CHO, CHON, CHOS, CHOP, CHN, CHS, CHP, CHNS, CHONS formulae

Summary $\cdot \mathrm{CHO}=$ sum $($ Data. $\mathrm{C}>0$ \& Data. $\mathrm{H}>0$ \& Data. $\mathrm{O}>0$ \& Data. $\mathrm{N}==0$ \& Data. $\mathrm{P}==0$ \& Data. $\mathrm{S}==0$ );

Summary. $\mathrm{CHON}=\operatorname{sum}($ Data. $\mathrm{C}>0$ \& Data. $\mathrm{H}>0$ \& Data. $\mathrm{O}>0$ \& Data. $\mathrm{N}>0$ \& Data.P==0 \& Data. $\mathrm{S}=0$ ) ) ;

Summary. $\mathrm{CHOS}=$ sum $($ Data. $\mathrm{C}>0$ \& Data. $\mathrm{H}>0$ \& Data. $0>0$ \& Data. $\mathrm{N}==0$ \& Data. $\mathrm{P}==0$ \& Data. $\mathrm{S}>0$ );

Summary $\cdot \mathrm{CHOP}=\operatorname{sum}($ Data. $\mathrm{C}>0$ \& Data.H $>0$ \& Data. $\mathrm{O}>0$ \& Data. $\mathrm{N}==0$ \& Data.P $>0$ \& Data. $\mathrm{S}==0$ );

Summary $\cdot \mathrm{CHN}=$ sum $($ Data. $\mathrm{C}>0$ \& Data. $\mathrm{H}>0$ \& Data. $\mathrm{O}==0$ \& Data. $\mathrm{N}>0$ \& Data. $\mathrm{P}==0$ \&

Data. $\mathrm{S}=0$ ) ;

Summary $\cdot \mathrm{CHS}=\operatorname{sum}($ Data. $\mathrm{C}>0$ \& Data. $\mathrm{H}>0$ \& Data. $\mathrm{O}==0$ \& Data. $\mathrm{N}==0$ \& Data. $\mathrm{P}==0$ \&

Data. S>0);

Summary. $\mathrm{CHNS}=$ sum (Data. $\mathrm{C}>0$ \& Data. $\mathrm{H}>0$ \& Data. $\mathrm{O}==0$ \& Data. $\mathrm{N}>0$ \& Data. $\mathrm{P}==0$ \&

Data. S>0);

Summary $\cdot \mathrm{CHNP}=\operatorname{sum}($ Data. $\mathrm{C}>0$ \& Data.H $>0$ \& Data. $\mathrm{O}==0$ \& Data.N $>0$ \& Data.P $>0$ \&

Data. $\mathrm{S}==0$ );

Summary $\cdot \mathrm{CHONS}=\operatorname{sum}($ Data $\cdot \mathrm{C}>0$ \& Data. $\mathrm{H}>0$ \& Data. $\mathrm{O}>0$ \& Data.N $>0$ \& Data.P==0 \&

Data. S>0);

Summary. $\mathrm{CHONP}=\operatorname{sum}($ Data. $\mathrm{C}>0$ \& Data. $\mathrm{H}>0$ \& Data. $\mathrm{O}>0$ \& Data.N $>0$ \& Data.P $>0$ \&

Data. $\mathrm{S}==0$ );

Summary. $\mathrm{CHONSP}=$ sum $($ Data. $\mathrm{C}>0$ \& Data. $\mathrm{H}>0$ \& Data. $\mathrm{O}>0$ \& Data.N $>0$ \& Data.P $>0$ \&

Data. $\mathrm{S}>0$ );

oCalculating aromaticity parameters: \# condensed aromatic (AI>=0.67),

aromatic $(\mathrm{AI}>=0.5)$,

onon-aromatic $(\mathrm{A} I<0.5)$

Summary. CondensedAromatics $=$ sum (Data.AI $>=0.67)$;

Summary. Aromatics $=$ sum (Data.AI $>=0.5)$;

Summary. NonAromatics=sum (Data.AI<0.5);

ocalculating other parameters: total \# of formulae, average molecular

oweight

Summary. TotalFormulae=height (Data);

Summary. AvgMW=mean (Data. NominalMass);

ostoring Summary Data

Common PreandPost ESI Pos SRFA Summary=struct2table (Summary); $\circ$ o $<<<<<<<<<<<<<<<<~$

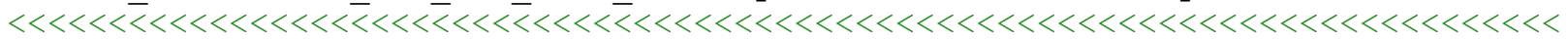

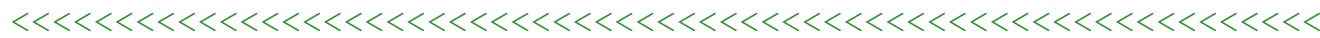

응 Comparing Pre- and Post-Oxidation GLSM for Positive mode ESI

onput Data so that the script can readily be applied to any data set

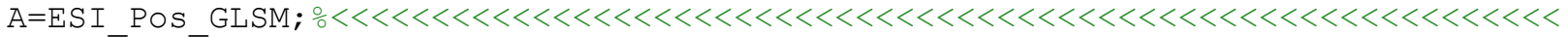

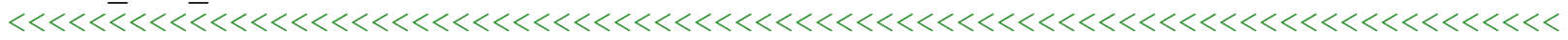
$<<<<<<<<<<<<<<<<<$

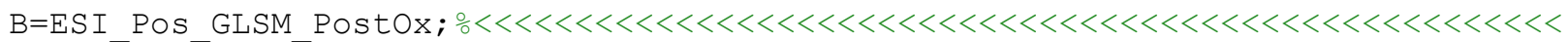

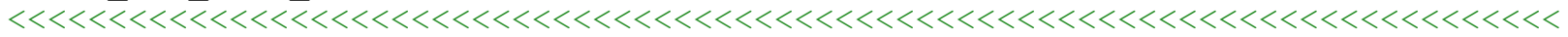
$<<<<<<<<<<<<<<<<<<<<<<<<~$

o Separate Data into those common and unique

Both $=[]$;

$\mathrm{k}=0$;

for $i=1$ : height (A)

for $j=1:$ height $(B)$

if $A \cdot C(i)==B \cdot C(j) \quad \& \& A \cdot H(i)==B \cdot H(j) \quad \& \& A \cdot N(i)==B \cdot N(j) \quad \& \& A \cdot O(i)==B \cdot O(j)$

$\& \& A \cdot P(i)==B \cdot P(j) \quad \& \& A \cdot S(i)==B \cdot S(j)$

$\mathrm{k}=\mathrm{k}+1$; 
$\operatorname{Both}(k,:)=[A . C(i), A . H(i), A . O(i), A . N(i), A . P(i)$,

A.S (i), A.NominalMass (i), A.HC_Ratio(i),

A.OC_Ratio(i),A.AI(i),A.DBE(i),A.AvgCOxState (i)] ;

$$
\text { A. C }(i)=0 \text {; }
$$

B. C $(j)=0$;

end

end

oCombining Data Sets

$A(A \cdot C==0,:)=[]$;

$A O n l_{Y}=[A . C, A . H, A . O, A . N, A . P, A . S$,

A. Nominalmass, A.HC Ratio,A.oC Ratio, A.AI, A.DBE, A.AvgCoxState];

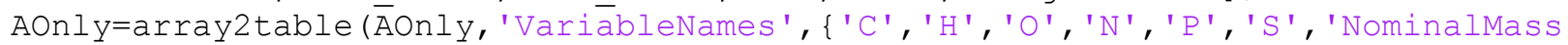
', 'HC_Ratio', 'OC_Ratio', 'AI', 'DBE', 'AvgCoxState'\});

$\mathrm{B}(\mathrm{B} \cdot \mathrm{C}==0,:)=[]$;

$\mathrm{BOnl} \mathrm{Y}_{\mathrm{B}}=[\mathrm{B} . \mathrm{C}, \mathrm{B} . \mathrm{H}, \mathrm{B} . \mathrm{O}, \mathrm{B} . \mathrm{N}, \mathrm{B} . \mathrm{P}$,

B.S,B.Nominalmass, B.HC_Ratio,B.OC_Ratio,B.AI, B.DBE, B.AvgCoxState] ;

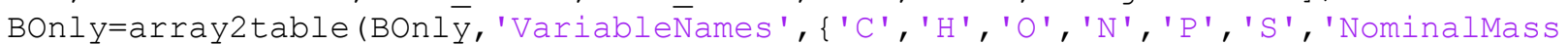
', 'HC_Ratio', 'OC_Ratio', 'AI', 'DBE', 'AvgCOxState'\}) ;

Both=array2table (Both, 'VariableNames', \{'C', 'H', 'O' , 'N', 'P' , 'S ', 'NominalMass', 'HC_Ratio', 'OC_Ratio', 'AI', 'DBE', 'AvgCOxState' \}) ;

ostoring Data with distinct names

Common PreandPost ESI Pos GLSM=unique (Both) ; $\circ<<<<<<<<<<<<<<<<<<<<<<<<<<<<<<<<<<<~$ $<<<<<<\overline{<}<<<<<<<<<<\overline{<}<<<\overline{<}<<<\overline{<}<<<<<<<<<<<<<<<<<<<<<<<<<<<<<<<<<<<<<<<<<<<<<<<<<<<~$

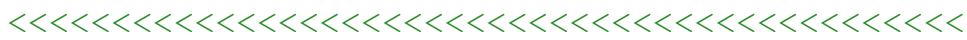

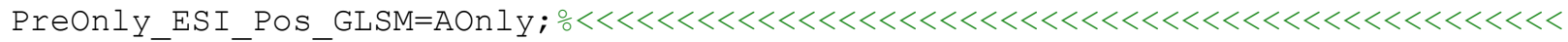
$<<<<<<\overline{<}<<<\overline{<}<<<\overline{<}<<<<<<<<<<<<<<<<<<<<<<<<<<<<<<<<<<<<<<<<<<<<<<<<<<<<<<<<<<<<<~$ $<<<<<<<<<<<<<<<<<<<<<<<<<<<<<~$

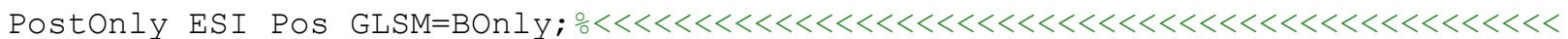

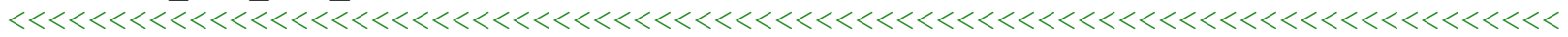
$<<<<<<<<<<<<<<<<<<<<<<<<<<<<<<~$

\% Summarizing Data

Data=AOnly;

oCalculating formulae type parameters: \# CHO, CHON, CHOS, CHOP, CHN, CHS, CHP, CHNS, CHONS formulae

Summary. $\mathrm{CHO}=\operatorname{sum}($ Data. $\mathrm{C}>0$ \& Data. $\mathrm{H}>0$ \& Data. $\mathrm{O}>0$ \& Data. $\mathrm{N}==0$ \& Data. $\mathrm{P}==0$ \& Data. $\mathrm{S}==0$ );

Summary. $\mathrm{CHON}=$ sum $($ Data. $\mathrm{C}>0$ \& Data. $\mathrm{H}>0$ \& Data. $\mathrm{O}>0$ \& Data. $\mathrm{N}>0$ \& Data.P==0 \&

Data. $\mathrm{S}=0$ ) ;

Summary $\cdot \mathrm{CHOS}=$ sum $($ Data. $\mathrm{C}>0$ \& Data.H $>0$ \& Data. O $>0$ \& Data. $\mathrm{N}==0$ \& Data. $\mathrm{P}==0$ \&

Data. S>0);

Summary. $\mathrm{CHOP}=\operatorname{sum}($ Data. $\mathrm{C}>0$ \& Data. $\mathrm{H}>0$ \& Data. $\mathrm{O}>0$ \& Data. $\mathrm{N}==0$ \& Data. $\mathrm{P}>0$ \&

Data. $\mathrm{S}==0$ ) ;

Summary. $\mathrm{CHN}=\operatorname{sum}($ Data. $\mathrm{C}>0$ \& Data. $\mathrm{H}>0$ \& Data. $\mathrm{O}==0$ \& Data. $\mathrm{N}>0$ \& Data. $\mathrm{P}==0$ \&

Data. $\mathrm{S}==0$ ) ;

Summary $\cdot \mathrm{CHS}=\operatorname{sum}($ Data $\cdot \mathrm{C}>0$ \& Data. $\mathrm{H}>0$ \& Data $\cdot \mathrm{O}==0$ \& Data. $\mathrm{N}==0$ \& Data. $\mathrm{P}==0$ \&

Data.s>0);

Summary. $\mathrm{CHNS}=\operatorname{sum}($ Data. $\mathrm{C}>0$ \& Data. $\mathrm{H}>0$ \& Data. $\mathrm{O}==0$ \& Data. $\mathrm{N}>0$ \& Data. $\mathrm{P}==0$ \&

Data. $\mathrm{S}>0$ );

Summary. $\mathrm{CHNP}=\mathrm{sum}($ Data. $\mathrm{C}>0$ \& Data.H $>0$ \& Data. $\mathrm{O}==0$ \& Data. $\mathrm{N}>0$ \& Data.P $>0$ \&

Data. $\mathrm{S}=0$ ) ;

Summary $\cdot \mathrm{CHONS}=\operatorname{sum}($ Data $\cdot \mathrm{C}>0$ \& Data. $\mathrm{H}>0$ \& Data. $\mathrm{O}>0$ \& Data. $\mathrm{N}>0$ \& Data.P==0 \&

Data. $\mathrm{S}>0$ );

Summary. $\mathrm{CHONP}=\operatorname{sum}($ Data. $\mathrm{C}>0$ \& Data. $\mathrm{H}>0$ \& Data. $\mathrm{O}>0$ \& Data.N $>0$ \& Data.P>0\&

Data. $\mathrm{S}=0$ ) ;

Summary. $\mathrm{CHONSP}=$ sum $($ Data. $\mathrm{C}>0$ \& Data.H $>0$ \& Data.O $>0$ \& Data.N $>0$ \& Data.P $>0$ \&

Data.S $>0$ ); 
oCalculating aromaticity parameters: \# condensed aromatic (AI>=0.67), aromatic $(\mathrm{AI}>=0.5)$,

onon-aromatic $($ AI $<0.5)$

Summary. CondensedAromatics $=$ sum (Data.AI $>=0.67)$;

Summary.Aromatics=sum (Data.AI $>=0.5)$;

Summary. NonAromatics=sum (Data.AI<0.5);

ocalculating other parameters: total \# of formulae, average molecular

oweight

Summary. TotalFormulae=height (Data);

Summary. AvgMW=mean (Data. NominalMass);

ostoring Summary Data

PreOnIy_ESI_Pos_GLSM_Summary=struct2table (Summary) ; 은 $<<<<<<<<<<<<<<<<<<<<<<<<<<~$

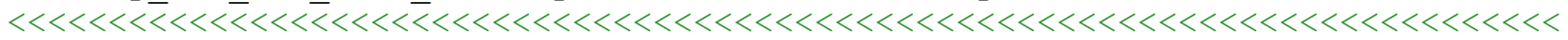

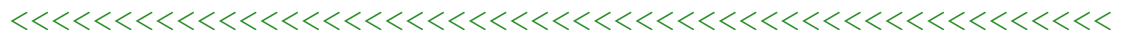

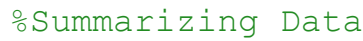

Data=BOnly;

oCalculating formulae type parameters: \# CHO, CHON, CHOS, CHOP, CHN, CHS, CHP, CHNS, CHONS formulae

Summary $\cdot \mathrm{CHO}=$ sum $($ Data. $\mathrm{C}>0$ \& Data. $\mathrm{H}>0$ \& Data. $\mathrm{O}>0$ \& Data. $\mathrm{N}==0$ \& Data. $\mathrm{P}==0$ \& Data. $\mathrm{S}=0$ ) ;

Summary $\cdot \mathrm{CHON}=$ sum $($ Data. $\mathrm{C}>0$ \& Data. $\mathrm{H}>0$ \& Data. $\mathrm{O}>0$ \& Data. $\mathrm{N}>0$ \& Data. $\mathrm{P}==0$ \& Data. $\mathrm{S}==0$ );

Summary. $\mathrm{CHOS}=$ sum $($ Data. $\mathrm{C}>0$ \& Data.H $>0$ \& Data. $\mathrm{O}>0$ \& Data. $\mathrm{N}==0$ \& Data. $\mathrm{P}==0$ \&

Data. $\mathrm{S}>0$ );

Summary $\cdot \mathrm{CHOP}=$ sum $($ Data. $\mathrm{C}>0$ \& Data. $\mathrm{H}>0$ \& Data. $\mathrm{O}>0$ \& Data. $\mathrm{N}==0$ \& Data. $\mathrm{P}>0$ \&

Data. $\mathrm{S}==0$ );

Summary $\cdot \mathrm{CHN}=$ sum $($ Data. $\mathrm{C}>0$ \& Data. $\mathrm{H}>0$ \& Data. $\mathrm{O}==0$ \& Data. $\mathrm{N}>0$ \& Data.P==0 \&

Data. $\mathrm{S}==0$ );

Summary. $\mathrm{CHS}=\mathrm{sum}($ Data. $\mathrm{C}>0$ \& Data. $\mathrm{H}>0$ \& Data. $\mathrm{O}==0$ \& Data. $\mathrm{N}==0$ \& Data. $\mathrm{P}==0$ \&

Data. $\mathrm{S}>0)$;

Summary. $\mathrm{CHNS}=\operatorname{sum}($ Data. $\mathrm{C}>0$ \& Data. $\mathrm{H}>0$ \& Data. $\mathrm{O}==0$ \& Data. $\mathrm{N}>0$ \& Data. $\mathrm{P}==0$ \&

Data. $\mathrm{S}>0$ );

Summary $\cdot \mathrm{CHNP}=\mathrm{sum}($ Data. $\mathrm{C}>0$ \& Data.H $>0$ \& Data. $\mathrm{O}==0$ \& Data. $\mathrm{N}>0$ \& Data. $\mathrm{P}>0$ \&

Data. $\mathrm{S}==0$ );

Summary. $\mathrm{CHONS}=\operatorname{sum}($ Data $. \mathrm{C}>0$ \& Data. $\mathrm{H}>0$ \& Data. $0>0$ \& Data.N>0 \& Data.P==0 \&

Data. S>0);

Summary. $\mathrm{CHONP}=$ sum $($ Data. $\mathrm{C}>0$ \& Data. $\mathrm{H}>0$ \& Data. $\mathrm{O}>0$ \& Data.N $>0$ \& Data.P $>0$ \&

Data. $\mathrm{S}==0$ ) ;

Summary. $\mathrm{CHONSP}=$ sum $($ Data. $\mathrm{C}>0$ \& Data. $\mathrm{H}>0$ \& Data. $\mathrm{O}>0$ \& Data.N $>0$ \& Data.P>0 \&

Data. $\mathrm{S}>0$ );

oCalculating aromaticity parameters: \# condensed aromatic (AI>=0.67),

aromatic $(A I>=0.5)$,

onon-aromatic $($ AI $<0.5)$

Summary. CondensedAromatics $=$ sum (Data.AI $>=0.67)$;

Summary. Aromatics $=$ sum (Data.AI $>=0.5)$;

Summary. NonAromatics=sum (Data.AI<0.5);

ocalculating other parameters: total \# of formulae, average molecular

oweight

Summary. TotalFormulae=height (Data);

Summary. AvgMW=mean (Data. NominalMass);

ostoring Summary Data

Postonly ESI Pos GLSM Summary=struct2table (Summary) ; $\circ<<<<<<<<<<<<<<<<<<<<<<<<<<$ $<<<<<<<<\overline{<}<<<\overline{<}<<<\overline{<}<<<<\overline{<}<<<<<<<<<<<<<<<<<<<<<<<<<<<<<<<<<<<<<<<<<<<<<<<<<<<<<<<~$

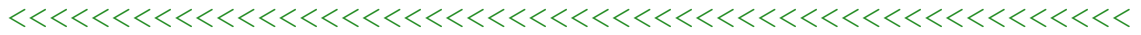

o Summarizing Data

Data=unique (Both); 
oCalculating formulae type parameters: \# CHO, CHON, CHOS, CHOP, CHN, CHS, CHP, CHNS, CHONS formulae

Summary $\cdot \mathrm{CHO}=$ sum $($ Data. $\mathrm{C}>0$ \& Data. $\mathrm{H}>0$ \& Data. $\mathrm{O}>0$ \& Data. $\mathrm{N}==0$ \& Data. $\mathrm{P}==0$ \& Data. $\mathrm{S}==0$ );

Summary. $\mathrm{CHON}=\operatorname{sum}($ Data. $\mathrm{C}>0$ \& Data. $\mathrm{H}>0$ \& Data. $\mathrm{O}>0$ \& Data. $\mathrm{N}>0$ \& Data.P==0 \& Data. $\mathrm{S}=0$ ) ) ;

Summary. $\mathrm{CHOS}=$ sum $($ Data. $\mathrm{C}>0$ \& Data. $\mathrm{H}>0$ \& Data. $0>0$ \& Data. $\mathrm{N}==0$ \& Data. $\mathrm{P}==0$ \& Data. $\mathrm{S}>0$ );

Summary $\cdot \mathrm{CHOP}=\operatorname{sum}($ Data. $\mathrm{C}>0$ \& Data.H $>0$ \& Data. $\mathrm{O}>0$ \& Data. $\mathrm{N}==0$ \& Data.P $>0$ \& Data. $\mathrm{S}==0$ );

Summary $\cdot \mathrm{CHN}=$ sum $($ Data. $\mathrm{C}>0$ \& Data. $\mathrm{H}>0$ \& Data. $\mathrm{O}==0$ \& Data. $\mathrm{N}>0$ \& Data. $\mathrm{P}==0$ \&

Data. $\mathrm{S}=0$ ) ;

Summary $\cdot \mathrm{CHS}=\operatorname{sum}($ Data. $\mathrm{C}>0$ \& Data. $\mathrm{H}>0$ \& Data. $\mathrm{O}==0$ \& Data. $\mathrm{N}==0$ \& Data. $\mathrm{P}==0$ \&

Data. S>0);

Summary. $\mathrm{CHNS}=$ sum (Data. $\mathrm{C}>0$ \& Data. $\mathrm{H}>0$ \& Data. $\mathrm{O}==0$ \& Data. $\mathrm{N}>0$ \& Data. $\mathrm{P}==0$ \&

Data. S>0);

Summary $\cdot \mathrm{CHNP}=\operatorname{sum}($ Data. $\mathrm{C}>0$ \& Data.H $>0$ \& Data. $\mathrm{O}==0$ \& Data.N $>0$ \& Data.P $>0$ \&

Data. $\mathrm{S}==0$ );

Summary $\cdot \mathrm{CHONS}=\operatorname{sum}($ Data $\cdot \mathrm{C}>0$ \& Data. $\mathrm{H}>0$ \& Data. $\mathrm{O}>0$ \& Data.N $>0$ \& Data.P==0 \&

Data. S>0);

Summary. $\mathrm{CHONP}=\operatorname{sum}($ Data. $\mathrm{C}>0$ \& Data. $\mathrm{H}>0$ \& Data. $\mathrm{O}>0$ \& Data.N $>0$ \& Data.P $>0$ \&

Data. $\mathrm{S}==0$ );

Summary. $\mathrm{CHONSP}=$ sum $($ Data. $\mathrm{C}>0$ \& Data.H $>0$ \& Data.O $>0$ \& Data.N $>0$ \& Data.P>0 \&

Data. $\mathrm{S}>0$ );

oCalculating aromaticity parameters: \# condensed aromatic (AI>=0.67),

aromatic $(\mathrm{AI}>=0.5)$,

onon-aromatic $(\mathrm{A} I<0.5)$

Summary. CondensedAromatics $=$ sum (Data.AI $>=0.67)$;

Summary.Aromatics $=$ sum (Data.AI $>=0.5)$;

Summary. NonAromatics=sum (Data.AI<0.5);

ocalculating other parameters: total \# of formulae, average molecular

oweight

Summary. TotalFormulae=height (Data);

Summary. AvgMW=mean (Data. NominalMass);

ostoring Summary Data

Common PreandPost ESI Pos GLSM Summary=struct2table (Summary); $\circ$ o $<<<<<<<<<<<<<<<<~$

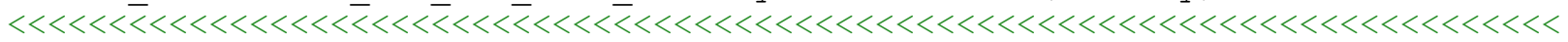

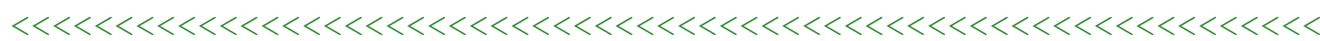

응 Comparing Pre- and Post-Oxidation SRFA for Positive mode LDI data onput Data so that the script can readily be applied to any data set

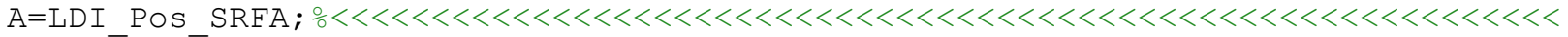

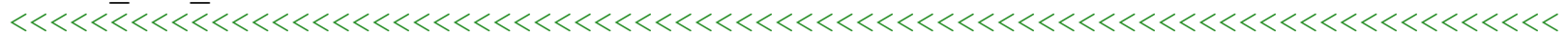
$<<<<<<<<<<<<<<<<<$

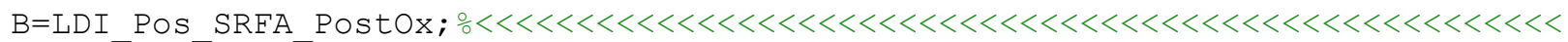
$<<<<<\overline{<}<<<\overline{<}<<<<\overline{<}<<<<<<<<<<<<<<<<<<<<<<<<<<<<<<<<<<<<<<<<<<<<<<<<<<<<<<<<<<<<<<~$ $<<<<<<<<<<<<<<<<<<<<<<<<~$

oseparate Data into those common and unique

Both $=[]$;

$\mathrm{k}=0$;

for $i=1:$ height $(A)$

for $j=1:$ height $(B)$

if $A \cdot C(i)==B \cdot C(j) \quad \& \& A \cdot H(i)==B \cdot H(j) \quad \& \& A \cdot N(i)==B \cdot N(j) \quad \& \& A \cdot O(i)==B \cdot O(j)$

$\& \& A \cdot P(i)==B \cdot P(j) \quad \& \& A \cdot S(i)==B \cdot S(j)$

$\mathrm{k}=\mathrm{k}+1$; 
$\operatorname{Both}(k,:)=[A . C(i), A . H(i), A . O(i), A . N(i), A . P(i)$,

A.S (i), A.NominalMass (i), A.HC_Ratio(i),

A.OC_Ratio(i),A.AI(i),A.DBE(i),A.AvgCOxState (i)] ;

$$
\text { A. C }(i)=0 \text {; }
$$

B. C $(j)=0$;

end

end

oCombining Data Sets

$A(A \cdot C==0,:)=[]$;

$A O n l_{Y}=[A . C, A . H, A . O, A . N, A . P, A . S$,

A. Nominalmass, A.HC Ratio,A.oC Ratio, A.AI, A.DBE, A.AvgCoxState];

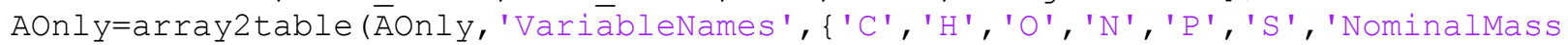
', 'HC_Ratio', 'OC_Ratio', 'AI', 'DBE', 'AvgCoxState'\});

$\mathrm{B}(\mathrm{B} \cdot \mathrm{C}==0,:)=[]$;

$\mathrm{BOnl} \mathrm{Y}_{\mathrm{B}}=[\mathrm{B} . \mathrm{C}, \mathrm{B} . \mathrm{H}, \mathrm{B} . \mathrm{O}, \mathrm{B} . \mathrm{N}, \mathrm{B} . \mathrm{P}$,

B.S,B.Nominalmass, B.HC_Ratio,B.OC_Ratio,B.AI, B.DBE, B.AvgCoxState] ;

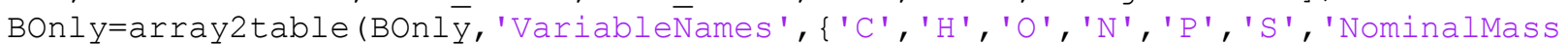
', 'HC_Ratio', 'OC_Ratio', 'AI', 'DBE', 'AvgCOxState'\}) ;

Both=array2table (Both, 'VariableNames', \{'C', 'H', 'O' , 'N', 'P' , 'S ', 'NominalMass', 'HC_Ratio', 'OC_Ratio', 'AI', 'DBE', 'AvgCOxState' \}) ;

ostoring Data with distinct names

Common PreandPost LDI Pos SRFA=unique (Both) ; $\circ<<<<<<<<<<<<<<<<<<<<<<<<<<<<<<<<<<<~$ $<<<<<<\overline{<}<<<<<<<<<<\overline{<}<<<\overline{<}<<<\overline{<}<<<<<<<<<<<<<<<<<<<<<<<<<<<<<<<<<<<<<<<<<<<<<<<<<<<~$

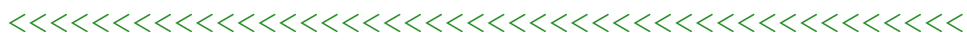

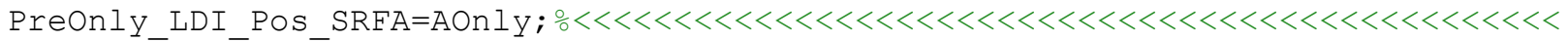
$<<<<<<\overline{<}<<<\overline{<}<<<\overline{<}<<<<<<<<<<<<<<<<<<<<<<<<<<<<<<<<<<<<<<<<<<<<<<<<<<<<<<<<<<<<<~$ $<<<<<<<<<<<<<<<<<<<<<<<<<<<<<~$

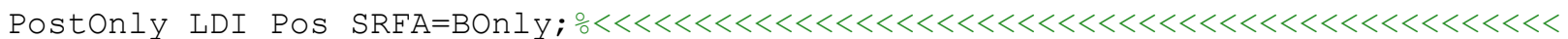

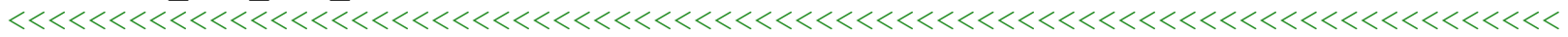
$<<<<<<<<<<<<<<<<<<<<<<<<<<<<<<~$

\% Summarizing Data

Data=AOnly;

oCalculating formulae type parameters: \# CHO, CHON, CHOS, CHOP, CHN, CHS, CHP, CHNS, CHONS formulae

Summary. $\mathrm{CHO}=\operatorname{sum}($ Data. $\mathrm{C}>0$ \& Data. $\mathrm{H}>0$ \& Data. $\mathrm{O}>0$ \& Data. $\mathrm{N}==0$ \& Data. $\mathrm{P}==0$ \& Data. $\mathrm{S}==0$ );

Summary. $\mathrm{CHON}=$ sum $($ Data. $\mathrm{C}>0$ \& Data. $\mathrm{H}>0$ \& Data. $\mathrm{O}>0$ \& Data. $\mathrm{N}>0$ \& Data.P==0 \&

Data. $\mathrm{S}=0$ ) ;

Summary $\cdot \mathrm{CHOS}=$ sum $($ Data. $\mathrm{C}>0$ \& Data.H $>0$ \& Data. O $>0$ \& Data. $\mathrm{N}==0$ \& Data. $\mathrm{P}==0$ \&

Data. S>0);

Summary. $\mathrm{CHOP}=\operatorname{sum}($ Data. $\mathrm{C}>0$ \& Data. $\mathrm{H}>0$ \& Data. $\mathrm{O}>0$ \& Data. $\mathrm{N}==0$ \& Data. $\mathrm{P}>0$ \&

Data. $\mathrm{S}==0$ ) ;

Summary. $\mathrm{CHN}=\operatorname{sum}($ Data. $\mathrm{C}>0$ \& Data. $\mathrm{H}>0$ \& Data. $\mathrm{O}==0$ \& Data. $\mathrm{N}>0$ \& Data. $\mathrm{P}==0$ \&

Data. $\mathrm{S}==0$ ) ;

Summary $\cdot \mathrm{CHS}=\operatorname{sum}($ Data $\cdot \mathrm{C}>0$ \& Data. $\mathrm{H}>0$ \& Data $\cdot \mathrm{O}==0$ \& Data. $\mathrm{N}==0$ \& Data. $\mathrm{P}==0$ \&

Data.s>0);

Summary. $\mathrm{CHNS}=\operatorname{sum}($ Data. $\mathrm{C}>0$ \& Data. $\mathrm{H}>0$ \& Data. $\mathrm{O}==0$ \& Data. $\mathrm{N}>0$ \& Data. $\mathrm{P}==0$ \&

Data. $\mathrm{S}>0$ );

Summary. $\mathrm{CHNP}=\mathrm{sum}($ Data. $\mathrm{C}>0$ \& Data.H $>0$ \& Data. $\mathrm{O}==0$ \& Data. $\mathrm{N}>0$ \& Data.P $>0$ \&

Data. $\mathrm{S}=0$ ) ;

Summary $\cdot \mathrm{CHONS}=\operatorname{sum}($ Data $\cdot \mathrm{C}>0$ \& Data. $\mathrm{H}>0$ \& Data. $\mathrm{O}>0$ \& Data. $\mathrm{N}>0$ \& Data.P==0 \&

Data. $\mathrm{S}>0$ );

Summary. $\mathrm{CHONP}=\operatorname{sum}($ Data. $\mathrm{C}>0$ \& Data. $\mathrm{H}>0$ \& Data. $\mathrm{O}>0$ \& Data.N $>0$ \& Data.P>0\&

Data. $\mathrm{S}=0$ ) ;

Summary. $\mathrm{CHONSP}=$ sum $($ Data. $\mathrm{C}>0$ \& Data.H $>0$ \& Data.O $>0$ \& Data.N $>0$ \& Data.P $>0$ \&

Data. S>0); 
oCalculating aromaticity parameters: \# condensed aromatic (AI>=0.67), aromatic $(\mathrm{AI}>=0.5)$,

onon-aromatic (AI<0.5)

Summary. CondensedAromatics $=$ sum (Data.AI $>=0.67)$;

Summary.Aromatics=sum (Data.AI $>=0.5)$;

Summary. NonAromatics=sum (Data.AI<0.5);

ocalculating other parameters: total \# of formulae, average molecular

oweight

Summary. TotalFormulae=height (Data);

Summary . AvgMW=mean (Data. NominalMass);

ostoring Summary Data

PreOnly_LDI_Pos_SRFA_Summary=struct2table (Summary); $\circ$ o $<<<<<<<<<<<<<<<<<<<<<<<<<<~$ $<<<<<<\overline{<}<<\overline{<}<<<\overline{<}<<<<\overline{<}<<<<<<<<<<<<<<<<<<<<<<<<<<<<<<<<<<<<<<<<<<<<<<<<<<<<<<<<$

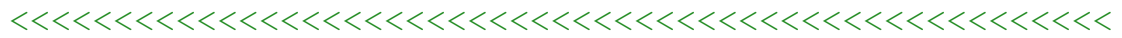

․ Summarizing Data

Data=BOnly;

oCalculating formulae type parameters: \# CHO, CHON, CHOS, CHOP, CHN, CHS, CHP, CHNS, CHONS formulae

Summary $\cdot \mathrm{CHO}=$ sum $($ Data. $\mathrm{C}>0$ \& Data. $\mathrm{H}>0$ \& Data. $\mathrm{O}>0$ \& Data. $\mathrm{N}==0$ \& Data. $\mathrm{P}==0$ \& Data. $\mathrm{S}==0$ );

Summary $\cdot \mathrm{CHON}=$ Sum $($ Data. $\mathrm{C}>0$ \& Data. $\mathrm{H}>0$ \& Data. $\mathrm{O}>0$ \& Data. $\mathrm{N}>0$ \& Data.P==0 \& Data. $\mathrm{S}==0$ );

Summary. $\mathrm{CHOS}=$ sum $($ Data. $\mathrm{C}>0$ \& Data. $\mathrm{H}>0$ \& Data. $\mathrm{O}>0$ \& Data. $\mathrm{N}==0$ \& Data. $\mathrm{P}==0$ \&

Data. $\mathrm{S}>0$ );

Summary $\cdot \mathrm{CHOP}=$ sum $($ Data. $\mathrm{C}>0$ \& Data.H $>0$ \& Data. $\mathrm{O}>0$ \& Data. $\mathrm{N}==0$ \& Data.P $>0$ \&

Data. $\mathrm{S}==0$ );

Summary $\cdot \mathrm{CHN}=$ sum $($ Data. $\mathrm{C}>0$ \& Data. $\mathrm{H}>0$ \& Data. $\mathrm{O}==0$ \& Data. $\mathrm{N}>0$ \& Data. $\mathrm{P}==0$ \&

Data. $\mathrm{S}==0$ );

Summary $\cdot \mathrm{CHS}=\operatorname{sum}($ Data $\cdot \mathrm{C}>0$ \& Data. $\mathrm{H}>0$ \& Data $\cdot \mathrm{O}==0$ \& Data. $\mathrm{N}==0$ \& Data. $\mathrm{P}==0$ \&

Data. $\mathrm{S}>0$ );

Summary. $\mathrm{CHNS}=$ sum $($ Data. $\mathrm{C}>0$ \& Data. $\mathrm{H}>0$ \& Data. $\mathrm{O}==0$ \& Data. $\mathrm{N}>0$ \& Data. $\mathrm{P}==0$ \&

Data. S>0);

Summary $\cdot \mathrm{CHNP}=\operatorname{sum}($ Data. $\mathrm{C}>0$ \& Data.H $>0$ \& Data. $\mathrm{O}==0$ \& Data. $\mathrm{N}>0$ \& Data.P $>0$ \&

Data. $\mathrm{S}==0$ );

Summary. $\mathrm{CHONS}=\operatorname{sum}($ Data $. \mathrm{C}>0$ \& Data. $\mathrm{H}>0$ \& Data. $0>0$ \& Data.N>0 \& Data.P==0 \&

Data. S>0);

Summary. $\mathrm{CHONP}=$ sum $($ Data. $\mathrm{C}>0$ \& Data. $\mathrm{H}>0$ \& Data. $\mathrm{O}>0$ \& Data.N $>0$ \& Data.P $>0$ \&

Data. $\mathrm{S}==0$ ) ;

Summary. $\mathrm{CHONSP}=$ sum $($ Data. $\mathrm{C}>0$ \& Data.H $>0$ \& Data.O $>0$ \& Data.N $>0$ \& Data.P>0 \&

Data. $\mathrm{S}>0$ );

ocalculating aromaticity parameters: \# condensed aromatic (AI>=0.67),

aromatic (AI>=0.5),

onon-aromatic $(\mathrm{A} I<0.5)$

Summary. CondensedAromatics $=$ sum (Data. AI $>=0.67)$;

Summary. Aromatics $=$ sum (Data.AI $>=0.5)$;

Summary. NonAromatics=sum (Data.AI<0.5);

ocalculating other parameters: total \# of formulae, average molecular

oweight

Summary. TotalFormulae=height (Data);

Summary. AvgMW=mean (Data. NominalMass);

ostoring Summary Data

Postonly LDI Pos SRFA Summary=struct2table (Summary); $\circ<<<<<<<<<<<<<<<<<<<<<<<<<<$ $<<<<<<<<\bar{L}<<<\overline{<}<<<\overline{<}<<<<\bar{L}<<<<<<<<<<<<<<<<<<<<<<<<<<<<<<<<<<<<<<<<<<<<<<<<<<<<<<<$

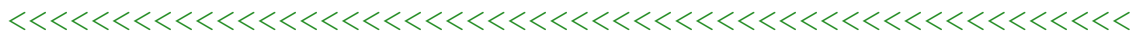

ㅇmmarizing Data

Data=unique (Both); 
oCalculating formulae type parameters: \# CHO, CHON, CHOS, CHOP, CHN, CHS, CHP, CHNS, CHONS formulae

Summary $\cdot \mathrm{CHO}=$ sum $($ Data. $\mathrm{C}>0$ \& Data. $\mathrm{H}>0$ \& Data. $\mathrm{O}>0$ \& Data. $\mathrm{N}==0$ \& Data. $\mathrm{P}==0$ \& Data. $\mathrm{S}==0$ );

Summary. $\mathrm{CHON}=\operatorname{sum}($ Data. $\mathrm{C}>0$ \& Data. $\mathrm{H}>0$ \& Data. $\mathrm{O}>0$ \& Data. $\mathrm{N}>0$ \& Data.P==0 \& Data. $\mathrm{S}=0$ ) ) ;

Summary. $\mathrm{CHOS}=$ sum $($ Data. $\mathrm{C}>0$ \& Data. $\mathrm{H}>0$ \& Data. $0>0$ \& Data. $\mathrm{N}==0$ \& Data. $\mathrm{P}==0$ \& Data. $\mathrm{S}>0$ );

Summary $\cdot \mathrm{CHOP}=\operatorname{sum}($ Data. $\mathrm{C}>0$ \& Data.H $>0$ \& Data. $\mathrm{O}>0$ \& Data. $\mathrm{N}==0$ \& Data.P $>0$ \& Data. $\mathrm{S}==0$ );

Summary $\cdot \mathrm{CHN}=$ sum $($ Data. $\mathrm{C}>0$ \& Data. $\mathrm{H}>0$ \& Data. $\mathrm{O}==0$ \& Data. $\mathrm{N}>0$ \& Data. $\mathrm{P}==0$ \&

Data. $\mathrm{S}=0$ ) ;

Summary $\cdot \mathrm{CHS}=\operatorname{sum}($ Data. $\mathrm{C}>0$ \& Data. $\mathrm{H}>0$ \& Data. $\mathrm{O}==0$ \& Data. $\mathrm{N}==0$ \& Data. $\mathrm{P}==0$ \&

Data. S>0);

Summary. $\mathrm{CHNS}=$ sum (Data. $\mathrm{C}>0$ \& Data. $\mathrm{H}>0$ \& Data. $\mathrm{O}==0$ \& Data. $\mathrm{N}>0$ \& Data. $\mathrm{P}==0$ \&

Data. S>0);

Summary $\cdot \mathrm{CHNP}=\operatorname{sum}($ Data. $\mathrm{C}>0$ \& Data.H $>0$ \& Data. $\mathrm{O}==0$ \& Data.N $>0$ \& Data.P $>0$ \&

Data. $\mathrm{S}==0$ );

Summary $\cdot \mathrm{CHONS}=\operatorname{sum}($ Data $\cdot \mathrm{C}>0$ \& Data. $\mathrm{H}>0$ \& Data. $\mathrm{O}>0$ \& Data.N $>0$ \& Data.P==0 \&

Data. S>0);

Summary. $\mathrm{CHONP}=\operatorname{sum}($ Data. $\mathrm{C}>0$ \& Data. $\mathrm{H}>0$ \& Data. $\mathrm{O}>0$ \& Data.N $>0$ \& Data.P $>0$ \&

Data. $\mathrm{S}==0$ );

Summary. $\mathrm{CHONSP}=$ sum $($ Data. $\mathrm{C}>0$ \& Data.H $>0$ \& Data.O $>0$ \& Data.N $>0$ \& Data.P>0 \&

Data. $\mathrm{S}>0$ );

oCalculating aromaticity parameters: \# condensed aromatic (AI>=0.67),

aromatic $(\mathrm{AI}>=0.5)$,

onon-aromatic $(\mathrm{A} I<0.5)$

Summary. CondensedAromatics $=$ sum (Data.AI $>=0.67)$;

Summary. Aromatics $=$ sum (Data.AI $>=0.5)$;

Summary. NonAromatics=sum (Data.AI<0.5);

ocalculating other parameters: total \# of formulae, average molecular

oweight

Summary. TotalFormulae=height (Data);

Summary. AvgMW=mean (Data. NominalMass);

ostoring Summary Data

Common PreandPost LDI Pos SRFA Summary=struct2table (Summary); $\circ$ o $<<<<<<<<<<<<<<<<$

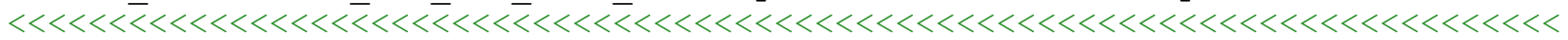

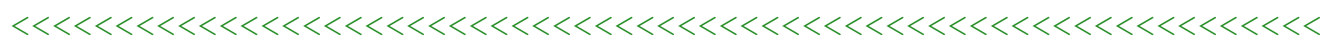

응 Comparing Pre- and Post-Oxidation GLSM for Positive mode LDI

oInput Data so that the script can readily be applied to any data set

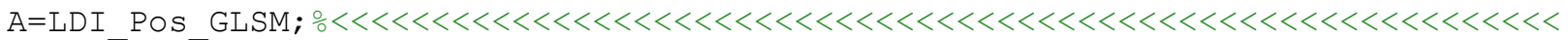

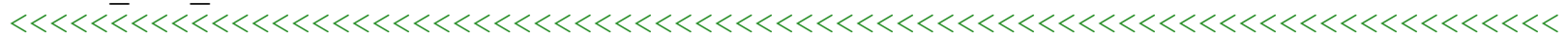
$<<<<<<<<<<<<<<<<<~$

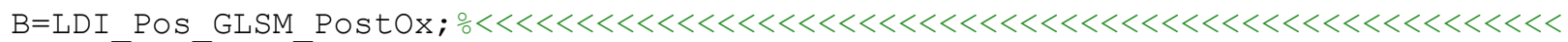

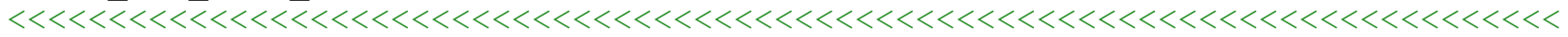
$<<<<<<<<<<<<<<<<<<<<<<<<~$

oSeparate Data into those common and unique

Both $=[]$;

$\mathrm{k}=0$;

for $i=1$ :height $(A)$

for $j=1:$ height $(B)$

if $A \cdot C(i)==B \cdot C(j) \quad \& \& A \cdot H(i)==B \cdot H(j) \quad \& \& A \cdot N(i)==B \cdot N(j) \quad \& \& A \cdot O(i)==B \cdot O(j)$

$\& \& A \cdot P(i)==B \cdot P(j) \quad \& \& A \cdot S(i)==B \cdot S(j)$

$\mathrm{k}=\mathrm{k}+1$; 
$\operatorname{Both}(k,:)=[A . C(i), A . H(i), A . O(i), A . N(i), A . P(i)$,

A.S (i), A.NominalMass (i), A.HC_Ratio(i),

A.OC_Ratio(i),A.AI(i),A.DBE(i),A.AvgCOxState (i)] ;

$$
\text { A. C }(i)=0 \text {; }
$$

B. C $(j)=0$;

end

end

oCombining Data Sets

$A(A \cdot C==0,:)=[]$;

$A O n l_{Y}=[A . C, A . H, A . O, A . N, A . P, A . S$,

A. Nominalmass, A.HC Ratio,A.oC Ratio, A.AI, A.DBE, A.AvgCoxState];

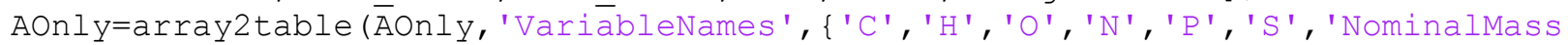
', 'HC_Ratio', 'OC_Ratio', 'AI', 'DBE', 'AvgCoxState'\});

$\mathrm{B}(\mathrm{B} \cdot \mathrm{C}==0,:)=[]$;

$\mathrm{BOnl} \mathrm{Y}_{\mathrm{B}}=[\mathrm{B} . \mathrm{C}, \mathrm{B} . \mathrm{H}, \mathrm{B} . \mathrm{O}, \mathrm{B} . \mathrm{N}, \mathrm{B} . \mathrm{P}$,

B.S,B.Nominalmass, B.HC_Ratio,B.OC_Ratio,B.AI, B.DBE, B.AvgCoxState] ;

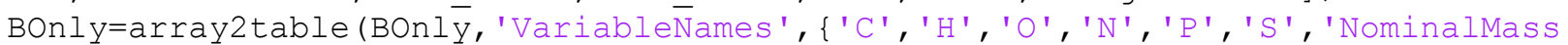
', 'HC_Ratio', 'OC_Ratio', 'AI', 'DBE', 'AvgCOxState'\}) ;

Both=array2table (Both, 'VariableNames', \{'C', 'H', 'O' , 'N', 'P' , 'S ', 'NominalMass', 'HC_Ratio', 'OC_Ratio', 'AI', 'DBE', 'AvgCOxState' \}) ;

ostoring Data with distinct names

Common PreandPost LDI Pos GLSM=unique (Both) ; $\circ<<<<<<<<<<<<<<<<<<<<<<<<<<<<<<<<<<<~$ $<<<<<<\overline{<}<<<<<<<<<<\overline{<}<<<\overline{<}<<<\overline{<}<<<<<<<<<<<<<<<<<<<<<<<<<<<<<<<<<<<<<<<<<<<<<<<<<<<~$

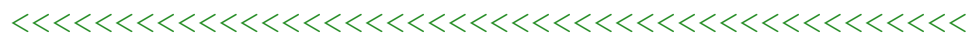

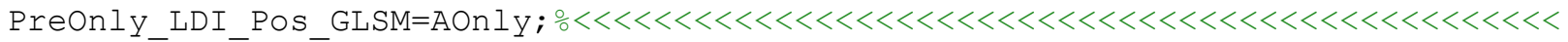
$<<<<<<\overline{<}<<<\overline{<}<<<\overline{<}<<<<<<<<<<<<<<<<<<<<<<<<<<<<<<<<<<<<<<<<<<<<<<<<<<<<<<<<<<<<<~$ $<<<<<<<<<<<<<<<<<<<<<<<<<<<<<~$

PostOnly LDI POS GLSM=BOnly; $\frac{\circ}{\circ}<<<<<<<<<<<<<<<<<<<<<<<<<<<<<<<<<<<<<<<<<<<<<<<<<<~$

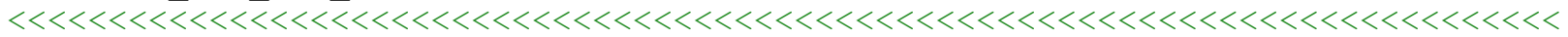
$<<<<<<<<<<<<<<<<<<<<<<<<<<<<<<~$

\% Summarizing Data

Data=AOnly;

oCalculating formulae type parameters: \# CHO, CHON, CHOS, CHOP, CHN, CHS, CHP, CHNS, CHONS formulae

Summary. $\mathrm{CHO}=\operatorname{sum}($ Data. $\mathrm{C}>0$ \& Data. $\mathrm{H}>0$ \& Data. $\mathrm{O}>0$ \& Data. $\mathrm{N}==0$ \& Data. $\mathrm{P}==0$ \& Data. $\mathrm{S}==0$ );

Summary. $\mathrm{CHON}=$ sum $($ Data. $\mathrm{C}>0$ \& Data. $\mathrm{H}>0$ \& Data. $\mathrm{O}>0$ \& Data. $\mathrm{N}>0$ \& Data.P==0 \&

Data. $\mathrm{S}=0$ ) ;

Summary $\cdot \mathrm{CHOS}=$ sum $($ Data. $\mathrm{C}>0$ \& Data.H $>0$ \& Data. O $>0$ \& Data. $\mathrm{N}==0$ \& Data. $\mathrm{P}==0$ \&

Data. S>0);

Summary. $\mathrm{CHOP}=\operatorname{sum}($ Data. $\mathrm{C}>0$ \& Data. $\mathrm{H}>0$ \& Data. $\mathrm{O}>0$ \& Data. $\mathrm{N}==0$ \& Data. $\mathrm{P}>0$ \&

Data. $\mathrm{S}==0$ ) ;

Summary. $\mathrm{CHN}=\operatorname{sum}($ Data. $\mathrm{C}>0$ \& Data. $\mathrm{H}>0$ \& Data. $\mathrm{O}==0$ \& Data. $\mathrm{N}>0$ \& Data. $\mathrm{P}==0$ \&

Data. $\mathrm{S}==0$ ) ;

Summary $\cdot \mathrm{CHS}=\operatorname{sum}($ Data $\cdot \mathrm{C}>0$ \& Data. $\mathrm{H}>0$ \& Data $\cdot \mathrm{O}==0$ \& Data. $\mathrm{N}==0$ \& Data. $\mathrm{P}==0$ \&

Data.s>0);

Summary. $\mathrm{CHNS}=\operatorname{sum}($ Data. $\mathrm{C}>0$ \& Data. $\mathrm{H}>0$ \& Data. $\mathrm{O}==0$ \& Data. $\mathrm{N}>0$ \& Data. $\mathrm{P}==0$ \&

Data. $\mathrm{S}>0$ );

Summary. $\mathrm{CHNP}=\mathrm{sum}($ Data. $\mathrm{C}>0$ \& Data.H $>0$ \& Data. $\mathrm{O}==0$ \& Data. $\mathrm{N}>0$ \& Data.P $>0$ \&

Data. $\mathrm{S}=0$ ) ;

Summary $\cdot \mathrm{CHONS}=\operatorname{sum}($ Data $\cdot \mathrm{C}>0$ \& Data. $\mathrm{H}>0$ \& Data. $\mathrm{O}>0$ \& Data. $\mathrm{N}>0$ \& Data.P==0 \&

Data. $\mathrm{S}>0$ );

Summary. $\mathrm{CHONP}=\operatorname{sum}($ Data. $\mathrm{C}>0$ \& Data. $\mathrm{H}>0$ \& Data. $\mathrm{O}>0$ \& Data.N $>0$ \& Data.P>0\&

Data. $\mathrm{S}=0$ ) ;

Summary. $\mathrm{CHONSP}=$ sum $($ Data. $\mathrm{C}>0$ \& Data.H $>0$ \& Data.O $>0$ \& Data.N $>0$ \& Data.P $>0$ \&

Data.S $>0$ ); 
oCalculating aromaticity parameters: \# condensed aromatic (AI>=0.67), aromatic $(\mathrm{AI}>=0.5)$,

onon-aromatic (AI<0.5)

Summary. CondensedAromatics $=$ sum (Data.AI $>=0.67)$;

Summary.Aromatics=sum (Data.AI $>=0.5)$;

Summary. NonAromatics=sum (Data.AI<0.5);

ocalculating other parameters: total \# of formulae, average molecular

oweight

Summary. TotalFormulae=height (Data);

Summary. AvgMW=mean (Data. NominalMass);

ostoring Summary Data

PreOnIy_LDI_Pos_GLSM_Summary=struct2table (Summary) ; 은 $<<<<<<<<<<<<<<<<<<<<<<<<<<~$

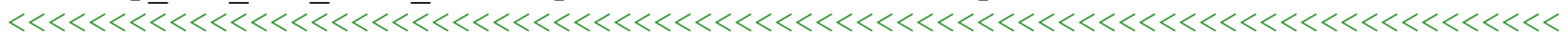

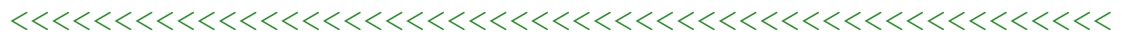

ㅇmmarizing Data

Data=BOnly;

oCalculating formulae type parameters: \# CHO, CHON, CHOS, CHOP, CHN, CHS, CHP, CHNS, CHONS formulae

Summary $\cdot \mathrm{CHO}=$ sum (Data. $\mathrm{C}>0$ \& Data. $\mathrm{H}>0$ \& Data. $\mathrm{O}>0$ \& Data. $\mathrm{N}==0$ \& Data. $\mathrm{P}==0$ \& Data. $\mathrm{S}==0$ );

Summary $\cdot \mathrm{CHON}=$ Sum $($ Data. $\mathrm{C}>0$ \& Data. $\mathrm{H}>0$ \& Data. $\mathrm{O}>0$ \& Data. $\mathrm{N}>0$ \& Data.P==0 \& Data. $\mathrm{S}==0$ );

Summary. $\mathrm{CHOS}=$ sum $($ Data. $\mathrm{C}>0$ \& Data. $\mathrm{H}>0$ \& Data. $\mathrm{O}>0$ \& Data. $\mathrm{N}==0$ \& Data. $\mathrm{P}==0$ \&

Data. $\mathrm{S}>0$ ) ;

Summary $\cdot \mathrm{CHOP}=\mathrm{sum}($ Data. $\mathrm{C}>0$ \& Data.H $>0$ \& Data. $\mathrm{O}>0$ \& Data. $\mathrm{N}==0$ \& Data.P $>0$ \&

Data. $\mathrm{S}==0$ );

Summary $\cdot \mathrm{CHN}=$ sum $($ Data. $\mathrm{C}>0$ \& Data. $\mathrm{H}>0$ \& Data. $\mathrm{O}==0$ \& Data. $\mathrm{N}>0$ \& Data.P==0 \&

Data. $\mathrm{S}==0$ );

Summary. $\mathrm{CHS}=\mathrm{sum}($ Data. $\mathrm{C}>0$ \& Data. $\mathrm{H}>0$ \& Data. $\mathrm{O}==0$ \& Data. $\mathrm{N}==0$ \& Data. $\mathrm{P}==0$ \&

Data. $\mathrm{S}>0$ );

Summary. $\mathrm{CHNS}=\operatorname{sum}($ Data. $\mathrm{C}>0$ \& Data. $\mathrm{H}>0$ \& Data. $\mathrm{O}==0$ \& Data. $\mathrm{N}>0$ \& Data. $\mathrm{P}==0$ \&

Data. S>0);

Summary $\cdot \mathrm{CHNP}=\mathrm{sum}($ Data. $\mathrm{C}>0$ \& Data.H $>0$ \& Data. $\mathrm{O}==0$ \& Data. $\mathrm{N}>0$ \& Data.P $>0$ \&

Data. $\mathrm{S}==0$ );

Summary. $\mathrm{CHONS}=\operatorname{sum}($ Data. $\mathrm{C}>0$ \& Data. $\mathrm{H}>0$ \& Data. $0>0$ \& Data.N>0 \& Data.P==0 \&

Data. S>0);

Summary. $\mathrm{CHONP}=$ sum $($ Data. $\mathrm{C}>0$ \& Data. $\mathrm{H}>0$ \& Data. $\mathrm{O}>0$ \& Data.N $>0$ \& Data.P $>0$ \&

Data. $\mathrm{S}==0$ ) ;

Summary. $\mathrm{CHONSP}=$ sum $($ Data. $\mathrm{C}>0$ \& Data.H $>0$ \& Data.O $>0$ \& Data.N $>0$ \& Data.P>0 \&

Data. $\mathrm{S}>0$ );

oCalculating aromaticity parameters: \# condensed aromatic (AI>=0.67),

aromatic $(A I>=0.5)$,

onon-aromatic $($ AI $<0.5)$

Summary. CondensedAromatics $=$ sum (Data. AI $>=0.67)$;

Summary. Aromatics $=$ sum (Data.AI $>=0.5)$;

Summary. NonAromatics=sum (Data.AI<0.5);

ocalculating other parameters: total \# of formulae, average molecular

oweight

Summary. TotalFormulae=height (Data);

Summary. AvgMW=mean (Data. NominalMass);

ostoring Summary Data

Postonly LDI Pos GLSM Summary=struct2table (Summary) ; $\circ<<<<<<<<<<<<<<<<<<<<<<<<<<$ $<<<<<<<<\overline{<}<<<\overline{<}<<<\overline{<}<<<<\overline{<}<<<<<<<<<<<<<<<<<<<<<<<<<<<<<<<<<<<<<<<<<<<<<<<<<<<<<<<~$

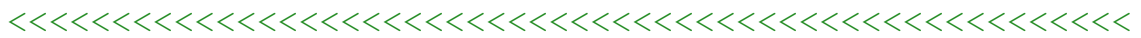

o Summarizing Data

Data=unique (Both); 
oCalculating formulae type parameters: \# CHO, CHON, CHOS, CHOP, CHN, CHS, CHP, CHNS, CHONS formulae

Summary $\cdot \mathrm{CHO}=$ sum $($ Data. $\mathrm{C}>0$ \& Data. $\mathrm{H}>0$ \& Data. $\mathrm{O}>0$ \& Data. $\mathrm{N}==0$ \& Data. $\mathrm{P}==0$ \& Data. $\mathrm{S}==0$ );

Summary. $\mathrm{CHON}=\operatorname{sum}($ Data. $\mathrm{C}>0$ \& Data. $\mathrm{H}>0$ \& Data. $\mathrm{O}>0$ \& Data. $\mathrm{N}>0$ \& Data.P==0 \& Data. $\mathrm{S}=0$ ) ) ;

Summary. $\mathrm{CHOS}=$ sum $($ Data. $\mathrm{C}>0$ \& Data. $\mathrm{H}>0$ \& Data. $0>0$ \& Data. $\mathrm{N}==0$ \& Data. $\mathrm{P}==0$ \& Data. $\mathrm{S}>0$ );

Summary $\cdot \mathrm{CHOP}=\operatorname{sum}($ Data. $\mathrm{C}>0$ \& Data.H $>0$ \& Data. $\mathrm{O}>0$ \& Data. $\mathrm{N}==0$ \& Data.P $>0$ \& Data. $\mathrm{S}==0$ );

Summary $\cdot \mathrm{CHN}=$ sum $($ Data. $\mathrm{C}>0$ \& Data. $\mathrm{H}>0$ \& Data. $\mathrm{O}==0$ \& Data. $\mathrm{N}>0$ \& Data. $\mathrm{P}==0$ \&

Data. $\mathrm{S}=0$ ) ;

Summary $\cdot \mathrm{CHS}=\operatorname{sum}($ Data. $\mathrm{C}>0$ \& Data. $\mathrm{H}>0$ \& Data. $\mathrm{O}==0$ \& Data. $\mathrm{N}==0$ \& Data. $\mathrm{P}==0$ \&

Data. S>0);

Summary. $\mathrm{CHNS}=$ sum (Data. $\mathrm{C}>0$ \& Data. $\mathrm{H}>0$ \& Data. $\mathrm{O}==0$ \& Data. $\mathrm{N}>0$ \& Data. $\mathrm{P}==0$ \&

Data. S>0);

Summary $\cdot \mathrm{CHNP}=\operatorname{sum}($ Data. $\mathrm{C}>0$ \& Data.H $>0$ \& Data. $\mathrm{O}==0$ \& Data.N $>0$ \& Data.P $>0$ \&

Data. $\mathrm{S}==0$ );

Summary $\cdot \mathrm{CHONS}=\operatorname{sum}($ Data $\cdot \mathrm{C}>0$ \& Data. $\mathrm{H}>0$ \& Data. $\mathrm{O}>0$ \& Data.N $>0$ \& Data.P==0 \&

Data. S>0);

Summary. $\mathrm{CHONP}=\operatorname{sum}($ Data. $\mathrm{C}>0$ \& Data. $\mathrm{H}>0$ \& Data. $\mathrm{O}>0$ \& Data.N $>0$ \& Data.P $>0$ \&

Data. $\mathrm{S}==0$ );

Summary. $\mathrm{CHONSP}=$ sum $($ Data. $\mathrm{C}>0$ \& Data. $\mathrm{H}>0$ \& Data. $\mathrm{O}>0$ \& Data.N $>0$ \& Data.P $>0$ \&

Data. $\mathrm{S}>0$ );

oCalculating aromaticity parameters: \# condensed aromatic (AI>=0.67),

aromatic $(\mathrm{AI}>=0.5)$,

onon-aromatic $(\mathrm{A} I<0.5)$

Summary. CondensedAromatics $=$ sum (Data.AI $>=0.67)$;

Summary. Aromatics $=$ sum (Data.AI $>=0.5)$;

Summary. NonAromatics=sum (Data.AI<0.5);

ocalculating other parameters: total \# of formulae, average molecular

oweight

Summary. TotalFormulae=height (Data);

Summary. AvgMW=mean (Data. NominalMass);

ostoring Summary Data

Common PreandPost LDI Pos GLSM Summary=struct2table (Summary); $\circ$ o $<<<<<<<<<<<<<<<<$

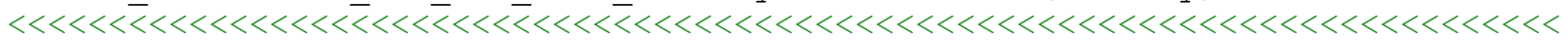

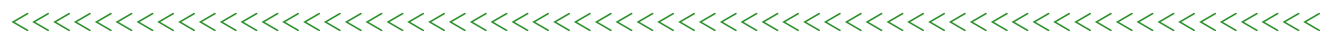

응 Comparing Pre- and Post-Oxidation SRFA for negative mode LDI data oInput Data so that the script can readily be applied to any data set

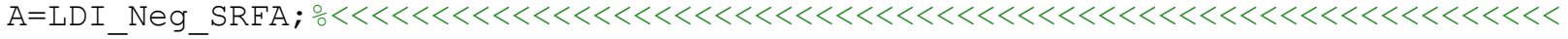

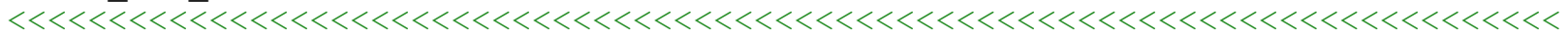
$<<<<<<<<<<<<<<<<<$

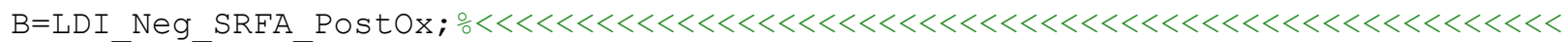

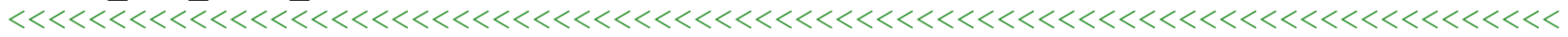
$<<<<<<<<<<<<<<<<<<<<<<<<~$

o Separate Data into those common and unique

Both=[] ;

$\mathrm{k}=0$;

for $i=1$ : height $(A)$

for $j=1:$ height $(B)$

if $A \cdot C(i)==B \cdot C(j) \quad \& \& A \cdot H(i)==B \cdot H(j) \quad \& \& A \cdot N(i)==B \cdot N(j) \quad \& \& A \cdot O(i)==B \cdot O(j)$

$\& \& A \cdot P(i)==B \cdot P(j) \quad \& \& A \cdot S(i)==B \cdot S(j)$

$\mathrm{k}=\mathrm{k}+1$; 
$\operatorname{Both}(k,:)=[A . C(i), A . H(i), A . O(i), A . N(i), A . P(i)$,

A.S (i), A.NominalMass (i), A.HC_Ratio(i),

A.OC_Ratio(i),A.AI(i),A.DBE(i),A.AvgCOxState (i)] ;

$$
\text { A. C }(i)=0 \text {; }
$$

B. C $(j)=0$;

end

end

oCombining Data Sets

$A(A \cdot C==0,:)=[]$;

$A O n l_{Y}=[A . C, A . H, A . O, A . N, A . P, A . S$,

A. Nominalmass, A.HC Ratio,A.oC Ratio, A.AI, A.DBE, A.AvgCoxState];

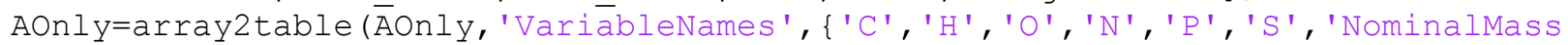
', 'HC_Ratio', 'OC_Ratio', 'AI', 'DBE', 'AvgCoxState'\});

$\mathrm{B}(\mathrm{B} \cdot \mathrm{C}==0,:)=[]$;

$\mathrm{BOnl} \mathrm{Y}_{\mathrm{B}}=[\mathrm{B} . \mathrm{C}, \mathrm{B} . \mathrm{H}, \mathrm{B} . \mathrm{O}, \mathrm{B} . \mathrm{N}, \mathrm{B} . \mathrm{P}$,

B.S,B.Nominalmass, B.HC_Ratio,B.OC_Ratio,B.AI, B.DBE, B.AvgCoxState] ;

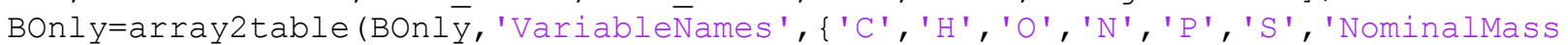
', 'HC_Ratio', 'OC_Ratio', 'AI', 'DBE', 'AvgCOxState'\}) ;

Both=array2table (Both, 'VariableNames', \{'C', 'H', 'O' , 'N', 'P' , 'S ', 'NominalMass', 'HC_Ratio', 'OC_Ratio', 'AI', 'DBE', 'AvgCOxState' \}) ;

ostoring Data with distinct names

Common PreandPost LDI Neg SRFA=unique (Both) ; $\circ<<<<<<<<<<<<<<<<<<<<<<<<<<<<<<<<<<~$ $<<<<<<\overline{<}<<<<<<<<<<\overline{<}<<<\overline{<}<<<\overline{<}<<<<<<<<<<<<<<<<<<<<<<<<<<<<<<<<<<<<<<<<<<<<<<<<<<<~$

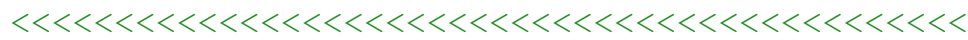

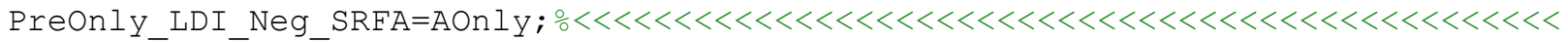
$<<<<<<\overline{<}<<<\overline{<}<<<\overline{<}<<<<<<<<<<<<<<<<<<<<<<<<<<<<<<<<<<<<<<<<<<<<<<<<<<<<<<<<<<<<<~$ $<<<<<<<<<<<<<<<<<<<<<<<<<<<<<~$

PostOnly LDI Neg SRFA=BOnly; $\frac{\circ}{\circ}<<<<<<<<<<<<<<<<<<<<<<<<<<<<<<<<<<<<<<<<<<<<<<<<<~$

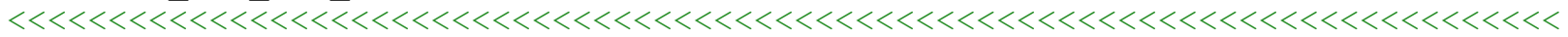
$<<<<<<<<<<<<<<<<<<<<<<<<<<<<<<~$

\% Summarizing Data

Data=AOnly;

oCalculating formulae type parameters: \# CHO, CHON, CHOS, CHOP, CHN, CHS, CHP, CHNS, CHONS formulae

Summary. $\mathrm{CHO}=\operatorname{sum}($ Data. $\mathrm{C}>0$ \& Data. $\mathrm{H}>0$ \& Data. $\mathrm{O}>0$ \& Data. $\mathrm{N}==0$ \& Data. $\mathrm{P}==0$ \& Data. $\mathrm{S}==0$ );

Summary. $\mathrm{CHON}=$ sum $($ Data. $\mathrm{C}>0$ \& Data. $\mathrm{H}>0$ \& Data. $\mathrm{O}>0$ \& Data. $\mathrm{N}>0$ \& Data.P==0 \&

Data. $\mathrm{S}=0$ ) ;

Summary $\cdot \mathrm{CHOS}=$ sum $($ Data. $\mathrm{C}>0$ \& Data.H $>0$ \& Data. O $>0$ \& Data. $\mathrm{N}==0$ \& Data. $\mathrm{P}==0$ \&

Data. S>0);

Summary. $\mathrm{CHOP}=\operatorname{sum}($ Data. $\mathrm{C}>0$ \& Data. $\mathrm{H}>0$ \& Data. $\mathrm{O}>0$ \& Data. $\mathrm{N}==0$ \& Data. $\mathrm{P}>0$ \&

Data. $\mathrm{S}==0$ );

Summary $\cdot \mathrm{CHN}=$ sum $($ Data. $\mathrm{C}>0$ \& Data. $\mathrm{H}>0$ \& Data. $\mathrm{O}==0$ \& Data. $\mathrm{N}>0$ \& Data. $\mathrm{P}==0$ \&

Data. $\mathrm{S}==0$ ) ;

Summary $\cdot \mathrm{CHS}=\operatorname{sum}($ Data $\cdot \mathrm{C}>0$ \& Data. $\mathrm{H}>0$ \& Data $\cdot \mathrm{O}==0$ \& Data. $\mathrm{N}==0$ \& Data. $\mathrm{P}==0$ \&

Data.s>0);

Summary. $\mathrm{CHNS}=\operatorname{sum}($ Data. $\mathrm{C}>0$ \& Data. $\mathrm{H}>0$ \& Data. $\mathrm{O}==0$ \& Data. $\mathrm{N}>0$ \& Data. $\mathrm{P}==0$ \&

Data. $\mathrm{S}>0$ );

Summary. $\mathrm{CHNP}=\mathrm{sum}($ Data. $\mathrm{C}>0$ \& Data.H $>0$ \& Data. $\mathrm{O}==0$ \& Data. $\mathrm{N}>0$ \& Data.P $>0$ \&

Data. $\mathrm{S}=0$ ) ;

Summary $\cdot \mathrm{CHONS}=\operatorname{sum}($ Data $\cdot \mathrm{C}>0$ \& Data. $\mathrm{H}>0$ \& Data. $\mathrm{O}>0$ \& Data.N $>0$ \& Data.P==0 \&

Data. $\mathrm{S}>0$ );

Summary. $\mathrm{CHONP}=\operatorname{sum}($ Data. $\mathrm{C}>0$ \& Data. $\mathrm{H}>0$ \& Data. $\mathrm{O}>0$ \& Data.N $>0$ \& Data.P>0\&

Data. $\mathrm{S}=0$ ) ;

Summary. $\mathrm{CHONSP}=$ sum $($ Data. $\mathrm{C}>0$ \& Data.H $>0$ \& Data.O $>0$ \& Data.N $>0$ \& Data.P $>0$ \&

Data.S $>0$ ); 
oCalculating aromaticity parameters: \# condensed aromatic (AI>=0.67), aromatic $(\mathrm{AI}>=0.5)$,

onon-aromatic $($ AI $<0.5)$

Summary. CondensedAromatics $=$ sum (Data.AI $>=0.67)$;

Summary.Aromatics=sum (Data.AI $>=0.5)$;

Summary. NonAromatics=sum (Data.AI<0.5);

ocalculating other parameters: total \# of formulae, average molecular

oweight

Summary. TotalFormulae=height (Data);

Summary. AvgMW=mean (Data. NominalMass);

ostoring Summary Data

PreOnly_LDI_Neg_SRFA_Summary=struct2table (Summary); $\circ$ o $<<<<<<<<<<<<<<<<<<<<<<<<<<~$ $<<<<<<<\overline{<}<<<\overline{<}<<<\overline{<}<<<<\overline{<}<<<<<<<<<<<<<<<<<<<<<<<<<<<<<<<<<<<<<<<<<<<<<<<<<<<<<<<<~$

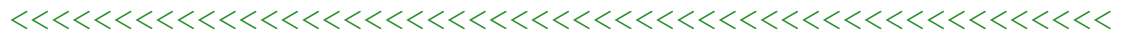

ㅇmmarizing Data

Data=BOnly;

oCalculating formulae type parameters: \# CHO, CHON, CHOS, CHOP, CHN, CHS, CHP, CHNS, CHONS formulae

Summary $\cdot \mathrm{CHO}=$ sum $($ Data. $\mathrm{C}>0$ \& Data. $\mathrm{H}>0$ \& Data. $\mathrm{O}>0$ \& Data. $\mathrm{N}==0$ \& Data. $\mathrm{P}==0$ \& Data. $\mathrm{S}=0$ ) ;

Summary $\cdot \mathrm{CHON}=$ sum $($ Data. $\mathrm{C}>0$ \& Data. $\mathrm{H}>0$ \& Data. $\mathrm{O}>0$ \& Data. $\mathrm{N}>0$ \& Data. $\mathrm{P}==0$ \& Data. $\mathrm{S}==0$ );

Summary. $\mathrm{CHOS}=$ sum $($ Data. $\mathrm{C}>0$ \& Data.H $>0$ \& Data. $\mathrm{O}>0$ \& Data. $\mathrm{N}==0$ \& Data. $\mathrm{P}==0$ \&

Data. $\mathrm{S}>0$ );

Summary $\cdot \mathrm{CHOP}=$ sum $($ Data. $\mathrm{C}>0$ \& Data. $\mathrm{H}>0$ \& Data. $\mathrm{O}>0$ \& Data. $\mathrm{N}==0$ \& Data. $\mathrm{P}>0$ \&

Data. $\mathrm{S}==0$ );

Summary $\cdot \mathrm{CHN}=$ sum $($ Data. $\mathrm{C}>0$ \& Data. $\mathrm{H}>0$ \& Data. $\mathrm{O}==0$ \& Data. $\mathrm{N}>0$ \& Data.P==0 \&

Data. $\mathrm{S}==0$ );

Summary. $\mathrm{CHS}=\mathrm{sum}($ Data. $\mathrm{C}>0$ \& Data. $\mathrm{H}>0$ \& Data. $\mathrm{O}==0$ \& Data. $\mathrm{N}==0$ \& Data. $\mathrm{P}==0$ \&

Data. $\mathrm{S}>0)$;

Summary. $\mathrm{CHNS}=\operatorname{sum}($ Data. $\mathrm{C}>0$ \& Data. $\mathrm{H}>0$ \& Data. $\mathrm{O}==0$ \& Data. $\mathrm{N}>0$ \& Data. $\mathrm{P}==0$ \&

Data. $\mathrm{S}>0$ );

Summary $\cdot \mathrm{CHNP}=\mathrm{sum}($ Data. $\mathrm{C}>0$ \& Data.H $>0$ \& Data. $\mathrm{O}==0$ \& Data. $\mathrm{N}>0$ \& Data. $\mathrm{P}>0$ \&

Data. $\mathrm{S}==0$ );

Summary. $\mathrm{CHONS}=\operatorname{sum}($ Data. $\mathrm{C}>0$ \& Data. $\mathrm{H}>0$ \& Data. $0>0$ \& Data.N>0 \& Data.P==0 \&

Data. S>0);

Summary. $\mathrm{CHONP}=$ sum $($ Data. $\mathrm{C}>0$ \& Data. $\mathrm{H}>0$ \& Data. $\mathrm{O}>0$ \& Data.N $>0$ \& Data.P $>0$ \&

Data. $\mathrm{S}==0$ ) ;

Summary. $\mathrm{CHONSP}=$ sum $($ Data. $\mathrm{C}>0$ \& Data. $\mathrm{H}>0$ \& Data. $\mathrm{O}>0$ \& Data.N $>0$ \& Data.P>0 \&

Data. $\mathrm{S}>0$ );

oCalculating aromaticity parameters: \# condensed aromatic (AI>=0.67),

aromatic $(A I>=0.5)$,

onon-aromatic $($ AI $<0.5)$

Summary. CondensedAromatics $=$ sum (Data.AI $>=0.67)$;

Summary. Aromatics $=$ sum (Data.AI $>=0.5)$;

Summary. NonAromatics $=$ sum (Data.AI<0.5);

ocalculating other parameters: total \# of formulae, average molecular

oweight

Summary. TotalFormulae=height (Data);

Summary. AvgMW=mean (Data. NominalMass);

ostoring Summary Data

Postonly LDI Neg SRFA Summary=struct2table (Summary) ; $\circ<<<<<<<<<<<<<<<<<<<<<<<<<~$ $<<<<<<<<\overline{<}<<<\overline{<}<<<\overline{<}<<<<\overline{<}<<<<<<<<<<<<<<<<<<<<<<<<<<<<<<<<<<<<<<<<<<<<<<<<<<<<<<<~$

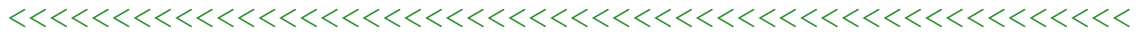

o Summarizing Data

Data=unique (Both); 
oCalculating formulae type parameters: \# CHO, CHON, CHOS, CHOP, CHN, CHS, CHP, CHNS, CHONS formulae

Summary $\cdot \mathrm{CHO}=$ sum $($ Data. $\mathrm{C}>0$ \& Data. $\mathrm{H}>0$ \& Data. $\mathrm{O}>0$ \& Data. $\mathrm{N}==0$ \& Data. $\mathrm{P}==0$ \& Data. $\mathrm{S}==0$ );

Summary. $\mathrm{CHON}=\operatorname{sum}($ Data. $\mathrm{C}>0$ \& Data. $\mathrm{H}>0$ \& Data. $\mathrm{O}>0$ \& Data. $\mathrm{N}>0$ \& Data.P==0 \& Data. $\mathrm{S}=0$ ) ) ;

Summary. $\mathrm{CHOS}=$ sum $($ Data. $\mathrm{C}>0$ \& Data. $\mathrm{H}>0$ \& Data. $0>0$ \& Data. $\mathrm{N}==0$ \& Data. $\mathrm{P}==0$ \& Data. $\mathrm{S}>0$ );

Summary $\cdot \mathrm{CHOP}=\operatorname{sum}($ Data. $\mathrm{C}>0$ \& Data.H $>0$ \& Data. $\mathrm{O}>0$ \& Data. $\mathrm{N}==0$ \& Data.P $>0$ \& Data. $\mathrm{S}==0$ );

Summary $\cdot \mathrm{CHN}=$ sum $($ Data. $\mathrm{C}>0$ \& Data. $\mathrm{H}>0$ \& Data. $\mathrm{O}==0$ \& Data. $\mathrm{N}>0$ \& Data. $\mathrm{P}==0$ \&

Data. $\mathrm{S}=0$ ) ;

Summary $\cdot \mathrm{CHS}=\operatorname{sum}($ Data. $\mathrm{C}>0$ \& Data. $\mathrm{H}>0$ \& Data. $\mathrm{O}==0$ \& Data. $\mathrm{N}==0$ \& Data. $\mathrm{P}==0$ \&

Data. S>0);

Summary. $\mathrm{CHNS}=$ sum (Data. $\mathrm{C}>0$ \& Data. $\mathrm{H}>0$ \& Data. $\mathrm{O}==0$ \& Data. $\mathrm{N}>0$ \& Data. $\mathrm{P}==0$ \&

Data. S>0);

Summary $\cdot \mathrm{CHNP}=\operatorname{sum}($ Data. $\mathrm{C}>0$ \& Data.H $>0$ \& Data. $\mathrm{O}==0$ \& Data.N $>0$ \& Data.P $>0$ \&

Data. $\mathrm{S}==0$ );

Summary $\cdot \mathrm{CHONS}=\operatorname{sum}($ Data $\cdot \mathrm{C}>0$ \& Data. $\mathrm{H}>0$ \& Data. $\mathrm{O}>0$ \& Data.N $>0$ \& Data.P==0 \&

Data. S>0);

Summary. $\mathrm{CHONP}=\operatorname{sum}($ Data. $\mathrm{C}>0$ \& Data. $\mathrm{H}>0$ \& Data. $\mathrm{O}>0$ \& Data.N $>0$ \& Data.P $>0$ \&

Data. $\mathrm{S}==0$ );

Summary. $\mathrm{CHONSP}=$ sum $($ Data. $\mathrm{C}>0$ \& Data.H $>0$ \& Data.O $>0$ \& Data.N $>0$ \& Data.P>0 \&

Data. $\mathrm{S}>0$ );

oCalculating aromaticity parameters: \# condensed aromatic (AI>=0.67),

aromatic $(\mathrm{AI}>=0.5)$,

onon-aromatic $(\mathrm{A} I<0.5)$

Summary. CondensedAromatics $=$ sum (Data.AI $>=0.67)$;

Summary.Aromatics $=$ sum (Data.AI $>=0.5)$;

Summary. NonAromatics=sum (Data.AI<0.5);

ocalculating other parameters: total \# of formulae, average molecular

oweight

Summary. TotalFormulae=height (Data);

Summary. AvgMW=mean (Data. NominalMass);

ostoring Summary Data

Common PreandPost LDI Neg SRFA Summary=struct2table (Summary); $\circ$ o $<<<<<<<<<<<<<<<<$

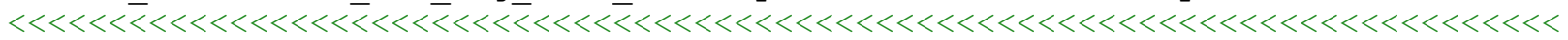

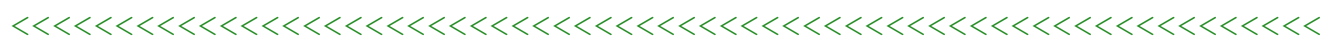

응 Comparing Pre- and Post-Oxidation GLSM for negative mode LDI

oInput Data so that the script can readily be applied to any data set

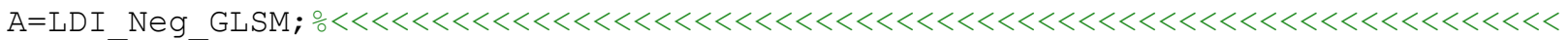

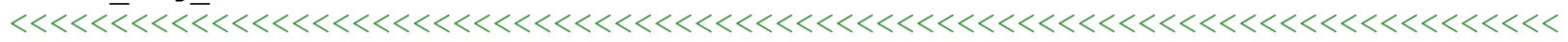
$<<<<<<<<<<<<<<<<<$

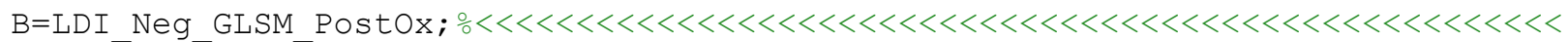

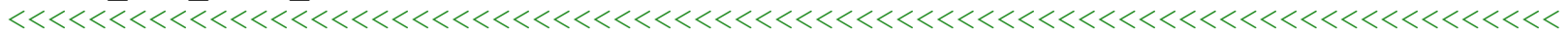
$<<<<<<<<<<<<<<<<<<<<<<<<~$

oSeparate Data into those common and unique

Both $=[]$;

$\mathrm{k}=0$;

for $i=1$ :height $(A)$

for $j=1:$ height $(B)$

if $A \cdot C(i)==B \cdot C(j) \quad \& \& A \cdot H(i)==B \cdot H(j) \quad \& \& A \cdot N(i)==B \cdot N(j) \quad \& \& A \cdot O(i)==B \cdot O(j)$

$\& \& A \cdot P(i)==B \cdot P(j) \quad \& \& A \cdot S(i)==B \cdot S(j)$

$\mathrm{k}=\mathrm{k}+1$; 
$\operatorname{Both}(k,:)=[A . C(i), A . H(i), A . O(i), A . N(i), A . P(i)$,

A.S (i), A.NominalMass (i), A.HC_Ratio(i),

A.OC_Ratio(i),A.AI(i),A.DBE(i),A.AvgCOxState (i)] ;

$$
\text { A. C }(i)=0 \text {; }
$$

B. C $(j)=0$;

end

end

oCombining Data Sets

$A(A \cdot C==0,:)=[]$;

$A O n l_{Y}=[A . C, A . H, A . O, A . N, A . P, A . S$,

A. Nominalmass, A.HC Ratio,A.oC Ratio, A.AI, A.DBE, A.AvgCoxState];

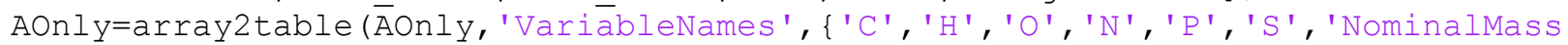
', 'HC_Ratio', 'OC_Ratio', 'AI', 'DBE', 'AvgCoxState'\});

$\mathrm{B}(\mathrm{B} \cdot \mathrm{C}==0,:)=[]$;

$\mathrm{BOnl} \mathrm{Y}_{\mathrm{B}}=[\mathrm{B} . \mathrm{C}, \mathrm{B} . \mathrm{H}, \mathrm{B} . \mathrm{O}, \mathrm{B} . \mathrm{N}, \mathrm{B} . \mathrm{P}$,

B.S,B.Nominalmass, B.HC_Ratio,B.OC_Ratio,B.AI, B.DBE, B.AvgCoxState] ;

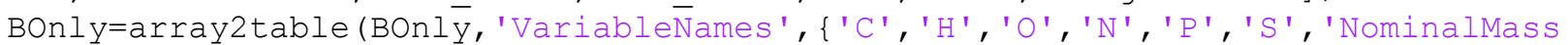
', 'HC_Ratio', 'OC_Ratio', 'AI', 'DBE', 'AvgCOxState'\}) ;

Both=array2table (Both, 'VariableNames', \{'C', 'H', 'O' , 'N', 'P' , 'S ', 'NominalMass', 'HC_Ratio', 'OC_Ratio', 'AI', 'DBE', 'AvgCOxState' \}) ;

ostoring Data with distinct names

Common PreandPost LDI Neg GLSM=unique (Both) ; $\circ<<<<<<<<<<<<<<<<<<<<<<<<<<<<<<<<<<~$ $<<<<<<\overline{<}<<<<<<<<<<\overline{<}<<<\overline{<}<<\overline{<}<<<<<<<<<<<<<<<<<<<<<<<<<<<<<<<<<<<<<<<<<<<<<<<<<<<<~$

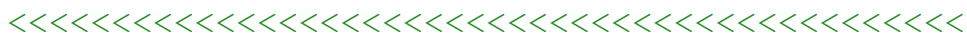

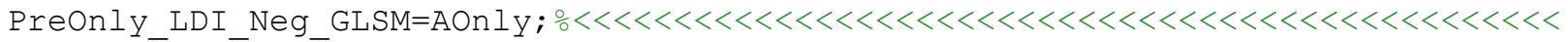
$<<<<<<\overline{<}<<<\overline{<}<<<\overline{<}<<<<<<<<<<<<<<<<<<<<<<<<<<<<<<<<<<<<<<<<<<<<<<<<<<<<<<<<<<<<<~$ $<<<<<<<<<<<<<<<<<<<<<<<<<<<<<~$

PostOnly LDI Neg GLSM=BOnly; $\frac{\circ}{\circ}<<<<<<<<<<<<<<<<<<<<<<<<<<<<<<<<<<<<<<<<<<<<<<<<<~$

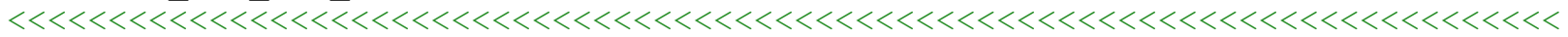
$<<<<<<<<<<<<<<<<<<<<<<<<<<<<<<~$

\% Summarizing Data

Data=AOnly;

oCalculating formulae type parameters: \# CHO, CHON, CHOS, CHOP, CHN, CHS, CHP, CHNS, CHONS formulae

Summary. $\mathrm{CHO}=\operatorname{sum}($ Data. $\mathrm{C}>0$ \& Data. $\mathrm{H}>0$ \& Data. $\mathrm{O}>0$ \& Data. $\mathrm{N}==0$ \& Data. $\mathrm{P}==0$ \& Data. $\mathrm{S}==0$ );

Summary. $\mathrm{CHON}=$ sum $($ Data. $\mathrm{C}>0$ \& Data. $\mathrm{H}>0$ \& Data. $\mathrm{O}>0$ \& Data. $\mathrm{N}>0$ \& Data.P==0 \&

Data. $\mathrm{S}=0$ ) ;

Summary $\cdot \mathrm{CHOS}=$ sum $($ Data. $\mathrm{C}>0$ \& Data.H $>0$ \& Data. O $>0$ \& Data. $\mathrm{N}==0$ \& Data. $\mathrm{P}==0$ \&

Data. S>0);

Summary. $\mathrm{CHOP}=\operatorname{sum}($ Data. $\mathrm{C}>0$ \& Data. $\mathrm{H}>0$ \& Data. $\mathrm{O}>0$ \& Data. $\mathrm{N}==0$ \& Data. $\mathrm{P}>0$ \&

Data. $\mathrm{S}==0$ ) ;

Summary. $\mathrm{CHN}=\operatorname{sum}($ Data. $\mathrm{C}>0$ \& Data. $\mathrm{H}>0$ \& Data. $\mathrm{O}==0$ \& Data. $\mathrm{N}>0$ \& Data. $\mathrm{P}==0$ \&

Data. $\mathrm{S}==0$ ) ;

Summary $\cdot \mathrm{CHS}=\operatorname{sum}($ Data $\cdot \mathrm{C}>0$ \& Data. $\mathrm{H}>0$ \& Data $\cdot \mathrm{O}==0$ \& Data. $\mathrm{N}==0$ \& Data. $\mathrm{P}==0$ \&

Data.s>0);

Summary. $\mathrm{CHNS}=\operatorname{sum}($ Data. $\mathrm{C}>0$ \& Data. $\mathrm{H}>0$ \& Data. $\mathrm{O}==0$ \& Data. $\mathrm{N}>0$ \& Data. $\mathrm{P}==0$ \&

Data. $\mathrm{S}>0$ );

Summary. $\mathrm{CHNP}=\mathrm{sum}($ Data. $\mathrm{C}>0$ \& Data.H $>0$ \& Data. $\mathrm{O}==0$ \& Data. $\mathrm{N}>0$ \& Data.P $>0$ \&

Data. $\mathrm{S}=0$ ) ;

Summary $\cdot \mathrm{CHONS}=\operatorname{sum}($ Data $\cdot \mathrm{C}>0$ \& Data. $\mathrm{H}>0$ \& Data. $\mathrm{O}>0$ \& Data. $\mathrm{N}>0$ \& Data.P==0 \&

Data. $\mathrm{S}>0$ );

Summary. $\mathrm{CHONP}=\operatorname{sum}($ Data. $\mathrm{C}>0$ \& Data. $\mathrm{H}>0$ \& Data. $\mathrm{O}>0$ \& Data.N $>0$ \& Data.P>0\&

Data. $\mathrm{S}=0$ ) ;

Summary. $\mathrm{CHONSP}=$ sum $($ Data. $\mathrm{C}>0$ \& Data.H $>0$ \& Data.O $>0$ \& Data.N $>0$ \& Data.P $>0$ \&

Data.S $>0$ ); 
oCalculating aromaticity parameters: \# condensed aromatic (AI>=0.67), aromatic $(\mathrm{AI}>=0.5)$,

onon-aromatic (AI<0.5)

Summary. CondensedAromatics $=$ sum (Data.AI $>=0.67)$;

Summary.Aromatics=sum (Data.AI $>=0.5)$;

Summary. NonAromatics=sum (Data.AI<0.5);

ocalculating other parameters: total \# of formulae, average molecular

oweight

Summary. TotalFormulae=height (Data);

Summary.AvgMW=mean (Data. NominalMass);

ostoring Summary Data

PreOnly_LDI_Neg_GLSM_Summary=struct2table (Summary); $\circ$ o $<<<<<<<<<<<<<<<<<<<<<<<<<<~$ $<<<<<<<\overline{<}<<<\overline{<}<<<\overline{<}<<<\overline{<}<<<<<<<<<<<<<<<<<<<<<<<<<<<<<<<<<<<<<<<<<<<<<<<<<<<<<<<<<~$

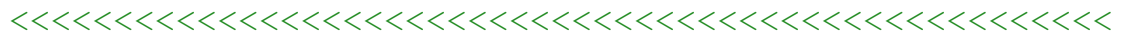

․ Summarizing Data

Data=BOnly;

oCalculating formulae type parameters: \# CHO, CHON, CHOS, CHOP, CHN, CHS, CHP, CHNS, CHONS formulae

Summary $\cdot \mathrm{CHO}=$ sum $($ Data. $\mathrm{C}>0$ \& Data. $\mathrm{H}>0$ \& Data. $\mathrm{O}>0$ \& Data. $\mathrm{N}==0$ \& Data. $\mathrm{P}==0$ \& Data. $\mathrm{S}==0$ );

Summary $\cdot \mathrm{CHON}=$ Sum $($ Data. $\mathrm{C}>0$ \& Data. $\mathrm{H}>0$ \& Data. $\mathrm{O}>0$ \& Data. $\mathrm{N}>0$ \& Data.P==0 \& Data. $\mathrm{S}==0$ );

Summary. $\mathrm{CHOS}=$ sum $($ Data. $\mathrm{C}>0$ \& Data. $\mathrm{H}>0$ \& Data. $\mathrm{O}>0$ \& Data. $\mathrm{N}==0$ \& Data. $\mathrm{P}==0$ \&

Data. $\mathrm{S}>0$ );

Summary $\cdot \mathrm{CHOP}=$ sum $($ Data. $\mathrm{C}>0$ \& Data.H $>0$ \& Data. $\mathrm{O}>0$ \& Data. $\mathrm{N}==0$ \& Data.P $>0$ \&

Data. $\mathrm{S}==0$ );

Summary $\cdot \mathrm{CHN}=$ sum $($ Data. $\mathrm{C}>0$ \& Data. $\mathrm{H}>0$ \& Data. $\mathrm{O}==0$ \& Data. $\mathrm{N}>0$ \& Data. $\mathrm{P}==0$ \&

Data. $\mathrm{S}==0$ );

Summary $\cdot \mathrm{CHS}=\operatorname{sum}($ Data $\cdot \mathrm{C}>0$ \& Data. $\mathrm{H}>0$ \& Data $\cdot \mathrm{O}==0$ \& Data. $\mathrm{N}==0$ \& Data. $\mathrm{P}==0$ \&

Data. $\mathrm{S}>0$ );

Summary. $\mathrm{CHNS}=$ sum $($ Data. $\mathrm{C}>0$ \& Data. $\mathrm{H}>0$ \& Data. $\mathrm{O}==0$ \& Data. $\mathrm{N}>0$ \& Data. $\mathrm{P}==0$ \&

Data. S>0);

Summary $\cdot \mathrm{CHNP}=\operatorname{sum}($ Data. $\mathrm{C}>0$ \& Data.H $>0$ \& Data. $\mathrm{O}==0$ \& Data. $\mathrm{N}>0$ \& Data.P $>0$ \&

Data. $\mathrm{S}==0$ );

Summary. $\mathrm{CHONS}=\operatorname{sum}($ Data $. \mathrm{C}>0$ \& Data. $\mathrm{H}>0$ \& Data. $0>0$ \& Data.N>0 \& Data.P==0 \&

Data. S>0);

Summary. $\mathrm{CHONP}=$ sum $($ Data. $\mathrm{C}>0$ \& Data. $\mathrm{H}>0$ \& Data. $\mathrm{O}>0$ \& Data.N $>0$ \& Data.P $>0$ \&

Data. $\mathrm{S}==0$ ) ;

Summary. $\mathrm{CHONSP}=$ sum $($ Data. $\mathrm{C}>0$ \& Data.H $>0$ \& Data.O $>0$ \& Data.N $>0$ \& Data.P>0 \&

Data. $\mathrm{S}>0$ );

ocalculating aromaticity parameters: \# condensed aromatic (AI>=0.67),

aromatic (AI>=0.5),

onon-aromatic $(\mathrm{A} I<0.5)$

Summary. CondensedAromatics $=$ sum (Data. AI $>=0.67)$;

Summary. Aromatics $=$ sum (Data.AI $>=0.5)$;

Summary. NonAromatics=sum (Data.AI<0.5);

ocalculating other parameters: total \# of formulae, average molecular

oweight

Summary. TotalFormulae=height (Data);

Summary. AvgMW=mean (Data. NominalMass);

ostoring Summary Data

Postonly LDI Neg GLSM Summary=struct2table (Summary); $\circ<<<<<<<<<<<<<<<<<<<<<<<<<<$ $<<<<<<<<\overline{<}<<<\overline{<}<<<\overline{<}<<<<\overline{<}<<<<<<<<<<<<<<<<<<<<<<<<<<<<<<<<<<<<<<<<<<<<<<<<<<<<<<<$

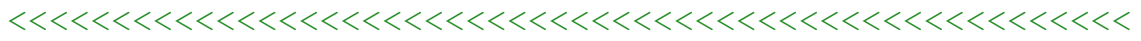

ㅇmmarizing Data

Data=unique (Both); 
oCalculating formulae type parameters: \# CHO, CHON, CHOS, CHOP, CHN, CHS, CHP, CHNS, CHONS formulae

Summary $\cdot \mathrm{CHO}=$ sum $($ Data. $\mathrm{C}>0$ \& Data. $\mathrm{H}>0$ \& Data. $\mathrm{O}>0$ \& Data. $\mathrm{N}==0$ \& Data. $\mathrm{P}==0$ \& Data. $\mathrm{S}==0$ );

Summary. $\mathrm{CHON}=\operatorname{sum}($ Data. $\mathrm{C}>0$ \& Data. $\mathrm{H}>0$ \& Data. $\mathrm{O}>0$ \& Data. $\mathrm{N}>0$ \& Data.P==0 \& Data. $\mathrm{S}=0$ ) ) ;

Summary. $\mathrm{CHOS}=$ sum $($ Data. $\mathrm{C}>0$ \& Data. $\mathrm{H}>0$ \& Data. $0>0$ \& Data. $\mathrm{N}==0$ \& Data. $\mathrm{P}==0$ \& Data. $\mathrm{S}>0$ );

Summary $\cdot \mathrm{CHOP}=\operatorname{sum}($ Data. $\mathrm{C}>0$ \& Data.H $>0$ \& Data. $\mathrm{O}>0$ \& Data. $\mathrm{N}==0$ \& Data.P $>0$ \& Data. $\mathrm{S}==0$ );

Summary $\cdot \mathrm{CHN}=$ sum $($ Data. $\mathrm{C}>0$ \& Data. $\mathrm{H}>0$ \& Data. $\mathrm{O}==0$ \& Data. $\mathrm{N}>0$ \& Data. $\mathrm{P}==0$ \&

Data. $\mathrm{S}=0$ ) ;

Summary $\cdot \mathrm{CHS}=\operatorname{sum}($ Data. $\mathrm{C}>0$ \& Data. $\mathrm{H}>0$ \& Data. $\mathrm{O}==0$ \& Data. $\mathrm{N}==0$ \& Data. $\mathrm{P}==0$ \&

Data. S>0);

Summary. $\mathrm{CHNS}=$ sum (Data. $\mathrm{C}>0$ \& Data. $\mathrm{H}>0$ \& Data. $\mathrm{O}==0$ \& Data. $\mathrm{N}>0$ \& Data. $\mathrm{P}==0$ \&

Data. S>0);

Summary $\cdot \mathrm{CHNP}=\operatorname{sum}($ Data. $\mathrm{C}>0$ \& Data.H $>0$ \& Data. $\mathrm{O}==0$ \& Data.N $>0$ \& Data.P $>0$ \&

Data. $\mathrm{S}==0$ );

Summary $\cdot \mathrm{CHONS}=\operatorname{sum}($ Data $\cdot \mathrm{C}>0$ \& Data. $\mathrm{H}>0$ \& Data. $\mathrm{O}>0$ \& Data.N $>0$ \& Data.P==0 \&

Data. S>0);

Summary. $\mathrm{CHONP}=\operatorname{sum}($ Data. $\mathrm{C}>0$ \& Data. $\mathrm{H}>0$ \& Data. $\mathrm{O}>0$ \& Data.N $>0$ \& Data.P $>0$ \&

Data. $\mathrm{S}==0$ );

Summary. $\mathrm{CHONSP}=$ sum $($ Data. $\mathrm{C}>0$ \& Data. $\mathrm{H}>0$ \& Data. $\mathrm{O}>0$ \& Data.N $>0$ \& Data.P $>0$ \&

Data. $\mathrm{S}>0$ );

ocalculating aromaticity parameters: \# condensed aromatic (AI>=0.67),

aromatic $(\mathrm{AI}>=0.5)$,

onon-aromatic $(\mathrm{A} I<0.5)$

Summary. CondensedAromatics $=$ sum (Data.AI $>=0.67)$;

Summary. Aromatics $=$ sum (Data.AI $>=0.5)$;

Summary. NonAromatics=sum (Data.AI<0.5);

ocalculating other parameters: total \# of formulae, average molecular

oweight

Summary. TotalFormulae=height (Data);

Summary. AvgMW=mean (Data. NominalMass);

oStoring Summary Data

Common PreandPost LDI Neg GLSM Summary=struct2table (Summary); $\circ$ o $<<<<<<<<<<<<<<<<$

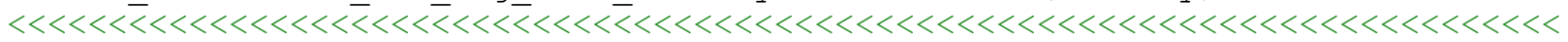

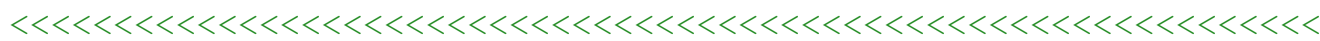

응 Comparing SRFA and GLSM isolates

oInput Data so that the script can readily be applied to any data set

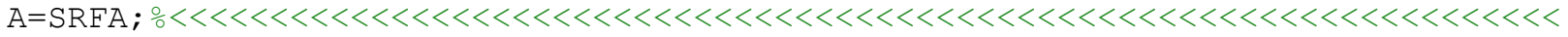

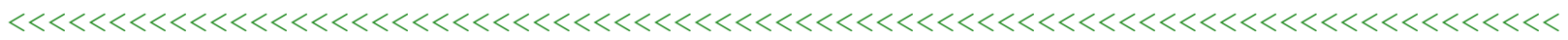
$<<<<<<<<<$

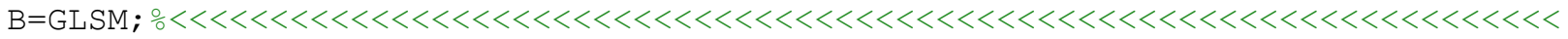

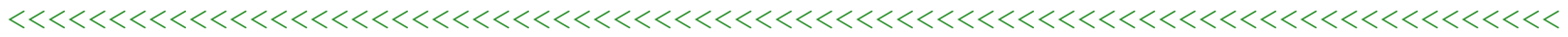
$<<<<<<<<<$

o Separate Data into those common and unique

Both=[] ;

$\mathrm{k}=0$;

for $i=1$ :height $(A)$

for $j=1$ :height $(B)$

if $A \cdot C(i)==B \cdot C(j) \quad \& \& A \cdot H(i)==B \cdot H(j) \quad \& \& A \cdot N(i)==B \cdot N(j) \quad \& \& A \cdot O(i)==B \cdot O(j)$

$\& \& A \cdot P(i)==B \cdot P(j) \quad \& \& A \cdot S(i)==B \cdot S(j)$

$\mathrm{k}=\mathrm{k}+1$; 
$\operatorname{Both}(k,:)=[A . C(i), A . H(i), A . O(i), A . N(i), A . P(i)$,

A.S (i), A.NominalMass (i), A.HC_Ratio(i),

A.OC_Ratio(i),A.AI (i),A.DBE(i),A.AvgCoxState (i) ] ;

A. $C(i)=0$;

B. C $(j)=0$;

end

end

end

oCombining Data Sets

$\mathrm{A}(\mathrm{A} \cdot \mathrm{C}==0,:)=[]$;

$A O n l_{y}=[A . C, A . H, A . O, A . N, A . P, A . S$,

A.NominalMass, A.HC Ratio,A.oC Ratio,A.AI, A.DBE, A.AvgCoxState];

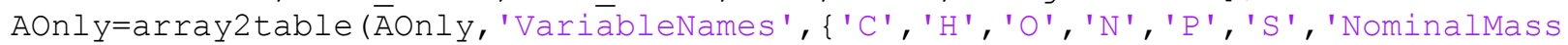
', 'HC_Ratio', 'OC_Ratio', 'AI', 'DBE', 'AvgCOxState'\}) ;

$\mathrm{B}(\mathrm{B} \cdot \mathrm{C}==0,:)=[]$;

$B O n l_{Y}=[B . C, B . H, B . O, B . N, B . P$,

B.S,B.NominalMass, B.HC_Ratio,B.OC_Ratio,B.AI, B.DBE, B.AvgCOxState] ;

Bonly=array2table (BOnlȳy, 'Variable Names ', \{'C', 'H', 'O', 'N ', 'P' ', 'S ', 'NominalMass ', 'HC_Ratio', 'OC_Ratio', 'AI', 'DBE', 'AvgCOxState'\}) ;

Both=array2table (Both, 'VariableNames', \{'C', 'H', 'O' , 'N', 'P' , 'S ', 'NominalMass', 'HC_Ratio', 'OC_Ratio', 'AI', 'DBE', 'AvgCOxState'\}) ;

ostoring Data with distinct names

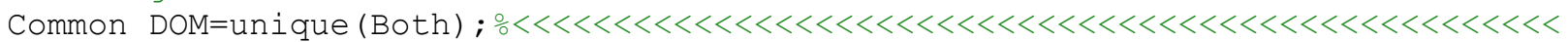

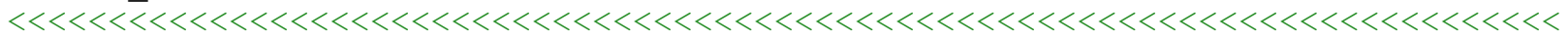
$<<<<<<<<<<<<<<<<<<<<<<<<<<~$

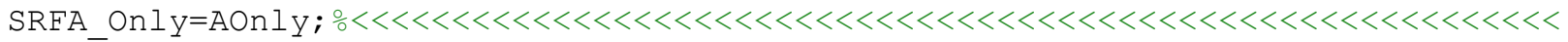

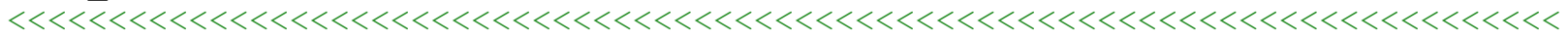
$<<<<<<<<<<<<<<<<<<~$

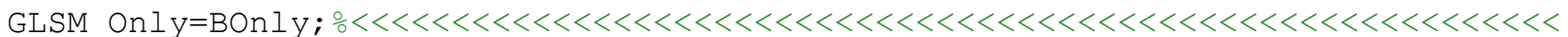

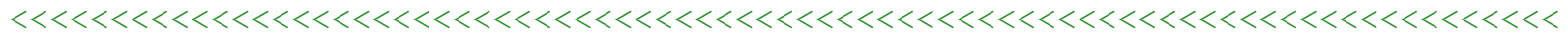
$<<<<<<<<<<<<<<<<<~$

\% Summarizing Data

Data=AOnly;

oCalculating formulae type parameters: \# CHO, CHON, CHOS, CHOP, CHN, CHS, CHP, CHNS, CHONS formulae

Summary $\cdot \mathrm{CHO}=\operatorname{sum}($ Data. $\mathrm{C}>0$ \& Data. $\mathrm{H}>0$ \& Data. $\mathrm{O}>0$ \& Data. $\mathrm{N}==0$ \& Data. $\mathrm{P}==0$ \& Data. $\mathrm{S}==0$ );

Summary $\cdot \mathrm{CHON}=\operatorname{sum}($ Data. $\mathrm{C}>0$ \& Data.H $>0$ \& Data. O>0 \& Data.N $>0$ \& Data.P==0\&

Data. $\mathrm{S}=0$ ) ;

Summary $\cdot \mathrm{CHOS}=$ sum $($ Data. $\mathrm{C}>0$ \& Data.H $>0$ \& Data. O $>0$ \& Data. $\mathrm{N}==0$ \& Data.P==0 \&

Data. S>0);

Summary. $\mathrm{CHOP}=\operatorname{sum}($ Data. $\mathrm{C}>0$ \& Data. $\mathrm{H}>0$ \& Data. $\mathrm{O}>0$ \& Data. $\mathrm{N}==0$ \& Data. $\mathrm{P}>0$ \&

Data. $\mathrm{S}==0$ );

Summary. $\mathrm{CHN}=$ sum $($ Data. $\mathrm{C}>0$ \& Data. $\mathrm{H}>0$ \& Data. $\mathrm{O}==0$ \& Data. $\mathrm{N}>0$ \& Data. $\mathrm{P}==0$ \&

Data. $\mathrm{S}==0$ );

Summary. CHS $=$ sum $($ Data $\cdot \mathrm{C}>0$ \& Data. $\mathrm{H}>0$ \& Data $\cdot \mathrm{O}==0$ \& Data. $\mathrm{N}==0$ \& Data. $\mathrm{P}==0$ \&

Data. S>0);

Summary. $\mathrm{CHNS}=\operatorname{sum}($ Data. $\mathrm{C}>0$ \& Data. $\mathrm{H}>0$ \& Data. $\mathrm{O}==0$ \& Data. $\mathrm{N}>0$ \& Data. $\mathrm{P}==0$ \&

Data. S>0);

Summary. $\mathrm{CHNP}=\operatorname{sum}($ Data. $\mathrm{C}>0$ \& Data. $\mathrm{H}>0$ \& Data. $\mathrm{O}==0$ \& Data.N $>0$ \& Data.P $>0$ \&

Data. $\mathrm{S}==0$ );

Summary $\cdot \mathrm{CHONS}=\operatorname{sum}($ Data $\cdot \mathrm{C}>0$ \& Data. $\mathrm{H}>0$ \& Data.O $>0$ \& Data.N $>0$ \& Data.P==0 \&

Data. $\mathrm{S}>0$ ) ;

Summary. $\mathrm{CHONP}=$ sum $($ Data. $\mathrm{C}>0$ \& Data.H $>0$ \& Data.O $>0$ \& Data.N $>0$ \& Data.P $>0$ \&

Data. $\mathrm{S}=0$ ) ;

Summary. $\mathrm{CHONSP}=$ sum $($ Data. $\mathrm{C}>0$ \& Data. $\mathrm{H}>0$ \& Data. $\mathrm{O}>0$ \& Data.N $>0$ \& Data.P $>0$ \&

Data. S>0); 
oCalculating aromaticity parameters: \# condensed aromatic (AI>=0.67), aromatic $(\mathrm{AI}>=0.5)$,

onon-aromatic $(\mathrm{AI}<0.5)$

Summary. CondensedAromatics $=$ sum (Data. AI $>=0.67)$;

Summary.Aromatics=sum (Data.AI $>=0.5)$;

Summary. NonAromatics=sum (Data.AI<0.5);

ocalculating other parameters: total \# of formulae, average molecular

oweight

Summary. TotalFormulae=height (Data);

Summary.AvgMW=mean (Data. NominalMass);

ostoring Summary Data

SRFA_OnIy_Summary=struct2table (Summary) ; 은 $<<<<<<<<<<<<<<<<<<<<<<<<<<<<<<<<<<<<<<~$

$<<<\overline{<}<<<<\overline{<}<<<<<<<<<<<<<<<<<<<<<<<<<<<<<<<<<<<<<<<<<<<<<<<<<<<<<<<<<<<<<<<<<<<~$

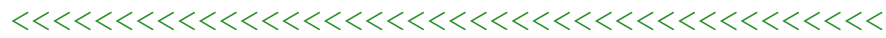

․ Summarizing Data

Data=BOnly;

oCalculating formulae type parameters: \# CHO, CHON, CHOS, CHOP, CHN, CHS, CHP, CHNS, CHONS formulae

Summary $\cdot \mathrm{CHO}=$ sum (Data. $\mathrm{C}>0$ \& Data. $\mathrm{H}>0$ \& Data. $\mathrm{O}>0$ \& Data. $\mathrm{N}==0$ \& Data. $\mathrm{P}==0$ \&

Data. $\mathrm{S}==0$ );

Summary $\cdot \mathrm{CHON}=$ Sum $($ Data. $\mathrm{C}>0$ \& Data. $\mathrm{H}>0$ \& Data. $\mathrm{O}>0$ \& Data. $\mathrm{N}>0$ \& Data.P==0 \&

Data. $\mathrm{S}==0$ );

Summary. $\mathrm{CHOS}=$ sum $($ Data. $\mathrm{C}>0$ \& Data. $\mathrm{H}>0$ \& Data. $\mathrm{O}>0$ \& Data. $\mathrm{N}==0$ \& Data. $\mathrm{P}==0$ \&

Data. $\mathrm{S}>0$ );

Summary $\cdot \mathrm{CHOP}=$ sum $($ Data. $\mathrm{C}>0$ \& Data.H $>0$ \& Data. $\mathrm{O}>0$ \& Data. $\mathrm{N}==0$ \& Data.P $>0$ \&

Data. $\mathrm{S}==0$ );

Summary $\cdot \mathrm{CHN}=$ sum $($ Data. $\mathrm{C}>0$ \& Data. $\mathrm{H}>0$ \& Data. $\mathrm{O}==0$ \& Data. $\mathrm{N}>0$ \& Data. $\mathrm{P}==0$ \&

Data. $\mathrm{S}==0$ );

Summary $\cdot \mathrm{CHS}=\operatorname{sum}($ Data $\cdot \mathrm{C}>0$ \& Data. $\mathrm{H}>0$ \& Data. $\mathrm{O}==0$ \& Data. $\mathrm{N}==0$ \& Data. $\mathrm{P}==0$ \&

Data. $\mathrm{S}>0$ );

Summary. $\mathrm{CHNS}=$ sum $($ Data. $\mathrm{C}>0$ \& Data. $\mathrm{H}>0$ \& Data. $\mathrm{O}==0$ \& Data. $\mathrm{N}>0$ \& Data. $\mathrm{P}==0$ \&

Data. S>0);

Summary $\cdot \mathrm{CHNP}=\operatorname{sum}($ Data. $\mathrm{C}>0$ \& Data.H $>0$ \& Data. $\mathrm{O}==0$ \& Data. $\mathrm{N}>0$ \& Data.P $>0$ \&

Data. $\mathrm{S}==0$ );

Summary. $\mathrm{CHONS}=\operatorname{sum}($ Data $. \mathrm{C}>0$ \& Data. $\mathrm{H}>0$ \& Data. $0>0$ \& Data.N>0 \& Data.P==0 \&

Data. S>0);

Summary. $\mathrm{CHONP}=$ sum $($ Data. $\mathrm{C}>0$ \& Data. $\mathrm{H}>0$ \& Data. $\mathrm{O}>0$ \& Data.N $>0$ \& Data.P $>0$ \&

Data. $\mathrm{S}==0$ ) ;

Summary. $\mathrm{CHONSP}=$ sum $($ Data. $\mathrm{C}>0$ \& Data.H $>0$ \& Data.O $>0$ \& Data.N $>0$ \& Data.P>0 \&

Data. $\mathrm{S}>0$ );

oCalculating aromaticity parameters: \# condensed aromatic (AI>=0.67),

aromatic $(A I>=0.5)$,

onon-aromatic $($ AI $<0.5)$

Summary. CondensedAromatics $=$ sum (Data. AI $>=0.67)$;

Summary. Aromatics $=$ sum (Data.AI $>=0.5)$;

Summary. NonAromatics=sum (Data.AI<0.5);

ocalculating other parameters: total \# of formulae, average molecular

oweight

Summary. TotalFormulae=height (Data);

Summary. AvgMW=mean (Data. NominalMass);

ostoring Summary Data

GLSM Only Summary=struct2table (Summary) ; $\circ<<<<<<<<<<<<<<<<<<<<<<<<<<<<<<<<<<<<<<<~$

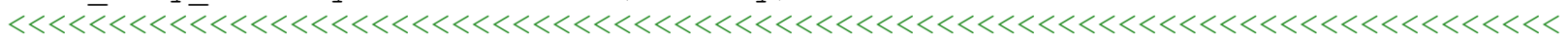

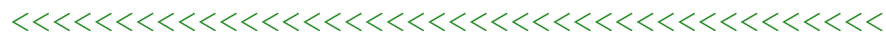

oSummarizing Data

Data=unique (Both) ; 


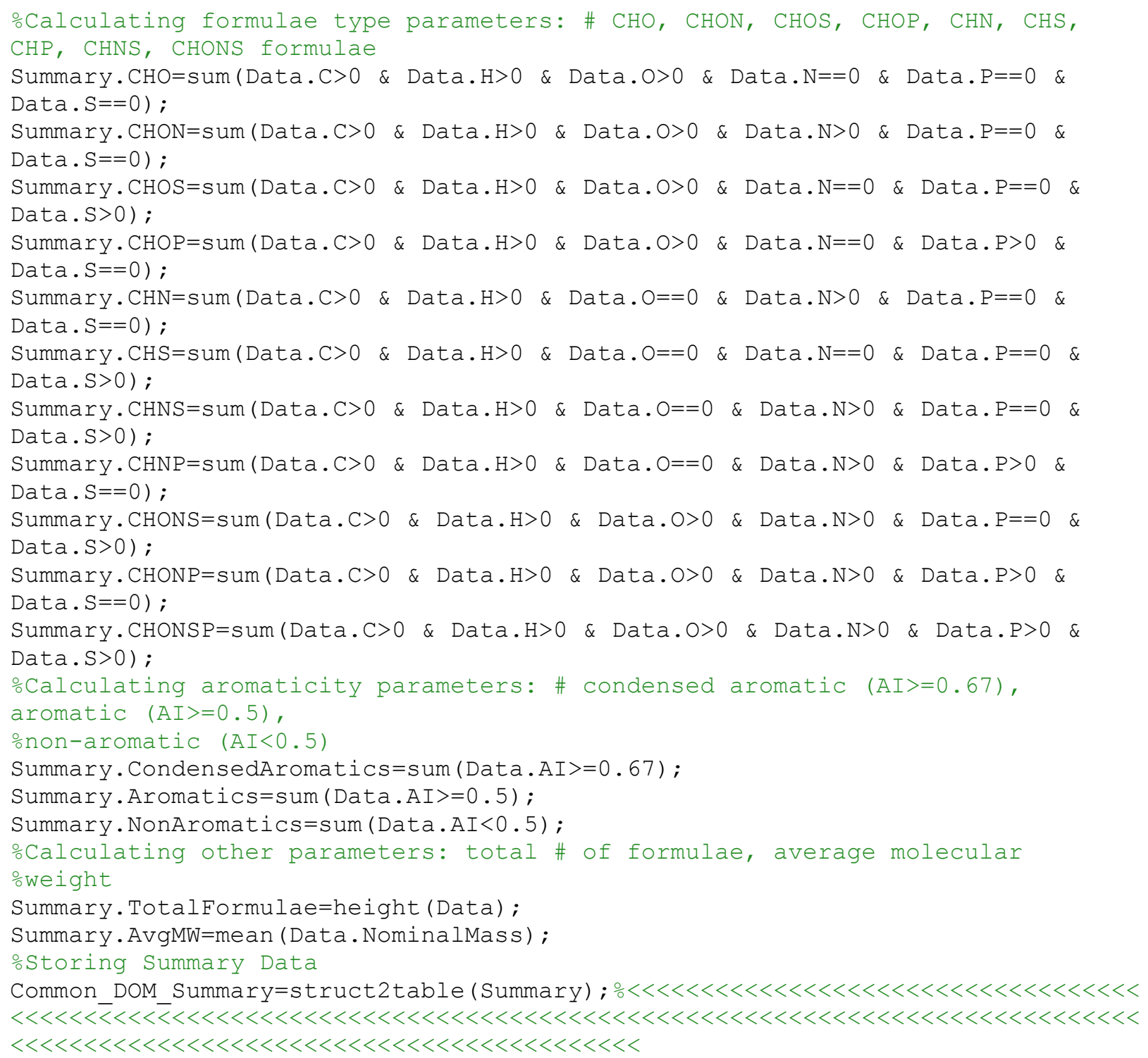

